
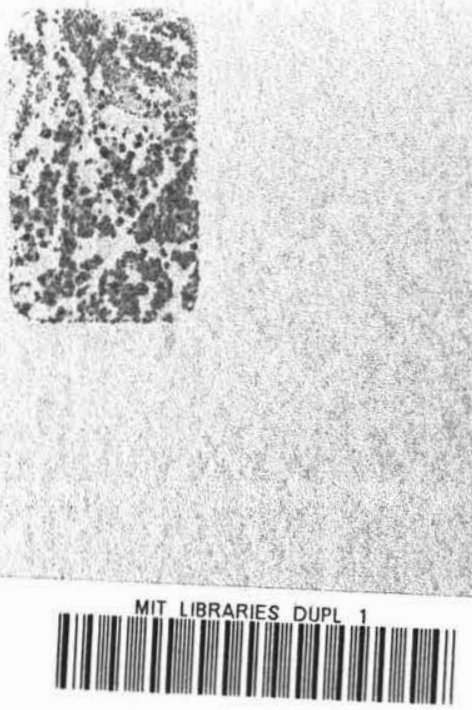

39080 00601143 8

UNSTEADY RADIAL TRANSPORT IN A

TRANSONIC COMPRESSOR STAGE

by

Petros A. Kotidis

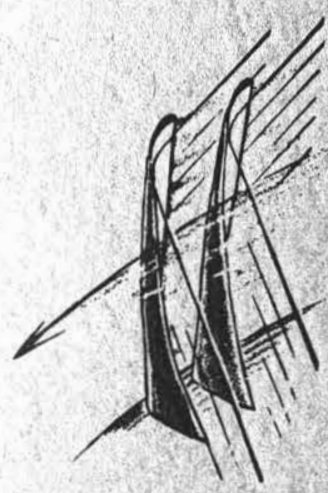

\title{
APR 241990
}

V. Unaseres

GAS TURBINE LABORATORY

MASSACHUSETTS INSTITUTE OF TECHNOLOGY

CAMBRIDGE, MASSACHUSETTS 


\title{
UNSTEADY RADIAL TRANSPORT IN A TRANSONIC COMPRESSOR STAGE
}

by

\author{
Petros A. Kotidis
}

GTL Report \#199

September 1989

This research was supported by the Air Force Office of Scientific Research, Dr. J.D. Wilson, Technical Monitor. 


\author{
UNSTEADY RADIAL TRANSPORT IN A TRANSONIC \\ COMPRESSOR STAGE \\ by \\ PETROS ANESTIS KOTIDIS
}

\title{
ABSTRACT
}

A technique, based on the operation of a dual-hot wire-aspirating probe with frequency response of at least $18 \mathrm{kHz}$ and uncertainty less than 0.005 to full scale in mass fraction units, has been developed to measure time resolved concentration in unsteady, compressible flows.

The goal of the experimental part of this research effort was to obtain time resolved measurements of spanwise transport in a transonic compressor. This was achieved by injecting a circumferentially oriented, thin sheet of tracer gas in front of the compressor and detecting the foreign fluid molecules at the rotor exit The experiments were conducted at the MIT Blowdown Facility using the Air Force High Through Flow Compressor Stage as the test article.

During a preliminary data reduction procedure, it was discovered that the signals from the probe's hot wires lag in time with respect to the signal from the companion total pressure probe. A correction incorporated in the data reduction schemes to account for this, eliminated most of the negative entropy regions observed in previous experiments with this probe.

Several conclusions have been drawn from the experimental observations. First, up to $5 \%$ of the compressor mass flow moved along the blade span. Second, the migrating fluid was found primarily in the blade wakes at the measurement location. Third, this fluid moved towards both hub and tip in the blade wakes. Fourth, the radially convected fluid had high entropy, much higher than that of the average flowfield. Fifth, the "inviscid core" fluid moves

preferentially towards the suction side of the blade passage and away from the pressure side.

A simple model was developed to explain the spanwise fluid transport. Gertz's 2-D wake vortex street model was extended into a quasi 3-D form. The 2-D model was fitted to the data at four spanwise locations and the spanwise variation of the parameters of the vortex street (such as vortex strength and core size) were determined. The model fit showed the shedding frequencies to be the same [17 (+/-) $0.4 \mathrm{kHz}]$ at all four spanwise locations, suggesting that the vortex shedding is coherent along the span. The spanwise pressure gradient created by the variation of vortex strength led to substantial spanwise transport in the vortex cores. The model predicted the transport to the hub, but underestimated the transport to the tip by a factor of five.

The measured spanwise transport can explain the previously observed discrepancy between predicted (viscoustnormal shock losses) and measured spanwise distributions of adiabatic efficiency in the tip regions of transonic compressors (assuming the radial outflow to be primarily in regions of separated flow on the blades). 


\section{ACKNOWLEHGMIFNTS}

Going through the MIT doctoral program is a challenge to one's endurance and determination. I believe I have been quite fortunate to be surrounded by a number of people who helped me "minimize" my losses and survive the past five years at MIT.

First and foremost I would like to express my sincere and deep appreciation to my thesis advisor. Professor A. H. Epstein. His objective judgment and quick and direct solutions to hard problems have made this work possible. His guidance, patience, enthusiasm and trust in my abilities will always be remembered and appreciated. I hope I have lived up to his expectations.

I would also like to thank Professor J. L. Kerrebrock for his invaluable contributions. Working with Professor Kerrebrock has been one of the great pleasures of conducting this research. He always found time in his busy schedule as Associate Dean of Engineering for technical and personal discussions. His ability to simplify extremely complicated problems and guide me into solving them was instrumental. Since most of the ideas in this thesis were suggested by him, the completion of this work would have been impossible without his help.

I have also been very fortunate to have Professor E. M. Greitzer in my doctoral committee. His patience in listening to my endless questions and his ability to "read" through my words are sincerely appreciated. His deep understanding and solid knowledge of fundamentals were key factors in the success of this research.

Thanks are most sincerely extended to Professor J. E McCune. His suggestions and comments on my research were very helpful. Professor McCune has a unique ability to communicate his ideas to students and his efforts to transfer some of these skills to me are deeply appreciated.

Sincere appreciation is also extended to Professor $E$ Covert, who served as a member of my doctoral committee for a short while. His insight and guidance during the first, but crucial steps of this research were quite valuable. Professor F. Marble at the California Institute of Technology is also to be thanked for his helpful suggestions during his MIT visits.

Dr. C. S. Tan deserves special thanks for his willingness to read my thesis and clarify most of my questions. Dr. G Guenette has been an inspiring friend and contributor to my research. His enthusiasm, personal and technical support and "late night" informal discussions will always be remembered. Thanks are also extended to Dr. J. Gertz for his assistance in the analysis of the data and the development of the necessary computer software. The suggestions of Professors M. Giles and M. Drela were very helpful during the course of this work. 
Many members of the research staff have also contributed to the experimental part of this work and deserve thanks: Mr. Victor Dubrowski for his patience and exceptional machining skills, Mr. Al Supple for his help in running the Facility and Mr. J. Nash and Mr. Roy Andrew for their willingness to help me with the "nuts and bolts" part of my experiment Special thanks are also extended to Mr. Bob Haimes for his help with the computer systems. Ms. Diana Park is to be commended for providing word processing assistance.

Many officemates and coworkers helped make my stay at MIT a pleasant experience. Dr. Mark Lewis has been a good friend and an endless bank of information on any subject. Gwo-Tung Chen, Lou Cattafesta, Andrew Crook, Knox Millsaps, Doug Loose, Dan Gysling, Judy Pinsley, Craig Seymour have all been fine companions during quick lunches and loud discussions in the "social hour". Special thanks are extended to the members of the Greek Intramural Soccer Team for all the unique and exciting moments that we shared. My roommate, Tasos Aslidis, is to be thanked for all the moral support and understanding. Our midnight dinners will always be remembered. Thanks are also extended to my friend and captain of the Soccer Team, Jason Papastavrou, for all the friendly discussions during lunch time.

Deep and sincere appreciation is due to my family and, especially, my mother. Her encouragement and unfailing love helped me make the transition from a small town in the mountains of Greece to an educational institution like MIT, and a country like United States. I will always be grateful to her for the unique upbringing and the constant moral support that she provided throughout my life.

However, this work would never be completed without the unending love, patience, faith and encouragement of Nina. I will always remember her ability to make things look brighter than I thought, her willingness to take the time and listen to my problems and her sharing both "bad" and "good" times with me. She really made me happy. She was the reason I survived MIT.

This research was supported by the Air Force Office of Scientific Research with technical monitor Dr. J. D. Wilson. 


\section{TABLE OF CONTENTS}

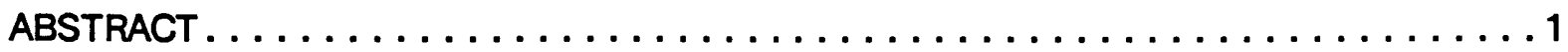

ACKNOMLDGMENTS . . . . . . . . . . . . . . . . . . . .

TABLE OF CONTENTS $\ldots \ldots \ldots \ldots \ldots \ldots \ldots \ldots \ldots \ldots \ldots \ldots \ldots$

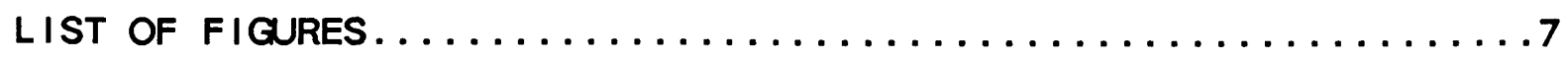

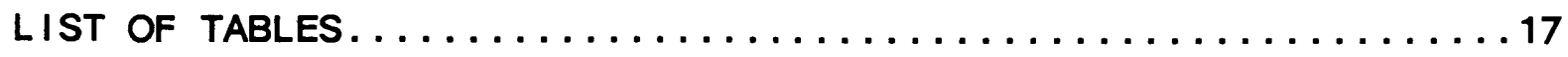

CHAPTER 1

INTRODUCTION. . . . . . . . . . . . . . . . . . . . . . 19

CHAPTER 2

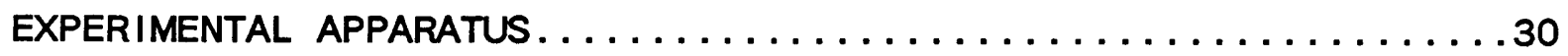

2.1 - Description of the Experiment.............. 30

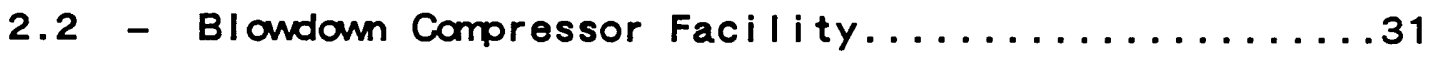

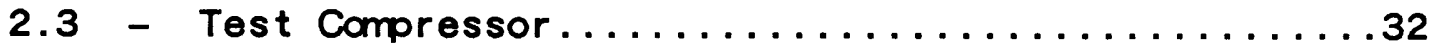

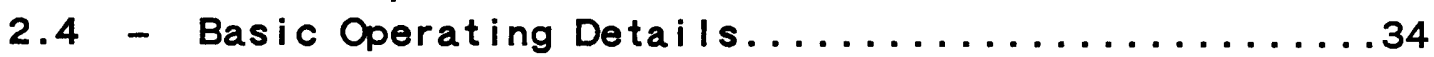

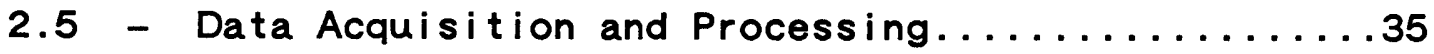

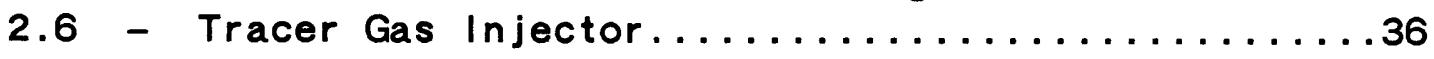

CHAPTER 3

I NSTRUMENTATION. .......................... 41

3.1 - The Dual-Hot-Wire Aspirating Probe............41

3.1.1 - Brief Description of the Aspirating Probe..41

3.1.2 - Time Lag Corrections in Probe Data.......46

3.1.2.1 - Analytical Model of the Flow in the Probe................47

3.1.2.2 - Implications of the Time Lag in the Probe Signal............51

3.2 - Description of the Concentration Measurement

Technique........................ . . . . . . .

3.3 - Tracer Gas Selection................... 58

3.4 - Static Calibration of the Aspirating Probe.......65

3.5 - Data Reduction Scheme...................80

3.6 - Dynamic Calibration-Shock Tube Tests...........82

3.7 - Probe Error Analysis...................93 
CHAPTER 4

PROOF OF DONCEPT TESTING. . . . . . . . . . . . . . . 100

4.1 - Tets with the Rotor and Stator Removed......... 100

4.2 - Tets with the Rotor and Stator in Place......... 102

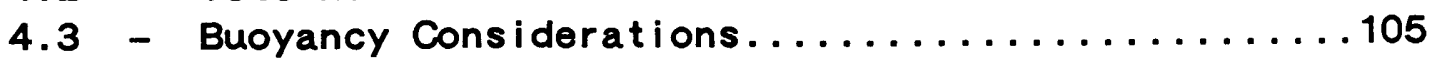

4.4 - Centrifugal Action of the Rotor..............109

4.5 - Turbulence Level in the Blowdown Facility.......110

CHAPTER 5

MEASUREMENTS OF TIME RESOLVED CONCENTRATION,

TOTAL TEMPERATURE AND TOTAL PRESSURE $\ldots \ldots \ldots \ldots \ldots \ldots \ldots \ldots \ldots$

5.1 - Consistency Checks....................111

5.2 - Instantaneous Data-High Frequency Results...... 112

5.3 - Time Averaged Data and General Trends..........116

5.4 - Ensemble Averaged Results................116

5.5 - Summary of Experimental Results.............119

CHAPTER 6

TIME AVERAGED SPANWISE FLUID TRANSPORT . . . . . . . . . . . 120

6.1 - Estimate of Radial Fluid Migration..........120

6.2 - Mixing Levels with and wi thout the Rotor.......128

CHAPTER 7

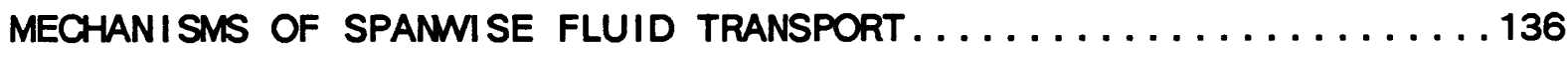

7.1 - Estimates of the Spanwise Migration Due to Various Mechan isms. . . . . . . . . . . . . . . . . . 136

7.1.1 - Spanwise Flows in the Blade Boundary

Layer.......................136

7.1 .2 - Spanwise Flows due to the Tip Clearance...138

7.1.3 - Spanwise Flows due to "Classical"

Secondary Flows..................141

7.1 .4 - Spanwise Flows due to the Relative Eddy...143

7.1.5 - Spanwise Flows in Regions of Separation...144

7.2 - Modeling of the Spanwise Vortex Street Wake...... 149

7.2.1 - Vortex Shedding and 2-D Model ing of the

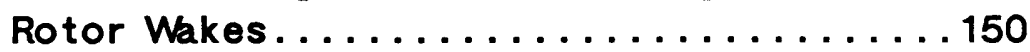

7.2.2 - A Review of Spanwise Vortices Shed Behind

Bluff Bodies..................... 152

7.2.3 - Quasi 3-D Modeling of Rotor Wakes

Vortices....................... 160 
7.2.4 - Fitting the Vortex Model to the

Experimental Data....................161

7.2.5 - Pressure Distribution in the Vortes Cores. 166

7.2.6 - Shedding Frequency and Strouhal Number

in the Spanwise Direction............167

7.2.7 - Spanwise Flow in the Vortex Cores........173

7.2.8 - Quasi-viscous Calculation of the Vortex

Core Growth.....................180

CHAPTER 8

CONSEQUENCES OF SPANWISE FLUID TRANSPORT $\ldots \ldots \ldots \ldots \ldots \ldots \ldots 188$

8.1 - Effect of Spanwise Fluid Transport on the Spanwise

Distribution of Adiabatic Efficiency.........188

8.1.1 - Spanwise Fluid Transport within the

Blade Passage.....................190

8.1.2 - Spanwise Fluid Transport after the Trailing

Edge of the Blade.................200

8.1.3 - Spanwise Fluid Transport both before and afte the Trailing Edge of the Blade.......203

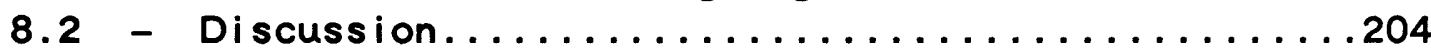

\section{CHAPTER 9}

CONCLUSIONS AND RECOMMENDATIONS FOR

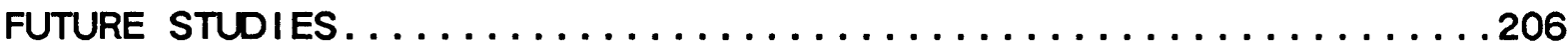

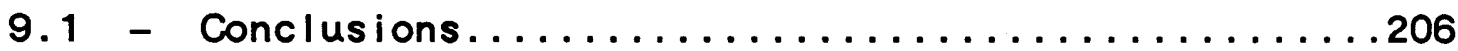

9.2 - Recommendations for Future Studies............209

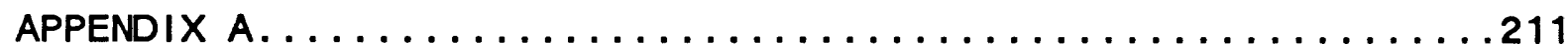

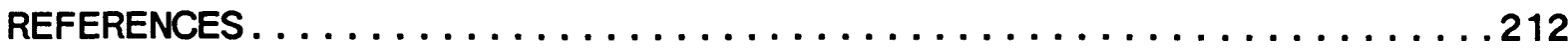

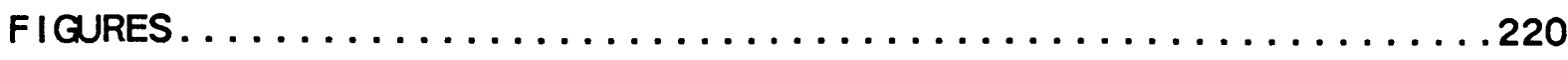




\section{LIST OF FIGURES}

\section{CHAPTER 2}

Figure 2.1: Scale drawing of the Blowdown Compressor Facility

Figure 2.2: Scale drawing of the test section

Figure 2.3: Top view of the experimental arrangement

Figure 2.4: Compressor stage performance map

Figure 2.5: Fractional corrected speed (a) and fractional corrected mass flow (b), during a typical Blowdown test

Figure 2.6: Blowdown Compressor Facility data acquisition system

Figure 2.7: Scale drawing of the Tracer Gas Injector

Figure 2.8: Schematic drawing of the injection system

Figure 2.9: Mach number distribution on the surface of the injector

Figure 2.10: Comparison of total pressure decay traces between injector and main Blowdowns

Figure 2.11: Enlarged view of the total pressure decay traces of Fig. 2.10 during test time and comparison with the curve fit of the total pressure trace of the main Blowdown

\section{CHAPTER 3}

Figure 3.1: Geometry of the dual wire aspirating probe

Figure 3.2: Mounting arrangement of aspirating probe and companion total pressure probe

Figure 3.3: Enlarged view of the probe inlet geometry with the captured streamtube included

Figure 3.4: Inlet geometry of the aspirating probe, used in the 1-D flow calculations of the time lag in the probe data

Figure 3.5: Experimental determination of the time lag - Comparison between total 
pressure measured by the pressure probe vs calculated using the signals of the two hot wires only

Figure 3.6: Comparison between entropy change (nondimensionalized with constant pressure specific heat) as calculated using the shifted (corrected) and unshifted (original) probe data for tip, midspan and hub

Figure 3.7: Comparison between entropy change (nondimensionalized with constant pressure specific heat) as calculated using the shifted (corrected) and unshifted (original) probe data

Figure 3.8: (a) Voltage unshifted (original) measurement, (b) Pressure ratio measurement, (c) Entropy change (nondimensionalized with constant pressure specific heat), as calculated using the unshifted (original) signals

Figure 3.9: Simulation of the probe data as $5 \mathrm{kHz}$ (blade passing frequency) sine waves

(a) Shifted (corrected) and unshifted (original) voltage

(b) Total pressure ratio

(c) Total temperature ratio as calculated using shifted (corrected) and unshifted (original) signals

(d) Entropy change (nondimensionalized with constant specific heat) as calculated using shifted (corrected) and unshifted (original) signals

Figure 3.10: Aspirating probe calibration curves for $0 \%$ concentration

Figure 3.11: Aspirating probe calibration curves for $\mathrm{CO}_{2}$ tracer gas at $400 \mathrm{mmHg}$

Figure 3.12: Aspirating probe calibration curves for Helium tracer gas at 400 $\mathrm{mmHg}$

Figure 3.13: Aspirating probe calibration curves for Helium and Freon 12 tracer mixture at $400 \mathrm{mmHg}$

Figure 3.14: Schematic drawing of the probe calibration facility

Figure 3.15: Aspirating probe calibration curves for Helium, Helium-Xenon and Helium-Freon 12 tracer mixtures

Figure 3.16: Aspirating probe calibration curve for Helium-Freon 12 at reference conditions (room temperature and $0 \%$ concentration) and comparison between calibration and prediction at two other conditions 
(104\% ${ }^{\circ}-20 \%$ conc. and $140^{\circ} \mathrm{F}-10 \%$ conc.)

Figure 3.17: Aspirating probe calibration curve for Helium at reference conditions (room temperature and $0 \%$ concentration) and comparison between calibration and prediction at two other conditions $\left(104^{\circ} \mathrm{F}-20 \%\right.$ conc. and $140^{\circ} \mathrm{F}-10 \%$ conc.)

Figure 3.18: Schematic drawing of the shock tube facility

Figure 3.19: Total pressure history in the shock tube facility as measured by the total pressure probe

Figure 3.20: Voltage history in the shock tube facility as measured by the aspirating probe hot wire

Figure 3.21: Shock tube wave diagram

Figure 3.22: (a) Total temperature and concentration when the first shock hits the aspirating probe and its companion, total pressure probe (point $A$ in Fig. 3.19, 3.20, 3.21)

Figure 3.22: (b) Total temperature and concentration when the contact surface hits the aspirating probe and its companion, total pressure probe (point $B$ in Fig. 3.19, 3.20, 3.21)

Figure 3.23: Aspirating probe model for frequency response prediction [48]

Figure 3.24: Aspirating probe step response in Argon-Freon 12 mixture

Figure 3.25: Second order system step response

Figure 3.26: Second order system frequency response

(a) Amplitude-ratio curves

(b) Phase-angle curves

Figure 3.27: Typical time resolved error in mass fraction units of full scale for the measurement of concentration

(a) Helium-Freon 12 tracer mixture

(b) Helium tracer gas

Figure 3.28: Time resolved measurement of concentration in mass fraction units with error bars

Figure 3.29: Typical time resolved error in ${ }{ }_{K}$ for the measurement of total temperature 

(a) Helium-Freon 12 tracer mixture
(b) Helium tracer gas

Figure 3.30: Time resolved measurement of total temperature in ${ }^{\mathrm{K}}$ with error bars

Figure 3.31: Typical time resolved error in atms for the measurement of total pressure

(a) Helium-Freon 12 tracer mixture

(b) Helium tracer gas

Figure 3.32: Time resolved measurement of total pressure in atms with error bars

\section{CHAPTER 4}

Figure 4.1: Time resolved tracer gas concentration (mass fraction) during Blowdown test with no rotor in place. Determination of basic mixing level in the Facility and jet spreading rate

Figure 4.2: Comparison between total temperature measured by the aspirating probe and calculated from isentropic expansion in the supply tank (rotor removed)

Figure 4.3: Comparison between total pressure measured by the total pressure probe at the downstream surveying location and by a pressure transducer in the Blowdown supply tank (rotor removed)

Figure 4.4: Comparison of time averaged total pressure ratio for various test conditions

Figure 4.5: Comparison of time averaged total temperature ratio for various test conditions

Figure 4.6: Demonstration of the repeatability of the Blowdown Facility. Comparison of time averaged total pressure ratio during two separate Blowdown tests under the same conditions

Figure 4.7: Demonstration of the repeatability of the Blowdown Facility.

Comparison of time averaged total temperature ratio during two separate Blowdown tests under the same conditions

Figure 4.8: Comparison of time averaged tracer gas concentration (a), total pressure ratio (b) and total temperature ratio (c) for tests with Helium and Helium-Freon as tracer gas 


\section{CHAPTER 5}

Figure 5.1: Comparison of instantaneous total pressure ratio during Blowdown tests with (a) and without (b) injection

Figure 5.2: Comparison of instantaneous total temperature ratio during Blowdown tests with (a) and without (b) injection

Figure 5.3: Comparison of time averaged total pressure ratio during Blowdown tests with and without injection

Figure 5.4: Comparison of time averaged total temperature ratio during Blowdown tests with and without injection

Figure 5.5: Time resolved tracer gas concentration (mass fraction) during tests without (a) and with (b) injection

Figure 5.6: Scale drawing of the rotor geometry showing the three injection locations

Figure 5.7: Time resolved total temperature (a) and total pressure (b) ratio behind the rotor

Figure 5.8: Time resolved tracer gas concentration (mass fraction) during injections at the tip (a), midspan (b) and hub (c)

Figure 5.9: Time resolved total pressure ratio (a), tracer concentration (mass fraction) (b) and total temperature ratio (c), at tip, midspan and hub, during injection near the tip

Figure 5.10: Time resolved total pressure ratio (a), tracer concentration (mass fraction) (b) and entropy change (c), at tip, midspan and hub, during injection near the tip

Figure 5.11: Time resolved total pressure ratio (a), tracer concentration (mass fraction) (b) and total temperature ratio (c), at tip, midspan and hub, during injection near the midspan

Figure 5.12: Time resolved total pressure ratio (a), tracer concentration (mass fraction) (b) and entropy change (c), at tip, midspan and hub, during injection near the midspan

Figure 5.13: Time resolved total pressure ratio (a), tracer concentration (mass 
fraction) (b) and total temperature ratio (c), at tip, midspan and hub, during injection near the hub

Figure 5.14: Time resolved total pressure ratio (a), tracer concentration (mass fraction) (b) and entropy change (c), at tip, midspan and hub, during injection near the hub

Figure 5.15: Spanwise distribution of time averaged adiabatic efficiency

Figure 5.16: Spanwise distribution of time resolved adiabatic efficiency

Figure 5.17: Time averaged tracer gas concentration (mass fraction) during tip, midspan, hub and "no rotor" injections

Figure 5.18: Comparison of time averaged total pressure ratio for tests with injection at tip, midspan and hub

Figure 5.19: Comparison of time averaged total temperature ratio for tests with injection at tip, midspan and hub

Figure 5.20: 10 cycle ensemble averaged total pressure ratio (a), tracer gas concentration (mass fraction) (b), total temperature ratio (c) and entropy change (d) at tip, midspan and hub for tests with injection at the tip

Figure 5.21: 10 cycle ensemble averaged total pressure ratio (a), tracer gas concentration (mass fraction) (b), total temperature ratio (c) and entropy change (d) at tip, midspan, and hub for tests with injection at the midspan

Figure 5.22: 10 cycle ensemble averaged total pressure ratio (a), tracer gas concentration (mass fraction) (b), total temperature ratio (c) and entropy change (d) at tip, midspan and hub for tests with injection at the hub

Figure 5.23: Comparison of 10 cycle ensemble averaged tracer gas concentration (mass fraction) contours for "no rotor", tip, midspan and hub injections

Figure 5.24: 10 cycle ensemble averaged contour plots of total pressure ratio and tracer gas concentration (mass fraction) during tests with injection at tip, midspan and hub

Figure 5.25: Contour plots of total pressure ratio, tracer gas concentration (mass fraction), total temperature ratio and entropy change for injection 
at the tip

Figure 5.26: Contour plots of total pressure ratio, tracer gas concentration (mass fraction), total temperature ratio and entropy change for injection at the midspan

Figure 5.27: Contour plots of total pressure ratio, tracer gas concentration (mass fraction), total temperature ratio and entropy change for injection at the hub

\section{CHAPTER 6}

Figure 6.1: Scale drawing of the rotor geometry showing the inlet and outlet regions

Figure 6.2: Spanwise fluid transport as percentage of the mass flow of each individual inlet region

Figure 6.3: Spanwise distribution of time averaged normalized mixing coefficient

Figure 6.4: Schematic drawing of the coordinates system used in the calculation of the mixing coefficient

Figure 6.5: Typical concentration contours used in the calculation of the turbulent mixing coefficient

\section{CHAPTER 7}

Figure 7.1: Schematic drawing of rotor geometry, showing the development of secondary flow in the blade passage (streamwise vorticity)

Figure 7.2: Mean rotor exit absolute velocity distribution for NASA Lewis rotor, measured by the laser anemometer at $60 \%$ span, $140 \%$ chord in a plane 42 degrees from the axial direction (from [2])

Figure 7.3: Probability density distributions of velocity measured by the L.A. at points A-F in Fig. 7.2 (from [2])

Figure 7.4: Geometry of rotor blade vortex street (from [2])

Figure 7.5a: Demonstration of the model fitting procedure at the selected spanwise locations (a), (b), (c) and (d)

Figure 7.5b: Scaled perspective of the spanwise vortices shed by the rotor blade, as predicted by the vortex model 
Figure 7.6: Velocity field of blade wake vortex street in the frame of reference moving with the street

Figure 7.7: Pressure coefficient distribution from vortex model at the four spanwise locations

Figure 7.8a: Static pressure distribution nondimensionalized with inlet total pressure from vortex model at four spanwise locations

Figure 7.8b: Static pressure distribution in the vortex cores nondimensionalized with inlet total pressure from vortex model at four spanwise locations

Figure 7.9: Schematic demonstration of the various coordinate systems used in the vortex model

Figure 7.10: Velocity vectors in the core of the vortex

Figure 7.11: Distribution of absolute tangential velocity, absolute axial velocity and relative tangential velocity in the core of the vortex

Figure 7.12: Distribution of spanwise velocities in the cores of the vortices at four spanwise locations, as predicted by the vortex model

Figure 7.13: Typical distribution of spanwise velocities in the vortex core, as predicted by the vortex model

Figure 7.14: Evolution of spanwise velocities in the vortex core, during its convection from the trailing edge of the blade to the probe location (calculation at $r / r_{t i p}=0.95$ )

Figure 7.15: Evolution of spanwise velocities in the vortex core, during its convection from the trailing edge of the blade to the probe location (calculation at $r / r_{t i p}=0.88$ )

Figure 7.16: Evolution of spanwise velocities in the vortex core, during its convection from the trailing edge of the blade to the probe location (calculation at $r / r_{t i p}=0.81$ )

Figure 7.17: Evolution of spanwise velocities in the vortex core, during its convection from the trailing edge of the blade to the probe location (calculation at $r / r_{t i p}=0.75$ )

Figure 7.18: Perspective of the evolution of spanwise velocities in the vortex 
cores, during their convection from the trailing edge of the blade to the probe location (calculation at $r / r_{t i p}=0.95$ )

Figure 7.19: Perspective of the evolution of spanwise velocities in the vortex cores, during their convection from the trailing edge of the blade to the probe location (calculation at $r / r_{t i p}=0.88$ )

Figure 7.20: Perspective of the evolution of spanwise velocities in the vortex cores, during their convection from the trailing edge of the blade to the probe location (calculation at $r / r_{t i p}=0.81$ )

Figure 7.21: Perspective of the evolution of spanwise velocities in the vortex cores, during their convection from the trailing edge of the blade to the probe location (calculation at $r / r_{t i p}=0.75$ )

Figure 7.22: Perspective of the spanwise velocities in the vortex cores at four spanwise locations

\section{CHAPTER 8}

Figure 8.1: Spanwise distribution of adiabatic efficiency for several transonic rotors. Theoretical estimates of losses (from [3]) are given for comparison

Figure 8.2: Streamtube exchange during spanwise transport within the blade passage (shown only for region 1)

Figure 8.3: Effect of spanwise transport on the spanwise distribution of time averaged adiabatic efficiency, when the transport occurs within the blade passage (4 regions included in the calculations)

Figure 8.4: Effect of spanwise transport on the spanwise distribution of time averaged adiabatic efficiency, when the transport occurs within the blade passage (3 regions included in the calculations)

Figure 8.5: Ideal representation of wake and free stream regions for modeling purposes

Figure 8.6: Effect of isentropic spanwise redistribution of the flow after the trailing edge of the blade, on the spanwise distribution of adiabatic efficiency

Figure 8.7: Comparison between measured, predicted and corrected spanwise distributions of time averaged adiabatic efficiency 


\section{APPENDIX A}

Figure A.1: Distribution of spanwise velocities in the cores of the vortices at four spanwise locations, as predicted by the vortex model

Figure A.2: Typical distribution of spanwise velocities in the vortex core, as predicted by the vortex model 


\section{LIST OF TABLES}

Table 2.1: Test Compressor characteristics

Table 3.1: 1-D calculation of the time delay between the pressure transducer and the hot wires for various gases

Table 3.2: Changes in the results after correcting for the time delay

Table 3.3: Probe sensitivity to various tracer gases

Table 3.4: Calibration coefficients

Table 3.5: Shock tube conditions

Table 3.6: Step response parameters as in Fig. 3.24 and 3.25

Table 3.7: Measured frequency response parameters

Table 3.8: Calculated frequency response parameters

Table 3.9: Maximum percentage error in the measurement

Table 3.10: Average percentage error in the measurement

Table 3.11: Sensitivity values for the aspirating probe hot wires for various tracer gases and test conditions

Table 4.1: Froude number, length of non-buoyant and intermediate regions and buoyancy origin

Table 6.1: Matrix of migrating fluid in the rotor. All the numbers are percentages of the total mass flow through the compressor

Table 6.2: Values of mixing coefficient defined in eq. (6.16)

Table 7.1: Vortex street parameters for the NASA LeRC Stage 67

Table 7.2: Vortex model parameters

Table 7.3: Shedding frequencies in the spanwise direction

Table 7.4: Strouhal number based on the wake width 
Table 7.5: Strouhal number based on the trailing edge boundary layer momentum thickness

Table 7.6: Strouhal number based on the trailing edge thickness

Table 7.7: Development of the vortex cores

Table 7.8: Measured and predicted maximum spanwise displacement and migrating mass flow 


\section{CHAPTER 1 INTRODUCTION}

Since the invention of the gas turbine engine, several decades ago, researchers have been investing enormous amounts of time and effort trying to understand these complicated devices. As the demand for engines with decreased fuel consumption and higher thrust increased, so did the complexity of the engines. Today the big thrust is towards light, high pressure ratio compressors and heavily loaded, extremely high temperature turbines.

One of the most fascinating products of intensive research is the transonic compressor. It is an axial flow compressor in which the inlet flow relative to the rotating blades varies from subsonic at the hub to fully supersonic at the tip. The flowfield in a transonic compressor is highly unsteady, three dimensional and clearly compressible. Unsteadiness is an inherent property of turbomachinery, since energy is transferred from the moving blades to the fluid through a series of unsteady periodic compressions. A major simplifying assumption commonly used in the design of these engines is that the flow in the frame moving with the blades (relative frame) is steady and similar in each blade passage. Recently, however, it has been found that the flow is unsteady even in the relative frame. $\mathrm{Ng}$ [1] attributed the observed unsteadiness to the motion of the blade passage shock, which is driven by the shedding of vortices from the blades. Later, Gertz [2] refined the concept by modeling the flow in the blade wakes as a two-dimensional von Karman vortex street

Another feature of great importance to the compressor design is the three dimensionality of the flowfield. The design of most of the compressors is based 
on the assumption that the flow remains on axisymmetric stream surfaces as it passes through the machine. Over the past decade, however, researchers have accumulated experimental data indicating that significant spanwise and cross-passage velocities exist, which result to considerable levels of spanwise mixing.

Kerrebrock [3] observed that many transonic compressors have unexpectedly low adiabatic efficiency near the tip. A 2-D calculation of the spanwise distribution of adiabatic efficiency was performed including viscous effects (in terms of diffusion factors [4] and normal shock losses loblique shock losses are generally lower). The results showed [3] that there is a 5-10\% drop in tip efficiency that cannot be explained from these calculations. Kerrebrock suggested that the high tip losses could be attributed to spanwise migration of "lossy" fluid from the hub to the tip. Therefore the poor performance of the tip might not be associated with mechanisms that generate additional loss, but rather with redistribution of "lossy" fluid and accumulation at the tip through spanwise migration.

Thompkins and Usab [5] developed a quasi 3-D, viscous computer code and calculated the spanwise velocities in the blade boundary layer and the location of the three dimensional separation line. Their calculations showed that unless there is early separation causing migration of fluid to the tip, the spanwise velocities in the boundary layers cannot account for the 5-10\% drop in tip efficiency.

In addition, adiabatic efficiency measurements performed by Wennerstrom [6] at the Air Force Aero-propulsion Laboratory showed regions of higher than one efficiency near the rotor hub of a transonic compressor. The same compressor 
was tested at the MIT Blowdown Facility by $\mathrm{Ng}$ [1], who confirmed that experimental finding.

Since it was suggested that spanwise fluid transport might lead to an explanation of these observations, we initiated a series of experiments to measure the spanwise fluid transport Hence, this research effort is an extension of the work by Gertz to include three dimensional effects and an investigation of the coupling between the mechanisms of relative frame unsteadiness and spanwise fluid transport.

In general, the spanwise flows that may be present in an axial compressor or fan are:

1) In the blade boundary layer.

2) Associated with the end-wall boundary layers.

3) Due to the relative vorticity in the rotor frame (relative eddy).

4) Caused by the tip clearance vortex.

5) Due to the trailing vorticity, shed behind the blade.

6) In the blade wakes.

7) In regions of separation.

8) Associated with motion in the cores of spanwise coherent vortices shed by the rotor blades

Spanwise flows in the blade boundary layer are generally caused by the imbalance between spanwise pressure gradients and centrifugal forces. The rotor blade boundary layer fluid has lower relative streamwise velocity than the core or free stream one, due to the viscous action of the blade. Therefore, a simple velocity triangle argument shows that the boundary layer fluid has larger swirl 
velocity in the absolute frame than does the free stream. The swirl velocity of the core flow in the passage balances the spanwise pressure gradient so that ideally no spanwise flow occurs here. The boundary layer fluid experiences the same pressure gradient, but having a larger swirl velocity, it moves outwards towards the rotor tip.

Spanwise flows associated with the end wall boundary layers occur because of the oncoming or passage generated streamwise vorticity. The classical approach to secondary flows of this type is described by Hawthorne [7], Squire and Winter [8] and Horlock [9]. Over the years considerable progress has been reported in this field and the secondary flow equations have been modified to include compressibility, inlet temperature gradients and rotation of the Bernoulli surfaces. Predictions of the spanwise flows due to the streamwise vorticity have been made and the agreement is generally good for single stage compressors, but not for multistage ones. One reason for that is the inviscid character of all these secondary flows calculations, when viscous effects probably dominate the flow in multistage engines.

Spanwise flows due to the "relative eddy" are generated even in the absence of vorticity in the oncoming flow. Their origin can be seen if one realizes that the flow in the relative rotor frame has vorticity equal to $-2 \Omega$, where $\Omega$ is the angular velocity of rotation. The induced spanwise velocities due to this mechanism have been investigated by [10], [11] Spanwise velocities up to $17 \%$ of wheel speed have been reported. However, in general it is believed that this is a negligible mechanism as far as spanwise flows are concerned.

Tip clearance flows have been the subject of extensive research for many 
years. There are basically two mechanisms that cause fluid to flow through the gap between rotor blade tip and outer casing. First, the pressure difference between suction and pressure side of the blade and, second, the viscous action of the rotating blade on the end-wall fluid, which is being dragged through the gap by the relative motion of the blade and the wall. $A$ number of models with satisfactory predictive capabilities have been proposed [12], [13], [14], but the phenomena associated with tip clearance flow are not yet fully understood.

Spanwise flows are also induced when there is a spanwise variation of the blade circulation, that causes the creation of a trailing vortex sheet behind the blade. The existence of a vortical structure like this induces spanwise velocities of opposite direction on each side of the wake [15]. This is basically an inviscid phenomenon and its effect can be calculated using 3-D Euler codes. Thompkins [16] computed the distortion of the blade-to-blade stream surfaces due to this mechanism and reported maximum spanwise displacements of about $20 \%$ of the blade height The streamsurfaces are deflected towards the hub near the suction side of the blade and towards the tip near the pressure side.

Spanwise flows in the viscous blade wakes are a result of a mechanism similar to the one that causes spanwise flows in the blade boundary layers. The difference is that the wake spanwise flows occur after the trailing edge of the blade and, therefore, the structure of the wake might influence their magnitude and direction.

Regions of separation near the trailing edge of the rotor blade are also a source of strong spanwise flows, since the detached fluid experiences no resistance from the blade wall and can move very quickly towards the rotor tip, 
under the action of centrifugal forces. Viscous three dimensional codes have been employed to predict the location and shape of the separation line in the spanwise direction [17], [18], and hence the boundary layer displacement thickness at the separation point, but their extreme sensitivity to the turbulence model they use, puts severe limitations to their predictive capabilities.

Spanwise flows in the cores of spanwise vortices shed behind the rotor blades have not been reported in the literature and is part of the experimental findings and modeling attempts of this thesis. The mechanism that causes these flows will be described later in this section, when a preview of the experimental results is presented.

Time averaged measurements of spanwise mixing have been obtained before in low speed multistage compressors. Recently two fundamentally different approaches to the modeling of spanwise mixing in these engines appeared in the literature.

The first one came from Adkins and Smith [19], who proposed that secondary flows are primarily responsible for both the spanwise mixing of flow properties and the deviation of blade row turnings from two- dimensional cascade theory. Their analysis was based on the fact that secondary flows can be associated with spanwise velocities. These velocities in turn can be associated with mixing in the spanwise direction. They modeled the spanwise mixing as a typical diffusion process, where the mixing coefficient is determined from spanwise velocities calculated after superposing the contributions from main flowfield secondary flow, end wall boundary layer flow, tip clearance flow, blade end shroud, blade boundary layer and wake centrifugation. They also incorporated 
this mixing model into throughflow calculations. The results showed considerably better agreement with experimental data for spanwise distributions of stagnation temperature and pressure than the ones obtained without including the mixing model.

A few years later Gallimore and Cumpsty [20] performed a series of time averaged tracer gas experiments concluded that the idea of deterministic, spanwise secondary flows being the primary cause of spanwise mixing in axial compressors was inaccurate. Instead they proposed that a random, turbulent type of diffusion process is the dominant mechanism. They also developed a method to estimate the value of the spanwise mixing coefficient in terms of stage geometry, loss and flow coefficient This model was introduced in their through-flow calculations [21] and their results were as impressive as the ones from Adkins and Smith.

More recently Wisler, Bauer and Okiishi [22] conducted a detailed study of the flow in the General Electric Low Speed Research Compressor (LSRC). They used the ethylene tracer gas technique and 3-D, slanted hot wire anemometry to measure secondary flows, fluid migration, diffusion rates, ty velocities and turbulence intensities for two loading levels. By doing that they were able to determine the relative magnitude of the contribution of the secondary flow to the spanwise mixing versus the one from the turbulent diffusion. They concluded that both mechanisms are equally important In the free-stream region, turbulent diffusion appeared to be the dominant mechanism, while closer to the walls the mixing effects of secondary flow were of the same order of magnitude as, and in some cases greater than, the diffusive effects from turbulence. 
However, all these research efforts were performed in low speed, multistage compressors and the applicability of their conclusions in high speed transonic compressors is questionable. Therefore, direct measurements of spanwise mixing in transonic compressors are needed.

The basic concept of our experiment is quite simple: inject a thin circumferentially oriented sheet of tracer gas in front of a transonic compressor and detect the foreign fluid molecules at the rotor exit If there were no 3-D effects the sheet would spread uniformly under the action of viscosity and turbulence only. Therefore any non-uniformity in the sheet behind the rotor could be associated with 3-D effects and in particular with spanwise motion of the tracer molecules. In Chapter 2 a description and detailed analysis of the major components of the experiment will be presented.

One of the most challenging problems faced during the experimental phase of this project was the measurement of the concentration of the tracer gas with sufficient accuracy, spatial resolution and frequency response (high frequency response is required to permit high spatial accuracy relative frame measurement to be made with Laboratory frame probes). As it will be explained in Chapter 2, a typical test lasts only 50 msec and the blade passing frequency is approximately $5 \mathrm{kHz}$. The Flame lonization Technique (FID) that is commonly used in low speed compressors for tracer gas experiments [20], [21], [22], [23] has very low frequency response (approximately $300 \mathrm{~Hz}$ ), a factor of 16 less than the blade passing frequency of our transonic compressor. Therefore it was necessary to develop a technique capable of measuring concentration of tracer with frequency response of at least $15 \mathrm{kHz}$. Details for this method will be presented in Chapter 3. 
In the same Chapter a detailed description and complete results of both dynamic and static calibrations of the probe will be presented. In addition, a detailed experimental and analytical demonstration is given of an artifact of the probe measurement. When the data reduction schemes were suitably corrected, most of the negative entropy regions previously measured in this compressor disappeared.

The spreading of the injection jet and its effect on the main rotor flowfield were directly measured in order to verify that the injector operates properly during the Blowdown test and has no substantial influence on the flowfield of the rotor. In addition, a series of preliminary tests and calculations were performed in order to ensure that the probe and the data reduction scheme gave consistent measurements. The details of these tests will be presented in Chapter 4.

The majority of the experimental data will be given in Chapter 5 . Several experimental observations have been made. First, there is significant spanwise transport (up to $5 \%$ of the compressor mass flow). Second, the spanwise motion occurs primarily in the blade viscous wakes. Third, the fluid moves towards both hub and tip in the blade wakes. Fourth, the spanwisely convected fluid has high entropy, much higher than that of the "inviscid core". Fifth, the "inviscid core" fluid moves preferentially towards the suction side of the blade passage and away from the pressure side.

A comparison of our time averaged data with related time averaged measurements available in the literature was also performed and is presented in Chapter 6. A discussion and rough estimate of the contribution of all possible 
mechanisms to spanwise migration will be presented in Chapter 7.

In addition, in this Chapter the details of a new mechanism will be discussed. This mechanism is associated with the shedding of vorticity in the form of more or less regular von Karman type vortex streets from the blade, as proposed by Gertz [2]. These vortices are very strong and can have large pressure drop in their cores. Since this pressure drop is a function of the strength of the vortex, a spanwise variation of vortex strength could lead to a strong pressure gradient in the core of this vortex in the spanwise direction.

A simple model has been developed in Chapter 7 to estimate the strength of the vortex in the spanwise direction and from that the pressure gradient in the core. A calculation based on the parameters of the model, gave an almost uniform shedding frequency in the spanwise direction, which is a prerequisite for spanwise coherence of the vortices. This model predicted the measured spanwise transport towards the hub, but underestimated the measured transport to the tip by a factor of 5 .

The effect of the spanwise fluid transport on the spanwise distribution of adiabatic efficiency was also investigated and the results will be presented in Chapter 8. Two extreme cases were considered in order to bound the effect. First, we assumed that the spanwise fluid transport occurs within the blade row and is completed before the trailing edge of the blade. Second, we assumed that the fluid transport is a simple redistribution of flow parameters and occurs only after the trailing edge of the blade. The first case had a substantial effect on the spanwise distribution of adiabatic efficiency (change of about $9 \%$ near the tip), while the second had a smaller effect (change of about $2 \%$ near the tip). 
However, when the prediction of the vortex model for the fluid transport after the trailing edge was used, an increase of about $13 \%$ in efficiency was predicted in the tip region. Therefore, the discrepancy observed by Kerrebrock between predicted (viscous+normal shock losses) and measured spanwise distributions of adiabatic efficiency near the tip of transonic compressors can be fully attributed to the measured spanwise fluid transport 


\section{CHAPTER 2 \\ EXPERIMENTAL APPARATUS}

\section{1 - Description of the experiment}

This series of tracer experiments were conducted at the MIT Blowdown Compressor Facility. The test article was a transonic fan stage (Air Force High Through Flow Compressor). A specially designed injector was constructed to inject a thin planar sheet of tracer gas in front of the fan. The foreign molecules of tracer gas were then detected by a sampling probe, called Aspirating Probe, located behind the rotor blades. A new technique was developed to measure the concentration of the tracer gas with sufficient accuracy, spatial resolution and frequency response. Once the time resolved concentration measurements were obtained, the radial transport of fluid in the fan could be determined, since any nonuniformity in the concentration trace can be associated with the action of three dimensional mechanisms on the tracer gas fluid particles.

In summary, some of the major components of the experimental part of this research effort were:

1) The Blowdown Compressor Facility, which was the test facility.

2) The Air Force High Through Flow Compressor, the test article.

3) The Tracer Gas Injector and Injection System, which were responsible for the injection of the thin planar sheet of tracer gas.

4) The Aspirating probe, which was employed for the simultaneous, time resolved measurement of total pressure, total temperature and concentration of tracer gas.

5) A high speed analog-to-digital converter, which was used as the data acquisition system. 


\section{2 - Blowdown Compressor Facility}

The details of the construction and operation of the Blowdown Compressor Facility can be found in numerous references [24] The facility consists of a supply tank, initially separated from the compressor test section by an aluminum diaphragm (Fig. 2.1). The test section is followed by a dump tank into which the compressor discharges. The supply tank is filled up to 0.53 atms., while the dump tank and the test section are kept in vacuum. Before a test the rotor is brought up to speed, but because it is in vacuum the power requirement is very small. When the diaphragm ruptures, the gas expands from the supply tank into the dump tank through the test section. As the gas expands, the rotor is driven only by its own inertia and therefore it slows down, because it does work on the flow. In order to be at the right point on the compressor map (the design point for our experiments), the axial and tangential Mach numbers must be kept constant. The axial Mach number determines the corrected mass flow through the rotor, while the tangential Mach number determines the corrected speed. An orifice downstream of the rotor, that remains choked during the useful test time, fixes the axial Mach number in the facility lapproximately 0.5 at the face of the rotor). The total temperature and total pressure in the supply tank decrease during the test, due to the expansion of the gas. In order to keep the inlet tangential Mach number constant, the change in the supply tank temperature and the deceleration of the rotor must be related by the expression: 


$$
\left[\frac{\omega(t)}{\omega(0)}\right]^{2}-\frac{T_{t}(t)}{T_{t}(0)}
$$

where $\omega$ is the rotational speed of the rotor and $T_{t}$ is the temperature in the supply tank. However it was found that the rotating inertia of the Air Force High Through Flow Stage was too small and the corrected speed during the useful test time was not constant Therefore a tungsten flywheel was built, which increased the inertia of the rotating assembly by $60 \%$, resulting in a variation of corrected speed from $100.3 \%$ to $99.6 \%$ during the test

An important feature, however, of the Blowdown Facility is its repeatability. Total temperature and total pressure measurements in the facility were found to vary from test to test by less than $1.0 \%$, which allows good comparison among tests.

\section{3 - Test Compressor}

The transonic compressor tested is the Air Force High Through Flow Stage (AFHTF) designed by Dr. Arthur Wennerstrom at the Air Force Aero-Propulsion Laboratory (AFAPL). Detailed description of the rotor can be found in [25], [26]. The following table gives the basic characteristics of the fan. 
Table 2.1: Test Compressor characteristics

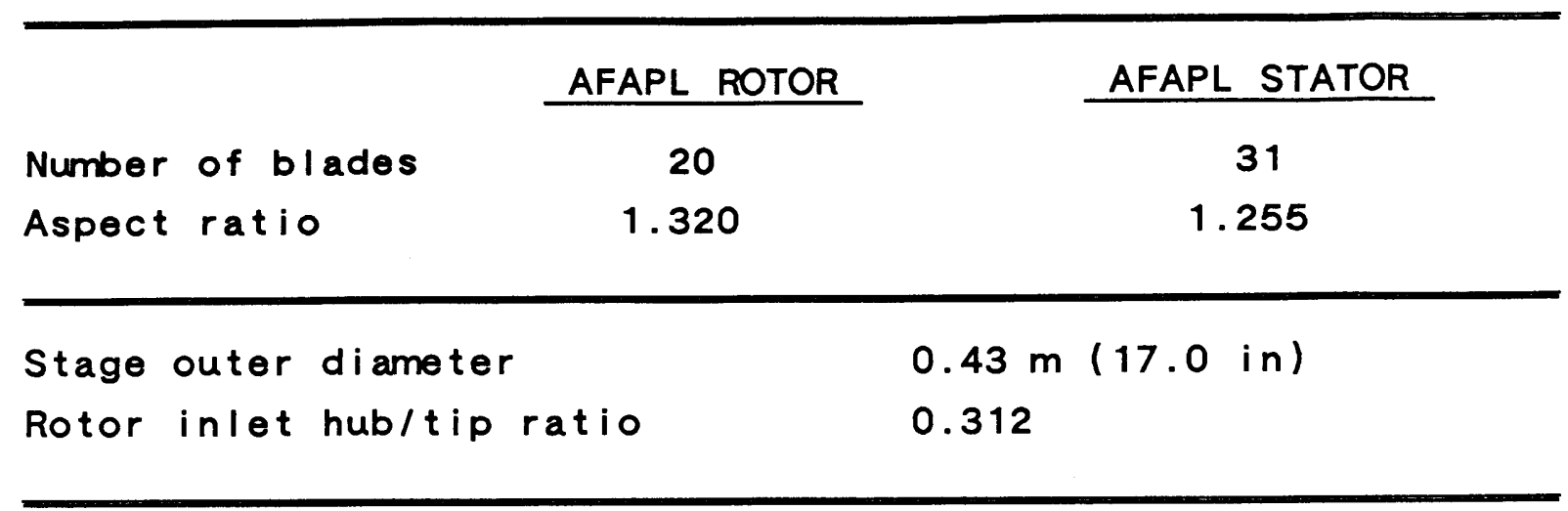

At design condition (100\% corrected speed)

Corrected mass flow

$28.41 \mathrm{~kg} / \mathrm{sec}(62.64 \mathrm{lbm} / \mathrm{sec})$

AFAPL ROTOR

AFAPL STAGE

Mass avg. total press. ratio

2.1

2.065

Adiabatic efficiency

$90.4 \%$

$88.2 \%$

A complete set of test results of this stage are given in [26] Fig. 2.2 and 2.3 show a scale drawing of the stage mounted in the Blowdown Facility test section and a top view of the relative position of the injector on front of the rotor. Location of instrumentation ports and stage flow path are also shown. 


\section{4 - Basic operating details}

In order to be able to compare the results from test to test, it was important to operate at the same point on stage performance map. That operating point is indicated in Fig. 2.4 and was kept the same for all the tests that will be discussed in this report At this point on the map the rotor has a mass averaged total pressure ratio of 2.1 and an adiabatic efficiency of $90.4 \%$.

The rotational speed of the rotor was monitored with an optical encoder, while the mass flow through the stage was calculated using the slope of the total pressure decay in the supply tank. These were used to calculate the corrected mass flow and the corrected speed during each test Fig. 2.5a and b show typical traces of fractional corrected speed and fractional corrected mass flow vs time, during a Blowdown test The fractions are refered to the design point conditions, as reported in [25] [26]. The corrected speed changes by less than $1 \%$ during the test and the corrected mass flow by less than $0.2 \%$. These changes are satisfactory for our purposes.

For a typical Blowdown run the useful test time starts at approximately 90 msec and lasts until $130 \mathrm{msec}$. During this time the probe is traversed from tip to hub, a total distance of about 2.5 in $(6.35 \mathrm{~cm})$, using a pneumatic traverser. For the design point operation the blade passing frequency is $5 \mathrm{kHz}$ and the wheel speed is $15000 \mathrm{rpm}$.

All the measurements were taken behind the rotor at a plane that approximately coincides with the stator's leading edge. The axial distance from the rotor trailing edge is 0.2 rotor chords (see Fig. 2.3). 


\section{5 - Data Acquisition and Processing}

The data acquisition system is shown in Fig. 2.6. The CAMAC units consist of eight 10 bit analog input channels with a maximum sampling rate of $1 \mathrm{MHz}$, total of 64,000 samples of memory and thirty-two 12 bit channels with maximum sampling rate of $5 \mathrm{kHz}$, total of 32,000 samples of memory on the CAMAC bus. Therefore a total of 276,000 data points can be taken during a typical blowdown test. This $A / D$ system is implemented with a four speed programmable clock with sampling frequencies ranging from $20 \mathrm{~Hz}$ to $1 \mathrm{MHz}$. The units have internal memory which was read back by a DEC computer, model PDP 11/70 after the test.

For the set of experiments presented in this report, the sampling rate of the high speed units was $100 \mathrm{kHz}$ per channel and of the low speed $5 \mathrm{kHz}$. This sampling rate gives approximately 20 data points per blade passage (blade passing frequency was $5 \mathrm{kHz}$ ). The low frequency signals were filtered through anti-aliasing 4-pole Bessel filters with a cut-off frequency of $1.4 \mathrm{kHz}$. The noise of the electronics was less than one digital count The A/D system was checked periodically to ensure proper operation. 


\section{6 - Tracer Gas Injector}

The success of this tracer gas experiment depends heavily on two factors. First, the sensitivity of the aspirating probe to the concentration levels of the experiment and second the ability of the injector to create a thin, compared to the fan's span, sheet of tracer gas. The probe sensitivity issue will be brought up later when we talk about the choice of tracer gas. In this chapter we will discuss the design and operation of the injector.

A detailed description of the injector and its design specifications and constraints can be found in [27] A brief summary will be given here. According to Fig. 2.7, the injector is basically a hollow airfoil, type NACA 654-021. Two hollow streamline shape struts support the airfoil. When the injector is assembled, a thin slot (dimensions $0.030 \times 3.300 \mathrm{in}$ ) is formed at the trailing edge that is fed from the cavity inside the airfoil. The cavity is designed to have dimensions large compared to the slot, so that it acts as a stagnation plenum. The stagnation pressure in the cavity is measured by a Kulite pressure transducer mounted within the cavity. The transducer wires are fed through a vacuum feedthrough.

The tracer gas is stored in a small supply tank (4 It). During the test, the tracer gas flows through the hollow supporting struts and a fast acting valve before reaching the injector cavity. Fig. 2.8 shows a schematic drawing of the injection system. This arrangement gives the injector freedom to move radially in and out of the test section. The slope and level of the total pressure decay in the cavity of the injector are essentially determined by the volume and initial pressure of the tracer gas tank. Therefore the tank was designed to have adjustable volume and pressure. 
The operation of the injector can be summarized as following. At a certain point in time after the main Blowdown Facility diaphragm ruptures, the solenoid valve that connects the tracer gas tank with the injector cavity opens, letting the tracer gas flow through the pipes and the final exit slot at the trailing edge of the injector, 0.2 blade chords upstream of the rotor. The stagnation pressure of the stream is measured by the Kulite pressure transducer in the injector's cavity. The relative location and size of the injector and the rotor tip blade section can be seen in Fig. 2.3.

The injection can be treated as a "small" blowdown process, compared to the main facility blowdown. In order to create a thin sheet of tracer gas, the momentum of the injection jet should match as close as possible with the momentum of the free stream that flows over the airfoil. Hidden behind this argument are a number of observations and requirements:

1) A thick wake or an early separation on the surface of the injector airfoil could lead to rapid spreading of the injection jet Since the Reynolds number at the location of the injector is quite small (approximately $3.5 \times 10^{5}$ ) there was a concern during the design process, of a shock induced separation on the airfoil. We were also interested to predict as close as we could the separation point in the case of shock free flow. A 2-D, viscous computer code was used, [28], to predict the surface Mach number and the separation location on the injector airfoil. Fig. 2.9 shows the results of this calculation. As it can be seen in the figure, the local Mach number is less than one and the separation location is at approximately $80 \%$ blade chord, giving a wake of approximately $10 \%$ of the airfoil's maximum thickness. These estimates show that we should not expect 
anything dramatic in terms of separation on the injector, except from the normal separation close to the trailing edge.

2) The turbulence level in the Blowdown tunnel is quite low and essentially fixed.

3) During the 'no injection' blowdown tests, the injector cavity is at a pressure approximately equal to the free stream static pressure at the point of injection. Therefore, this pressure can be measured by the pressure transducer that is mounted in the cavity. However, during the 'with injection' tests the same pressure transducer picks up the stagnation pressure of the tracer gas, while the repeatability of the blowdown process ensures that the free stream static pressure at the injection point is still the same with the 'no injection' case. Using these measurements, the Mach number at the injector exit was found to be less than 0.7. Therefore the exit flow is clearly subsonic and adjusts smoothly to the pressure of the free stream.

From all the above, we concluded that matching the velocities of the two streams would give the minimum injection jet spreading.

The temperature of the injection jet was not measured directly, since the frequency response of most of the common temperature measuring instruments is much lower than the time scale of the test time. However, an estimate of the injection jet temperature was made. The mass of the gas that flows through the injector is quite small, while the inside wall surface of the injector is large. Therefore we can expect the gas in the injection system to become colder due to the blowdown expansion, but at the same time heat up, due to the hotter 
walls of the cavity. The wall temperature of the injector does not change during the main blowdown, since there is not enough time for heat transfer from the cold free stream gas (approximately $240^{\circ} \mathrm{K}$ ) to the inside surfaces of the injector. Therefore for design purposes, we considered the temperature of the injection jet to be equal to the metal temperature (room temperature) at the start of the test and to remain constant during the blowdown. Since the injector cavity can be considered as a stagnation plenum, the total temperature of the injection jet will be equal to the temperature of the cavity walls (room temperature). Therefore the temperature difference between the jet and the free stream must be taken into consideration, during the design.

For the tracer gas that we finally selected, a mixture of Helium and Freon-12, there is no difference in molecular weight between injectant and main gas, but there is a difference in the ratio of specific heats. This difference is also taken into consideration during the injector design process.

The total pressure of the jet and free stream are indicators of the velocity matching. Since the temperatures and ratios of specific heats of the two flows are slightly different, the requirement of equal velocities means that the injector jet total pressure must be slightly lower than the supply tank one. We assumed that the total pressure losses from the measurement location in the cavity to the slot exit are negligible, so that the total pressure we measured in the cavity is the same with the total pressure of the jet

Fig. 2.10 shows typical total pressure histories of the main and injector blowdowns. Fig. 2.11 is an expanded view of Fig. 2.10, during the useful test time. The third curve in Fig. 2.11 is a curve fit of the total pressure decay trace 
in the supply tank. The slope of this curve fit function is also used to calculate the total mass flow through the fan during the main blowdown. The form of this function is $f(t)-(a+b t)^{c}$, where $t$ is the time in seconds, $f(t)$ is in atms and $a, b$ and c are determined from the curve fit For a typical blowdown experiment a-1.086, $b=0.890 \mathrm{c}=-7.821$. An important point is that the matching requirements were imposed at both the beginning and end of the useful test time (90 and $125 \mathrm{~ms}$ ). Since acoustic fluctuations in the supply tank total pressure trace are observed (see Fig. 2.10), the injector design was based on the curve fit, rather than the fluctuating actual signal. Based on these measurements the difference between the jet and the mean free stream velocities is less than $0.5 \%$. Notice that the mean free stream velocity was calculated using the values of total pressure as given by the curve fit

The window of close matching was adjusted to be coincident with the constant corrected speed and mass flow test time, by firing the solenoid valve at the appropriate time. 


\section{CHAPTER 3}

\section{INSTRUMENTATION}

\section{1 - The Dual-Hot-Wire Aspirating Probe}

The aspirating probe was originally [1] constructed to measure time resolved total pressure and total temperature in highly fluctuating, compressible flowfields. In this report, its application to the measurement of time resolved concentration will be discussed. More details can also be found in [27] The most important features of this probe are its high frequency response (over $15 \mathrm{kHz}$ ) and its ability to give simultaneous time resolved measurements of total pressure, total temperature and concentration.

\subsection{1 - Brief Description of the Aspirating Probe}

Although a full and detailed description of the aspirating probe is given in [1] and [27], a brief one will be presented here. The probe consists of two coplanar hot wires placed in a $1.5 \mathrm{~mm}$ diameter, constant cross sectional area channel, as it is shown in Fig. 3.1. A choked orifice behind the wires' plane provides constant Mach number of 0.2 at that plane. The hot wires are operated at different overheat ratios and thus, different wire temperatures, by using a conventional TSI Model 1050 anemometer. A total pressure impact probe incorporating a Kulite model XCQ-093, silicon diaphragm pressure transducer is mounted "piggy-back" to the aspirating probe, giving a simultaneous measurement of the total pressure (see Fig. 3.2). The hot wires are made of platinum plated tungsten and the sensing length is isolated by copper plating the wires at their ends, thus eliminating 
thermal boundary layer interference. For this set of experiments 0.0002 in. diameter wires were used with a sensing length of 0.050 in.

As far as reliability of the hot wires readings was concerned, two major problems had to be overcome. The first was associated with the frequent damage of the wires during the blowdown test Very often, small aluminum particles, part of the broken aluminum diaphragm, were ingested by the probe, resulting in complete or partial damage of the hot wires. Therefore, quick and reliable replacement of the hot wires was of vital importance. Special care was taken to ensure that after their replacement the wires were taut enough. A manifestation of a loose wire was the appearance of a signal modulating frequency of as high as $18 \mathrm{kHz}$ in the hot wire readings, which disappeared when they were taut. In order to avoid the problem, the aspirating probe was protected in a cavity during the transient period of the blowdown test, when most of the aluminum particles move through the rotor. At about $80 \mathrm{msec}$ after the initial diaphragm explosion, the pneumatic probe traverser was activated and the probe moved from tip to hub in about 50 msec.

The second problem was associated with the contamination of the hot wires during the blowdown tests. Comparisons between before and after the test calibrations of the hot wires, showed dramatic differences in their readings. In addition, extensive observation of the wires under the microscope revealed the presence of a coating around them. It was felt that this contamination was a result of the smoke that normally filled the facility right after the diaphragm explosion. In order to eliminate the problem, a fast acting valve was installed in the suction line of the aspirating probe. The valve was triggered at about 20 msec after the diaphragm explosion and was fully open after approximately 50 
msec. Since the useful test time is from 90 to $130 \mathrm{msec}$ and most of the smoke goes by the probe during the transient period ( 0 to $80 \mathrm{msec}$ ), the wire contamination was eliminated, resulting in good agreement between before and after calibrations.

The well known equation [1] for the anemometer bridge voltage output of a constant temperature hot wire is:

$$
\frac{v^{2}}{\left(T_{w}-r T_{t} T\right.}=\frac{\left(R_{s}+R_{w}\right)^{2}}{R_{w}} \pi \mid k N u
$$

where $V$ is the voltage output, $T_{w}$ is the hot wire temperature, which remains constant during the test, $R_{\mathbf{z}}$ is the anemometer resistance in series with the hot wire, $R_{w}$ is the wire operating resistance, set by the anemometer, $r$ is a recovery factor, 1 is the wire length, $k$ is the thermal conductivity of the gas that is being sampled and $\mathrm{Nu}$ is the Nusselt number.

The form of the relationship between the Nusselt and Reynolds number has been the subject of extensive research. We picked the semi-empirical formula, eq. (3.2), of Collis and Williams [29] for our application.

$$
N u=\left(\frac{T_{m}}{T_{\infty}}\right)^{0.17}\left[a\left(R e_{d}\right)^{m}+b\right]
$$

where $\mathrm{Re}_{\mathrm{d}}$ is the Reynolds number based on wire diameter, $\mathrm{a}, \mathrm{b}, \mathrm{m}$ are empirically determined constants, $T_{\infty}$ is the free stream fluid temperature and $T_{m}$ is the so called "film temperature", which is the mean of the free stream fluid temperature 
and the hot wire temperature. According to [29] this equation is valid for Reynolds numbers ranging from 0.01 to 140 and therefore it is applicable in our case, since a typical value for the Reynolds number, based on the wire diameter, is 20. In [29] it is also argued that at Reynolds numbers higher than about 44, a vortex street exists in the wake of the hot wire. Therefore two sets of $a, b, m$ coefficients are given, depending on whether the $R e$ is above or below 44. During this set of experiments, special care was taken to understand and predict the behavior of these coefficients throughout the range of test and calibration conditions.

Since the channel of the aspirating probe is choked, the mass flux at the wires' plane is given by the expression:

$$
\rho U=\frac{p_{t}}{\sqrt{T_{t}}} \frac{A^{*}}{A_{c}} \sqrt{\frac{\gamma}{R}}\left(\frac{2}{\gamma+1}\right)^{\frac{\gamma+1}{2(\gamma-1)}}
$$

where $A^{*}$ is the sonic area of the channel, $A_{c}$ the channel cross sectional area at the wires' plane, $\gamma$ the ratio of specific heats and $R$ the fluid gas constant The Reynolds number is given by the expression:

$$
R_{d}=\frac{\rho U d}{\mu}
$$

where $\mu$ is the fluid viscosity. Combining equations (3.1), (3.2), (3.3),(3.4) we obtain the final expression for the aspirating probe voltage output in terms of compressible flow parameters: 


$$
\begin{gathered}
\frac{v^{2}}{T_{w}^{-}-r T_{t}}=\frac{\left(R_{s}+R_{w}\right)^{2}}{R_{w}} \pi \mid k\left\{a \left[\frac{p_{t}}{\sqrt{T}_{t}} \frac{d A^{*}}{\mid A_{c}}\left(\frac{\gamma}{R}\right)^{1 / 2}\right.\right. \\
\left.\left.\left(\frac{2}{\gamma+1}\right)^{\frac{\gamma+1}{2(\gamma-1)}}\right]^{m}+b\right\}\left(\frac{T_{m}}{T_{\infty}}\right)^{0.17}
\end{gathered}
$$

Equation (3.5) can be written as:

$$
\frac{v^{2}}{T_{w}-r T_{t}}=C\left(\frac{P_{t}}{\sqrt{T_{t}}}\right)^{m}+D
$$

where $C$ and $D$ are functions of the geometry of the probe and the fluid properties, which are also strong functions of the temperature and composition of the gas mixture that is being sampled, but weak functions of its pressure. If the composition of the gas mixture is known and constant, then, by operating the wires at different temperatures, two simultaneous voltage measurements are made from which two equations like (3.6) can be formed, with two unknowns, namely total pressure, $p_{t}$, and total temperature, $T_{t}$. This system of two equations can then be solved to determine $p_{t}$ and $T_{t}$. 


\subsection{2 - Time Lag Corrections in Probe Data}

Previous data reduction schemes for the aspirating probe signals from the hot wires and the pressure transducer did not account for any phase shift due to convective time delays. However, as it will be shown in this section such a correction must be applied to the probe signals and its implications are very important

The physical explanation behind this correction is the following. Since the flow through the probe is choked, the stream tube area is a function of Mach number and the ratio of specific heats, $\gamma$. For this series of tests, the free stream Mach number is approximately 0.6 while the Mach number at the wires' plane is only 0.2. Therefore the cross section of the captured streamtube is quite smaller than the actual cross section of the probe (see Fig. 3.3). In order to minimize flow angle dependence of the probe measurement, the lip of the probe inlet is rounded (region B in Fig. 3.3). Therefore the flow has to diffuse down to a low Mach number (plane $A-A$ in Fig. 3.3) and then accelerate again up to $M=0.2$ as it reaches the constant diameter region (region C in Fig. 3.3). However, the total pressure transducer, which is mounted on top of the aspirating probe (see Fig. 3.3), has a different geometry and different flowfield. The pressure fluctuations are transmitted, rather than convected, through the cavity that is formed between the lips of the total pressure probe and the diaphragm (region D in Fig. 3.3). Thus the time for a fluid perturbation to reach the hot wire sensors is determined by a much lower convective velocity than in the case of the pressure transducer, which is determined by the speed of sound.

The conclusion from all this is that the hot wire voltage signals must be time 
shifted relative to that from the pressure transducer before any data reduction procedure is started. In the following sections we will determine the amount of time shift required using a simplified analytical model, present an experimental verification of that model and then discuss the relative importance of this shift on the measurement of entropy.

\subsubsection{1 - Analytical Model of the Flow in the Probe}

Fig. 3.4 presents a somewhat simplified, in scale drawing of the probe's inlet (dimensions are in $\mathrm{mm}$ ). The first and simplest attempt to estimate the time lag, was to consider one dimensional flow through the probe and calculate how long it takes for a fluid particle to travel from plane A to the wires' plane (see Fig. 3.4). The location of the stagnation point on the lip of the probe inlet can be determined by replacing the inlet by a set of sources and sinks in a conventional potential flow manner (see Kuchemann [30] for details). However, such a detailed calculation would not be justified by the simplicity of the 1-D approach. Therefore two locations for the stagnation point were arbitrarily chosen (points A and $B$ in Fig. 3.4), in order to simply demonstrate the sensitivity of the 1-D calculation to the stagnation point location. The following table gives the results of this calculation for various gases at typical pressure and temperature conditions. 
Table 3.1: 1-D calculation of the time delay between the pressure transducer and the hot wires for various gases

\begin{tabular}{|c|c|c|c|c|}
\hline & & & \multicolumn{2}{|c|}{$\begin{array}{l}\text { Time delay in } \mu \text { sec for } \\
\text { the stagnation point at }\end{array}$} \\
\hline & & & A & B \\
\hline 1. $18 \%$ & Freon-12, $82 \%$ & Argon & 46.4 & 33.4 \\
\hline 2. $43 \%$ & Freon-12, $57 \%$ & Hel i um & 48.0 & 34.5 \\
\hline 3. & Hel i um & & 11.8 & 8.5 \\
\hline 4. & $\mathrm{CO}_{2}$ & & 42.5 & 30.6 \\
\hline 5. & Air & & 33.6 & 24.2 \\
\hline
\end{tabular}

For comparison, the blade passing period is $200 \mu \mathrm{sec}$. Therefore the delay can be a substantial fraction of the blade passage period. The first gas in the table is the main flow mixture used in the Blowdown Facility and the second is the tracer. Since the delay is a function of the gas composition, the concentration must in general be known in advance. This introduces a complication, since the probe is being used to measure concentration. Fortunately, the difference between the Helium-Freon 12 tracer gas mixture and the Argon-Freon 12 main flow mixture is very small, less than $5 \%$.

Although the previous calculation captures the basic idea of the delay and gives an estimate of its magnitude, it is rather crude and cannot be used to actually correct the data for the following reasons. The flow in the probe's channel is not one dimensional and the streamlines are probably strongly curved near the walls. Therefore fluid particles originating at different points of plane 
A-A (see Fig. 3.4) take different amounts of time to reach the plane of the wires. Therefore the voltage output of the wires is not anymore a true representation of the flow conditions in front of the probe, because some of the fluid particles that occupied that region have already passed by the wires, while some of them have not reached yet However, even if we know exactly the velocity field in the channel, it is still nontrivial to calculate exactly when each fluid particle will start affecting the heat transfer from the wires or equivalently, the voltage output from the anemometer circuit Although the previous simple 1-D calculation does not include any viscous effects, we believe that, except from some blockage, the error introduced is not important, since the copper plated ends of the wires isolate the sensing length, eliminating any wall boundary layer effects.

In order to verify the results of the simple 1-D model calculations, we decided to measure the time delay experimentally. Since the number of flow parameters that can influence the value of the time delay is quite large, we decided to design an experiment, where we could simulate, as closely as possible, the real Blowdown conditions, thus minimizing extrapolation errors. One experiment that fitted these specifications stemed from the ability of the probe to independently check the total pressure measurement This can be seen as follows. If the gas composition is known, the probe gives an extra signal that is not needed, when the goal is to measure total temperature and total pressure only. In particular, the total pressure can be first calculated using the signals from the two wires only, and then compared to the one measured by the total pressure probe. If our theory about the delay is correct, the total pressure calculated using only the two wires, must lead the total pressure directly measured by the total pressure probe by the amount of the delay. We felt that this comparison was the best way to measure the lag, since it involved no extrapolations or assumptions. 
The fact that the delay was first measured in a gas of $0 \%$ tracer concentration and then used to correct the data from a gas of different concentration, introduced only a negligible error, since, as mentioned before, the delay is a very weak function of the tracer concentration (in the case of Helium-Freon 12 tracer). Fig. 3.5 shows this comparison. The total pressure from the total pressure probe leads the one calculated using the two hot wires only, by $50 \mu s e c$, which is close to the $47 \mu \mathrm{sec}$ calculated under the 1-D flow assumption for the stagnation point at $A$ (see Table 3.1).

Recently, Watts, Ng and Kurosaka [31], published the results of a series of experiments, where they used a similar probe to measure total temperature and pressure in the vortex street behind a cylinder. In their paper they also compared the total pressure measured by the pressure transducer with the one inferred from the two hot wires and found no time delay. However, there is no contradiction here, because their aspirating probe has the same dimensions with ours, but the Mach number at the plane of the wires is 0.4 instead of 0.2 that we have. For this higher Mach number the 1-D calculation for air gave a delay of $11.6 \mu \mathrm{sec}$ for the stagnation point at B (see Fig. 3.4) and $16.3 \mu \mathrm{sec}$ for the stagnation point at $A$. Therefore the delay in their probe was at least half of the one that ours would have in a similar experiment In addition, their sampling rate was approximately $20 \mathrm{kHz}$, which is not enough to resolve such a small time delay. Note that even our AVD with a sampling rate of $100 \mathrm{kHz}$ would not have been able to pick it up. On the other hand, the phenomena they are trying to measure have a frequency of about $1 \mathrm{kHz}$ and therefore a delay of, even 50 $\mu \mathrm{sec}$ would probably make no difference in their data reduction scheme. In our case the blade passing frequency is as high as $5 \mathrm{kHz}$ and this delay is a substantial fraction, almost 1/4, of the blade passing period. 


\subsubsection{2 - Implications of the Time Lag in the Probe Signal}

The voltage signals from the two hot wires were shifted in time by an amount equal to the delay we measured from the total pressure comparison, which was found to be $50 \mu \mathrm{sec}$ or $0.050 \mathrm{msec}$. The total temperature and concentration were then calculated using the shifted voltage signals and the original total pressure signal from the total pressure probe. The next step was to calculate the change in the averaged total temperature, concentration, efficiency and entropy. The following table gives the results.

Iable 3.2: Changes in the results after correcting for the time delay

\begin{tabular}{rlr}
\hline & $\begin{array}{c}\text { Percent change from the value } \\
\text { before the shift }\end{array}$ \\
\cline { 2 - 2 } Total temperature ratio : & -0.03 \\
Concentration : & 3.14 \\
Entropy : & 1.56 \\
Adiabatic efficiency : & 0.60 \\
\hline
\end{tabular}

The change in temperature ratio is due to a temperature change of $0.2^{\circ} \mathrm{C}$. The change in concentration is the largest and in terms of mole fraction units it is about 0.0011. Notice that all the changes are smaller than the error of the measurement itself (see section 3.1.8). Thus the quantitative impact of the 
correction is minor.

There are important qualitative implications of the time shift, however. Before the correction was applied, the entropy peaks were observed to occur either in the middle of the blade passage or close to the suction side of the core flow. The entropy traces calculated from the shifted signals show that the majority of the peaks have moved into the viscous wakes, which are conventionally recognized as regions of high entropy. Fig. 3.6 shows a comparison of the shifted and original entropy traces at the tip, middle and hub regions.

The second implication is related to the phenomenon of negative entropy that has been observed by a number of researchers [1], [32], [6]. The same phenomenon was also observed in our measurements. In particular, we found that a large number of negative entropy regions occured close to the hub. Unfortunately, no satisfactory explanation of this phenomenon was ever given and the subject was still under investigation. Since these negative entropy regions appeared in both types of tests, "with injection" and "without injection", we decided to work with the one with the smallest uncertainty and number of variables, namely the "no injection" one. In this case, the total temperature and, therefore the entropy, can be calculated using the voltage from one wire and the total pressure signal from the total pressure probe. When the time shift correction was applied to the hot wire signal most of the negative entropy regions diminished substantially or disappeared. Fig. 3.7 shows a comparison of the two entropy traces, shifted and original. From this figure it is rather obvious that the entropy traces are modified in two ways, when the signals are corrected. First, the peak to peak magnitude is reduced and second, the negative entropy regions become smaller and fewer. 
A simple explanation of this finding is that the entropy change level is closer to zero in the hub region than it is in the tip region. Therefore, if the magnitude of the entropy fluctuations is reduced, while the mean remains the same, the negative regions will disappear or at least shrink. However, a more rigorous demonstration of this was sought.

The voltage signals from the aspirating and total pressure probe were simulated as $5 \mathrm{kHz}$ sine waves. Their amplitude and mean value were matched to the data In addition, the unshifted signals were phase shifted by $180^{\circ}$, such that the peaks of voltage would correspond to the troughs of total pressure. This was necessary, since the same pattern was observed in the data, where the negative entropy regions were associated with peaks of voltage and troughs of total pressure (see Fig. 3.8). Fig. 3.9a shows the two voltage inputs. The solid line is the unshifted signal and the dashed is the shifted one. Fig. 3.9b shows the sine wave for the pressure. These signals were input to the standard data reduction programs. The total temperature ratio, calculated from this, is shown in Fig. 3.9c, while the entropy is given in Fig. 3.9d. From these two figures it is clear that the amplitude of the total temperature and entropy signals diminishes, when the time shift correction is applied. In addition, the corrected entropy trace is "more positive" than the original one, or in other words, the negative entropy regions become smaller. Although this model can predict the behavior of the negative entropy regions, it is not intended to explain all the changes in the measured entropy traces. However, it shows that the reduction in the amplitude of the entropy traces is primarily due to the reduction in the amplitude of the total temperature signals. 
The latter can be explained as follows. When we shift the voltage signals in time, the peaks of voltage move away from the troughs of total pressure and into regions of higher pressure levels. With reference to the probe calibration curves, for the same voltage, higher pressure corresponds to higher total temperature (follow the arrows in Fig. 3.10). Therefore the troughs in the corrected (time shifted) total temperature trace are at a higher level than the ones in the original (not time shifted) trace (see Fig. 3.9d). A reverse argument applies for the peaks of the total temperature trace. In summary, the result of the time shift in the signals is a reduction of the amplitude of the fluctuations in the total temperature trace. Consequently, the entropy fluctuations are also reduced and the negative entropy regions are eliminated. However, it is important to notice that the simulation of the data as regular sine waves is good only for the hub region, where the absolute total pressure traces are quite periodic and the wakes can be identified as regions of low absolute total pressure. At the tip, the wakes can have absolute total pressure higher than the free stream, and the flowfield is much more complicated. Therefore, at the tip, the result of the time shift in the input voltage signals is only a time shift of the entropy traces and no substantial amplitude change can be observed (see Fig. 3.6). Finally, it should also be pointed out that the purpose of this model is not to predict the absolute change in the magnitude of the negative entropy spots in a quantitative manner, but rather to give a qualitative demonstration of the mechanism that explains the negative entropy regions as artifacts of the data reduction procedure. 


\section{2 - Description of the concentration measurement technique}

In section 3.1.1 we explained how in the case of a flow of constant or known concentration, the signals from the two wires would yield two unknowns, namely total pressure, $p_{t}$, and total temperature, $T_{t}$. In this case, one has the option to use or not the signal from the total pressure probe. The total pressure and total temperature can be determined using either the two hot wire signals, or one hot wire signal and the total pressure reading from the total pressure probe [1].

If the concentration of the tracer gas in the flow is not known, then an additional unknown quantity is introduced, namely the mole fraction, $\psi$, or equivalently, the mass fraction, $\xi$, of the tracer gas. As we mentioned in section 3.1.1, the voltage output of the hot wires is a function of the tracer concentration. This can be seen in eq. (3.5), where the right hand side includes quantities like the thermal conductivity, $k$, the viscosity, $\mu$, the gas constant, $R$, and the ratio of specific heats, $\gamma$. All these fluid properties are strong functions of the composition and temperature of the test fluid, and weak functions of its pressure. Therefore, the unknowns are now three, namely total pressure, total temperature and concentration of tracer gas. If all three signals, two from the hot wires and one from the total pressure probe, are used, then the measurement is essentially reduced to the solution of a system of three equations and three unknowns.

Equation (3.5) must be separately written for each hot wire. However, if the two wires were identical, then the two resulting equations would also be 
identical, and the system of three equations would become a system of two equations with still three unknowns, resulting in an undetermined problem. This leads to the conclusion that this system of equations is capable of giving a unique solution for temperature and concentration, only if the two equations from the hot wires are substantially different from each other. Consequently, the resolution of the measurement depends heavily on the degree of difference between these two equations and increases as this difference increases. Notice that the total pressure measurement does not have the same problem, since it is directly determined from the total pressure probe.

One way of making the wires different is to operate them at different temperatures. Unfortunately, as the wire temperature drops, both its frequency response and the signal to noise ratio drop to unacceptable levels. In addition, there is a $300^{\circ} \mathrm{C}$ upper limit to the wire temperature, set by the oxidization problems of the tungsten wire. Although, there is no air in the facility during the test, we were not able to quarantee no contact with air, during test preparations and calibrations. Therefore we decided not to increase the wire temperature over the oxidization limit

Different diameters could also make the wires different, but stability problems of the anemometers limited our choice of different diameters. Once the wire diameter was fixed, its length was directly determined, since the Length/Diameter (I/d) must be large (above 200) in order to avoid serious thermal end effects. Although we could use wires made of different material, like Tungsten or Platinum or an alloy of Platinum and Iridium, the fragility of both the Platinum and the Platinum-Iridium wires prevented us from using them. In addition, the low temperature coefficient of resistance of Platinum and 
Platinum-Iridium hot wires would decrease the already low signal to noise ratio of the experiment. Notice that the Platinum wires can operate at very high wire temperatures $\left(800^{\circ} \mathrm{C}\right)$. 


\section{3 - Tracer Gas Selection}

As it was mentioned in section 2.5, one of the major design goals of the tracer gas injector was to match the velocities of the injectant and main gas as closely as possible, in order to minimize the spreading of the injection jet. However, the selection of the right tracer gas is an equally important factor for the success of this experiment The following are the primary requirements and constraints that had to be satisfied by the selected tracer gas:

1) Buoyancy considerations are among the top in the list. The centrifugal acceleration experienced by the fluid during its trajectory through the fan can be as high as $10,000 \mathrm{~g}$. In such an environment small density differences can lead to significant and difficult to predict fluid motion that would be extremely hard to distinguish from motion due to three dimensional effects in the fan. Therefore matching the molecular weight of the tracer gas to the main flow one, was of primary importance.

2) The voltage output of the aspirating probe hot wires is directly proportional only to the thermal conductivity of the fluid, while all the other terms are raised to the $\mathrm{m}$ power, which is approximately equal to 0.3 for this set of experiments (eq. (3.5)). Therefore the sensitivity of the probe is primarily determined by the thermal conductivity of the fluid that is being sampled. Thus high sensitivity can be achieved if a tracer gas, much different than the main flow, with as high as possible thermal conductivity is used. It can be argued that this experiment is essentially an attempt to measure thermal conductivity with frequency response higher than $15 \mathrm{kHz}$. 
More details about the tracer gas choice can be found in [27] Carbon Dioxide $\left(\mathrm{CO}_{2}\right)$ was chosen initially, because its molecular weight (44) was close to that of the main gas one (54.5). In addition, $\mathrm{CO}_{2}$ has 1.16 times higher thermal conductivity than the main mixture of Argon and Freon-12 and is inexpensive and easily available. Although first estimates of the probe sensitivity looked promising, the level of concentration in the real test was lower than expected, making the detection of the $\mathrm{CO}_{2}$ difficult The major reason for this problem can be seen in Fig. 3.11, 3.12, 3.13. The information in these figures can be used in a number of ways. Each figure presents a calibration at fixed pressure (typical level of 400 $\mathrm{mmHg}$ ) for various tracer gases, $\mathrm{CO}_{2}$ (Fig. 3.11), Helium (Fig. 3.12) and a mixture of Helium-Freon 12 (Fig. 3.11). Each figure gives two curves of constant concentration, where the variable is the temperature, and two curves of constant temperature, where the variable is the concentration. We chose the levels to be 0.0 and $20.0 \%$ in mole fraction units for the concentration and 300 and $340 \% \mathrm{~K}$ for the temperature. For the constant temperature curves each symbol corresponds to $5 \%$ increment in mole fraction units and for the constant concentration ones the symbols correspond to $10^{\circ} \mathrm{K}$ increments. The symbols cover a range from 0.0 to $100.0 \%$ in concentration and from 200 to $400 \%$ in temperature.

A sensitivity comparison can be made from these plots by comparing the relative distance between symbols on a constant property curve. For example, since the symbols on the T=340\% of the Helium plot (Fig. 3.12) are further apart than the ones on the same curve for $\mathrm{CO}_{2}$ (Fig. 3.11), we conclude that the sensitivity of the probe to Helium is higher than the one to $\mathrm{CO}_{2}$. Note that the sensitivity to temperature does not change very much when we change the tracer gas. It is only the sensitivity to concentration that makes the difference in our choice. This sensitivity is expressed as a partial derivative of voltage, V, with 
respect to mole fraction, $\psi$, that is $\partial V / \partial \psi$. Although more details can be found in section 3.6, typical values for the probe sensitivity to these tracer gases for both low and high overheat ratio (OHR) wires are given in the following table.

Table 3.3: Probe sensitivity to various tracer gases

\begin{tabular}{lll}
\hline & $\frac{O H R}{2.0}$ & $\frac{\partial V}{\partial \psi}$ \\
\cline { 2 - 3 }$\infty_{2}$ & $\frac{1.7}{2.0}$ & 4.2 \\
\hline \multirow{2}{*}{ Hel ium } & 2.0 & 33.8 \\
\hline \multirow{2}{*}{ Hel ium-Freon 12} & 1.7 & 28.1 \\
\cline { 2 - 3 } & 2.0 & 18.4 \\
\hline
\end{tabular}

The sensitivity is expressed in $\mathrm{mV}$ per 0.01 change in mole fraction units.

Another way of looking at these curves is in terms of the shaded area shown. The smaller this area the higher the uncertainty of the concentration and temperature measurement becomes. This, of course, implies that the uncertainty in the voltage signals remains the same. According to the probe error analysis the major source of uncertainty in the voltage signals is the A/D resolution $(2 \mathrm{mV})$. It can be clearly observed from Fig. 3.11, 3.12, 3.13 that the shaded area in the case of Helium is 3.4 times larger than the one in the case of $\mathrm{CO}_{2}$ and 1.1 times larger than that of Helium-Freon 12. This indicates that the $\mathrm{CO}_{2}$ gives significantly less resolution than the two other mixtures. 
Although the calibration curves are shown together, they do not have the same chronological order. The idea to use a mixture of Helium and Freon 12 came much later than the $\mathrm{CO}_{2}$ and Helium ones. The Helium tests were conducted immediately after the $\mathrm{CO}_{2}$ ones. The difference in signal level and probe response were encouraging. However, the buoyancy issue causes questions.

Clearly the tracer gas must have both high thermal conductivity and molecular weight almost equal to the main gas. Since we were unable to find a single component gas that has these properties, we decided to use a binary mixture. Helium was chosen as one of the components, because of its high thermal conductivity. That limited our choices immediately to very heavy gases, such that the low molecular weight of Helium would be balanced by the molecular weight of the heavy component Fortunately, the thermal conductivity of a binary mixture is a nonlinear function of the thermal conductivities of the components, while the molecular weight is a linear one, and this was used to good advantage.

Since the analytical calculation of the thermal properties of a binary mixture using the thermal properties of the components is approximate and semi-empirical, any attempt to calculate the probe's response, using analytical predictions of the thermal conductivity and viscosity of the binary tracer gas mixture, would be crude and probably misleading. In addition, most of the semi-empirical formulas are valid only for monoatomic components, giving large uncertainties when they are applied to mixtures of polyatomic components (like Freon-12 and $\mathrm{CO}_{2}$ ) [33], [34] Therefore we decided to actually measure the response of the aspirating probe to various binary mixtures. This immediately raised the following practical issue. The supply tank of the Blowdown Facility was 
previously used for the steady state calibrations. There we could independently change the gas temperature by heating the tank with a steam jacket, the pressure in the tank by using the pump and the concentration of the tracer gas by using the partial pressures method. One of the problems during the probe calibration was the suction through the body of the probe, which lowered the pressure in any portable pre-filled tank. The advantage of the supply tank was that it was so large that the pressure did not change during the calibrations, while the temperature could also be fixed by adjusting the steam supply to the steam jacket The major disadvantage of this procedure was the large amount of gas needed for each calibration. Therefore it seemed impractical to use that tank, for experimenting with various tracer gases. For example, the cost for one calibration with a mixture of Helium and Xenon could be as high as $\$ 5,000$.

This led us to construct a new steady state calibration facility where the basic features of the old one would be preserved, but the amount of gas required would be as small as possible. In addition to all the above, calibration time was also a factor, since electronic drift problems mandated a quick and reliable probe calibration shortly before and after every Blowdown test The large amount of binary mixtures we could test limited the time we could spend for each potential candidate. Therefore we wanted the new facility to be as quick, inexpensive and reliable as possible.

Fig. 3.14 shows a schematic drawing of the calibration facility. According to the figure, tank $A$ is filled with main gas (Argon and Freon) and tracer mixture. The volume of the tank is small (approximately 9 It). A fan in the tank (part E in Fig. 3.14) mixes the gas for about 2 hours before the calibration. Part $C$ in the figure is a simple copper tube that acts as a heat exchanger. Since the gas from 
tank A expands during the calibration, it cools down and therefore in order to calibrate versus concentration or pressure only, the gas must be reheated and maintained at a certain temperature level. This is exactly the purpose of the simple heat exchanger. Continous sturing of the water and careful monitoring of the temperature kept it constant within $1^{\circ} \mathrm{F}$, during the calibration. Part $\mathrm{B}$ is a small chamber where the probe (part $F$ in the figure) is mounted.

The gases are introduced through a manifold (see Fig. 3.14) with multiple valves to allow flexibility among mixture components. After the gases are fully mixed, valve $D$ is opened and the pump attached to the aspirating probe is turned on. The gas mixture rushes through the coil and through the smaller plenum B into the aspirating probe. During this expansion the total pressure in both $A$ and $B$ drops and goes through a range of values that are the same with the real Blowdown test The temperature in tank $B$ is monitored and is constant within $2^{\circ} \mathrm{F}$. In addition the total pressure of the gas at point $B$ is also measured. It is actually important to be able to measure the total pressure at plenum B and not just at plenum $A$, because of the total pressure losses that occur through the long heat exchanger coil. The total pressure probe mounted on the aspirating probe was used to measure the total pressure in B. The facility can also be used to independently calibrate the pressure transducer, by closing valve G (see Fig. 3.14) and changing the pressure in the whole facility, using of course an inexpensive gas, like Argon. Two sensitive pressure gauges are used to monitor the pressure in plenums $A$ and $B$. Since the calibration is rather fast (total time of about $50 \mathrm{sec}$ a CAMAC AVD converter is used with a sampling rate of $20 \mathrm{~Hz}$ to digitize and record the signals from the aspirating probe and the companion total pressure probe. This is the same A/D converter that is used for the Blowdown test, where the sampling rate changes from $5 \mathrm{kHz}$ to $100 \mathrm{kHz}$ during the test 
The construction and reliable operation of the new calibration facility eliminated the high cost and extremely tedious calibration procedure of the old one. Since testing the response of the probe was quick and inexpensive, we decided to base our selection of potential tracer gases only on the requirement that the molecular weight of the mixture should be equal to 54.5 (the molecular weight of the main mixture of Argon and Freon-12) and determine the probe sensitivity by testing it One of the heaviest gases available was Xenon (MW=131.3). The mole fraction of Xenon in the Helium-Xenon mixture that gave us the right molecular weight was $39.7 \%$. The results of the calibration are shown in Fig. 3.15. It is rather clear that the probe is much more sensitive to Helium and Xenon. Although these results were quite promising, the cost of Xenon was still quite high. Therefore we attempted to use other heavy gases, but all of them gave us responses similar to the $\mathrm{CO}_{2}$ ones. However, one of these gases (Freon-12 with molecular weight of 120.93) gave interesting results. Fig. 3.15 shows a comparison of the probe's response to this mixture with the response to previous ones. The Helium-Freon 12 mixture had the highest sensitivity than any of the other mixtures, even higher than the expensive Helium-Xenon one. The mole fraction of Freon-12 in the mixture that gave the right mixture molecular weight was $43 \%$. Given all the above we finally chose the mixture of $43 \%$ Freon-12 and $57 \%$ Helium to be our tracer gas. 


\section{4 - Static Calibration of the Aspirating Probe}

Details about the calibration procedure of the aspirating probe can also be found in [27]. The supply tank of the Blowdown Facility was used as a stagnation plenum. A steam jacket around the facility allowed us to increase the temperature up to $100^{\circ} \mathrm{C}\left(212^{\circ} \mathrm{F}\right)$ and keep it constant at desired levels by adjusting the steam supply. Various gases could be introduced in the tank and the concentration of these mixtures could be determined by the partial pressures law.

Equation (3.7) is the major equation used in the calibration procedure.

$$
\frac{v^{2}}{T_{w}-T_{t}}=C\left(\frac{P_{t}}{\sqrt{T_{t}}}\right)^{m}+D
$$

In this equation $C, D$ and $m$ are determined from the calibration data, by a simple curve fit Equations (3.8) and (3.9) give the full expression for C and D:

$$
\begin{aligned}
& C=A k\left(\frac{T_{m}}{T_{\infty}}\right)^{0.17}\left\{\frac{1}{\mu} \sqrt{\frac{/ \gamma}{R}}\left(\frac{2}{\gamma+1}\right)^{\frac{\gamma+1}{2(\gamma-1)}}\right\}^{m} \\
& D=B k\left(\frac{T_{m}}{T_{\infty}}\right)^{0.17}
\end{aligned}
$$

where $A$ and $B$ are given by the following expressions:

$$
\begin{aligned}
& A=a \pi l \frac{\left(R_{s}+R_{w}\right)^{2}}{R_{w}} \\
& B=b \pi I \frac{\left(R_{s}+R_{w}\right)^{2}}{R_{w}}
\end{aligned}
$$


where $a, b$ are the coefficients from the Collis and Williams expression, eq. (3.2).

In order to be able to convert the raw voltage data into meaningful quantities, like pressure, temperature and concentration, we need to know the behavior of the probe as these quantities change independently. This can be achieved either, by calibrating for all possible combinations of pressure, temperature and concentration, or, by finding a functional form, like the Collis and Williams expression, which contains certain parameters that can be determined from only a few calibrations and remain constant as the conditions change.

Our first attempt towards a reliable calibration scheme was to vary the pressure keeping the temperature and concentration of the gas at fixed reference levels (room temperature and $0 \%$ concentration of tracer). The results were curve fit and certain values for the coefficients $C, D$ and $m$ (see eq. (3.7)) were

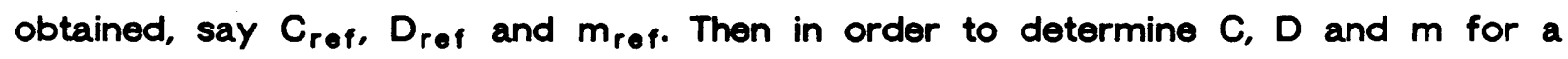
different set of temperature and concentration, a correction must be applied, because these coefficients are also functions of both temperature and concentration (also negligibly weak functions of pressure) (see eq. (3.8),(3.9)). Since the thermal properties of the gas mixture appear in both eq. (3.8) and (3.9), we must be able to predict them for various combinations of temperature and tracer concentration levels.

The specific heat or the specific enthalpy of a mixture of a number of non-reacting, perfect gases is a simple weighted average of the corresponding properties of its components. However, this is not true for the thermal conductivity and viscosity of the mixture. The reason is that theses properties are functions of the mean free path of the molecules. In a mixture of gases, the 
mean free path of the molecules of each component changes as a result of the presence of molecules of the other gases. The complexity of the problem led researchers to develop semi-empirical formulas for the calculation of thermal conductivity and viscosity. Since the values of these properties for mixtures are needed for many practical applications, a great number of publications, semi-empirical formulas and measurements of these properties can be found in the literature.

Binary mixtures of monoatomic gases are the most benign situations. Sutherland [35] derived a formula for the viscosity of binary mixtures, based on kinetic theory arguments, that is:

$$
\mu_{m}=\frac{\mu_{1}}{1+A_{1} \frac{\psi_{2}}{\psi_{1}}}+\frac{\mu_{2}}{1+A_{2} \frac{\psi_{1}}{\psi_{2}}}
$$

where $\mu_{m}, \mu_{1}, \mu_{2}$ are the viscosities of the mixture, component 1 and 2 respectively, $\psi_{1}, \psi_{2}$ are the mole fractions of the components and $A_{1}, A_{2}$ are coefficients that are complicated functions (see [35]) of the molecular weights of the components and the diameters of their molecules. Many authors [37], [38], [39] have proposed different types of equations for determining the viscosity of binary mixtures and also some modifications of (3.12). Wilke [36] proposed a modification of (3.12) which simplified the expressions for $A_{1}, A_{2}$ and is given by the following equations: 


$$
\mu_{m}=\frac{\mu_{1}}{1+\phi_{12} \frac{\psi_{2}}{\psi_{1}}}+\frac{\mu_{2}}{1+\phi_{21} \frac{\psi_{1}}{\psi_{2}}}
$$

where $\phi_{12}, \phi_{21}$ are coefficients that are now relatively simple functions of the molecular weights and the viscosities of the individual components, and are given by the following equations:

$$
\begin{aligned}
& \phi_{12}=\frac{\left[1+\left(\frac{\mu_{1}}{\mu_{2}}\right)^{1 / 2}\left(\frac{M_{2}}{M_{1}}\right)^{1 / 4}\right]^{2}}{\left(8+8 \frac{M_{1}}{M_{2}}\right)^{1 / 2}} \\
& \phi_{21}=\frac{\left[1+\left(\frac{\mu_{2}}{\mu_{1}}\right)^{1 / 2}\left(\frac{M_{1}}{M_{2}}\right)^{1 / 4}\right]^{2}}{\left(8+8 \frac{M_{2}}{M_{1}}\right)^{1 / 2}}
\end{aligned}
$$

where $M_{1}, M_{2}$ are the molecular weights of the components. The same formulas can be extended to multicomponent mixtures and the formulas for a n-component mixture are: 


$$
\begin{aligned}
& \mu_{m}=\sum_{i=1}^{E_{1}} \frac{\mu_{i}}{1+\frac{1}{\psi_{i}} \sum_{\{\neq j}^{\sum_{j} \psi_{j} \phi_{i j}}} \\
& \phi_{i j}=\frac{\left[1+\left(\frac{\mu_{i}}{\mu_{j}}\right)^{1 / 2}\left(\frac{M_{j}}{M_{i}}\right)^{1 / 4}\right]^{2}}{\left(8+8 \frac{M_{i}}{M_{j}}\right)^{1 / 2}}
\end{aligned}
$$

The same arguments apply for the thermal conductivity. Wassiljewa [37] derived an equation similar to (3.12) with conductivity replacing the viscosity, that is:

$$
k_{m}=\frac{k_{1}}{1+B_{1} \frac{\psi_{2}}{\psi_{1}}}+\frac{k_{2}}{1+B_{2} \frac{\psi_{1}}{\psi_{2}}}
$$

where again $B_{1}, B_{2}$ are complicated functions of the molecular weights and the molecular diameters of the individual components. Since then, most of the research on the calculation of the viscosity of gas mixtures [38], [40], [41], [42], [43], [44], [45], [46] has been focusing on deriving simpler formulas for the coefficients $B_{1}$ and $B_{2}$. Following [38], for a $n$-component mixture an expression identical to (3.16) and (3.17) was chosen and is given by the following equations: 


$$
\begin{aligned}
& k_{m}=\sum_{i=1}^{\sum_{1}} \frac{k_{i}}{1+\frac{1}{\psi_{i}} \sum_{i j j_{j} \sigma_{i j}}^{k_{j}}} \\
& \sigma_{i j}=\frac{\left[1+\left(\frac{k_{i}}{k_{j}}\right)^{1 / 2}\left(\frac{M_{j}}{M_{i}}\right)^{1 / 4}\right]^{2}}{\left(8+8 \frac{M_{i}}{M_{j}}\right)^{1 / 2}}
\end{aligned}
$$

where the notation is the same as before.

These expressions were used to calculate the thermal properties of the test gas at various temperature and tracer concentration levels. 
For the calculation of the thermal properties of the individual components (like Freon 12, Argon or Helium), we used the formulas given in [39], [46], [47]. As we mentioned before, the thermal conductivity and viscosity are weak functions of pressure and, since the pressure in our experiments does not change very much, we neglected its effect (a maximum error of $0.01 \%$ or $0.4 \mathrm{mV}$ ). However, the pressure effect was included in the calculation of the constant pressure specific heat An important point is that the thermal properties of the mixture being sampled, were calculated at the "film temperature", $T_{m}$. However, in order to calculate $T_{m}$, the value of the "free stream" static temperature $T_{\infty}$ must be known. Given the "free stream" total temperature of the flow (it is taken to be equal to the total temperature outside the probe), $T_{\infty}$ can be calculated if the ratio of specific heats $\gamma$ is known. But $\gamma$ must be calculated at the "film temperature", just like all the other thermal properties. Therefore, an iterative scheme was involved every time there was a need to calculate either the thermal properties of the mixture or the voltage output from the probe or both.

The following equations give the form of the corrections we applied to the coefficients $C$ and $D$, to account for changes in the temperature and concentration of the mixture: 


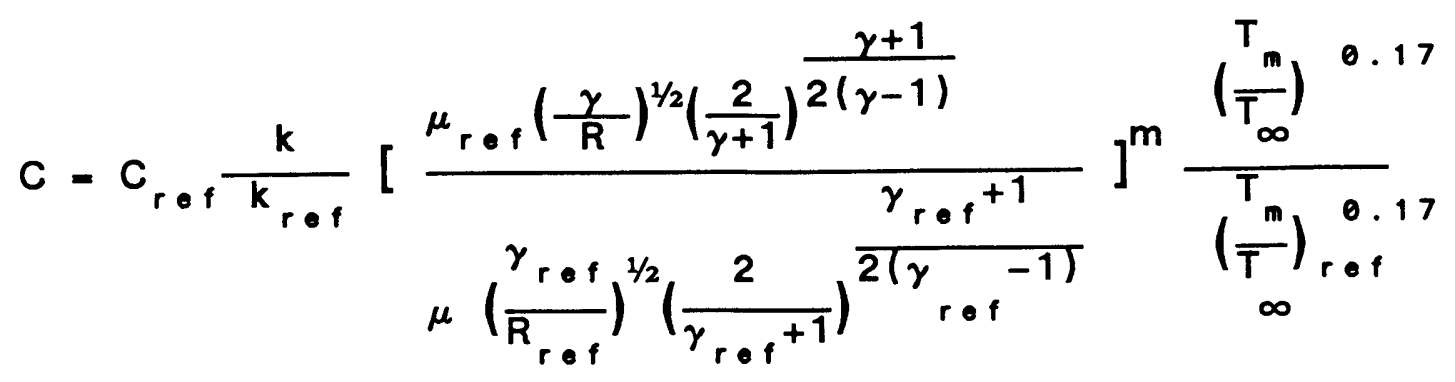

$D=D_{r \in f} \frac{k}{k_{r \in f}} \frac{\left(\frac{T_{m}}{T_{\infty}}\right)^{0.17}}{\left(\frac{T_{m}}{T_{\infty}}\right)_{r \bullet 1}^{0.17}}$

where the subscript "ref" denotes reference conditions of $0 \%$ concentration and room temperature. $C_{\text {ref }}$ and $D_{\text {rof }}$ were obtained by curve fitting the data at the reference conditions. These equations were derived applying equations (3.8) and (3.9) for the reference and the test conditions. Hidden behind these corrections is the assumption that the coefficients $a, b$ and the exponent $m$ from the Collis and Williams expression remain constant for all values of temperature, pressure and concentration. The last assumption simplified things considerably, since only one calibration versus pressure at reference conditions was required in order to determine their value. The predictive ability of this method was exhaustively tested for the case of $\mathrm{CO}_{2}$ as tracer gas (see Fig. 3.3, 3.4, 3.5 in [27]), by comparing the measured voltage output from the probe with the prediction, based on the constants from the reference calibration. The maximum error was less than the resolution of the analog-to-digital converter (2 $\mathrm{mV})$. 
However, this assumption was found to be invalid in the case of Helium-Freon 12 tracer mixture and considerable variation of the coefficients a and $b$ was observed. In order to keep things as simple as possible, we decided to keep the exponent $m$ constant and try to resolve the problem using $a$ and $b$ as variables. A great amount of effort was spent to modify these coefficients such that their variation with temperature and concentration would be predictable. As it turned out, we could predict them, within the experimental error, for various conditions of temperature and concentration, by taking into account only the changes in concentration. In other words, they seemed to be very weak functions of temperature, but strong ones of concentration. The simplest function we could find that gave us satisfactory results, was a quadratic, that is:

$$
\begin{aligned}
& \frac{a}{a_{r \in f}}=Z_{1} \psi^{2}+Z_{2} \psi+1 \\
& \frac{b}{b_{r \in f}}=Y_{1} \psi^{2}+Y_{2} \psi+1
\end{aligned}
$$

where $\psi$ is the mole fraction of the tracer gas and $Z_{1}, Z_{2}, Y_{1}$ and $Y_{2}$ are constants that depend on the type of tracer gas we used. $Z_{1}, Z_{2}, Y_{1}$ and $Y_{2}$ are determined through calibration at two different concentration levels (reference conditions not included).

Since the anemometer's output is pure voltage, the determination of the coefficients $C$ and $D$ in equation (3.7) depends on the value of the wire temperature, $T_{w}$ Under the constant temperature mode of operation, the hot wire anemometer keeps this temperature constant during both calibration and test. Equation (3.25) gives the relationship between the specified overheat ratio (OHR) 
and the wire temperature:

$$
O H R=\frac{R_{h}}{R_{c}}=1+\alpha\left(T_{w}-T_{\bullet}\right)
$$

where $R_{h}$ is the operating (hot) resistance of the sensor, $R_{c}$ is the resistance of the sensor at the reference (cold) temperature, usually environmental temperature, OHR is the overheat ratio, $T_{w}$ is the sensor operating temperature, $T_{e}$ is the environmental reference (cold) temperature and $\alpha$ is the temperature coefficient of resistance of the material. For Tungsten hot wires a typical value for $\alpha$ is $0.0042 /{ }^{\circ} \mathrm{C}$. The coefficient $\alpha$ is given only for the cold resistance $R_{c}$ at the

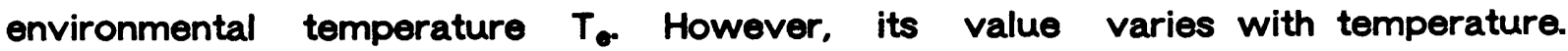
Therefore, in order to calculate the wire operating temperature using eq. (3.25), an actual curve of resistance versus temperature must be available. In other words, if we use equation (3.25) to calculate the wire temperature, we essentially assume a linear relation between temperature change and resistance change, which is not true.

If the fluid temperature were the same during both calibration and test, then the actual value of the wire temperature would not be needed, since by design the anemometer would keep it constant However, in our case the temperature of the fluid is unknown. In fact, it is one of the quantities that we want to measure. But even in this case, if we could calibrate for all possible temperature, pressure and tracer concentration combinations that might appear during the test, then again the actual value of the wire temperature would not be needed. Unfortunately, this calibration scheme would be extremely tedious and impractical. In fact, the only reason for all the predictive schemes developed for the coefficients C and D (see eq. (3.21), (3.22), (3.23), (3.24)), was to avoid this time 
consuming calibration procedure.

The first step to resolve this problem was to see if eq. (3.25) gave the right value for the wire temperature. Through extensive calibrations at a large number of temperature, pressure and tracer concentration levels, we found that the value of the wire temperature, as given by equation (3.25), was not correct As we mentioned before, this was a result of the nonlinear variation of the temperature coefficient of resistance, $\alpha$, with temperature. Therefore, one more complication was added to the calibration procedure, in order to determine the actual wire temperature. A number of combinations of temperature, pressure and tracer concentration levels were chosen and the voltage output from the probe was recorded for each one of them. For convenience, these combinations will be called "random conditions". Typically 20 such combinations per calibration were chosen, resulting in 20 data points. Using an initial guess for the value of the wire temperature, the coefficients $C_{\text {ref }}$ and $D_{\text {ref }}$ were determined from a basic calibration at reference conditions. These coefficients were then corrected using equations (3.21) and (3.22), for each one of the "random conditions" and the corresponding probe voltage outputs were predicted and compared with the measured ones. This process was repeated for a wide range of values of the wire temperature and the final choice for the wire temperature was the one that gave the minimum deviation between predicted and measured voltage outputs. This procedure is described in a schematic form in the following computer flow chart 


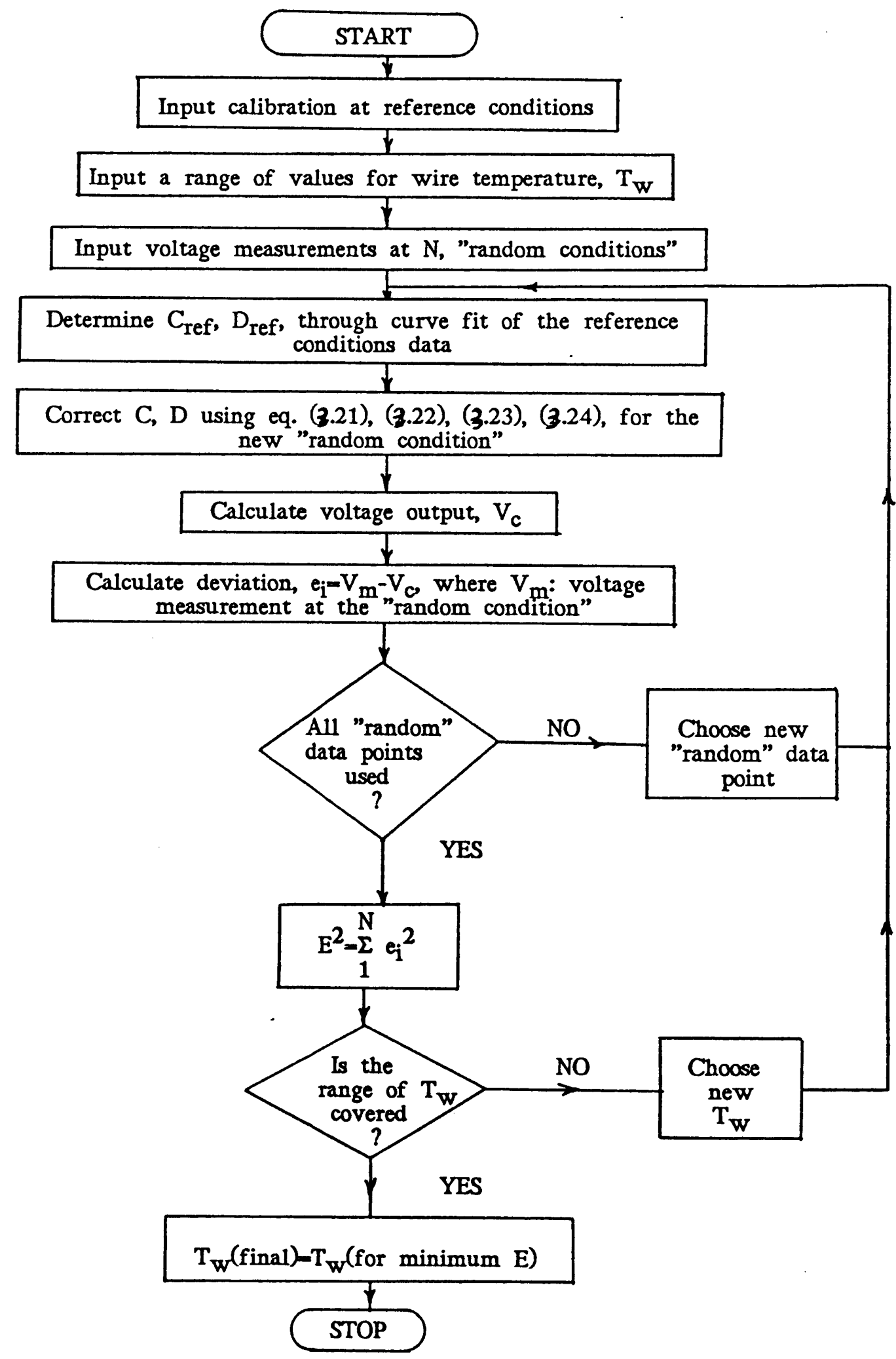


As it turned out, the final value for the wire temperature gave a maximum deviation of $2 \mathrm{mV}$ for all the "random conditions" that we chose. It should be pointed out that, if we had used equation (3.25) to determine $T_{w}$, we would have made an error of about $30^{\circ} \mathrm{C}$ in the value of the wire temperature for each wire, giving a $30 \mathrm{mV}$ error in voltage prediction. Such an error in voltage could lead to a $3 \%$ error in temperature or a $10 \%$ error in concentration measurement (in the case of Helium-Freon 12).

In summary, the following calibration procedure was followed immediately before and after each Blowdown test First, we calibrated at the reference conditions (room temperature, $0 \%$ concentration). This calibration was also used as a quick indicator of possible substantial malfunction of the wires. Then we calibrated at constant temperature (like room temperature) but at two different concentration levels, as close as possible to typical test levels, like at $15 \%$ and $30 \%$ in mole fraction. Finally, we picked approximately 20 "random conditions" for pressure, temperature and concentration and measured the voltage output from the probe. The first step gave us the coefficients $C_{\text {ref, }} D_{\text {ref, }} a_{\text {ref }}$ and $b_{\text {ref. The }}$ second determined the $Z_{1}, Z_{2}, Y_{1}$ and $Y_{2}$ coefficients of equations (3.23) and (3.24). The final step gave us the exact value of the wire temperature $T_{w}$

Typical values for some of these parameters are given in the following Table: 
Table 3.4: Calibration coefficients

\begin{tabular}{|c|c|c|c|}
\hline \multirow{3}{*}{ OHR } & \multirow[b]{2}{*}{ wire 1} & \multirow{2}{*}{$\frac{\text { Helium }+ \text { Freon } 12}{2.0}$} & \multirow{2}{*}{$\frac{\mathrm{Hel} \text { ium }}{2.0}$} \\
\hline & & & \\
\hline & wire 2 & 1.7 & 1.7 \\
\hline \multirow{2}{*}{$T_{w}\left({ }^{O_{K}}\right)$} & wire 1 & 558 & 558 \\
\hline & wire 2 & 490 & 490 \\
\hline \multirow{2}{*}{$C_{r e f}$} & wire 1 & 0.004897 & 0.004897 \\
\hline & wire 2 & 0.008743 & 0.008743 \\
\hline \multirow{2}{*}{$D_{r e f}$} & wire 1 & -0.013419 & -0.013419 \\
\hline & wire 2 & -0.021681 & -0.021681 \\
\hline \multirow{2}{*}{$m$} & wi re 1 & 0.318 & 0.318 \\
\hline & wi re 2 & 0.285 & 0.285 \\
\hline \multirow{2}{*}{$z_{1}$} & wire 1 & -0.70 & -0.73 \\
\hline & wire 2 & -0.29 & -0.68 \\
\hline \multirow{2}{*}{$z_{2}$} & wire 1 & 0.96 & 1.65 \\
\hline & wire 2 & 0.91 & 1.59 \\
\hline \multirow{2}{*}{$Y_{1}$} & wire 1 & 0.67 & -1.37 \\
\hline & wire 2 & 1.69 & -0.95 \\
\hline \multirow{2}{*}{$Y_{2}$} & wire 1 & 0.51 & 3.20 \\
\hline & wire 2 & 0.39 & 2.61 \\
\hline
\end{tabular}

This calibration scheme was repeatedly tested and Fig. 3.16 and 3.17 present a very small representative part of the results. Fig. 3.16 is for the final choice of 
tracer gas (43\% Freon-12, 57\% Helium) and Fig. 3.17 is for pure Helium. The circles represent calibration at reference conditions (room temperature, $0 \%$ concentration) and the line through them is a curve fit that gave us $C_{r e f}, D_{\text {ref }}$ and $m_{\text {ref. }}$ The triangles represent data points at $140^{\circ} \mathrm{F}\left(60^{\circ} \mathrm{C}\right)$ and $10 \%$ concentration of the tracer gas and the crosses data points at $104^{\circ} \mathrm{F}\left(40^{\circ} \mathrm{C}\right)$ and $20 \%$ concentration. The solid lines through the crosses and triangles are predicted using all the constants from the previously described calibration procedure. The total temperature behind the rotor in a Blowdown test varies from approximately $68^{\circ} \mathrm{F}\left(20^{\circ} \mathrm{C}\right)$ to $176^{\circ} \mathrm{F}\left(80^{\circ} \mathrm{C}\right)$, the concentration of tracer from $0 \%$ to $30 \%$ and the total pressure from approximately 0.4 to 0.65 atms. Therefore the conditions, chosen in Fig. 3.16 and 3.17 to validate the calibration procedure, can be considered representative of the ones in a typical test. For the data in Fig. 3.16 and 3.17, the maximum error in the prediction occurs away from the test window (0.4-0.65 atms) and is equal to $8 \mathrm{mV}$. In the useful region the error is about 2-3 mV, almost equal to the $A / D$ resolution of $2 \mathrm{mV}$. In terms of the final output of the measurement, namely concentration (mole fraction) and temperature, this calibration error leads to errors of about $10 \%$ in concentration and $0.30 \%$ in temperature for Helium-Freon 12 as tracer. The same errors for the case of Helium tracer gas are $1.5 \%$ in concentration and $0.32 \%$ in temperature. 


\section{5 - Data Reduction Scheme}

Based on the calibration procedure described above, the following data reduction scheme was constructed. The three signals, two from the hot wires and one from the total pressure probe, mounted on the aspirating probe, were input to a computer code. Additional inputs were the coefficients $C_{\text {ref, }} D_{\text {ref }}$ and $m_{\text {ref }}$, the wire temperature $T_{w}$ and the coefficients $Z_{1}, Z_{2}, Y_{1}, Y_{2}$. The total pressure was directly calculated from the reading of the total pressure probe. Therefore a system of two equations with two unknowns, temperature and concentration, was left to be solved. A simple Newton-Raphson scheme was incorporated to solve this system. It should be pointed out that, for each data point that was input to the code, a number of additional iterations were involved in order to calculate the fluid thermal properties. This was necessary, because their values are functions of static and not total temperature and pressure, and these static quantities cannot be calculated unless the ratio of specific heats, $\gamma$, is known, which in turn requires knowledge of the temperature and concentration of the fluid. Notice that the Mach number was constant and equal to 0.2 at the plane of the hot wires, since the flow through the probe orifice was choked. The following flow chart gives a description of the steps that the data reduction scheme goes through in order to calculate temperature, concentration and pressure from the three voltage inputs. 


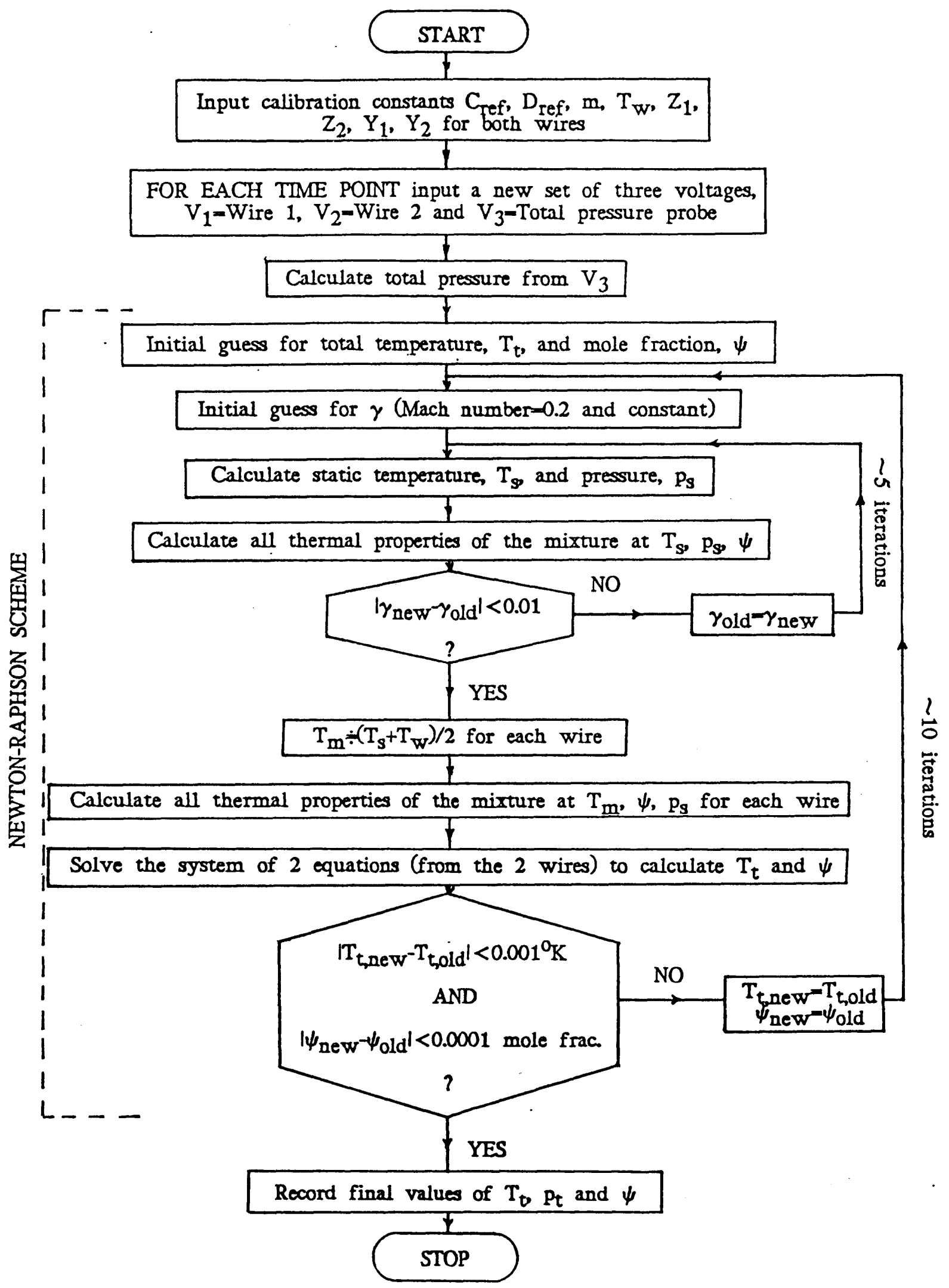




\section{6 - Dynamic Calibration - Shock Tube Tests}

In section 3.4 we talked about the static calibration of the probe. However, a complete calibration scheme should always include a dynamic calibration in addition to the static one.

A dynamic calibration is commonly used to determine the frequency response of a probe or a transducer in general. The dominant frequency in the probe measurements was the rotor blade passing frequency $(5 \mathrm{kHz})$. Since this frequency was quite high, it was very important to prove that the frequency response of the probe was substantially higher that the input dominant frequency.

In addition, this dynamic calibration could also serve as an excellent test of the data reduction scheme. As it was explained in the previous sections, the conversion of the three raw voltage inputs from the two hot wires and the total pressure probe into temperature, concentration and pressure, was based on a semi-empirical model. It was therefore felt that this model should be exhaustively tested under as many different test conditions as possible, in order to prove its validity. As it will be described in this section, the dynamic calibration gave us this opportunity.

A shock tube experiment would satisfy all the goals we set above. Fig. 3.18 shows a schematic drawing of the shock tube facility. Details about the construction and operation of the facility can be found in [48], while a short description and some modifications will be given here. The facility consists of two sections. The one on the left of Fig. 3.18 is the low pressure side ("driven section") and the other one is the high pressure side ("driver section"). A 
diaphragm, made of Cellophane (DuPont MSD-60, 220 gauge, 22.8 $\mu \mathrm{m}$ thick), separates the two sides, while the high pressure one is being charged. For simplicity no breaking mechanism is used. The pressure ratio across the diaphragm right before bursting was approximately equal to 1.5. It was also observed that the pressure difference across the diaphragm during bursting was repeatable within 0.2 psi.

With reference to Fig. 3.18, the probe was mounted between two flush mounted high frequency response Kulite pressure transducers. The Camac A/D system, used during the Blowdown tests, was also used here to digitize the signals. Occasional damage of the probe's hot wires was also observed, due to release of particles from the Cellophane diaphragm upon bursting.

As the shock propagates into the driven section, it increases the pressure of the gas behind it and induces a mass motion with certain velocity $u_{c}$. The interface between the driver and driven gases is called the contact surface, which also moves with velocity $u_{c}$. This contact surface is somewhat like the conventional slip lines during shock intersection phenomena, since the entropy changes discontinuously across it However, the pressure and velocity are continuous [49]

The characteristics of the travelling shock and the contact surface can be fully determined, if the initial conditions in the driven and driver sections are known [49]. On the other hand, if the initial pressure difference is not known, one can calculate the same characteristics using a measurement of the shock speed. If. both parameters are measured, then a comparison can be made to validate the calculations. In our case, it was felt that a direct measurement of the shock 
speed was needed, since the lack of a diaphragm breaking mechanism caused uncertainty in the measurement of the initial pressure difference between driven and driver section. The shock speed was measured by the two flush mounted transducers, located as Fig. 3.18 shows. In addition, the first pressure transducer (transducer $A$ in Fig. 3.18) was used to trigger the Camac A/D. Therefore, every time information is referenced to the trigger time of transducer $A$. The shock formation process immediately downstream from the diaphragm is clearly a three dimensional, quite complicated one and we felt that it would be unnecessary to include it in our measurements.

The sequence of events during a shock tube test can now be clearly seen in Fig. 3.19 and 3.20 , as points $A, B, C$ and $D$. The first figure presents the response of the total pressure probe and the second the response of one of the hot wires on the aspirating probe. With reference to Fig. 3.19, the first normal shock hits the probe at approximately $1 \mathrm{msec}$ after transducer $A$ is triggered. Associated with this shock, is a step change in total temperature and total pressure, but no change in concentration, since the contact surface has not reached the probe yet. At approximately 7.5 msec the contact surface hits the probe (point B in Fig. 3.19 and 3.20) and brings with it changes in total temperature, total pressure and concentration. The total temperature drops, because the driver side expands, the concentration increases, because the tracer gas reaches the probe, and the total pressure shows a small change, because of the different speed of sound in the driven and driver gases. Notice the dramatic increase in the wire voltage output at point B (contact surface), which is a demonstration of the high sensitivity of the probe to Helium. At approximately 13.5 msec (point C in Fig. 3.19 and 3.20) the reflected normal shock reaches the probe and then expansion waves (point D in Fig. 3.19 and 3.20) mark the end of the useful test time. Extra care was taken, 
in terms of probe location and initial conditions in the shock tube, to make sure that the contact surface reaches the probe before the reflected shock and the expansion waves. Otherwise the outcome of the interaction of the moving reflected shock or the expansion waves with the contact surface would have been impossible to evaluate with satisfactory accuracy. The following table presents some of the shock tube conditions during a typical dynamic calibration, like the one presented in Fig. 3.19.

Table 3.5: Shock tube conditions

Driver section gamma (Helium)

Driven section gamma (Argon-Freon 12)

Initial temperature in both sections $\left({ }^{\circ} \mathrm{K}\right)$

Diaphragm pressure ratio

Shock total pressure ratio

Shock static pressure ratio

Shock velocity (m/sec)

Reflected shock velocity $(\mathrm{m} / \mathrm{sec})$

Contact surface velocity $(\mathrm{m} / \mathrm{sec})$
$=1.66$

$=1.38$

$=300$

$-2.07$

$=1.91$

$=1.72$

$=324.35$

$=238.33$

$=106.20$

Fig. 3.21 presents a wave diagram for the shock tube that corresponds to the conditions of Table 3.5. The locations of the two pressure transducers and the aspirating probe are indicated. The time points $A, B$ and $C$ from Fig. 3.19 and 3.20 are also shown in the wave diagram. Notice that the contact surface (point B) reaches the probe location before the reflected shock (point C). The $x$-axis is non-dimensionalized with the total length of the driven section of the shock tube.

From all the above it is rather clear that during a shock tube test, there are instants, like points $A$ and $B$ in Fig. 3.19 and 3.20, where the total temperature, 
total pressure and concentration change in a pre-described manner. In particular at point $A$ the concentration is zero, while the total pressure and total temperature increase, while at point $B$ the concentration increases, the total temperature drops and the total pressure experiences a rather small drop. Notice that for the set of tests presented here the driver gas was pure Helium and the driven one Argon-Freon 12 mixture. Since one of the goals of these tests was to validate the model used in the data reduction scheme, the choice of tracer gas (gas in the driver section) was immaterial. Therefore we decided to use the one that gives the highest probe sensitivity, in order to be able to clearly distinguish the location of the contact surface.

The results of these tests were fed into the data reduction computer code and the total temperature and concentration were calculated. Fig. 3.22 presents the results at points $A$ and $B$. As it can be seen, at point $A$ there is a small jump in the concentration measurement, while in reality there was no tracer gas present, but it is small enough to be neglected. At point $B$ the concentration and total temperature move in opposite directions, which is exactly what happens in reality. We believe that these results clearly resolve the question of concentration and total temperature coupling and prove that the "apparent coupling" between these two quantities is not an artifact of the probe or the measurement in general.

In addition to all the above, the shock tube experiments can be used to measure the frequency response of the probe, which is basically determined by the response of the cavity in front of the choked orifice (see Fig. 3.1 or 3.3). Since the blade passing frequency in our experiments is $5 \mathrm{kHz}$, the probe must have a frequency response well above that, in order to resolve any blade to blade 
flow features. According to [48], the probe can be modelled as a constant area duct with mass flow (see Fig. 3.23) and its damped natural frequency, $f_{d}$, is given in $\mathrm{Hz}$ by Whitehead [50], as:

$$
f_{d}=\frac{(2 m-1)\left(1-M^{2}\right) \alpha}{4 L}
$$

where $m$ is an integer value defining the frequency mode shape, $M$ is the Mach number of the mean flow, $\alpha$ is the speed of sound for the particular gas and $L$ is the length of the duct In addition, the logarithmic decrement of the duct, $\delta$ is given by Whitehead [50], as:

$$
\delta \frac{(2 m-1)}{2}=\ln \frac{(1+M)\left(1+\frac{\gamma-1}{2} M\right)}{(1-M)\left(1-\frac{\gamma-1}{2} M\right)}
$$

where $\gamma$ is the ratio of specific heats. Once the damped natural frequency $f_{d}$ and logarithmic decrement $\delta$ are known, the undamped natural frequency $f_{n}$ and damping ratio $\zeta$ can be calculated from the following equations.

$$
\begin{aligned}
& \zeta=\frac{\delta}{\left(4 \pi^{2}+\delta^{2}\right)^{1 / 2}} \\
& f_{n}=\frac{f_{d}}{\left(1-\xi^{2}\right)^{1 / 2}}
\end{aligned}
$$

These equations give a prediction of the natural frequency, logarithmic decrement and damping ratio of a constant area duct, but there are many assumptions involved in the simulation of the probe as a simple constant area duct with flow. Therefore it was felt that the probe's frequency response should 
be measured first and the results should then be compared with the predictions of equations (3.26) to (3.29). If the comparison is satisfactory, these equations can be used to predict $f_{n}$ and $\zeta$ in a different gas, eliminating additional tedious shock tube tests.

Fig. 3.24 shows an enlarged view of a typical probe response. For this set of tests both driver and driven sections were filled with a mixture of $82 \%$ Argon and $18 \%$ Freon 12, identical to the mixture used in the Blowdown Facility. From this figure we inferred that the system behaves very much like a conventional second order system (for comparison see Fig. 3.25). The frequency response of any second order system is generally governed by two parameters, the undamped natural frequency $\omega_{n}$ and the damping ratio $\zeta$ [51] Fig. 3.26 shows frequency response curves of second order systems. It is clear from Fig. 3.26a that an increase in $\omega_{n}$, will increase the range of frequencies for which the amplitude ratio curve is relatively flat Therefore a high $\omega_{n}$ is needed to accurately measure high frequency input From the same curves it can be seen that an optimum range of values for $\zeta$ exists for both the amplitude ratio and phase angle curves of about 0.6 to 0.7 . For these values of $\zeta$ the amplitude ratio curves (Fig. 3.26a) exhibit the widest flat behavior, while the phase angle ones are essentially linear, giving good reproduction of the shape of the input signal.

The following expressions are given in [51], in order to calculate the $\omega_{n}$ and $\zeta$ parameters from the step response of a second order system, like the one in Fig. 3.25: 


$$
\begin{aligned}
& \zeta=\left\{\frac{1}{\left(\frac{\pi}{\ln \left(\frac{a}{A}\right)}\right)^{2}+1}\right\}^{1 / 2} \\
& \omega_{n}=\frac{2 \pi}{T\left(1-\zeta^{2}\right)^{1 / 2}}
\end{aligned}
$$

where $a, A$ and $T$ are explained in Fig. 3.25. These expressions were used to calculate the $\omega_{n}$ and $\zeta$, using $a, A$ and $T$ as can be measured from Fig. 3.24. According to [51], if several cycles of oscillation appear in the step response signal, like in Fig. 3.24, it is more accurate to determine the period $T$ as the average of as many distinct cycles as are available rather than from a single cycle. Typical values for a, $A$ and $T$ as measured from Fig. 3.24, are given in the following Table: 
Table 3.6: Step response parameters as in Fig. 3.24 and 3.25

$\begin{array}{ll}\text { Steady state value, A, (Volts) } & -4.04 \\ \text { Maximum overshoot of step response } & =0.14 \\ \text { over the steady state value, a, (Volts) } & =0.03 \\ \text { Minimum period of oscillations, Tmin. (msec) } & 0.08 \\ \text { Maximum period of oscillations, Tmax, (msec) } & 0.05 \\ 20 \text { cycles averaged period of oscillations, Tavg, (msec) } & =0.05\end{array}$

Using equations (3.30) and (3.31) $f_{n}$ and $\zeta$ were calculated and the results are given in the following table:

Iable 3.7: Measured frequency response parameters

\begin{tabular}{|c|c|}
\hline $\begin{array}{l}\text { Damping ratio, } \zeta \\
\text { Maximum undamped natural frequency, f } n \cdot m a x \\
\text { corresponding to Tmin. from Table } 3.6 \\
\text { Minimum undamped natural frequency, fn.min } \\
\text { corresponding to Tmox, from Table } 3.6 \\
\text { Average undamped natural frequency, fn. ovg } \\
\text { corresponding to Tava, from Table } 3.6\end{array}$ & $\begin{array}{l}=0.725 \\
=48.4(\mathrm{kHz}) \\
=18.2(\mathrm{kHz}) \\
=27.9(\mathrm{kHz})\end{array}$ \\
\hline
\end{tabular}

Notice that in general:

$$
\omega_{n}=2 \pi f_{n}
$$

As it can be seen the average natural frequency is calculated to be 27.9 $\mathrm{kHz}$ in Argon-Freon 12, under the assumption that the probe is a true second 
order system. However, in order to be on the safe side, we quote the probe's natural frequency to be $18.2 \mathrm{kHz}$ Notice also that the damping ratio turned out to be very close to the optimal range of 0.6 to 0.7 .

Equations (3.26), (3.27), (3.28) and (3.29) were then used to predict $f_{n}$ and $\zeta$ for the conditions of the shock tube tests. The results are given in the following Table:

Table 3.8: Calulated frequency response parameters

Damping ratio, $\zeta$

Logarithmic decrement, $\delta$

Undamped natural frequency, $f_{n}$
$=0.15$

$=0.96$

$=15.9(\mathrm{kHz})$

It is rather obvious that there is a substantial discrepancy between the predicted and measured values. However, the Whitehead model is based on a number of assumptions, like one-dimensional flow, perfect gas, small flow oscillations and negligible fluid friction. In addition, it is assumed that the duct is fed at constant total pressure through a short contraction. Although no thorough investigation of the validity of these assumptions was made, it was felt that the Whitehead model could not be safely used to predict the frequency response parameters of the probe in an arbitrarily chosen gas. Therefore we concluded that direct dynamic calibration and measurement, rather than prediction, of the frequency response parameters of the probe is required, every time the test gas is changed. 
In summary, the dynamic calibration showed that the measured frequency response of the probe in Argon-Freon 12 mixture is at least $18.2 \mathrm{kHz}$, which is well above the $5 \mathrm{kHz}$ blade passing frequency. In addition, the data reduction scheme successfully converted the three raw voltage outputs of the probe into temperature, pressure and tracer concentration throughout the sequence of events in the shock tube. 


\section{7 - Probe Frror analysis}

An important and crucial aspect of any experiment is the determination of the uncertainty level in the measurements. In general, the error analysis starts with the identification of the sources of error and proceeds with the calculation of the combined influence of all these error sources on the measured quantities, which in our case are total temperature, total pressure and concentration of tracer gas.

The noise level in the voltage output from the anemometers used, is typically $2 \mathrm{mV}$ peak to peak. The resolution of the analog-to-digital converter used is also $2 \mathrm{mV}$. Therefore the uncertainty of the measurement is equal to 2 $\mathrm{mV}$ in terms of voltage measurement

Some general aspects of the error analysis will be described first The uncertainty in the measurement of total temperature, concentration and total pressure is calculated using a root-mean square (rms) formula:

$\Delta T_{t}=\left\{\left(\frac{\partial T_{t}}{\partial V_{1}} \Delta V_{1}\right)^{2}+\left(\frac{\partial T_{t}}{\partial V_{2}} \Delta V_{2}\right)^{2}+\left(\frac{\partial T_{t}}{\partial V_{3}} \Delta V_{3}\right)^{2}\right\}^{1 / 2}$

$\Delta \xi=\left\{\left(\frac{\partial \xi}{\partial V_{1}} \Delta V_{1}\right)^{2}+\left(\frac{\partial \xi}{\partial V_{2}} \Delta V_{2}\right)^{2}+\left(\frac{\partial \xi}{\partial V_{3}} \Delta V_{3}\right)^{2}\right\}^{1 / 2}$

$\Delta p_{t}=\frac{\partial p_{t}}{\partial V_{3}} \Delta V_{3}$ 
where $\Delta V_{1}, \Delta V_{2}$ are the uncertainties from the two hot wires on the aspirating probe and $\Delta V_{3}$ the uncertainty from the total pressure probe, $\Delta T_{t}$ is the total temperature uncertainty, $\Delta p_{t}$ is the total pressure uncertainty and $\Delta \xi$ the uncertainty in concentration (mass fraction units). Notice that equation (3.35) does not include the uncertainties from $V_{1}$ and $V_{2}$, because the total pressure is measured directly from the total pressure probe and therefore there is no influence from the uncertainties in the voltages of the two wires. It should also be pointed out that the choice of the rms formula to calculate the uncertainty is not a trivial one (for details see Ref. [52] [53] [54], [55]). It is generally believed that when the individual uncertainties are not considered as absolute limits of error but rather as statistical bounds, equations (3.33), (3.34), (3.35) are probably the most appropriate to use.

During a typical Blowdown test, the flow properties change with time. Since the uncertainty in the measurement of concentration, total pressure and total temperature depends on the properties of the flow, its level will also vary during the test If we did not inject tracer gas, the uncertainty in the total temperature and total pressure measurements would still vary with time for a particular test, but would be constant from test to test The same would be true, if the tracer gas was injected at a fixed radius for all the tests. However, in this series of tests the injection occurs at different radii. Therefore the distribution of tracer gas is different in each test, while the total temperature and pressure distributions are approximately the same. This introduces an additional complication in the determination of the uncertainty level, since at each point in time the probe samples fluid of different total temperature, pressure and concentration, not only during one test, but from test to test as well. Therefore an error analysis was performed for each data point and not for a representative 
sample only. In other words the final result of the error analysis was a time resolved uncertainty distribution, which changes as the injection radius changes. A computer code was employed to do that and the time resolved uncertainty traces were generated for each injection test and for three tracer gases, namely $\mathrm{CO}_{2}$, Helium and Helium-Freon 12.

The results of these calculations are presented in figures 3.27 to 3.32 . In Fig. 3.27 the time resolved distribution of uncertainty in mass fraction units is plotted vs. \% blade span from the hub for the cases of Helium and Helium-Freon 12 tracer gases. Notice that the uncertainty in the case of Helium is three times less than that of Helium-Freon 12. Fig. 3.28 shows the measured mass fraction distribution during the tip injection vs. \% blade span. In the same figure the error bars for both Helium and Helium-Freon 12 are given, for several radial locations. Fig. 3.29 presents similar distributions of uncertainty for total temperature, while the relative magnitude of the error bars is given in Fig. 3.30. The total pressure uncertainty is constant with time (see Fig. 3.31), because the total pressure is directly measured by the pressure probe and the flow properties do not affect the level of uncertainty. The relative magnitude of the error bars is presented in Fig. 3.32 along with a typical total pressure trace.

The calculation of the uncertainty distribution with time, resolved two important issues. First, it helped identify regions of high or low measurement uncertainty during the test time. Second, and most important, it gave the magnitude of the uncertainty fluctuations during the test and proved that it is small enough to be considered negligible compared to its mean value.

Table 3.9 presents the results of the error analysis in terms of maximum 
error for total pressure, total temperature, mass fraction and mole fraction. Table 3.10 presents the average error for the same quantities.

Table 3.9: Maximum percentage error in the measurement

\begin{tabular}{|c|c|c|c|c|c|}
\hline $\begin{array}{l}\text { tracer } \\
\text { gas }\end{array}$ & $\begin{array}{l}\text { injection } \\
\text { location }\end{array}$ & $\begin{array}{c}\text { total } \\
\text { pressure }\end{array}$ & $\begin{array}{c}\text { MAXIMUM PERC } \\
\text { ERROR } \\
\text { total } \\
\text { temperature }\end{array}$ & $\begin{array}{l}\text { NTAGE } \\
\text { mass } \\
\text { fraction } \\
\text { (to full } \\
\text { scale) }\end{array}$ & $\begin{array}{c}\text { mole } \\
\text { fraction } \\
\text { (to full } \\
\text { scale) }\end{array}$ \\
\hline \multirow{3}{*}{ HEL IUM } & tip & 0.08 & 0.33 & 0.10 & 0.12 \\
\hline & midspan & 0.08 & 0.33 & 0.10 & 0.12 \\
\hline & hub & 0.08 & 0.32 & 0.09 & 0.10 \\
\hline \multirow{3}{*}{$\begin{array}{l}\text { HEL IUM } \\
\stackrel{+}{\text { FREON } 12}\end{array}$} & tip & 0.08 & 0.23 & 0.37 & 0.37 \\
\hline & midspan & 0.08 & 0.23 & 0.37 & 0.37 \\
\hline & hub & 0.08 & 0.23 & 0.33 & 0.33 \\
\hline
\end{tabular}


Iable 3.10: Average percentage error in the measurement

\begin{tabular}{|c|c|c|c|c|c|}
\hline $\begin{array}{l}\text { tracer } \\
\text { gas }\end{array}$ & $\begin{array}{l}\text { injection } \\
\text { location }\end{array}$ & $\begin{array}{c}\text { total } \\
\text { pressure }\end{array}$ & $\begin{array}{c}\text { AVERAGE PER } \\
\text { ERROR } \\
\text { total } \\
\text { temperature }\end{array}$ & $\begin{array}{l}\text { NTAGE } \\
\text { mass } \\
\text { fraction } \\
\text { (to full } \\
\text { scale) }\end{array}$ & $\begin{array}{c}\text { mole } \\
\text { fraction } \\
\text { (to full } \\
\text { scale) }\end{array}$ \\
\hline \multirow{3}{*}{ HEL I UM } & tip & 0.08 & 0.30 & 0.026 & 0.031 \\
\hline & midspan & 0.08 & 0.30 & 0.026 & 0.031 \\
\hline & hub & 0.08 & 0.29 & 0.023 & 0.026 \\
\hline \multirow{3}{*}{$\begin{array}{l}\text { HEL IUM } \\
\stackrel{+}{\text { FREON } 1}\end{array}$} & tip & 0.08 & 0.21 & 0.330 & 0.330 \\
\hline & midspan & 0.08 & 0.21 & 0.330 & 0.330 \\
\hline & nub & 0.08 & 0.21 & 0.290 & 0.290 \\
\hline
\end{tabular}

As it was mentioned in section 3.3 , one of the major problems we faced during this series of tests, was the low sensitivity of the aspirating probe to concentration changes. This was one of the primary reasons for changing the tracer gas from $\mathrm{CO}_{2}$ to the mixture of Helium-Freon 12. In this section an overview of the sensitivity issue will be given.

The sensitivity of the aspirating probe hot wires, like the uncertainty in the measurement, changes during the test and from test to test In addition, its value depends on the overheat ratio of the wire. Therefore, two sensitivity values will be obtained for each test since the two hot wires of the aspirating probe are operated at different overheat ratios. A computer code was developed to calculate the time resolved sensitivity distribution during the blowdown test and pick the minimum and maximum values. Table 3.11 gives the results of the 
sensitivity analysis for all three injection radii and for both tracer gases.

Table 3.11: Sensitivity values for the aspirating probe hot wires for various tracer gases and test conditions.

\begin{tabular}{|c|c|c|c|c|c|c|}
\hline $\begin{array}{l}\text { tracer } \\
\text { gas }\end{array}$ & $\begin{array}{l}\text { injection } \\
\text { location }\end{array}$ & OHR & $\begin{array}{r}\text { HOT } \\
\text { total } \\
\text { pressure } \\
\text { (V/atms) }\end{array}$ & $\begin{array}{l}\text { IIRE SENS I } \\
\text { IN } \\
\text { tota I } \\
\text { temp } 8_{K j} \text {. } \\
(\mathrm{mV} /\end{array}$ & $\begin{array}{l}\text { IVITY } \\
\text { mole } \\
\text { fraction } \\
(\mathrm{mV} / 1 \% \mathrm{m.f})\end{array}$ & \\
\hline \multirow{3}{*}{ HEL I UM } & tip & $\begin{array}{l}2.0 \\
1.7\end{array}$ & $\begin{array}{l}0.95 \\
2.58 \\
0.81 \\
2.34\end{array}$ & $\begin{array}{r}-5.95 \\
-11.97 \\
-8.17 \\
-15.39\end{array}$ & $\begin{array}{l}22.15 \\
45.39 \\
18.41 \\
37.74\end{array}$ & $\begin{array}{l}(\min ) \\
(\max ) \\
(\min ) \\
(\max )\end{array}$ \\
\hline & midspan & $\begin{array}{l}2.0 \\
1.7\end{array}$ & $\begin{array}{l}1.01 \\
2.23 \\
0.87 \\
2.00\end{array}$ & $\begin{array}{r}-5.95 \\
-9.92 \\
-8.17 \\
-13.02\end{array}$ & $\begin{array}{l}22.64 \\
35.48 \\
18.70 \\
32.17\end{array}$ & $\begin{array}{l}(\min ) \\
(\max ) \\
(\min ) \\
(\max )\end{array}$ \\
\hline & hub & $\begin{array}{l}2.0 \\
1.7\end{array}$ & $\begin{array}{l}0.95 \\
2.32 \\
0.81 \\
2.09\end{array}$ & $\begin{array}{r}-6.13 \\
-9.06 \\
-8.41 \\
-12.18\end{array}$ & $\begin{array}{l}22.81 \\
37.12 \\
19.29 \\
33.70\end{array}$ & $\begin{array}{l}(\min ) \\
(\max ) \\
(\min ) \\
(\max )\end{array}$ \\
\hline \multirow{3}{*}{$\begin{array}{l}\text { HEL I UM } \\
\stackrel{+}{\text { FREON } 12}\end{array}$} & tip & $\begin{array}{l}2.0 \\
1.7\end{array}$ & $\begin{array}{l}0.91 \\
1.87 \\
0.76 \\
1.67\end{array}$ & $\begin{array}{r}-5.73 \\
-7.73 \\
-8.27 \\
-11.26\end{array}$ & $\begin{array}{l}16.74 \\
20.02 \\
14.04 \\
17.40\end{array}$ & $\begin{array}{l}(\min ) \\
(\max ) \\
(\min ) \\
(\max )\end{array}$ \\
\hline & midspan & $\begin{array}{l}2.0 \\
1.7\end{array}$ & $\begin{array}{l}0.91 \\
1.87 \\
0.76 \\
1.67\end{array}$ & $\begin{array}{r}-5.73 \\
-7.75 \\
-8.26 \\
-11.29\end{array}$ & $\begin{array}{l}17.37 \\
19.72 \\
14.06 \\
17.03\end{array}$ & $\begin{array}{l}(\min ) \\
(\max ) \\
(\min ) \\
(\max )\end{array}$ \\
\hline & hub & $\begin{array}{l}2.0 \\
1.7\end{array}$ & $\begin{array}{l}0.91 \\
1.87 \\
0.76 \\
1.67\end{array}$ & $\begin{array}{r}-5.89 \\
-7.74 \\
-8.50 \\
-11.26\end{array}$ & $\begin{array}{l}17.46 \\
19.76 \\
14.06 \\
17.05\end{array}$ & $\begin{array}{l}(\min ) \\
(\max ) \\
(\min ) \\
(\max )\end{array}$ \\
\hline
\end{tabular}

An example to clarify the units of the concentration sensitivity is the following. If at a certain point in time, the tracer concentration is 0.2 in mole 
99

fraction, then a sensitivity level of 4 , means that if the concentration becomes 0.21 or 0.19 the voltage will change by +4 or $-4 \mathrm{mV}$ respectively.

According to Table 3.11 the concentration sensitivity for Helium is higher than that for Helium-Freon 12, while the total temperature sensitivities are almost equal. However, the sensitivity of the Helium- Freon 12 mixture is still high enough for our application, while its density is the same with the main mixture of Argon-Freon 12. In summary, the Helium-Freon 12 mixture gives adequate probe sensitivity, while at the same time, it eliminates the buoyancy problem that Helium has. As it was also explained in section 3.3, these were the primary reasons for the selection of Helium-Freon 12 as tracer gas mixture. 


\section{CHAPTER 4}

\section{PROOF OF CONCEPT TESTING}

The spreading of the injection jet and its effect on the main rotor flowfield were directly measured in order to verify that the design goals of the injector were successfully achieved. This Chapter describes the experimental procedure and results of this effort under two basic categories. The first includes the experiments with the rotor and stator completely removed from the test section of the Blowdown Facility. Note that in this case the flow path contours in terms of hub and tip geometry remained the same. The second category includes all the tests with the rotor and stator in place.

\section{1 - Tests with the rotor and stator removed}

In order to determine the basic mixing level in the Blowdown Facility during a typical test, Blowdown tests with both rotor and stators removed from the test section were conducted. The flow path contours were kept the same. The initial pressure level in the supply tank was appropriately adjusted to keep the flow choked during the test. The volume and the initial pressure of the injectant supply tank were adjusted to match the free stream and injection jet velocities. Note that for these tests the tracer gas was $\mathrm{CO}_{2}$.

The aspirating probe was traversed during the test from tip to hub and stopped at $R / R_{t i p}$ of 0.7 . The voltage outputs from the two hot wires and the total pressure probe were then input into the data reduction codes and the calculated time resolved concentration of tracer gas and total temperature vs. 
nondimensional radius are shown in Fig. 4.1 and 4.2 respectively. In Fig. 4.2 the total temperature in the supply tank is superimposed. Since no work is done on the fluid (no rotor) and there is heat transfer to or from the fluid, the total temperature in the supply tank and the one measured by the aspirating probe must be the same. The agreement is within $3^{\circ} \mathrm{C}$ (see Fig. 4.2 ). Fig. 4.3 presents a comparison between total pressure measured by the companion total pressure probe and by the pressure transducer in the Blowdown supply tank. The agreement is within the repeatability level of the Blowdown Facility.

The high frequency, small amplitude fluctuations in the total temperature trace from the aspirating probe and in the total pressure trace from the companion total pressure probe are due to both the free stream turbulence in the Facility and the $A / D$ resolution. It should also be kept in mind that the signal from the supply tank was recorded using a low sampling rate A/D channel (5 $\mathrm{kHz}$ ), while the one from the aspirating probe was sampled at $50 \mathrm{kHz}$. Therefore no high frequency fluctuations can be apparent in the supply tank total temperature trace. The injection jet has a thickness of approximately $0.35 \mathrm{in}$. 0.89 $\mathrm{cm}$ ) at the plane of the aspirating probe (see Fig. 4.1). Since the height of the passage at that location is about 4 in. $(10.2 \mathrm{~cm})$, it was felt that the jet was thin enough to give usable data.

The centerline of the jet at the plane, where the probe is traversed, can be estimated to be approximately at $R / R_{t i p}=0.83$. The point of injection is at $R / R_{t i p}=0.76$ and the change in radius is simply due to the contraction of the flow path and the high ramp of the hub in the downstream direction. 


\section{2 - Tests with the rotor and stator in place}

The goal of these tests was to estimate the effect of the injection and the injector itself on the flowfield of the compressor. The interaction between injector and compressor is an extremely complicated phenomenon. A brief discussion will be given first in order to illustrate some aspects of it.

The surfaces of both the injector and the supporting struts are sources of vorticity in the radial and circumferential direction. These vortical structures are convected and turned through the compressor blade row, inducing secondary flows in the spanwise and circumferential direction. However, the lower surface of the injector generates vorticity of equal magnitude but of opposite sign from that of the upper surface. The same is true for the supporting struts. Therefore secondary velocities of opposite sign, but equal magnitude are induced in the blade row, with the net effect being negligibly small.

The density difference between injectant and main gas, due to either molecular weight or temperature difference, is also a source of secondary flow. On the other hand the interaction of this density gradient with the shock at the rotor's leading edge is hard to predict

One additional effect of the presence of the injector in front of the compressor, is the change in blade incidence angle that results from a simple velocity triangles argument at the fan's inlet The injector creates a wake behind it or, in other words, a decrease in the absolute axial velocity of the rotor. This is translated into an increase in the absolute magnitude of the relative flow angle. 
However, the velocity defect in the wake is considerably smoothed out by the time it reaches the leading edge of the rotor. The increase of the wake centerline velocity downstream of the injector was calculated using wake decay formulas given in Schlichting [56] and the results showed that the maximum change in incidence angle was negative, but less than $1.5^{\circ}$ in magnitude. Therefore the change in incidence angle is quite small and it does not play a major role in modifying the rotor's flowfield.

Vortex shedding from the body of the injector can also influence the flowfield of the rotor. The outcome of the interaction of the shed vortices with the rotor is quite hard to predict.

The effect of the blade passing frequency on the formation and stability of the injection jet can also be significant In addition, the upstream influence of the compressor flowfield is quite strong and its effect on the injection characteristics is unpredictable.

For all these reasons an analytical approach to the quantitative prediction of the effect of both the injector and the injection on the rotor performance is hopeless. However, a measurement of this effect is quite feasible. Therefore, a series of blowdown tests were conducted in order to isolate and quantify the result of the interaction between injector, injection and rotor flowfield. The following list gives a summary of these tests: 


\section{Eull Stage Tests at Design Point:}

Iest A - Injector body completely removed

Test B - Injector in place, but no injection

Iest C - Injector in place and full injection near the tip

Test D - Injector in place and full injection at midspan

Test E - Injector in place and full injection near the hub

Fig. 4.4 shows a comparison of the pressure ratio vs. \% blade span from the hub for these five cases. Fig. 4.5 presents the comparison for the total temperature ratio for the same five cases measured with the aspirating probe. In the same figures, the total pressure and total temperature ratios measured in a conventional steady state test rig with stator leading edge instrumentation are also presented (only symbols). There is some discrepancy between the steady state and blowdown measurements, which may be due to a differing casing boundary layer thickness. For comparison purposes, the distribution of total pressure and total temperature vs. \% blade span from the hub for two blowdown tests with identical conditions are presented in Fig. 4.6 and 4.7. The data from the five blowdown tests (Fig. 4.4 and 4.5), compare very well with each other, the maximum difference being less than the repeatability of the facility, which is less than 1\% (see Fig. 4.6 and 4.7). Therefore, on a time average sense, the injector and the injection have little effect on the compressor average performance. 


\section{3 - Buoyancy considerations}

Buoyancy is expected to play some role in this experiment, since the density of the injectant is in general different from that of the main gas. Depending on what tracer gas is used, this difference is due to either molecular weight difference (Helium, $\mathrm{CO}_{2}$ ) or temperature difference (Helium-Freon 12, Helium, $\mathrm{CO}_{2}$ ) or both (Helium, $\mathrm{CO}_{2}$ ). However, it should be pointed out that gravity acts in the plane of the injection sheet, since the injector is mounted on the side of the test section (see Fig. 2.2) and the injection slot is in the vertical direction. Therefore the effect of buoyancy, if any, would be to deflect the injection jet in the circumferential, rather than the radial direction of the rotor, before entering the blade row.

With reference to Gebhart's work on buoyant jets [57], an indication of how buoyant a jet is, can be given by the densimetric Froude number, Fr, defined as:

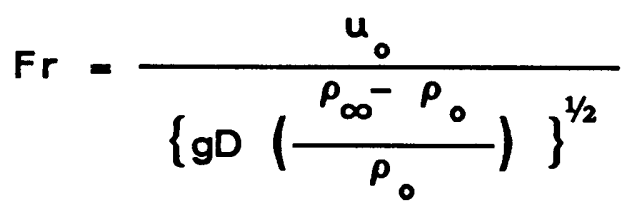

where $u_{0}$ is the jet velocity, $D$ is the thickness of the injection slot, $\rho_{0}$ and $\rho_{\infty}$ are the densities of the jet and free stream respectively. For low values of $\mathrm{Fr}$ number, buoyancy dominates the behavior of the jet, while for large ones the buoyancy can be neglected. For Fr above 500 the jet can be considered non-buoyant Another interesting point from [57] is that, even for the buoyant jets, there is a region, right after the orifice, in which the behavior of the jet is essentially a non-buoyant one, and an intermediate region immediately afterwards, 
where the flow progresses from non-buoyant jetlike toward plumelike behavior.

The buoyancy of the injection jet has different magnitude and origin for each of the tracer gases that were used. However, in all the cases the contribution to jet buoyancy from the temperature difference between injectant and main gas is the same. The following table gives the Froude number, the length of the non-buoyant and intermediate regions and a quick description of the buoyancy inducing mechanisms for the three tracer gases that were used.

Iable 4.1: Froude number, length of non-buoyant and intermediate regions, and buoyancy origin

\begin{tabular}{|c|c|c|c|c|}
\hline Tracer gas & $\begin{array}{l}\text { Froude } \\
\text { number }\end{array}$ & $\begin{array}{c}\text { Non-buoyant } \\
\text { region }\end{array}$ & $\begin{array}{c}\text { Intermediate } \\
\text { region }\end{array}$ & $\begin{array}{l}\text { Buoyancy } \\
\text { mechanism }\end{array}$ \\
\hline Hel i um & 400 & $z<0.4 \mathrm{in}$ & $z<4$ in & $\begin{array}{l}\text { temp. }+ \text { mol. } \\
\text { weight diff. }\end{array}$ \\
\hline$\infty_{2}$ & 2100 & $z<2.2 \mathrm{in}$ & $z<22$ in. & $\begin{array}{l}\text { temp. }+ \text { mol. } \\
\text { weight diff. }\end{array}$ \\
\hline Hel-Fre. 12 & 3100 & $z<3.0 \mathrm{in}$ & $z<30$ in. & temp. diff. \\
\hline
\end{tabular}

The distance from the injection point to the rotor leading edge is approximately 0.7 in. According to Table 4.1, the Froude number is generally high enough for buoyancy to be neglected in the cases of $\mathrm{CO}_{2}$ and Helium-Freon 12. The Helium case is probably the most questionable, as far as buoyancy is concerned. However, even in this case the Froude number is quite high and the non-buoyant region extends almost up to the rotor leading edge. Further investigation of the Helium case led us to use the information presented by 
Gebhart in [58]. He calculated the trajectory of a buoyant jet for several Froude number values and ratios of free stream to jet velocities. For a Froude number of 200 (highest we could find in that report) and ratio of free stream to jet velocity of 0.8 , the jet centerline deflects only by 0.015 in. $(0.38 \mathrm{~mm})$ at a distance of 25 orifice diameters from the injection point and by 0.03 in 10.76 $\mathrm{mm})$ at a distance of 130 orifice diameters. These two downstream locations correspond to the leading and trailing edge of the rotor. In the Helium case the Froude number is higher than 200 and the ratio of velocities is close to one. Therefore, the results of the jet deflection presented in [58] correspond to a case worse than the Helium one. In conclusion, buoyancy can be safely neglected for all three tracer gases.

Experimental verification of these predictions was also sought The first came from the "no rotor" case presented in section 4.1, where $\mathrm{CO}_{2}$ was injected and, as predicted, no substantial deflection of the injection jet was observed. In addition, a comparison between injection tests with Helium-Freon 12 and Helium was attempted with the rotor and stator in place. Fig. $4.8 \mathrm{a}, \mathrm{b}$ and $\mathrm{c}$ show the results of this comparison in terms of time averaged concentration, total pressure and total temperature ratios, respectively. The concentration is presented in mass fractions units. Notice that the measured level of Helium concentration is lower than the Helium-Freon 12 one. This is a result of the smaller injection mass flow of Helium, which is necessary in order to keep the slope of the total pressure decay traces for both tracer gases equal to each other and to the main Blowdown one. However, it is important to notice from the same figure that the peak of concentration occurs at the same radial location in both cases. In addition, the shape of the concentration, total pressure and total temperature traces are almost identical. It is therefore fair to conclude that the buoyancy 
effect on the motion of the fluid particles is the same for both tracers. However, with reference to Table 4.1, buoyancy has a very negligible effect in the case of Helium-Freon 12. Therefore, the same must be true for the tests with Helium. 


\section{4 - Centrifugal action of the rotor}

The similarity of the concentration measurement for Helium and Helium-Freon 12 tracer gases presented in Fig. 4.8 can also be used to prove that the strong centrifugal action of the rotor has a negligible effect on the measurement of radial migration, since in the Helium-Freon 12 case, no such effect should be expected.

This experimental finding is probably due to the quick decay of the jet centerline concentration. With reference to decay formulas given by Gebhart in [57] for a plane Helium jet with its density being 13.5 times less than the free stream, the centerline concentration drops to $10 \%$ of the initial one $(100 \%)$ by the time the jet reaches the rotor leading edge. For a $\mathrm{CO}_{2}$ jet, the centerline concentration drops to $25 \%$ of the initial. However, it is interesting to notice (see [57]) that the decay is very abrupt in a region that extends for about 20-30 slot thicknesses downstream from the injection orifice. After that the centerline concentration decays very slowly and for all practical purposes can be considered constant Therefore, the decay of the centerline concentration of the Helium jet is so fast, that by the time it reaches the leading edge of the rotor, its density is so close to the free stream one that the effect of the centrifugal action of the rotor is negligible. Further downstream the decay rate decreases substantially and the centerline concentration remains almost constant Therefore in the Helium case, the concentration at the rotor leading edge is probably much lower than the one in the Helium-Freon 12 case. However, in both cases the

motion of the fluid particles is not affected by the centrifugal forces of the rotor, since in the former the concentration is already too low, while in the latter there is no density difference to begin with. 


\section{5 - Turbulence level in the Blowdown Facility}

The turbulence intensity in the Blowdown Facility was measured during the "no rotor" blowdown tests. Single straight hot wires were used to record fluctuations in the tunnel during the test They were typically placed at an axial location that corresponds to the immediate upstream region of the rotor. The turbulence intensity $T$ is generally given as in equation (4.2):

$$
T=\frac{\left(\overline{u^{2}}\right)^{1 / 2}}{\bar{U}}
$$

where the numerator is the traditional rms value of the velocity fluctuation, $u$, and $U$ is the average velocity. This definition assumes that the turbulence is isotropic in nature and therefore, all three fluctuating components of velocity are equal. In addition, it does not take into account any fluctuations due to temperature turbulence. In the presence of both types of turbulence, a single hot wire cannot distinguish between them, since its voltage output is a function of mass flux $\rho U$, rather than only velocity $U$. For simplicity, we are going to neglect any temperature turbulence and attribute all the hot wire voltage fluctuations to velocity turbulence, as it is given by equation (4.2).

A typical hot wire calibration was used to convert the voltage fluctuations into velocity fluctuations. Their $\mathrm{rms}$ value and the mean velocity were then calculated. The final result was a turbulence intensity, given by eq. (4.2), of 0.0057 or $0.57 \%$. According to Schlichting [56], this turbulence intensity is considered to be quite low for typical wind tunnels. 


\section{CHAPTER 5}

\section{MEASUREMIENTS OF TIME RESOLVED CONCENIRATION, TOTAL THMPERATURE AND TOTAL PRESSURE}

In this Chapter the experimental results will be presented. It should be kept in mind that all the measurements were taken in the absolute (laboratory) frame. Details about the operation of the facility were presented in section 2.4 . In the light of the discussion in Chapter 4, the concentration results for Helium and Helium-Freon 12 tracer gases will be indiscriminately presented.

\section{1 - Consistency checks}

A series of blowdown tests were conducted in order to determine the consistency of both the probe and the data reduction scheme and the repeatability of the Blowdown Facility. The results and measurements for the latter were presented in Chapter 4 (Fig. 4.5 and 4.6). The consistency of the probe measurements was checked by comparing "no injection" and "with injection" tests. The total temperature and total pressure measurements during both types of tests must be the same except from uncertainty associated with the repeatability of the tunnel operation from test to test Fig. 5.1 and 5.2 present a comparison of instantaneous total pressure and total temperature ratios between the "with injection" and the "without injection" cases. These correspond to the time averaged traces presented in Fig. 5.3 and 5.4. The agreement is within the repeatability range of the tunnel (see Fig. 4.5 and 4.6) in both time averaged and time resolved terms. Fig. 5.5 presents instantaneous concentration traces for the same tests. 
Notice that the concentration in the "rio injection" case is zero, except from a few noise related peaks that are isolated and of no significance. The difference in the signal between the two cases can be clearly seen in Fig. 5.5 .

Another consistency check was to compare the mass of tracer gas sampled by the probe behind the rotor with the mass of tracer gas that was injected. The latter was determined from the slope of the total pressure decay in the injector. This comparison is based on the assumption that, on time average, the concentration measured is a representative sample of the concentration distribution in the circumferential and spanwise directions. Typically the mass flow of the upstream injected tracer gas is $1 / 1000$ of the total compressor mass flow. Using the assumption above, the probe measures $80 \%$ of the injected tracer flow. Given the complexity of the flow, this agreement is quite satisfactory. It should also be pointed out that the object of the mass balance calculation was to identify any dramatic experimental discrepancies rather than provide a precise comparison.

\section{2 - Instantaneous data - High frequency results}

As it was mentioned before, the major contribution of this experimental effort is the acquisition of high frequency, time resolved, simultaneous measurements of concentration, total pressure and total temperature. These are presented below.

Three sets of experiments were basically conducted. All the operating parameters (corrected speed and mass flow, pressure ratio, injectant mass flow) were kept the same for all the tests. Only the spanwise location of the injection 
was changed. Three locations were chosen at $R / R_{t i p}=0.6$ (hub injection), $R / R_{t i p}=0.76$ (midspan injection) and $R / R_{t i p}-0.9$ (tip injection). Fig. 5.6 presents a schematic scale drawing of the rotor geometry with the three injection locations. The mass balance calculation was repeated for each test to confirm that tracer mass continuity was satisfied.

Fig. 5.7 presents typical traces of absolute total pressure and total temperature ratio vs. nondimensional radius behind the rotor. A low frequency modulation of the signal can be easily observed in the total pressure ratio measurement This phenomenon has already been observed by $\mathrm{Ng}$ [1] several years ago. In addition, Owen [59] proposed an explanation based on the motion of the separation point on the blade. However, although it is still an open question, we believe that its in depth investigation is beyond the scope of this research effort It is also clear that the blade wakes (low total pressure region in the hub region, but not necessarily so in the tip region) are deeper at the hub, while at the tip the picture is not clear and it is quite difficult to identify any structure in the trace that might correspond to blade passing. The same trends in the traces of total temperature and total pressure were also observed by $\mathrm{Ng}$ [1]. However, it should be pointed out that in his tests the total temperature was measured using only one hot wire and the total pressure probe, instead of the two hot wires that we used.

In Fig. 5.8, instantaneous traces of concentration are presented vs. nondimensional radius, as measured in the three injection tests (tip, midspan, hub). The high level of fluctuations in the trace can be clearly observed from hub to tip. 
Figures 5.9 to 5.14 present short time segments of the instantaneous data at three different radii, close to the tip, the midspan and the hub. In particular, Fig. 5.9 and 5.10 show such traces for total pressure ratio, concentration, total temperature ratio and total pressure ratio, concentration, entropy production respectively, for the tip injection. Similarly, Figures 5.11 and 5.12 present the same information for the midspan injection and figures 5.13, 5.14 for the hub injection.

It should be pointed out that the calculation of entropy production $\left(\Delta s / c_{p}\right)$ is based on the following perfect gas equation:

$$
\frac{\Delta s}{c_{p}}-\ln \left(\frac{T_{t 2}}{T_{t 1}}\right)-\frac{\gamma-1}{\gamma} \ln \left(\frac{P_{t 2}}{P_{t 1}}\right)
$$

where $\Delta s=s_{2}-s_{1}, c_{p}$ is the constant pressure specific heat and subscripts 2 and 1 correspond to downstream and upstream conditions respectively. Since $\gamma$ is a function of both temperature and concentration, its value was calculated for each data point, using an iterative scheme.

A few observations from these figures can be made. First, in the case of tip or even midspan injection (Fig. 5.9 or 5.11) the peaks of concentration in the hub region are random in nature and correspond to the blade wakes, defined as regions of low absolute total pressure. This is not the case in the regions, where the injection jet is expected to emerge from the rotor. There the peaks can be found everywhere, in the core flow and in the wakes. Second, the majority of the concentration peaks correspond to the entropy ones, which indicates that, if the migration is isentropic, then the high entropy fluid (e.g. wake fluid) is the one that 
migrates the most The implications of this observation will be clear during the discussion of the experimental results in Chapter 6 and 7.

In addition to all the flow quantities presented before, adiabatic efficiency was also calculated, using the following equation:

$\eta=\frac{\left(\frac{p_{t 2}}{p_{t 1}}\right)^{\frac{\gamma-1}{\gamma}}-1}{\frac{T_{t 2}}{T_{t 1}}-1}$

where subscripts 2 and 1 respresent downstream and upstream conditions, respectively. However, it should be pointed out that in a general unsteady flowfield this definition of adiabatic efficiency is not appropriate. Detailed discussion of this is given by Gertz in [2] Although we are aware of this problem, we are going to use equation (5.2) to calculate adiabatic efficiency only for comparison purposes with the results of other researchers.

Fig. 5.15 presents the spanwise time averaged variation of adiabatic efficiency, while Fig. 5.16 shows the corresponding instantaneous trace. Notice that the efficiency varies from close to one at the hub down to approximately 0.75 at the tip. In Fig. 5.15 the results from the Air Force Aero-propulsion Laboratory (AFAPL) are superimposed. The agreement is quite good. It should however be pointed out that in the AFAPL data there is a region $\left(R / R_{t i p}\right.$ less than 0.6$)$ of time averaged adiabatic efficiency higher than one. Unfortunately our measurements do not include that region, because the probe could not safely traverse that close to the hub. Therefore we have no way of proving or disproving this seemingly surprising result 


\section{3 - Time averaged data and general trends}

Fig. 5.17 presents the results of the concentration measurements in mass fraction units vs. nondimensional radius for the three locations as well as for injection with no rotor present. The injection locations and their corresponding ones downstream, estimated using a streamline curvature calculation, are indicated.

The peak of the concentration profile in each case is close to the one predicted by the streamline curvature calculation. However, there is a significant amount of fluid that has moved spanwise, primarily towards the tip. In addition, some fluid has moved towards the hub, contrary to conventional expectations based on centrifugal forces arguments alone. The shape of the concentration trace changes drastically with injector location.

Fig. 5.18 and 5.19 present a comparison of the time averaged total pressure and total temperature ratios for the injections at tip, midspan and hub. The differences between these three tests are smaller than the repeatability of the Blowdown Facility, which is determined from Fig. 4.5 and 4.6.

\section{4 - Ensemble average results}

In order to look at flow patterns the data were ensemble averaged and a 2-D map was assembled from ensemble averages of traces. 
Since the probe traversing velocity is much lower than the rotor or gas velocities (approximately $1 / 100$ of the gas velocity), each short time segment of the measurements is a time history of the flow at that point The width of this time segment is determined by the time it takes for the probe to traverse a distance equal to its spatial resolution. Given the dimensions and traversing velocity of the probe, this time interval was estimated to be 2 msec. All the blade passages (equal to 10) that correspond to this time segment can now be ensemble averaged and the result is a time history at that radial location.

One advantage of the ensemble average technique is that it makes it easier to identify any circumferential variations in the flowfield. In addition, any periodic phenomena locked on the blade passing frequency are amplified and, therefore, clearly observed. However, the interpretation of the ensemble averaged data can be misleading, since any phenomena that are either random or not phase locked with blade passing are suppressed and various artifacts are introduced.

Fig. 5.20 presents ensemble averaged data for total pressure, total temperature, mass fraction and entropy change in the case of injection near the tip. Fig. 5.21 presents the same flow quantities for injection at the midspan and Fig. 5.22 for injection near the hub.

By using similar ensemble averaged traces for a number of radii (in the previous figures only three radii are presented) 2-D maps or contour plots were assembled. Fig. 5.23 to 5.27 present these contour plots as they appear to an observer looking upstream and located behind the rotor in the absolute frame of reference. 
The circumferential extent of these plots is equal to two blade passages, starting at the middle of one passage and ending at the middle of the second consecutive one.

In Fig. 5.23 the shape and thickness of the injection jet in the case of "no rotor" is compared to the concentration traces measured behind the rotor during the tip, midspan and hub injections. It is rather clear that the rotor has a substantial effect on the distortion of the injection jet An important point to note is that the fluid has moved radially away from the injector in both directions, towards the hub and towards the tip.

Fig. 5.24 presents a comparison of absolute total pressure ratio and concentration traces, as measured in the three cases of injection at the tip, midspan and hub. The low pressure regions in the pressure contour plots mark the approximate location of the blade wakes. The majority of the radial migration occurs in the blade wakes. In addition, it can be observed that in the case of hub and midspan injections the tracer gas experienced a substantial migration, while in the tip injection it remained almost uniform in the circumferential direction, at least in the core region. It is also important to point out that the tracer gas has almost completely disappeared from the pressure side of the blade and has moved to either the suction side of the blade or into the wake region. The latter information is an indication of the usefulness of the ensemble average technique.

Fig. 5.25, 5.26 and 5.27 present contour plots of total pressure ratio, concentration, total temperature ratio and entropy production for injection at the tip, midspan and hub respectively. An important point to notice is the high total temperature region near the tip of the passage, which may represent high work 
fluid.

All these experimental observations will be the subject of discussion in Chapters 6, 7 and 8.

\section{5 - Summary of experimental results}

1. The injector seems to be working as it was expected and the injection jet was measured to be thin enough to give reliable data.

2. A series of diagnostic tests have been conducted to assess the effect of the injector's body and injection jet to the flow properties of the rotor. No substantial interference with the rotor flowfield has been detected.

3. Random peaks of tracer concentration have been detected away from the injection locations. These peaks correspond to regions of high entropy.

4. The tracer gas has moved away from the pressure side of the blade and into the wakes or the suction side of the blade.

5. Substantial radial migration occurs towards the tip, during the hub and midspan injection cases. The migrating fluid was found primarily in the blade wakes at the measurement location.

6. Very few negative entropy or higher than one efficiency regions have been observed in both the instantaneous and time averaged data. 


\section{CHAPTHR 6}

\section{TIME AVERAGED SPANWISE FLUID TRANSPORT}

\section{1 - Estimate of radial fluid migration}

The radial migration of fluid in the test compressor can be qualitatively visualized in the concentration plots presented in Chapter 5. However, the ultimate goal of this research effort is to give, not only a qualitative measurement of the migration, but a quantitative one as well. Therefore this section addresses the following questions: How much fluid migrates in the compressor as a percentage of the total mass flow and, how can this be inferred from the measurements of concentration?

The tracer gas concentration measured and presented in Chapter 5, is expressed as a fraction of the mass flow sampled by the aspirating probe, which is not the total mass flow of the compressor. In addition, the amount of tracer gas that was measured behind the rotor represents the migration only from the region occupied by the injection jet Fig. 6.1 shows a schematic drawing of the rotor. The points of upstream injection are also indicated. For the purpose of this calculation, the span of the rotor was divided in four regions, 1 to 4 (dashed lines in Fig. 6.1) with full circumferential extent From now on, the upstream regions will be called "inlet regions" and the downstream ones "outlet regions". Tracer gas was injected approximately in the middle of each of the first three inlet regions (tip, midspan, hub injections). However, this was not done for the fourth region, but its contribution to the total radial migration was also included, as it will be shown later in this section. 
The following assumptions were made in order to extrapolate from the measurements to an estimate of the amount of total radial fluid migration:

1) In order to describe the mutual interactions between inlet or outlet regions, we introduced a set of indices. Index $k$ is used for inlet regions and index $j$ for the outlet ones. In our case with equal number of inlet and outlet regions both indices take values from 1 to 4 .

2) We assumed that the injection is uniform and the injector extends over the whole rotor circumference. Since the actual circumferential extent of the injector is approximately equal to two blade passages, the mass flow of the injectant must be multiplied by a factor, $\alpha_{k}$, to account for that The subscript $k$ is used here to denote the inlet region that the calculation refers to. This was necessary because the value of $\alpha_{k}$ depends on the radial location of the injection, since the circumference of the rotor changes with radius, while the circumferential extent of the injector does not The mass flow of the injectant during the test, $\dot{m}_{0}$ is calculated using the slope of the total pressure decay in the injector cavity. The corresponding mass flow with full circumferential injection in inlet region $k, m_{c, k}$ is given as:

$$
\dot{m}_{c, k}=\alpha_{k} \dot{m}_{\text {. }}
$$

3) We chose the combination of indices, $\mathrm{kj}$, to indicate radial migration from region $k$ into region j. Therefore, the tracer gas mass flow that is measured behind the blade in the $j_{t h}$ outlet region during injection in the $k_{t h}$ inlet region (see also Fig. 6.1), is denoted as $\dot{\mu}_{m, k j}$. Since we chose four inlet and four outlet 
regions, a $4 \times 4$ matrix of migrating fluid masses is directly formed.

4) The mass flow sampled by the probe during its traverse through the $j_{\text {th }}$ outlet region, $\dot{m}_{p, j}$, is a fraction of the total mass flow, $\dot{m}_{j}$, that goes through the $j_{t h}$ outlet region. This fraction, $\lambda_{\mathrm{f}}$, is given as:

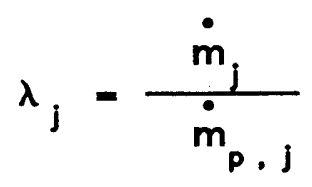

The mass flow through the probe is always choked and can be calculated using the measured total pressure, total temperature and concentration of the flow.

5) The tracer mass flow that is detected in the $j_{\mathrm{th}}$ outlet region during injection in the $k_{t h}$ inlet region, $\dot{\mu}_{m, k j}$, is a fraction of the mass flow sampled by the probe in the same region. We assume that the magnitude and direction of radial transport remain constant for all blade passages. Under this assumption, if the probe sampled all the mass flow of the $j_{t h}$ outlet region, $\dot{m}_{j}$, then the total tracer mass flow detected would be a fraction, $\dot{\mu}_{t, \mathrm{kj}}$, of it, which is equal to the one above. This assumption is expressed as:

$$
\dot{\mu}_{t, k j}=\lambda_{j} \dot{\mu}_{m, k j}
$$

6) However, one more correction must be applied in order to calculate the total mass of radial fluid migration. This is necessary, since $\dot{\mu}_{t, \mathrm{kj}}$ is a fraction of the total injected mass flow, $\dot{m}_{c, k}$, which is only a small fraction of the total mass flow $\dot{m}_{k}$ that goes through the $k_{t h}$ inlet region. Therefore, in order to calculate the total contribution to radial migration from the $k_{t h}$ inlet region, an 
extrapolation must be made. We assume that the measured radial fluid migration of tracer gas, due to injection in the middle of the $k_{t h}$ inlet region, is an exact representation of the rest of that region. Under this assumption, the mass of tracer gas that migrates into the $j_{t h}$ outlet region and originated anywhere in inlet region $k$ is larger than $\dot{\mu}_{t, k j}$ by a factor $f_{k}$, equal to:

$$
f_{k}=\frac{\dot{m}_{k}}{\dot{m}_{c, k}}
$$

Therefore, the total mass flow that migrates from region $k$ to region $j, \dot{\mu}_{k j}$, is given as:

$$
\dot{\mu}_{k j}=f_{k} \dot{\mu}_{t, k j}
$$

In summary, if we combine equations (6.5) and (6.3) the total mass flow that migrates from region $k$ to region $j$ is given as:

$$
\dot{\mu}_{k j}=\lambda_{j} f_{k} \dot{\mu}_{m, k j}
$$

Using equations (6.2), (6.1) and (6.4) the total mass flow that migrates from region $k$ to region $j$ can be expressed as a fraction of the total compressor mass flow, $\dot{m}$, in terms of measured quantities only:

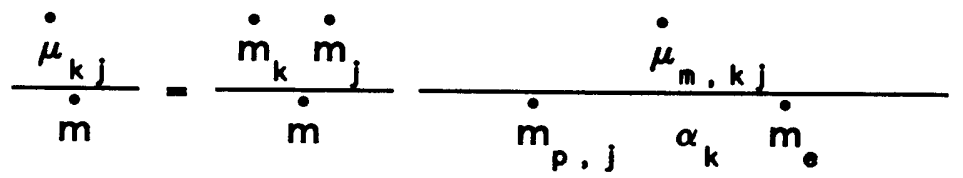

The nomenclature used in eq. (6.7) is repeated below: 


$$
\begin{array}{ll}
\dot{\mu}_{k j} & =\text { total mass flow that moves from region } k \text { into region } j \\
\dot{\mu}_{m, k j} & =\text { total tracer gas mass flow that was measured in region } \\
& j \text { during injection in region } k \\
\dot{m}_{k} \text { or } \dot{m}_{j} & =\text { total mass flow that goes through region } k \text { or } j \\
\dot{m} & =\text { total mass flow of the compressor } \\
\alpha_{k} & =\text { factor that accounts for the limited circumferential extent of } \\
\dot{m}_{e} & \text { the injector and is defined in eq. }(6.1) \\
& =\text { injectant mass flow during the test }
\end{array}
$$

The total mass $\dot{\mu}_{\mathrm{in}, \mathrm{j}}$ that migrates into the $j_{\mathrm{th}}$ region, due to contributions from all the $k$ regions, is given by the following equation:

$$
\dot{\mu}_{i n, j}=\sum_{\substack{k=1 \\ k \neq j}}^{4} \dot{\mu}_{k j}
$$

Similarly the total mass that migrates away from a region $j$ is the sum of the masses that move into the other regions and is expressed as:

$$
\dot{\mu}_{\text {out . j }}=\sum_{\substack{k=1 \\ k \neq j}}^{4} \dot{\mu}_{j k}
$$

Notice the difference in the order of the $k$ and $j$ indices in eq. (6.8) and (6.9).

As we mentioned before, no tracer gas was injected in region 4 (see Fig. 6.1). However, the contribution of this region to the total migration is included in the calculation by linear extrapolation from the measurements in the other three 
regions.

It should also be pointed out that both the total mass flow through the compressor and the mass flow of the injector change with time during the Blowdown tests. This cannot be avoided, if we want to keep the corrected mass flow through the compressor constant and at the same time match the injection jet velocity to the free stream one. A typical deviation from a mean value for both mass flows during the useful test time is less than 5\%. However, the calculation described in this section is not expected to give results more accurate than $5 \%$. Therefore we believe that using a mean value for both mass flows introduces only a negligible additional uncertainty.

Equation (6.7) was applied for all $k$ and $j(1$ to 4$)$ and a matrix of migrating mass flows was formed. The following Table 6.1 presents the results of this calculation in compact form: 
Table 6.1: Matrix of migrating fluid in the rotor. All the numbers are percentages of the total mass flow through the compressor.

\begin{tabular}{|c|c|c|c|c|c|}
\hline \multirow[b]{2}{*}{$\begin{array}{l}\text { inlet } \\
\text { region } \\
k\end{array}$} & \multicolumn{4}{|c|}{ outlet region, $j$} & \multirow[b]{2}{*}{$\begin{array}{l}\text { mass } \\
\text { out }\end{array}$} \\
\hline & 1 & 2 & 3 & 4 & \\
\hline 1 & - & $1.1 \%$ & $0.8 \%$ & $0.5 \%$ & $2.4 \%$ \\
\hline 2 & $1.8 \%$ & - & $0.8 \%$ & $0.6 \%$ & $3.2 \%$ \\
\hline 3 & $1.7 \%$ & $1.9 \%$ & - & $0.6 \%$ & $4.2 \%$ \\
\hline 4 & $1.7 \%$ & $1.8 \%$ & $1.9 \%$ & - & $5.4 \%$ \\
\hline mass in & $5.2 \%$ & $4.8 \%$ & $3.5 \%$ & $1.7 \%$ & \\
\hline
\end{tabular}

The column on the right and the row in the bottom of the table present the mass flows that move in or out from the four regions. These numbers were obtained using equations (6.8) and (6.9). It should be pointed out that mass continuity is satisfied, since the total amount of fluid that migrates away from all the regions (sum of the numbers in the fifth column of Table 6.1) is equal to the total amount of fluid that migrates into them (sum of the numbers in the fifth row of Table 6.1).

Fig. 6.2 presents a schematic drawing of the migration process. Notice that the numbers are percentages of the mass that enters the compressor at each individual region, and not of the total mass flow of the compressor. This was 
done in order to give a better graphic presentation of the migration process in the compressor. 


\section{2 - Mixing levels with and without the rotor}

A number of researchers [19], [20], [21], [22] have attempted to measure the contribution to time average mixing in multistage, axial compressors, from either convection by secondary flows or pure diffusion by turbulence. A comparison of our time averaged results with the published data would serve as a check on the validity of our measurements.

In general, following Hinze [60] the turbulent diffusion of matter or heat emitted continuously from a fixed source in a turbulent flow of mean velocity $U$, can be described by the following differential equation:

$$
U \frac{\partial P}{\partial x_{1}}=\epsilon \frac{\partial^{2}}{\partial x_{i} \partial x_{i}} P
$$

where $x_{1}$ is in the direction of the mean flow, $\epsilon$ is a diffusion constant (coefficient of eddy diffusion in a turbulent flow) or, as shown later, a mixing coefficient, and $P$ is the mean concentration of fluid particles at a point $\left(x_{1}, x_{2}, x_{3}\right)$, which is identical with the probability of finding a marked fluid particle at that point.

Adkins and Smith [19] followed this idea and modeled the spanwise mixing as a diffusion process. The calculation of the mixing coefficient $\epsilon$ was based on a semi-empirical evaluation of the spanwise velocities. In particular, they calculated $\epsilon$ using the following equation: 


$$
\epsilon=\frac{z}{a} \int_{0}^{a} \frac{w^{2}}{u^{2}} d x
$$

where $w, u$ are the velocities in the spanwise $(y)$ and axial ( $z$ ) direction, respectively, $x$ is the cross passage direction and $a$ is the passage width at the blade exit

Gallimore and Cumpsty calculated the same normalized mixing coefficient using the observed spreads of the concentration contours of a tracer gas (ethylene in this case). They assumed that the tracer gas diffused from a point source in a uniform flow with velocity $U$. In this case, a solution of equation (6.10) can be found as in the following equation:

$$
P\left(x_{1}, x_{2}, x_{3}\right)=\frac{S}{4 \pi r \epsilon} \exp \left[-U\left(r-x_{1}\right) / 2 \epsilon\right]
$$

where $S$ is the volume flow rate of the source and $r^{2}-x_{j} x_{i}$. This model did not account for any radial variation in the value of $\epsilon$ and its constant normalized value was found to be equal to $1.8 \times 10^{-3}$ for the first test compressor and $3.8 \times 10^{-3}$ for the second one. In addition, they proposed an approximate method for calculating the mixing coefficient for a multistage compressor. From their results they concluded that a random, turbulent type of diffusion process is the dominant mechanism of spanwise mixing and that the physical model of spanwise mixing by Adkins and Smith, based on deterministic, radial secondary flows was inaccurate and invalid.

An additional comment on this came from Wisler, Bauer and Okiishi [22] who 
performed an extensive and detailed experimental investigation, using both ethylene injection and hot wire measuremetns to determine the relative importance of convection by secondary flows and diffusion by turbulence as potential mechanisms of mixing. They used both the Adkins-Smith and Gallimore-Cumpsty models to evaluate the normalized mixing coefficient for their compressor. The results of these calculations are shown in Fig. 6.3. In the same figure the results of their calculations of the normalized mixing coefficient, using the spreading of the ethylene contours from their experiments and equations (6.10) and (6.12), are also presented. It can be observed that convection by secondary flows (Adkins-Smith) dominates the mixing process close to the endwalls, while turbulent diffusion (Gallimore-Cumpsty) takes over in the midspan region. Therefore it was concluded that both secondary flow and turbulent diffusion must be included in order to evaluate correctly the mixing process in multistage, axial compressors.

A basic mixing level through our transonic fan can be obtained by applying the same ideas discussed above. In particular, equation (6.10) can be used in the same way that was used in the Gallimore-Cumpsty model. The only difference is that we solve this partial differential equation, not for a point source of strength $S$, but rather for a line source of strength $F$ per unit length. The solution in this case is given by Hinze again as follows:

$$
P\left(x_{1}, x_{2}\right)-\frac{F}{2 \pi \epsilon} K_{0}\left[\frac{U\left(x_{1}^{2}+x_{2}^{2}\right)^{1 / 2}}{2 \epsilon}\right] \exp \left(\frac{U x_{1}}{2 \epsilon}\right)
$$

where $x_{1}$ is in the flow direction, $x_{2}$ is in the normal direction and the line source is placed along the $x_{3}$ direction (see Fig. 6.4). $K_{0}$ is the modified Bessel function of the second kind and of zero order. Since, in general, $K_{0}(t) \rightarrow \sqrt{ }(\pi / 2 t) \exp (-t)$ as 
$t \rightarrow \infty$, the solution (6.13) can be simplified for small $\epsilon / \mathrm{UX}_{1}$ and not large values of $x_{2} / x_{1}$ (which is true for our case), into:

$$
P\left(x_{1}, x_{2}\right)=\frac{F}{2\left(\pi \epsilon U \mid x_{1} 1\right)^{1 / 2}} \quad \exp \left[-\frac{U x_{2}^{2}}{4 \epsilon\left|x_{1}\right|}\right]
$$

Following similar procedure with [20] we can finally give an expression for the nondimensional spread $x_{2} / L$ (spanwise direction in our case) of the tracer gas as a function of downstream streamwise nondimensional distance $x_{1} / L$, normalized mixing coefficient $\epsilon / U L$ and $C-P\left(x_{1}, x_{2}\right) / P_{\max }$ :

$\frac{x_{2}}{L}=\left[-4 \frac{\epsilon}{U L} \frac{\left|x_{1}\right|}{L} \ln C\right]^{1 / 2}$

where $L$ was chosen to be the axial length of the rotor and $P_{\max }$ was assumed to occur along the $x_{1}$ axis (flow direction).

Equation (6.15) was used to estimate the radial variation of mixing coefficient from the observed tracer gas spreads presented in Chapter 5. A typical concentration contour plot is presented in Fig. 6.5 in order to illustrate the details of this calculation. The value of $x_{2}$ for the tracer gas contours was taken to be the minimum distance from the core to the $20 \%$ of the peak value contours (Fig. 6.5). Although this choice cannot be rigorously justified, it was necessary in order to be consistent with that of Wisler, Bauer and Okiishi. This calculation was performed for the three injection tests (tip, middle,hub) and for the case without the rotor in place. The results are shown in Fig. 6.3, as filled circles for the three injections and as an open circle for the "no rotor" case. Notice that these values 
of the mixing coefficient represent only the turbulent diffusion contribution to the mixing process.

From this figure it is clear that the average mixing level in the compressor due to turbulent diffusion, is slightly higher than the one measured by Wisler (curve W\&B\&O in Fig. 6.3) in a low speed multistage compressor. This higher level of turbulent diffusion can be attributed to the action of the rotor only, since the free stream oncoming turbulence intensities in the Blowdown Facility is quite lower than the one used by Wisler $(2.6 \%$ in Wisler's tunnel [22] and $0.6 \%$ in the Blowdown Facility).

In addition, to this calculation we used the method proposed by Gallimore and Cumpsty [20] to predict the turbulent mixing coefficient its value can be estimated from:

$$
\frac{\epsilon}{U L}=\frac{A t}{L}\left[\frac{2 \omega\left(\frac{t}{L}\right)}{3 \phi^{2}}\right]^{1 / 3}
$$

where $t$ is the blade thickness, $\omega$ is the loss coefficient, $\phi$ the flow coefficient and $A$ is given by:

$$
A=\left[\frac{\overline{u^{\prime} v^{\prime}}}{q^{2}}\right]^{1 / 2}
$$

where $u^{\prime}, v^{\prime}$ are the fluctuating components of the velocity and $q$ is defined as: 


$$
q^{2}=\frac{\overline{u^{\prime 2}}+\overline{v^{\prime 2}}+\overline{w^{\prime 2}}}{3}
$$

The quantity $u^{\prime} v^{\prime} / q^{2}$ in eq. (6.17) has been measured in the turbulent flow near walls and found to be approximately 0.2 [61] which gives $A$ as approximately equal to 0.4. Using the values for $\omega, \phi$ and $t$ for our compressor, along with the value of 0.4 for $A$, the turbulent mixing coefficient for the fan was found to be equal to approximately $3.2 \times 10^{-3}$. Since the model does not predict any variation of the mixing coefficient in the spanwise direction this value is assumed constant radially and it is shown in Fig. 6.3 with a dashed line. As it can be seen from this figure, the values of the turbulent mixing coefficient during our tests are relatively close to the prediction from the Gallimore-Cumpsty model (dashed line). However, the uncertainty involved in the determination of the value of $A$ in the compressor limits the credibility of this prediction.

The following table summarizes values of the turbulent mixing coefficient as calculated or measured, from [20] and this work in the Blowdown Facility: 
Table 6.2: Values of mixing coefficient defined in eq. (6.16).

\section{Value of $\epsilon / U L$}

Turbulent flat plate boundary layer mixing, as calculated by Gallimore and Cumpsty [20] using parameters from Compressor A $3.2 \times 10^{-4}$ and from Compressor B $7.8 \times 10^{-4}$

Two dimensional wake mixing calculated by Gallimore and Cumpsty [20] for both Compressors $2.7 \times 10^{-3}$

Measured ethylene spreads by Gallimore and Cumpsty [20] in Compressor $\mathbf{A}$ $1.8 \times 10^{-3}$

in Compressor B $3.8 \times 10^{-3}$

Predicted from equation (6.16) by Gallimore and Cumpsty [20] for Compressor A

$1.6 \times 10^{-3}$

for Compressor B

$2.1 \times 10^{-3}$

Predicted from equation (6.16) for the Compressor tested by Wisler [22]

$1.75 \times 10^{-3}$

Spanwise average of the

Adkins-Smith prediction

for the Compressor in [22]

$2.57 \times 10^{-3}$

Predicted from equation (6.16) for the Blowdown tests

$3.2 \times 10^{-3}$

Measured during the "no rotor" tests in the Blowdown - two dimensional jet mixing $5.5 \times 10^{-4}$

The important information that can be extracted from Fig. 6.3 is that the turbulent mixing coefficient in a transonic fan has values higher than the ones in 
conventional low speed compressors by a factor of approximately two. (To our knowledge, this is the first time that the value and the spanwise distribution of the turbulent mixing coefficient was experimentally evaluated in a high speed, transonic compressor.) Note that in the Blowdown Facility the wall boundary layers are bled off approximately 4 rotor chords upstream from the rotor leading edge. Therefore the inflow is uniform and the effect of secondary vorticity generated in the passage due to inlet vorticity is negligible. This implies that the mixing in the facility is dominated by other mechanisms. One of them, the turbulent diffusion, was investigated in this section and its magnitude was presented in Fig. 6.3. However, the structure evident in the concentration contours presented in Fig. 6.5, is clearly not due to diffusion alone. For uniform inflow, the shape of the concentration contours can be attributed to deterministic transport phenomena, such as boundary layer cross flows, radial flows induced by the rotation (relative eddy), flows in separated regions or flows in the cores of spanwise coherent vortices shed by the blades. These will be discussed in the section 7.1 with the exception of the latter which will be discussed in section 7.2. 


\section{CHAPTHR 7}

\section{MECHANISMS OF SPANWISE FLUID TRANSPORT}

\section{1 - Estimates of the spanwise migration due to various mechanisms}

In this section we will attempt to estimate the contribution to spanwise flows from a number of possible mechanisms, such as boundary layer flows, tip clearance vortex flows, secondary flows, relative eddy flows and flows in regions of separation. These calculations are only approximate and their purpose is not to give an exact prediction of the induced spanwise flows, but rather to identify which mechanism may dominate. In addition, it is highly desirable to compare the spanwise flows induced by all these mechanisms to the measured spanwise migration from the tracer gas experiment.

\subsection{1 - Spanwise flows in the blade boundary layer}

A great number of researchers have been involved with blade boundary layer flows. We chose to adopt the method proposed by Adkins and Smith [19]. Their approach is based on the assumption that the viscous stresses can be neglected. The spanwise acceleration of a small mass of fluid in the boundary layer is then calculated at a representative point along the blade chord. If this acceleration is assumed to act over the time it takes for the small mass to travel a representative distance downstream, then its spanwise velocity can be calculated. This velocity is then compared with experimental data and a constant is chosen. Their model uses the momentum equation in the rotating frame for 
both the free stream and the boundary layer fluid. After some manipulation their final expression for the spanwise velocity in the boundary layer is:

$$
\begin{aligned}
\frac{D w_{r}}{W_{z}}=\frac{1}{k_{w}} & \left\{\frac{D W_{r}}{W_{z}}-\frac{D z}{r}\left[\tan ^{2} \beta_{z}\left(1-k_{w}^{2}\right)+\right.\right. \\
& \left.\left.+2 \tan \beta_{z} \frac{\omega r}{W_{z}}\left(1-k_{w}\right)\right]\right\}
\end{aligned}
$$

where the operator $D$ denotes substantial derivative, $w_{r}, w_{z}$ are the spanwise and axial velocities in the boundary layer, $W_{r}, W_{z}$ are the spanwise and axial velocities in the free stream, $\omega r$ is the wheel speed, $\beta_{z}$ is the relative flow angle and $k_{w}$ is a constant defined as in the following equation:

$$
k_{w}=\frac{w_{2}}{w_{z}}
$$

This constant is selected in the model such that equation (7.1) gives a spanwise velocity that is in reasonable agreement with experimental measurements of the peak spanwise velocity in the wake.

Equation (7.1) can be further simplified if we neglect the first term on the right hand side. This can be safely done if there are no pronounced spanwise shifts of the streamlines. The axial distance over which the spanwise acceleration is assumed to act, Dz, was taken to be approximately equal to $50 \%$ of the axial chord projection. This distance is assumed to extend from the middle of the chord up to the trailing edge of the blade. We chose to use mean values between mid-chord and trailing edge for $\beta_{2}$ and $W_{2}$. One of the most important 
inputs in this model is the value of $k_{w}$ Lack of detailed measurements in the blade boundary layer for our rotor, led us to adopt the same value that Adkins and Smith did, that is $k_{w}-0.5$.

All these quantities were input in equation (7.1) and the spanwise velocity in the boundary layer was found to be equal to approximately $12 \%$ of the axial free stream velocity $\left(W_{2}\right)$. Given the time available for a small mass fluid to move from mid-chord to the trailing edge, a spanwise migration results of about $6 \%$ of the blade span at the trailing edge of the rotor, which is insignificant compared to the measured migration towards the tip of about $40 \%$ of the blade span in the case of hub injection.

Thompkins and Usab [5] calculated the spanwise velocity in the blade boundary layer of a similar transonic rotor (NASA Low Aspect Ratio stage), using a quasi-three dimensional computer code. They found that the spanwise velocity was approximately equal to $15 \%$ of the free stream axial velocity. Their result is very close to the prediction of the Adkins and Smith model. The important point, however, is that the spanwise velocity in the blade boundary layer is not strong enough to account for the spanwise migration that we observed in our measurements, which amounts to approximately $40 \%$ of the blade span at the rotor trailing edge.

\subsection{2 - Spanwise flows due to the tip clearance} vortex

An enormous number of publications exist in the literature about the tip clearance vortex. In addition, many models have been proposed to predict the 
behaviour of this vortex and its effect on the stage performance. Since the subject of this Chapter is not an exact calculation of the velocities induced by the tip clearance flow, but rather an approximate estimate of them, we felt that any model would serve this purpose. In particular, we decided to adopt the Lakshminarayana model, described in [12], for the prediction of spanwise and pitchwise velocities.

First, the hot tip clearance at design speed was predicted using the information from the Air Force design report of the rotor. An approximate value of 0.015 in $(0.38 \mathrm{~mm})$ was obtained, giving a clearance to blade spacing ratio at the tip of approximately 0.006. In the Lakshminarayana model an empirical factor, $K$, is introduced and an empirical expression is given for it, under the assumption that $K$ is only a function of the clearance to blade spacing ratio. According to [12] this expression is valid for values of clearance to blade spacing ratios from 0.01 to 0.1. Although this ratio is smaller than 0.01 in our case, we felt that the model would still give us satisfactory estimates of the induced velocities.

The procedure described in [12] was then followed step by step and analytical expressions were obtained for the spanwise and pitchwise velocities. In addition, the tip clearance vortex core was calculated to be located at about $25 \%$ of the blade pitch away from the suction side of the blade in the pitchwise direction and $5 \%$ of the span away from the tip casing. The spanwise velocity induced by this vortex on the suction side of the blade was found to have a maximum value at the same spanwise distance from the tip with the core of the vortex and a direction towards the tip. Its magnitude was found to be approximately equal to $23 \%$ of the tip blade speed. This velocity is quite high, but its action is only local and limited. For example, at a spanwise location $90 \%$ of 
the blade span from the hub the induced spanwise velocity on the suction side is reduced to $0.7 \%$ of the tip blade speed, which is almost negligible. On the pressure side of the blade the maximum induced spanwise velocity was found to be towards the tip and equal to $0.9 \%$ of the tip blade speed, hence almost negligible.

The presence of the tip clearance vortex might be responsible for the substantial difference between the concentration traces for tip and midspan or hub injections, as shown in Fig. 5.30. We believe that the absence of tracer gas next to the pressure side of the blade passage is an indication of the removal action of the tip vortex, which moves the fluid from the pressure side through the gap into the suction side of the adjacent blade passage. In addition, in the case of the tip injection the action of the tip leakage vortex is indicated by the small spreading of the concentration contours. The fluid particles, marked as tracer gas, are trapped into the strong tip vortex and remain there, until they are sampled by the aspirating probe.

In conclusion, we believe that the tip vortex induces quite strong spanwise and pitchwise velocities. The action of the tip vortex might be responsible for the absence of tracer gas from the pressure side of the blade. In addition, it is the main reason for the limited spreading of the tracer gas contours for the tip injection. However, we do not think that it is responsible for the strong spanwise transport observed in the measurements, since the action of the tip leakage vortex is limited in the tip region only and both spanwise and pitchwise induced velocities become less than $1 \%$ of the tip blade speed (a negligible magnitude) at approximately $85 \%$ of the blade span from the hub $\left(R / R_{t}=0.93\right)$. 


\subsection{3 - Spanwise flows due to "classical" secondary flows}

The term "classical" is used in this case to characterize the secondary flows due to both the original inlet streamwise vorticity and the subsequently developed one, due to the turning in the blade passage. Fig. 7.1 shows a schematic drawing of the vorticity vectors and the blade arrangement at the inlet and exit of the blade row. The streamwise vorticity at the exit is composed of two parts. The first is due to the inlet vorticity and is simply given by the following equation:

$$
\omega_{: 2}^{\prime}=\omega_{s 1} \frac{W_{2}}{W_{1}}
$$

where the notation is explained in Fig. 7.1. The second part of the exit streamwise vorticity is due to the turning in the blade passage of the normal component of inlet vorticity $\omega_{n 1}$. Since the turning in a compressor blade is generally small, the streamwise vorticity developed by this mechanism can be given by the approximate formula of Squire and Winter [8], as in the following equation:

$$
\omega_{: 2}^{\prime}=-2 \in \omega_{n 1}
$$

where $\epsilon$ is the turning in the blade row lapproximately equal to the camber of the blade airfoil). The total streamwise vorticity is given then as the sum of the two components as follows: 


$$
\omega_{: 2}=\omega_{82}^{\prime}+\omega_{s 2}^{\prime \prime}
$$

The induced velocities due to this streamwise vorticity can be calculated using a conventional stream function $\Psi$, which is the solution of the following Poisson equation:

$$
\nabla^{2} \Psi=-\omega=2
$$

In the Blowdown Facility the casing boundary layer is bled off right before the entrance to the rotor. However, there is still a distance left from the bleed-off point to the blade location, giving rise to a boundary layer on the tip casing. An additional boundary layer is developed at the hub. Both boundary layers were taken into account in order to determine the inlet vorticity. Then equation (7.6) was solved using a conventional Fourier method. The maximum spanwise velocity estimated from this calculation was less than $4 \%$ of the blade tip speed. However, the spanwise velocities induced by this mechanism are essentially local and concentrated near the tip and hub regions. Outside these regions the magnitude of these velocities drops to very low, almost negligible values. Therefore, except from the case of tip injection, their effect is expected to be negligible. In the tip injection case the magnitude of the "classical" secondary flow due to the casing boundary layer is expected to be of second order compared to the effect of the tip clearance vortex. We believe that the flow pattern near the tip region is dominated by the presence of the tip clearance vortex 


\subsection{4 - Spanwise flows due to the relative eddy}

The term "relative eddy" is used here to describe the presence of relative vorticity in the rotating frame of reference. The relative vorticity, $\zeta$, is generally given by the following expression:

$$
\vec{\zeta}=\vec{\omega}-2 \vec{\Omega}
$$

where $\vec{\omega}$ is the vorticity vector in the absolute frame and $\vec{\Omega}$ is the angular velocity of the rotor. The streamwise component of this vector equation gives the relative streamwise vorticity, $\zeta_{8}$. Notice that even in the case of no absolute vorticity, there is still vorticity in the rotor frame equal to $-2 \vec{\Omega}$. Therefore secondary flows can develop due to this vorticity.

The existence of the relative eddy has been known for many years and its effect on the three dimensionality of the flow in axial machines was found to be negligible. However, fairly recently Dring and Joslyn [10] demonstrated both experimentally and theoretically that this mechanism could be responsible for strong spanwise flows on the pressure and suction surface of an axial turbine rotor blade. Maximum spanwise velocities of about $13 \%$ of the wheel speed were reported in [10] Since these velocities were quite high, it was felt that an estimate of the spanwise velocities induced in the passage due to the "relative eddy" was necessary.

A similar procedure with the one followed in [10] was adopted to calculate the induced spanwise velocities with all the appropriate inputs adjusted to fit the fan characteristics. The maximum spanwise velocities were found to occur at 
midspan next to the pressure and suction sides of the blade passage with directions towards the tip on the suction side and towards the hub on the pressure side. At that spanwise location these velocities were found to be approximately equal to $10 \%$ of the blade tip speed. If this velocity is assumed to act upon the fluid particle throughout its travel through the blade row, the maximum expected spanwise migration would be approximately $15 \%$ of the blade span at the rotor trailing edge, which is less than the measured one $140 \%$ of the span), but still a significant spanwise displacement of the fluid particles on the pressure and suction sides of the blade. It should also be pointed out that on the pressure side the motion due to the relative eddy is opposite to the one due to the centrifugation of the boundary layer. Therefore on the pressure side the two mechanisms compete with each other, while on the suction side they reinforce each other.

\subsection{5 - Spanwise flows in regions of separation}

This kind of spanwise flows are probably the hardest to predict, since the location and behavior of the three dimensional separation line on the blade is still an open question among researchers. In the case of a transonic rotor the separation issue becomes more complicated, since its onset and strength depend heavily on the location and strength of the shock system that exists in the rotor. Therefore research efforts like the ones presented in [62] that are limited to low speed machines, may not be extendable to our case. Fortunately, a great number of both experimental and computational results are also available for this particular Air Force High Through Flow transonic rotor [63], [64] and for similar ones like the NASA Lewis transonic fan rotor [17], [65] 
Three dimensional, viscous, steady computer codes are generally available to calculate the location of the blade separation line in the spanwise direction [18], [5] In addition, 2-D, unsteady, viscous codes are common and have been widely used to calculate the flowfield in transonic compressors [66], [67] However, the two dimensional calculations for the separation line are substantially different [5] from the three dimensional ones (3-D separation region two to three times larger than the 2-D one). On the other hand, even in the case of a full 3-D, viscous calculation the turbulence model that is being used is probably the dominant factor as far as spanwise location and shape of the three dimensional separation line is concerned. Hence, it would be pointless to embark into a complete 3-D, viscous calculation and realize in the end that it is still a crude approximation of the real picture.

The spanwise velocity that can be experienced by a fluid particle that enters a region of separated flow can be estimated as follows. The radial (spanwise) momentum equation is given below:

$V_{r} \frac{\partial V_{r}}{\partial r}+V_{z} \frac{\partial V_{r}}{\partial z}+\frac{V_{\theta}}{r} \frac{\partial V_{r}}{\partial \theta}-\frac{V_{\theta}^{2}}{r}=-\frac{1}{\rho} \frac{\partial p}{\partial r}+F_{r}$

(3)

(4)

(5)

(6)

where $r, \theta, z$ are cylindrical coordinates, $V_{r}, V_{\theta}, V_{z}$ the corresponding velocities, and $F_{r}$ the blade force. This force is in general composed of normal and tangential components. However, in most applications the normal component of $F_{r}$ can be neglected [68], while in the case of a separated flow the same is also true for the tangential one. Therefore no blade force (term (6)) in eq. (7.8) will be included in the calculations. We now assume that the fluid particle acquired blade velocity, equal to $\Omega r$. Term (3) in eq. (7.8) can be neglected if we consider an 
axisymmetric case. In addition, term (2) can be neglected if we assume that the axial velocity of the fluid particle is very small. The radial pressure gradient, term (5), is taken to be equal to the free stream one, since we assumed that the pressure in the separated region is almost constant A simple order of magnitude argument can now be applied for the terms that are left in equation (7.8) and a typical spanwise velocity is calculated.

Although this velocity came out to be very high, it should be noted that the time interval during which it acts upon the fluid particle is of primary importance as far as the total spanwise migration of this particle is concerned. However, this is quite hard to evaluate since neither the axial velocity in the separated region, nor the location of the separation line are accurately known. According to most of the available data from transonic rotors [5], [18], the pressure side boundary layer is very thin and does not separate before approximately $90 \%$ of the blade chord, giving almost no time for the fluid particles to move in the spanwise direction. On the suction side the boundary layer is quite thick and tends to separate much earlier. In the case of a transonic rotor the presence of the shock makes things more complicated. The shock impinges on the suction side of the blade in a direction almost normal to the blade surface [17], [65]. According to [69], [5], [17] for a rotor similar to ours at approximately the same operating conditions, the point of shock impingement on the suction side changes from the front part of the blade near the hub (10\% of the chord from the leading edge) to the aft portion of the blade near the tip lapproximately $85 \%$ of the chord from the leading edge). In addition, the shock strength was found to be high enough to cause separation of the boundary layer at the point of impingement. This can also be observed in the computational results presented in [18] from a 3-D, steady, viscous computer code, where the boundary layer separates at the point of shock 
impingement.

In order to calculate the total spanwise displacement of a small mass of fluid in the region of separation, the time interval during which the pressure gradient (term (5) in eq. (7.8)) acts upon it, must be known. Consequently, the point of separation and the convective velocity of this small mass of fluid must be known. Lack of this kind of information, led us to assume that the convective velocity of the fluid in the region of separation is equal to the free stream one. This assumption gave an upper bound for the convective velocity and a lower bound for the convection time and the spanwise displacement of the small mass of fluid. Under this assumption, if the flow near the hub separates at $10 \%$ of the chord from the leading edge, the spanwise velocity, estimated through eq. (7.8), resulted in a spanwise displacement equivalent to $65 \%$ of the blade span at the trailing edge, which is 1.6 times higher than the one we measured. On the other hand, if the flow separates at $65 \%$ of the chord from the leading edge, as it might happen in the midspan region, a spanwise migration of $46 \%$ of the blade span would be feasible. As a reminder, the measured spanwise displacement towards the tip, was as high as $40 \%$ of the blade span in the case of hub injection.

This order of magnitude analysis shows the strong effect that the regions of separation can have on the spanwise migration in transonic fans. The presence of the shock might be responsible for early separation on the suction side of the blade. However, on the pressure side the separation is very small and its effect on the spanwise migration can be practically neglected. Therefore, we believe that the strong migration to the tip can be fully explained, if the flow separates early enough on the suction side of the blade. The strong migration to the hub cannot 
obviously be explained by this mechanism, but it will be the subject of discussion for the next section. 


\section{2 - Modeling of the spanwise vortex street wake}

One additional mechanism of radial transport is associated with the shedding of vortices in the rotor blade wakes. Assuming these vortices are coherent along the blade span, a spanwise variation of their strength leads to a pressure gradient along the span. This can be explained as follows. At each spanwise location the pressure drop in the core of the spanwise vortex is a function of the vortex strength. Therefore, if the strength of the vortex varies in the spanwise direction, the pressure drop in its core will vary too, thus generating a spanwise pressure gradient Under the action of this pressure gradient, fluid entering the vortex cores moves radially. Prediction of the direction of this motion (towards the hub or towards the tip) requires knowledge of the characteristics of the vortex (as will be presented later in this section). This phenomenon is similar to the axial flow in the cores of trailing vortices behind three dimensional wings. 


\subsection{1 - Vortex shedding and 2-D modeling of the rotor wakes}

Von Karman type vortices have been observed to occur behind bodies with blunt trailing edges, due to intermittent separation of the surface boundary layers in the vicinity of the body's trailing edge. This phenomenon is quite well known and a great number of researchers have investigated the characteristics of the vortex street that is usually formed behind these bodies.

Similar structures have also been observed in the wakes of flat plates ([70], [71], [72], [73]) and airfoils with blunt trailing edges ([74], [75], [76], [77]), both isolated and in cascade. However, Gertz [2] was the first to identify regular arrays of vortex streets in the wakes of a transonic rotor. This was achieved by using the results of Laser Anemometry (LA) measurements of that rotor as presented in [78], [79]. These measurements revealed the presence of a regular array of vortices through the bi-modal character of the velocity probability density distribution (PDD). Fig. 7.2 and 7.3 from [2], present this experimental finding. Gertz was able to infer the characteristics of the von Karman street from the LA measurements. He modeled the rotor blade wakes as modified ideal von Karman vortex streets consisting of two staggered rectilinear rows of Rankine vortices of opposite sign in a uniform free stream. The flow in the cores was considered to be solid body rotation, while in the region outside the cores a potential free vortex flow was chosen. Although a complete and detailed description of the model fitting procedure is given in [2], a brief one will be given here since familiarity with the basics of that model is important in understanding our model, which is an extension of the model proposed by Gertz. Fig. 7.4 is a schematic drawing of the simplified von Karman vortex street behind the blade 
and shows important parameters used in this model. Three basic parameters were chosen to characterize the vortex street, the core-radius ratio, $r_{0} / h$, the vortex strength, $k$, and the spacing ratio, $h / a$. The core-radius ratio, $r_{0} / h$ and vortex strength, $k$, were chosen in order to match the shape and depth of the average wake profile as measured by the LA. The spacing ratio was then chosen such that the probability density distribution of the velocity at the wake centerline approximates the bi-modal distribution measured by the LA. This was accomplished by matching both the upper and lower most-probable velocities. The core-radius ratio was chosen to be equal to 0.5 , in which case the edges of the vortex cores on both upper and lower rows of the street coincide with the wake centerline. The final values of the model parameters for the NASA LeRC Stage 67 at $60 \%$ span (modeled by Gertz) are given in Table 7.1.

Table 7.1: Vortex Street Parameters for the NASA LeRC Stage 67

$\begin{array}{ll}\text { Spacing Ratio }(\mathrm{h} / \mathrm{a}) & -0.635 \\ \text { Core-radius Ratio }\left(\mathrm{r}_{0} / \mathrm{h}\right) & -0.5 \\ \text { Pressure Defect Velocity Ratio }\left(U_{p} / U_{\infty}\right) & -0.2205 \\ \text { Vortex Strength }\left(k / 2 \pi a U_{\infty}\right) & -0.07\end{array}$

where $U_{\infty}$ is the free stream velocity in the relative blade frame and $U_{p}$ is the pressure defect velocity, which is the angular velocity at the edge of the vortex core and is equal to $\omega_{\circ} r_{0}$. The pressure defect velocity can also be expressed in terms of the vortex strength, $k$ : 


$$
u_{p}=\frac{k}{2 \pi r_{0}}
$$

Once the model parameters are chosen the vortex street flowfield is completely determined. A shedding frequency can also be determined from the model and a typical value of $15.8 \mathrm{kHz}$ was found in [2] for that particular spanwise location of the NASA Fan. The next step in Gertz's analysis was to "insert" a simulated probe, stationary in the absolute frame, behind the rotor blade and calculate what the "measured" instantaneous traces of various flow parameters would look like, if the probe cut through the vortex street of each blade in a random fashion. A comparison with the measurements from a real 4-way probe are given in detail in [2] This idea of a simulated probe will be very useful in our model.

\subsection{2 - A review of spanwise vortices shed behind bluff bodies}

The explanation for the motion in the cores of spanwise coherent vortices was given in the beginning of this Chapter. Several aspects of their structure, like the variation of their strength in the spanwise direction and their coherence in the same direction need some discussion. As the vortex core starts forming behind a separation point on the blade, a thin vortex sheet provides a flux of vorticity from the boundary layer to the core. For a steady separation, this flux is equal to one half of the square of the free stream velocity at the point of separation. If we assume that the vortex cores are regions of solid body rotation flow, then an indication of the vortex strength is the value of the circulation in the core. 
Due to vorticity cancellation, the amount of core circulation in the near wake is in general less than the original one in the boundary layers. According to Cantwell and Coles [80], the measured circulation for a vortex in the near wake of a circular cylinder never exceeded $44 \%$ of the total circulation discharged from one side of the cylinder during a shedding cycle. Therefore the strength of the vortex or the circulation in the core cannot be determined just from the flow conditions on the blade. However, even if no vorticity cancellation took place, the strength of the vortex would vary in the spanwise direction, since the free stream velocity at the separation point, and hence the flux of vorticity into the core, varies in the spanwise direction. The radial twist and variation of turning in the blade row, the presence of a shock with radially variable strength, and the substantial variation in the spanwise location of the separation point all contribute to the spanwise variation of the free stream velocity at the separation point. An analytical approach to the problem is beyond the scope of this research effort In addition, for a complete calculation of the flux of vorticity from the blade boundary layer, the velocity of the separation point itself must be included in the calculation [81] To our knowledge, no reliable analytical or experimental information exists for this velocity. A 3-D, viscous, unsteady code may yield some information about the motion and location of the separation point, but this kind of calculation is still beyond the capabilities of today's supercomputers.

The spanwise coherence and structure of the vortices is an issue of great importance and interest A large number of publications on this subject exist in the literature. Before we give a brief literature survey, some basic definitions are needed. A useful quantity in the analysis of unsteady, periodic phenomena, like vortex shedding, is the Strouhal number defined as: 


$$
S t=\frac{f d}{U}
$$

where $f$ is the frequency of the phenomenon (shedding frequency), $d$ is a characteristic length of the flow (diameter in the case of shedding behind a circular cylinder) and $U$ a characteristic velocity (free stream velocity). In the case of vortex shedding from circular cylinders, it is observed that the Strouhal number based on the diameter has a value of approximately 0.2 . The Strouhal number is in general a weak function of the Reynolds number of the flow. Therefore for all practical purposes we can assume that its value is constant and equal to 0.2. This means that for fixed free stream velocity the shedding frequency of a circular cylinder with small diameter must be higher than the one with large diameter.

The wake behind a circular cylinder is basically three dimensional. Two-dimensional flow can be found only at very low Reynolds numbers, at which a periodic wake is formed. As the Reynolds number is increased, the three dimensional character becomes more and more prominent Tritton [82] was the first to discover that at $\operatorname{Re} \sim 90$ a transition occurs, where the vortices are no longer produced only as a result of wake instability, but the body itself starts playing a substantial role in their formation. An indication of the three dimensionality of the wake is the spanwise inclination of the vortex lines with respect to the axis of the cylinder. However, this phenomenon seems to depend on the flow and test conditions. Kovasznay [83] and later Phillips [84] found that the vortex lines were straight and parallel to the cylinder axis for at least 30 diameters. In both cases the Reynolds number was much below 90. On the other hand, for similar values of Re, Tritton found a tilted vortex configuration in which 
the vortex lines were sometimes inclined to the cylinder axis at angles up to $30^{\circ}$. Hama [85] found parallel vortices at Re=117, while Taneda [86] found parallel configuration at $\operatorname{Re}$ less than 60 , but inclined one at Re=75. According to [84] the parallel vortices could be observed at higher $\mathrm{Re}$, if the water was allowed to settle so as to be free from disturbances. Therefore, it was concluded that the straight and parallel vortex configuration can be observed only when the fluid is free from disturbances. However, Gerrard [87] suggested that the three dimensionality of the free stream close to the side walls of the tunnel might play a role in the formation of the vortices. Berger [88] showed in a wind tunnel that the vortex lines were inclined at about $19^{\circ}$ to the cylinder. When the cylinder was forced to oscillate the vortex lines became parallel to the cylinder axis

At Reynolds numbers between 90 and 150, many researchers find significant three dimensionality in the cylinder wake. A waviness in the spanwise direction is also observed. For Reynolds numbers around 200 we enter the irregular range, where vortices are composed of turbulent fluid. The three dimensional structure exhibits a chaotic nature and it is usually described in terms of correlation length. Some spanwise periodicity has been found by Mattingley [89] and Humphreys [90] in the form of a cellular pattern. Despite the transition to turbulence, periodic vortex shedding can be observed up to the highest Reynolds numbers (about 107) at which measurements have been made. There are only two ranges for the Reynolds number [91] from 200 to 400 and from $3 \times 10^{5}$ to $3 \times 10^{6}$, in which the regularity of shedding decreases. In the former the Strouhal number shows scatter, while in the latter, the periodicity is lost except very close behind the cylinder [92], [93] For Reynolds numbers higher than 106, Jones, Cincotta and Walker [94] gave important clues on the reappearing of regular vortex shedding. 
As far as the spanwise structure of the vortices is concerned, an additional complication arises when the cylinder is tapered in the spanwise direction or when the free stream velocity changes in the same direction. We will first discuss the results for the low Reynolds number flows (less than 200). If the two dimensional theory for the vortex shedding is correct, then the shedding frequency would be that appropriate to a two-dimensional body of diameter equal to the local diameter. As quoted by Gerrard in [87], this was Abernathy's (1964) conclusion from the photographs that he presented at the Ann Arbor IUTAM Conference. Gerrard [87] gave an explanation of the structure of the vortex lines behind a cylinder with variable diameter. It is obvious that in this case the shedding frequency at the larger diameters will be lower than the one at the smaller diameters. Therefore in the high shedding frequency region there will be more vortex lines than in the low frequency one. Continuity of the vortex filaments will not be violated, if the extra vortex lines loop over and join with the vortex lines of the opposite sign from the other side of the cylinder.

As we mentioned before, Tritton [82] reported a change in the flow mode at about Re=90 and was able to distinguish between the two modes. A low speed mode $(40<\operatorname{Re}<110)$, which is the result of an instability of the wake, developing from the flow around the cylinder with its attached pair of vortices, and a high speed one $(80<\operatorname{Re}<160)$, where the periodic shedding comes directly from the cylinder. Serious controversy arose when Gaster [95] reported on vortex shedding from slender cones. He found that the periodic hot wire signals in the wake had a superimposed beat that was constant along the span, whereas the vortex frequency changed and was related to a Reynolds number based on the local diameter of the cone. In addition, he was not able to detect the change in the flow mode, that was observed by Tritton. He concluded that the change from one 
vortex pattern to the other, might be related to nonuniformities in the flow. Tritton [96] repeated his experiments with new apparatus and found the same results with a circular cylinder as in his original work. To prove his point, Gaster [97] repeated his experiments with both weakly tapered and circular cylinders. In the case of the tapered cylinder he found that vortex shedding occurred in cells along the span of the cylinder, the frequency and amplitude being constant within a cell and changing from cell to cell. These cells appeared again when he introduced an artificial nonuniformity in the oncoming flow. Therefore he concluded that slight nonuniformities in the flow are responsible for the jumps in flow modes and street configuration that Tritton had observed earlier.

Coming back to Gaster's experiments, it is interesting to notice the coherence of the spanwise vortices during the tests of various models. In the case of slender cones there is strong coupling between flows at neighboring spanwise locations and the vortices are coherent over many cylinder diameters. The shedding motion expected from the quasi-cylindrical approach is very sharply defined by a near line spectrum with a center frequency that varies continuously along the cone. However, the coupling between regions of different characteristic frequency introduces a certain amount of amplitude modulation. This modulation frequency was found [95] to be independent of spanwise station. The vortices are shed in patches with the predominant frequency in each patch varying continuously along the model, while the repetition frequency of the packet remains constant In the case of a slightly tapered cylinder the shedding frequency is adjusted along the span so that it remains roughly compatible with the local diameter. This adjustment, however, unlike the slender cone case, was observed to occur through cells of finite size. The coherence of the vortices was lost in the transition regions between cells, but within each cell the vortices were coherent 
and the shedding frequency was constant These cells were found to be about 15 cylinder diameters long and their spanwise position was found to be insensitive to changes in the velocity of the tunnel flow. It is interesting that the dominant frequency in the transition regions was found to be precisely the one that would result from the summation of the two regular signals in neighboring cells. When the flow velocity was increased the structure became similar to that observed on slender cones. The same cell structure was also found in the case of a straight circular cylinder, when the oncoming velocity was artificially distorted. In this case, spanwise wandering of the transition regions between cells was observed to occur. However, the addition of small disks at the ends of the cylinder eliminated this wandering.

Gaster [98] repeated the experiments with slender cones in the case of high Reynolds number in the subcritical regime of $10^{3}$ to $5 \times 10^{4}$. In this case the vortices are expected to be turbulent and the use of correlation lengths is more appropriate in order to determine spanwise coherence. An important finding of [98] was that the frequency spectra showed a broadening tendency, although its center line was always found to correspond to a Strouhal number of 0.2 . This broadening was attributed to the strong three dimensional coupling. In order to answer the fundamental vortex filament continuity question, Gaster suggested that the frequency of shedding is adjusted through a pairing process, where a large number of weak vortices join to form stronger, but fewer new vortices, as they peel off into the wake.

The basic conclusion from the previous brief literature review is that vortex coherence is possible, but the conditions under which it happens and its detailed structure is still a subject of discussion and disagreement Note that the Reynolds 
number based on the blade chord in our test rotor varies from $3.8 \times 10^{6}$ at the tip to $1.3 \times 10^{6}$ at the hub. If the same number is based on the trailing edge thickness the Reynolds number becomes $0.9 \times 10^{4}$ at the tip and $0.5 \times 10^{4}$ at the hub. However, in [2] it was argued that the correct length scale in the case of a compressor blade is the boundary layer momentum thickness at the trailing edge. Based on that the Reynolds number becomes $3.7 \times 10^{4}$ at the tip and $1.7 \times 10^{4}$ at the hub of the rotor. Therefore it seems reasonable to argue that the only experiments with cylinders or cones that might be relevant to our case, are the ones reported in the last paper by Gaster [981, where the Reynolds number varied from $10^{3}$ up to $5 \times 10^{4}$.

However, the flowfield behind the rotor is extremely complicated and the well controlled test conditions of all the previous experiments with cones and cylinders are not even closely repeated in the case of the rotor. In addition, both cylinders and cones have zero loading, while the fan blades are heavily loaded. The boundary layers of a cylinder or a cone are thin compared to the local diameter. However, this is far not true in the case of a compressor rotor blade, which is usually very thin compared to its boundary layer. In addition, the contribution of the cylinder or cone wake to the flowfield around the body is quite large compared to that of a rotor wake. Therefore the structure observed in the wakes of cylinders and cones cannot be directly linked to the case of a rotor blade. On the other hand, even if the two cases were comparable, the significant disagreement among them would prevent solid conclusions in terms of the structure and coherence of the vortices in our case. Therefore we believe that it is beyond the scope of this project to attempt a detailed 3-D modeling of the spanwise vortices shed by the blade. We chose instead the simple quasi two-dimensional approach given below. 


\subsection{3 - Quasi 3-D modeling of rotor wakes vortices}

The model proposed and used by Gertz was also employed here to model the vortices in the spanwise direction. This was achieved by fitting it at four appropriately chosen spanwise locations. Each one of them was treated separately in a 2-D manner, since the Gertz model is inherently 2-D.

Gertz determined the characteristics of the vortex street by fitting the model to detailed laser anemometry (LA) measurements. Unfortunately we were not able to obtain similar measurements for the Air Force rotor. However, as Gertz [2] pointed out, the NASA rotor, where he fitted his model, and the Air Force rotor, tested in the MIT Blowdown Facility, are very similar. The flow path of the NASA rotor has a slightly decreasing tip radius and increasing hub radius, while the Air Force has constant tip radius and very steep radius contour at the hub. The pressure ratio for the NASA stage is 1.6, while the Air Force one is 2.0. Both stages have very high adiabatic efficiency close to $90 \%$. In addition, they are both transonic, although the Air Force rotor has a slightly higher inlet relative Mach number and the sonic line is at a lower $r / r_{t i p}$ location. The loading distribution in the NASA rotor is constant across the span, while the Air Force rotor has slightly higher loading at the tip. According to [2], the measurements of total pressure, static pressure and flow angle in both facilities are very similar, even though the NASA Facility is a steady state one, while the MIT Facility operates at a blowdown (short duration) mode. The latter result of Gertz's measurements is extremely important as far as data interpretations and conclusions from the Blowdown Facility are concerned.

All these arguments led us to conclude that regular vortex streets similar to 
the ones detected behind the NASA rotor, might also exist in the Air force rotor. In order to determine the parameters of the Gertz model, we decided to use the idea of an simulated probe, used by Gertz to compare the real measurements to the ones predicted by the model. In simpler terms, we inverted the fitting procedure relative to the one Gertz used. We first assumed that regular vortex streets exist behind the rotor blades and then used the simulated probe to survey the flowfield created by these vortices. The traces obtained by this simulated probe were compared to the data and the characteristics of the vortex street were appropriately adjusted so that both sets of traces would match as closely as possible. Vortex parameters were successfully determined with satisfactory level of uncertainty, as will be shown.

\subsection{4 - Fitting the vortex model to the experimental data}

The flow quantities that were measured during our experiments were time resolved total pressure, total temperature and concentration of species. It was felt that in order to be able to verify the predictions of the model, no more than two of the measured quantities should be included in the fitting procedure. We chose the total pressure and total temperature to be used for that purpose.

The model was fitted at four spanwise locations, $r / r_{t i p}=0.95,0.88,0.81$ and 0.75, covering the distance that the aspirating probe traverses during a typical Blowdown Test For simplicity, the core-radius ratio, $r_{0} / h$, was fixed and set equal to 0.5 at all four locations. In this case, the edges of the vortex cores on both rows of the street coincide with the wake centerline. After eliminating the core-radius ratio as a variable parameter, the fitting procedure was focused on 
determining the spacing ratio $(\mathrm{h} / \mathrm{a})$ and the pressure defect velocity ratio $\left(U_{\mathrm{p}} / U_{\infty}\right)$, as defined in section 7.2.1.

Although $\mathrm{h} / \mathrm{a}$ and $U_{\mathrm{p}} / U_{\infty}$ were the primary model parameters, a number of quantities that described the free stream in the relative frame were also needed. These were the relative tangential flow angle, $\beta_{\text {rel, }}$ the relative total Mach number, $M_{\text {rel }}$, the ratio of static pressure to absolute inlet total pressure, $p_{s} / p_{t 1}$, the wake to pitch ratio, $w / s$, and the wheel speed $U_{w}$ The first three of them were determined from Ng's [1] data on the same stage (we did not measure them during this set of experiments). However, given the excellent repeatability of the Facility, the similarities between our measurements and $\mathrm{Ng}$ 's in terms of total pressure and total temperature and the fact that the operating conditions were the same in both cases, we felt that most of the other flow quantities would be similar too and could be safely used in our case. The wake to pitch ratio was determined using the ensemble averaging technique described in Chapter 5, while the wheel speed was directly measured.

Although originally both total pressure and total temperature were considered as fitting parameters, the latter was not used for the following reasons. First, according to Gertz [2], the absolute total temperature comparison between data and model predictions were quite different as far as magnitude of the fluctuations are concerned. As it will be described later, the magnitude of these fluctuations is one of the matching requirements between data and model predictions. Second, the Gertz model uses constant static temperature in the cores of the vortices. Although this assumption satisfies the energy equation, it is quite far from a realistic representation of the flow. The invalidity of this assumption was recently supported by Mandella and Bershader [99], who measured the static 
temperature distribution of a compressible vortex. In their report a very steep static temperature gradient was observed in the vortex core, leading to a temperature drop in the core of about $80^{\circ} \mathrm{C}$ or $27 \%$ of the free stream temperature. Since in our case the Mach numbers of the vortices appear to be on the high subsonic regime, they must be considered as compressible ones and, hence, similar to those described in [99]

The fitting procedure follows a number of steps. First, a segment from the instantaneous total pressure ratio data is chosen at each of the four radial locations that the model is fitted. A typical data segment extends for about 3 msec or 15 blade passages. The relative frame free stream flow parameters, along with the wake to pitch ratio and the wheel speed for that radius, are then specified. A first guess is then given for $h / a$ and $U_{p} / U_{\infty}$. The total pressure ratio as predicted by the model is calculated and compared with the data segment from the measurements. There are a number of criteria that must be fulfilled in order to consider the matching satisfactory. First, the deepest trough and highest peaks in this data segment must match with the predicted ones from the model. Second, a qualitative similarity in shape must be achieved between model prediction and data. For example, in some cases the peaks of total pressure are more prominent than the troughs or in other cases there are very few peaks of total pressure and troughs tend to dominate the trace. Two additional complications arise during this procedure. First, the relative frame free stream flow parameters change from blade to blade and, second, the free stream region is hard to be precisely identified. Therefore, we decided not to chose single values for the free stream flow parameters, but rather a range of values for each parameter. Consequently an iterative scheme is employed that scans through these ranges and after fitting for $h / a$ and $U_{p} / U_{\infty}$ at each point in the range of input values, choses the best fit 
as a final answer. Although this procedure seems to be very tedious, intuition and experience quickly eliminated many combinations of input values, simplifying things considerably. In practice, the final values of $h / a$ and $U_{p} / U_{\infty}$ appear to be unique.

An uncertainty analysis was performed in order to determine the sensitivity of the model fitting procedure. The uncertainty is primarily due to uncertainty in the determination of the free stream flow conditions, such as relative flow angle, relative Mach number and wake-to-pitch ratio. Ensemble averaged values are used for all these quantities. There are two major sources of uncertainty in the free stream conditions. First, since the data segment used for the model fit is quite long (15-20 blade passages), some variation in the value of the free stream conditions from blade to blade is observed. Second, the flow angle and the Mach number are measured with some level of probe inherent uncertainty. These two uncertainties were combined for a total uncertainty of $+/-4$ degrees in relative flow angle, $+/-0.02$ in relative Mach number and $+/-10 \%$ in wake-to-pitch ratio. The inputs to the model were then perturbed by the amount of individual total uncertainty and the worst case was considered, when all the uncertainties contribute in the same direction to the total uncertainty (e.g. +4 degrees for relative flow angle and -0.02 for the Mach number). The parameters $h / a$ and $U_{p} / U_{\infty}$ were then adjusted such that the criteria for the model fitting procedure, like distance between lowest to highest peak and qualitative similarity of shape, were again satisfied. The difference between the original and perturbed values of $h / a$ and $U_{p} / U_{\infty}$ is the uncertainty of the model fit and it was approximately $5 \%$ for both quantities. Note that the uncertainty in the measurement of the wake-to-pitch ratio has negligible effect on the model fitting procedure. 
Fig. 7.5a presents the results of the fitting procedure for the four radii. Although the agreement is not excellent, we believe that it is quite satisfactory, given the simplicity of the model. Table 7.2 gives the final model parameters for the four spanwise locations, along with the uncertainty of the model fitting procedure for each location.

Table 72: Vortex model parameters

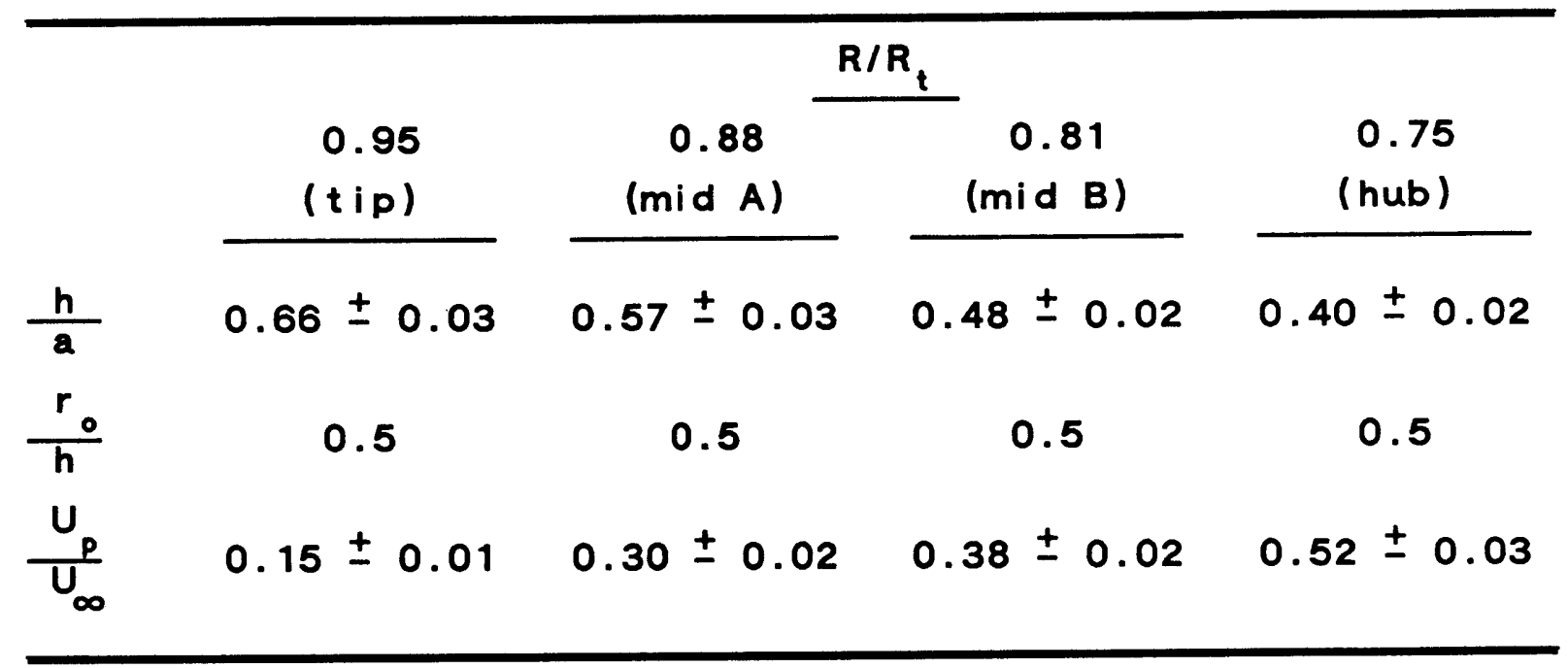

Fig. 5.b presents a scaled perspective of the spanwise vortices as they are predicted by the vortex model. The vortex cores grow as they move downstream, towards the aspirating probe. This evolution will be described in detail in section 7.2.8. 


\subsection{5 - Pressure distribution in the vortex cores}

The next step in the modeling of the spanwise vortices is the calculation of the pressure distribution in the cores of the vortices at the four radial locations. This can be done once the parameters of the vortex street are determined. Following [2], a pressure coefficient, $C_{p}$ can be defined as:

$$
C_{p}=\frac{p-p_{\infty}}{p_{\infty} U_{p}^{2}}
$$

where the subscript $\infty$ denotes the free stream conditions, and $U_{p}$ is the pressure defect velocity. Fig. 7.6 (from [2]), shows the velocity field of a typical blade wake vortex street in the frame moving with the street The letters $A$ through $E$ indicate cross sections through the street at various distances (y/a) from the centerline of the street The distribution of the pressure coefficient, defined above, at each section is presented in Fig. 7.7 for the four spanwise fitting locations. The pressure in the core can now be calculated from eq. (7.11), given the value of $U_{p}$ from the model. We will come back to this point later in section 7.2.7. 


\subsection{6 - Shedding frequency and Strouhal number in the spanwise direction}

Once the vortex street parameters are determined, the shedding frequency can be calculated from the model. From [2] the shedding frequency, $f$, is given as:

$$
f=\frac{U_{\infty}}{a}\left(1+\frac{U_{i}}{U_{\infty}}\right)
$$

where $U_{i}$ is the velocity induced at the center of a vortex by the opposite row and is called the induced velocity. Its magnitude can be related to the other vortex street parameters through the following equation:

$$
U_{i}=\frac{-k}{2 a} \tanh \left(\pi \frac{h}{a}\right)
$$

where $k$ is the strength of the vortex. Gertz [2] reported a shedding frequency of $15.8(+/-12 \mathrm{kHz}$ or approximately 2.8 times blade passing. Using (7.12) and (7.13) the shedding frequency at each radial location was calculated and its values are given in Table 7.3. 
Table 7.3: Shedding frequencies in the spanwise direction

\begin{tabular}{|c|c|c|}
\hline$R / R_{t}$ & & Shedding frequency $(\mathrm{kHz})$ \\
\hline 0.95 & $(t i p)$ & $16.9 \pm 1.5$ \\
\hline 0.88 & $(\operatorname{midspan} A)$ & $16.7 \pm 1.5$ \\
\hline 0.81 & (midspan $B)$ & $17.3 \pm 1.5$ \\
\hline 0.75 & (hub) & $16.4 \pm 1.5$ \\
\hline
\end{tabular}

From this Table it is clear that the shedding frequency is almost constant throughout the spanwise distance that we chose.

An error analysis of eq (7.11) and (7.12) was performed in order to determine the confidence level of the calculation. The uncertainties due to the varying free stream flow conditions and the model fitting procedure, as described in Table 7.2, were the primary sources of uncertainty in the calculation of the shedding frequency. The total uncertainty was estimated to be no higher than $+1-$ $1.5 \mathrm{kHz}$. Therefore the discrepancies in the spanwise distribution of the shedding frequency are well within the uncertainty level of the calculation.

This finding is quite important, since uniformity of shedding frequency is a prerequisite for spanwise coherence of the vortices. However, we do not argue that the vortices are coherent for the total blade span. What we are suggesting is that at least for the spanwise distance for which data are available labout 75\% of the blade span), the uniformity of the shedding frequency might be a manifestation of a coherent vortex structure that covers the investigated part of the blade span. This finding is similar to the one reported by Gaster [97], where 
discrete cells of constant shedding frequency were detected in the spanwise direction of a tapered cylinder. The spanwise length of these cells was found to be about 15 cylinder diameters and their number was about 4 . However, no analogy can be drawn with our experiments as far as the length and number of these coherent cells are concerned, since the flowfield in our case is different and much more complicated. We can only argue that, since the presence of discrete coherent cells with constant shedding frequency within each cell has been observed before, the possibility of appearing again in our case cannot be excluded.

It is interesting to calculate the distribution of Strouhal number that results from these shedding frequencies. A problem that immediately arises is the choice of a length scale to be used in the calculation. Since the shedding phenomenon is a local one, several length scales in the trailing edge region might be used, like the trailing edge blade thickness, the wake width or the boundary layer momentum thickness at the blade trailing edge. Gertz [2] argued that the latter is the appropriate length scale for compressor rotor blades. Table 7.4 gives the Strouhal number, Stw, based on the wake width. 
Table 7.4: Strouhal numbers based on the wake width

\begin{tabular}{|c|c|c|}
\hline$R / R_{t}$ & & $S t_{w}$ \\
\hline 0.95 & $(t i p)$ & 1.11 \\
\hline 0.88 & $(\operatorname{midspan} A)$ & 0.855 \\
\hline 0.81 & (midspan B) & 0.715 \\
\hline 0.75 & (hub) & 0.574 \\
\hline
\end{tabular}

Notice that Gertz [2] found a value of 1.008 for $S t_{w}$, while the Strouhal number in the case of vortex shedding from a cylinder is equal to 0.21 , but is based on the cylinder diameter.

In order to calculate the Strouhal number based on momentum thickness, $S t_{m}$, the value of the boundary layer momentum thickness at the trailing edge, $\theta$ is needed. The calculation was based on the work of Koch and Smith [100] The calculation was performed for the streamtubes that correspond to the four locations where the model was fitted. The values of boundary layer momentum thickness to chord ratios, $\theta / c$, were found to be equal to $0.00978,0.0099,0.0103$ and 0.0127 for the tip, midspan A, midspan B and hub locations respectively. The corresponding Strouhal numbers were equal to $0.086,0.084,0.091$ and 0.100 , still less than the value for the case of cylinder vortex shedding. However, these values of $\theta / c$ do not include the correction for blade surface roughness. The blade surface roughness was estimated and a correction factor of 1.8 was found from Koch and Smith (Fig. 5 in [100]). The corrected Strouhal numbers are presented in Table 7.5. 
Iable 7.5: Strouhal numbers based on the trailing edge boundary layer momentum thickness

\begin{tabular}{|c|c|c|}
\hline$R / R_{t}$ & & $S t_{m}$ \\
\hline 0.95 & $(t$ ip $)$ & 0.155 \\
\hline 0.88 & (midspan A) & 0.151 \\
\hline 0.81 & (midspan B) & 0.164 \\
\hline 0.75 & (hub) & 0.180 \\
\hline
\end{tabular}

These values are certainly much closer to the value of Strouhal number for cylinders. Therefore it might be argued that, for flow similarity with the shedding from circular cylinders, the right length scale to use in this case is the boundary layer momentum thickness at the trailing edge.

For comparison purposes the values of the Strouhal number based on the blade trailing edge thickness, St $\mathrm{c}_{\mathrm{c}}$ are also given in the following Table 7.6. 
Table 7.6: Strouhal numbers based on the trailing edge thickness

\begin{tabular}{|c|c|c|}
\hline$R / R_{t}$ & & $S t_{c}$ \\
\hline 0.95 & $(t \mathbf{i p})$ & 0.022 \\
\hline 0.88 & (midspan A) & 0.022 \\
\hline 0.81 & (midspan B) & 0.024 \\
\hline 0.75 & (hub) & 0.022 \\
\hline
\end{tabular}

The Strouhal number based on the trailing edge thickness is much lower than the previous ones. Note also that this Strouhal number is almost constant throughout the blade span. 


\subsection{7 - Spanwise flow in the vortex cores}

The pressure level in the cores of the spanwise vortices at the four spanwise locations, is not only a function of the local flow quantities in the core, but also of the free stream. If we assume that in the free stream, the centrifugal forces are completely balanced by the free stream radial pressure gradient, then no significant radial flows can occur in that region. This is evident from Ng's measurements [1] of the radial Mach number behind this rotor. For simplicity, we are going to neglect the centrifugal forces due to the curvature of the meridional streamlines and consider only the centrifugal forces caused by conventional rotation about the axis of the machine. Thus the radial momentum equation becomes:

$$
\frac{1}{\rho_{\infty}} \frac{\partial P_{\infty}}{\partial r}=\frac{V_{\theta, \infty}^{2}}{r}
$$

where the subscript $\infty$ denotes free stream conditions.

Fig. 7.8a presents the pressure distribution in the cores of the vortices for the four spanwise locations. Fig. $7.8 \mathrm{~b}$ gives the pressure distribution only in the vortex cores (cross sections A) in Fig. 7.8a. The pressure is nondimensionalized with the inlet total pressure. Note that the inlet total pressure drops slightly during the useful test time. Therefore the values of the inlet total pressure at each of the four spanwise locations are also given in Fig. 7.8a. It is clear that the pressure in the cores is quite low near the hub. The maximum pressure difference between the pressure in the vortex cores at the tip and at the hub is approximately 0.14 atms. 
It is highly desirable to estimate the radial velocities that are being induced by this pressure gradient Before we proceed, the coordinates in the various reference frames that will be involved are given in Fig. 7.9. The spanwise flows are in the $r$-direction in the absolute frame or in the $x$-direction in the vortex frame.

A number of simplifying assumptions are needed in order to attack the problem. First, we will neglect the radial velocities ( $\sigma$-direction in Fig. 7.9 ) in the vortex cores. It is clear that the presence of spanwise (x-direction) velocities in the cores is in conflict with this assumption, as far as the continuity equation is concerned. However, the spanwise length of these vortices is quite large compared to their cross-section and, therefore very small radial velocities distributed in the spanwise direction could account for the velocities induced in the $x$-direction (see Fig. 7.9). Second, we will assume that the vortex does not tilt or distort under the action of the tangential velocity of the swirling flow behind the rotor. Third, the end walls will be considered to have no effect on the formation and development of the vortex.

The first attempt to calculate the induced spanwise velocity in the core of the vortex is based on the modeling of the vortex as a "pipe" or "straw". In this case the pressure gradient at the ends of the "pipe" is the driving force for the fluid motion. An approximate value for this pressure gradient can be obtained from Fig. 7.8, using the pressure drop in the vortex cores of two radial locations, e.g. hub and tip. A quick, but rather crude, order of magnitude calculation gives the velocity in the "pipe", $V_{r}$, as a function of the imposed pressure gradient at the ends, $\Delta p$, as following: 


$$
v_{r} \sim\left(\frac{2 \Delta p}{\rho}\right)^{1 / 2}
$$

where the assumption is made that the fluid is allowed to accelerate freely under the action of the pressure gradient This velocity is towards the hub and, if it acted for an interval of time equivalent to the flow time from the rotor trailing edge to the location of the probe (or stator leading edge), it would lead to a radial displacement of about $55 \%$ of the blade span. If this were true, then the total measured radial displacement towards the hub lapproximately equal to $40 \%$ of blade span) could be explained by this mechanism. In addition, the random nature of the concentration peaks detected near the hub, when we injected at the tip, could be a manifestation of the fact that these velocities are induced only in the cores of the vortices, which are, in turn, sampled by the aspirating probe in a random manner.

However, it was soon realized that the previous calculations oversimplified the problem and a more rigorous estimate of the spanwise velocities was sought The complications arise from two basic reasons. First, the frame of the vortex is noninertial and, therefore, some additional body force terms must be included in the equations of motion. Second, the rotation of the vortex itself must be taken into account when calculating the tangential and axial velocities in the core. The relative (rotating) blade frame is the most appropriate for this calculation, since the vortex is actually spinning with the blades. In this frame, the equation of motion in vector form is: 


$$
\frac{D \vec{w}}{D t}+2 \vec{\omega} \times \vec{w}=\omega^{2} \vec{r}-\frac{\nabla p}{\rho_{c}}
$$

where $\omega$ is the angular velocity of the rotor, $\vec{r}$ is the radial location of the fluid particle, $\rho_{c}$ the density in the vortex core and D/Dt denotes substantial derivative. Equation $(7.16)$ is written for the core region, where the velocity is $\vec{w}=\left(w_{z}, w_{\theta}\right.$, $\left.w_{r}\right)$. The same equation can also be written for the free stream region in the relative frame, where the velocity is $\vec{W}-\left(W_{z}, W_{\theta}, W_{r}\right)$ (see Fig. 7.9 for the coordinates), as following:

$$
\frac{D \vec{W}}{D t}+2 \vec{\omega} \times \vec{W}=\omega^{2} \vec{r}-\frac{\nabla P}{\rho_{\infty}}
$$

where $P$ is the static pressure and $\rho_{\infty}$ is density of the free stream. If we assume that the vortex core and the free stream are at the same radial location, then subtracting eq. (7.17) from (7.16) eliminates the centrifugal force terms and the combined equation becomes:

$$
\frac{D \vec{w}}{D t}=\frac{D \vec{W}}{D t}+2 \vec{\omega} \times(\vec{W}-\vec{w})+\frac{\nabla P}{\rho_{\infty}}-\frac{\nabla P}{\rho_{c}}
$$

The $r$-component of this equation is quite useful for our purposes and simple vector manipulation, leads to the following equation:

$$
\Delta w_{r}=-\Delta t\left[\left(w_{\theta}-w_{\theta}\right)\left(W_{\theta}+w_{\theta}+2 \omega\right)-\frac{1}{\rho_{\infty}} \frac{\partial P}{\partial r}+\frac{1}{\rho_{c}} \frac{\partial P}{\partial r}\right]
$$

In the process of deriving eq. (7.19) the spanwise velocity of the free stream was 
neglected, since, according to eq. (7.14), full radial equilibrium with no spanwise velocities was assumed for that region.

In order to calculate the components of $\mathbf{w}$ in the core we should translate the velocity vectors into the blade relative frame. If the velocity in the frame moving with the vortex is $\vec{q}$, then in the blade relative frame the velocity, $\vec{w}$, will be given by:

$$
\vec{w}=\vec{q}+\left(\vec{u}_{\infty}+\vec{u}_{i}\right)
$$

with reference to Fig. 7.9 and 7.10. In Fig. 7.10 it is also shown how the velocity in the core, $\vec{v}$, in the absolute (stationary) frame can be found by simple vector addition of the wheel speed, $U_{w}$ and the relative velocity $\vec{w}$.

Fig. 7.11 presents typical contour plots of the distribution of $w_{\theta}, v_{\theta}$ and $v_{z}$, which is the same with $w_{2}$ for clockwise and counterclockwise rotating vortex. Although this figure presents the results only for the hub region, the same trends have been observed at the other three radial locations.

In order to calculate the incremental radial velocity in eq. (7.19) the convection time, $\Delta t$ must be known. A simplifying assumption would be to use constant convection time for all the fluid particles in the vortex core. A convenient value for this would be that of the vortex center. However, the variation of axial and circumferential velocities in the core are substantial (see Fig. 7.11). Therefore it is not justifiable to assume that the convective velocity of all the fluid particles in the vortex core is the same with the vortex center. The calculation with constant convective velocity or $\Delta t$ is included in Appendix A for 
comparison.

Given the distribution of axial velocity in the cores and a typical distance between the rotor trailing edge and the location of the aspirating probe, the time interval $\Delta t$ in eq. (7.19) is calculated as a function of $\sigma$ and $\phi$ (see Fig. 7.10). The spanwise pressure gradients, that appear in eq. (7.19) are known at each point in the core region, since the pressure distribution in the core is known at four different radial locations from the Gertz model. Therefore eq. (7.19) can be used to estimate the spanwise velocities induced in the vortex cores. The calculation was performed for all four spanwise locations and for both clockwise and counterclockwise rotating vortices. Fig. 7.12 presents the results of this calculation. Note that the core radius is equal to $1.78 \mathrm{~mm}$ at the hub, $2.1 \mathrm{~mm}$ at midspan $B, 2.6 \mathrm{~mm}$ at midspan $A$ and $3.2 \mathrm{~mm}$ at the tip. However, it appears to be constant in this figure, because everything is nondimensionalized with the radius of the core at the corresponding spanwise location.

A perspective of the core structure is given in Fig. 7.13. The figure is given only for the hub, since the core structure is quite similar at the other three locations, namely midspan A, midspan B and tip.

The remarkable observation from these figure is that the velocity changes sign in the core. Negative spanwise velocities indicate motion to the hub, while positive ones to the tip. It is also clear that the magnitude of both positive and negative spanwise velocities increases substantially as we get closer to the hub. In the same figures the fraction of the core occupied by positive or negative velocity can also be seen. This fraction is larger near the hub than it is near the tip. Therefore it seems that the tendency of the fluid in the core to move in the 
spanwise direction is higher closer to the hub than it is closer to the tip. The net motion seems to be close to zero near the tip, but clearly towards the hub in regions away from the tip. If the mean negative spanwise velocity from the four spanwise locations is used, then a typical spanwise displacement during the travel of the fluid particles from the rotor trailing edge to the probe can be calculated. The result was a total spanwise displacement of about $48 \%$ of the rotor blade span at the trailing edge. This result compares well with the measured spanwise displacement of $40 \%$ of blade span.

The next step is to estimate the mass of fluid that can move along the spanwise direction due to the calculated spanwise velocities. A typical cross section of the vortex core at the midspan was chosen. From Fig. 7.12 the area occupied by the negative velocity was calculated. Given the density and spanwise velocity distribution in the core the mass flow towards the hub was calculated. However, this is the contribution of only one vortex. Given the shedding frequency of the phenomenon and a typical flow time interval, from rotor trailing edge to the probe location, the number of vortices was calculated. This number was then simply multiplied by the total number of blades. If we assume that fluid particles entering the vortex core near the tip or midspan regions remain in the core throughout its trajectory towards the probe and move only in the radial direction, then the total mass flow that move to the hub as calculated from the vortex model amounts to approximately $95 \%$ of the estimated one from the measurements, as presented in section 6.1. (see Table 6.1). The model also predicts transport towards the tip. The maximum spanwise displacement towards the tip was $70 \%$ of the blade span and the mass flow that migrates to the tip was $20 \%$ of the estimated one from the measurements (see Table 6.1). If the "pipe" model is used, then only motion to the hub can be predicted. The total 
mass flow towards the hub is approximately double the estimated one from Table 6.1.

The uncertainty of this calculation is primarily due to the uncertainty in the determination of the model parameters, as described in section 7.2.4 and Table 7.2. Based on those results, a $15 \%$ uncertainty in the calculation of the mass flow that migrates and a $1 \%$ uncertainty in the maximum spanwise displacement are expected. Given these uncertainty levels, the model predicts the measured spanwise transport to the hub, but not the measured migration towards the tip.

\subsection{8 - Quasi-viscous calculation of the vortex core growth}

An assumption inherent in the calculations of the previous section is that the size of the vortex core and the angular velocity at the edge of the core remain constant during the convection of the vortex from the blade trailing edge to the downstream probe location. This assumption will be relaxed in this section by allowing the viscosity of the fluid to contribute to the growth of the vortex core and the decay of its angular velocity.

The motion in the cores of the vortices is physically not very different from the flow in the cores of conventional trailing vortices behind three dimensional wings. Some of the differences between the two cases are the following. First, the vortices in our case develop in a noninertial rotating frame and therefore the effect of rotation must be included in the calculation. Second, in the case of wing trailing vortices the free stream flow direction is the same with the flow in the vortex cores, while in our case the flow in the core is in the spanwise direction 
and the free stream is almost normal to it However, for comparison purposes, we can consider a limiting case of the wing trailing vortex flowfield, where the free stream velocity goes to zero. Batchelor [101] gave an analytical solution for the axial (spanwise in our case) velocity in the vortex core. He gave the solution to a simple example of a vortex core of radius $\sigma_{0}$ rotating rigidly with angular velocity $\Omega$, where all the total pressure losses were neglected. The axial velocity, $w$, induced in the core was given by the following equation:

$$
w=\left[u^{2}+2 \Omega^{2}\left(\sigma_{0}^{2}-\sigma^{2}\right)\right]^{1 / 2}
$$

where $U$ is the free stream velocity. If we consider the limiting case, where $U \rightarrow 0$, then the distribution of axial velocity in the core can be calculated from eq. (7.21). An area averaged velocity was then found. Its value was $90 \%$ of the velocity given by the "pipe" model in eq. (7.15). The important conclusion from this estimate is that the spanwise velocities we measured or calculated with the model are not unusually high, since the axial velocities in the cores of wing trailing vortices are of similar, if not higher, magnitude.

Lamb [102] was the first to give an estimate of the growth of a vortex in laminar flow. According to [102], the circumferential velocity $w_{\theta}$ of the vortex is given by:

$$
w_{\theta}=\frac{K}{2 \pi \sigma}\left(1-e^{\frac{-\sigma^{2}}{4 \nu t}}\right)
$$

where $\sigma$ is the radius of the vortex core, $\nu$ the kinematic viscosity and $K$ the strength of the vortex at $t=0$. Squire [103] extended Lamb's solution for the case 
of turbulent flows. He suggested that the same formula can be used in this case with $\nu$ replaced by an eddy viscosity, $\nu_{t}$. Squire [103] argued that there is no justification for the introduction of complicated formulas for eddy viscosities or mixing lengths and that the simplest formula is usually as good as any other. Therefore he suggested that:

$$
\nu_{t}=\alpha K
$$

where $\alpha$ is an empirical constant The value of $\alpha$ has been the center of discussion by many researchers. Govindaraju and Saffman [104] report values for $\alpha$ ranging from $5 \times 10^{-5}$ to $7.6 \times 10^{-3}$ depending on the vortex Reynolds number (vortex circulation at infinity divided by laminar kinematic viscosity). We adopted the idea proposed by Squire to describe the decay of the angular velocity and the growth of the vortex core in our case. A typical value for the vortex Reynolds number in our case is $10^{5}$. Therefore a reasonable value for the constant $\alpha$ is $4 \times 10^{-4}$. However, in order to determine the function that describes the growth of the vortex core, the edge of the vortex core must be defined first. Squire defined it as the radius $\sigma_{0}$ at which the vorticity has dropped down to $5 \%$ of the value at $\sigma=0$. Following this argument, the vortex core at an instant $t$ is:

$$
\sigma_{0}(t)=\left(12 \nu_{t} t\right)^{1 / 2}
$$

An arbitrary time and space origin needs to be defined in order to calculate the time $t$ However, as it was shown before the properties of the vortex core are known at the probe location through the fitting procedure from the Gertz model. Therefore, the function that describes the growth of the vortex core is given by 
the following equation:

$$
\sigma_{0}(t)=\left(\sigma_{0}^{2}(0)+12 \nu_{t} \Delta t\right)^{1 / 2}
$$

where $\sigma_{0}(0)$ is the size of the core at a location close to the trailing edge of the blade, where the vortex is considered to be fully developed (similar to a solid body rotation), and $\Delta t$ the time interval from that point to the probe location.

The determination of the time origin (point of fully developed vortex) is quite complicated. Lack of detailed measurements close to the blade trailing edge, led us to use the available information from circular cylinders. According to [80] the vortex seems to be fully developed after about 3 diameters downstream. Since it was shown before that the relevant length scale for the shedding phenomenon behind the rotor blade is the boundary layer momentum thickness at the trailing edge, the vortex should be fully developed at about 3 momentum thicknesses downstream from the trailing edge. Given a typical velocity of convection the vortex development time was found to be approximately $1 / 5$ of the total convection time from the rotor trailing edge to the aspirating probe location. Therefore the time interval $\Delta t$ that appears in equation (7.25) is equal to $80 \%$ of the total flow time from the trailing edge of the blade to the probe. Using equations (7.22), (7.23) and (7.25) a similar equation can be derived for the circumferential velocity, $U_{p}$ at the edge of the vortex core, as:

$$
U_{p}(t)=\left[\frac{1}{U_{p}^{2}(0)}+\frac{524.9 \nu_{t}}{K^{2}} \Delta t\right]^{-1 / 2}
$$


where $U_{p}(0)$ is the circumferential velocity at the edge of the core at the time origin as defined before. Notice that this velocity is identical to the pressure defect velocity used in the fitting of the Gertz model.

The next step is to apply all these to our case. Equations (7.25) and (7.26) were used to calculate the core radius and pressure defect velocity at $t=0$. Assuming that the free stream properties do not change, the new parameters for the Gertz model were calculated at the time origin $(t=0)$. The following table gives the values for the pressure defect velocity, $U_{p}$ and the core radius $\sigma_{0}$ at the time origin and at the probe location where the model was fitted.

Table 7.7: Development of the vortex cores

\begin{tabular}{|c|c|c|c|}
\hline & & $\begin{array}{l}\text { At time } \\
\text { origin }\end{array}$ & $\begin{array}{l}\text { At probe } \\
\text { location }\end{array}$ \\
\hline \multirow{4}{*}{$U_{p}(m / s e c)$} & $\mathbf{T} \mathbf{I P}$ & 30.2 & 28.8 \\
\hline & MID A & 66.5 & 58.4 \\
\hline & MID B & 94.1 & 75.4 \\
\hline & HUB & 163.2 & 105.7 \\
\hline \multirow{4}{*}{$\sigma_{0}(\mathrm{~mm})$} & $\mathbf{T I P}$ & 3.0 & 3.2 \\
\hline & MID A & 2.3 & 2.6 \\
\hline & MID B & 1.7 & 2.1 \\
\hline & HUB & 1.2 & 1.8 \\
\hline
\end{tabular}

It is important to notice that there is a spanwise variation of circulation 
along the axis of the vortex. This variation is primarily due to the spanwise variation of the pressure defect velocity, $U_{p}$.

Equation (7.19) was then applied to calculate the incremental radial velocity, using a very short time interval. This process was repeated for a number of time intervals until a point in time was reached when the fluid had covered the total distance from the time origin to the probe location. Figures 7.14 to 7.17 present the growth of the spanwise velocities in the core with time for tip, midspan A, midspan B and hub, respectively. In addition, a perspective of the development of these velocities and the structure of the core can be seen in Figures 7.18 to 7.21 for tip, midspan A, midspan B and hub, respectively. It should be pointed out that the calculation of the development of these vortices uses constant time intervals for all particles in the vortex core. This was a necessary simplification, in order to be consistent with the Squire model for the growth of the vortex, which is based on the assumption that the vortex core is convected with the free stream velocity. In our case, the core convective velocity was chosen to be that of the vortex center. Fig. 7.22 presents a perspective of the spanwise velocities at the four selected spanwise locations as predicted by the vortex model.

Although this calculation is based on a viscous model for the growth of the vortex, viscosity is not included in the calculation of the induced spanwise velocities (eq. (7.19)). In other words, the calculation assumes that the vortex grows and decays under the action of viscosity, but the change in the vortex core properties occurs in finite jumps. In between them the viscosity is "turned off" and the growth of the spanwise velocities is given by an inviscid calculation. At the end of each of these jumps the new vortex properties are input to the Gertz model and the new flowfield is calculated. This simplification was necessary 
in order to avoid complications that could not be justified by the already approximate type of our approach.

The mass flow that can move in the spanwise direction and the maximum spanwise displacement were also calculated for this case. The maximum possible displacement was found to be approximately $44 \%$ of the blade span towards the hub and $57 \%$ towards the tip. The migrating mass flow to the hub was approximately $84 \%$ of the estimated one from the measurements (Table 6.1). The model also predicts transport towards the tip equal to approximately $18 \%$ of the estimated one (Table 6.1). Given the uncertainties in the calculation (15\% in mass flow and $1 \%$ in spanwise displacement) the model predicts the motion towards the hub, but underpredicts the transport to the tip.

The following table summarizes the results of these calculations. 
Iable 7.8: Measured and predicted maximum spanwise displacement and migrating mass flow

\begin{tabular}{|c|c|c|c|c|c|}
\hline \multirow[b]{2}{*}{$\begin{array}{l}\text { Mesured } \\
(\text { Tab. 6.1) }\end{array}$} & \multicolumn{2}{|c|}{$\begin{array}{c}\text { Max. span. } \\
\text { displac. } \\
\text { towards } \\
\text { hub tip } \\
(\% \text { blade span })\end{array}$} & \multicolumn{3}{|c|}{$\begin{array}{l}\text { Migrating } \\
\text { mass f I ow } \\
\text { towards }\end{array}$} \\
\hline & $40 \%$ & $40 \%$ & & $1.6 \%$ & $3.5 \%$ \\
\hline $\begin{array}{l}\text { "Pipe" } \\
\text { model } \\
(e q .(7.15))\end{array}$ & $55 \%$ & - & & $3.3 \%$ & - \\
\hline $\begin{array}{l}\text { Inviscid } \\
\text { detailed } \\
\text { calcul. } \\
(\text { eq. }(7.19))\end{array}$ & $48 \%$ & $70 \%$ & & $1.5 \%$ & $0.7 \%$ \\
\hline $\begin{array}{l}\text { Quas i-visc. } \\
\text { calcul. } \\
(\text { sec. } 7.2 .8)\end{array}$ & $44 \%$ & $57 \%$ & & $1.3 \%$ & $0.6 \%$ \\
\hline
\end{tabular}




\section{CHAPTHR 8}

\section{CONSEQUENCES OF SPANWISE FLUID TRANSPORT}

\section{1 - Effect of spanwise fluid transport on the spanwise distribution of adiabatic efficiency}

It has been observed that transonic compressors experience a very low adiabatic efficiency close to the tip region. Fig. 8.1 (from [3]) presents a collection of adiabatic efficiency distributions as measured behind a number of quite different transonic compressors. The basic similarity among these data points is the low adiabatic efficiency near the tip region. According to [3], viscous effects and normal shock losses could not account for this drop in efficiency. Therefore Kerrebrock [3] hypothesized that strong spanwise flows might be a mechanism that selectively moves high entropy fluid (like wake or boundary layer fluid) towards the tip region. If this is the case, then the tip will appear in the measurements as a low efficiency region. However, this drop in efficiency is not due to a "lossy" tip, since it is caused by a redistribution of high entropy fluid and not by a local entropy producing mechanism.

One of the major motivations behind this experimental effort was to measure the amount of fluid that moves in the spanwise direction. In addition, it was highly desirable to estimate the effect of this migration on the spanwise distribution of adiabatic efficiency. This is the subject of this section.

It is important to distinguish between spanwise migration before and after the trailing edge of the blade. In the former the blade exerts forces on the fluid 
particles and any fluid motion in the spanwise direction is associated with work done by the rotor on the fluid particle (or vice versa). The work done in either direction is usually given by the Euler turbine equation [105], as follows:

$$
c_{p}\left(T_{t b}-T_{t a}\right)=\omega\left(r_{b} V_{\theta_{b}}-r_{a} V_{\theta_{a}}\right)
$$

where $T_{t}$ is the total temperature of the fluid, $c_{p}$ its specific heat at constant pressure, $\omega$ the angular velocity of the blade, $r$ the radius of the streamtube and $V_{\theta}$ the tangential velocity of the fluid in the absolute (stationary) frame. The subscripts $a$ and $b$ refer to upstream and downstream locations respectively. From this equation it is clear that if the inflow has no tangential component of velocity (our experimental setup), we need not identify the inlet streamtube radius. Only the tangential velocity and spanwise location is required in order to calculate the work done on the fluid. This last point will be very useful throughout the calculations of this section.

If the spanwise migration occurs after the trailing edge of the blade, then there is no work done by the rotor or on the rotor during the migration of the fluid. A simple redistribution of flow properties occurs as a result of this motion. However, if the fluid that moves has high entropy, then the region that it moves into will experience a drop in adiabatic efficiency.

In section 6.1 the spanwise migration was derived from the experimental measurements. Given this matrix of migrating mass flows, the effect of this migration on the spanwise distribution of adiabatic efficiency can be estimated. Since the phenomena associated with the spanwise migration are quite different, depending on whether the migration occurs before or after the trailing edge of 
the blade, these two extreme cases will be investigated separately.

\subsection{1 - Spanwise fluid transport within the blade passage}

Fig. 8.2 presents a schematic drawing of the blade and the basic parameters and notation that will be used throughout this section. For simplicity we will deal only with one region (tip region or region 1). The notation for the migrating mass flow is the same with section 6.1. For example, $\dot{\mu}_{31}$ denotes the mass flow that migrates from region 3 (hub) into region 1 (tip), while $\dot{\mu}_{13}$ is the mass flow that moves from region 1 into region 3 . In addition, $\dot{m}_{1}$ is the total mass flow that enters region 1, before any migration occurs. For simplicity, we also omitted the subscript $t$ for the total temperature.

Due to the complexity of this phenomenon some simplifying assumptions will be made. First, we will assume that the migration occurs instantaneously at some axial location in the blade row (point A) in Fig. 8.2. The streamlines exchange positions as it is shown in the same figure. However, it is quite difficult to estimate what a realistic location for point A would be. In addition, it is not clear what the effect of the location of point $A$ is on the final calculation. Therefore we decided to perform the calculations for two points, shown as dashed lines $A$ and $B$ in Fig. 8.2, and compare the results. The choice of the two points was based on the availability of information in the blade region from the streamline curvature calculation [26] Locations A and B correspond to thru-blade stations 8 and 10 in the Air Force report [26] The need to chose an axial location where the migration occurs and the way we used the streamline curvature results will be clear in the next paragraphs. 
A simple energy balance calculation was performed for the shaded region in Fig. 8.1. However, in order to do that the total temperatures of the fluid right before entering the shaded region are needed. The fluid that came from region 1 , mass flow $\dot{m}_{1}$, has already experienced a certain amount of turning or, in other words, some work is done on it Its total temperature is definitely higher that the inlet total temperature $T_{i n}$. The question is what fraction of the total work done on the fluid was completed up to location A or B. Since we had no experimental results for the flowfield in the blade row, we used the information from the streamline curvature calculation [26] We assumed that the fraction of the total work given in [26] denoted $\alpha_{1}$ remains the same during our experiments. (This fraction varies in the spanwise direction and thus each region (1 to 4) will have a different value). Therefore the change in the total temperature of the flow from inlet up to point $A$ or $B$ in the blade row, is given as:

$$
T_{\ldots, 1^{-}} T_{i n}=\alpha_{1}\left(T_{\text {out }, 1^{-}}^{(-)} T_{\text {in }}\right)
$$

where $T_{0,1}$ is the total temperature of the fluid at point $A$ or $B, T_{\text {out }, 1}$ is the exit total temperature of the fluid in region 1 and $\alpha_{1}$ is the fraction of total work done on the fluid from inlet to point A or B and is calculated using the results of streamline curvature calculation [26] The superscript (-) denotes the ideal case where no migration occurs.

We also assumed that the fluid that leaves region 1 (this is equal to $\dot{\mu}_{14}+$ $\left.\dot{\mu}_{13}+\dot{\mu}_{12}\right)$ has total temperature approximately equal to $T_{0,1}$. Since the right hand side of eq. (8.2) depends on the region under question (1 to 4), the value of T. will also vary in the spanwise direction. Therefore we assumed that the fluid 
that leaves each region has total temperature equal to the value of $T_{0}$ of that region.

The work done on (or by) the fluid due to the radius change (as expressed by the Euler turbine equation (8.1)) must be taken into account The total temperature of the fluid right before its interaction with the fluid of region $j$ and immediately after the completion of the radius change from region $k$ to region $j$, is denoted as $T_{k j}$. For example, the migrating mass flows from regions 4,3 and 2 into 1, denoted as $\dot{\mu}_{41}, \dot{\mu}_{31}$ and $\dot{\mu}_{21}$ respectively, will have total temperatures equal to $T_{41}, T_{31}$ and $T_{21}$ respectively right before entering the interaction area of region 1 (shaded area in Fig. 8.2).

Since work is done on the fluid during the radius exchange process, total temperatures $T_{k j}$ are generally different from $T_{e, k}$ (total temperature at the point of departure from the $k_{t h}$ region). An important point is that $T_{k j}$ are not necessarily higher than $T_{0, k}$. For example, when fluid moves from a higher radius to a lower one (fluid of mass $\dot{\mu}_{13}$ moves from $r_{1}$ to $r_{3}$ ), then there is work done on the rotor by the fluid and therefore the fluid total temperature drops. All these arguments can be expressed in mathematical form in the following equation, which is written for $T_{41}$ only:

$$
T_{41}-T_{i n}=\alpha_{4}\left(T_{0 u t .4}^{(-)}-T_{i n}\right)+\frac{\omega}{c_{p}} V_{\theta, . .4}\left(r_{1}-r_{4}\right)
$$

where $V_{\theta, \Theta, 4}$ is the tangential velocity at the point of departure $A$ or $B$ in region 4. Equation (8.3) can be simplified by calculating $V_{\theta, e, 4}$ using the Euler turbine equation (8.1) between the inlet and point $A$ in region 4. The final form of equation (8.3) is as follows: 


$$
T_{41}-T_{i n}=\alpha_{4}\left(T_{\text {out.4 }}^{(-)}-T_{\text {in }}\right) \frac{r_{1}}{r_{4}}
$$

Notice the dependence of $T_{41}$ on the ratio of radii. In this case $r_{1}$ is higher than $r_{4}$ and therefore there is work done on the migrating fluid. However, there are cases where this ratio of radii is less than one, indicating that the fluid particle does work to the rotor.

Two important assumptions are inherent in these calculations. The first is that the tangential velocity of the fluid was constant during its migration from one radius to another. The second is that all the mixing processes were neglected during the motion from one region to another. However, mixing was included in the shaded region (see Fig. 8.2).

A simple energy balance was performed for the shaded region in Fig. 8.2. The algebraic form of this is given below:

$$
\dot{Q}_{i n}-\dot{Q}_{m}+\dot{Q}_{p}=\dot{Q}_{o u t}
$$

where $\dot{Q}_{i n}$ is the power coming in, $\dot{Q}_{m}$ is the power leaving the region in the form of migrating mass flow, $\dot{Q}_{\text {out }}$ is the power at the exit of the rotor and $\dot{Q}_{p}$ is the power input from the rotor as the fluid moves from point $A$ to the trailing edge. Note that, for a perfect gas, $\dot{Q}$ is equal to $\dot{m} c_{p} \Delta T_{t}$. 
194

Equation (8.5) can be written for all four regions in the spanwise direction.

Thus a system of four equations is formed with four unknowns as:

$$
\begin{aligned}
& \left(\dot{m}_{1}-\dot{\mu}_{14}-\dot{\mu}_{13}-\dot{\mu}_{12}\right) \alpha_{1} T_{\circ u t, 1}^{(-)}+\dot{\mu}_{21} \alpha_{2} \frac{r_{1}}{r_{2}} T_{0 u t, 2}^{(-)}+\dot{\mu}_{31} \alpha_{3} \frac{r_{1}}{r_{3}} T_{o u t, 3}^{(-)}+ \\
& +\dot{\mu}_{41} \alpha_{4} \frac{r_{1}}{r_{4}} T_{\text {out.4 }}^{(-)}=\left(\dot{m}_{1}+\dot{\mu}_{41}+\dot{\mu}_{31}+\dot{\mu}_{21}-\dot{\mu}_{14}-\dot{\mu}_{13}-\dot{\mu}_{12}\right) \mathrm{T}_{\text {out, } 1}^{(+)} \alpha_{1}+ \\
& +T_{i n}\left[\dot{\mu}_{41}\left(\alpha_{4} \frac{r_{1}}{r_{4}}-\alpha_{1}\right)+\dot{\mu}_{31}\left(\alpha_{3} \frac{r_{1}}{r_{3}}-\alpha_{1}\right)+\dot{\mu}_{21}\left(\alpha_{2} \frac{r_{1}}{r_{2}}-\alpha_{1}\right)\right]
\end{aligned}
$$

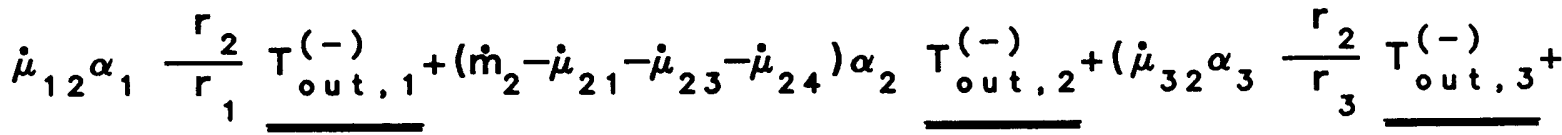

$$
\begin{aligned}
& +\dot{\mu}_{42} \alpha_{4} \frac{r_{2}}{r_{4}} T_{o u t, 4}^{(-)}=\left(\dot{m}_{2}+\dot{\mu}_{42}+\dot{\mu}_{32}+\dot{\mu}_{12}-\dot{\mu}_{21}-\dot{\mu}_{23}-\dot{\mu}_{24}\right) T_{0 u t, 2}^{(+)} \alpha_{2}+ \\
& +T_{i n}\left[\dot{\mu}_{42}\left(\alpha_{4} \frac{r_{2}}{r_{4}}-\alpha_{2}\right)+\dot{\mu}_{32}\left(\alpha_{3} \frac{r_{2}}{r_{3}}-\alpha_{2}\right)+\dot{\mu}_{12}\left(\alpha_{1} \frac{r_{2}}{r_{1}}-\alpha_{2}\right)\right]
\end{aligned}
$$

(8.7) 


$$
\begin{aligned}
& \dot{\mu}_{13} \alpha_{1} \frac{r_{3}}{r_{1}} \underbrace{(-)}_{o u t, 1}+\dot{\mu}_{23} \alpha_{2} \frac{r_{3}}{r_{2}} T_{0 u t, 2}^{(-)}+\left(\dot{m}_{3}-\dot{\mu}_{31}-\dot{\mu}_{32}-\dot{\mu}_{34}\right) \alpha_{3} T_{o u t, 3}^{(-)} \\
& +\dot{\mu}_{43} \alpha_{4} \frac{r_{3}}{r_{4}} \frac{T_{0 u t, 4}^{(-)}}{r^{(-)}}=\left(\dot{m}_{3}+\dot{\mu}_{43}+\dot{\mu}_{23}+\dot{\mu}_{13}-\dot{\mu}_{31}-\dot{\mu}_{32}-\dot{\mu}_{34}\right) T_{0 u t, 3}^{(+)} \alpha_{3}+ \\
& +T_{i n}\left[\dot{\mu}_{43}\left(\alpha_{4} \frac{r_{3}}{r_{4}}-\alpha_{3}\right)+\dot{\mu}_{23}\left(\alpha_{2} \frac{r_{3}}{r_{2}}-\alpha_{3}\right)+\dot{\mu}_{13}\left(\alpha_{1} \frac{r_{3}}{r_{1}}-\alpha_{3}\right)\right]
\end{aligned}
$$

$$
\begin{aligned}
& \dot{\mu}_{14} \alpha_{1} \frac{r_{4}}{r_{1}} \underbrace{(-)}_{0 u t, 1}+\dot{\mu}_{24} \alpha_{2} \frac{r_{4}}{r_{2}} T_{0 u t, 2}^{(-)}+\dot{\mu}_{34} \alpha_{3} \frac{r_{4}}{r_{3}} T_{0 u t, 3}^{(-)} \\
& \left(\dot{m}_{4}-\dot{\mu}_{41}-\dot{\mu}_{42}-\dot{\mu}_{43}\right) \alpha_{4} \underbrace{T_{0 u t}^{(-)}}_{0 u t, 4}= \\
& =\left(\dot{m}_{4}+\dot{\mu}_{14}+\dot{\mu}_{24}+\dot{\mu}_{34}-\dot{\mu}_{43}-\dot{\mu}_{42}-\dot{\mu}_{41}\right) T_{0 u t, 4}^{(+)} \alpha_{4}+ \\
& +T_{i n}\left[\dot{\mu}_{14}\left(\alpha_{1} \frac{r_{4}}{r_{1}}-\alpha_{4}\right)+\dot{\mu}_{24}\left(\alpha_{2} \frac{r_{4}}{r_{2}}-\alpha_{4}\right)+\dot{\mu}_{34}\left(\alpha_{3} \frac{r_{4}}{r_{3}}-\alpha_{4}\right)\right]
\end{aligned}
$$

The unknown quantities (underlined in eq. (8.5) through (8.9)) are the total temperatures at the exit of each region in the case without migration, while the inputs are the migrating mass flows, the $\alpha$ factors, the radii of each region and the measured (with migration) total temperatures at the exit of each region. Again the superscript $(+)$ indicates the case with migration (measured) and the $(-)$ the hypothetical case without migration. This system of equations is solved and the no migration spanwise distribution of total temperature is determined. As we mentioned before, the contributions to spanwise transport of the fourth region 
(see Fig. 6.1) were estimated by extrapolation from the other three regions where the spanwise transport was directly measured. However, this analysis is still applicable, even if the fourth region is totally neglected. In this case the migrating mass flows leaving or entering the fourth region lessentially any variable with subscript 4) are set to zero in eq. (8.6) through (8.9) and a system of three equations and three unknowns is obtained.

The ultimate goal of this analysis is to calculate the spanwise distribution of adiabatic efficiency in the case with no migration and compare it with the one with migration. However, the adiabatic efficiency is also a function of total pressure or total pressure ratio and therefore the effect of the spanwise migration on total pressure must also be included.

The total pressure change due to the spanwise migration was treated as a constant area mixing process with injection and removal of mass. The influence coefficients from [106] were used to estimate the effect on the total pressure. The change in total pressure, again only for region 1, is given in algebraic form by:

$$
\frac{p_{o u t, 1}^{(+)}-p_{o u t, 1}^{(-)}}{p_{o u t, 1}^{(-)}}=-\gamma M_{m i x, 1}^{2}\left(\frac{\dot{\mu}_{i n}-\dot{\mu}_{o u t}}{m_{1}}\right)
$$

where Pout, 1 is the total pressure at the exit of region 1, the superscripts $(+)$ and (-) denoting the cases with and without migration, $M_{m i x, 1}$ is the Mach number at which the mixing occurs, $\dot{m}_{1}$ is the mass flow through region 1 and $\dot{\mu}_{i n}$, $\dot{\mu}_{\text {out }}$ are the total migrating mass flows entering or leaving region 1. 
Using this estimate of the total pressure losses due to mixing, the total pressure ratio for each region without the migration was estimated. We define the compressor total pressure ratio without migration of, say, region 1, as:

$$
\pi_{1}^{(-)}=\frac{p_{o u t, 1}^{(-)}}{p_{i n}}
$$

In addition, the compressor total temperature ratio for the same region will be defined as:

$$
\tau_{1}^{(-)}=\frac{T_{\text {out, }}^{(-)}}{T_{\text {in }}}
$$

The same definitions are applicable for all the regions and the subscript takes values from 1 to 4 .

The adiabatic efficiency in the case without migration for, say region $1, \eta^{(-)}$, is then calculated as:

$$
\eta_{1}^{(-)}=\frac{\left(\pi_{1}^{(-)}\right)^{\frac{\gamma-1}{\gamma}}-1}{\tau_{1}^{(-)}-1}
$$

where the superscript (-) indicates the case without migration. The same equation was also used to calculate the adiabatic efficiency distribution for the $(+)$ case (with migration). In this case the values for $\pi$ and $\tau$ are defined as in eq. (8.11) 
and (8.12) with the $(-)$ sign replaced by $(+)$. Note that the total pressure and total temperature ratios in the case with migration were directly measured during the tests and therefore are inputs to the calculations.

One additional assumption inherent in this calculation is that the mechanism of spanwise migration does not generate any entropy. Therefore the exchange of spanwise locations during the migration of fluid is considered an isentropic process.

The same calculation was repeated for another point in the blade row shown as point $B$ in Fig. 8.2. The two points are quite different in terms of the values of the $\alpha$ factors. For example, near the tip region the $\alpha$ factor for point $A$ is equal to 0.23 , while the one for point $B$ in the same region is equal to 0.71 . Two cases were investigated for each one of points $A$ and $B$. In the first the total pressure losses due to mixing were included and in the second they were not Fig. 8.3 shows the results of these calculations, where adiabatic efficiency is plotted vs. $r / r_{t i p}$. Five curves are shown in this figure. The first is the distribution of the measured adiabatic efficiency (with migration). The next four curves represent the results of the calculation, namely the "no migration" adiabatic efficiency. The conditions for each curve are as following:

1) Calculation assuming that the migration occurs at point $A$ and including total pressure mixing losses.

2) Calculation assuming that the migration occurs at point $A$ but not including total pressure mixing losses.

3) Calculation assuming that the migration occurs at point $B$ and including total pressure mixing losses.

4) Calculation assuming that the migration occurs at point $B$ but 
not including total pressure mixing losses.

The first observation from Fig. 8.3 is that the two calculations for points A and $B$ are not very different, which indicates that the calculation is not very sensitive to the choice of points $A$ and $B$. However, according to the same figure the effect of total pressure losses is quite substantial probably as high as $10 \%$ in adiabatic efficiency. It is also clear that if the total pressure losses are included in the calculation, the tip efficiency increases to about $90 \%$, while the hub efficiency drops to about $84 \%$. As we mentioned in the beginning of this Chapter and can be seen in Fig. 8.1, there is a discrepancy of about $13 \%$ between the predicted and measured adiabatic efficiency at the tip of transonic compressors. According to our calculations this discrepancy can be explained by the spanwise migration process.

In section 6.1 we estimated the percentages of the total mass flow that move in the spanwise direction. Since we did not have any information about the region close to the hub wall (region 4), we extrapolated from our measurements to include that region. However, it was felt that we should estimate the effect of this approximation. Therefore, we repeated the calculations described before, using only the three regions where we had measurements of the migration and ignoring completely the presence of the fourth region. The same cases were investigated and the results are shown in Fig. 8.4. The five curves presented in that plot correspond to the same conditions with the ones in Fig. 8.3. It is clear from this figure that the effect of spanwise migration is much smaller if the fourth region is not included. However, neglecting that region is not realistic. On the other hand, extrapolating the measurements to include it introduces uncertainty in the outcome of the calculation. Since these two cases represent the 
extreme input conditions for the calculation, we believe that the real effect lies somewhere between them.

\subsection{2 - Spanwise fluid transport after the trailing edge of the blade}

The calculation described in section 8.1.1 gave an estimate of the effect of the spanwise migration, when it occurs within the blade passage. However, as it was shown in section 7.2, there may be migration after the trailing edge too. In this section we will present the calculation to estimate the effect of spanwise migration, under the assumption that it happens only after the trailing edge. Although this calculation is not completely realistic, it gives another extreme condition to bound the effect of the migration.

According to the experimental results presented in Chapter 5, most of the migration occurs in the viscous blade wakes. Therefore we will assume that only wake fluid moves in the spanwise direction. This is quite important since the wake fluid is the one with the highest entropy level and its motion influences substantially the distribution of adiabatic efficiency.

In this calculation we will assume that there is no mixing after the fluid moves into a region. This was done, because we believe that the convection time from the rotor trailing edge to the stator leading edge is too small for mixing effects to take place. Therefore we will consider the spanwise migration as a simple redistribution of the flow properties behind the trailing edge of the rotor.

In order to calculate the adiabatic efficiency distribution with and without 
migration we need to know the total temperature and total pressure distributions or equivalently, the total temperature and entropy distributions. We chose to use the latter for this calculation. The flow behind the rotor was idealized as it is shown in Fig. 8.5. There is a wake and a free stream region. The wake region has higher entropy and total temperature than the free stream. Two factors $\lambda$ and $\kappa$ are used to relate the free stream values of entropy and total temperature to the wake ones. The values for these factors come from our measurements and vary in the spanwise direction. The same idealization of the flowfield can be applied to all the regions, obviously with different values of $s_{f}, T_{f}, \lambda$ and $\kappa$.

The calculation starts with a simple entropy and total temperature accounting argument for each spanwise region, given by the following equation for region 1 only:

$$
\begin{gathered}
\left(\dot{m}_{1}+\dot{\mu}_{21}+\dot{\mu}_{31}+\dot{\mu}_{41}-\dot{\mu}_{12}-\dot{\mu}_{13}-\dot{\mu}_{14}\right) T_{1}^{(+)}=\left(\dot{\mu}_{w 1} \kappa_{1} T_{f 1}^{(-)}+\dot{\mu}_{f 1} T_{f 1}^{(-)}\right)+ \\
\dot{\mu}_{21} \kappa_{2} T_{f 2}^{(-)}+\dot{\mu}_{31} \kappa_{3} T_{f 3}^{(-)}+\dot{\mu}_{41} \kappa_{4} T_{f 4}^{(-)}-\left(\dot{\mu}_{12}+\dot{\mu}_{13}+\dot{\mu}_{14}\right) \kappa_{1} T_{f 1}^{(-)}
\end{gathered}
$$

where the notation for the masses is given earlier in this Chapter. The new symbols are the wake and free stream mass flows of region $1 \dot{\mu}_{w 1}$ and $\dot{\mu}_{f 1}$ respectively, and the free stream total temperature of region $1, T_{f 1}$. The superscripts $(+)$ and $(-)$ represent again the cases with and without migration.

A similar equation can be written for the entropy, as follows: 


$$
\begin{gathered}
\left(\dot{\mathrm{m}}_{1}+\dot{\mu}_{21}+\dot{\mu}_{31}+\dot{\mu}_{41}-\dot{\mu}_{12}-\dot{\mu}_{13}-\dot{\mu}_{14}\right) \mathrm{s}_{1}^{(+)}=\left(\dot{\mu}_{w 1} \lambda_{1} \mathrm{~s}_{\mathrm{f} 1}^{(-)}+\dot{\mu}_{\mathrm{f} 1} \mathrm{~s}_{\mathrm{f} 1}^{(-)}\right)+ \\
\dot{\mu}_{21} \lambda_{2} \mathrm{~s}_{\mathrm{f} 2}^{(-)}+\dot{\mu}_{31} \lambda_{3} \mathrm{~s}_{\mathrm{f}}^{(-)}+\dot{\mu}_{41} \lambda_{4} \mathrm{~s}_{\mathrm{f} 4}^{(-)}-\left(\dot{\mu}_{12}+\dot{\mu}_{13}+\dot{\mu}_{14}\right) \lambda_{1} \mathrm{~s}_{\mathrm{f} 1}^{(-)}
\end{gathered}
$$

where the same notation as in equation (8.14) is used.

Similar equations can be written for the other three spanwise regions and two systems of equations are formed. One for the total temperature and one for the entropy. The unknown quantities are the free stream "no migration" total temperatures and entropy levels for the four regions. (variables with superscript $(-)$ in eq. (8.14) and (8.15)). All the other quantities that appear in equations (8.14) and (8.15) are determined from our measurements. Once the unknown "no migration" total temperature and entropy spanwise distributions are calculated, the adiabatic efficiency is given as:

$$
\eta=\frac{\tau e^{\left(-\frac{\Delta s}{c_{p}}\right)}-1}{\tau-1}
$$

In this equation $\tau, \Delta s$ and hence $\eta$, take different values for each region (1 to 4). The total temperature ratio is defined in equation (8.12) and has different values for the with or without migration cases. Therefore, using this equation, both the "with" and the "without migration" efficiency distribution can be calculated. The results of these calculations are presented in Fig. 8.6. The first curve in this figure represents the measured values of adiabatic efficiency or, in other words, the efficiency with the migration included. The other two curves give the results 
of the calculations described above for two cases. The first includes region 4 (close to the hub wall) in the calculation, while the second ignores it completely.

A comparison with Figures 8.3 and 8.4 shows that the effect of the spanwise migration after the trailing edge of the blade is small compared to that of the migration within the blade passage. However, even in the former case (presented in Fig. 8.6) a change of adiabatic efficiency of as much as $2 \%$ is possible.

In section 8.1.1 we postulated that the real effect of the spanwise migration lies somewhere between the curves shown in Figures 8.3 and 8.4 , if any spanwise transport is completed before the trailing edge of the blade. Since we expect part of the total spanwise migration to occur after the trailing edge, we finally conclude that the two cases presented in Fig. 8.3 or 8.4 and in Fig. 8.6, are the upper and lower bounds of the effect of spanwise migration on the adiabatic efficiency distribution.

\subsection{3 - Spanwise fluid transport both before and after the trailing edge of the blade}

The spanwise fluid transport towards both hub and tip, as predicted by the vortex model, was subtracted from the total measured spanwise transport It was then assumed that the remaining measured transport occured within the blade passage. The calculations presented in sections 8.1.1 and 8.1.2 were repeated for 
this case and a corrected distribution of adiabatic efficiency was obtained (solid line in Fig. 8.7). In the same figure the predicted, from viscoustoblique and viscous+normal shock losses, and the measured distributions of adiabatic efficiency are also given.

In summary, these calculations show that, if the data are corrected for the measured spanwise migration, before and after the trailing edge, then an increase of about $13 \%$ in the tip adiabatic efficiency can be achieved. Therefore, the discrepancy between predicted (viscoustoblique or viscous+normal shock losses) and measured adiabatic efficiency at the tip can be fully explained by the spanwise fluid transport.

The mass averaged adiabatic efficiency for each of the four curves in Fig. 8.7, is also given in the same figure. Note that both measured and corrected (by the vortex model) spanwise distributions have the same mass averaged adiabatic efficiency (within 0.2\%).

\section{2 - Discussion}

An important result of the calculation of adiabatic efficiency in the absence of spanwise transport (Fig. 8.7) was the low efficiency at the hub. Although no experimental evidence exists to support this observation, we believe that further investigation is needed in order to explain it.

The presence of vortical structures in the rotor blade wakes might have important implications as far as total rotor loss is concerned. The shed vortices have a certain amount of kinetic energy "locked in" them. The maximum entropy 
or loss production occurs, when all this kinetic energy is dissipated into heat Since all the vortex parameters (core circumferential velocity, core size etc.) are known, an order of magnitude calculation of the total loss can be performed. According to this calculation, the loss produced due to the complete dissipation of the "locked in" kinetic energy, was approximately equal to $1 \%$ of the total entropy production in the rotor. 


\section{CHAPTER 9}

\section{CONCLUSIONS AND RECOMMMENDATIONS \\ FOR FUTURE STUDIES}

\section{1 - Conclusions}

A technique has been developed to measure time resolved concentration in unsteady, compressible flows. It is based on the operation of the dual-hot wire-aspirating probe and has frequency response of at least $18 \mathrm{kHz}$ and uncertainty less than 0.005 to full scale in mass fraction units. This application is an extension of the previous use of the probe to measure time resolved total pressure and total temperature.

It has been demonstrated both analytically and experimentally that the signals from the two hot wires mounted on the aspirating probe lag in time with respect to the signal from the companion total pressure probe. This time lag has been both analytically estimated and experimentally measured to be approximately $1 / 4$ of the blade passing period. The incorporation of the time lag correction in the probe data reduction schemes eliminated most of the negative entropy regions observed in previous experiments with this probe.

For the first time ever, time resolved measurements of spanwise transport in a transonic compressor have been obtained. For that purpose, tracer gas experiments were conducted at the MIT Blowdown Facility. The test article was the Air Force High Through Flow Transonic Compressor. Simultaneous, time resolved, high frequency (at least $18 \mathrm{kHz}$ ) measurements of total pressure, total 
temperature and concentration of tracer were obtained, using the newly developed concentration measurement technique.

Several experimental observations of the transport were made. First, up to $5 \%$ of the compressor mass flow moved along the blade span. Second, the spanwise migrating fluid was found in the blade wakes at the measurement location. Third, this fluid moved towards both hub and tip in the blade wakes. Fourth, the radially convected fluid had high entropy, much higher than that of the average flowfield. Fifth, the "inviscid core" fluid moves preferentially towards the suction side of the blade passage and away from the pressure side.

Under certain assumptions, the time averaged turbulent mixing in the transonic compressor has been estimated from our time averaged data. The results compare closely (within 20\%) with the prediction of the model proposed by Gallimore and Cumpsty (based on low speed multistage compressors).

A simple model was developed to explain the spanwise fluid transport. Gertz's 2-D wake vortex street model was extended into a quasi 3-D form. The 2-D model was fitted to the data at four spanwise locations and the spanwise variation of the parameters of the vortex street (such as vortex strength and core size) were determined. The model fit showed the shedding frequencies to be the same [17 (+/-) $0.4 \mathrm{kHz}]$ at all four spanwise locations, suggesting that the vortex shedding is coherent along the span. The spanwise pressure gradient created by the variation of vortex strength led to substantial spanwise transport in the vortex cores. The model predicted the transport to the hub (approximately $2 \%$ of the total compressor mass flow), but not the transport to the tip, which was underestimated by a factor of 5 . 
Spanwise transport within the blade row is required in order to have a substantial effect on the spanwise distribution of adiabatic efficiency. In this case, a change of about $9 \%$ in adiabatic efficiency near the tip is possible. The total spanwise transport, before and after the trailing edge, can explain the characteristic low efficiencies observed near the tip of transonic compressors. The model predicts very low adiabatic efficiency near the hub. However, this result (see Fig. 8.7) represents the maximum effect of the spanwise transport on the distribution of adiabatic efficiency. To our knowledge, no experimental evidence of this unusually high hub entropy production exists. We believe that further investigation into this phenomenon is needed.

As we mentioned in the Introduction, the higher than one adiabatic efficiency measured by Wennerstrom (see Fig. 5.15, "Air Force Data") was one of the motivations for this research. A few years later, $\mathrm{Ng}$ [1] obtained time resolved measurements of adiabatic efficiency and found also higher than one efficiency (or negative entropy) regions. However, the time lag correction that we applied to the aspirating probe signals eliminated those negative entropy regions. On the other hand, no conclusive explanation exists for the higher than one time averaged efficiency measured by Wennerstrom. A possible probe artifact (probes give a time average reading) might be the reason for that paradox. 


\section{2 - Recommendations for future studies}

In regions of separation strong spanwise transport is possible, since the separated fluid can be centrifuged towards the tip under the action of the strong spanwise pressure gradient of the rotor. Prediction of the amount of fluid that can move requires knowledge of the location and shape of the separation line on the rotor blade. Unsteady phenomena in the relative frame (such as vortex shedding) can also be coupled with the unsteady motion of the separation point and its interaction with the compressor shock system. Therefore strong need exists for direct measurement of the motion and location of the separation line on the blade surface.

The presence of unsteadiness in the relative rotor frame in the form of regular vortex streets leads to the important question of what happens to the kinetic energy that is "locked" in these vortices. In particular, once the vortex parameters are determined from the vortex model, the energy of each individual vortex can be estimated. If all this energy is assumed to be lost or transformed into heat, then an efficiency penalty can be calculated. An order of magnitude calculation was actually performed and the results were presented in section 8.2. However, a more realistic approach would be to include the downstream stator row and try to estimate its effect on performance as the vortices interact with the blades. A possibility exists that by designing the stator blades appropriately some of the energy "locked" in the vortices can be transformed into useful work or pressure rise and/or the effects on performance can be optimized.

The measured spanwise fluid transport and its effect on the distribution of adiabatic efficiency have important implications as far as design criteria and 
procedures are concerned. Further investigation in this area is highly desirable.

Finally, a more rigorous 3-D model to describe the formation and evolution of the spanwise vortices is needed. In addition, the possibility of using optical techniques to observe these vortices as they are shed behind the blade is quite optimistic. 


\section{APPENDIX A}

The calculation of the spanwise velocities in the vortex core under the assumption of constant convective velocity for all the fluid particles in the core is presented here. This calculation uses eq. (7.19) with constant convection time $\Delta t$ Fig. A.1 shows the results of this calculation for four spanwise locations. Fig. A.2 presents a perspective of the vortex core and the spanwise velocities in the core.

The migrating mass flow and the maximum spanwise displacement were also calculated (as in section 7.2.7). The maximum spanwise displacement towards hub and tip were found to be equal to $36 \%$ and $54 \%$ of the blade span, respectively. The migrating mass flow towards hub and tip were approximately $70 \%$ and $17 \%$, respectively, of the estimated ones from the measurements (Table 6.1). A comparison with the results presented in Table 7.7 shows that this prediction underestimates the measured migration more than the ones described in sections 7.2.7 and 7.2.8 (inviscid based on eq. 7.19 with variable convective velocity and quasi-viscous). 


\section{REH'ERENCES}

[1] Ng, Wing-fai, "Time-Resolved Stagnation Temperature Measurement in a Transonic Compressor Stage," MIT-GTL Report, No. 177, 1983.

[2] Gertz, J.B., "Unsteady Design Point Flow Phenomena in Transonic Compressors," MIT-GTL Report, No. 188, September 1986.

[3] Kerrebrock, J.L., "Flow in Transonic Compressors," AlAA-80-0124, Dryden Lectureship in Research, January 14-16, 1980/Pasadena, California.

[4] Lieblein, S., "Experimental Flow in Two-Dimensional Cascades," Aerodynamic Design of Axial Flow Compressors, NASA SP-36, 1965, Chapter VI.

[5] Thompkins, W.T., Jr., Usab, W.J., Jr., "A Quasi-Three-Dimensional Blade Surface Boundary Layer Analysis for Rotating Blade Rows," ASME Paper No. 81-GT-126.

[6] Wennerstrom, A.J., private communication.

[7] Hawthorne, W.R., "Secondary Circulation in Fluid Flow," Proc. Royal Society of London (Series A), pp. 206, 1951.

[8] Squire, H.B. and Winter, K.G." The Secondary Flow in a Cascade of Aerofoils in a Non-Uniform Stream," Journal of Aeronautical Science, Vol. 18, April 1951, pp. 271-277.

[9] . Horlock, J.H., "Annulus Wall Boundary Layers in Axial Compressor Stages," Journal of Basic Engineering, Vol. 55, March 1963.

[10] Dring, R.P. and Joslyn, H.D., "The Relative Eddy in Axial Turbine Rotor Passages," ASME Paper No. 83-GT-22.

[11] Stanitz, J.D. and Ellis, GO., "Flow Surfaces in Rotating Axial- Flow Passages," NACA TN 2834, 1952.

[12] Lakshminarayana, B., "Methods of Predicting the Tip Clearance Effects in Axial Flow Turbomachinery," Journal of Basic Engineering, Transactions of the ASME, pp. 467-482, September 1970.

[13] Rains, D.A., "Tip Clearance Flow in Axial Flow Compressors and Pumps", California Institute of Technology, Hydrodynamics and Mechanical Engineering Laboratories Report No. 5, 1945.

[14] Inoue, M., Kuroumaru, M. and Fukuhara, M., "Behavior of Tip Leakage Flow 
Behind an Axial Flow Compressor Rotor", ASME Journal of Engineering for Gas Turbine and Power, Vol. 108, No. 1, Jan. 1986, pp. 7-13.

[15] McCune, J.E and Hawthorne, W.R., "The Effects of Trailing Vorticity on the Flow Through Highly Loaded Cascades," Journal of Fluid Mechanics, Vol. 74, part 4, pp. 721-740, 1976.

[16] Thompkins, W.T., Jr., "An Experimental and Computational Study of the Flow in a Transonic Compressor Rotor," MIT-GTL Report No. 129, May 1976.

[17] Pierzga, M.J. and Wood, J.R. "Investigation of the Three- Dimensional Flow Field Within a Transonic Fan Rotor: Experiment and Analysis," ASME Paper No. 84-GT-200.

[18] Choi, D. and Knight, C.J., "Computations of 3-D Viscous Flows in Rotating Turbomachinery Blades," to be presented at the AIAA Conference in Reno, Nevada, January 9-12, 1989.

[19] Adkins, GG, Jr. and Smith, L.H., Jr., "Spanwise Mixing in Axial- Flow Turbomachines," ASME Paper No. 81-GT-57.

[20] Gallimore, S.J. and Cumpsty, NA., "Spanwise Mixing in Multistage Axial Flow Compressors: Part I-Experimental Investigation," ASME Journal of Turbomachinery, Vol. 108, 1986, pp. 2-9.

[21] Gallimore, S.J., "Spanwise Mixing in Multistage Axial Flow Compressors: Part II-Throughflow Calculations Including Mixing," ASME Journal of Turbomachinery, Vol. 108, 1986, pp. 10-16.

[22] Wisler, D.C., Bauer, RC. and Okiishi, T.H., "Secondary Flow, Turbulent Diffusion, and Mixing in Axial-Flow Compressors," Journal of Turbomachinery, Vol. 109, pp.455-482, October 1987.

[23] Denton, J.D. and Usui, S., Use of a Tracer Gas Technique to Study Mixing in a Low Speed Turbine," ASME Paper No. 81-GT-86.

[24] Kerrebrock, J.L., et al., "The MIT Blowdown Compressor Facility," Journal of Engineering for Power, Vol. 46, No. 4, October 1974.

[25] Wennerstrom, A.J. and Frost, GR, "Design of a $1500 \mathrm{ft} / \mathrm{sec}$, Transonic, High-Through-Flow, Single-Stage Axial-Flow Compressor with Low Hub/Tip Ratio," AFAPL-TR-76-59, October 1976.

[26] Wennerstrom, A.J., et al., "Investigation of a $1500 \mathrm{ft} / \mathrm{sec}$, Transonic, High-Through-Flow, Single-Stage Axial-Flow Compressor with Low 
Hub/Tip Ratio," AFAPL-TR-76-92, October 1976.

[27] Kotidis, P.A., MIT S.M. Thesis, Gas Turbine Laboratory, August 1985.

[28] Drela, M., personal communication.

[29] Collis, D.C. and Williams, M.J., "Two-Dimensional Convection from Heated Wires at Low Reynolds Numbers," Journal of Fluid Mechanics, Vol. 6, 1959.

[30] Kuchemann D., "The Aerodynamic Design of Aircraft," Pergamon Press, 1978

[31] Watts, M.E, Ng, W.F. and Kurosaka, M., "Time-Resolved Stagnation Temperature and Pressure Measurements in the Vortex Street Behind a Cylinder," Virginia Polytechnic Institute and State University, Department of Mechanical Engineering Interim Report 85-147-05 \#1, February 1986.

[32] Kurosaka, M., et al., "Energy Separation in a Vortex Street," Journal of Fluid Mechanics, Vol. 178, pp. 1-29, 1987.

[33] Hirschfelder, J.O., Curtiss, C.F. and Bird, RB., "Molecular Theory of Gases and Liquids," John Wiley \& Sons, Inc., New York, 1964.

[34] Rohsenow, W.M., "Handbook of Heat Transfer Fundamentals," McGraw-Hill, 2nd ed., New York, 1985.

[35] Sutherland, W., "The Viscosity of Mixed Gases," Phil. Mag. 40, pp. 421-431, 1895.

[36] Wilke, C.R., "A Viscosity Equation for Gas Mixtures," The Journal of Chemical Physics, Vol. 18, Number 4, pp. 517-519, April 1950.

[37] Wassiljewa, A., "Warmeleitung in Gasgemischen, 1," Z Physik 5, pp. 737-742, 1904.

[38] Buddenberg, J.W. and Wilke, C.R, "Calculation of Gas-Mixture Viscosities," Ind. Eng. Chem. 41, pp. 1345-1347, 1949.

[39] Touloukian, Y.S., Liley P.E and Saxena, S.C., "Thermophysical Properties of Matter," The TPRC Data Series, Purdue University, Vol. 11, 1970.

[40] Saxena, S.C., "Thermal Conductivity of Binary Mixtures of Rare Gases and Wassiljewa Coefficients," Thermal Conductivity, Proceedings of the 8th Conference, Plenum Press, NY., 265-280, 1969. 
[41] Mason, EA. and Saxena, S.C., "Approximate Formula for the Thermal Conductivity of Gas Mixtures," Phys. Fluids, 1(5), pp. 361-369, 1958.

[42] Brokaw, RS., "Approximate Formulas for the Viscosity and Thermal

Conductivity of Gas Mixtures," J. Chem. Phys., 29(2), pp. 391-397, 1958.

[43] Brokaw, RS., "Approximate Formulas for the Viscosity and Thermal

Conductivity of Gas Mixtures, II.," J. Chem. Phys., 42(4), pp. 1140-1146, 1965.

[44] Cowling, T.G, Gray, P. and Wright, P.G. "The Physical Significance of Formulae for the Thermal Conductivity and Viscosity of Gaseous Mixtures," Proc. Roy. Soc., A276, pp. 69-82, 1963.

[45] Saxena, S.C. and Gambhir, RC., "Semi-Empirical Formulae for the Viscosity and Translational Thermal Conductivity of Gas Mixtures," Proc. Phys. Soc. (London), 81, pp. 788-789, 1963.

[46] Touloukian, Y.S., Liley P.E and Saxena, S.C., "Thermophysical Properties of Matter," The TPRC Data Series, Purdue University, Vol. 3, 1970.

[47] "Thermophysical Properties of Refrigerants," ASHRAE, New York, 1976.

[48] Holt, J.L., MIT Gas Turbine Laboratory, S.M. Thesis, June 1985.

[49] Liepmann, H.W. and Roshko, A., "Elements of Gasdynamics," John Wiley \& Sons, Inc., New York 1957.

[50] Whitehead, D.S., "The Vibration of Air in a Duct with a Subsonic Mean Flow," The Aeronautical Quarterly, February 1961, pp. 34-40.

[51] Doebelin, EO., "Measurement Systems," McGraw-Hill, Inc., 1975.

[52] Kline, S.J., "The Purposes of Uncertainty Analysis," Journal of Fluids Engineering, Vol. 107, pp. 153-160, June 1985.

[53] Moffat, R.J., "Using Uncertainty Analysis in the Planning of an Experiment," Journal of Fluids Engineering, Vol. 107, pp. 173-178, June 1985.

[54] Abernethy, RB., Benedict, R.P. and Dowdell, R.B., "ASME Measurement Uncertainty," Journal of Fluids Engineering, Vol. 107, pp. 161-164, June 1985.

[55] Kline, S.J., 1983 Symposium on Uncertainty Analysis Closure, Journal of Fluid Engineering, Vol. 107, pp. 181-182, June 1985. 
[56] Schlichting, H., "Boundary Layer Theory," 7th ed., McGraw-Hill, New York, 1979.

[57] Gebhart, B., et al., "Buoyancy Induced Flows and Transport," Hemisphere Pub., Washington DC, 1988.

[58] Gebhart, B., Hilder, D.S. and Kelleher M., "The Diffusion of Turbulent Buoyant Jets," Advances in Heat Transfer, Vol. 16, 1984.

[59] Owen, P.R., "Computational Simulation of Unsteady Flow in a Transonic Compressor Rotor," MIT-GTL Report No. 189, October 1986.

[60] Hinze, J.O., "Turbulence," McGraw-Hill, Now York, 1959.

[61] Launder, B.E and Spalding, D.B., Mathematical Models of Turbulence," Academic Press, New York, 1972.

[62] Hah, C., "A Numerical Study of Three-Dimensional Flow Separation and Wake Development in an Axial Compressor Rotor," ASME Paper No. 84-GT-34.

[63] Wennerstrom, A.J., "Experimental Study of a High-Throughflow Transonic Axial Compressor Stage," Journal of Engineering for Gas Turbines and Power, Transactions of the ASME, Vol. 106, pp. 552-560, July 1984.

[64] Wennerstrom, A.J. and Puterbaugh, S.L., "A Three-Dimensional Model for the Prediction of Shock Losses in Compressor Blade Rows," Journal of Engineering for Gas Turbines and Power, Transactions of the ASME, Vol. 106. pp. 295-299, April 1984.

[65] Strazisar, A.J., "Investigation of Flow Phenomena in a Transonic Fan Rotor Using lase Anemometry," Journal of Engineering for Gas Turbines and Power, Transactions of the ASME, Vol. 107, pp. 427-435, April 1985.

[66] Scott, J.N and Hankey, W.L., Jr., "Navier-Stokes Solutions of Unsteady Flow in a Compressor Rotor," ASME Paper No. 86-GT-226.

[67] Richardson, S.M., "A Numerical Study of Unsteady Flow Effects in a Supersonic Compressor Cascade," AGARD-CP-401, pp. 20-1 to 20-11, Munich, September 1986.

[68] Marble, F., in "Aerodynamics of Turbines and Compressors," ed. W.R. Hawthorne, Princeton Series, Vol. X, 1964.

[69] Epstein, A.H., "Quantitative Density Visualization in a Transonic Compressor Rotor," PhD. Thesis, MIT, September 1975. 
[70] Heinemann, H.J., Lawaczeck, O. and Butefisch, K.A., "Von Karman Vortices and their Frequency Determination in the Wakes of Profiles in the Sub- and Transonic Regimes," IUTAM Symposium, 1976, pp. 75-82.

[71] Greenway, M.E. and Wood, C.J., "The Effect of a Bevelled Trailing Edge on Vortex Shedding and Vibration," Journal of Fluid Mechanics, Vol. 61, part 2. 1973.

[72] Paterson, R.W. and Weingold, H.P., "Experimental Investigation of a Simulated Compressor Airfoil Trailing Edge Flowfield," AIAA-84-0101, 1984.

[73] Xing, W.F. and Marenbach, G, "Periodic Vortex Shedding in the Supersonic Wake of a Flat Plate," (German), A84-20842, 1983.

[74] Heinemann, H.J. and Butefisch, K.A., "Determination of the Vortex Shedding Frequency of Cascades with Different Trailing Edge Thicknesses," AGARD CP-227, 1977, pp. 35-1 to 35-10.

[75] Camus, J.J. and Bryanston-Cross, P.J., "One MHz Bandwidth, Real Time Schlieren Techniques in a Linear Cascade," Proceedings of Symposium on Measurement Techniques in Transonic and Supersonic Flows in Cascades and Turbomachines, Lyon, 1981.

[76] Young, W.H., Meyers, J.F. and Hepner, T.E. "Laser Velocimeter Systems Analysis Applied to a Flow Survey Aboved a Stalled Wing," NASA TN D-8408, 1977.

[77] Dunker, RJ., Flow Measurements in the Stator Row of a Single Stage Transonic Axial Compressor with Controlled Diffusion Stator Blades," AGARD CP-351, 1983.

[78] Strazisar, A.J. and Powell, J.A., "Laser Anemometer Measurements in a Transonic Axial Flow Compressor Rotor," ASME Journal of Engineering for Power, Vol. 103, №. 2, April 1981, pp. 430-437.

[79] Powell, J.A., Strazisar, A.J. and Seasholtz, RG, "Efficient Laser Anemometer for Intra-Rotor Flow Mapping in Turbomachinery," ASME Journal of Engineering for Power, Vol. 103, No. 2, April 1981, pp. 424-429.

[80] Cantwell, B. and Coles, D., "An Experimental Study of Entrainment and Transport in the Turbulent Near Wake of a Circular Cylinder," Journal of Fluid Mechanics, Vol. 136, pp. 321-374, 1983.

[81] Sears, W.R, "Unsteady Motion of Airfoils with Boundary-Layer Separation," 
AIAA Journal, Vol. 14, No. 2, pp. 216-220.

[82] Tritton, D.J., "Experiments on the Flow Past a Circular Cylinder at Low Reynolds Numbers," Journal of Fluid Mechanics, Vol. 6, pp. 547-567, 1959.

[83] Kovasznay, L.S.G. "Hot-Wire Investigation of the Wake Behind Cylinders at Low Reynolds Numbers," Proc. Roy. Soc., Series A, 198, pp. 174-190, 1949.

[84] Phillips, O.M., "The Intensity of Aeolian Tones," Journal of Fluid Mechanics, Vol. 1. pp. 607-624, 1956.

[85] Hama, F.R., "Three-Dimensional Vortex Pattern Behind a Circular Cylinder," Journal of Aerospace Science, Vol. 24, pp. 156-158, 1957.

[86] Taneda, S., "Studies on Wake Vortices," Rep. Res. Inst Appl. Mech., Kyushu Univ. 1, pp. 131-144, 1952.

[87] Gerrard, J.H., "The Three-Dimensional Structure of the Wake of a Circular Cylinder," Journal of Fluid Mechanics, Vol. 25, Part 1, pp. 143-164, 1966.

[88] Berger, E, "Bestimmung der Hydrodynamischen Groessen einer Karmanschen Wirbelstrasse aus Hitzdrahtmessungen bei Kleinen Reynoldsschen Zahlen," Z Flugwiss., 12, pp. 41-59, 1964.

[89] Mattingley, GE, Univ. of Maryland, TN No. BN-295, 1962.

[90] Humphreys, J.S., "On a Circular Cylinder in a Steady Wind at Transition Reynolds Numbers," Journal of Fluid Mechanics, Vol. 9, pp. 603, 1960.

[91] Tritton, D.J., "Physical Fluid Dynamics," Van Nostrand Reinhold Co., 1977.

[92] Berger, E and Wille, R, "Periodic Flow Phenomena," Annual Review of Fluid Mechanics, Vol. 4, pp. 313-340, 1972.

[93] Roshko, A., "Experiments on the Flow Past a Circular Cylinder at Very High Reynolds Number," Journal of Fluid Mechanics, Vol. 10, pp. 345-356, 1961.

[94] Jones, GW., Jr., Cincotta, J.J. and Walker, RW., "Aerodynamic Forces on a Stationary and Oscillating Circular Cylinder at High Reynolds Numbers," NASA Tech Rep. TR R-300. 
[95] Gaster, M., "Vortex Shedding from Slender Cones at Low Reynolds Numbers," Journal of Fluid Mechanics, Vol. 38, Part 3, pp. 565-576, 1969.

[96] Tritton, D.J., "A Note on Vortex Streets Behind Circular Cylinders at Low Reynolds Numbers," Journal of Fluid Mechanics, Vol. 45, pp. 203-208, 1971.

[97] Gaster, M., "Vortex Shedding from Circular Cylinders at Low Reynolds Numbers," Journal of Fluid Mechanics, Vol. 46, Part 4, pp. 749-756, 1971.

[98] Gaster, M., "Vortex Shedding from Slender Cones," Resent Research on Unsteady Boundary Layers, IUTAM Symposium, Vol. 2, pp. 1499-1534, 1971.

[99] Mandella, M. and Bershader, D., "Quantitative Study of the Compressible Vortex: Generation, Structure and Interaction with Airfoils," AIAA-87-0328, 1987.

[100] Koch, C.C. and Smith, L.H., "Loss Sources and Magnitudes in Axial Flow Compressors," Engineering for Power, Vol. 98, №. 3, 1976.

[101] Batchelor, GK., "Axial Flow in Trailing Line Vortices," Journal of Fluid Mechanics, Vol. 20, Part 4, pp. 645-658, 1964.

[102] Lamb, H.L., "Hydrodynamics," Dover Publications, Now York, 1945.

[103] Squire, H.B., "The Growth of a Vortex in Turbulent Flow," The Aeronautical Quarterly, Vol. XVI, pp. 302-306, August 1965.

[104] Govindaraju, S.P. and Saffman, P.G., "Flow in a Turbulent Trailing Vortex," The Physics of Fluids, Vol. 14, No. 10, pp. 2074-2080, October 1971.

[105] Kerrebrock, J.L., "Aircraft Engines and Gas Turbines," MIT Press, Cambridge, Massachusetts, 1977.

[106] Shapiro, A.H., "The Dynamics and Thermodynamics of Compressible Fluid Flow," Volume I, John Wiley \& Sons, Inc., 1953. 


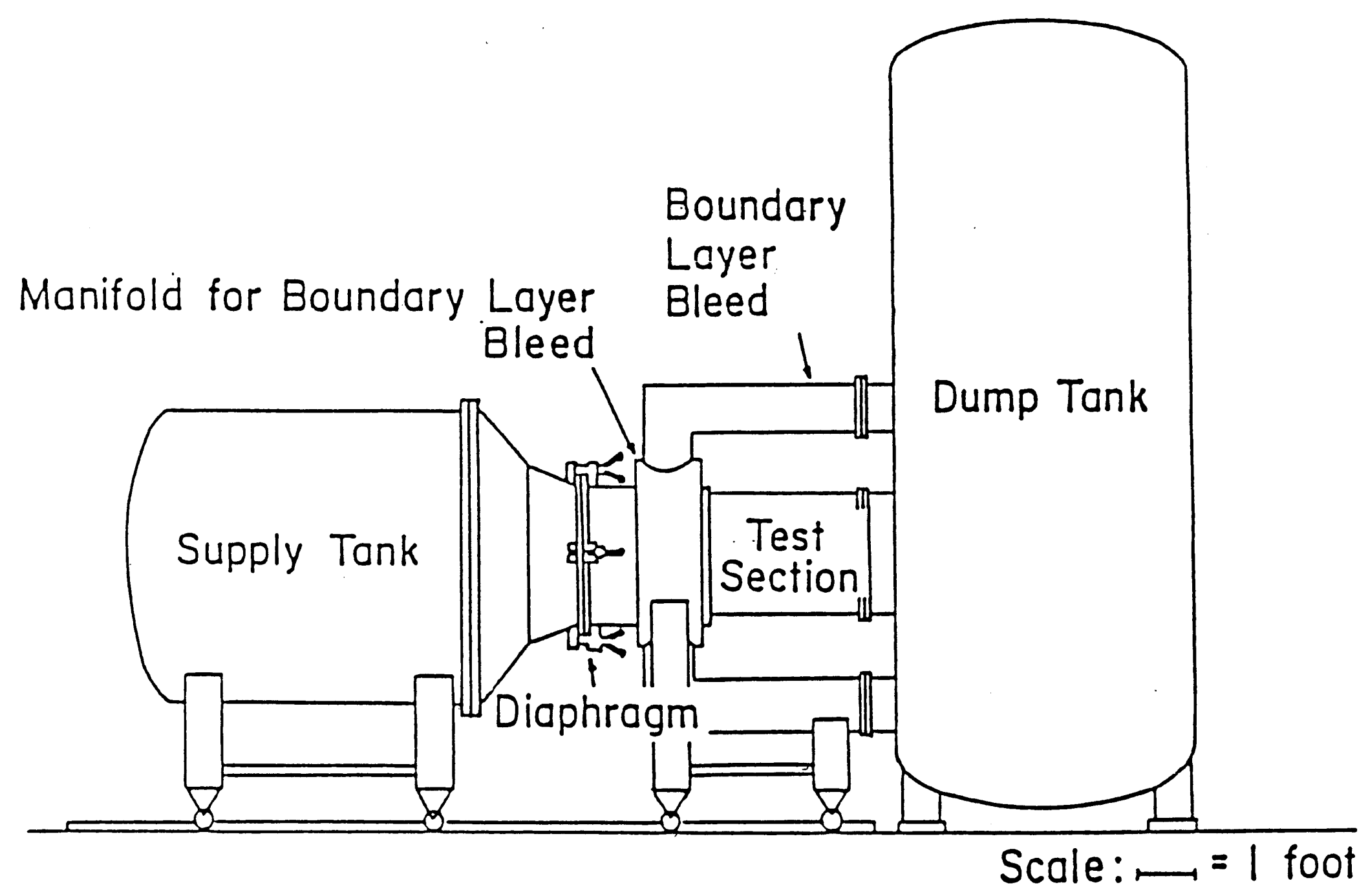

Figure 2.1: Scale drawing of the Blowdown Compressor Facility 

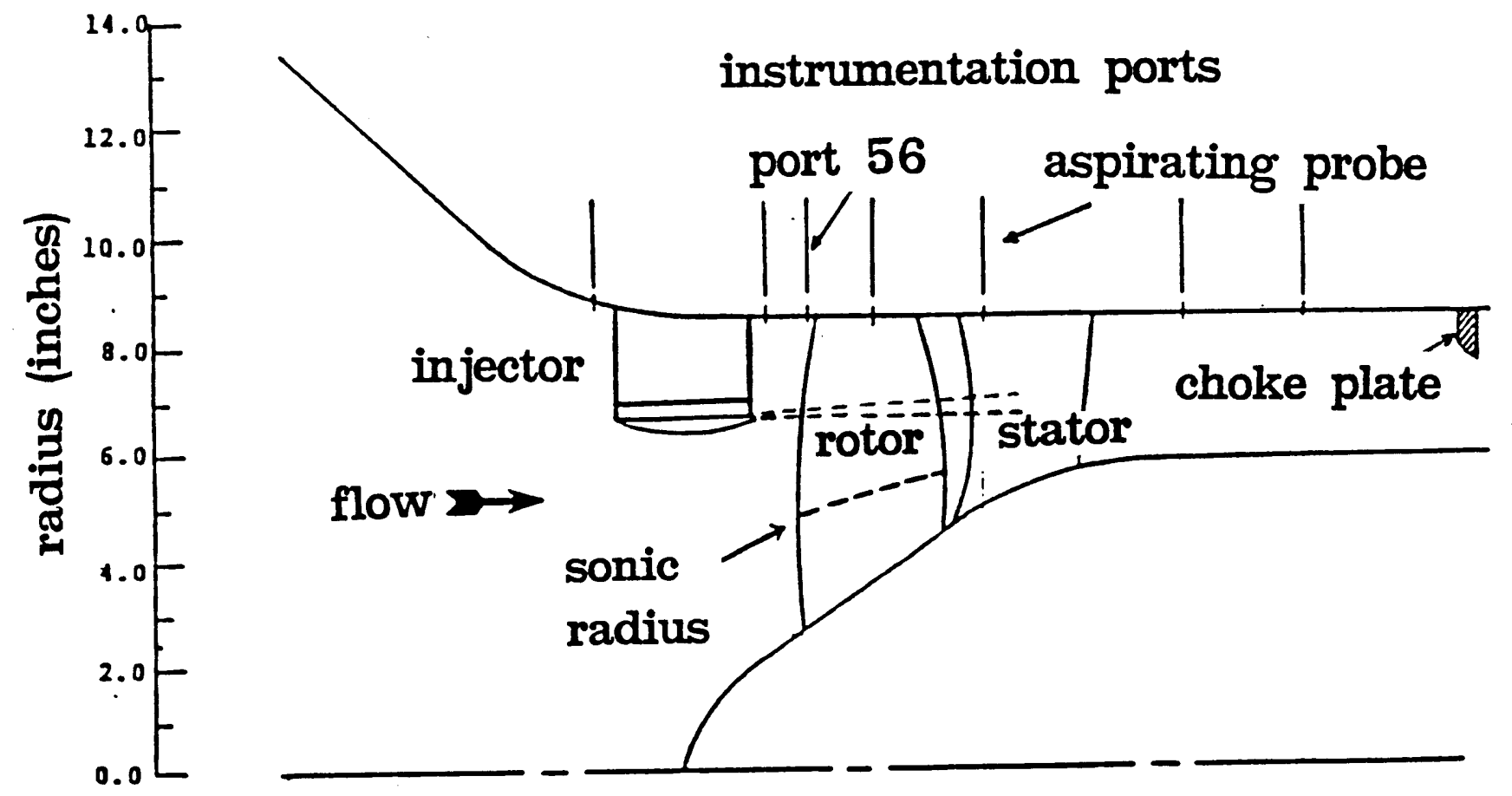

\section{Air Force High Thru Flow Stage (AFAPL)}

Figure 2.2: Scale drawing of the test section 


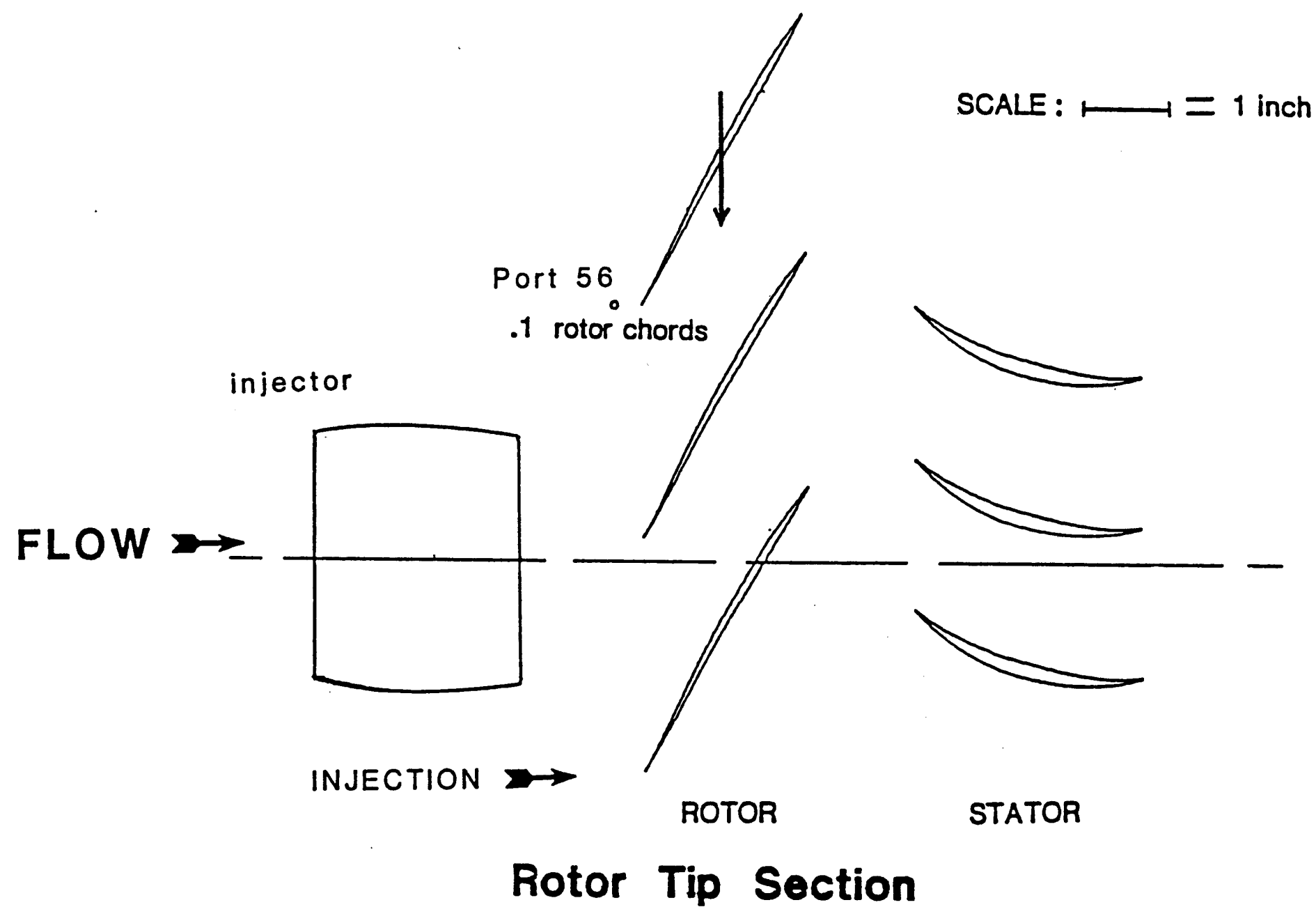

Figure 2.3: Top view of the experimental arrangement 


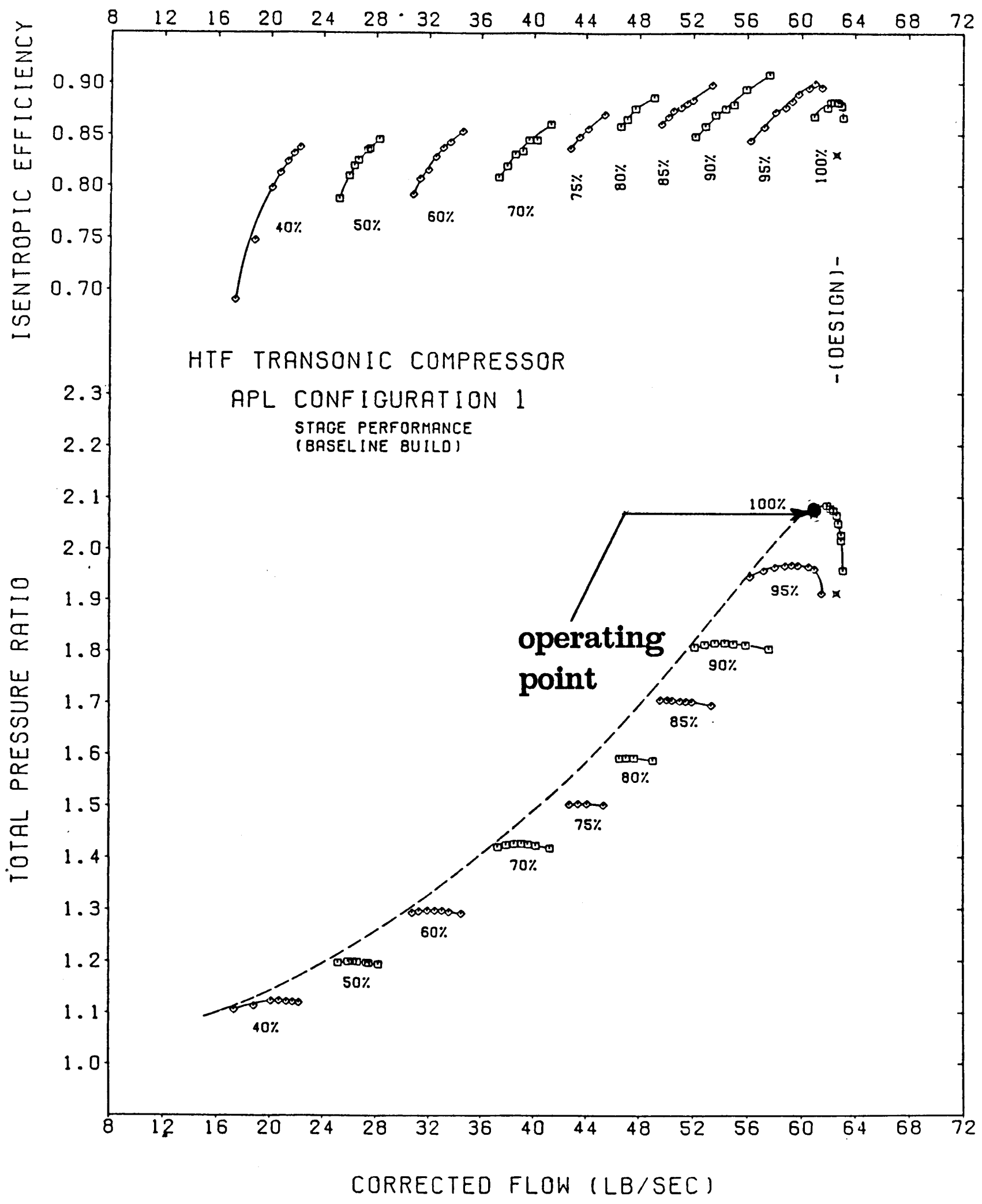

Figure 2.4: Compressor stage performance map 

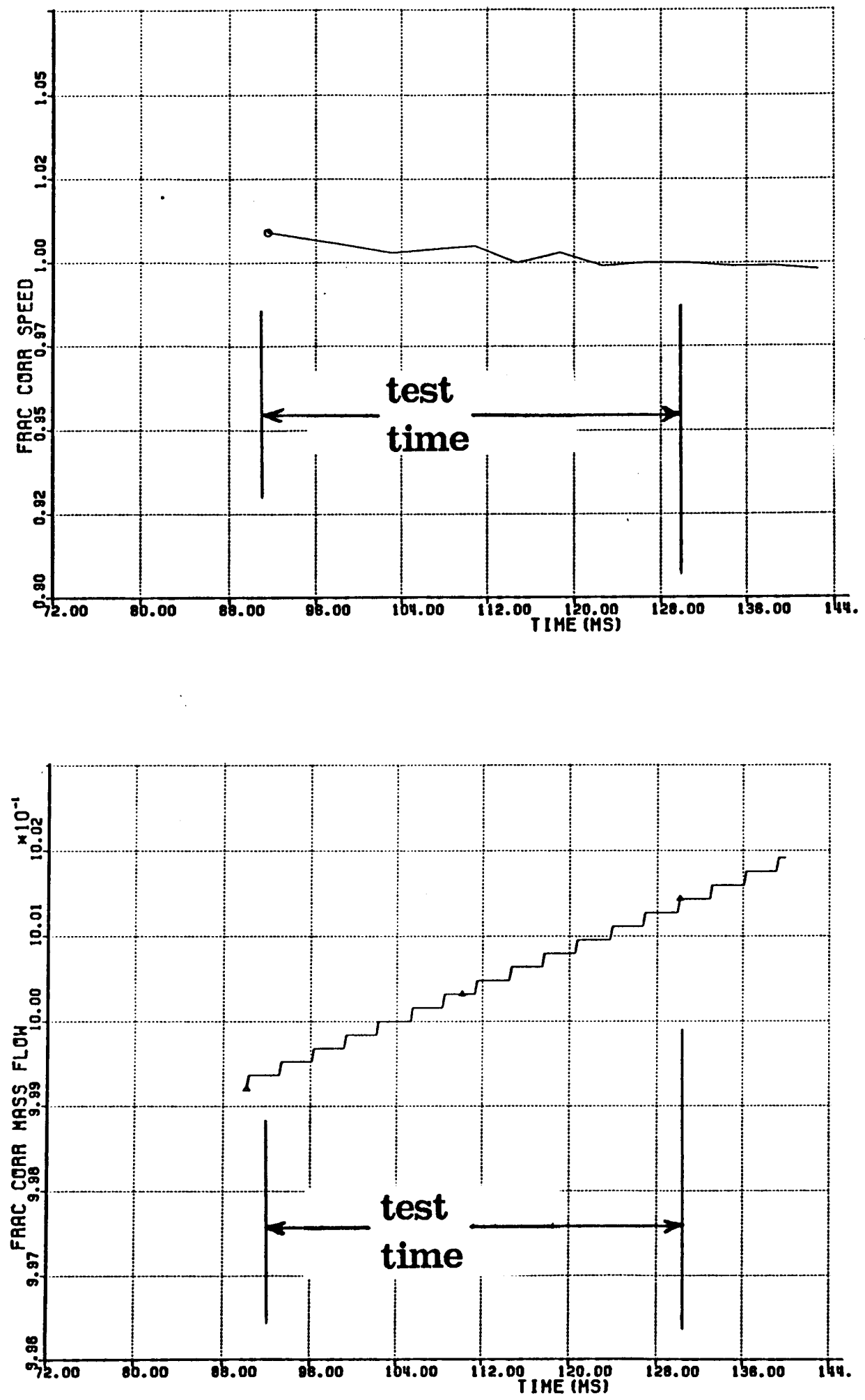

Figure 2.5: Fractional corrected speed (a) and fractional corrected mass flow (b), during a typical Blowdown test 


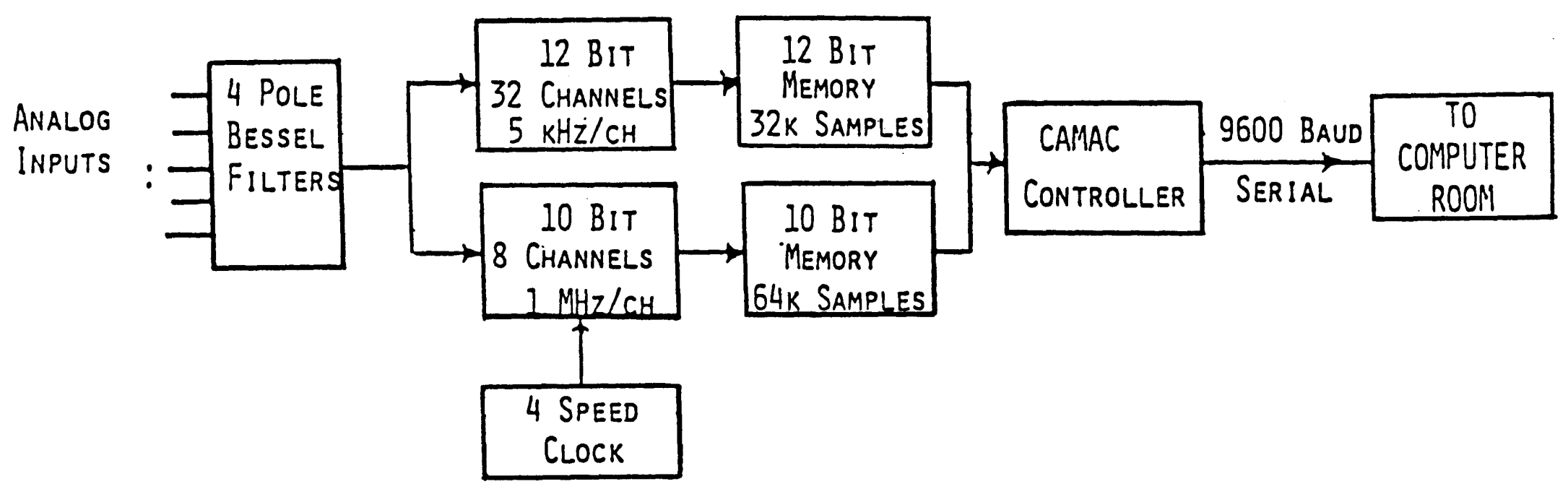

BLOWDOWN TUNNEL DATA ACQUISITION SYSTEM

Figure 2.6: Blowdown Compressor Facility data acquisition system 


\section{TRACER GAS INJECTOR}

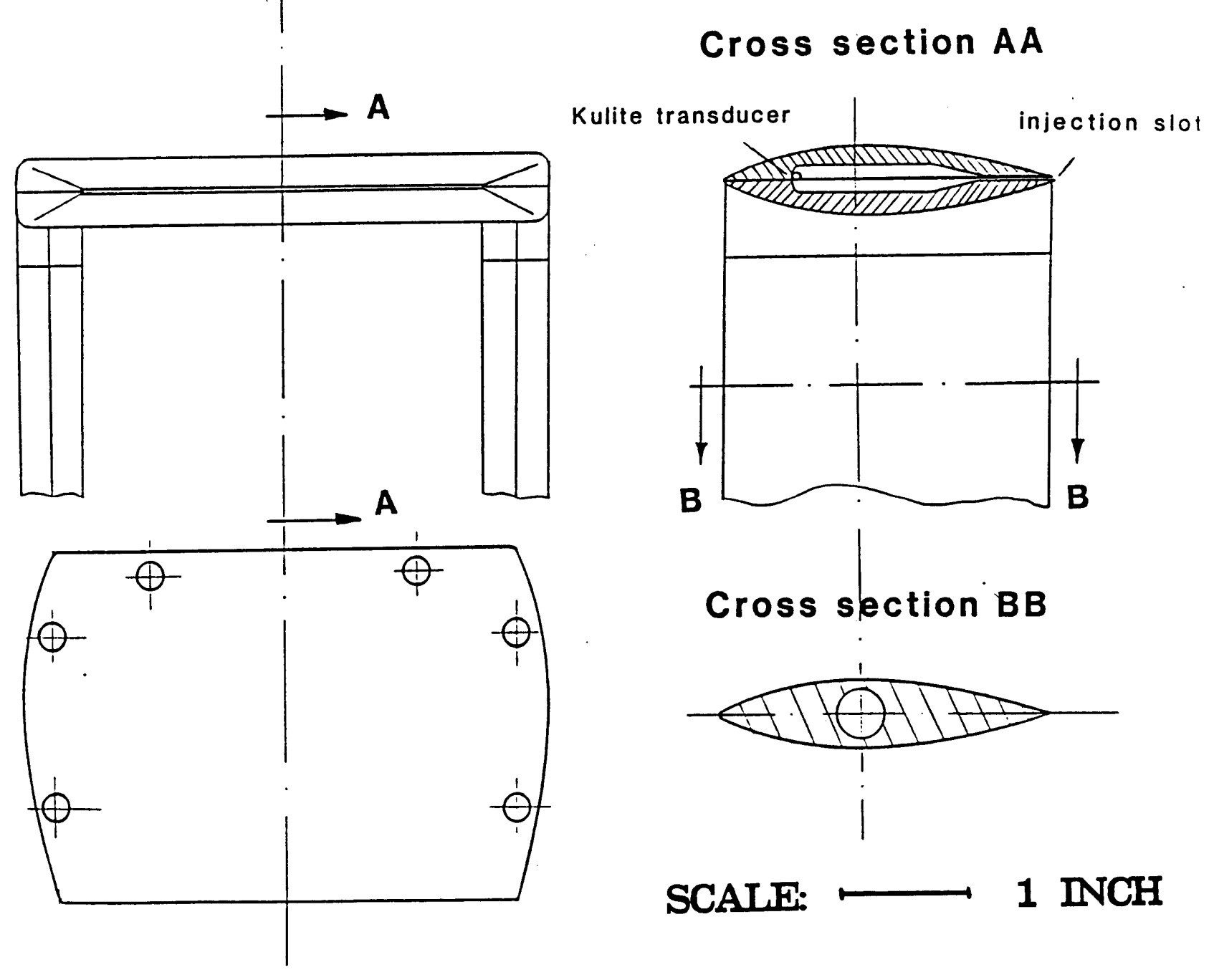

Figure 2.7: Scale drawing of the Tracer Gas Injector 


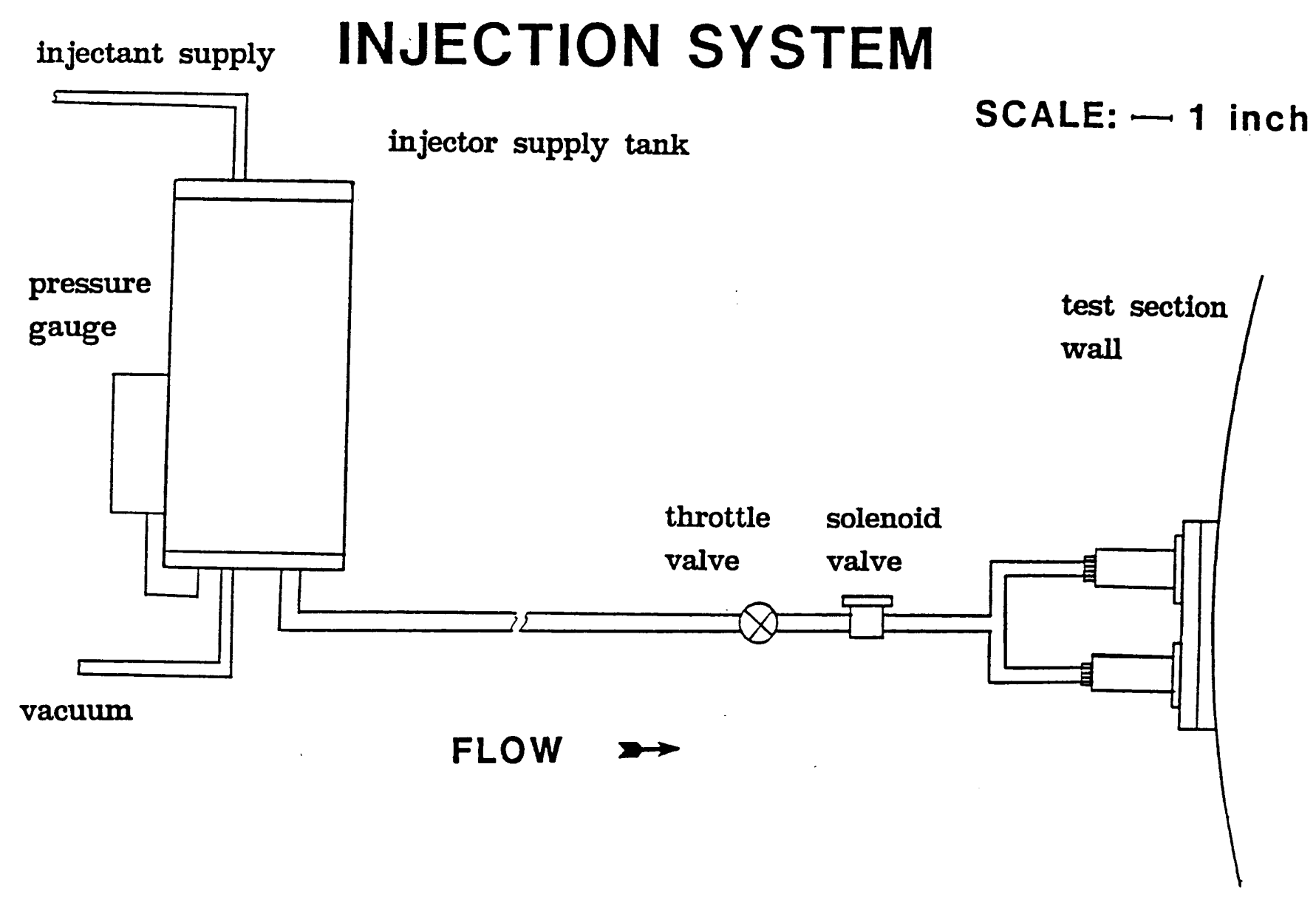

Figure 2.8: Schematic drawing of the injection system 


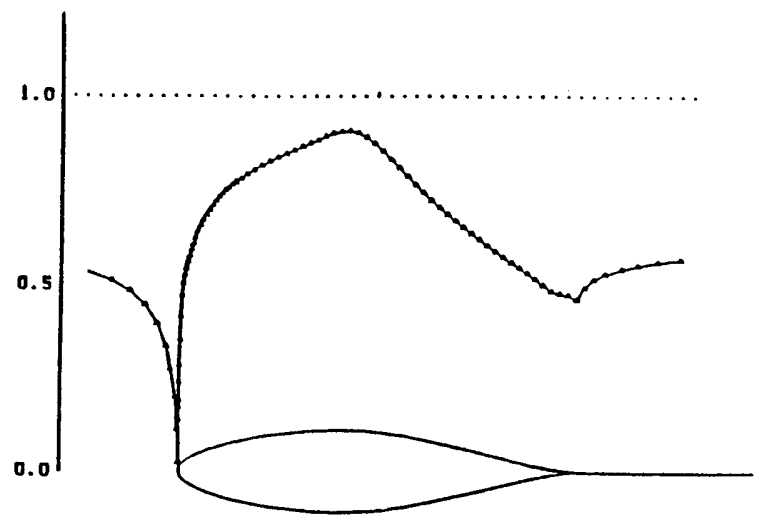

Surface Mach number

for NACA 654-021

INVISCID CASE

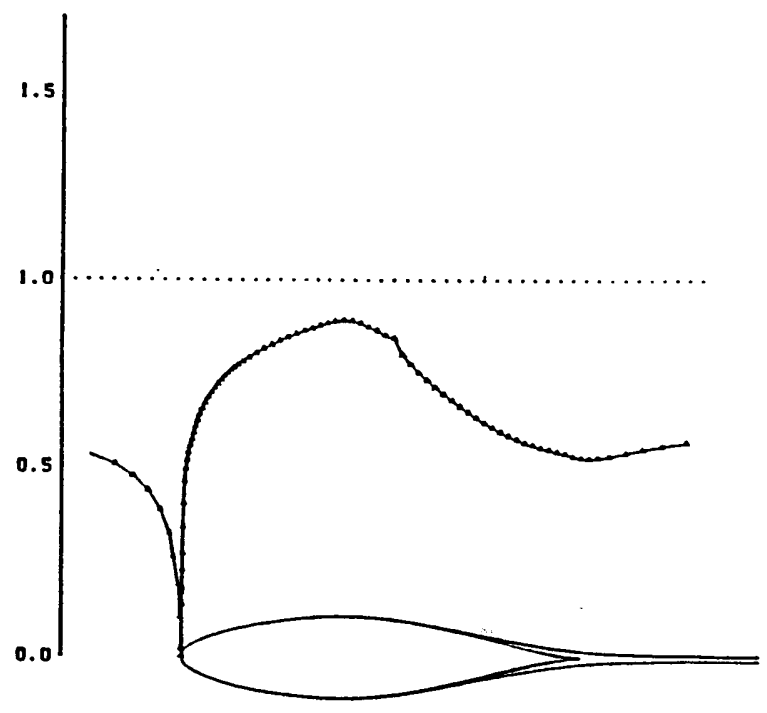

Surface Mach number

for NACA 654-021

VISCOUS CASE

Figure 2.9: Mach number distribution on the surface of the injector 


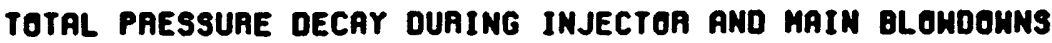

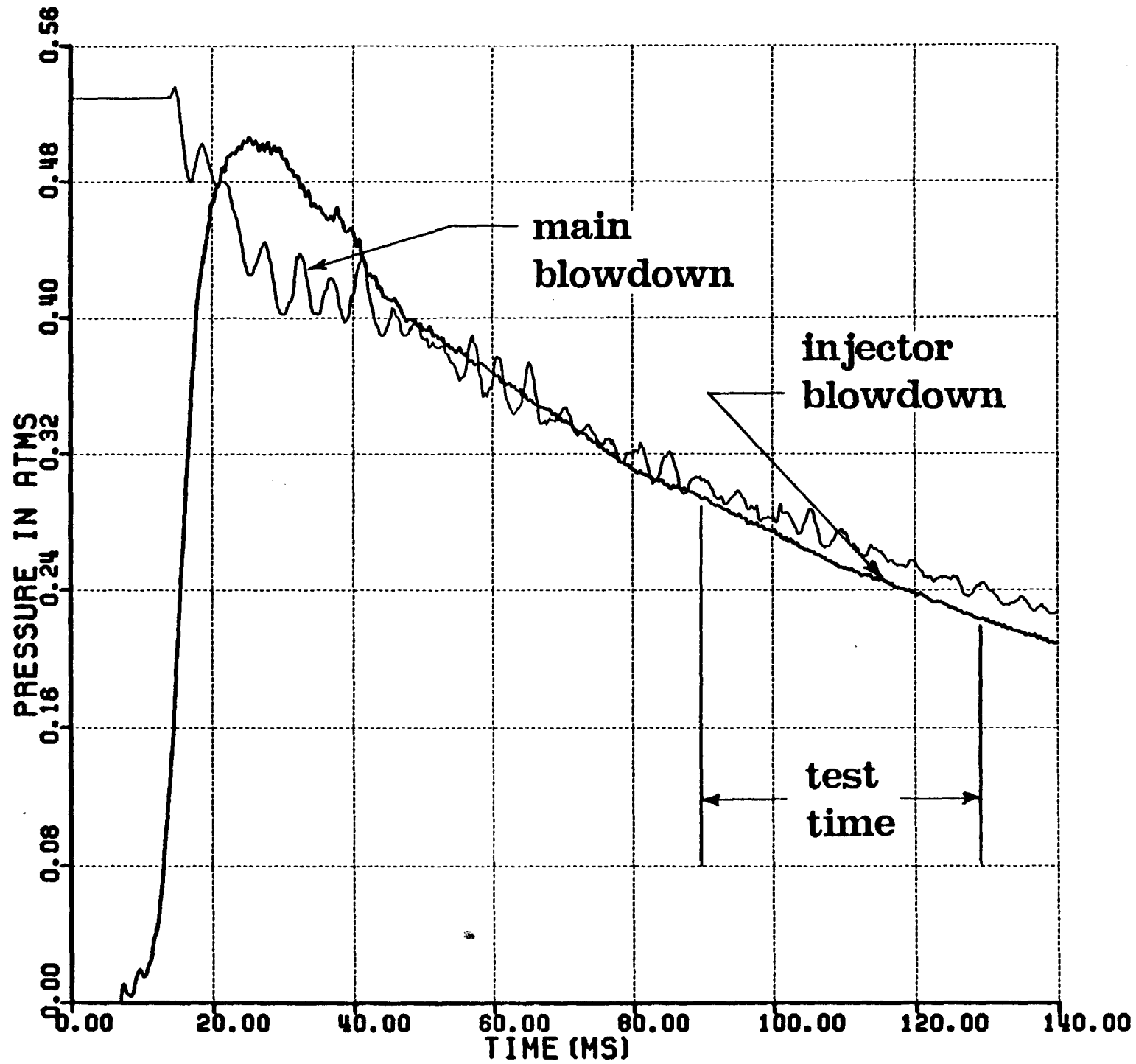

Figure 2.10: Comparison of total pressure decay traces between injector and main Blowdowns 
ENLARGED VIEH OF TOTAL PRESSURE TAACES DUAING the test TIME

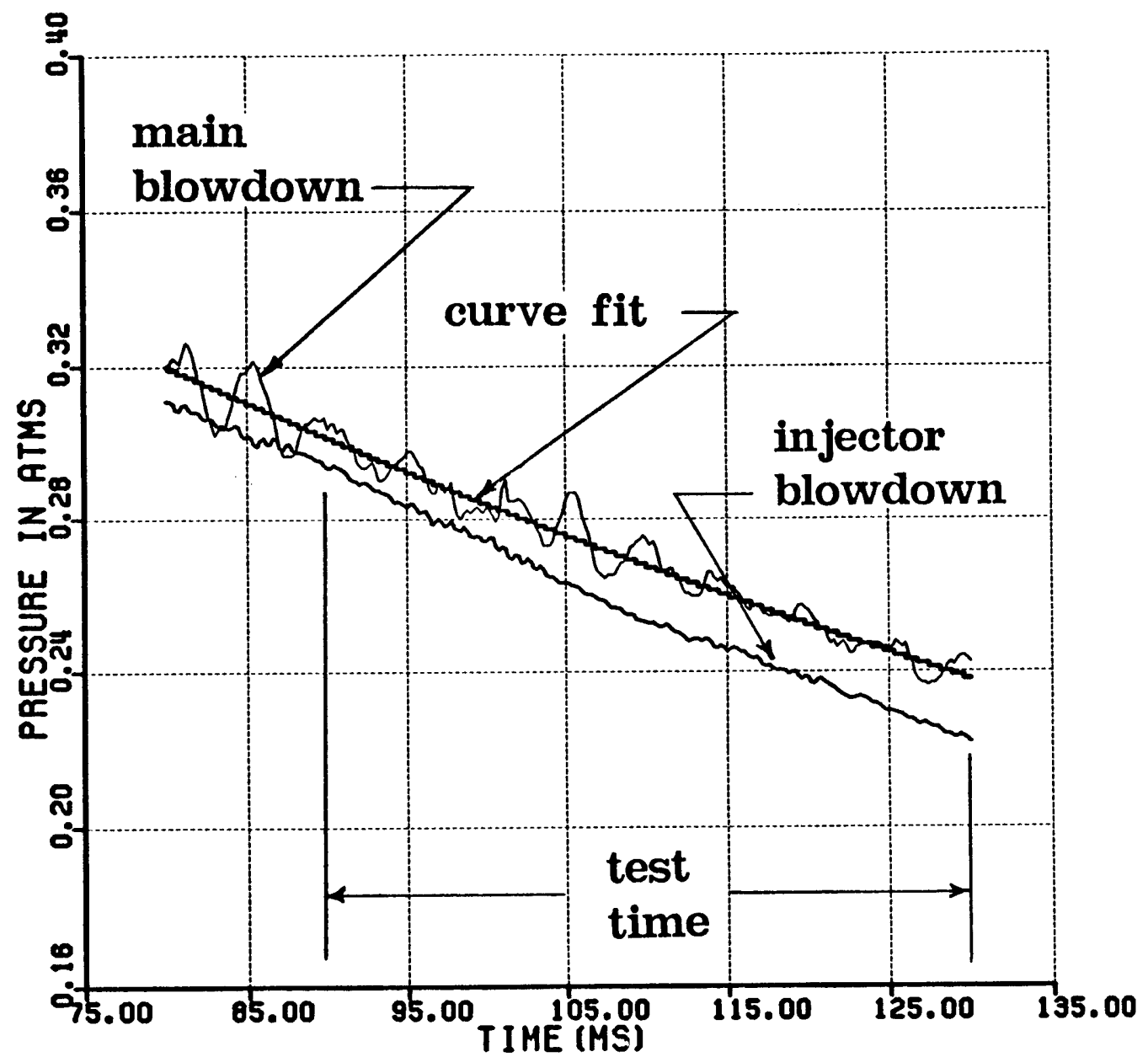

Figure 2.11: Enlarged view of the total pressure decay traces of Fig. 2.10 during test time and comparison with the curve fit of the total pressure trace of the main Blowdown 


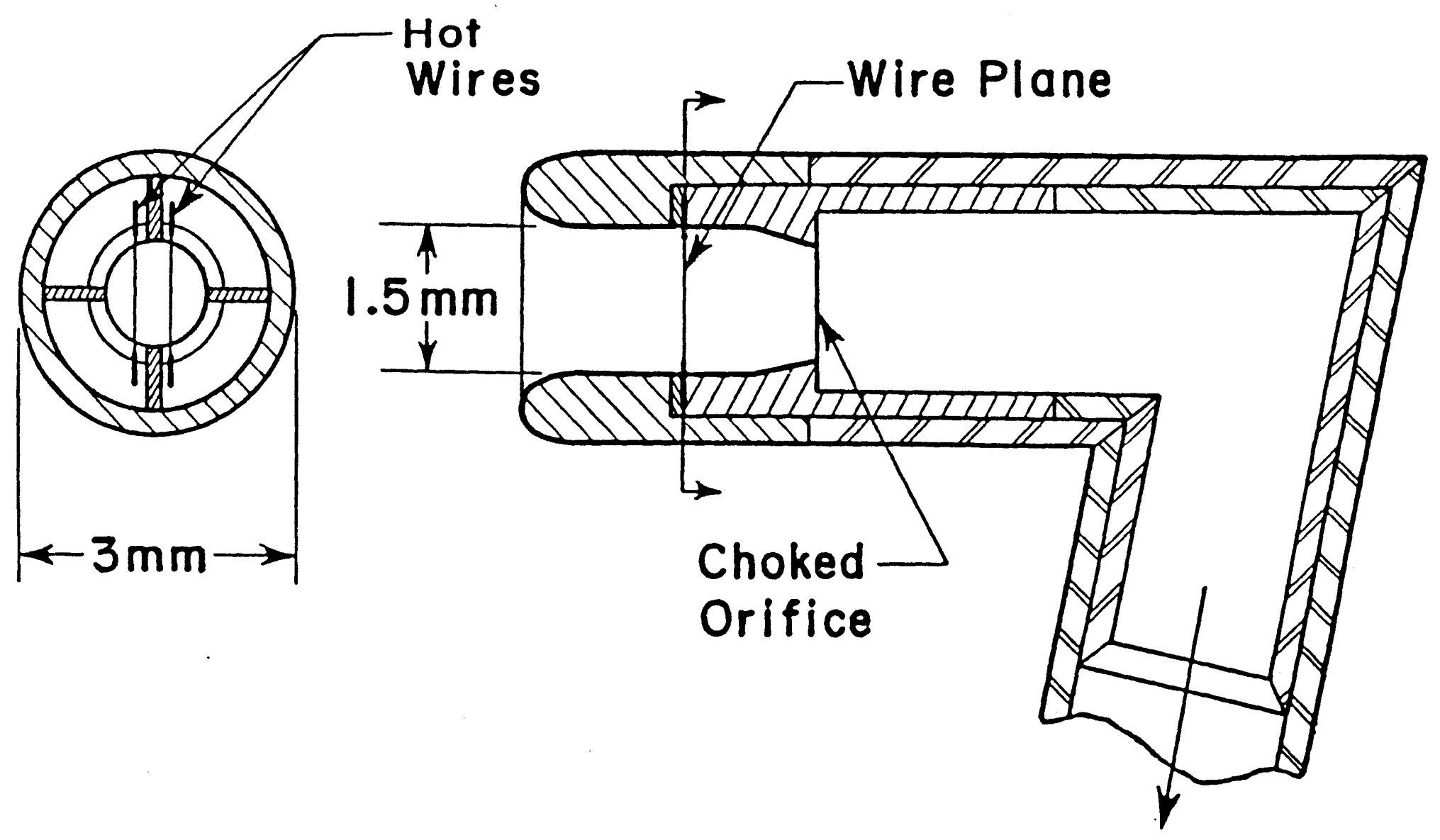

To Vacuum

Figure 3.1: Geometry of the dual wire aspirating probe 


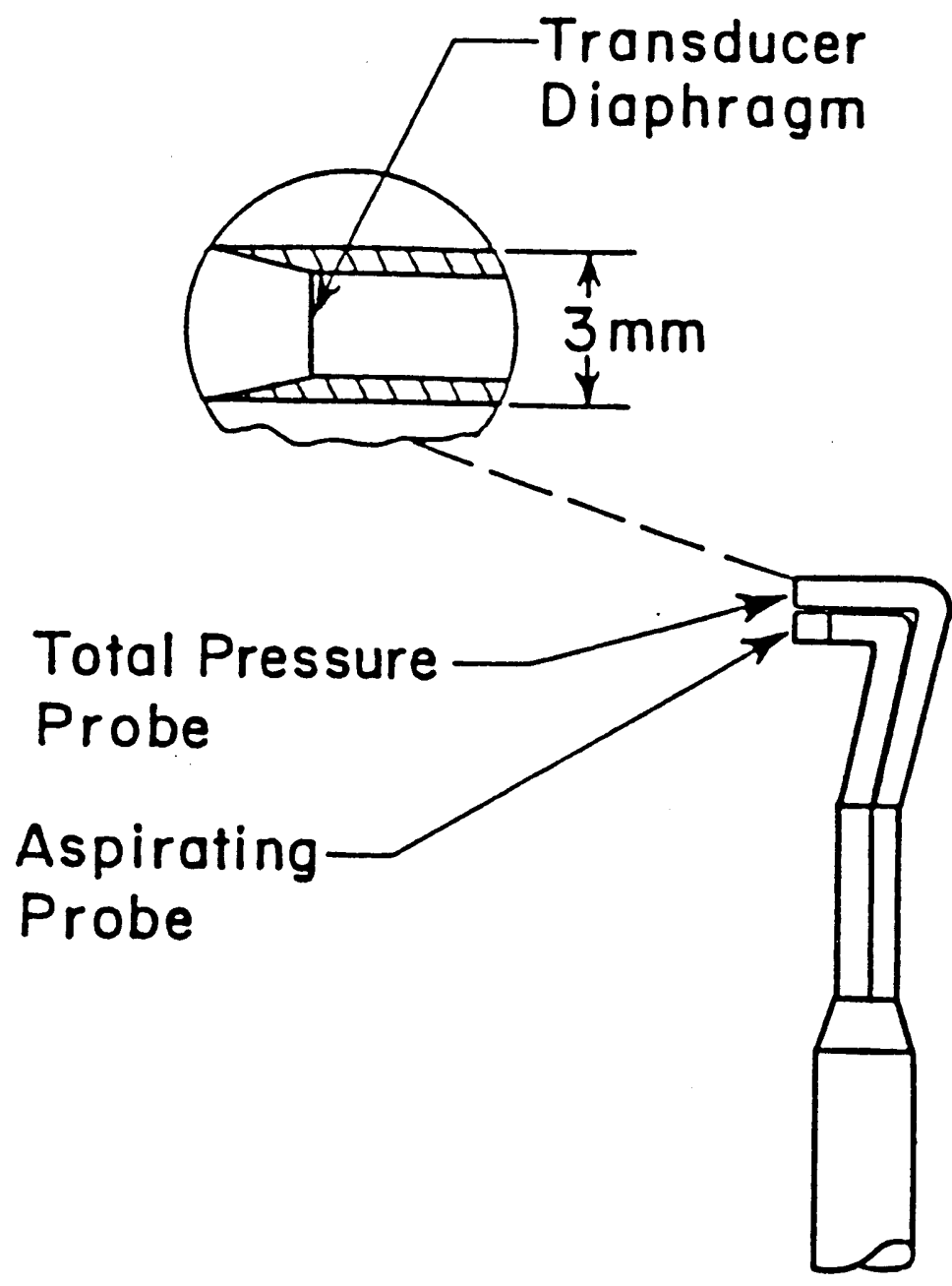

Figure 3.2: Mounting arrangement of aspirating probe and companion total pressure probe 


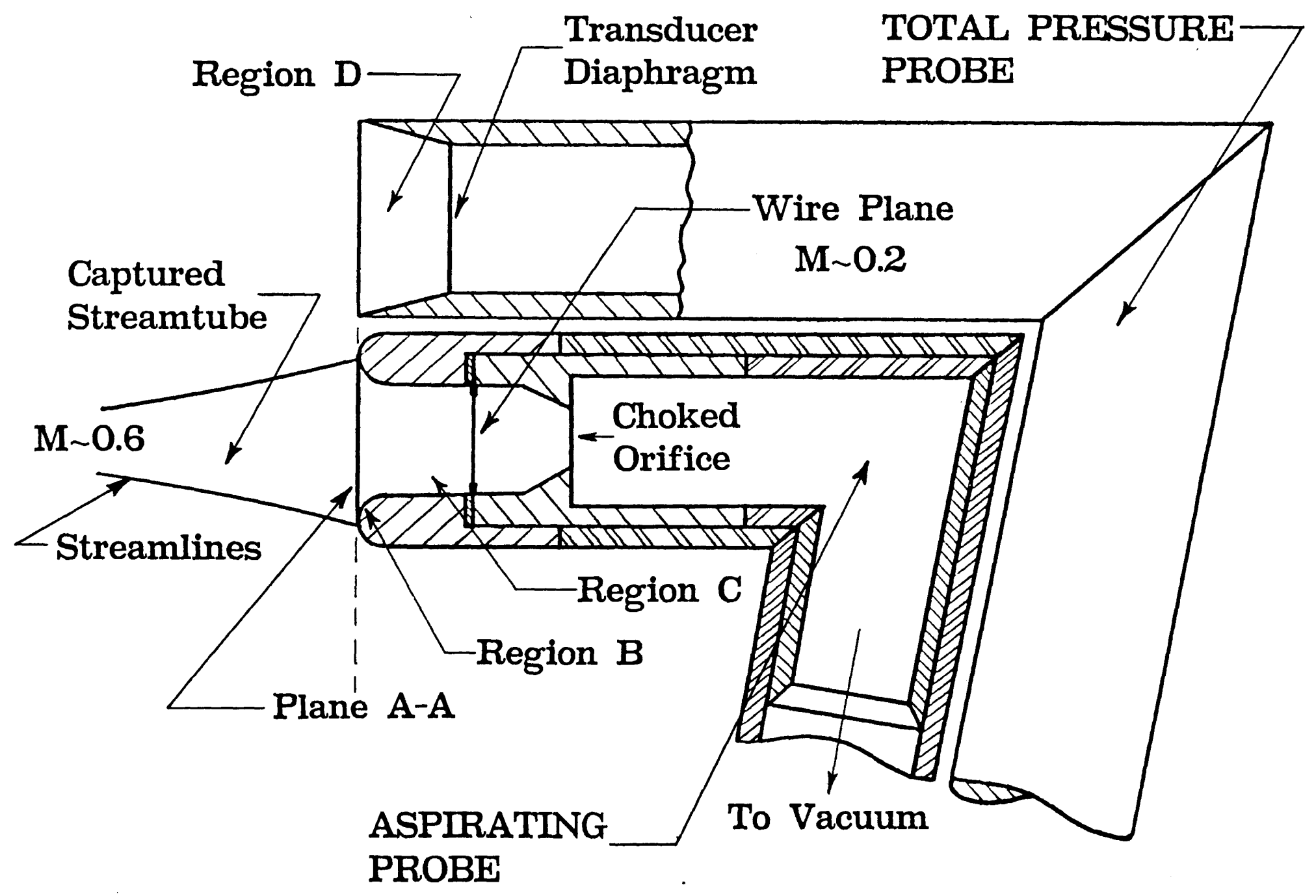

Figure 3.3: Enlarged view of the probe inlet geometry with the captured streamtube included 
PROBE INLET GEOMETRY FOR THE 1-D FLOW MODEL

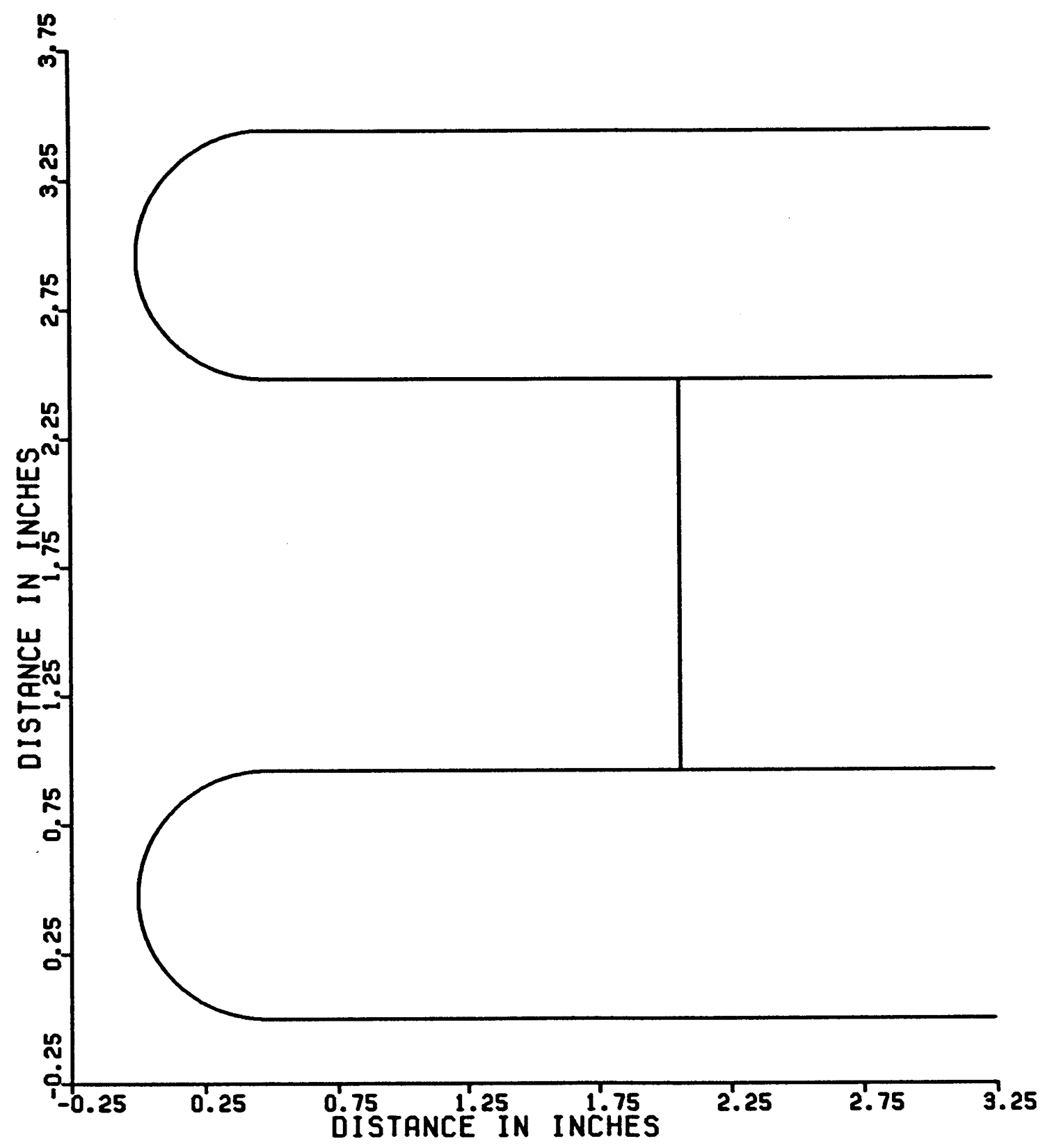

Figure 3.4: Inlet geometry of the aspirating probe, used in the 1-D flow calculations of the time lag in the probe data 
EXPERIMENTAL DETERMINATION OF THE TIME LAG IN THE PROBE DATA

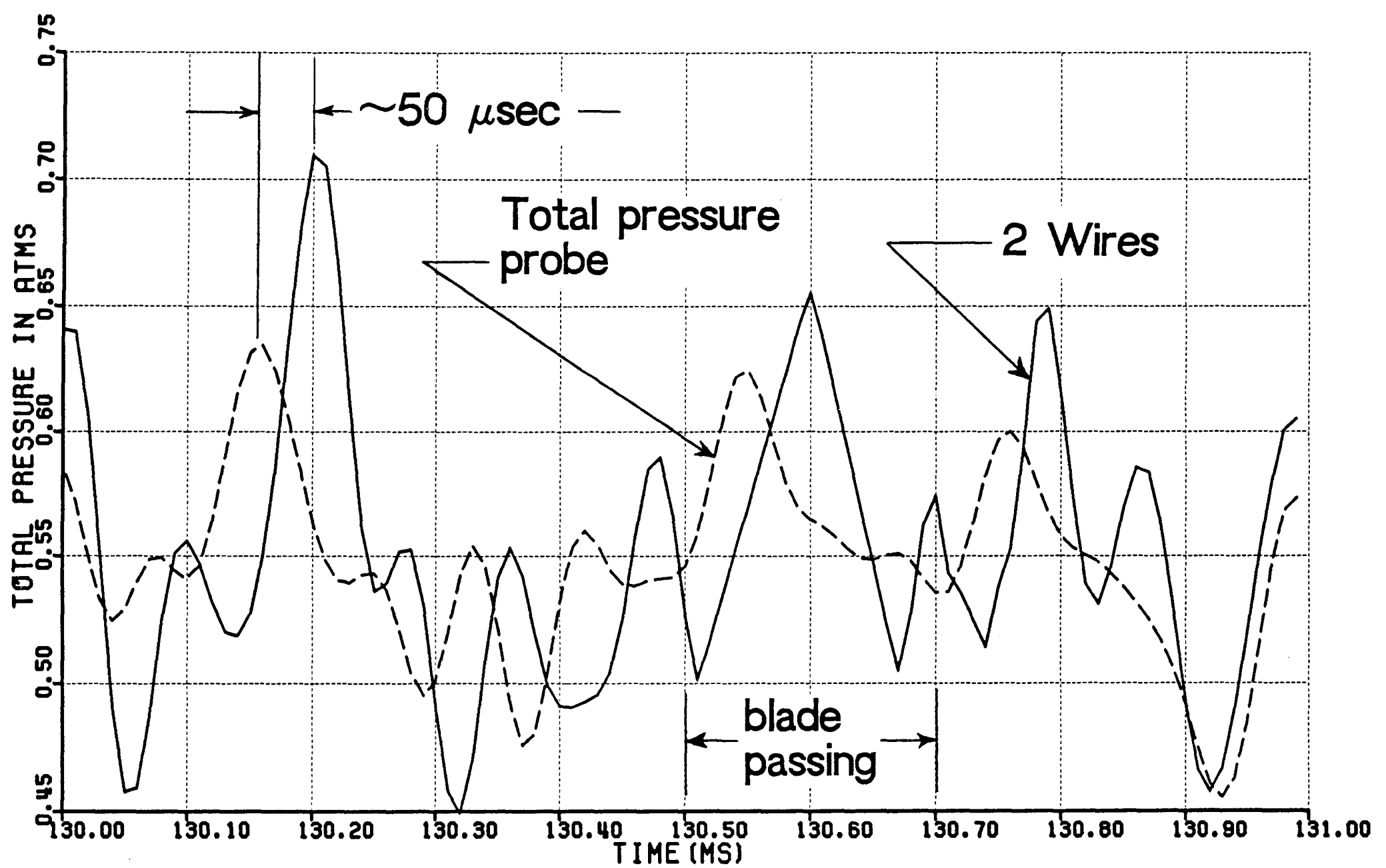

Figure 3.5: Experimental determination of the time lag - Comparison between total pressure measured by the pressure probe vs calculated using the signals of the two hot wires only 

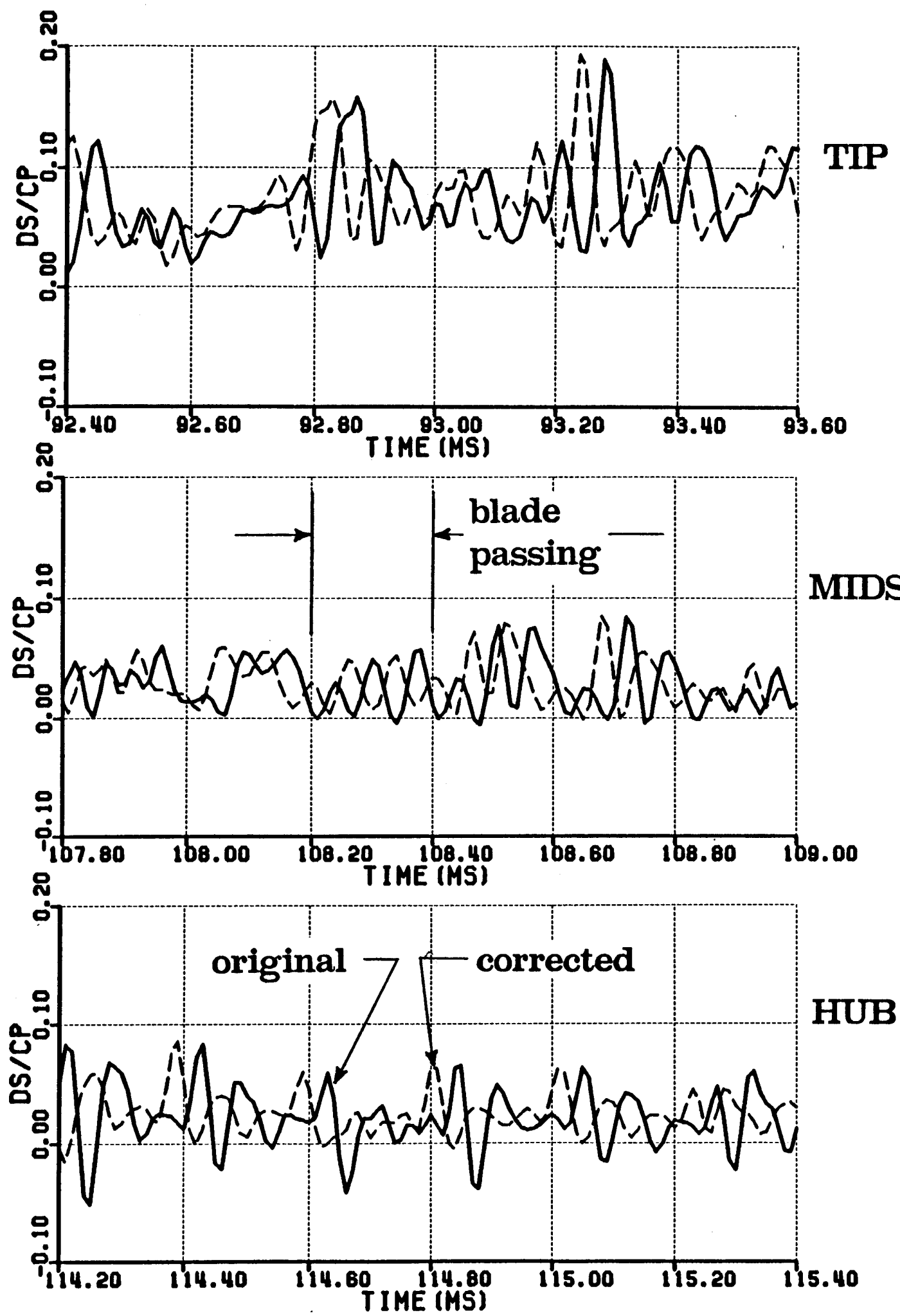

Figure 3.6: Comparison between entropy change (nondimensionalized with constant pressure specific heat) as calculated using the shifted (corrected) and unshifted (original) probe data for tip, midspan and hub 
COMPARISON BETMEEN SHIFTED AND UNSHIFTED ENTROPY TRACES

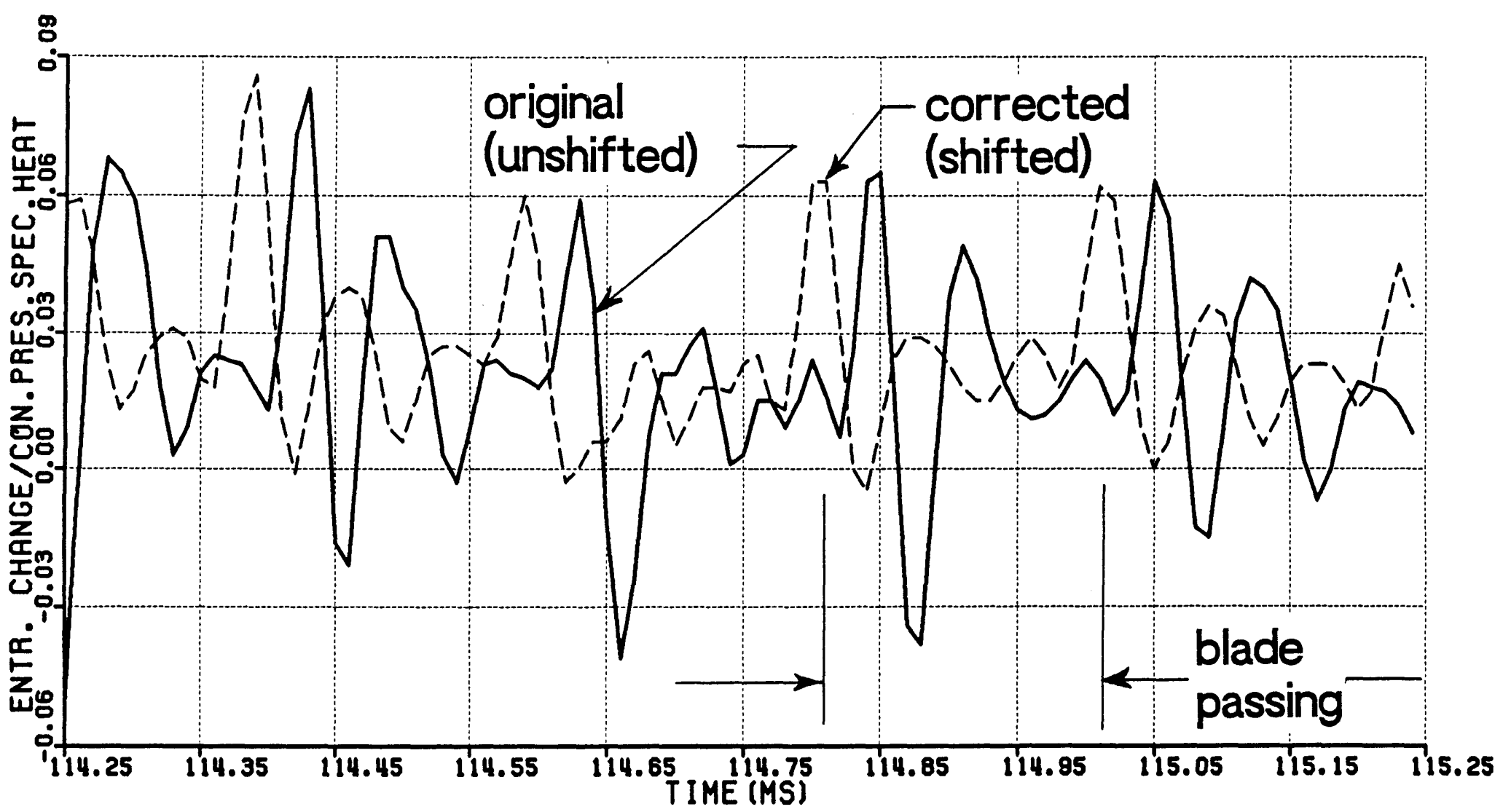

Figure 3.7: Comparison between entropy change (nondimensionalized with constant pressure specific heat) as calculated using the shifted (corrected) and unshifted (original) probe data 


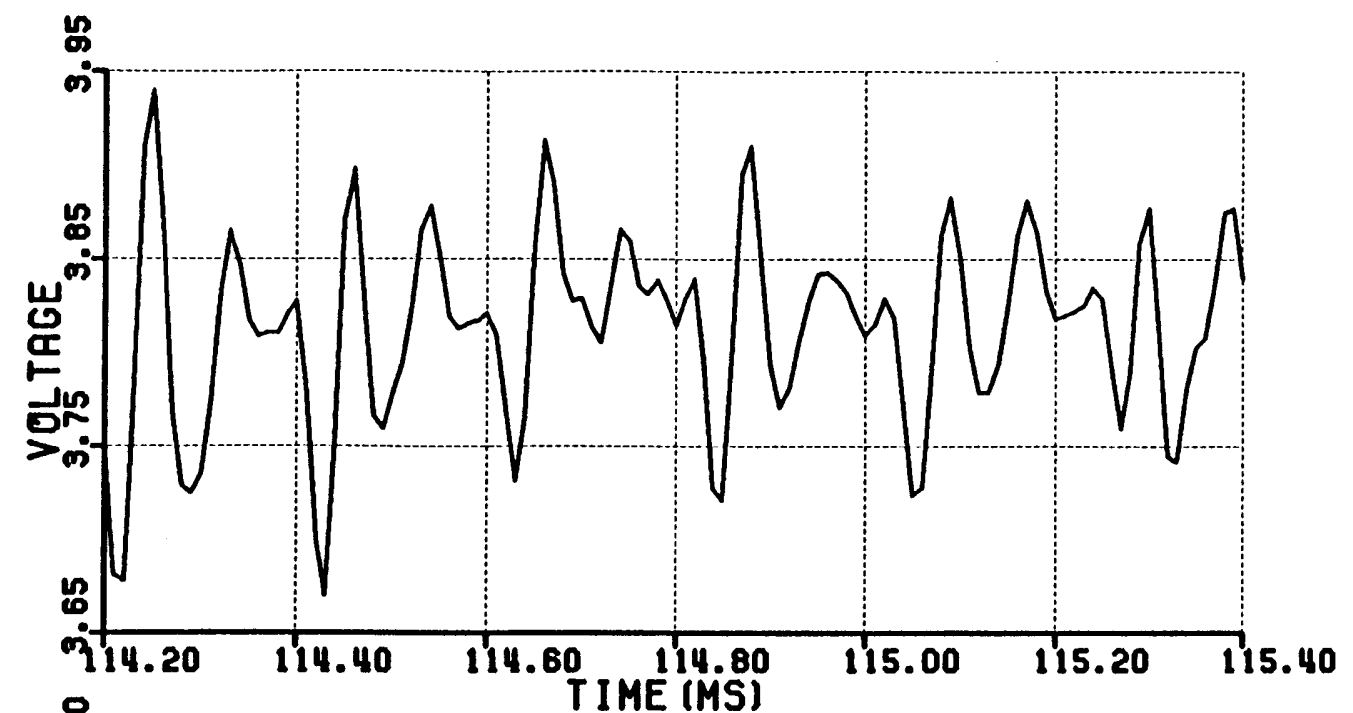

(a)

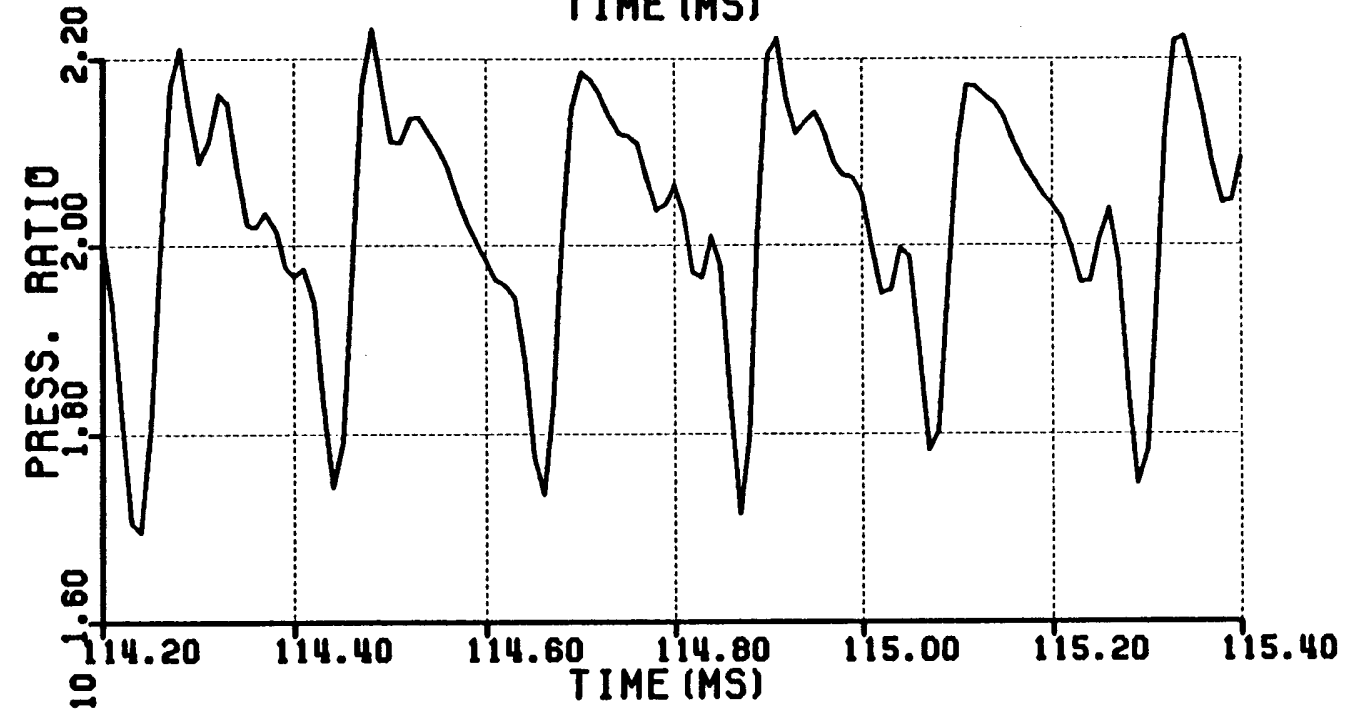

(b)

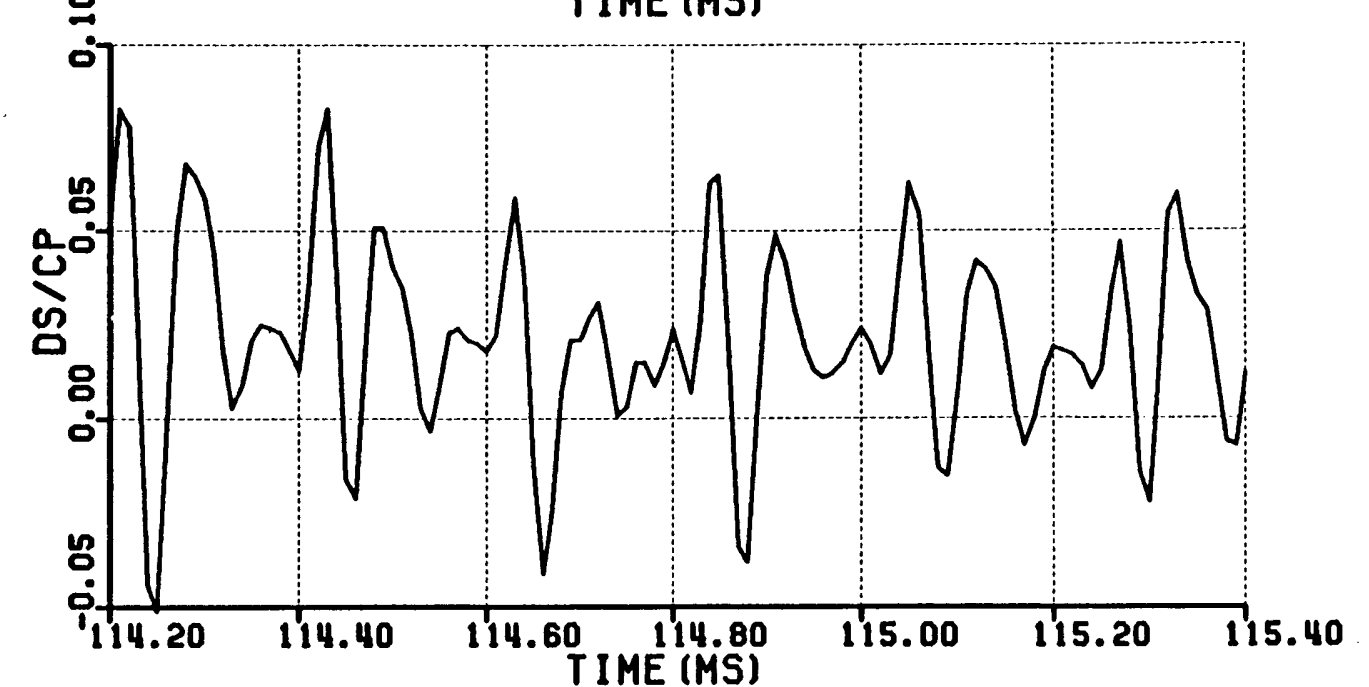

Figure 3.8: (a) Voltage unshifted (original) measurement

(b) Pressure ratio measurement

(c) Entropy change (nondimensionalized with constant pressure specific heat) as calculated using the unshifted (original) signals 


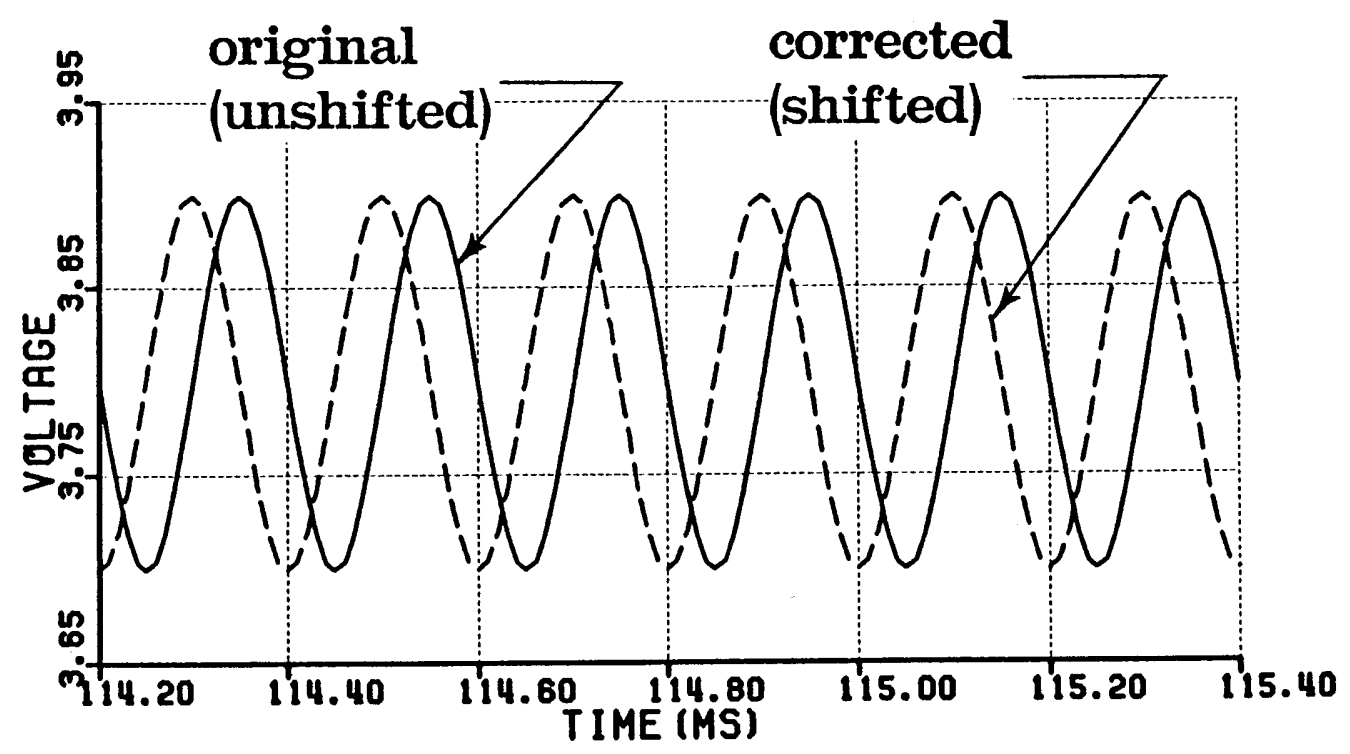

(a)

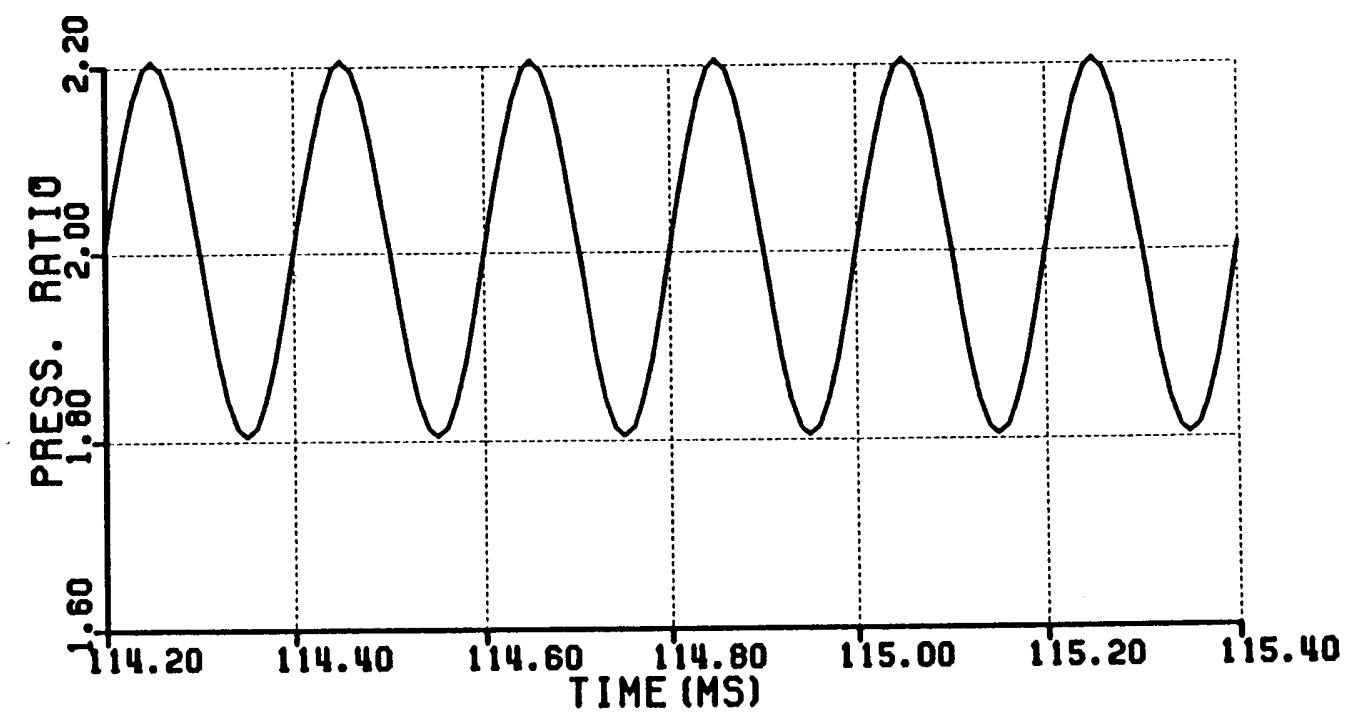

(b)

Figure 3.9: Simulation of the probe data as $5 \mathrm{kHz}$ (blade passing frequency) sine waves

(a) Shifted (corrected) and unshifted (original) voltage

(b) Total pressure ratio 


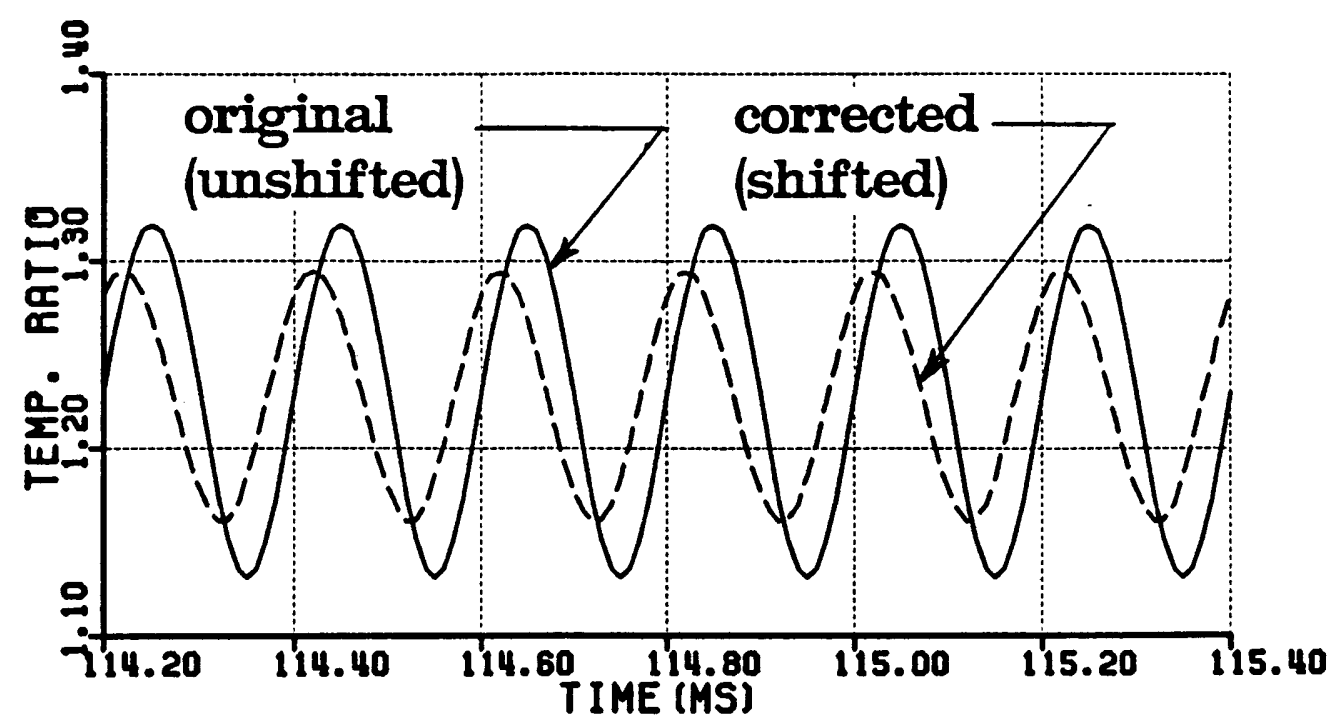

(c)

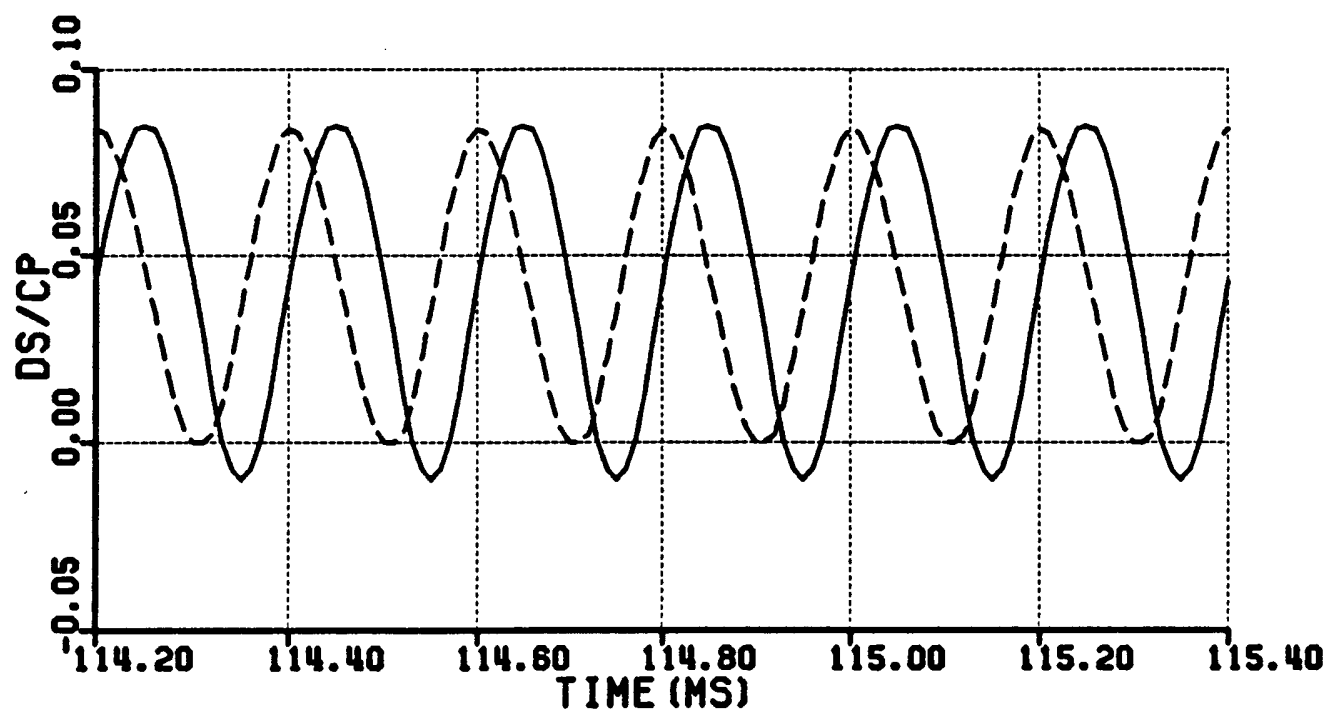

(d)

Figure 3.9: (c) Total temperature ratio as calculated using shifted (corrected) and unshifted (original) signals

(d) Entropy change (nondimensionalized with constant specific heat) as calculated using shifted (corrected) and unshifted (original) signals 
ASPIRATING PROBE CALIBRATION CURVES FOR O\% CONCENTRATION

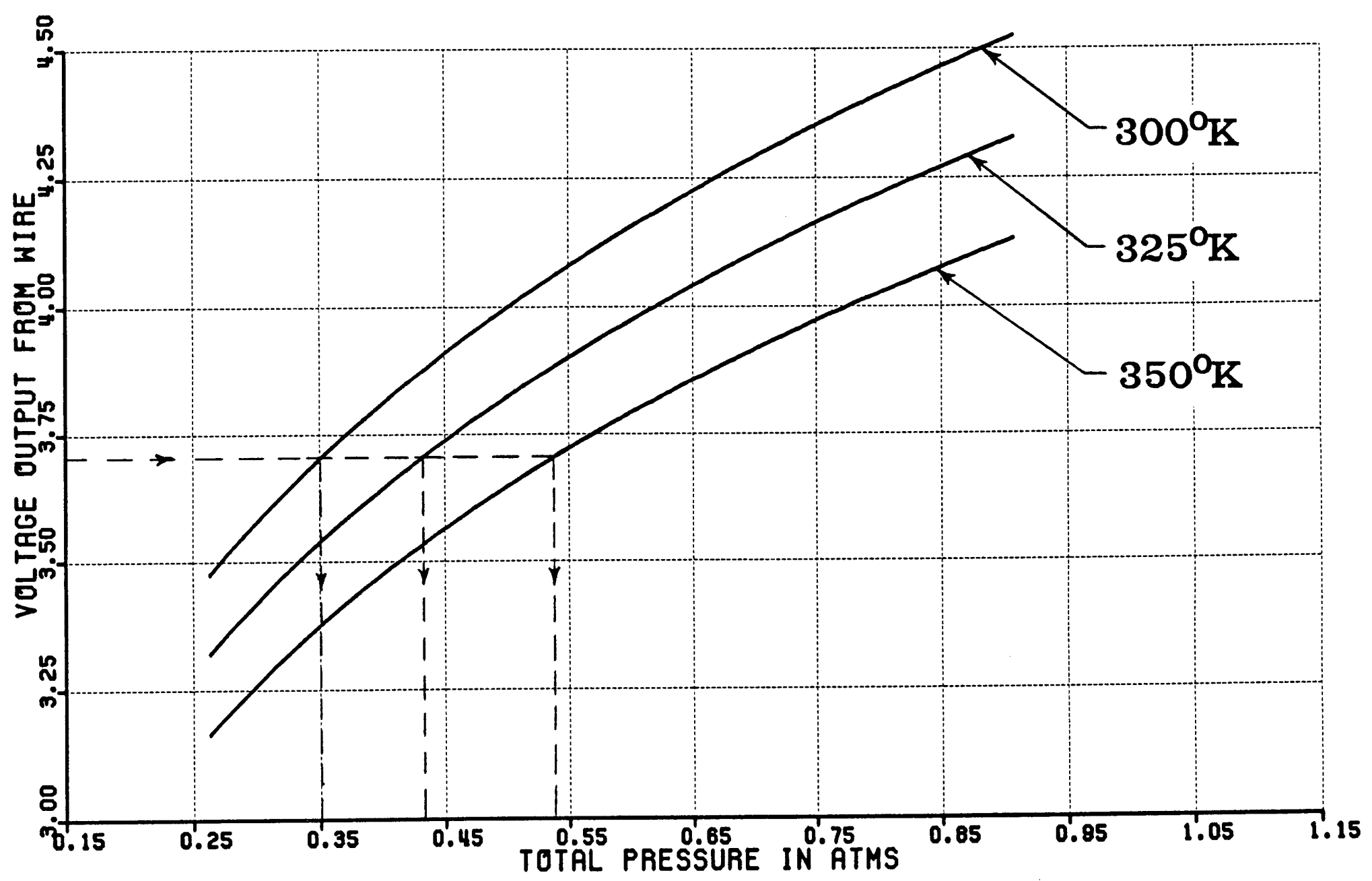

Figure 3.10: Aspirating probe calibration curves for $0 \%$ concentration 
CALIBRATION CURVES FOR CO2 AT 400 MMHG

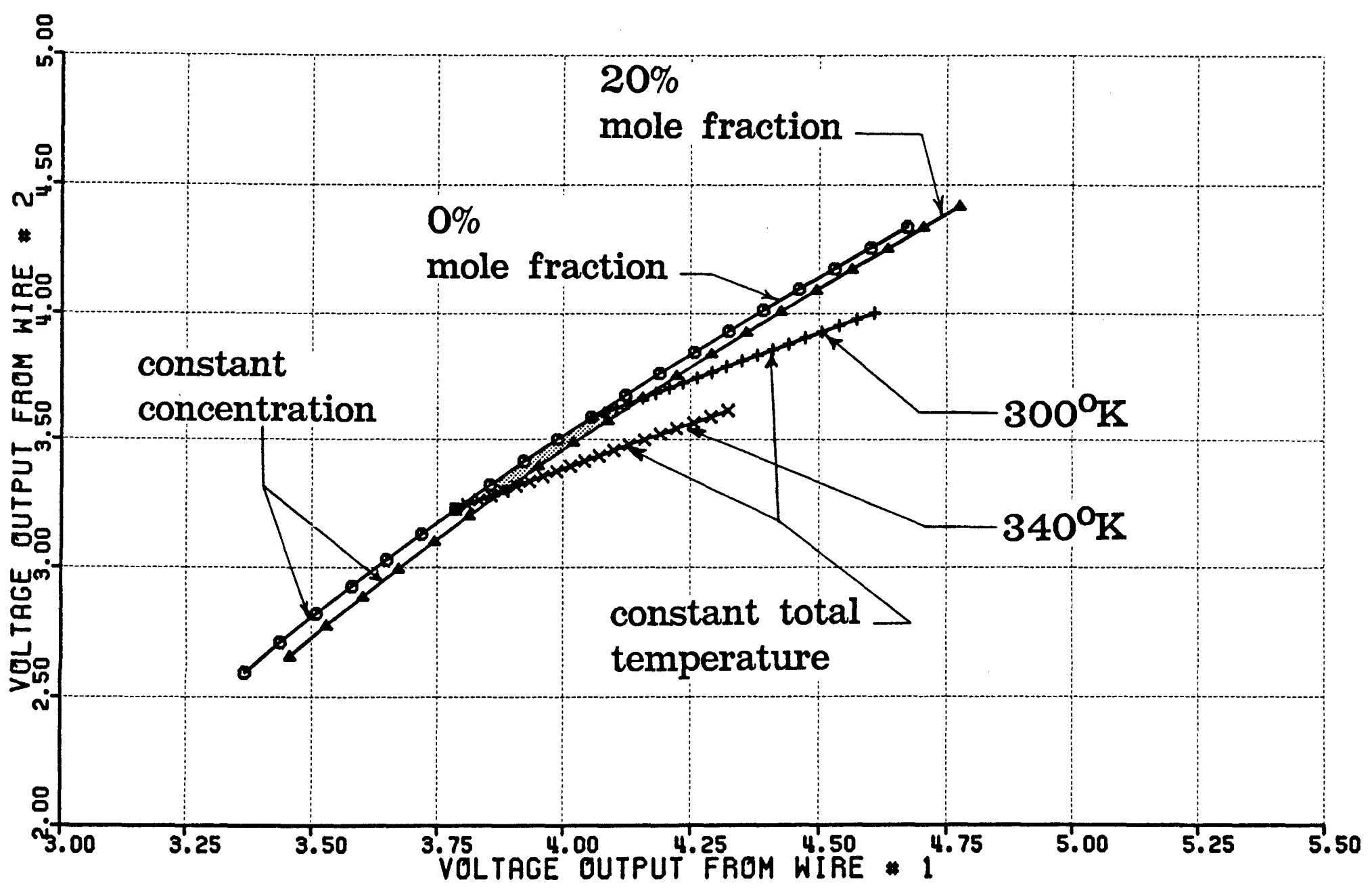

Figure 3.11: Aspirating probe calibration curves for $\mathrm{CO}_{2}$ tracer gas at $400 \mathrm{mmHg}$ 


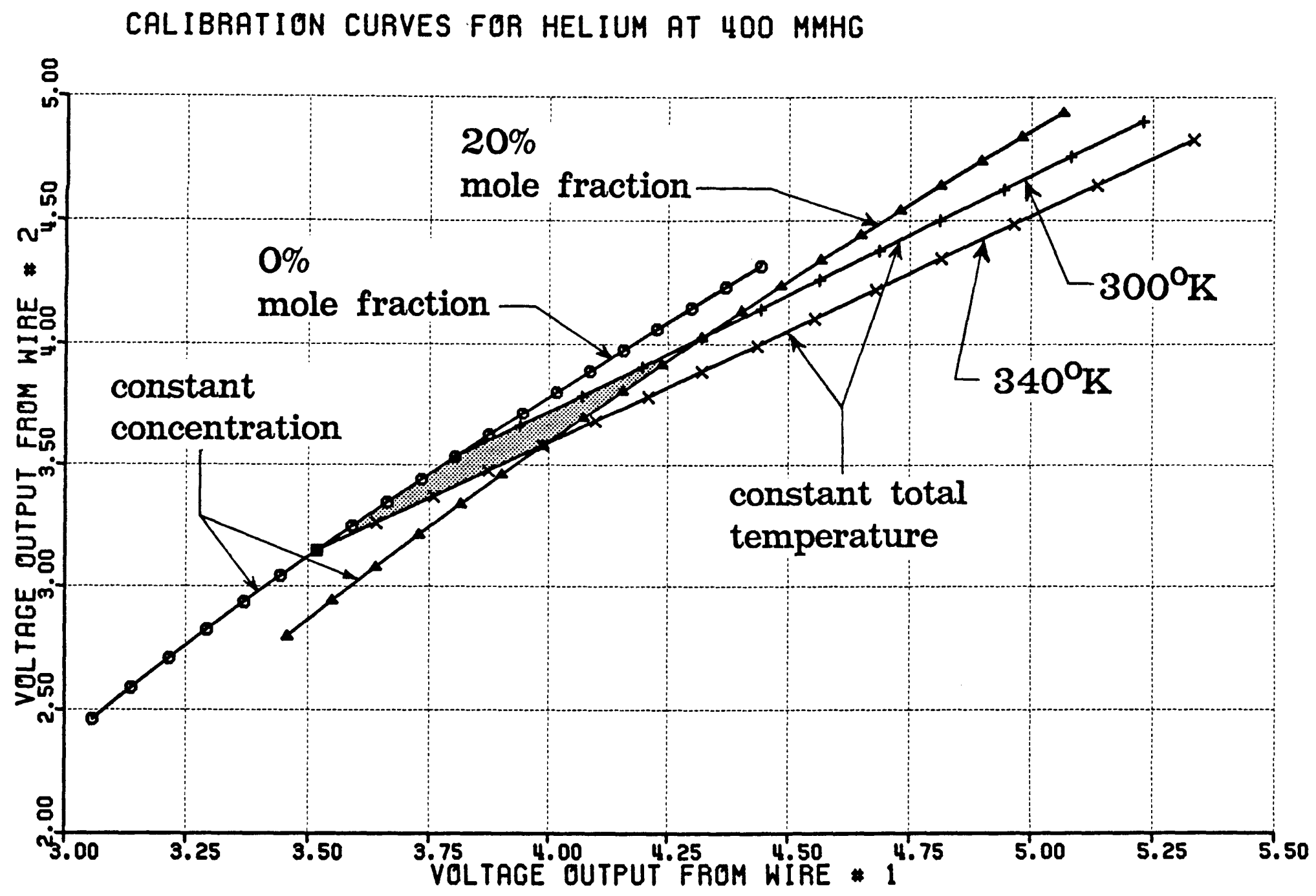

Figure 3.12: Aspirating probe calibration curves for Helium tracer gas at $400 \mathrm{mmHg}$ 
CALIBRATION CURVES FOR HELIUM-FREON 12 AT 400 MMHG

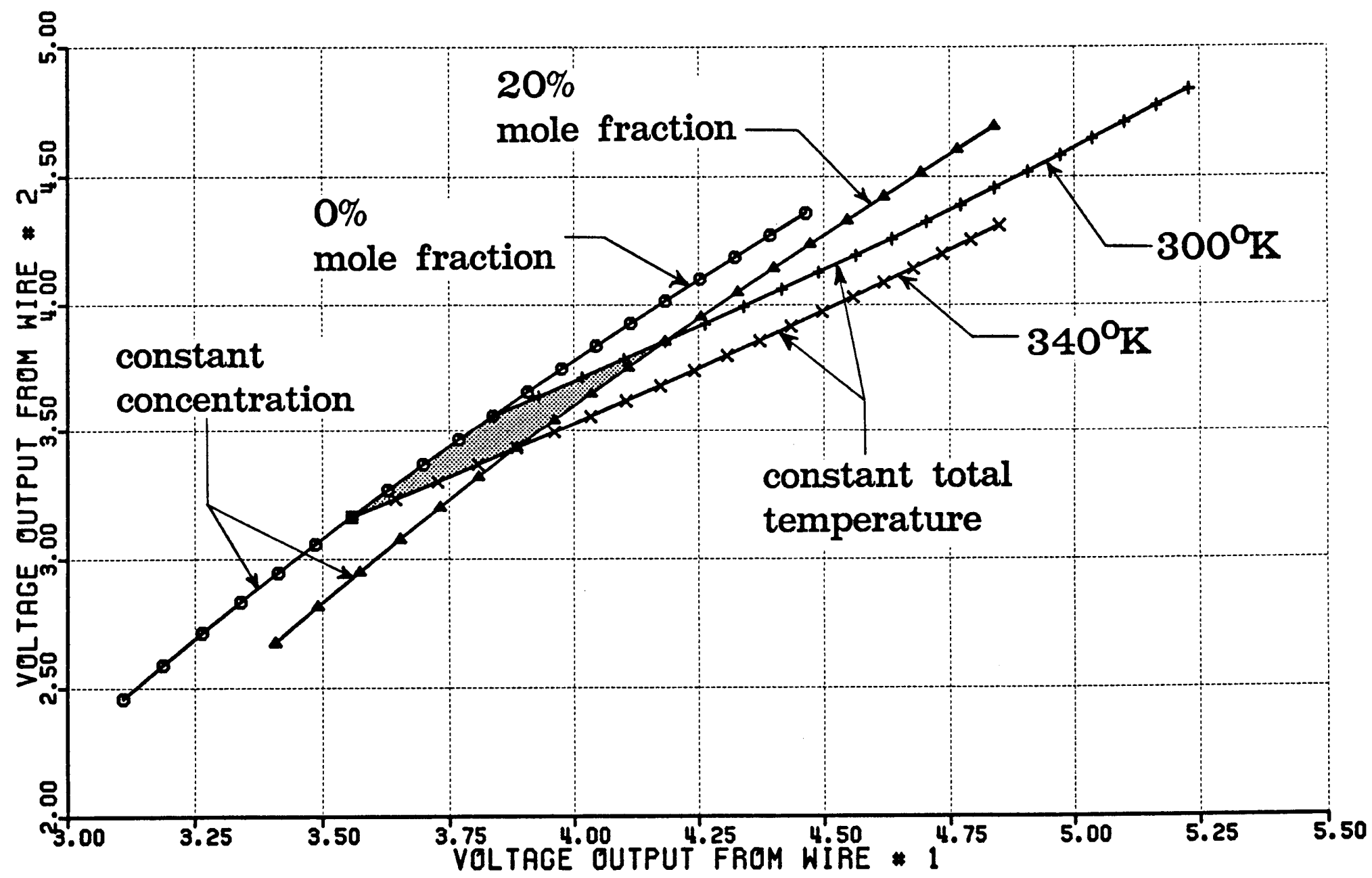

Figure 3.13: Aspirating probe calibration curves for Helium and Freon 12 tracer mixture at $400 \mathrm{mmHg}$ 
CALIBRATION FACILITY

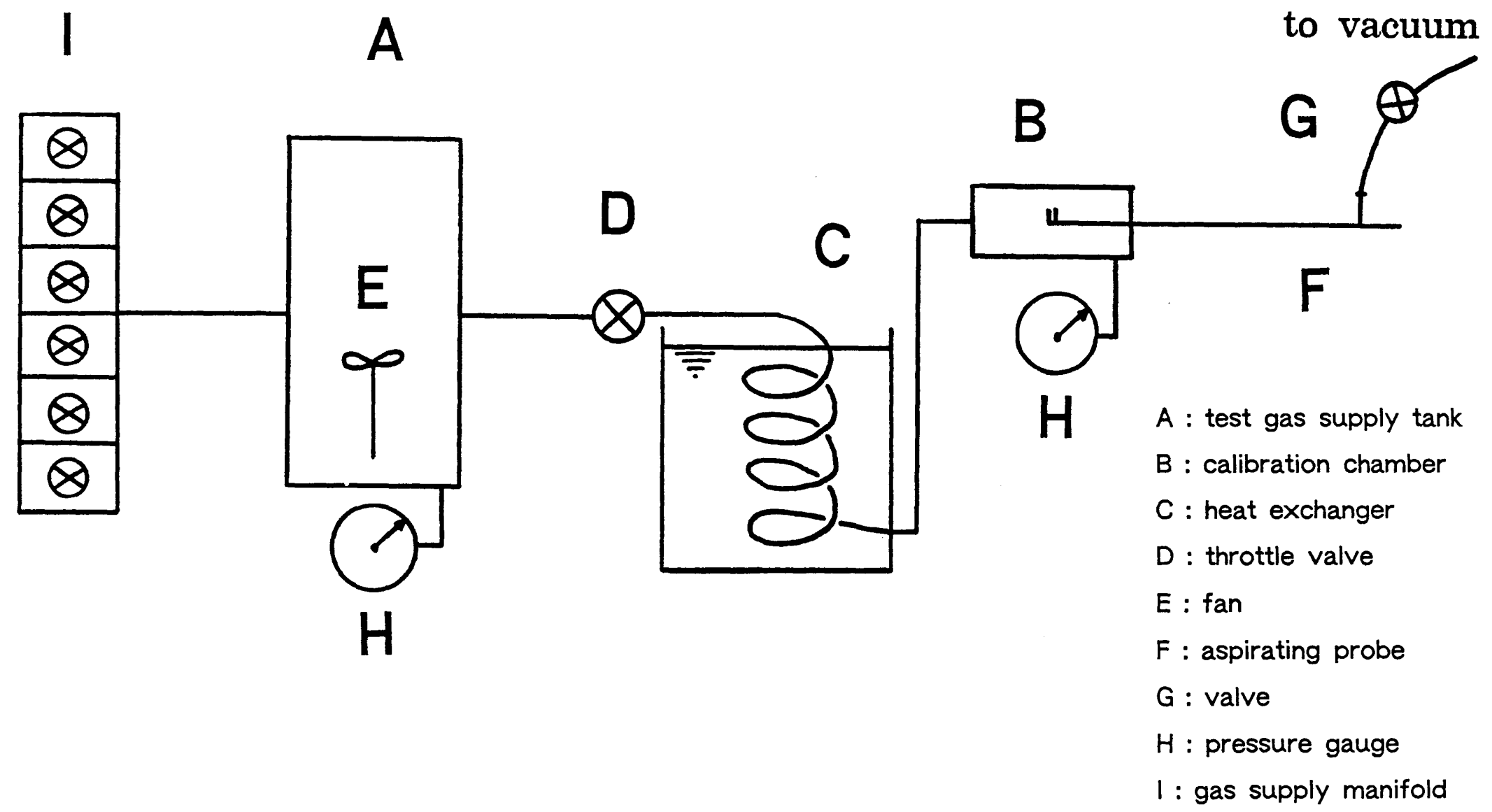

Figure 3.14: Schematic drawing of the probe calibration facility 


\section{CALIBRATION CURVES FOR VARIOUS TRACER GASES}

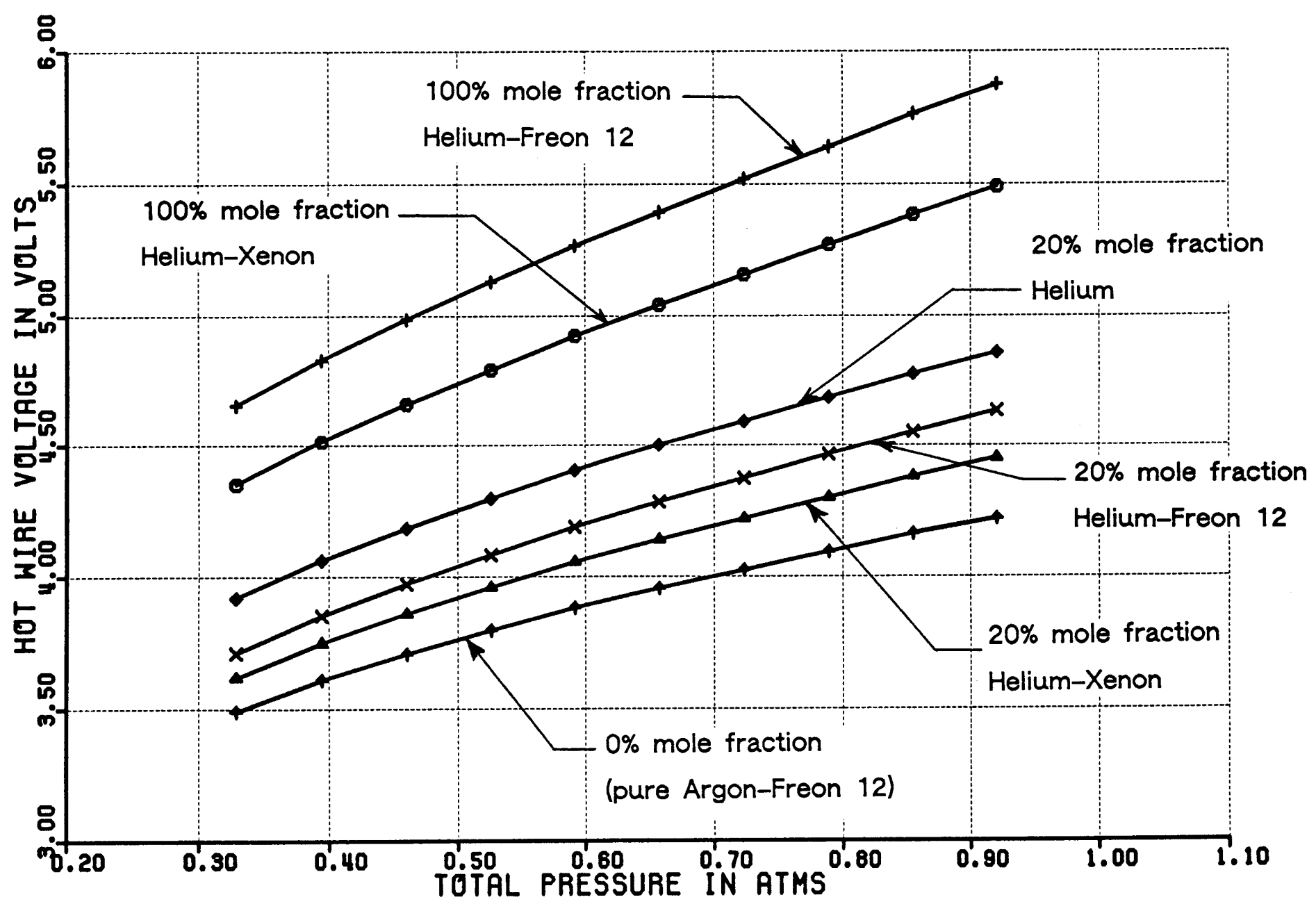

Figure 3.15: Aspirating probe calibration curves for Helium,

Helium-Xenon and Helium-Freon 12 tracer mixtures 


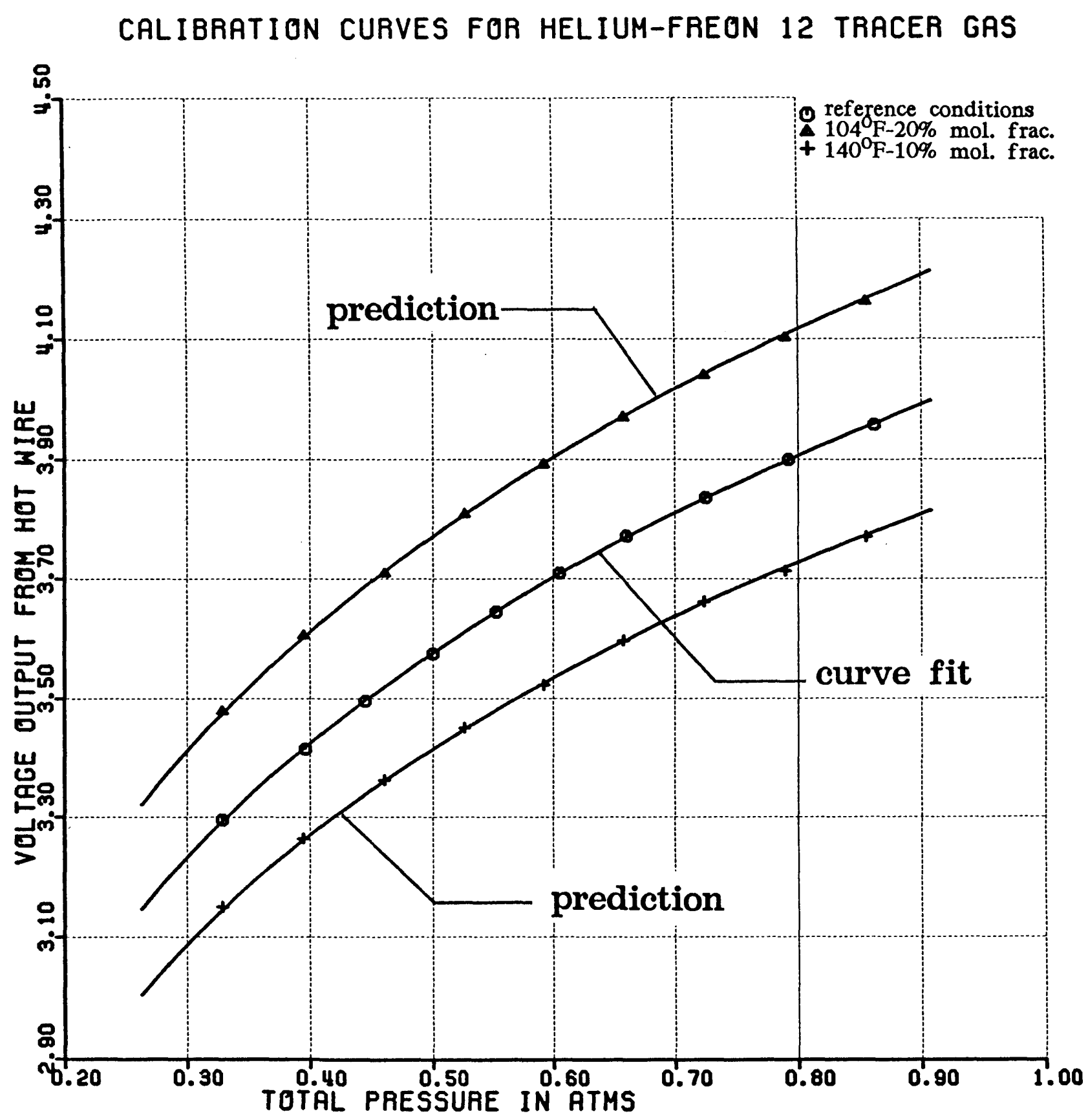

Figure 3.16: Aspirating probe calibration curve for Helium-Freon 12 at reference conditions (room temperature and $0 \%$ concentration) and comparison between calibration and prediction at two other conditions $\left(104^{\circ} \mathrm{F}-20 \%\right.$ conc. and $140^{\circ} \mathrm{F}-10 \%$ conc.) 


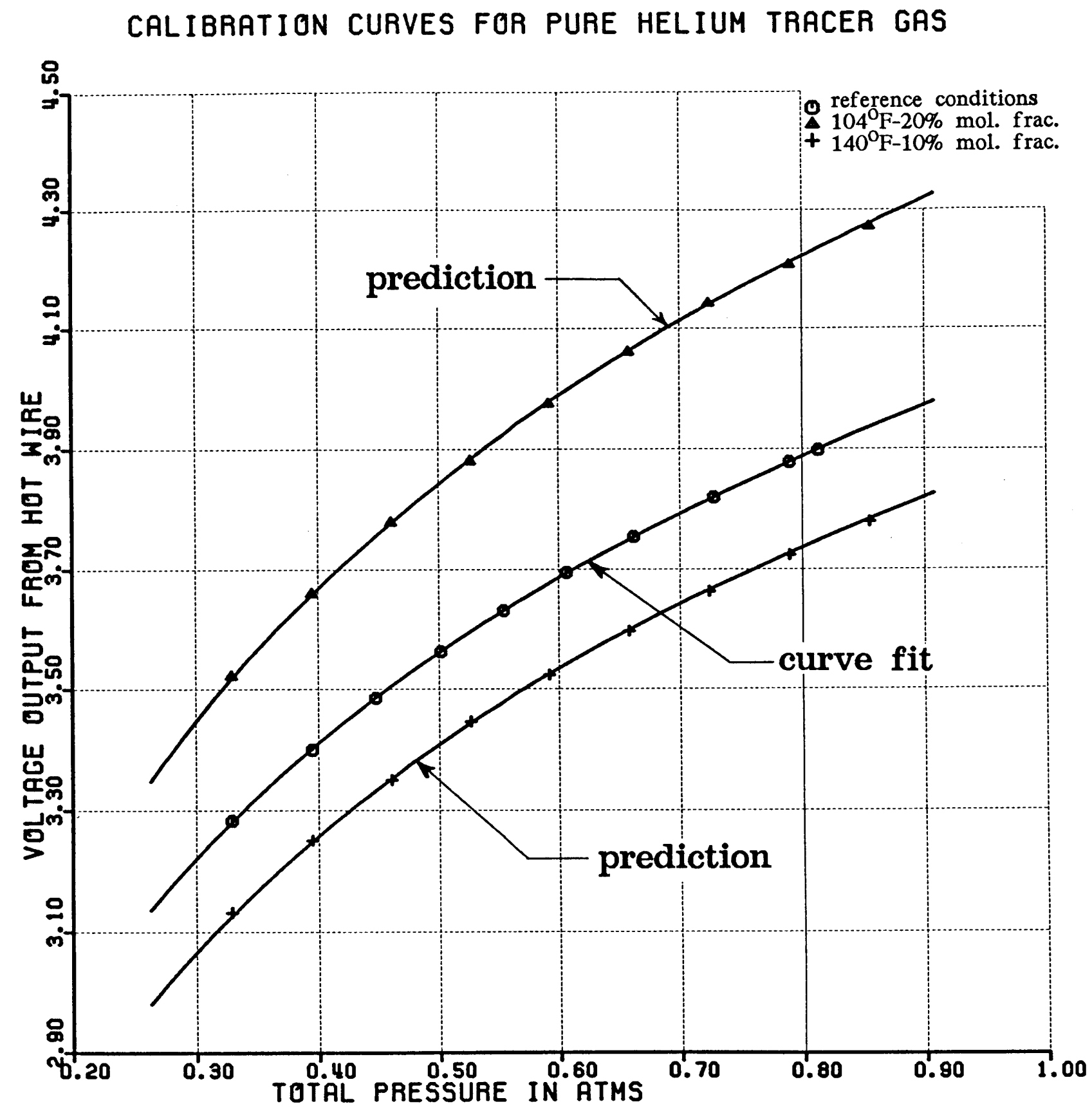

Figure 3.17: Aspirating probe calibration curve for Helium at reference conditions (room temperature and $0 \%$ concentration) and comparison between calibration and prediction at two other conditions ( $104^{\circ} \mathrm{F}-20 \%$ conc. and $140^{\circ} \mathrm{F}-10 \%$ conc.) 


\section{SHOCK TUBE FACILITY}

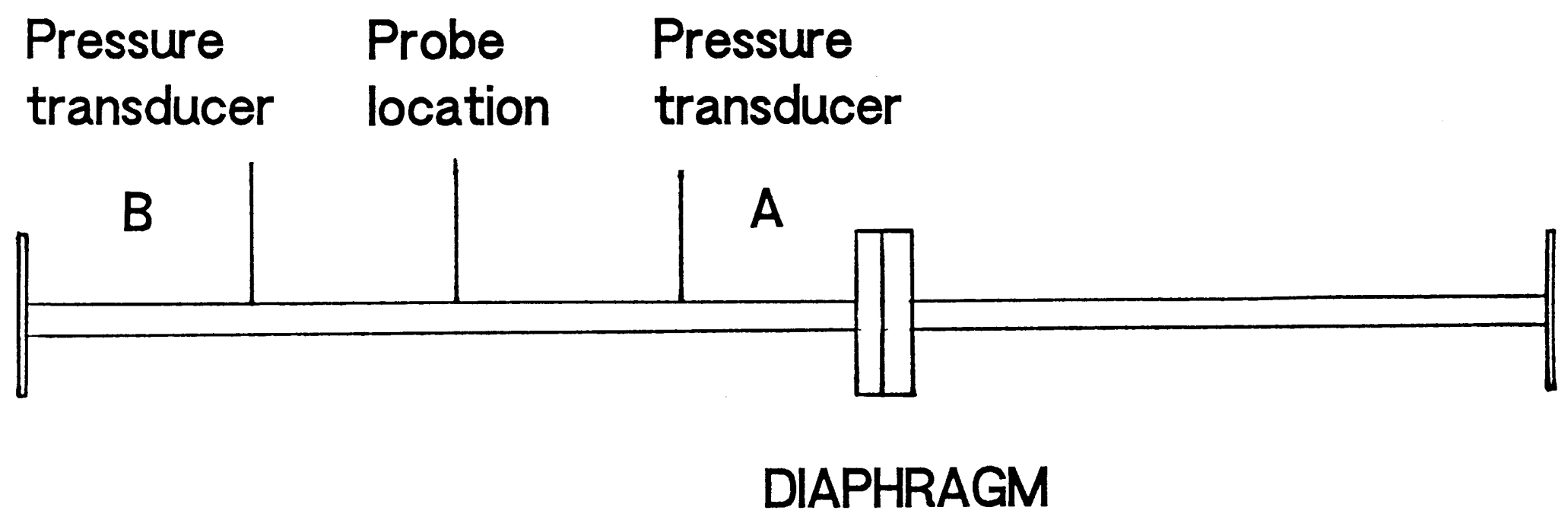

LOW PRESSURE SIDE
HIGH PRESSURE SIDE

Figure 3.18: Schematic drawing of the shock tube facility 
RSPIRATING PROBE RESPONSE TO STEP INPUT

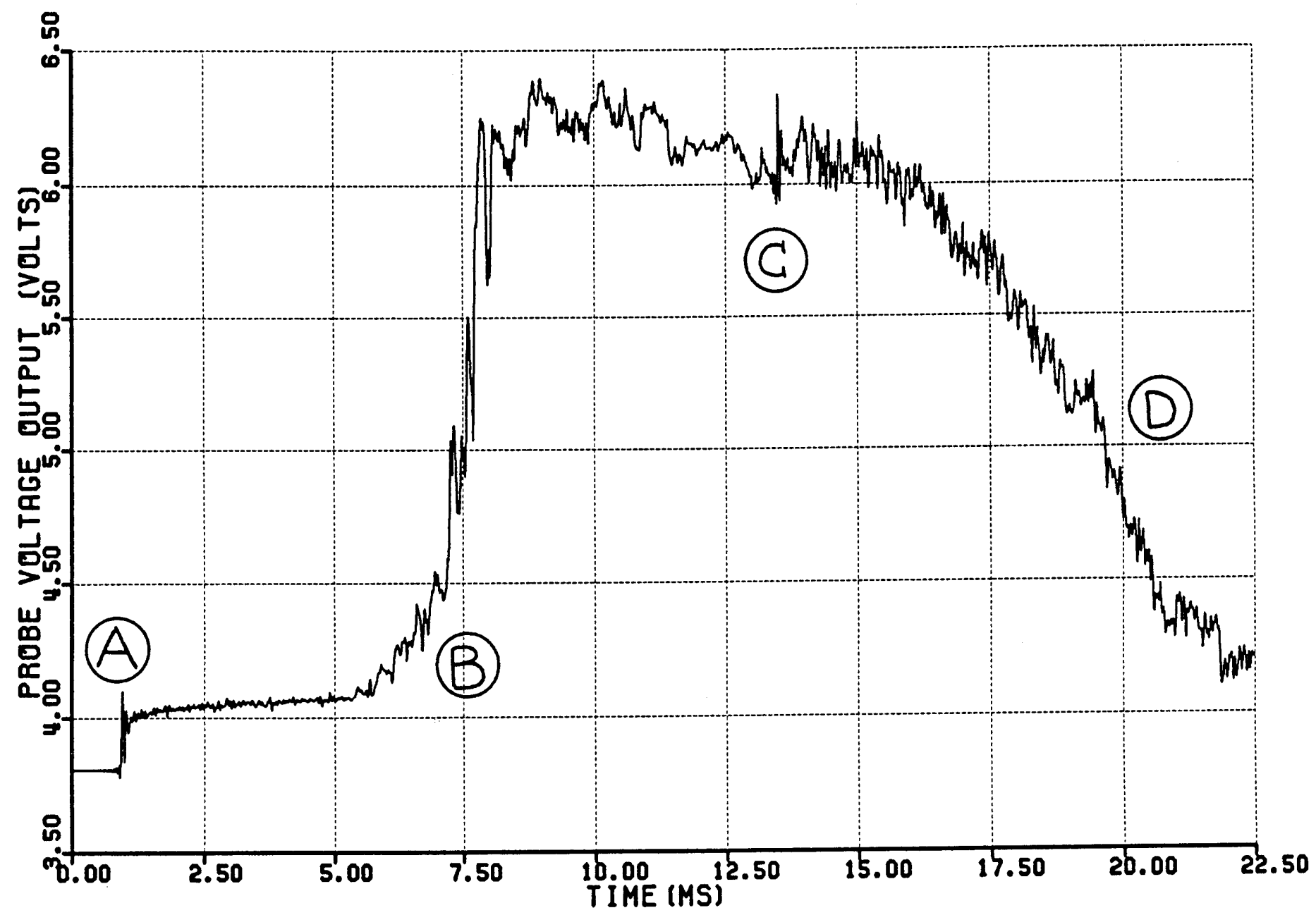

Figure 3.20: Voltage history in the shock tube facility as measured by the aspirating probe hot wire 


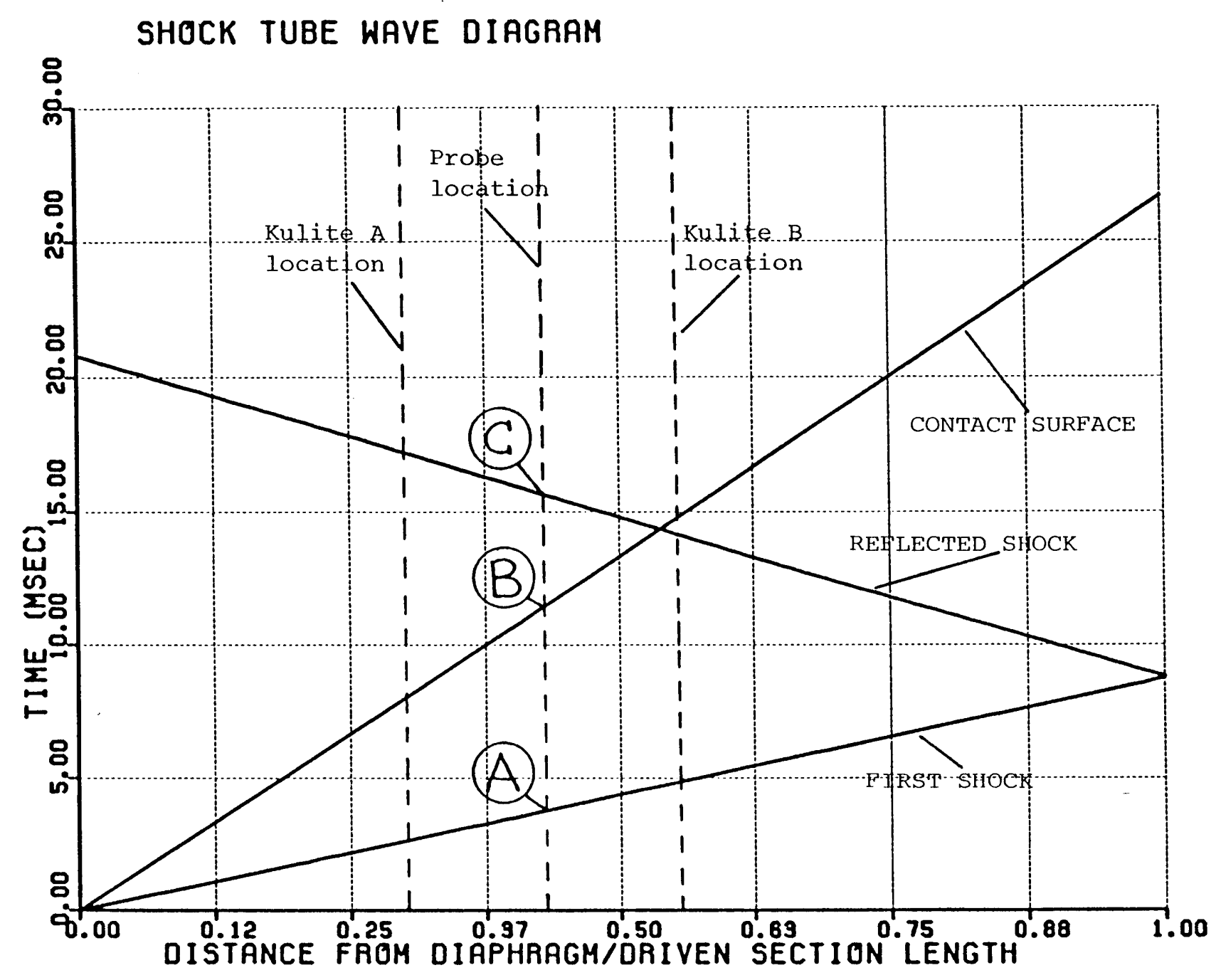

Figure 3.21: Shock tube wave diagram 


\section{POINT A}

CONCENTRATION AT POINT a

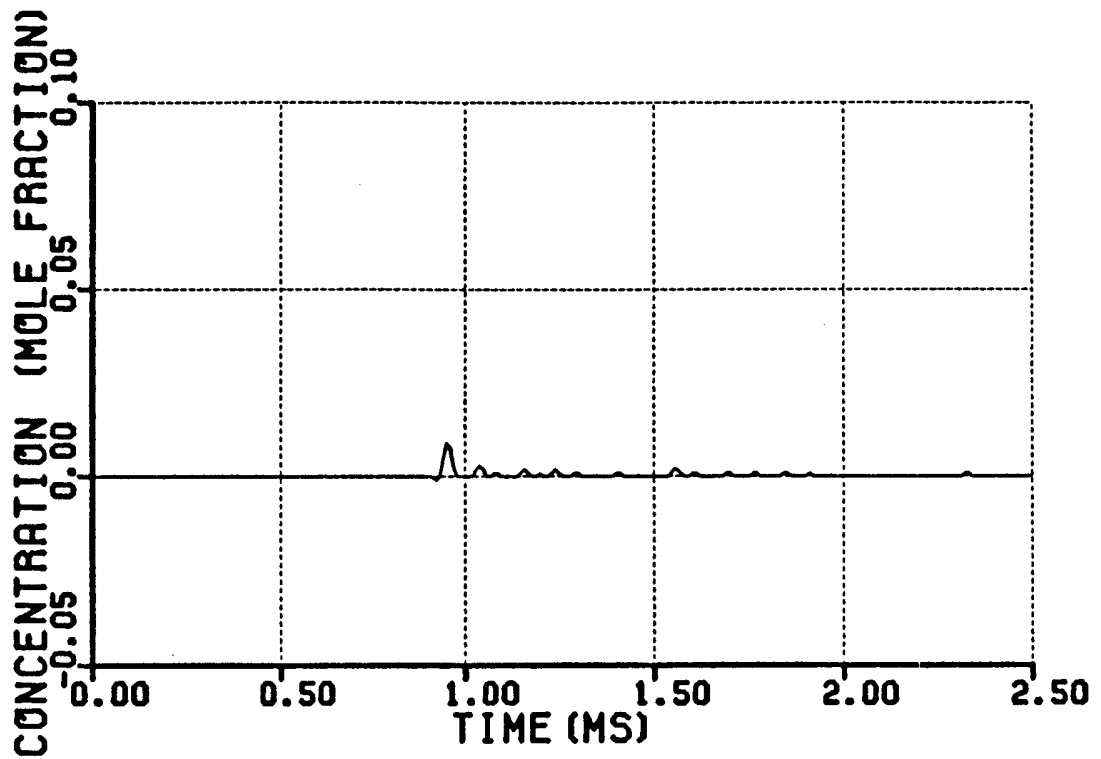

total temperatuae at point a

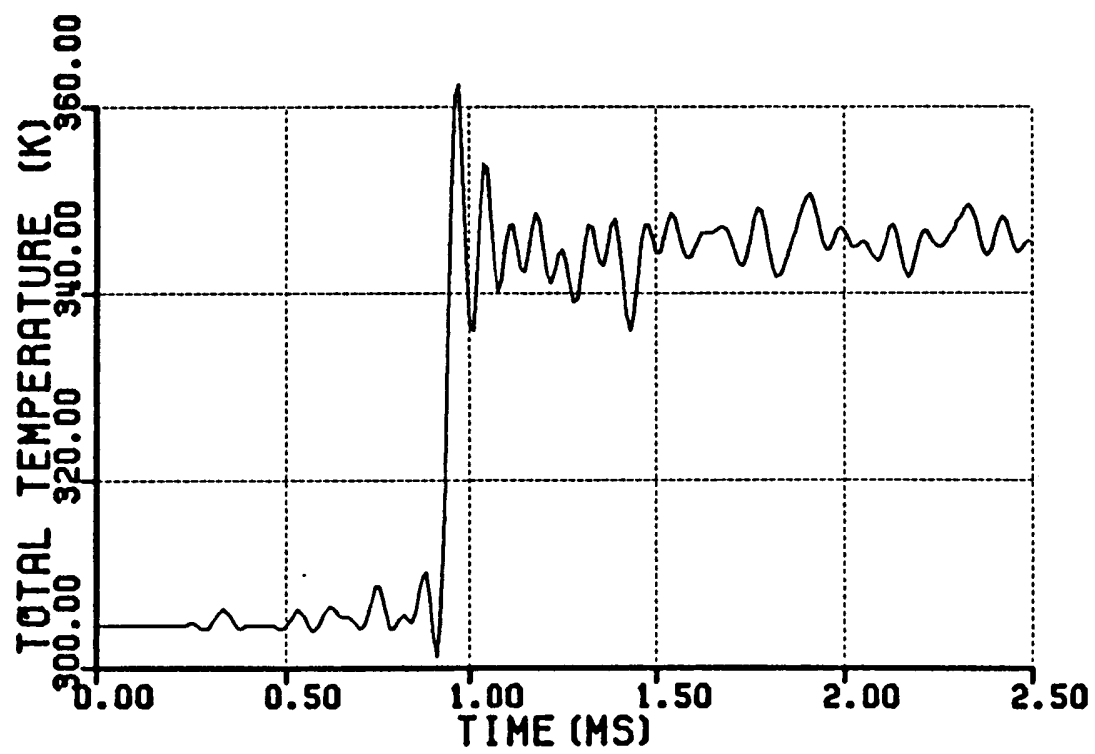

Figure 3.22: (a) Total temperature and concentration when the first shock hits the aspirating probe and its companion, total pressure probe (point $A$ in Fig. 3.19, 3.20, 3.21) 


\section{POINT B}

CONCENTRATION AT POINT B

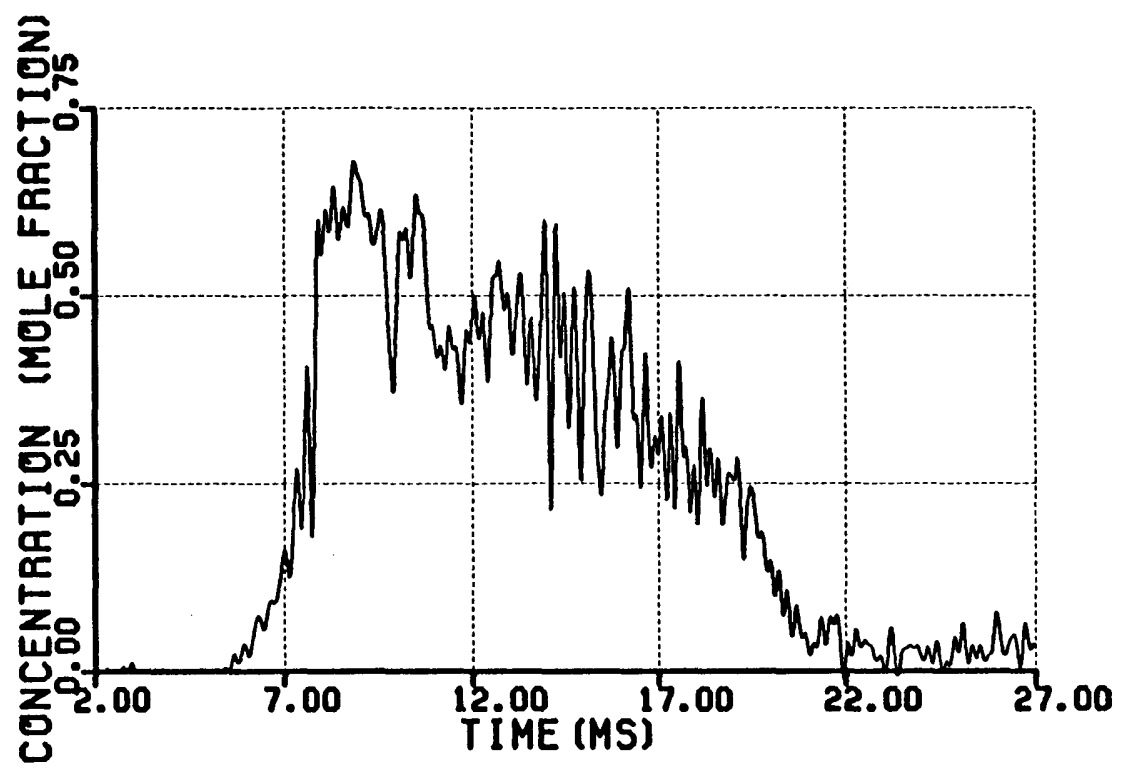

total tempeatutue at point B

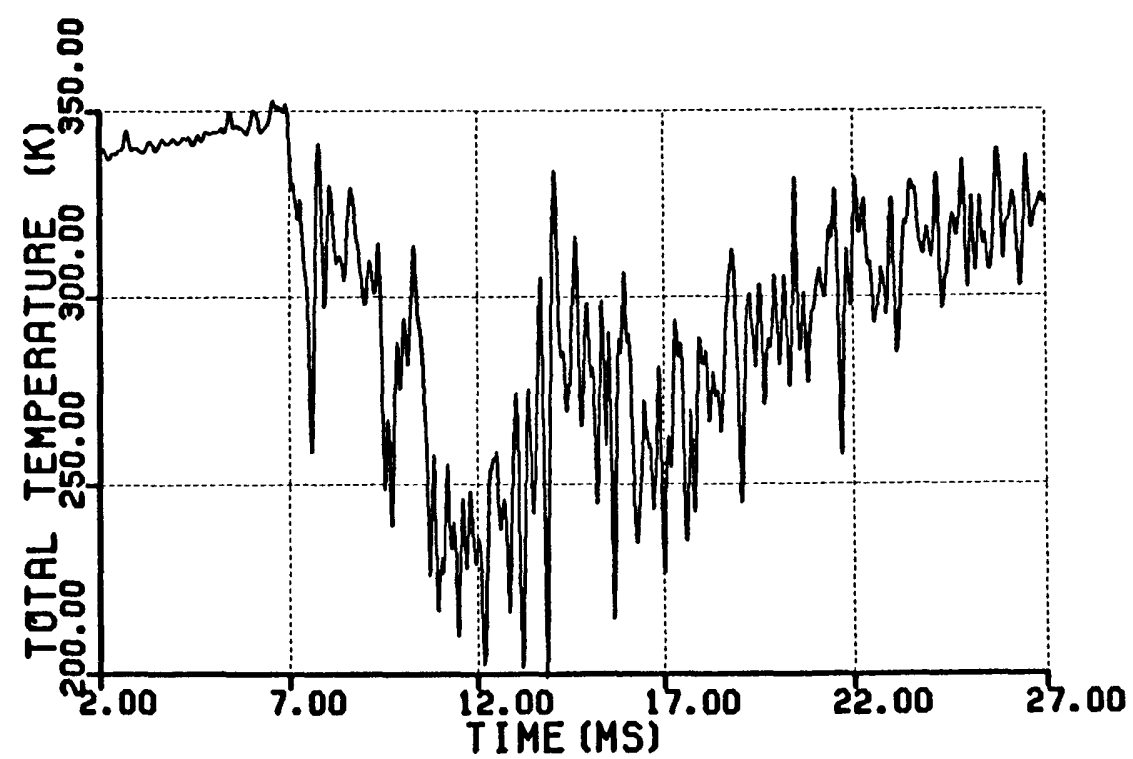

Figure 3.22: (b) Total temperature and concentration when the contact surface hits the aspirating probe and its companion, total pressure probe (point B in Fig. 3.19, 3.20, 3.21) 


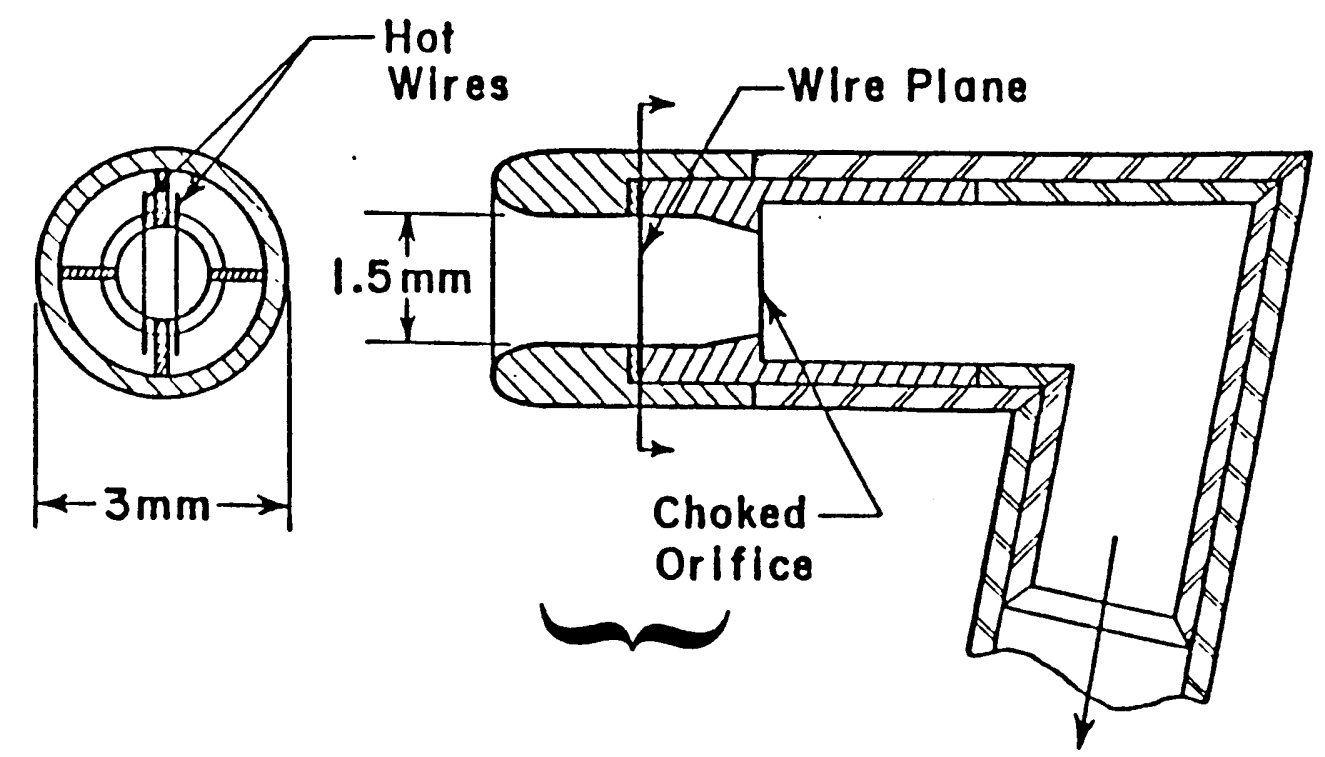

To Vacuum

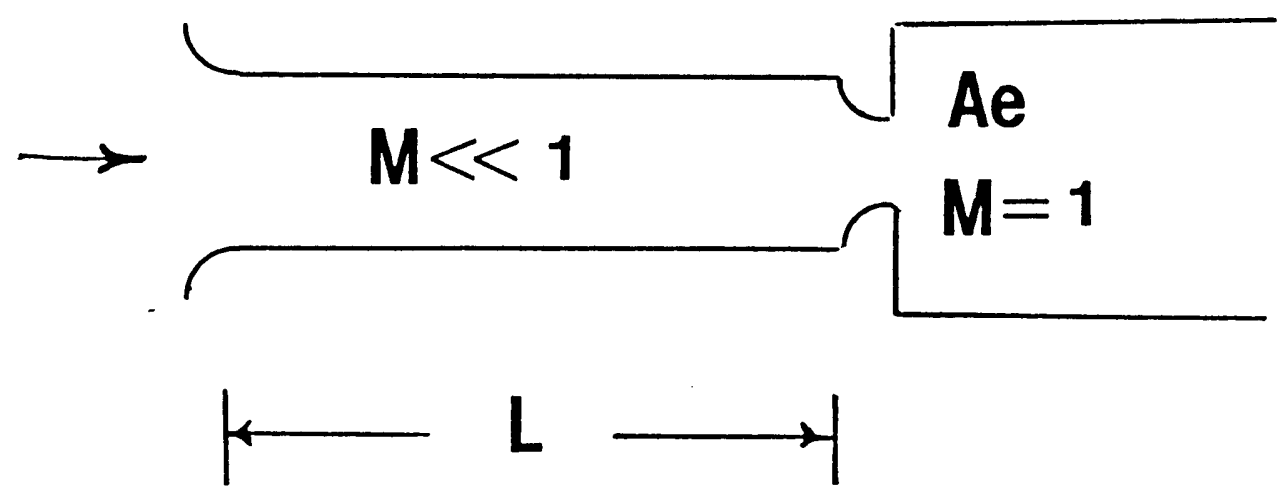

Figure 3.23: Aspirating probe model for frequency response prediction [48] 
ASPIRATING PROBE SteP RESPONSE IN ARGON-FREON 12 MIXTURE

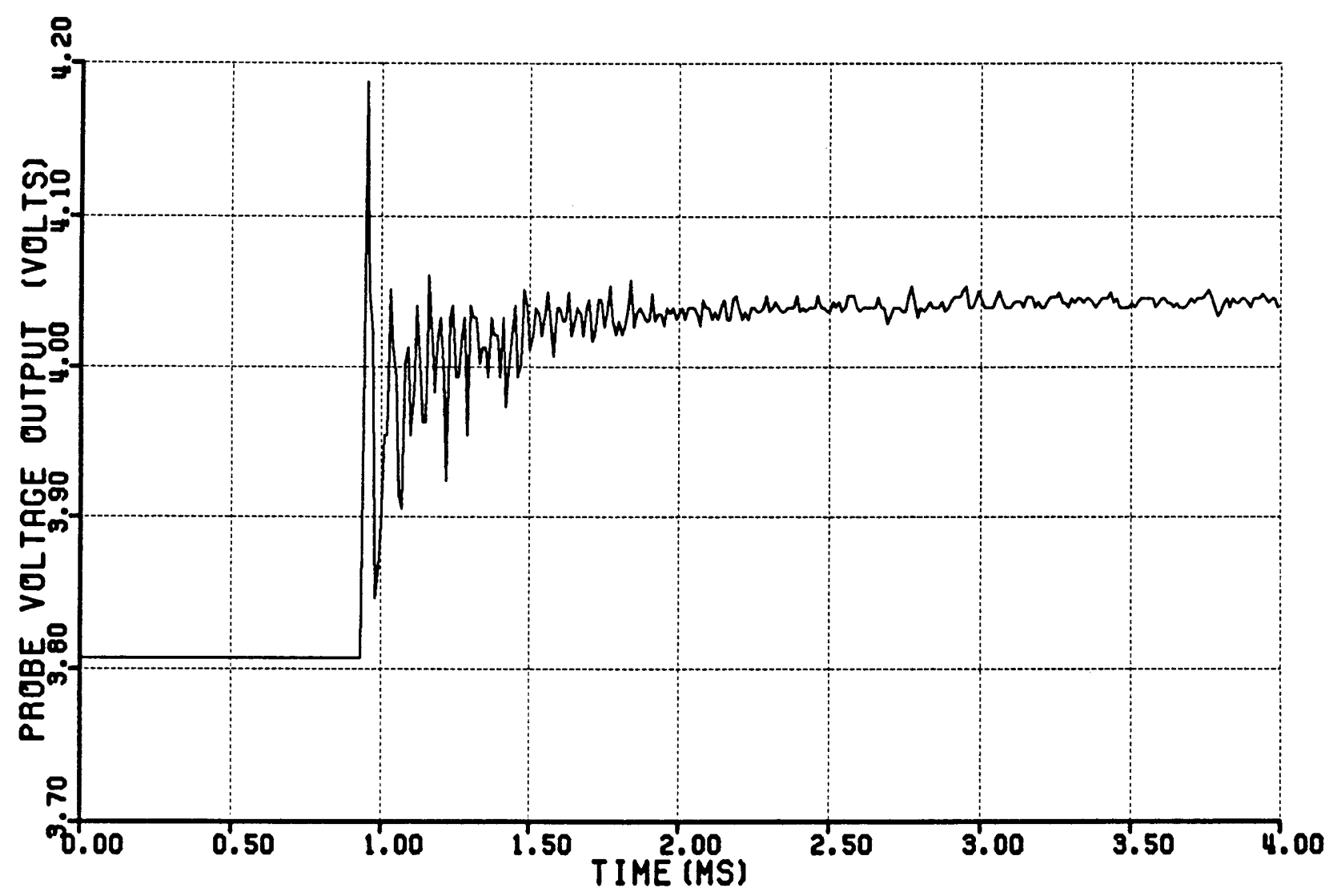

Figure 3.24: Aspirating probe step response in Argon-Freon 12 mixture 


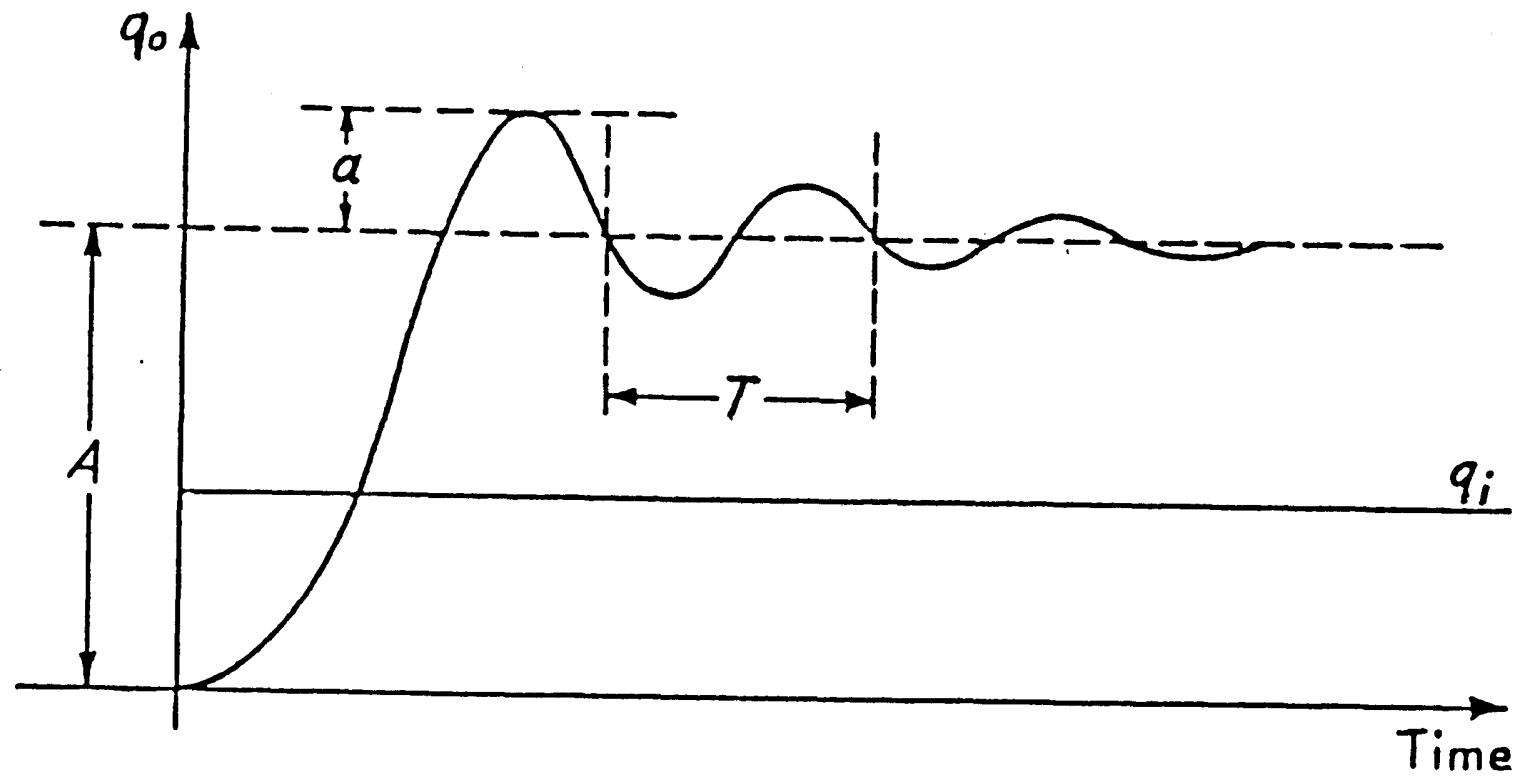

Figure 3.25: Second order system step response 


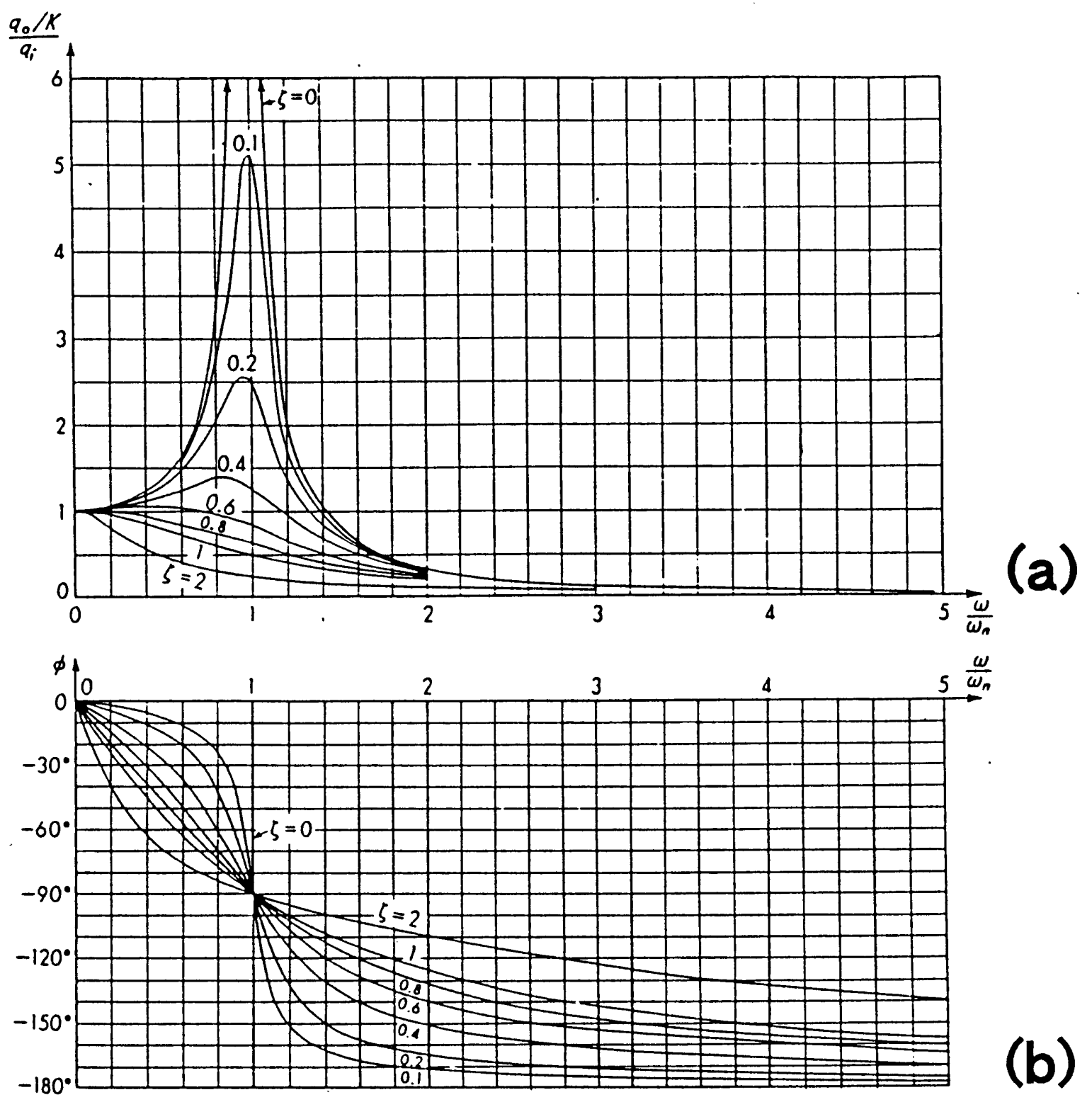

Figure 3.26: Second order system frequency response

(a) Amplitude-ratio curves

(b) Phase-angle curves 
MASS FRACTION ERROR FOR HELIUM-FREON 12 TRACER MIXTURE

7

$\underset{x}{\overrightarrow{0}}$

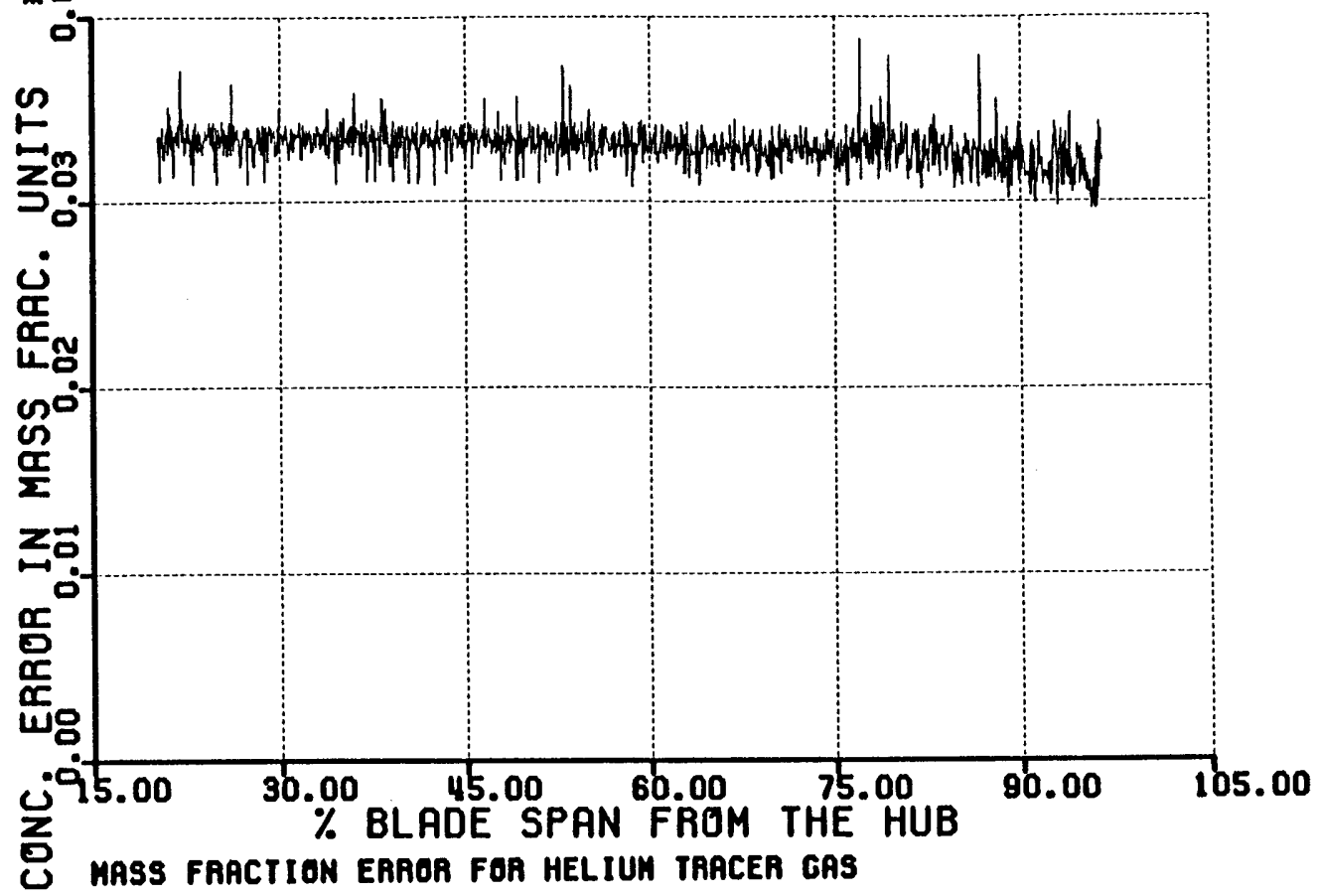

(a)

i

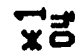

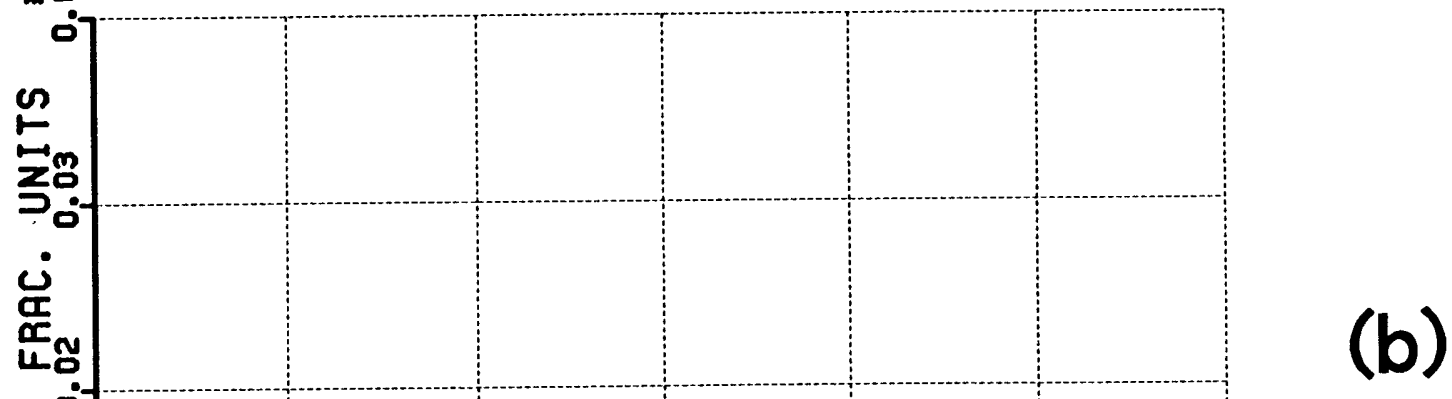

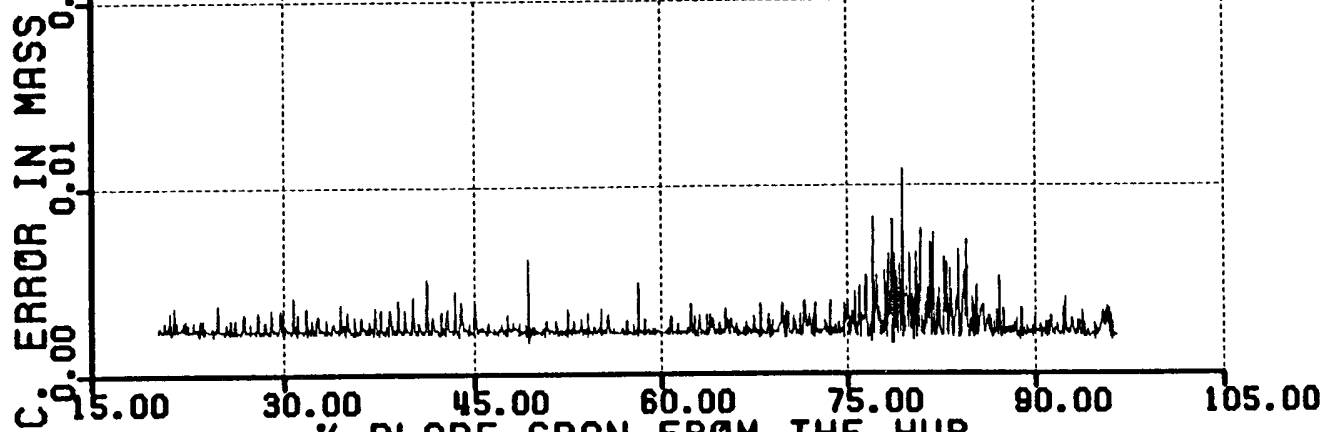

Z $\%$ BLADE SPAN FROM THE HUB

UFigure 3.27: Typical time resolved error in mass fraction units of full scale for the measurement of concentration

(a) Helium-Freon 12 tracer mixture

(b) Helium tracer gas 
TRACER MASS FRACTION FOR INJECTION AT THE TIP

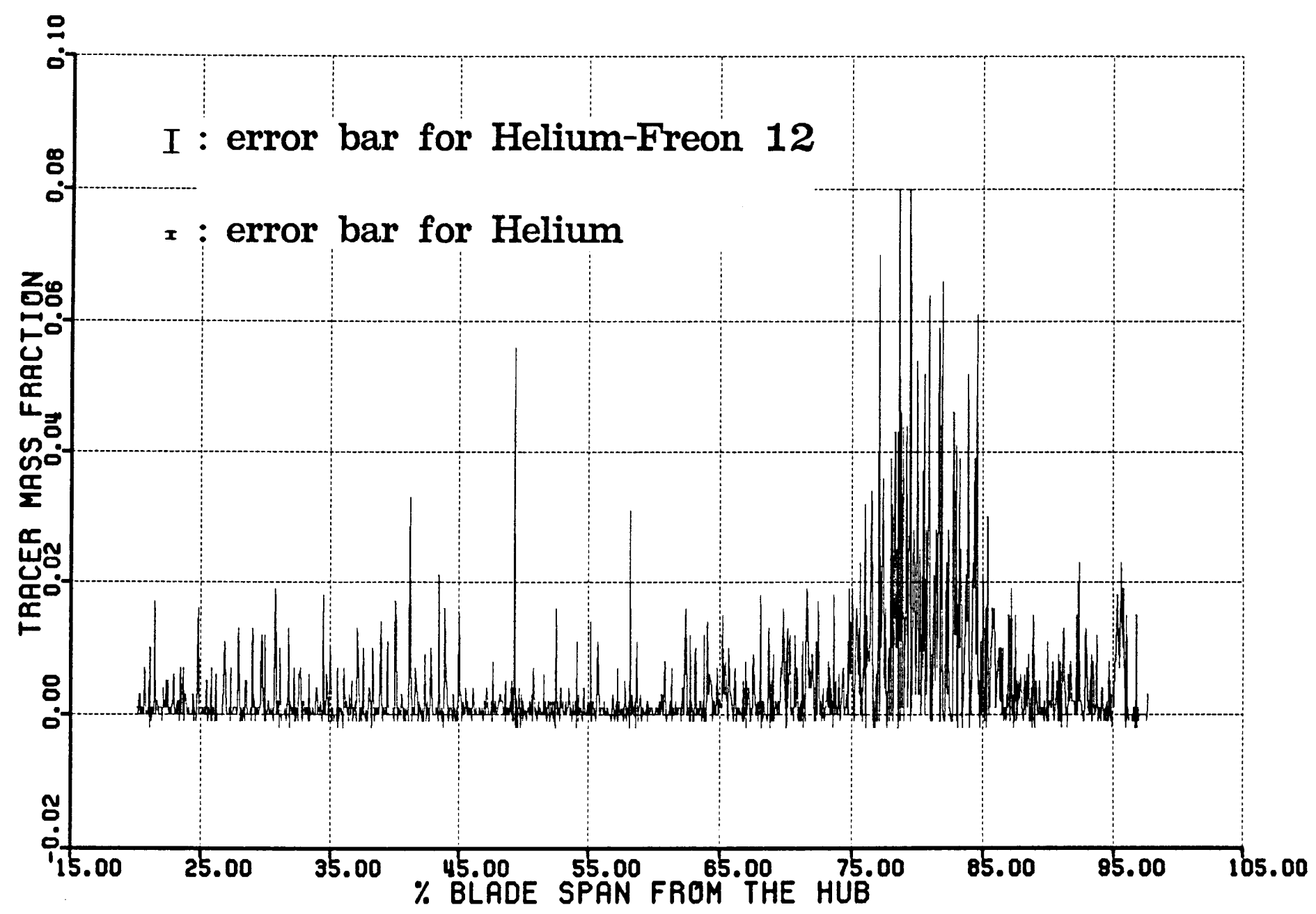

Figure 3.28: Time resolved measurement of concentration in mass 
TOTAL TEMPEAATURE ERROA FOR HELIUM-FREOM 12 TRACER MIXTURE

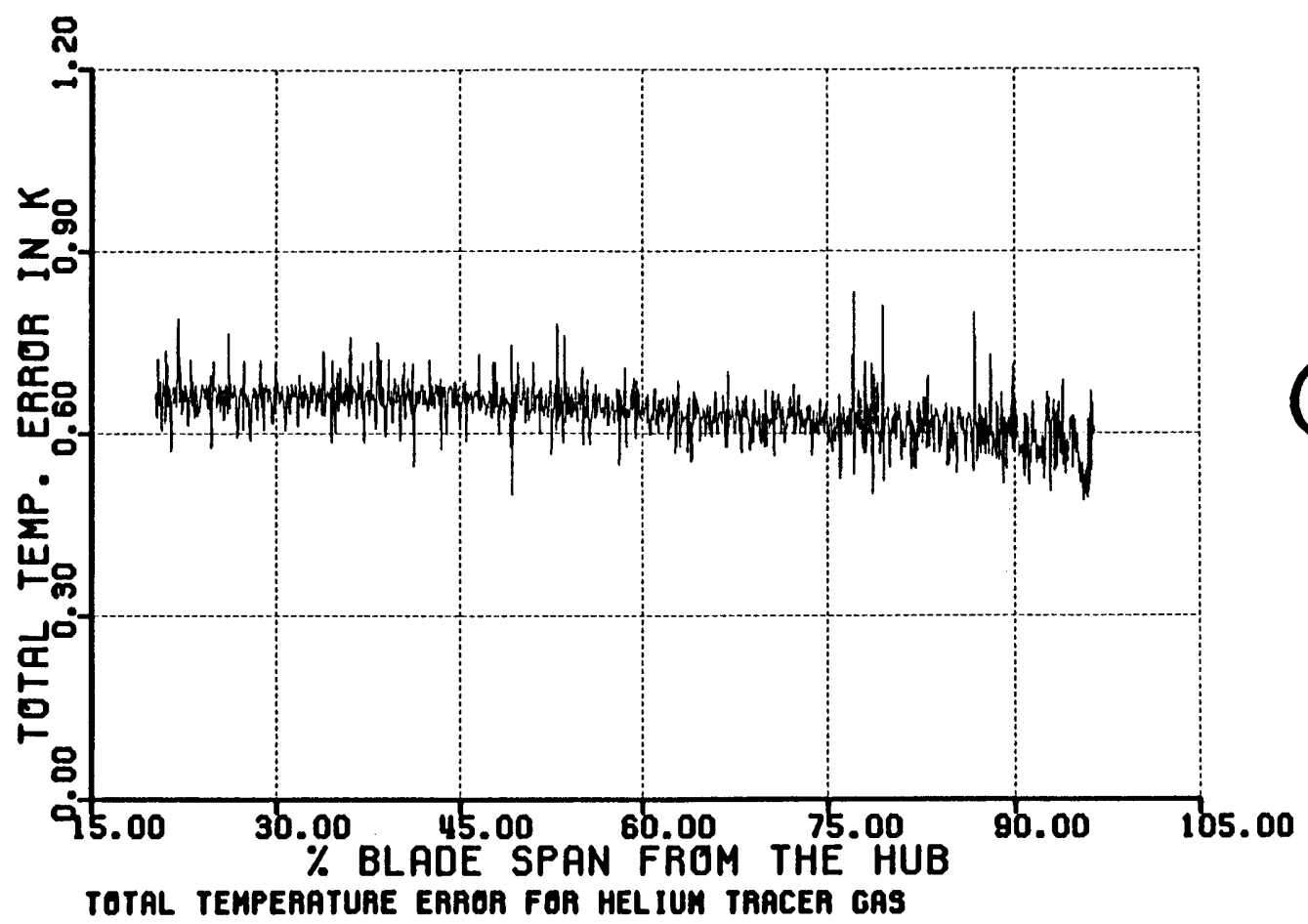

(a)

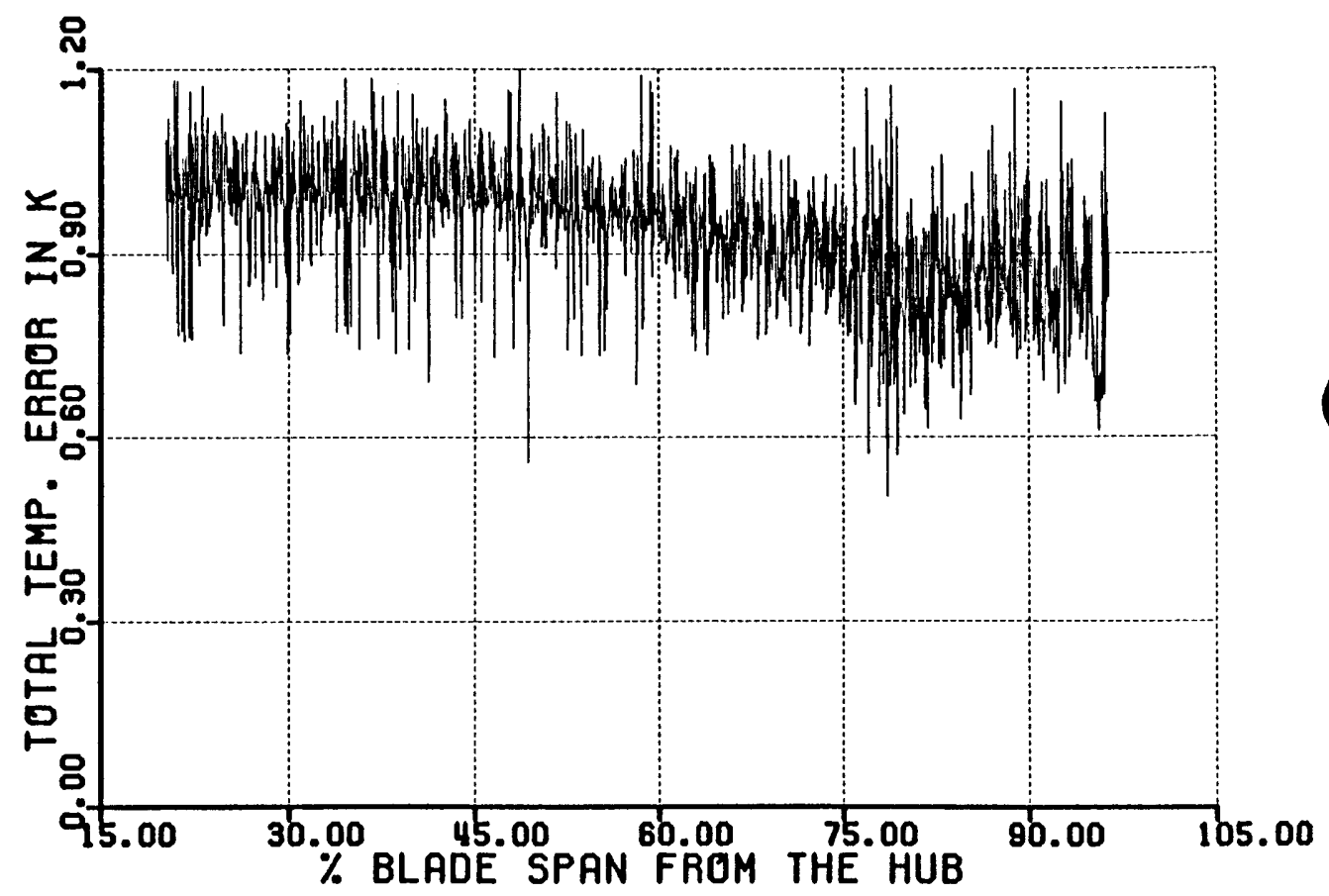

(b)

Figure 3.29: Typical time resolved error in $O_{K}$ for the measurement of total temperature

(a) Helium-Freon 12 tracer mixture

(b) Helium tracer gas 
TOTAL TEMPERATURE FOR INJECTION AT THE TIP

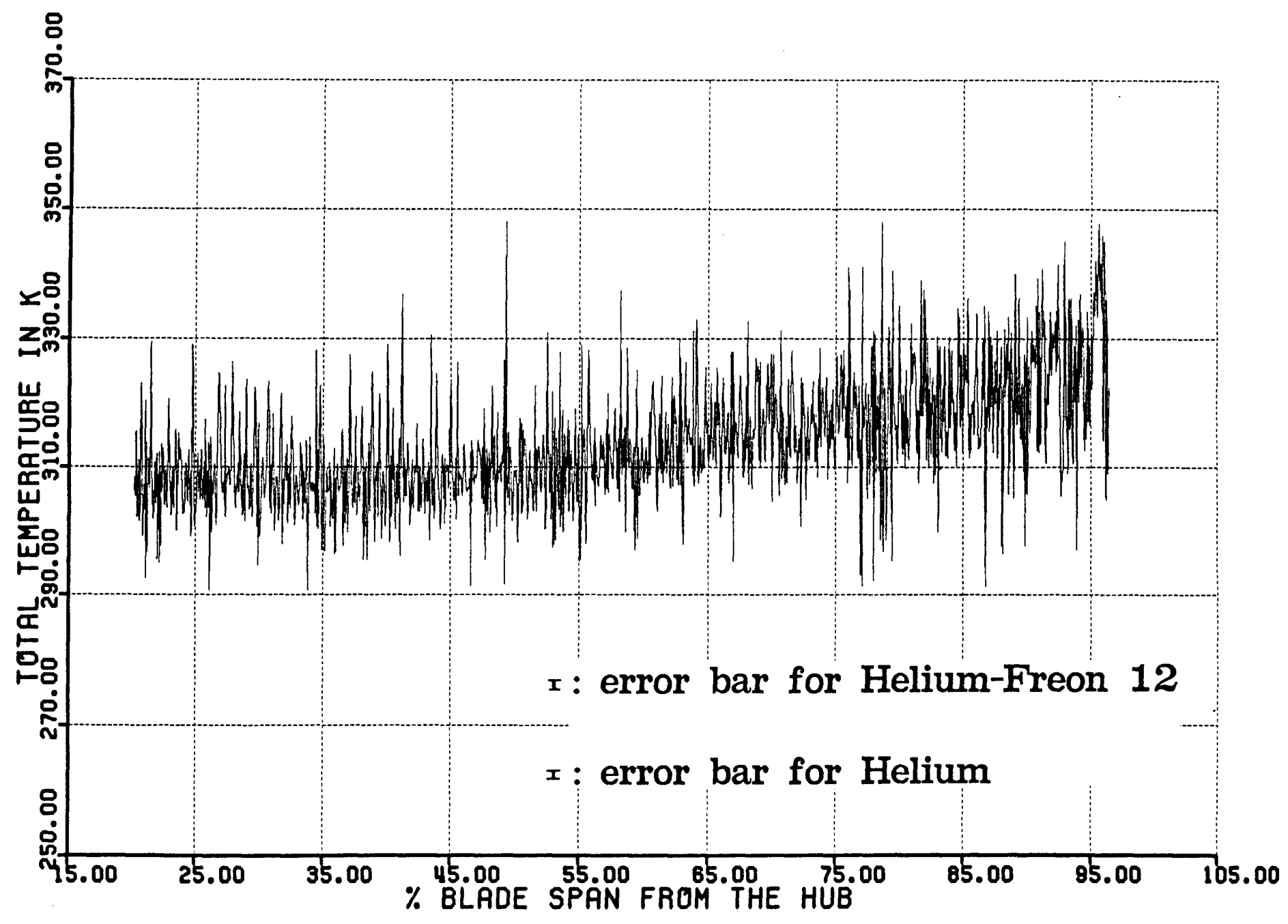

Figure 3.30: Time resolved measurement of total temperature in

${ }_{\mathrm{K}}$ with error bars 
TOTAL PRESSURE ERROR FOR HELIUM-FREON 12 TRACER MIXTURE

$$
\text { 임 }
$$

$\times$

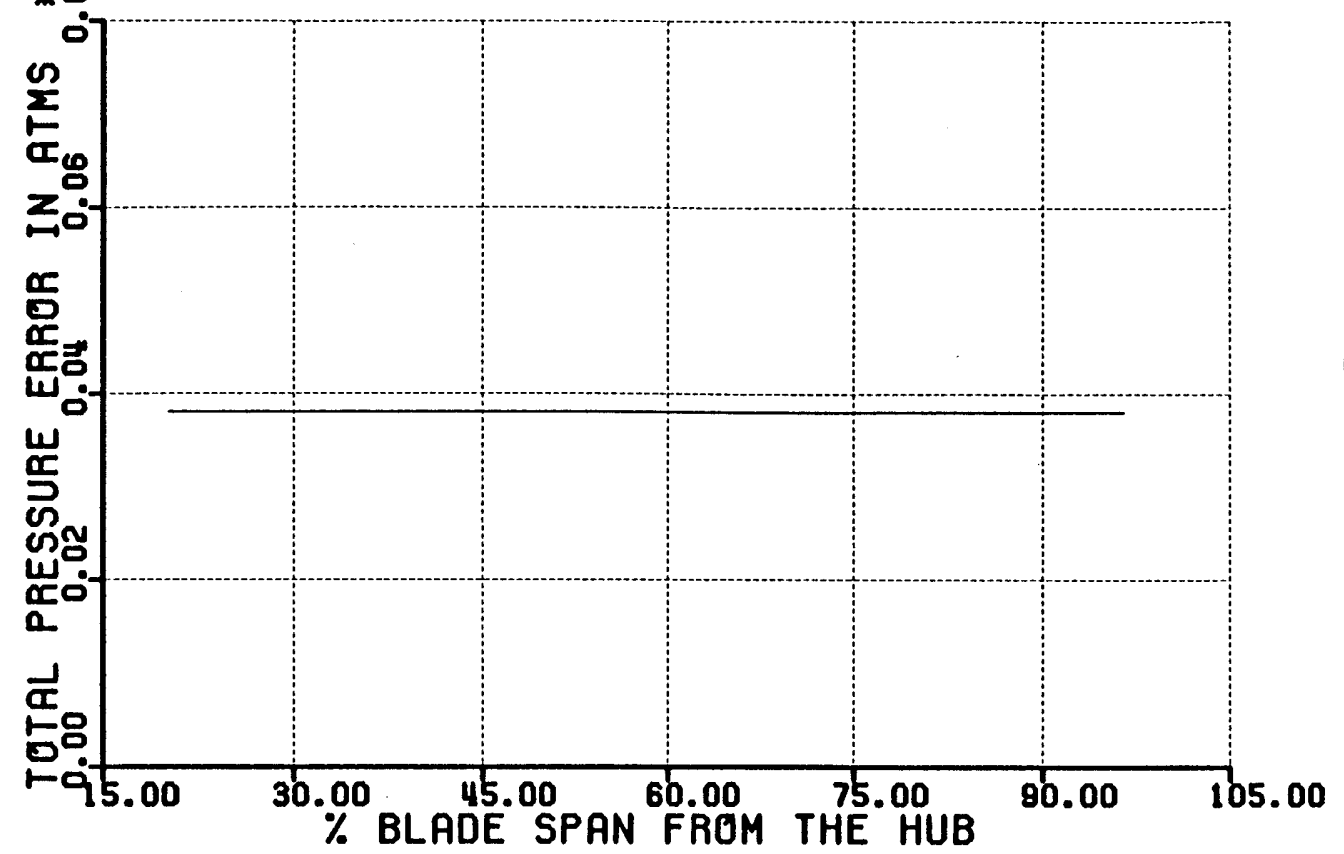

TOTAL PRESSURE ERROR FOR HELIUM TRACER GAS

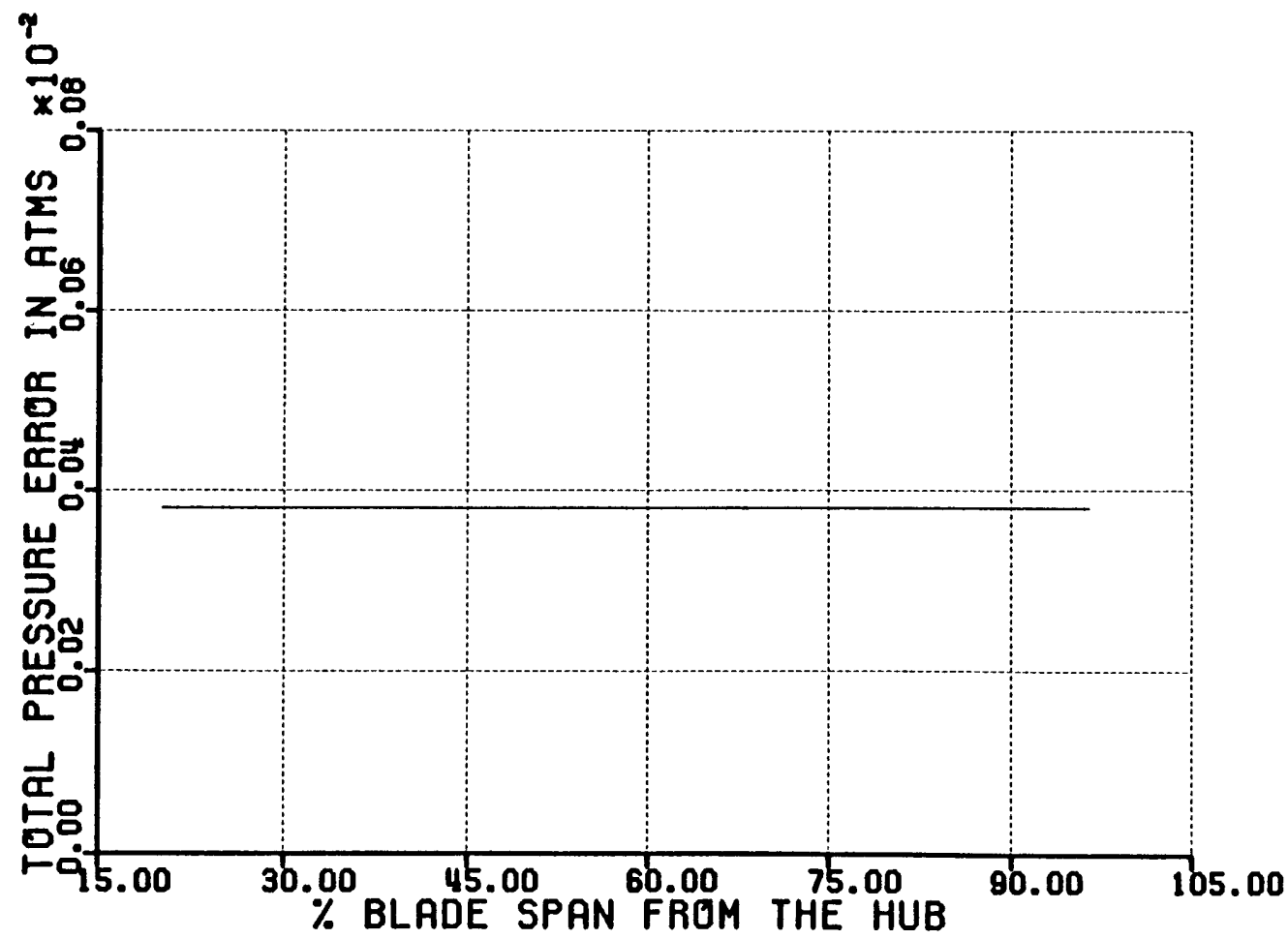

Figure 3.31: Typical time resolved error in atms for the measurement of total pressure

(a) Helium-Freon 12 tracer mixture

(b) Helium tracer gas 
TOTAL PRESSURE FOR INJECTION AT THE TIP

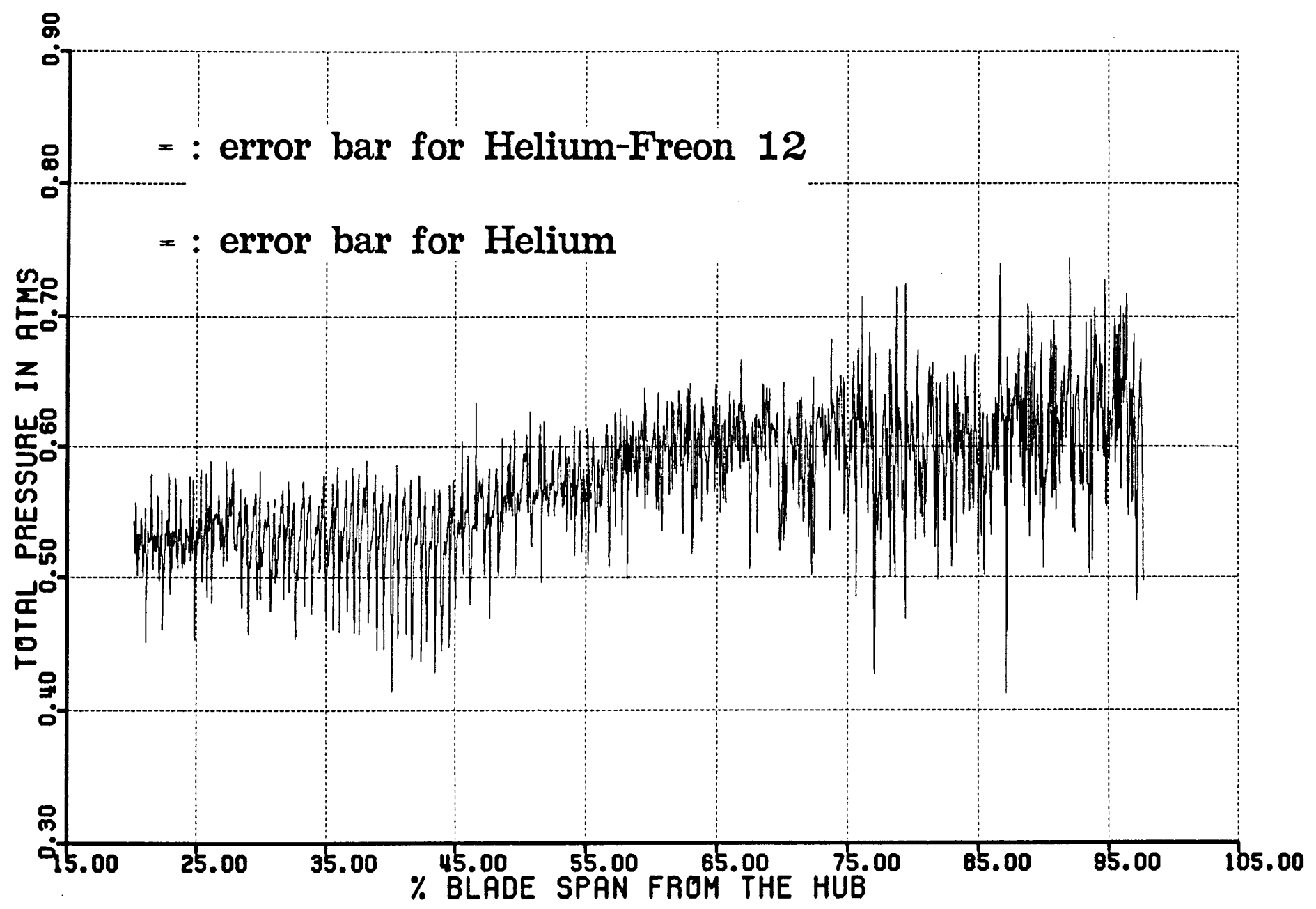

Figure 3.32: Time resolved measurement of total pressure in atms with error bars 
CONCENTRATION DURING NO ROTOR TEST

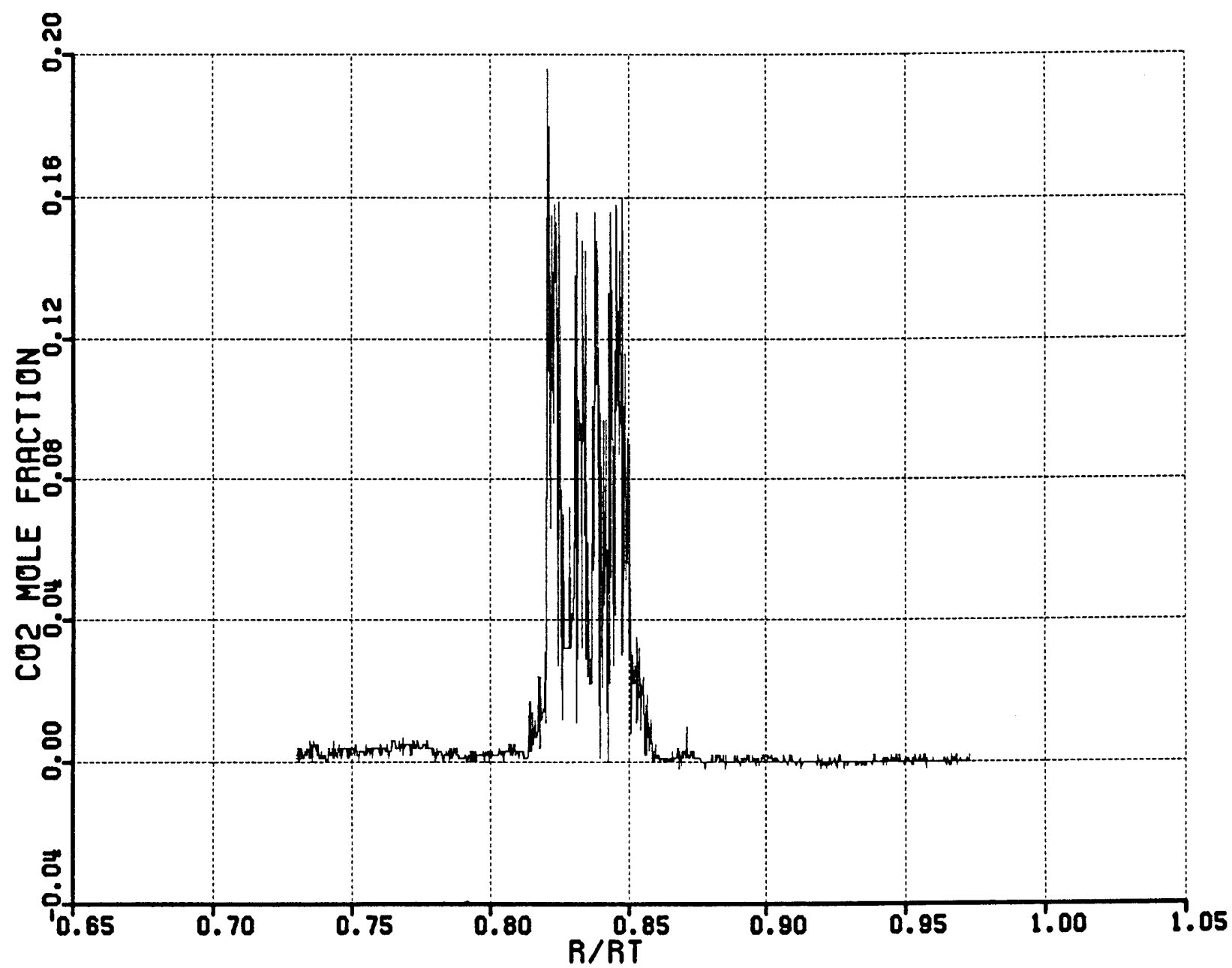

Figure 4.1: Time resolved tracer gas concentration (mass fraction) during Blowdown test with no rotor in place. Determination of basic mixing level in the Facility and jet spreading rate

N 
TOTAL TEMPERATUAE IM tHE MO hotOR CaSE

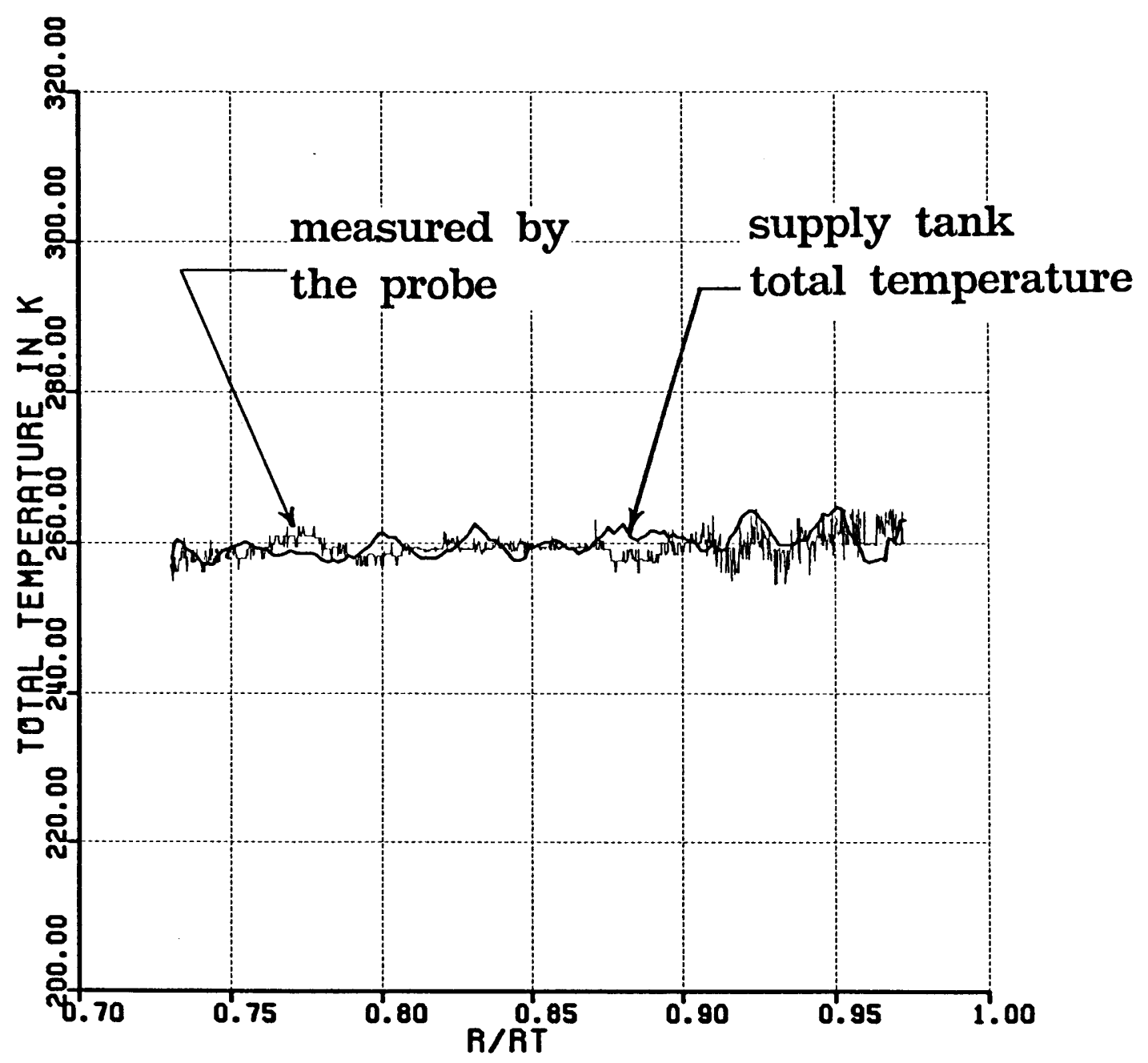

Figure 4.2: Comparison between total temperature measured by the aspirating probe and calculated from isentropic expansion in the supply tank (rotor removed) 
total paessure IN the nO rotor case

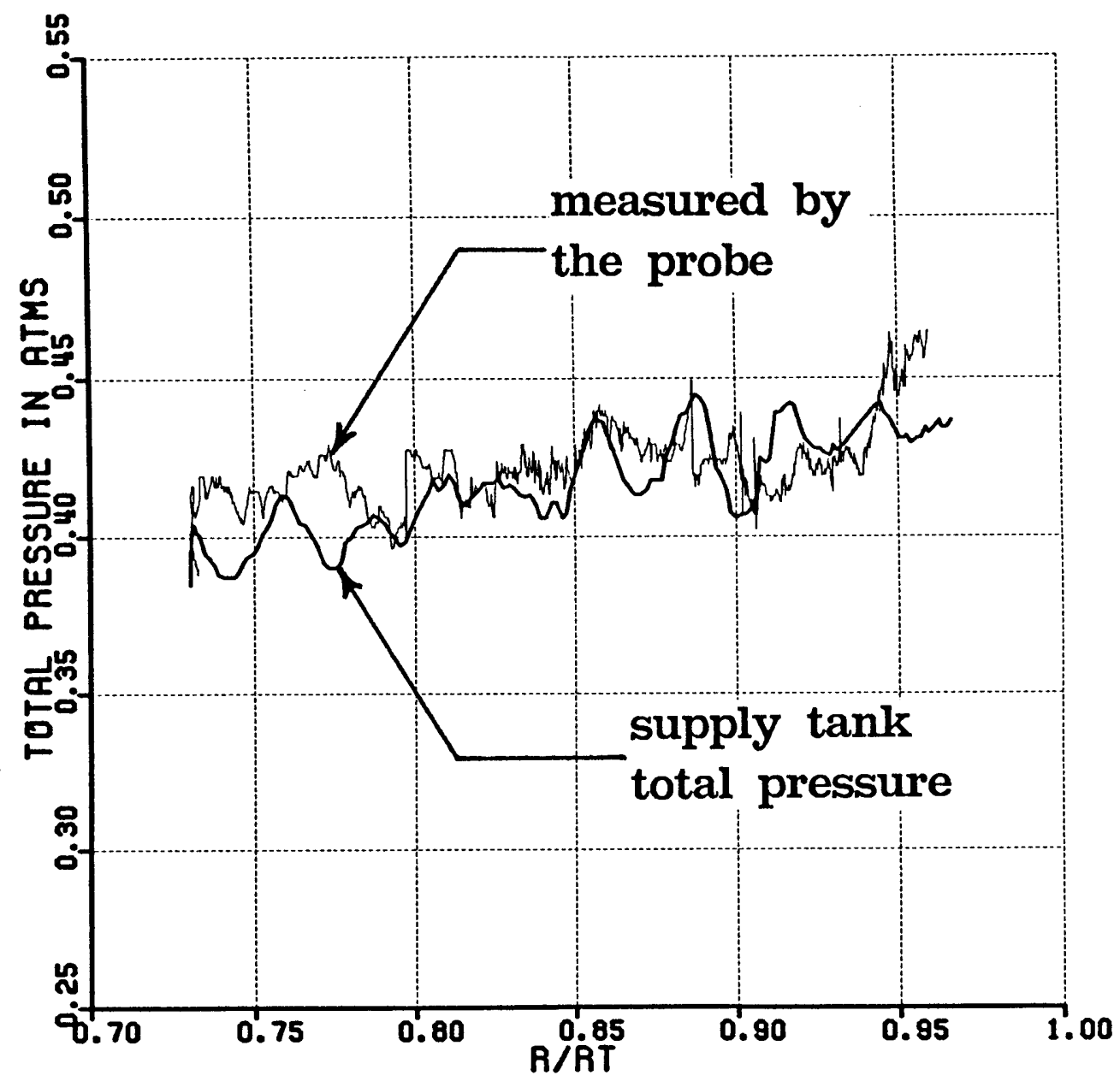

Figure 4.3: Comparison between total pressure measured by the total pressure probe at the downstream surveying location and by a pressure transducer in the Blowdown supply tank (rotor removed) 
TIME AVERAGED TOTAL PRESSURE RATIO

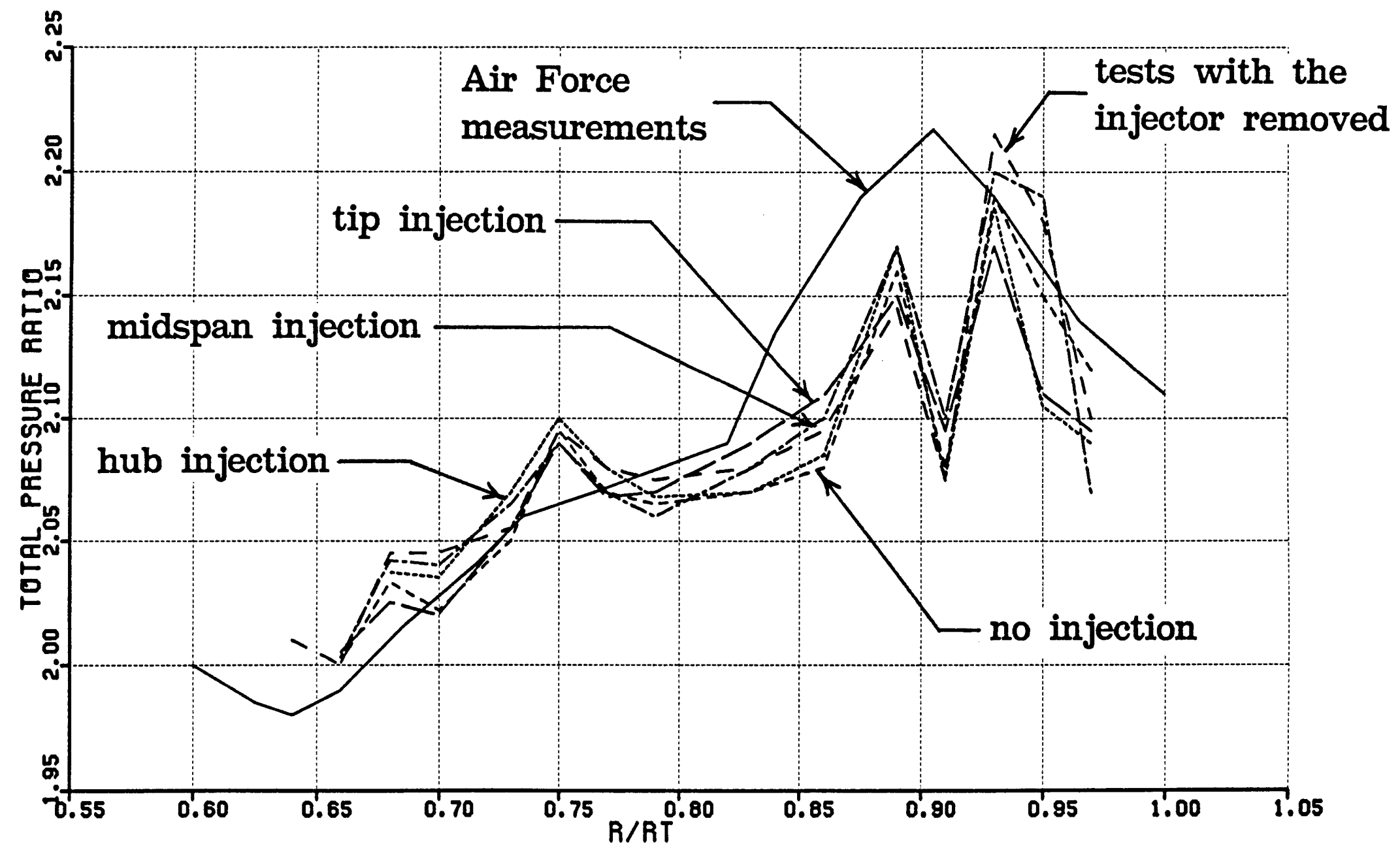

Figure 4.4: Comparison of time averaged total pressure ratio for various test conditions 
TIME AVERAGED TOTAL TEMPERATURE RATIO

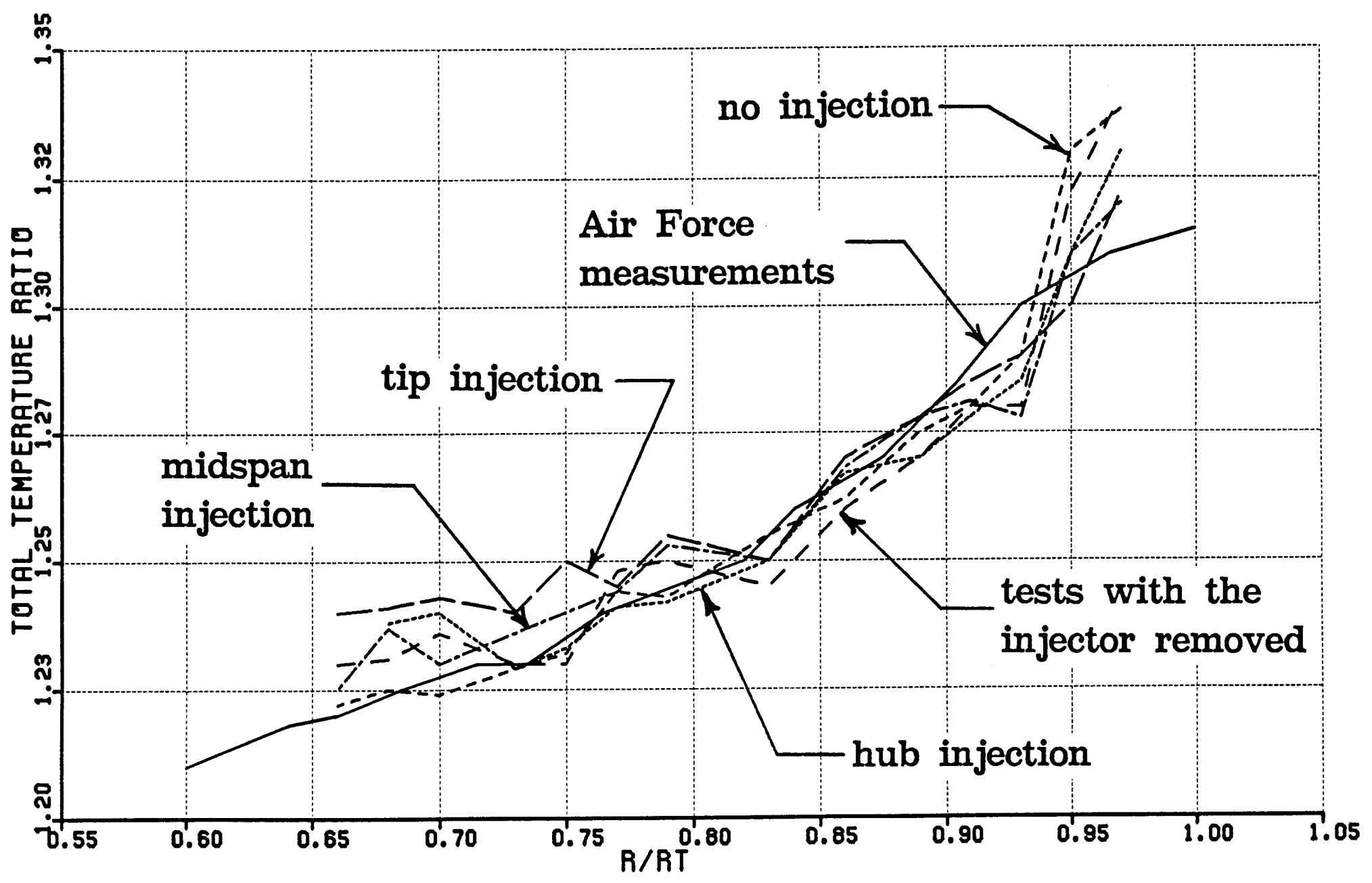

Figure 4.5: Comparison of time averaged total temperature ratio for various test conditions 


\section{TIME AVERAGED TOTAL PRESSURE RATIO}

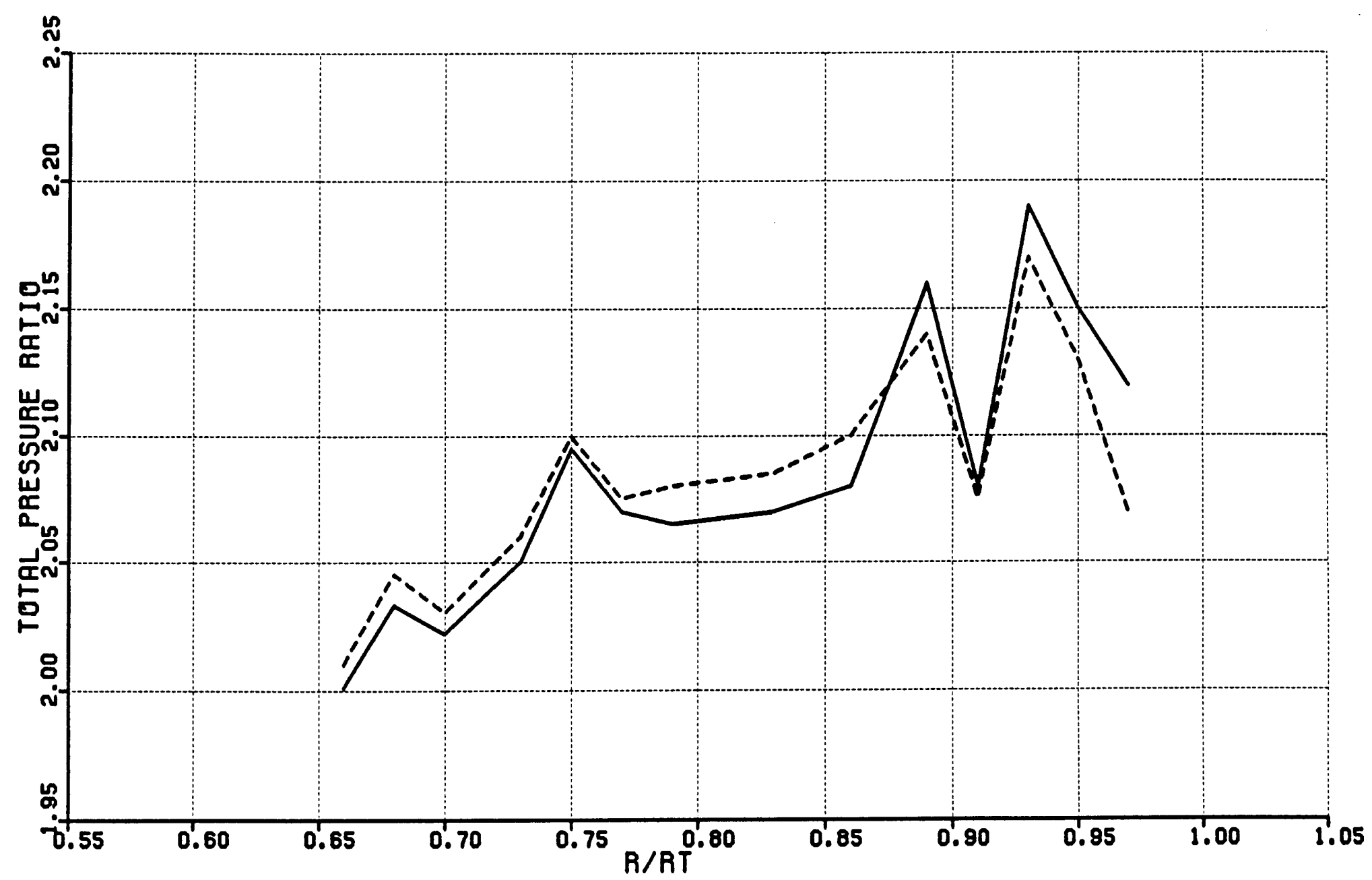

Figure 4.6: Demonstration of the repeatability of the Blowdown Facility. Comparison of time averaged total pressure ratio during two separate Blowdown tests under the same conditions 
TIME AVERAGED TOTAL TEMPERATURE RATIO

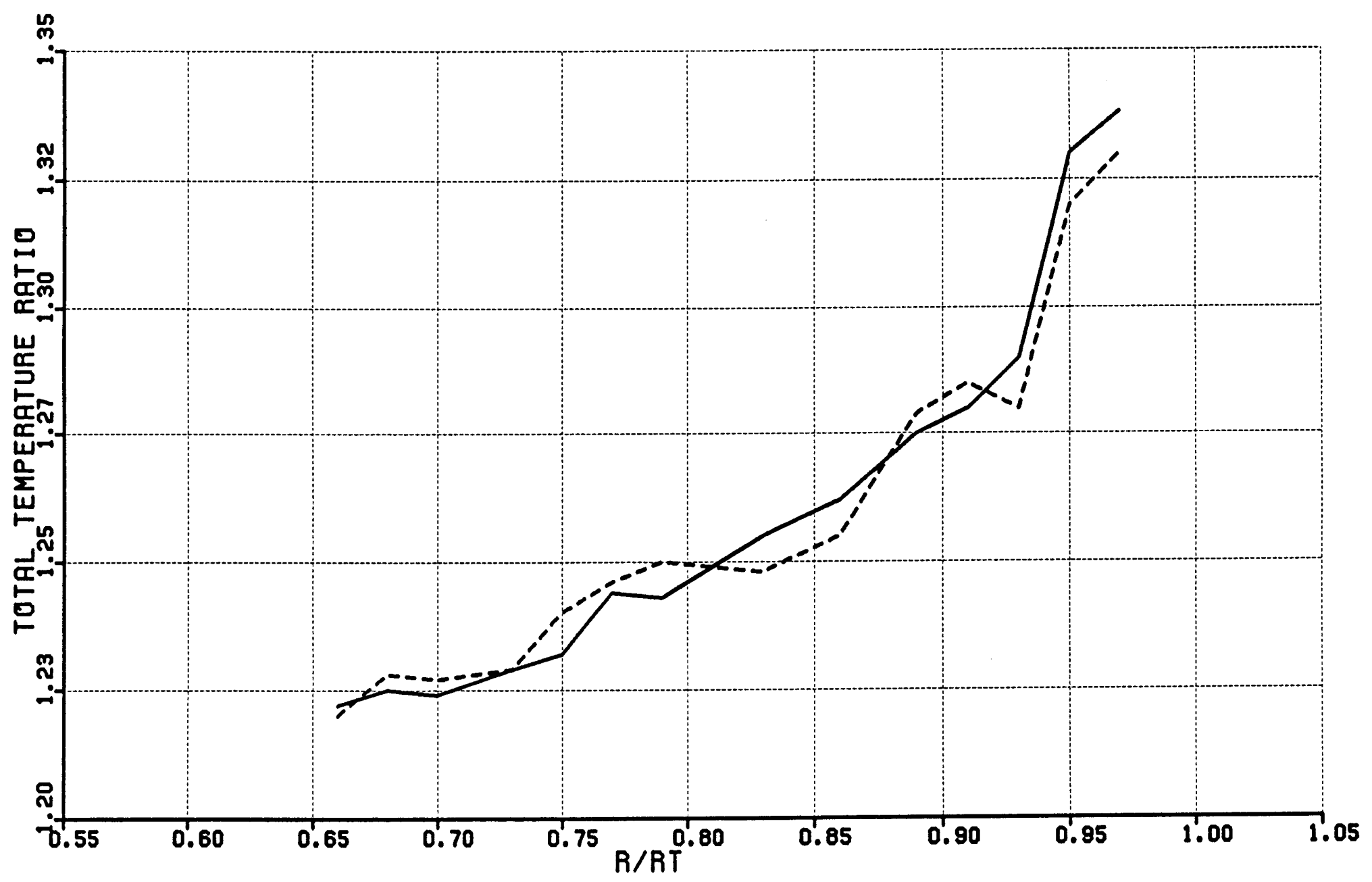

Figure 4.7: Demonstration of the repeatability of the Blowdown Facility. Comparison of time averaged total temperature ratio during two separate Blowdown tests under the same conditions 


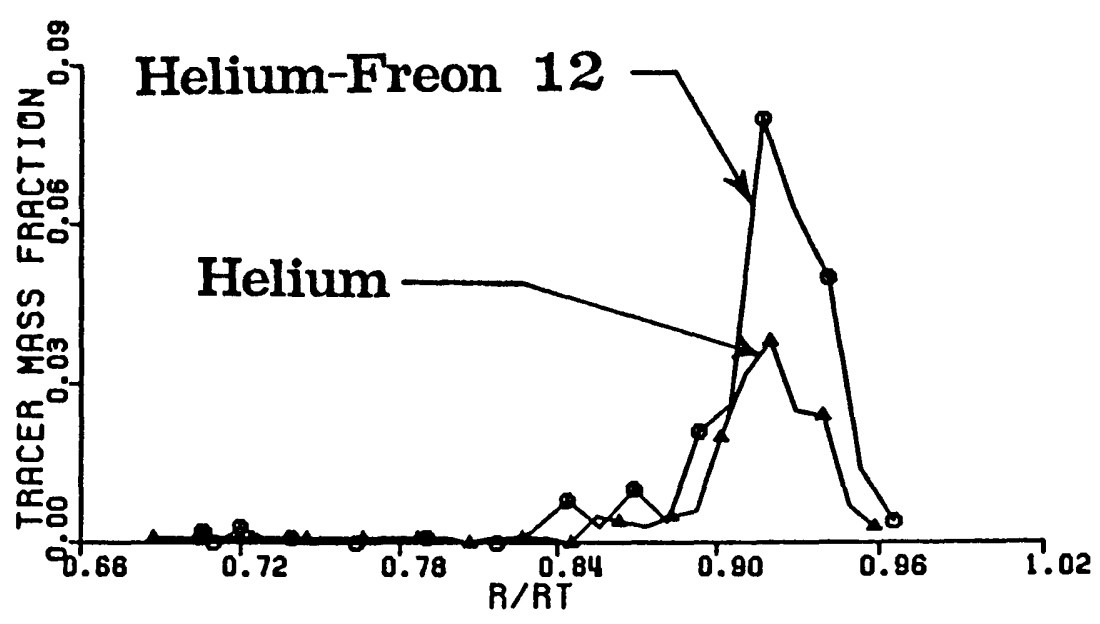

(a)

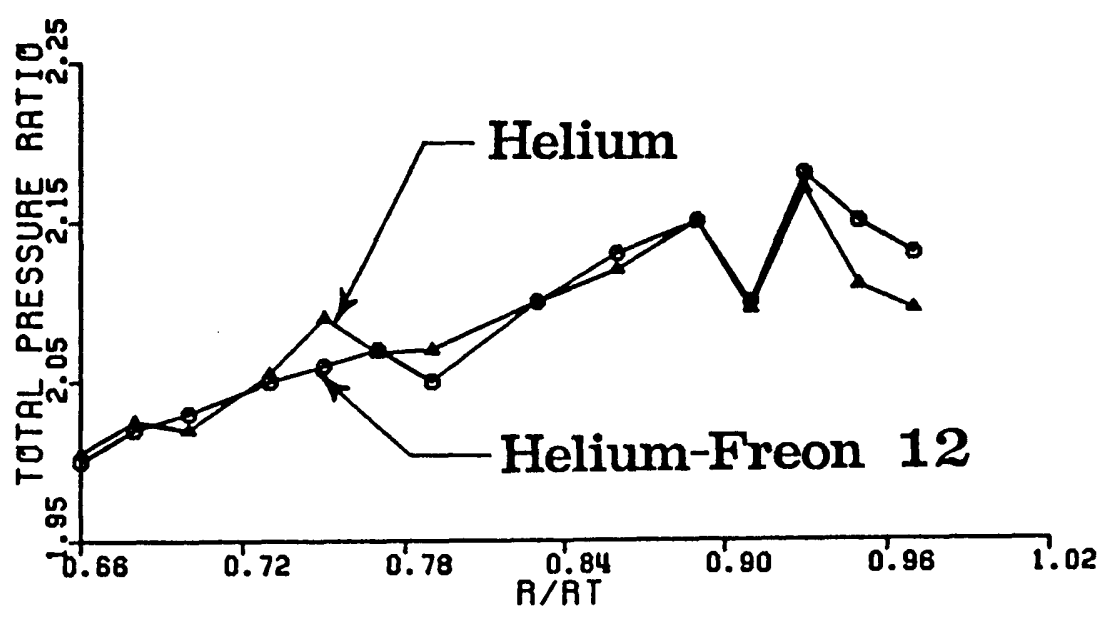

(b)

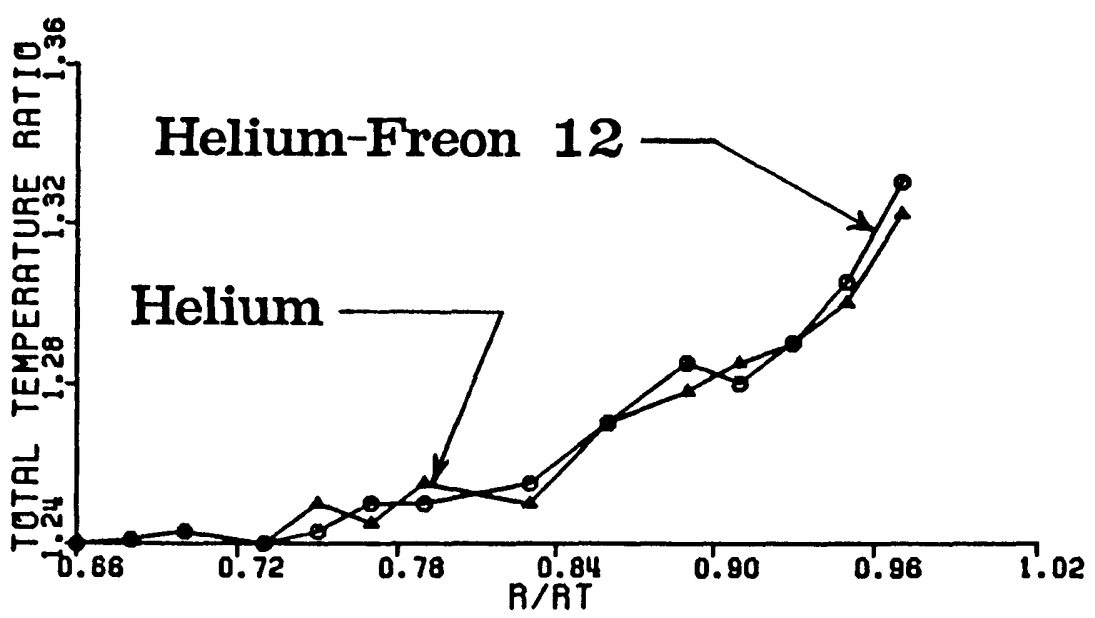

(c)

Figure 4.8: Comparison of time averaged tracer gas concentration (a), total pressure ratio (b) and total temperature ratio (c) for tests with Helium and Helium-Freon as tracer gas 


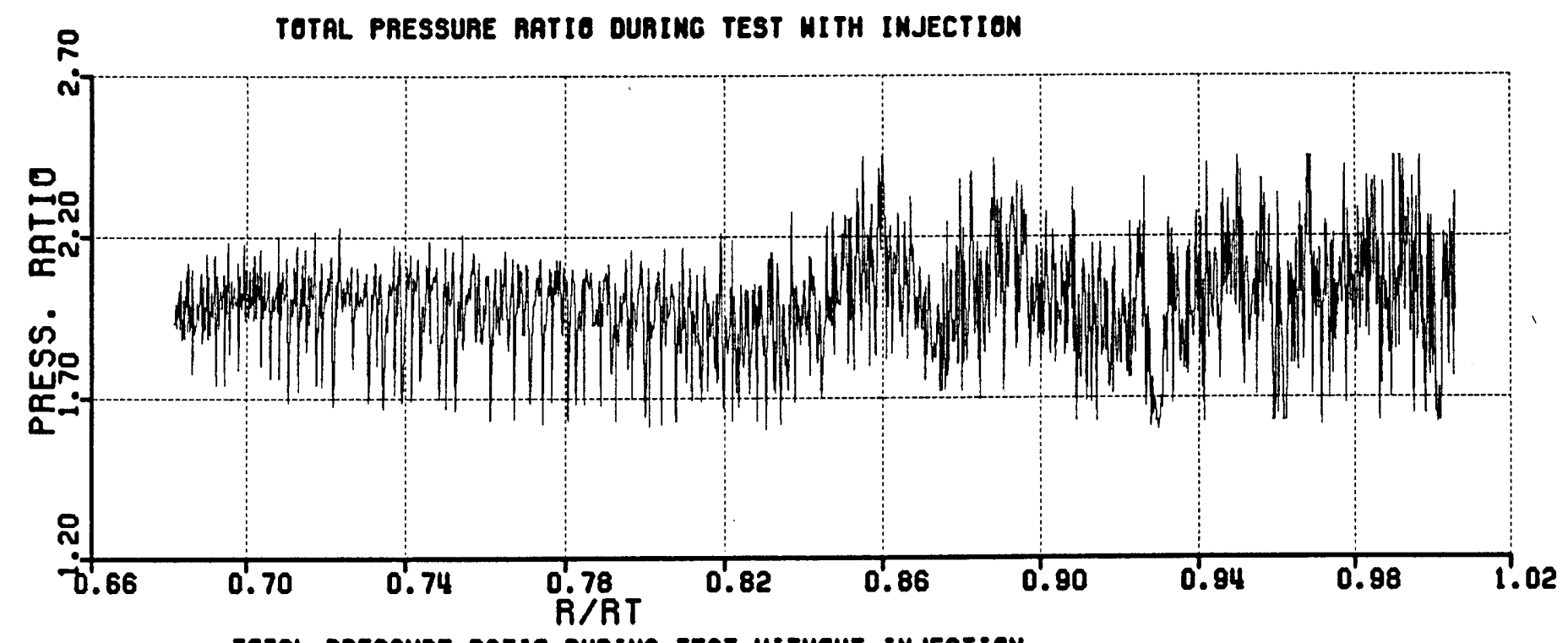

(a)

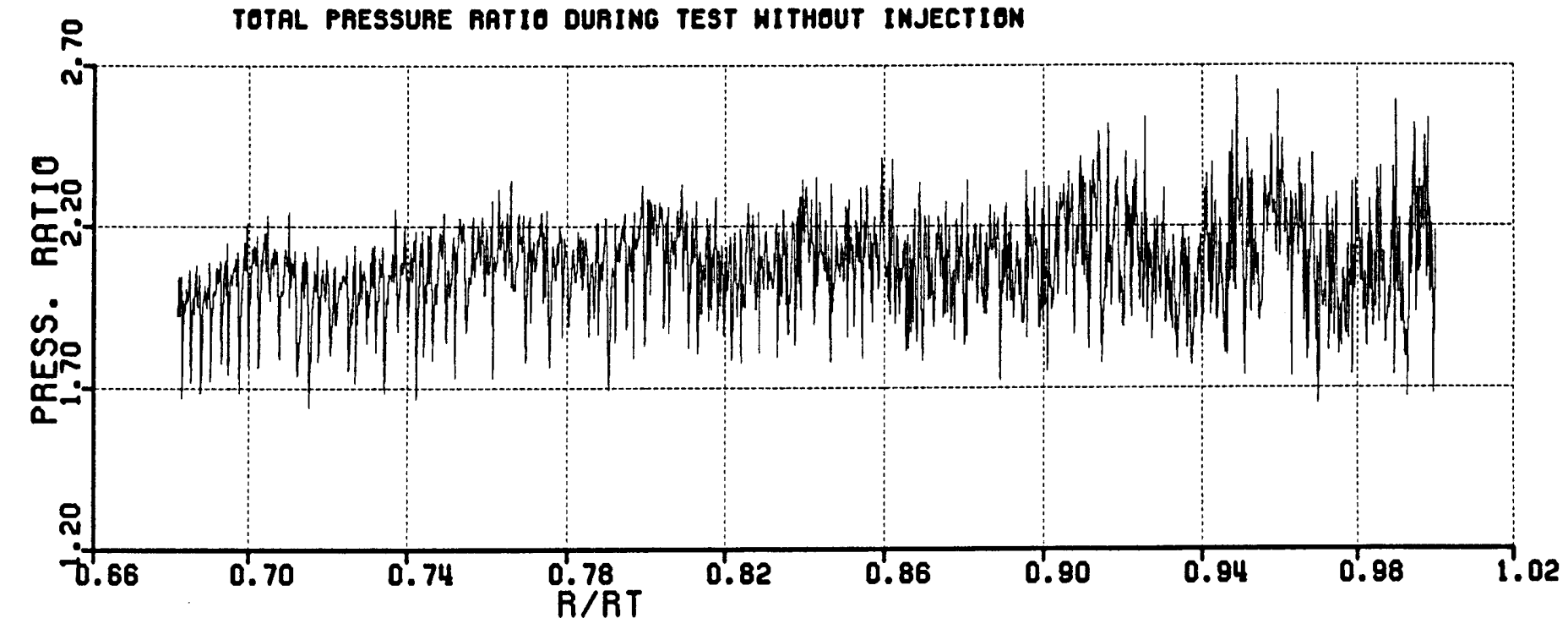

(b)

Figure 5.1: Comparison of instantaneous total pressure ratio during Blowdown tests with (a) and without (b) injection 


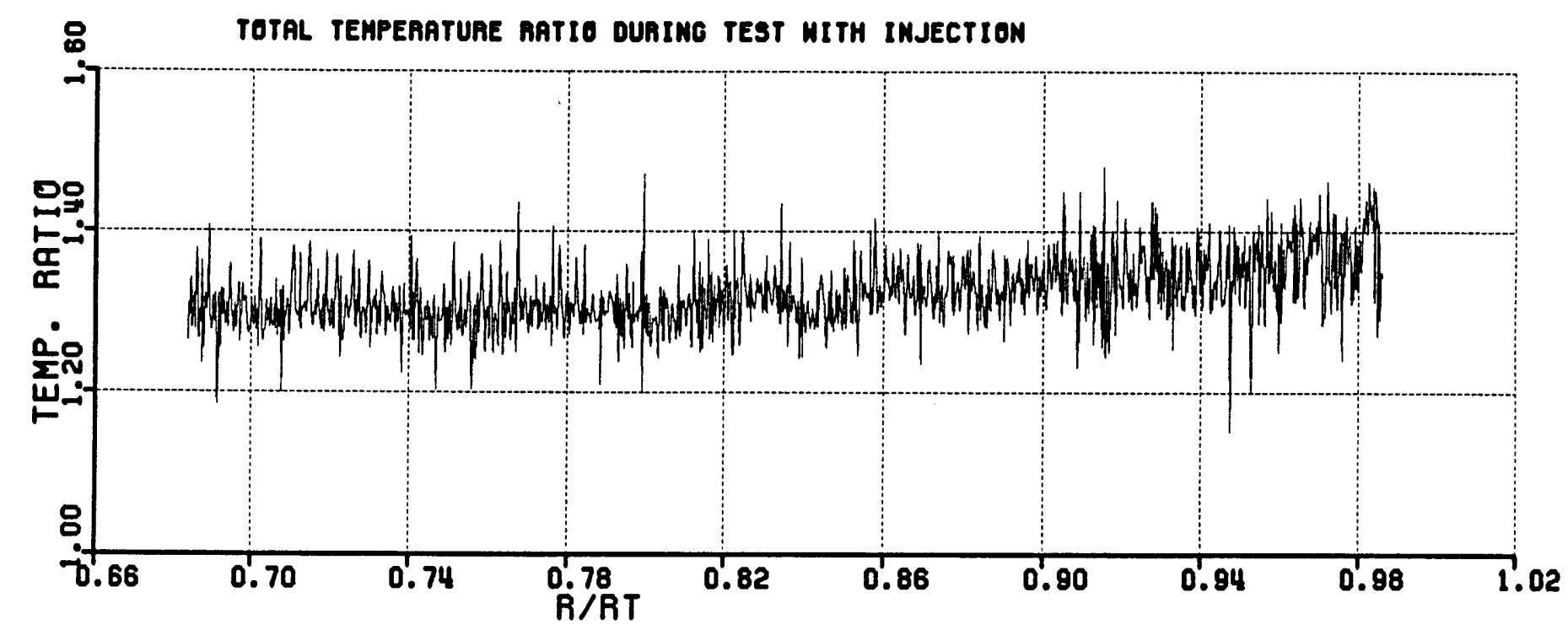

(a)

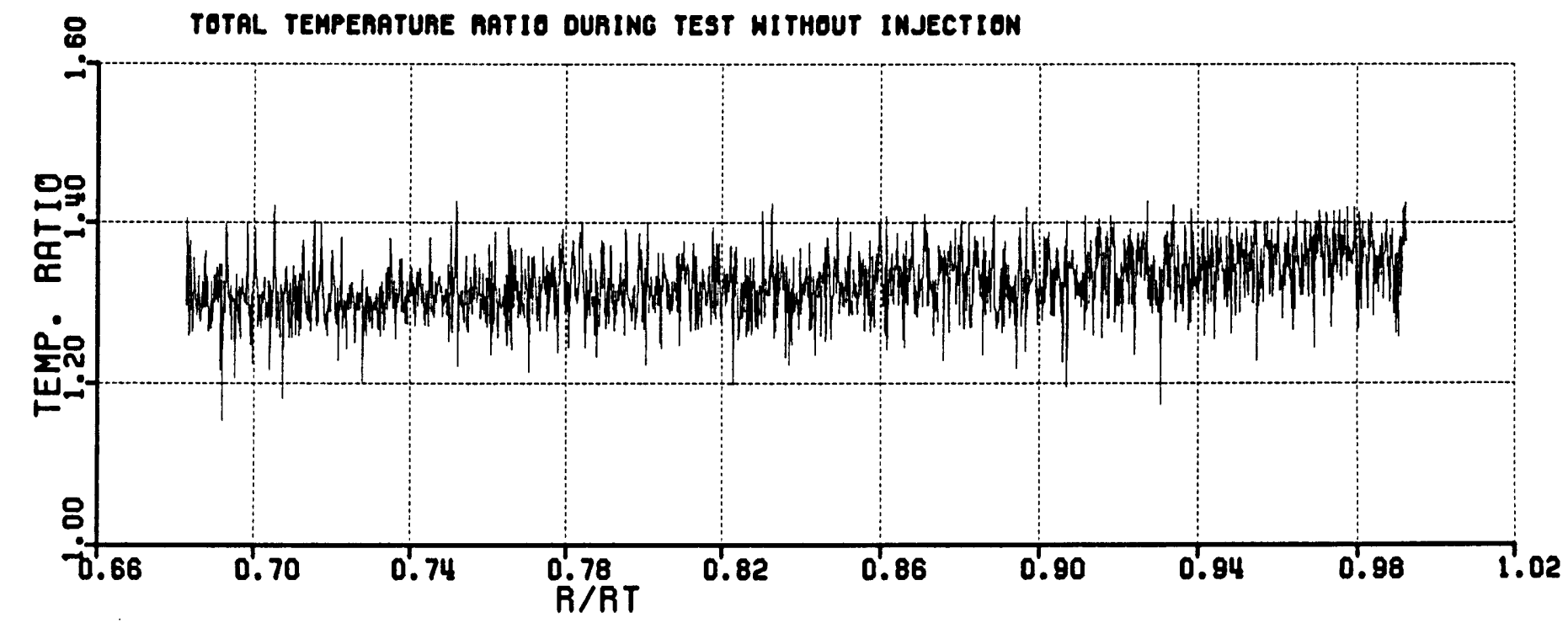

(b)

Figure 5.2: Comparison of instantaneous total temperature ratio during Blowdown tests with (a) and without (b) injection 
TIME AVERAGED TOTAL PRESSURE RATIO

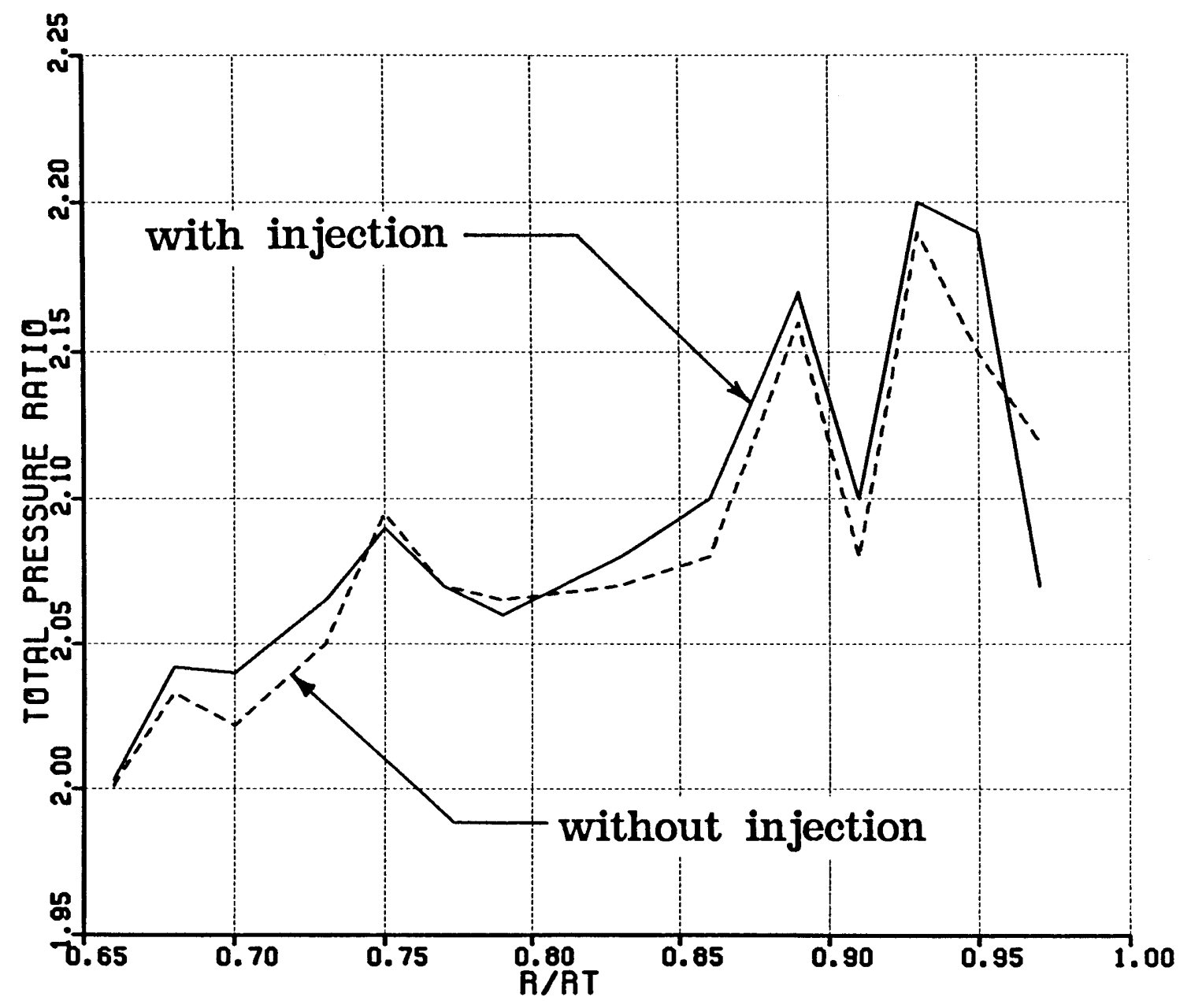

Figure 5.3: Comparison of time averaged total pressure ratio during Blowdown tests with and without injection 
TIME AVERAGED TOTAL TEMPERATURE RATIO

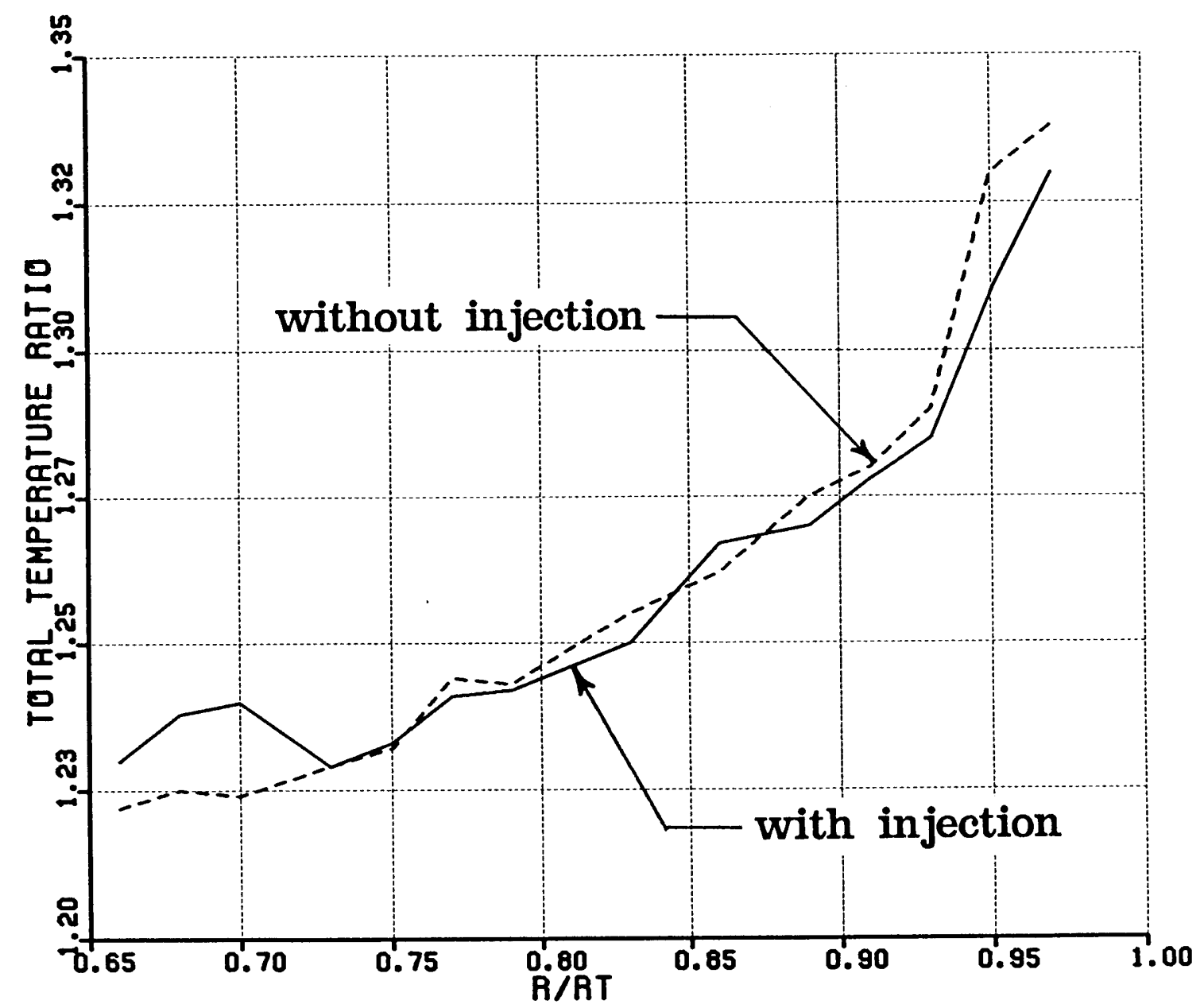

Figure 5.4: Comparison of time averaged total temperature ratio during Blowdown tests with and without injection 


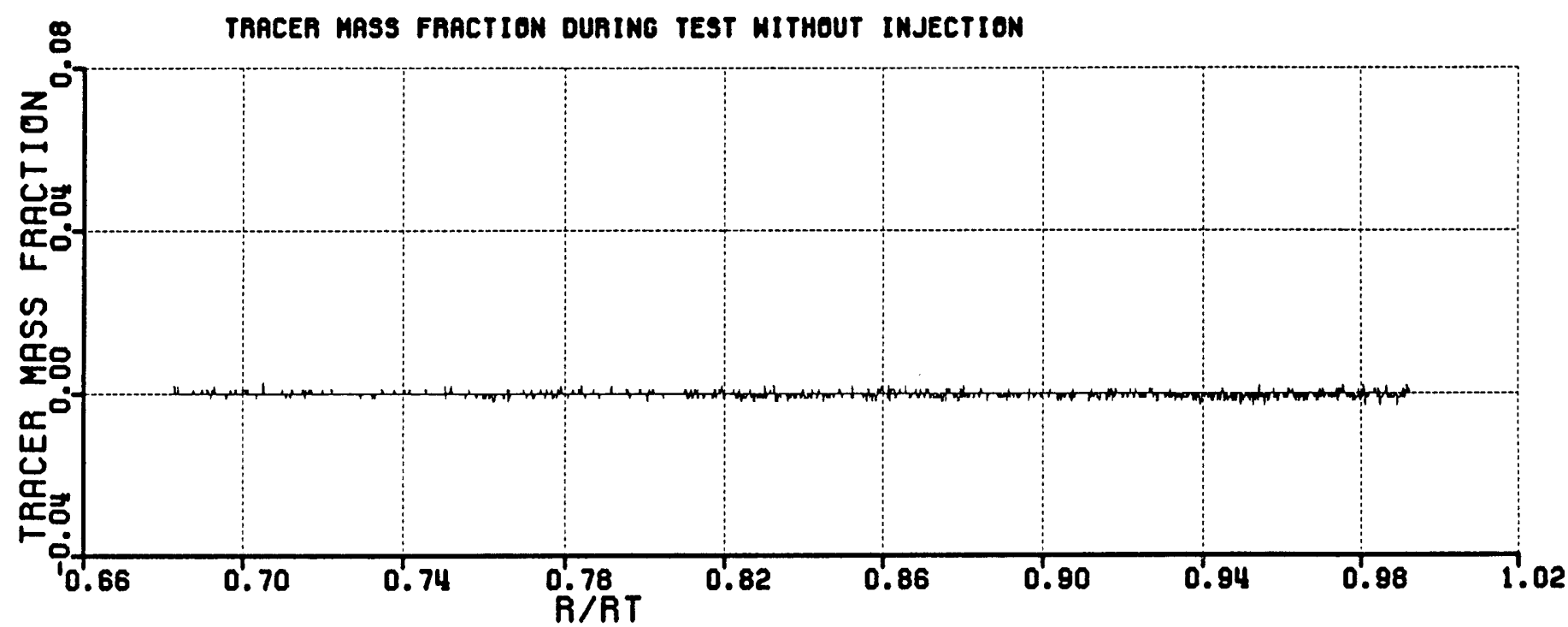

(a)

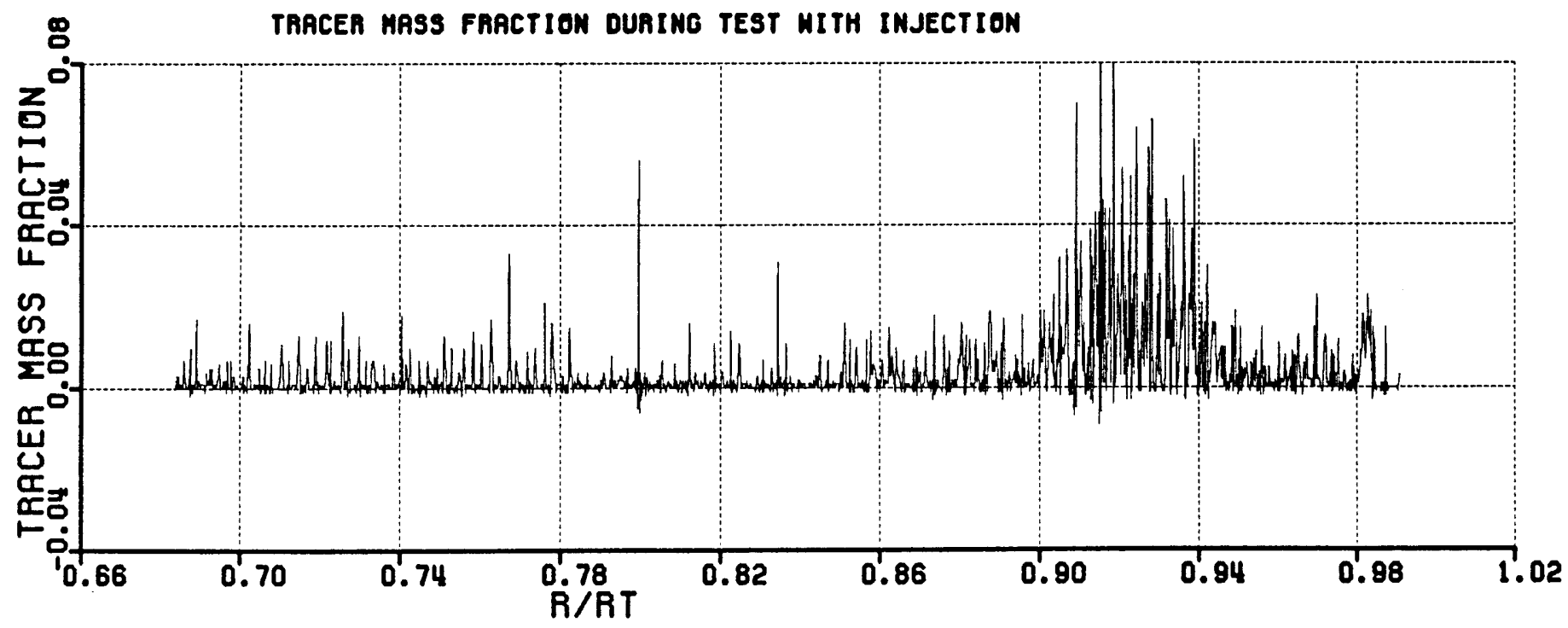

(b)

Figure 5.5: Time resolved tracer gas concentration (mass fraction) during tests without (a) and with (b) injection 
INJECTION LOCATIONS

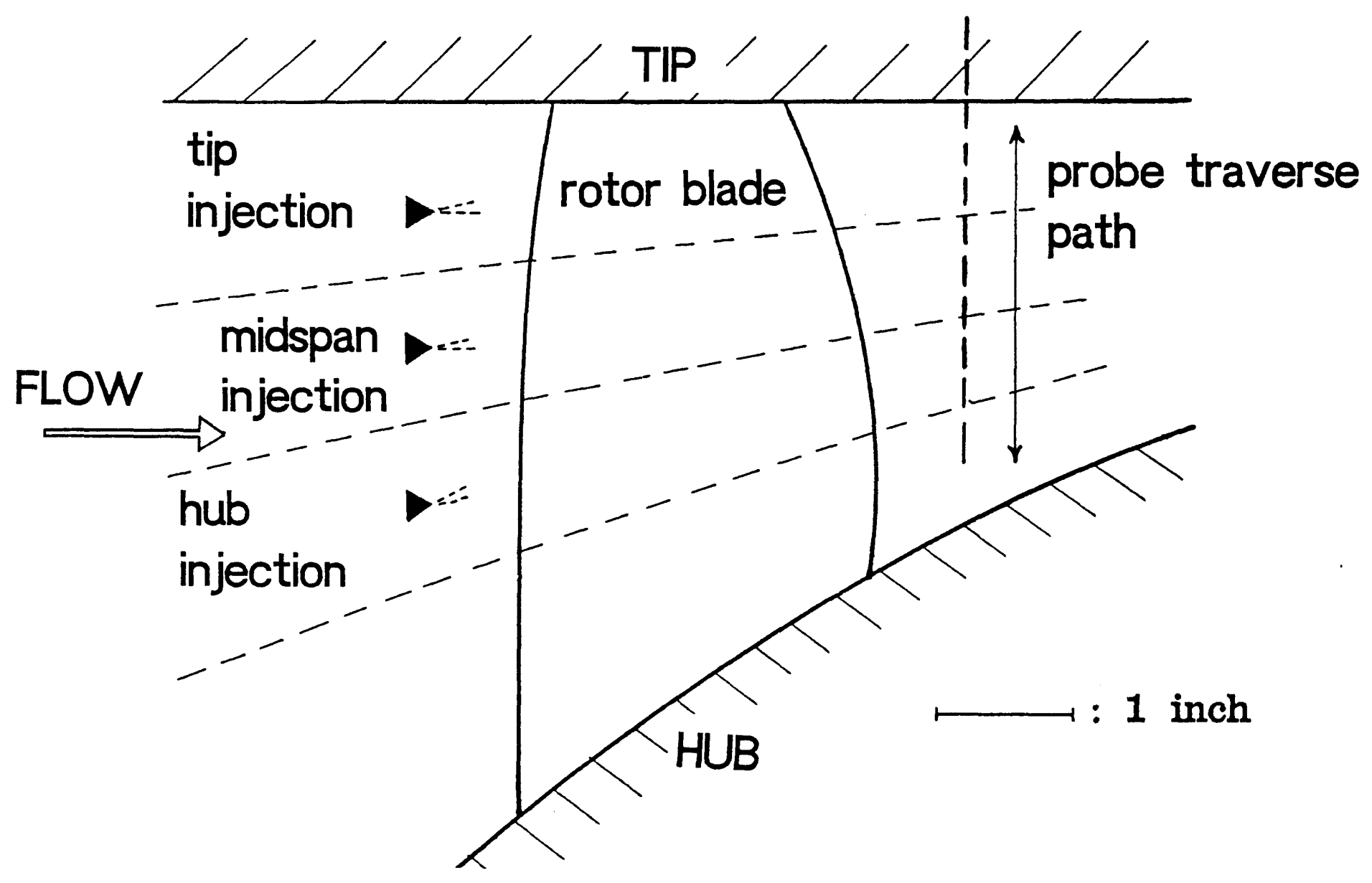

Figure 5.6: Scale drawing of the rotor geometry showing the three injection locations 


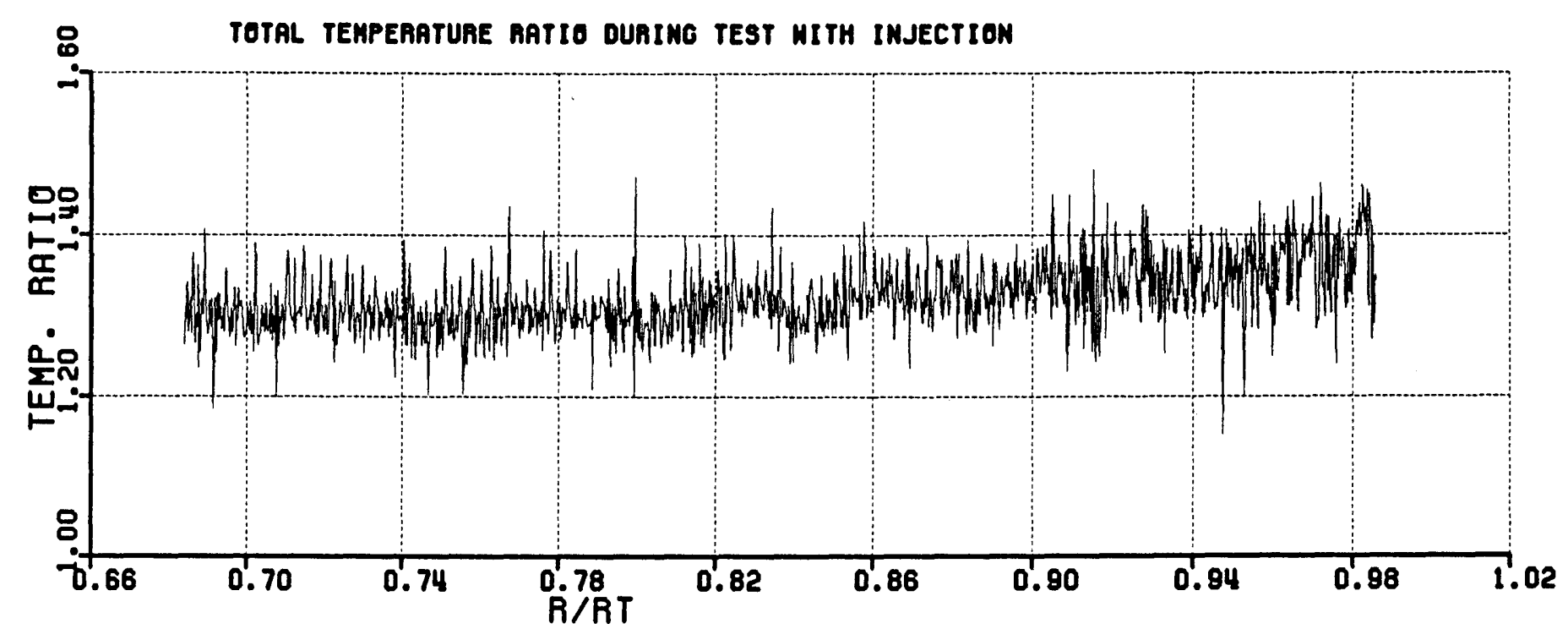

(a)

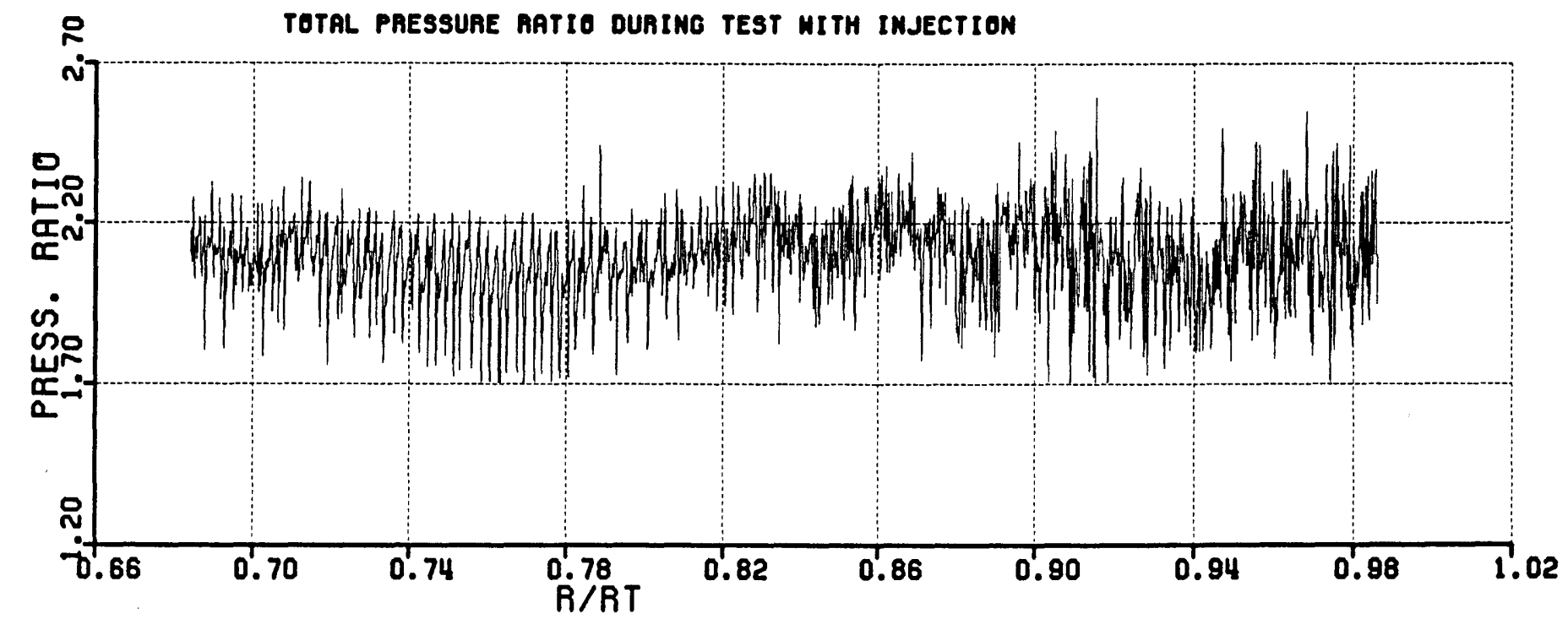

(b)

Figure 5.7: Time resolved total temperature (a) and total pressure (b) ratio behind the rotor 

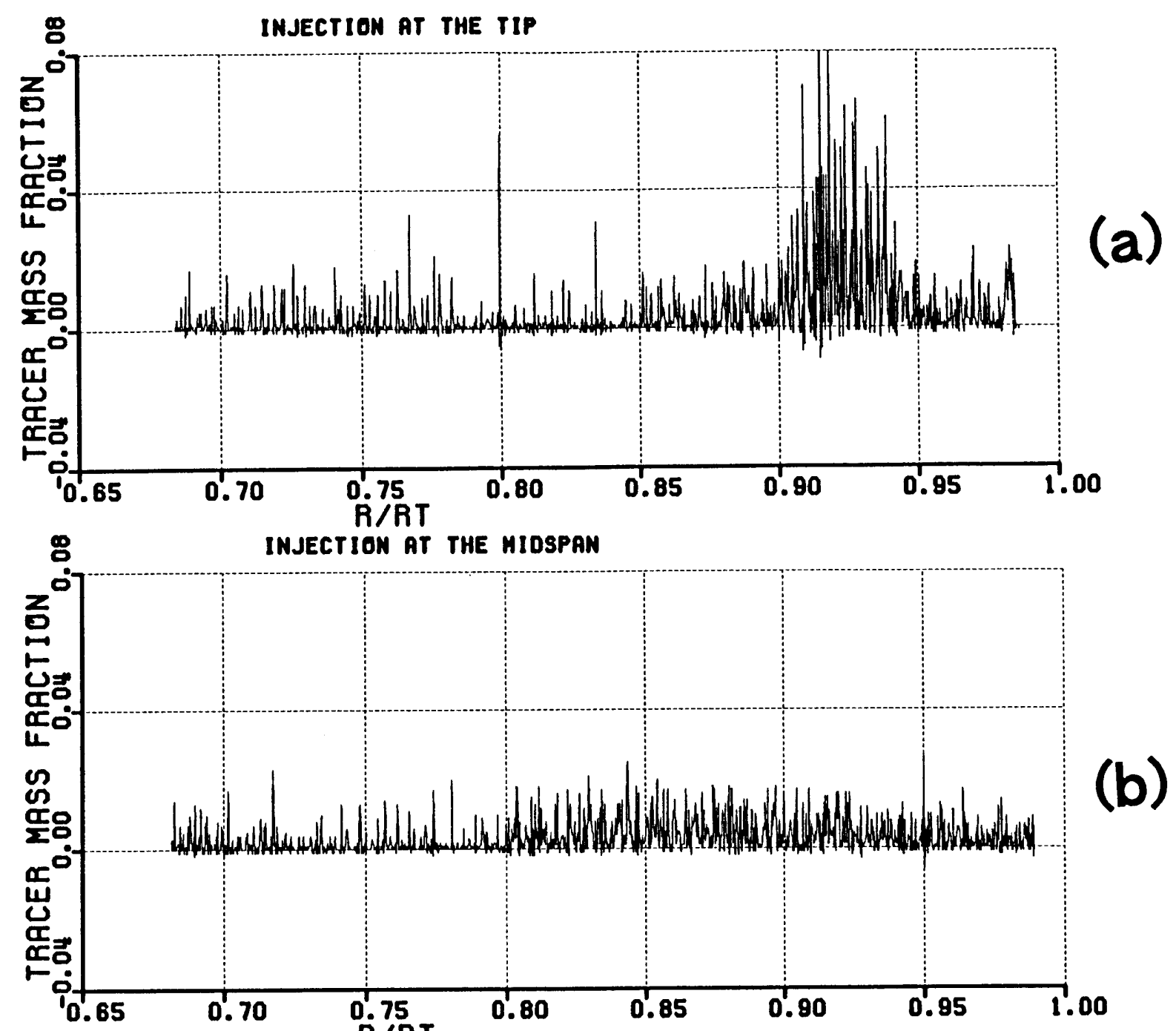

(b)

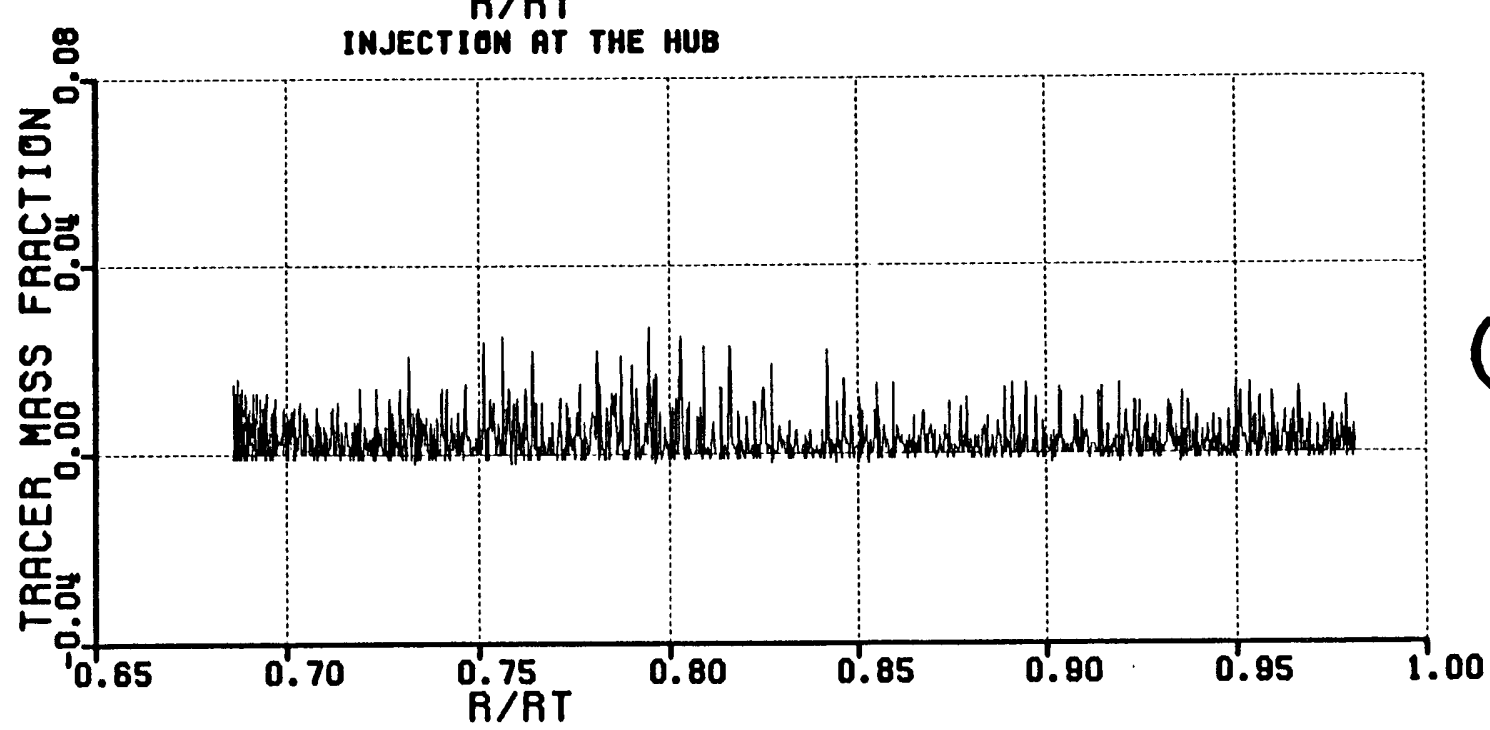

Figure 5.8: Time resolved tracer gas concentration (mass fraction) during injections at the tip (a), midspan (b) and hub (c) 
TIP INJECTION

TIP AEGION
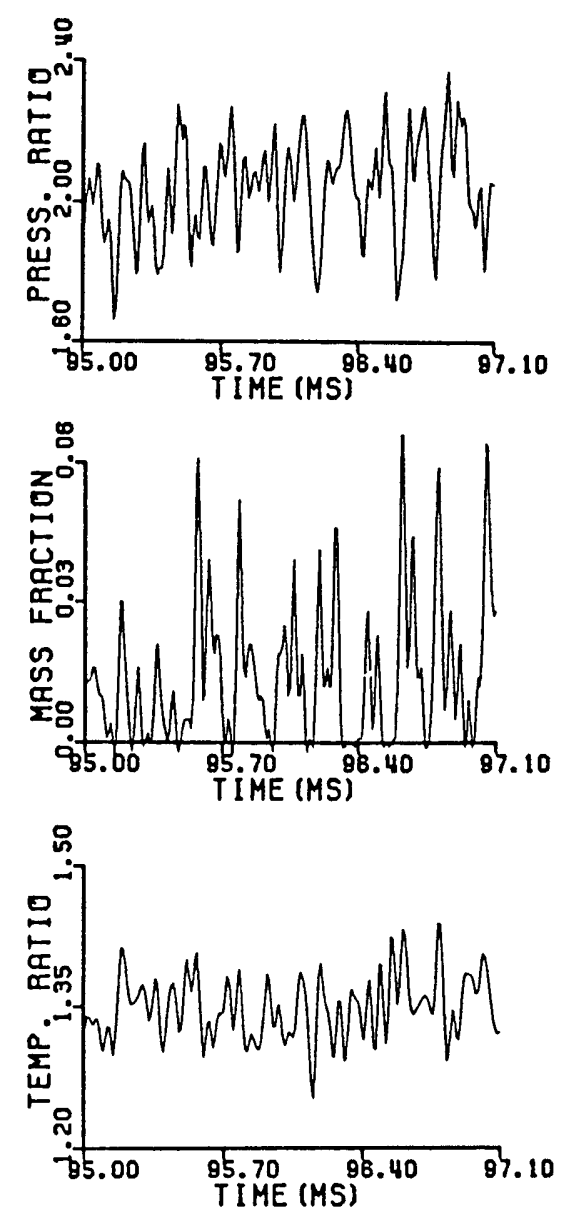

MIDSPAN REGIOK
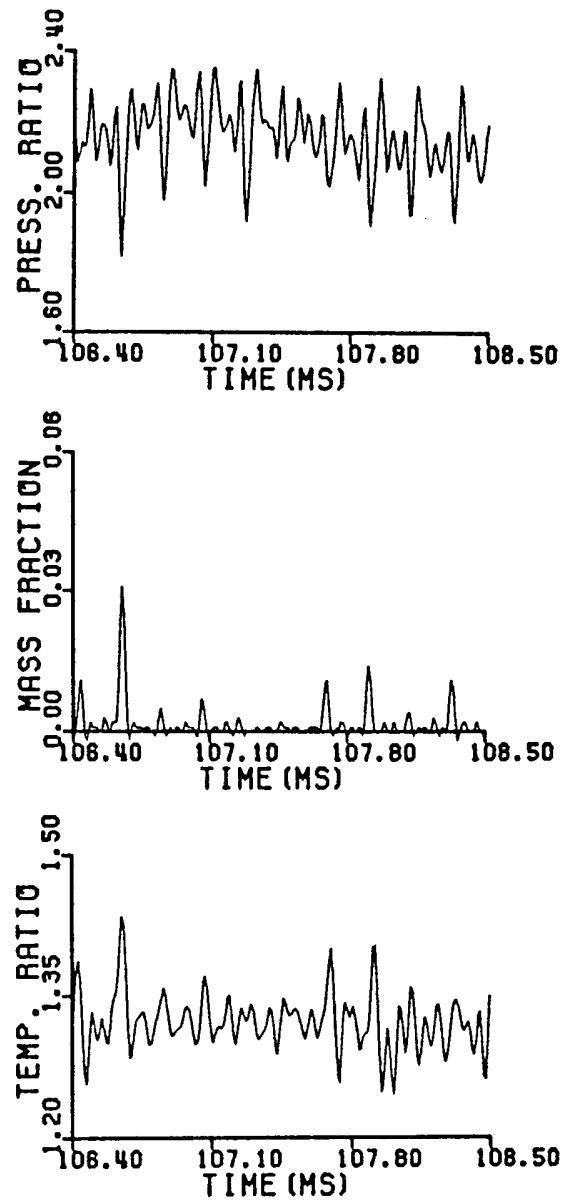

blade passing period

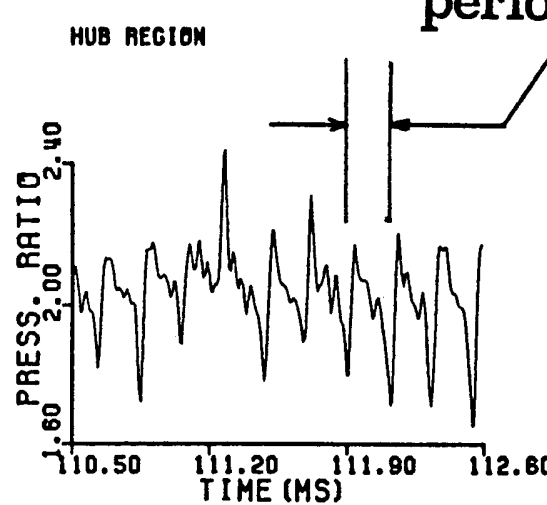

(a)

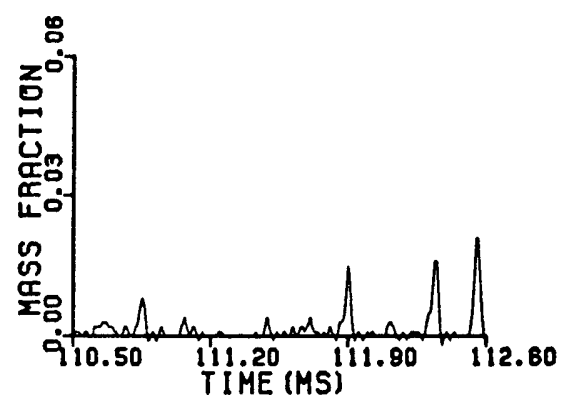

(b)

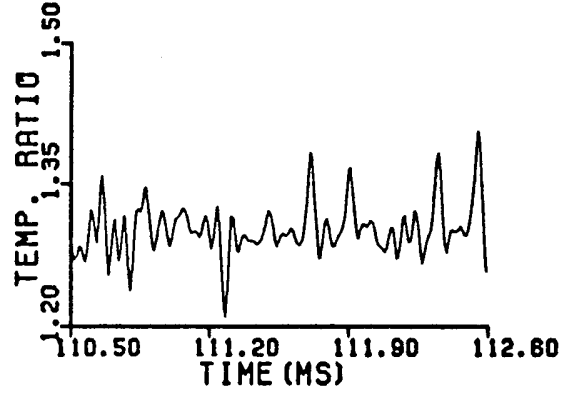

Figure 5.9: Time resolved total pressure ratio (a), tracer concentration (mass fraction) (b) and total temperature ratio (c), at tip, midspan and hub, during injection near the tip 


\section{TIP INJECTION}

IIP REGION
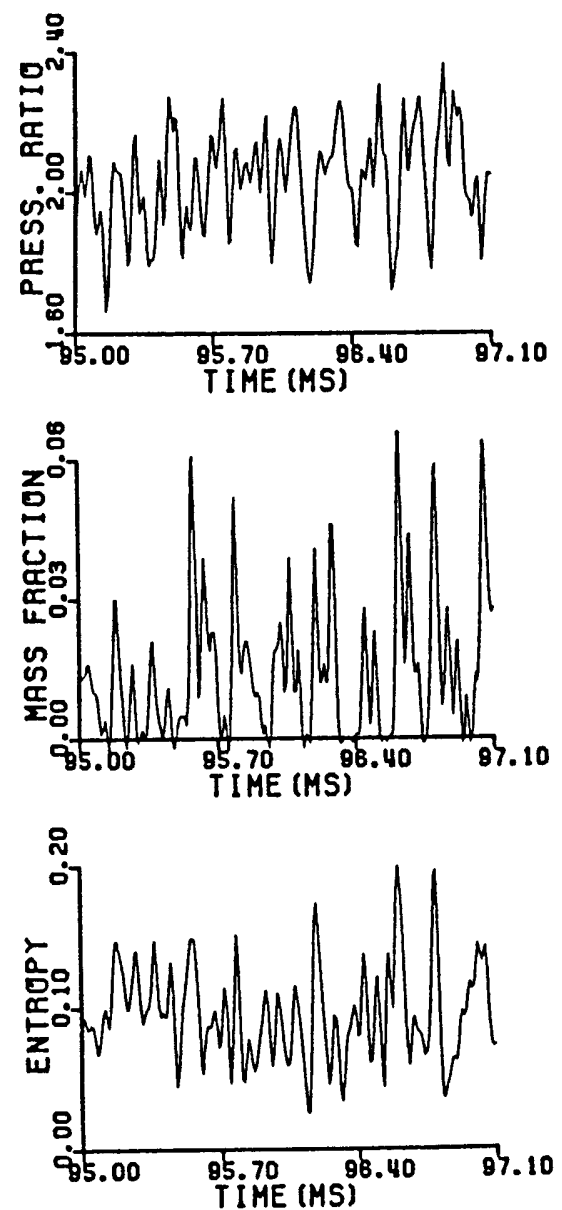

NIDSPAN REGIOK
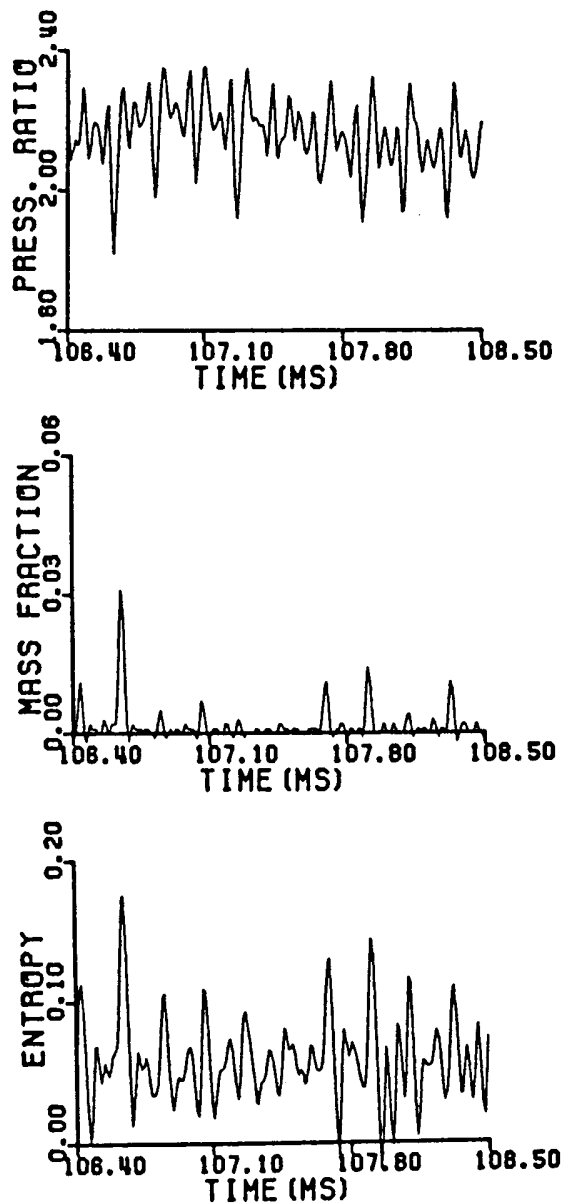

blade passing period
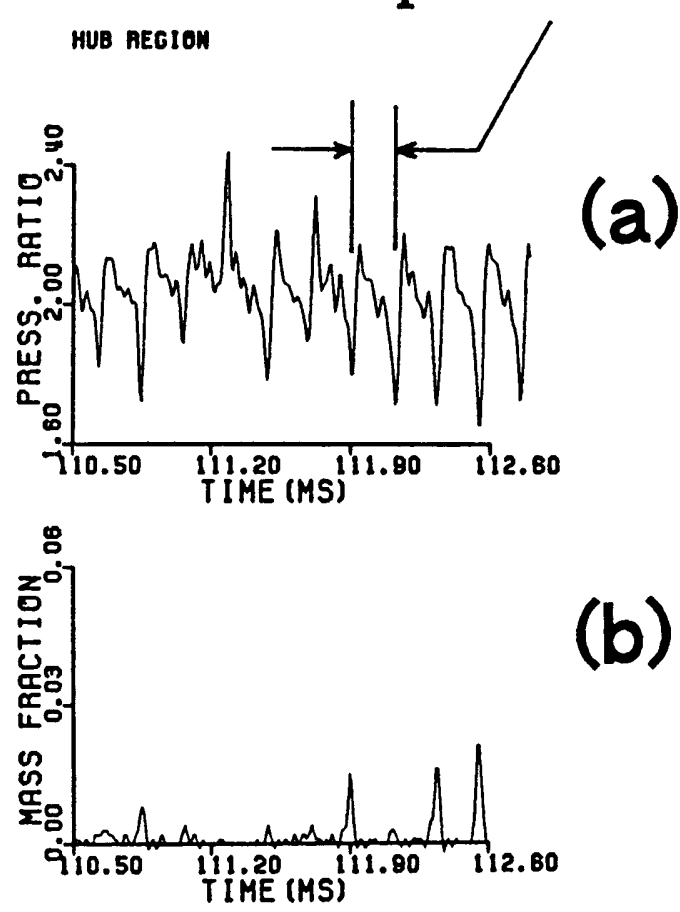

(b)

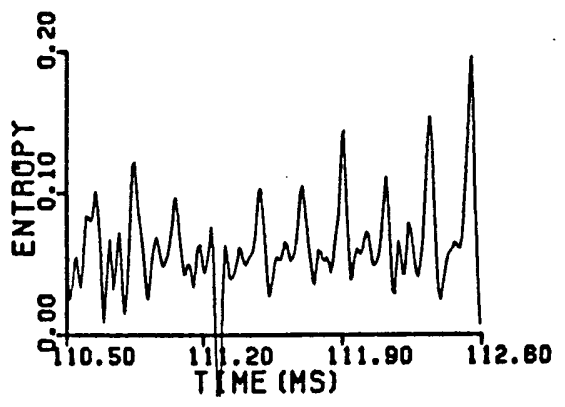

(c)

Figure 5.10: Time resolved total pressure ratio (a), tracer concentration (mass fraction) (b) and entropy change (c), at tip, midspan and hub, during injection near the tip 


\section{MIDSPAN INJECTION}
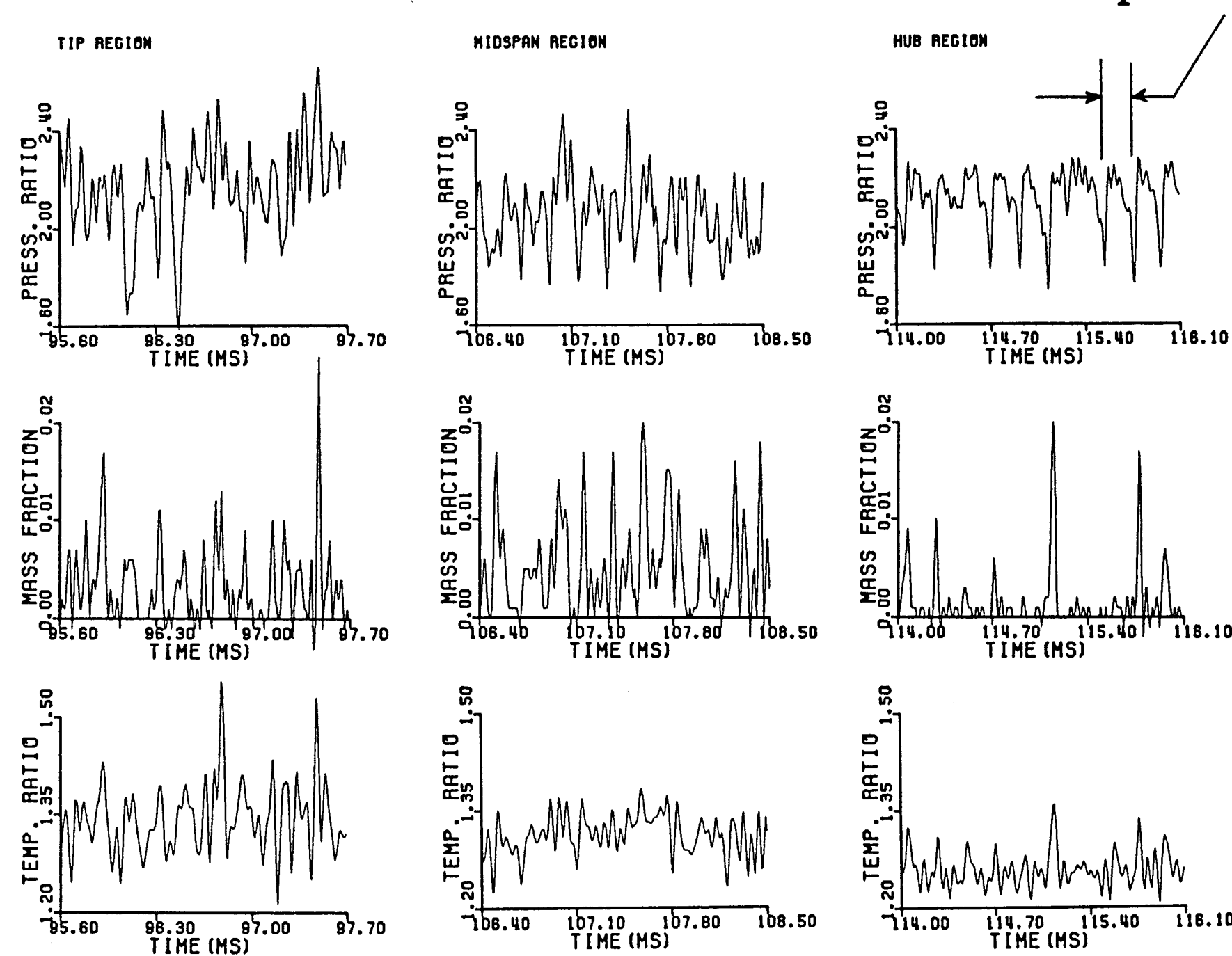

(a)
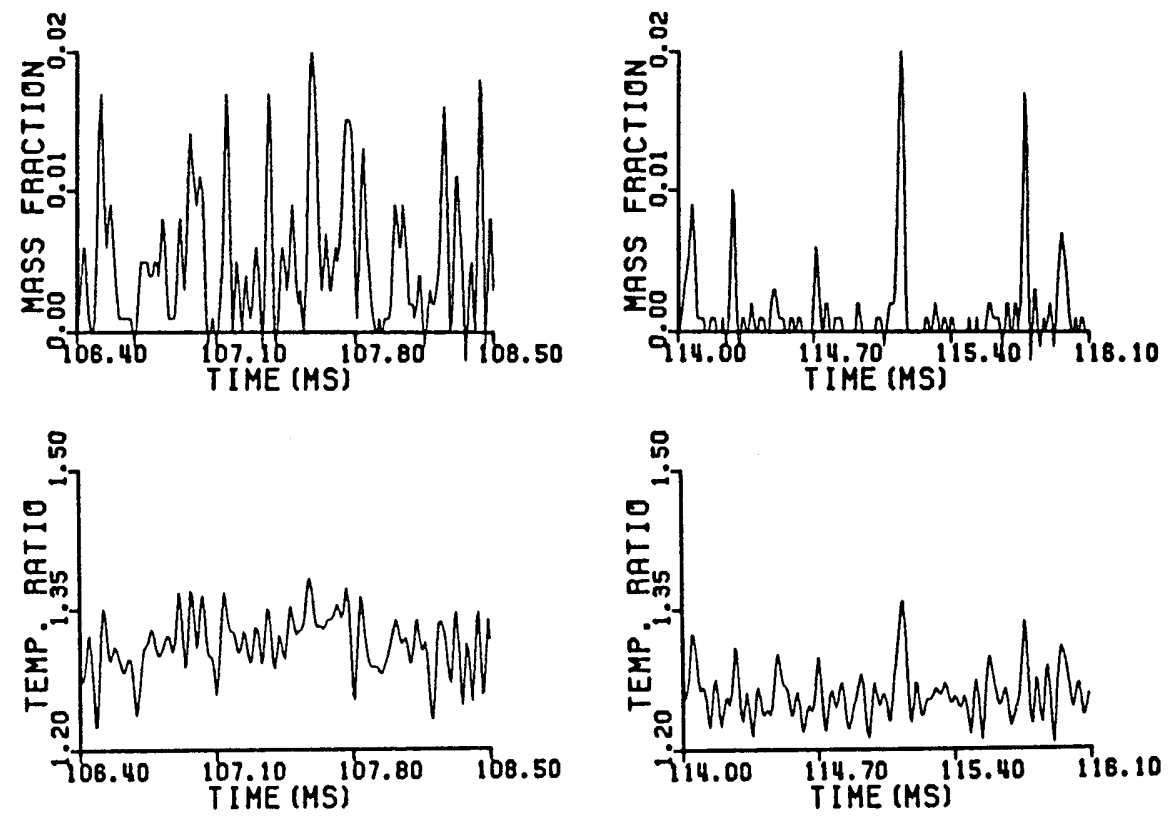

(c)

Figure 5.11: Time resolved total pressure ratio (a), tracer concentration (mass fraction) (b) and total temperature ratio (c), at tip, midspan and hub, during injection near the midspan 


\section{MIDSPAN INJECTION}
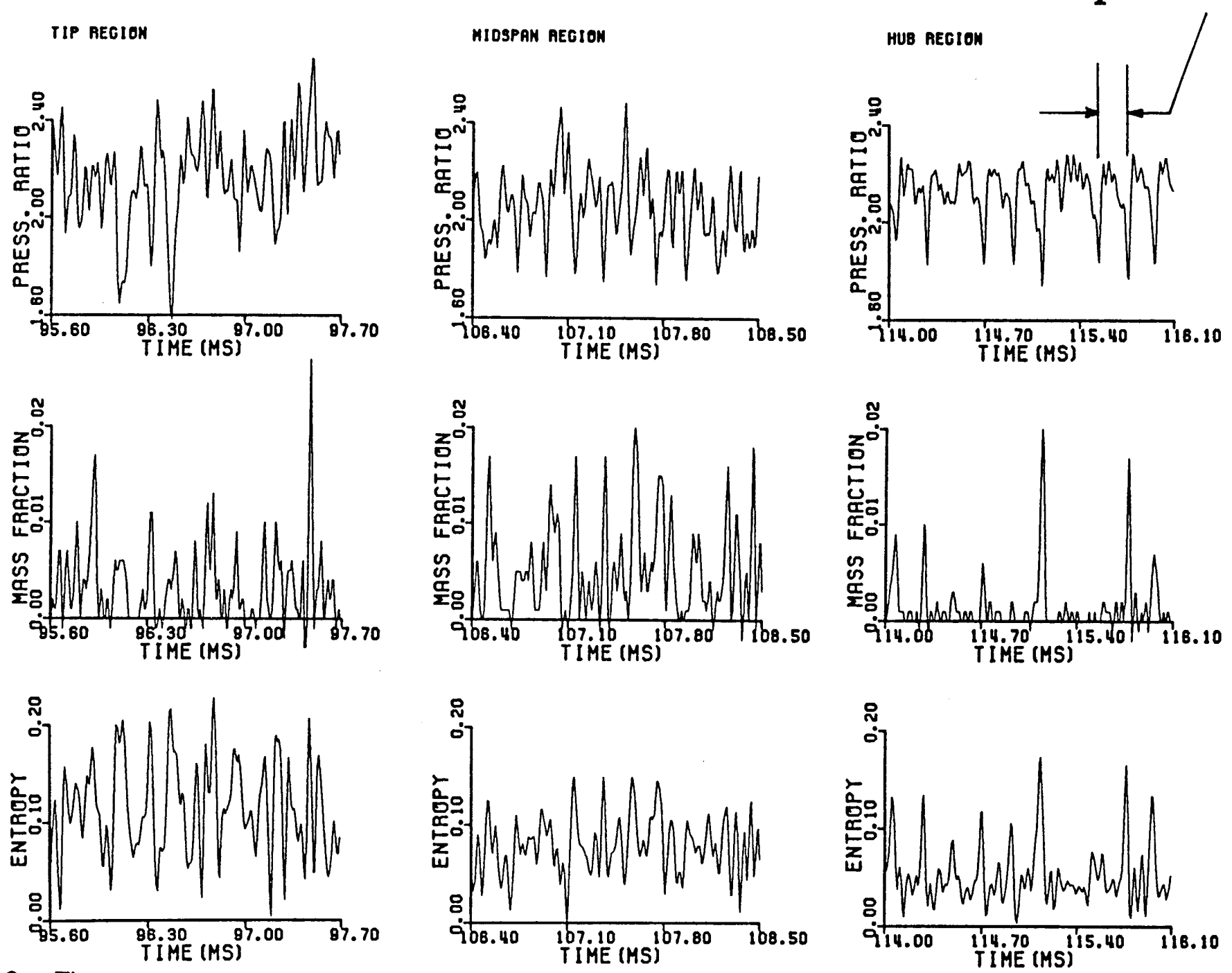

Figure 5.12: Time resolved total pressure ratio (a), tracer concentration
at tip, midspan and hub, during injection near the midspan

(a)

(b)

(c) 
HUB INJECTION

TIP REgION
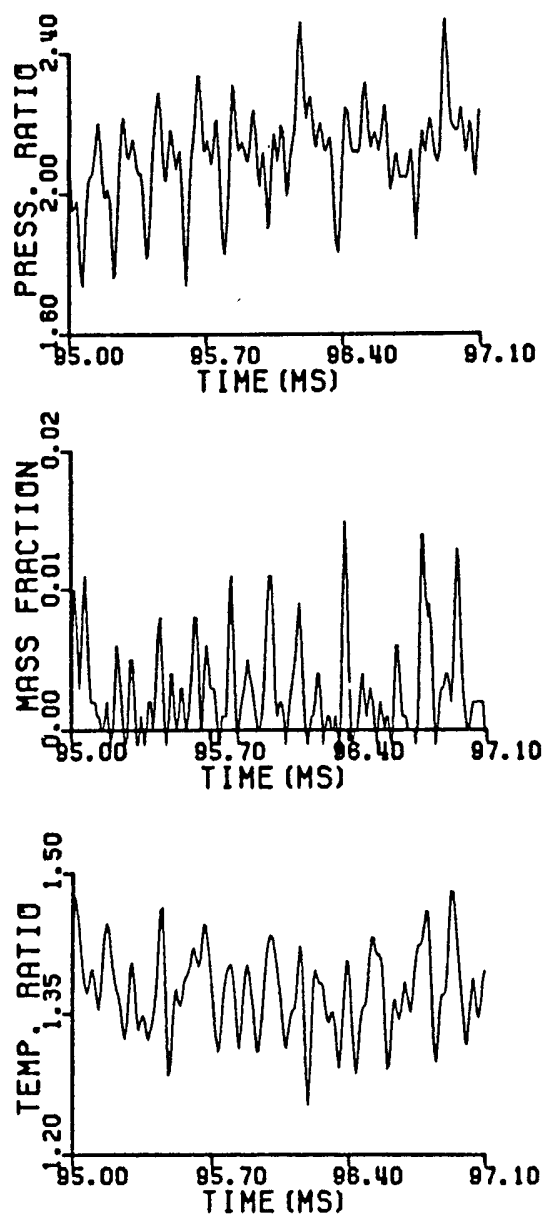

MIOSPAK REGION
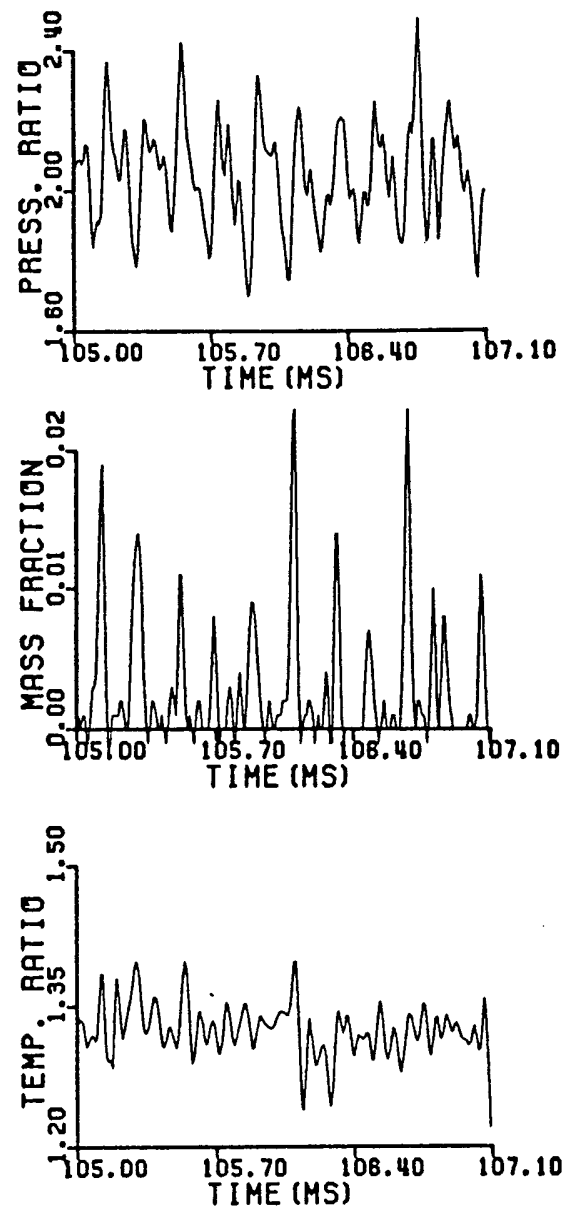

blade passing period

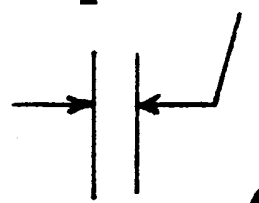

HUB REGION
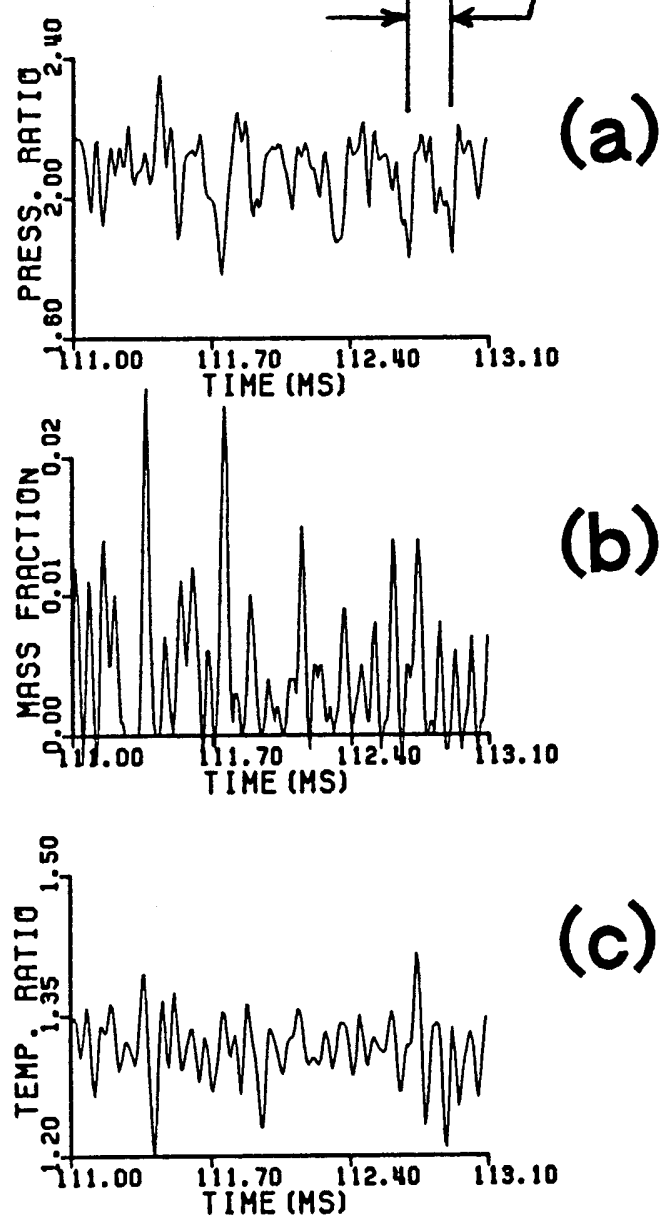

Figure 5.13: Time resolved total pressure ratio (a), tracer concentration (mass fraction) (b) and total temperature ratio (c), at tip, midspan and hub, during injection near the hub

$\stackrel{N}{\infty}$ 


\section{HUB INJECTION}

TIP REgION
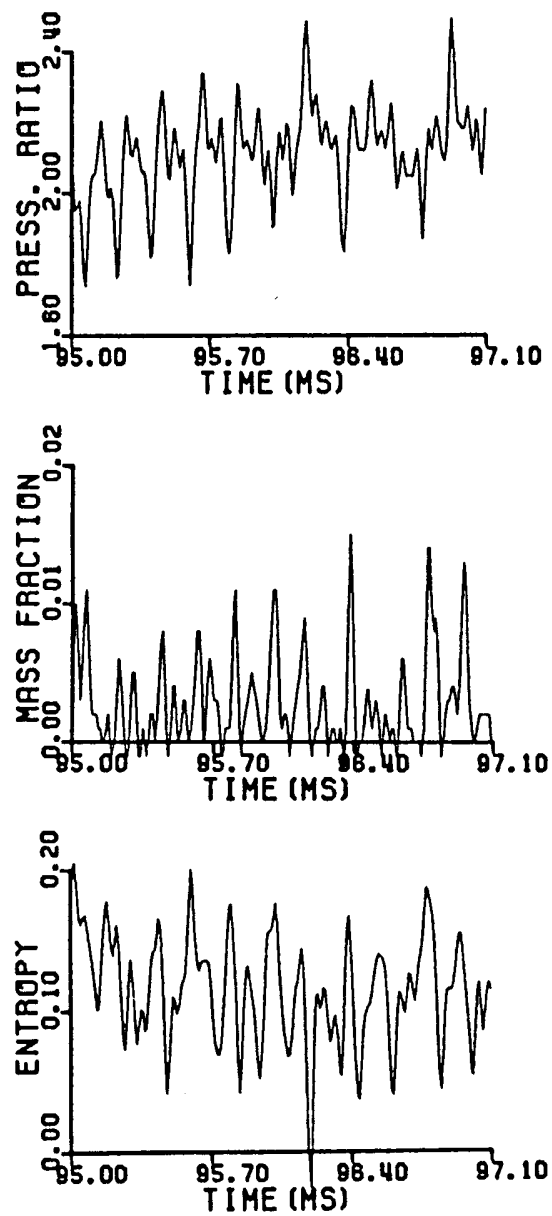

MIDSPAN AEGIOM
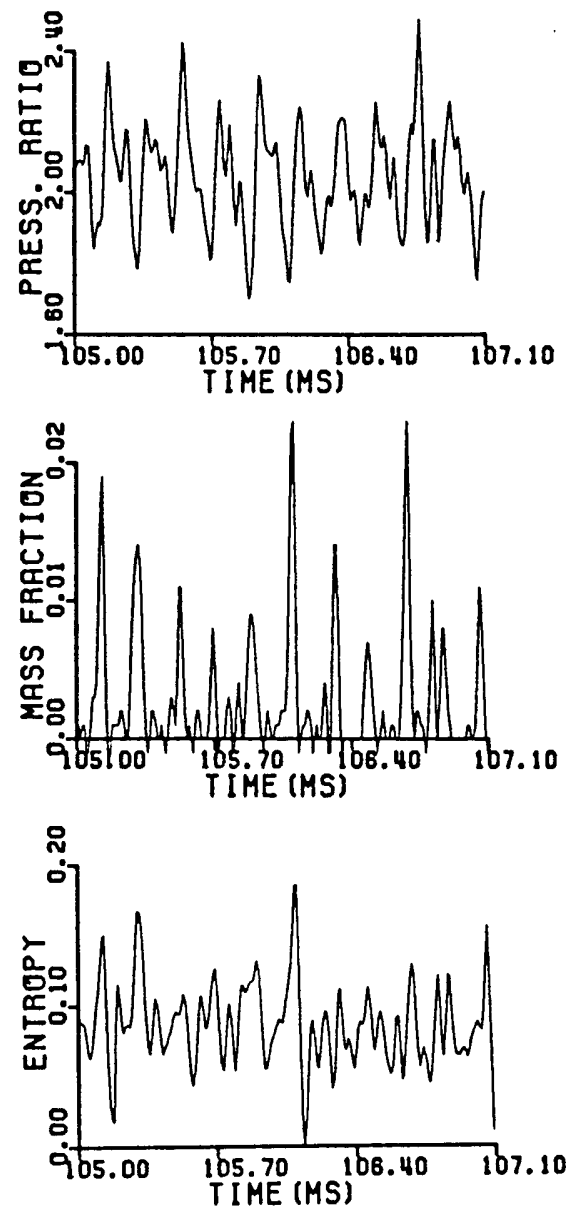

blade passing period

HUB REGION
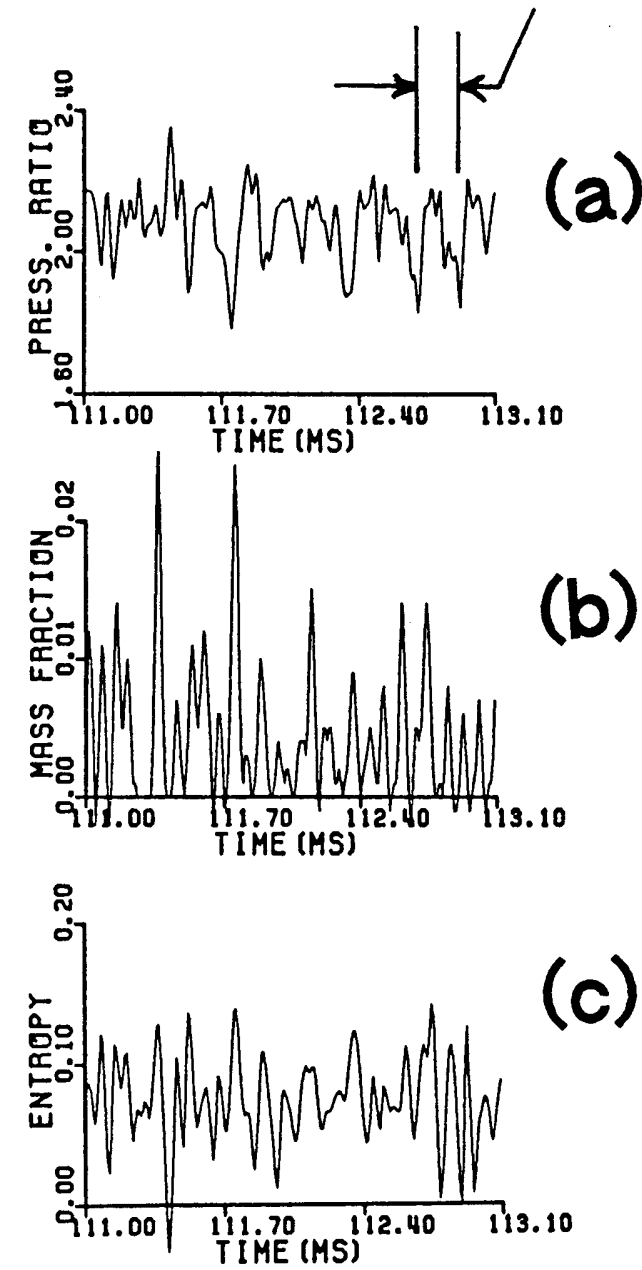

Figure 5.14: Time resolved total pressure ratio (a), tracer concentration (mass fraction) (b) and entropy change (c), at tip, midspan and hub, during injection near the hub 


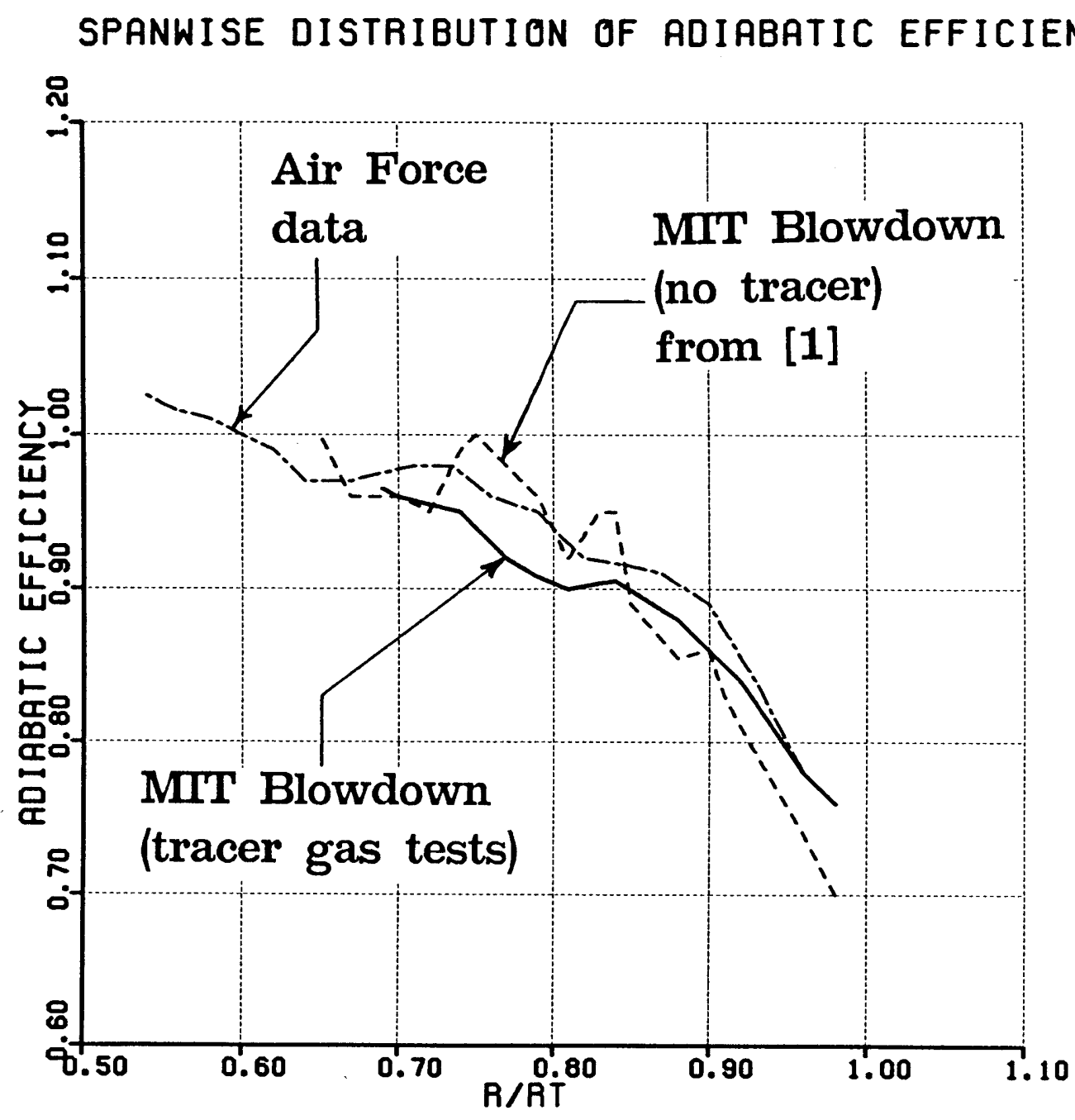

Figure 5.15: Spanwise distribution of time averaged adiabatic efficiency 


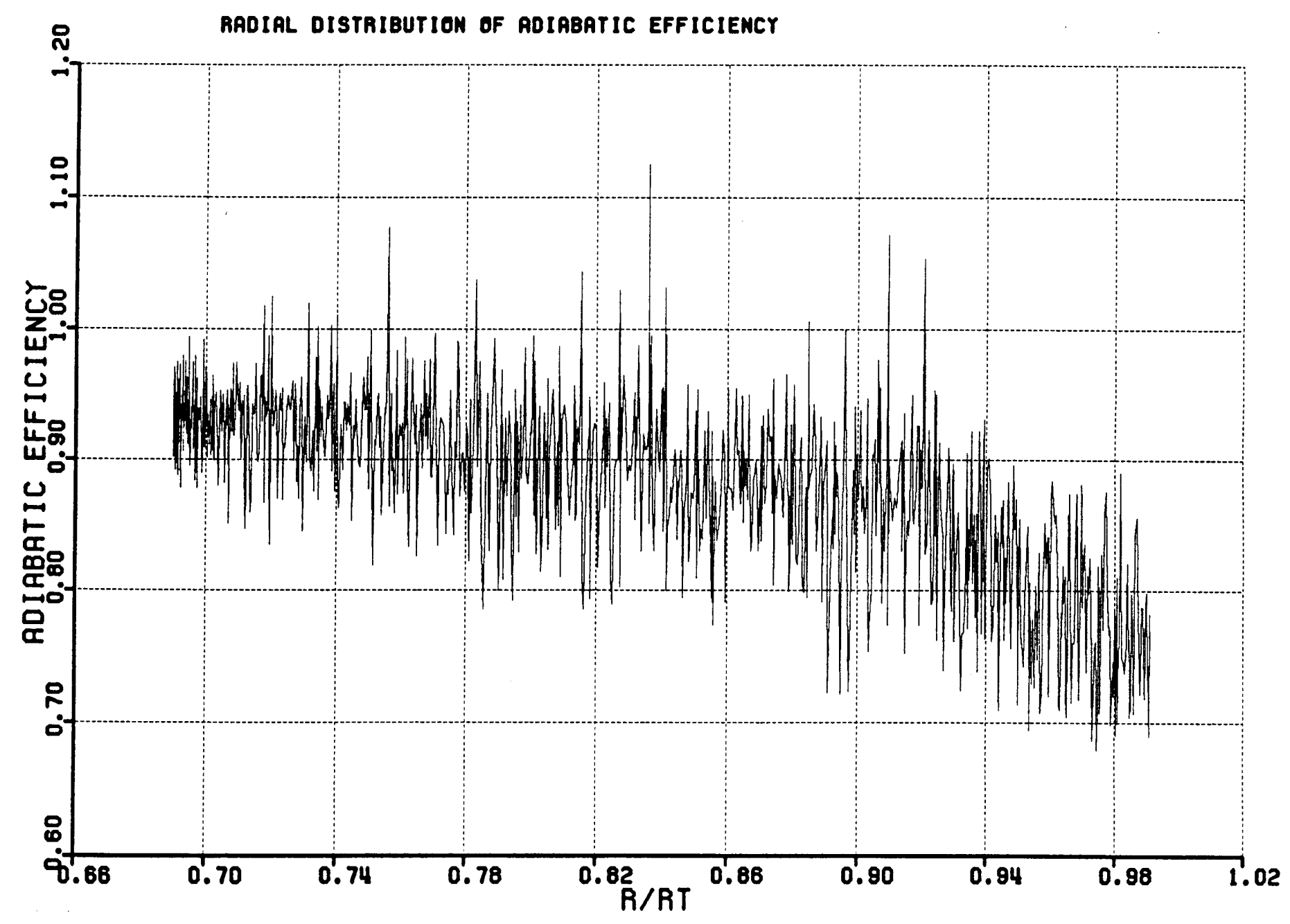

Figure 5.16: Spanwise distribution of time resolved adiabatic efficiency 


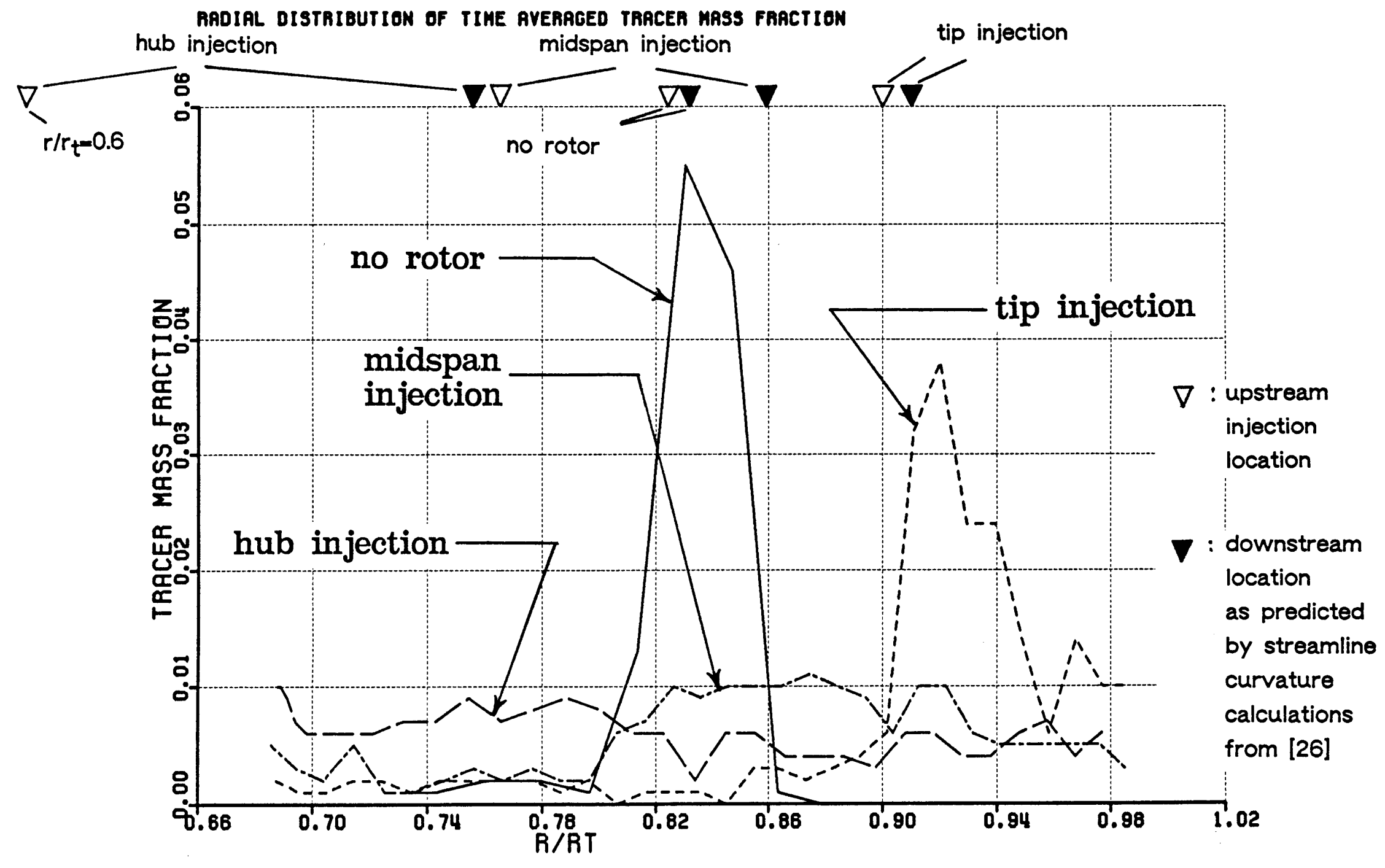

Figure 5.17: Time averaged tracer gas concentration (mass fraction) during tip, midspan, hub and "no rotor" injections 
TIME AVERAGED TOTAL PRESSURE RATIO

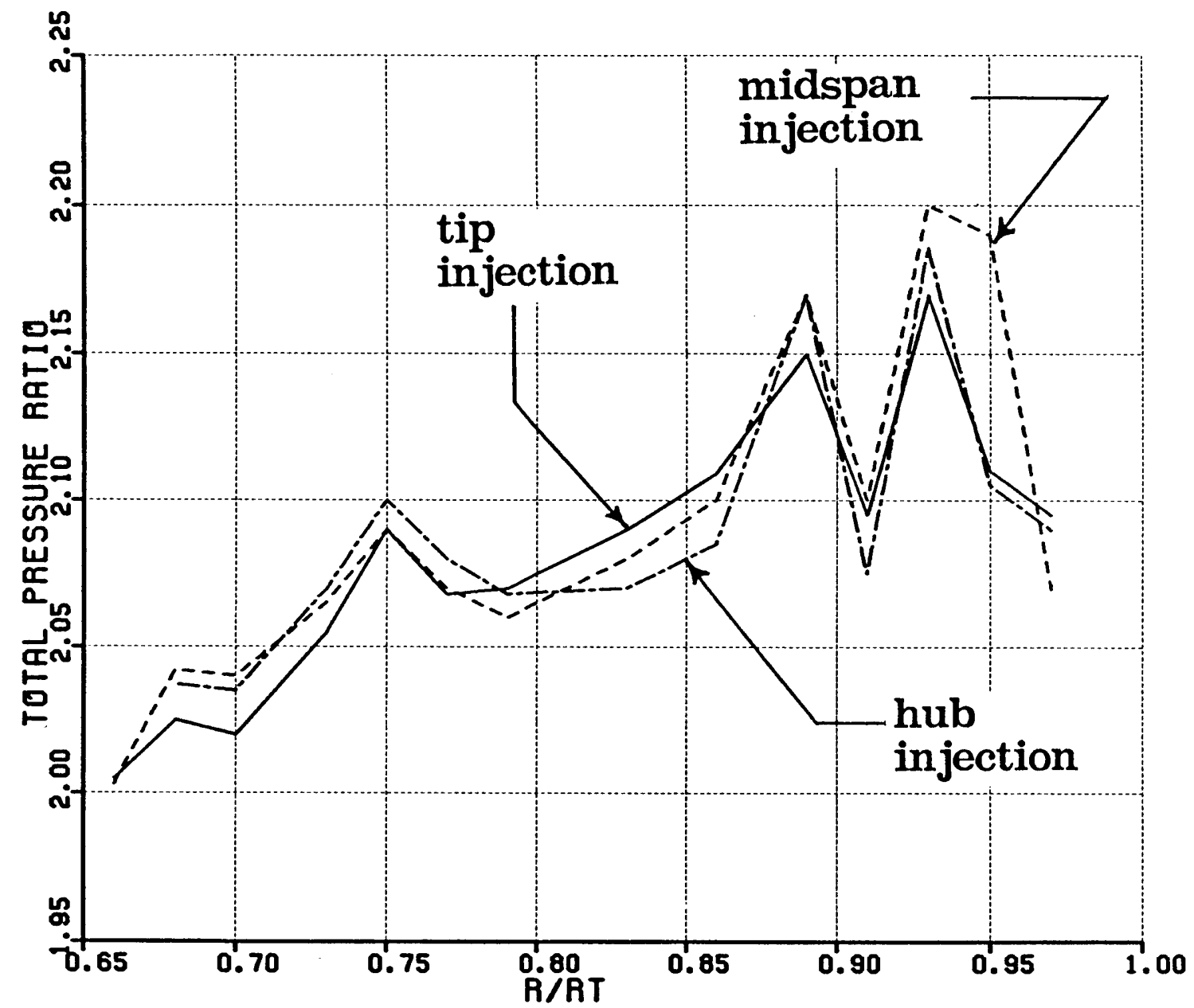

Figure 5.18: Comparison of time averaged total pressure ratio for tests with injection at tip, midspan and hub 


\section{TIME AVERAGED TOTAL TEMPERATURE RATIO}

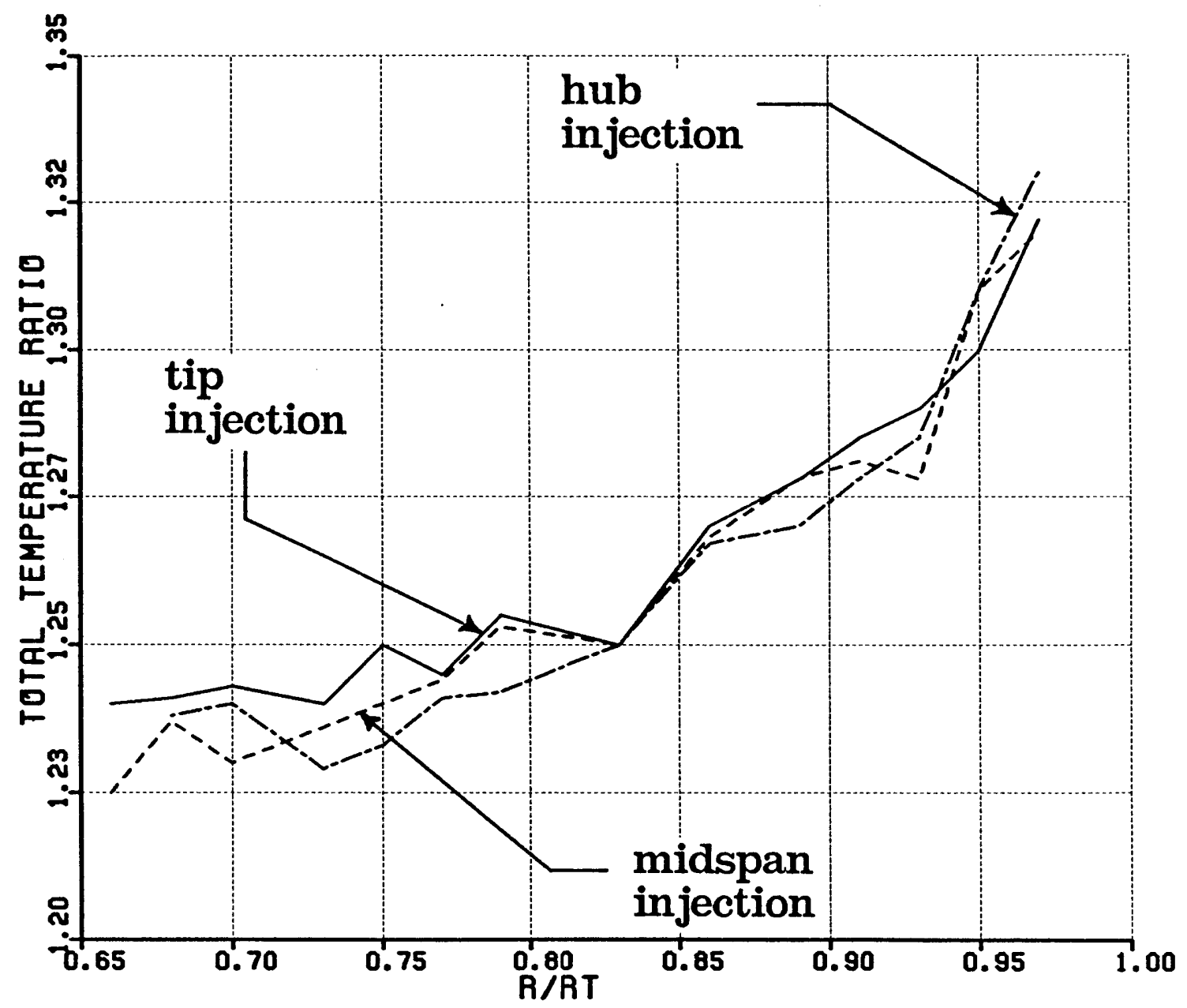

Figure 5.19: Comparison of time averaged total temperature ratio for tests with injection at tip, midspan and hub 


\section{TIP INJECTION}

tip region
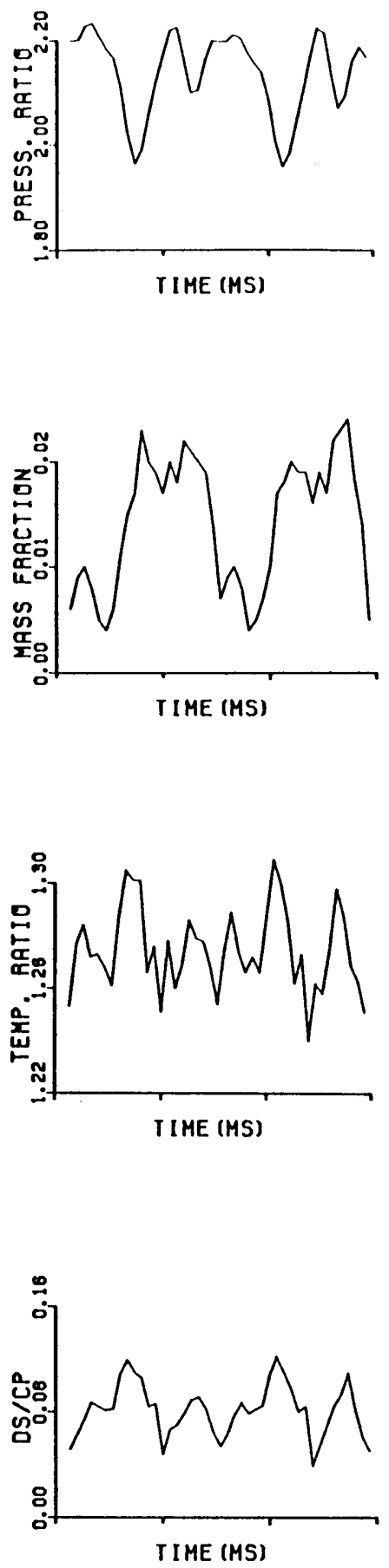
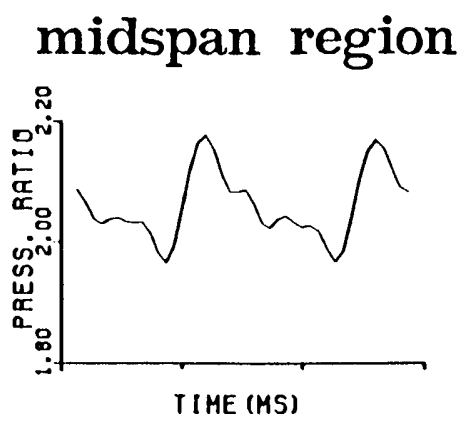

blade passing period
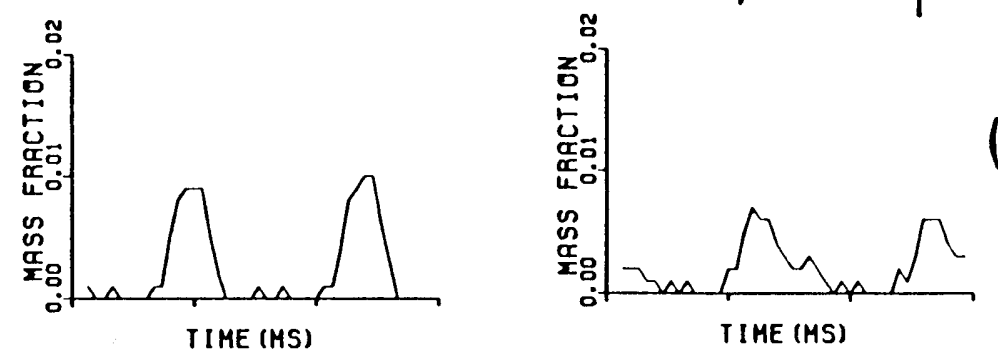

(b)
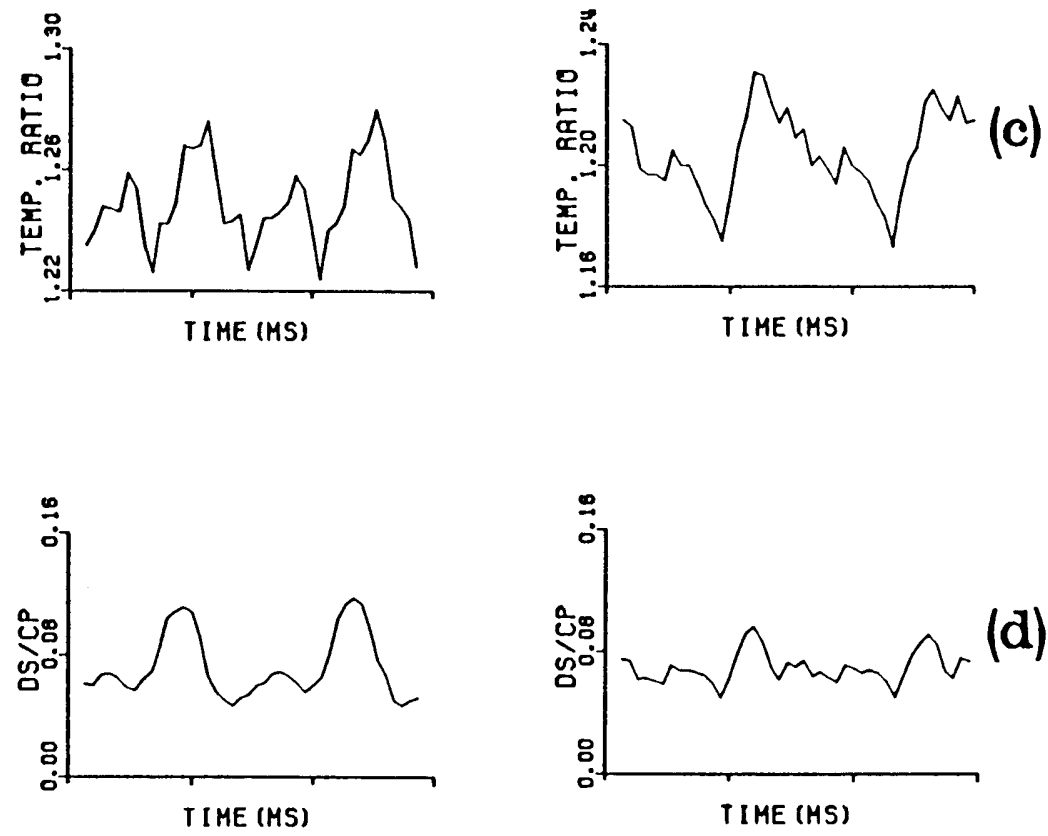

Figure 5.20: 10 cycle ensemble averaged total pressure ratio (a), tracer gas concentration (mass fraction) (b), total temperature ratio (c) and entropy change (d) at tip, midspan and hub for tests with injection at the tip 


\section{MIDSPAN INJECTION}

tip region
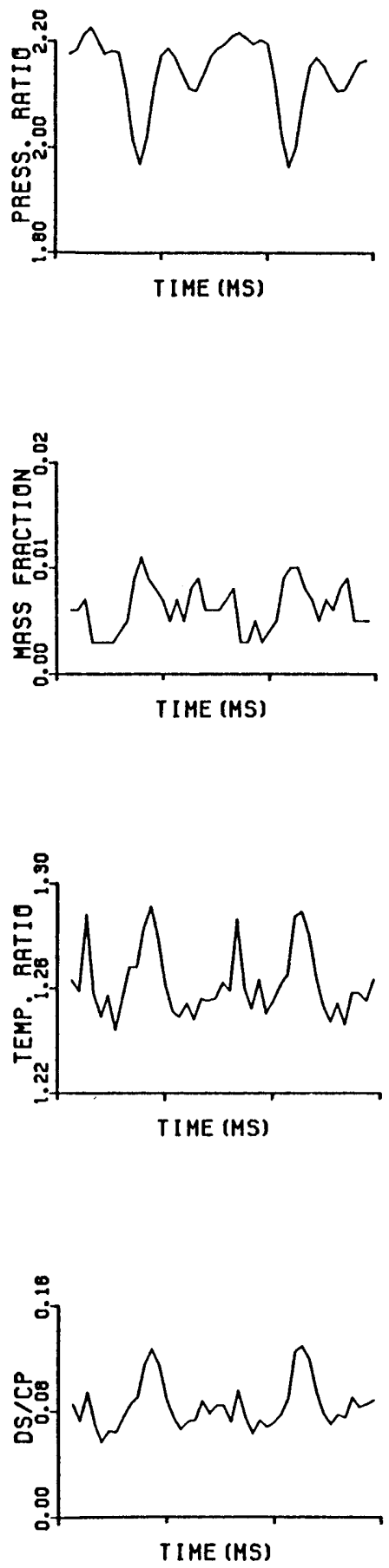
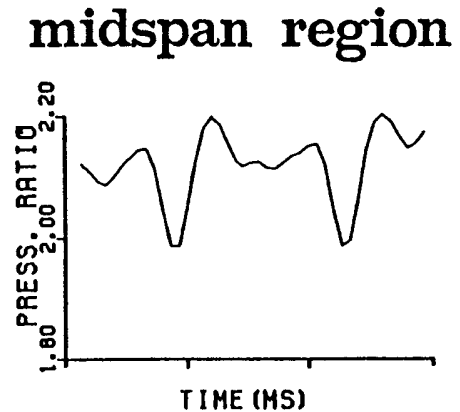

blade passing period
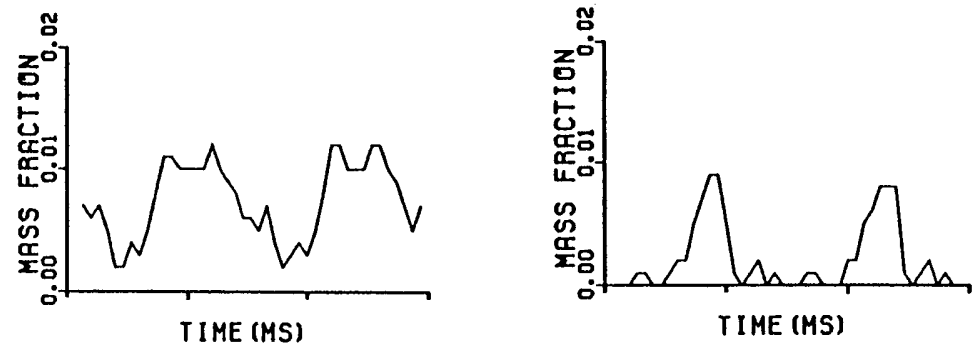

(b)

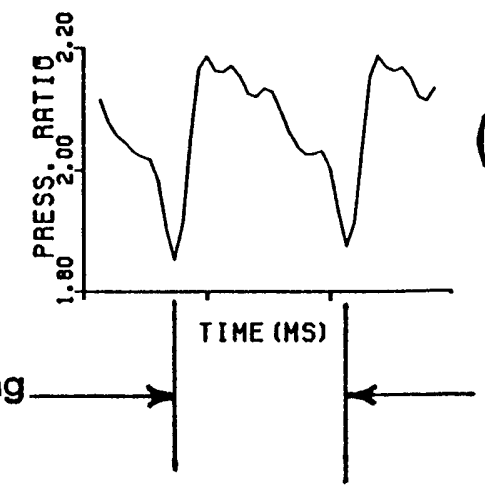

(a)

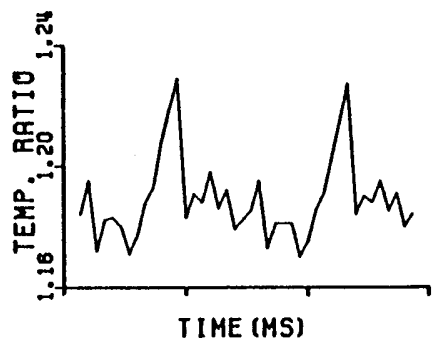

(c)
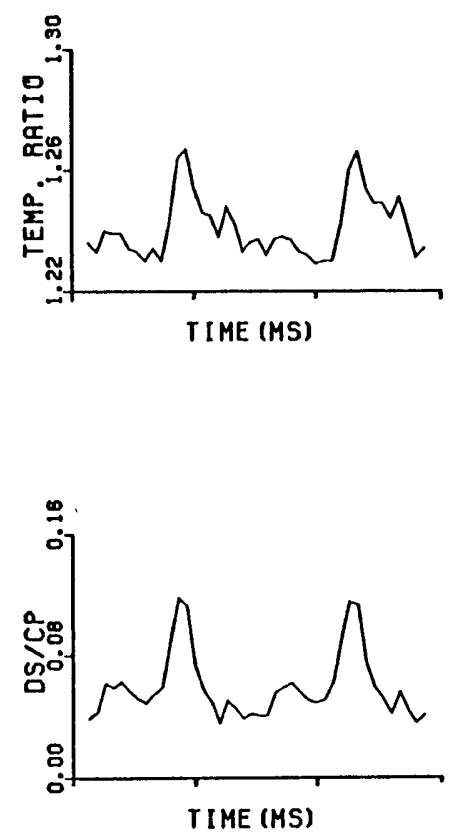

Figure 5.21: 10 cycle ensemble averaged total pressure ratio (a), tracer gas concentration (mass fraction) (b), total temperature ratio (c) and entropy change (d) at tip, midspan and hub for tests with injection at the midspan 


\section{HUB INJECTION}
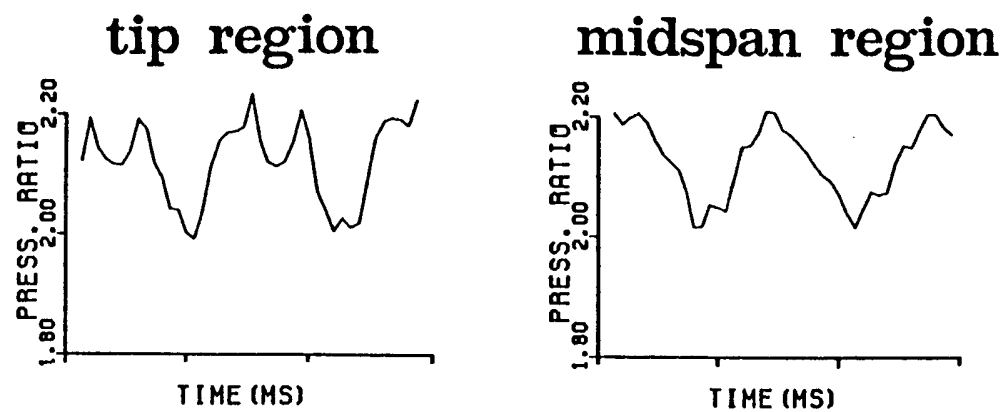

hub region
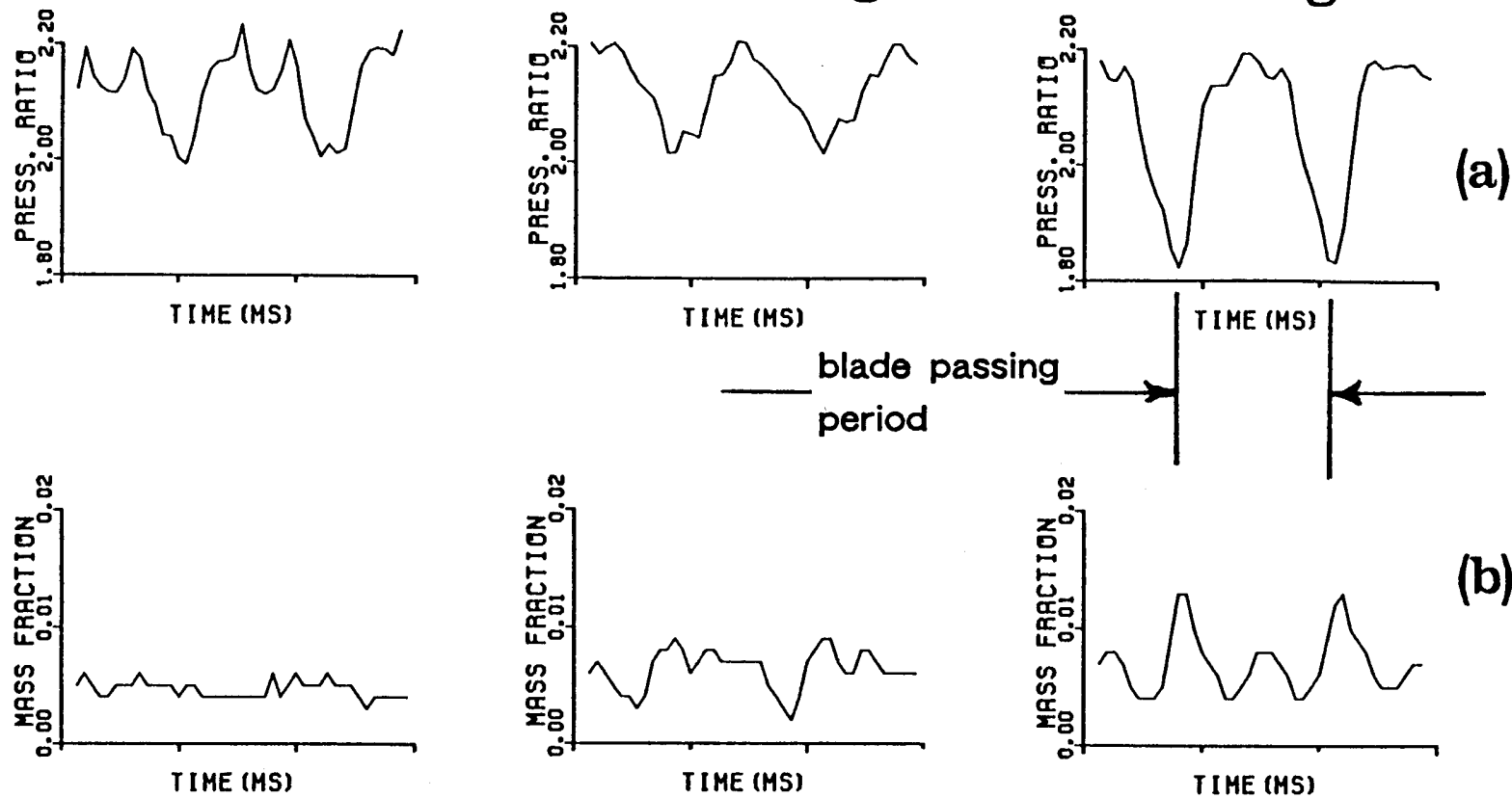

(b)
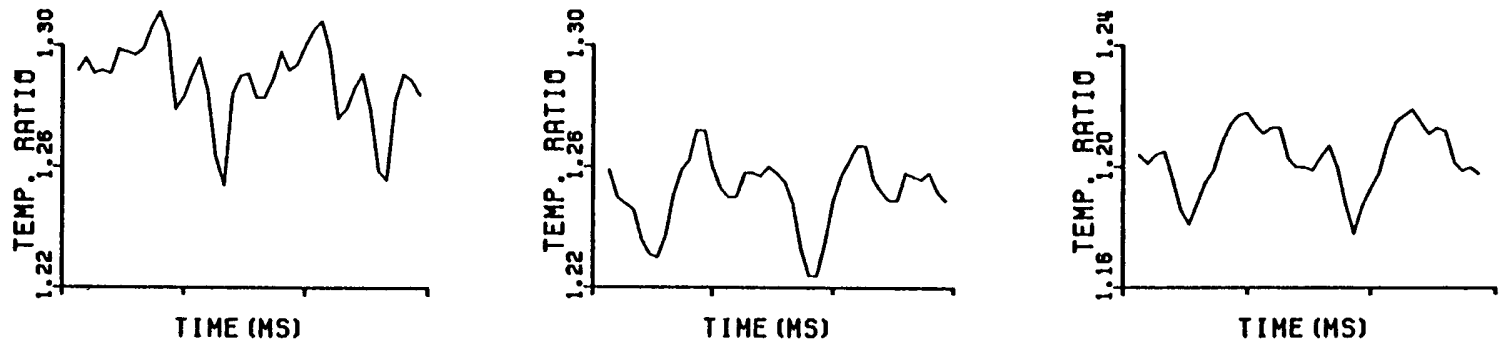

(c)
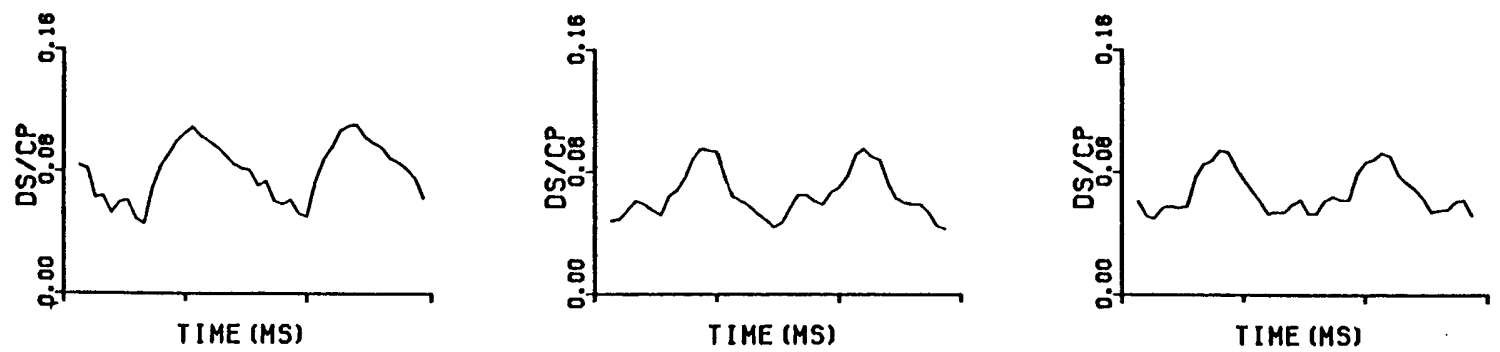

(d)

Figure 5.22: 10 cycle ensemble averaged total pressure ratio (a), tracer gas concentration (mass fraction) (b), total temperature ratio (c) and entropy change (d) at tip, midspan and hub for tests with injection at the hub 


\section{TRACER GAS MASS FRACTION}

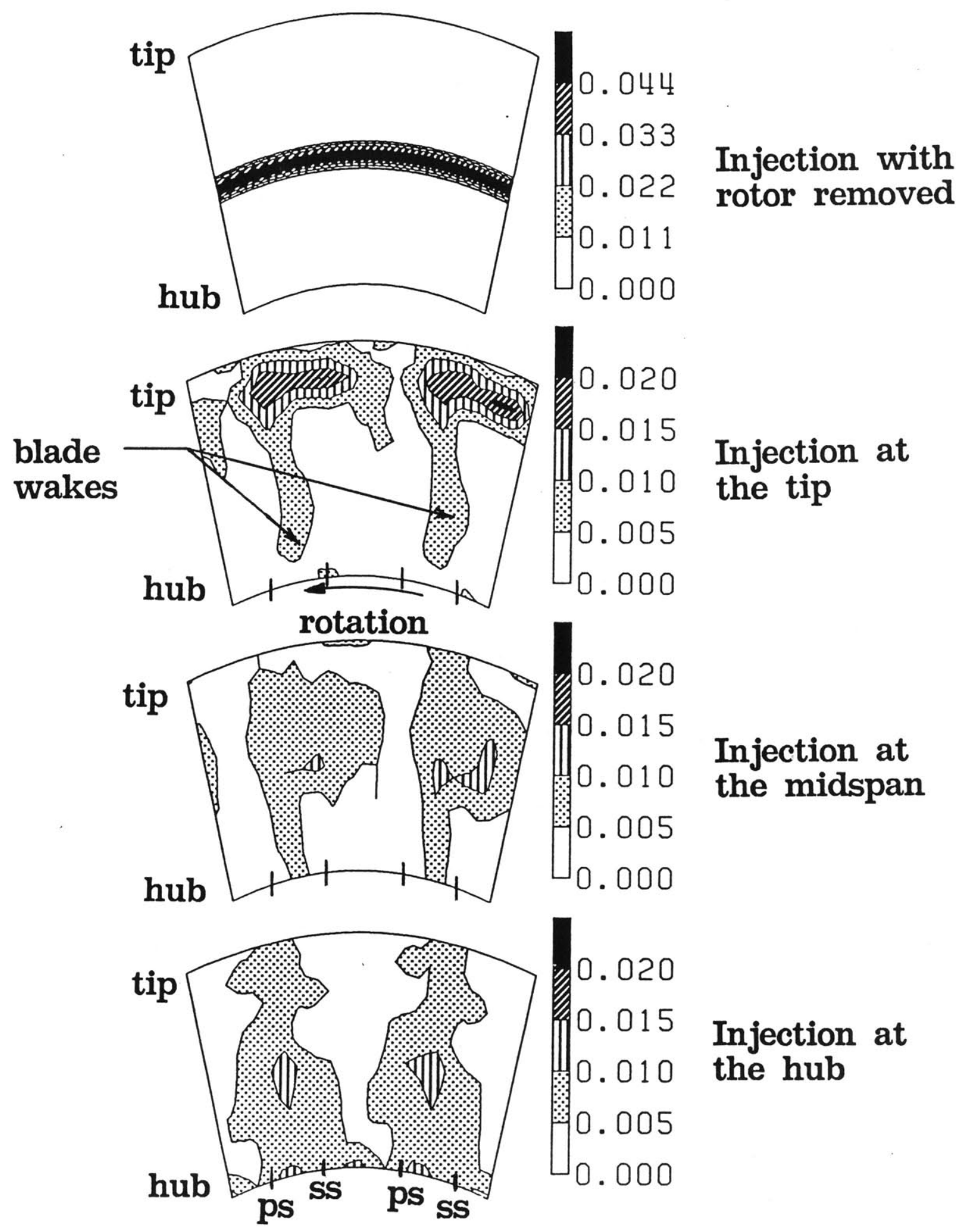

Figure 5.23: Comparison of 10 cycle ensemble averaged tracer gas concentration (mass fraction) contours for "no rotor", tip, midspan and hub injections 


\section{INJECTION AT}
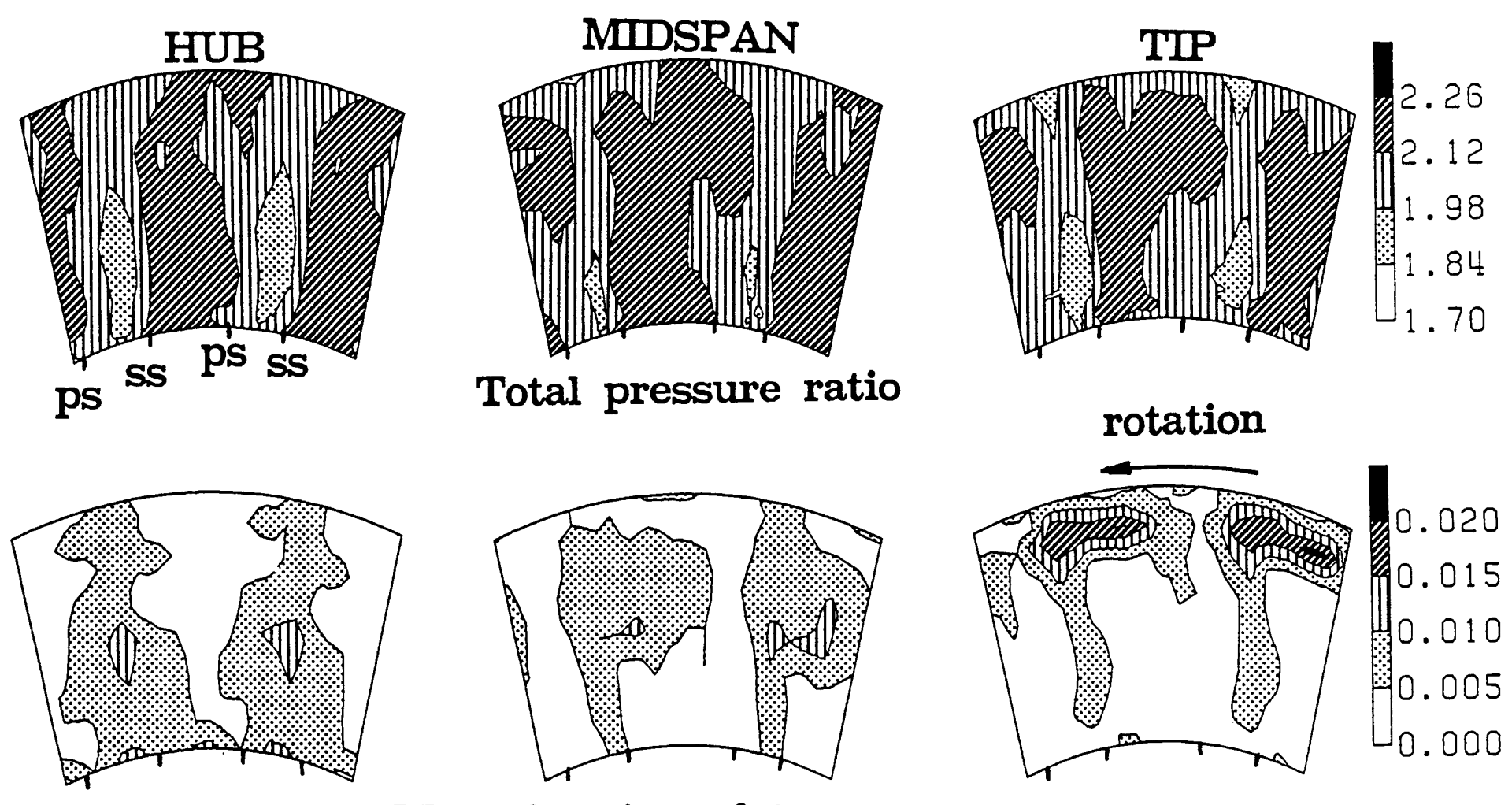

Mass fraction of tracer gas

Figure 5.24: 10 cycle ensemble averaged contour plots of total pressure ratio and tracer gas concentration (mass fraction) during tests with injection at tip, midspan and hub 


\section{TIP INJECTION}
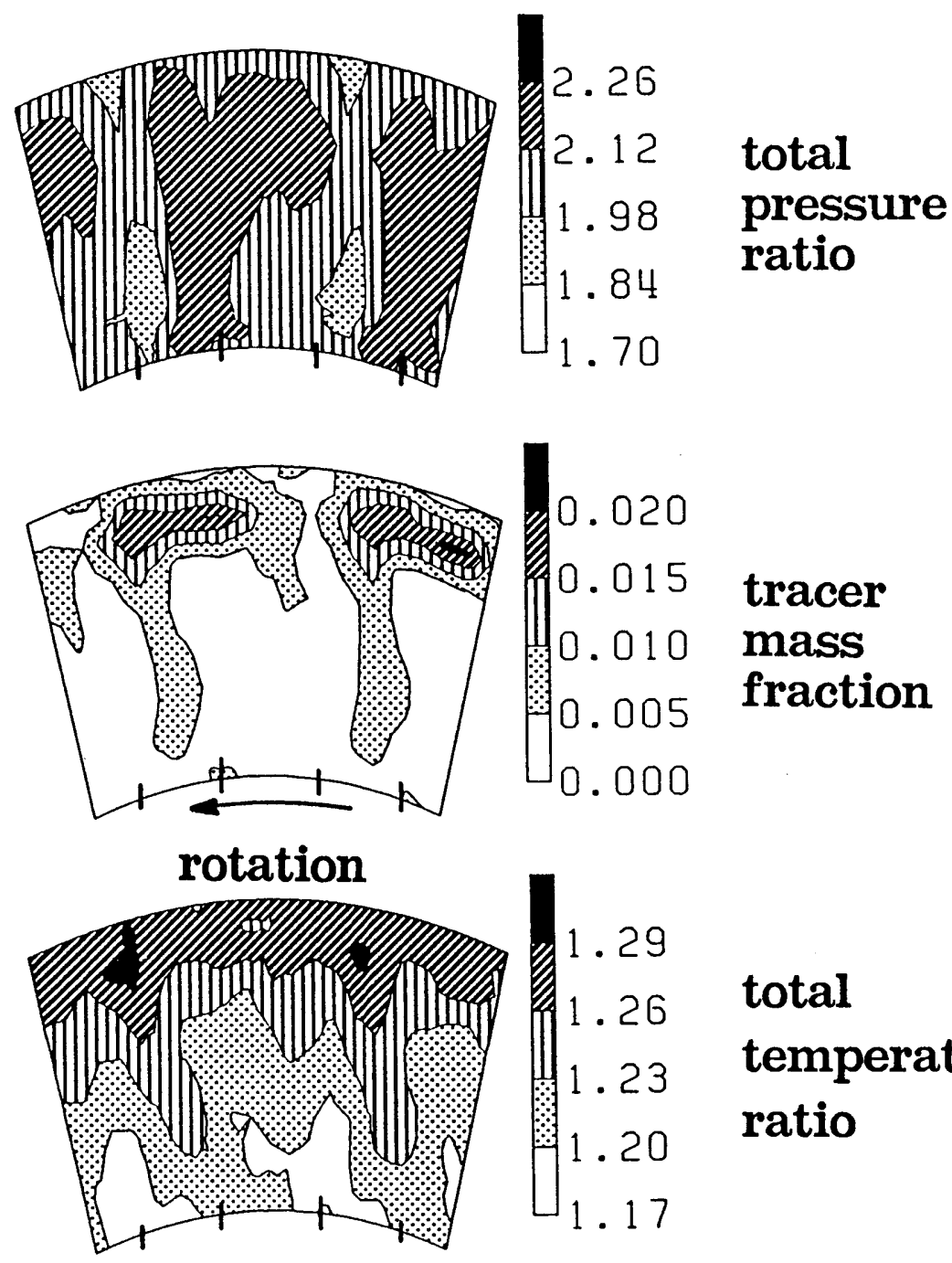

total temperature ratio

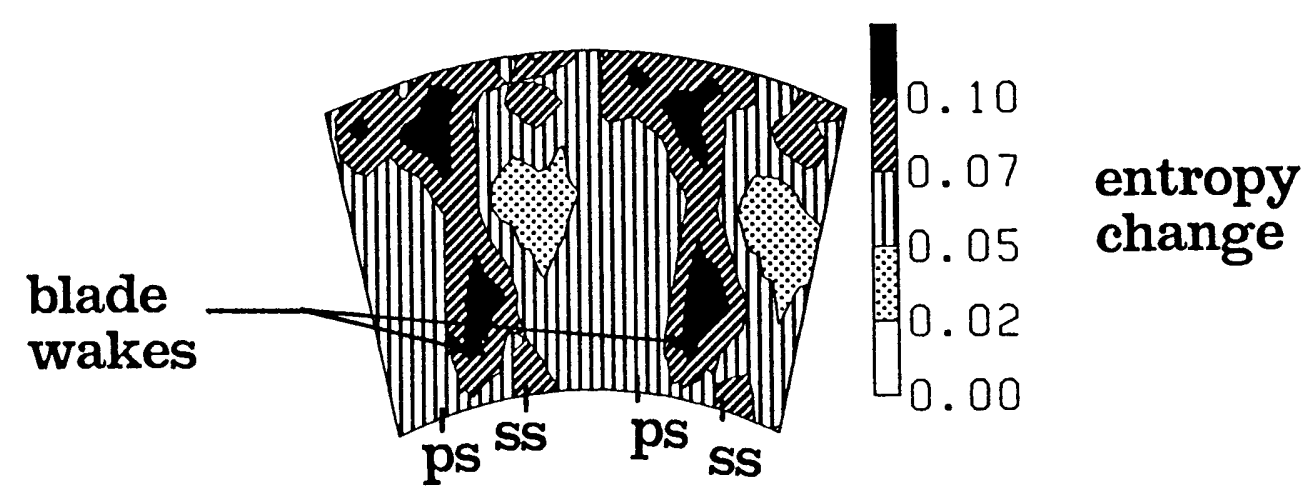

Figure 5.25: Contour plots of total pressure ratio, tracer gas concentration (mass fraction), total temperature ratio and entropy change for injection at the tip 


\section{MIDSPAN INJECTION}
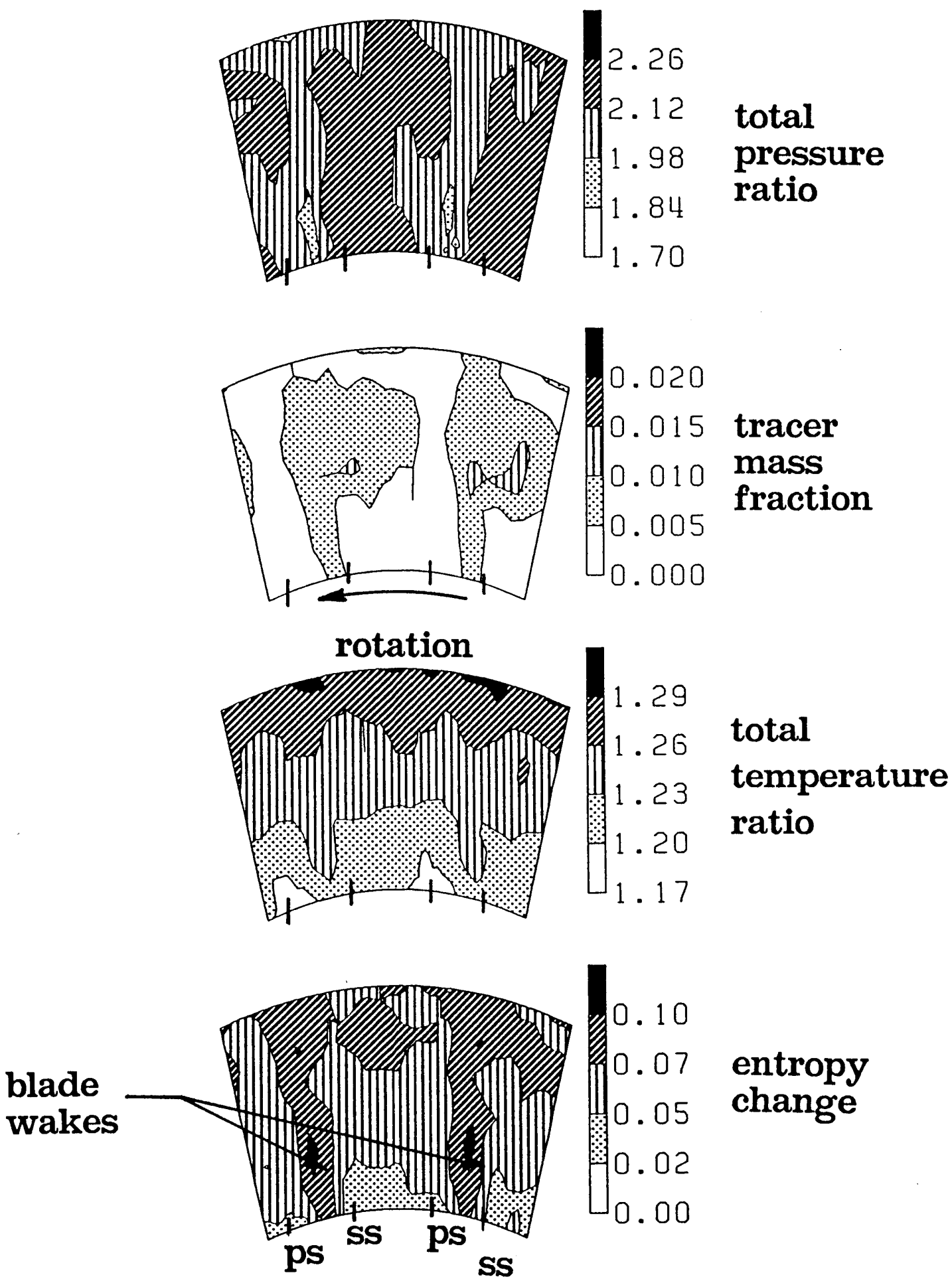

Figure 5.26: Contour plots of total pressure ratio, tracer gas concentration (mass fraction), total temperature ratio and entropy change for injection at the midspan 


\section{HUB INJECTION}
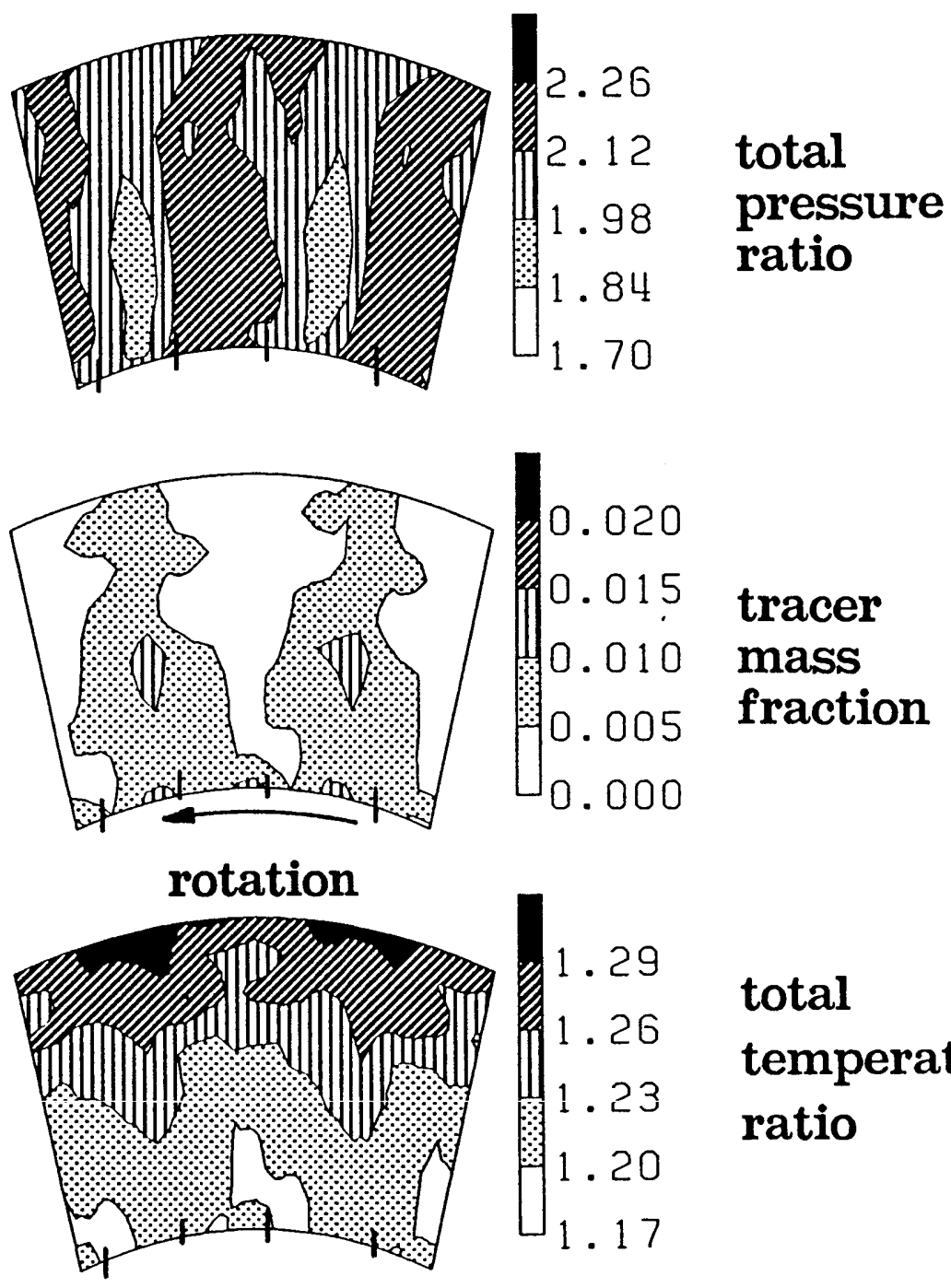

total temperature ratio

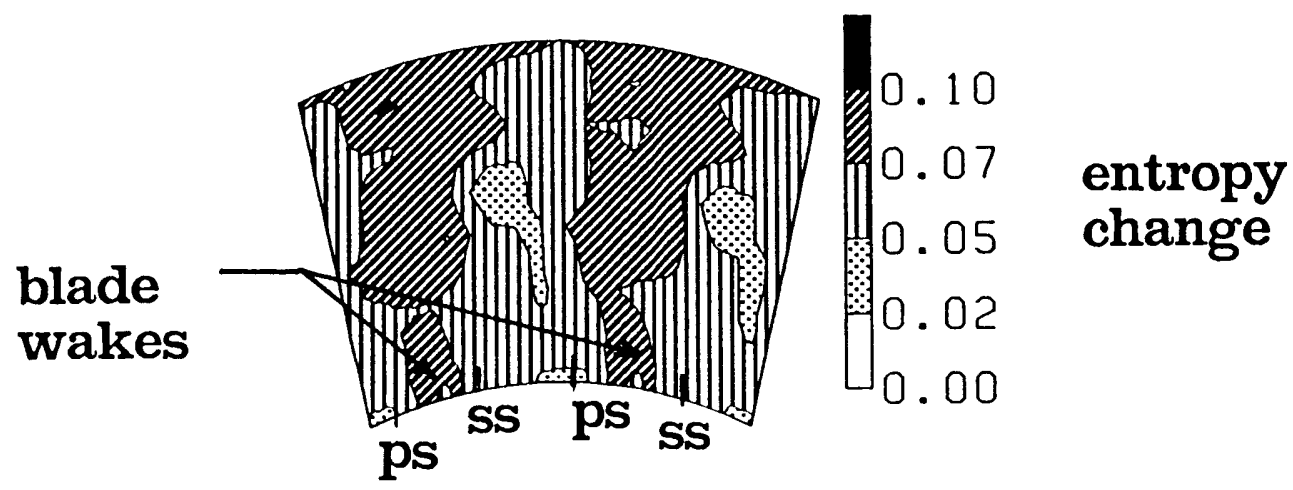

Figure 5.27: Contour plots of total pressure ratio, tracer gas concentration (mass fraction), total temperature ratio and entropy change for injection at the hub 


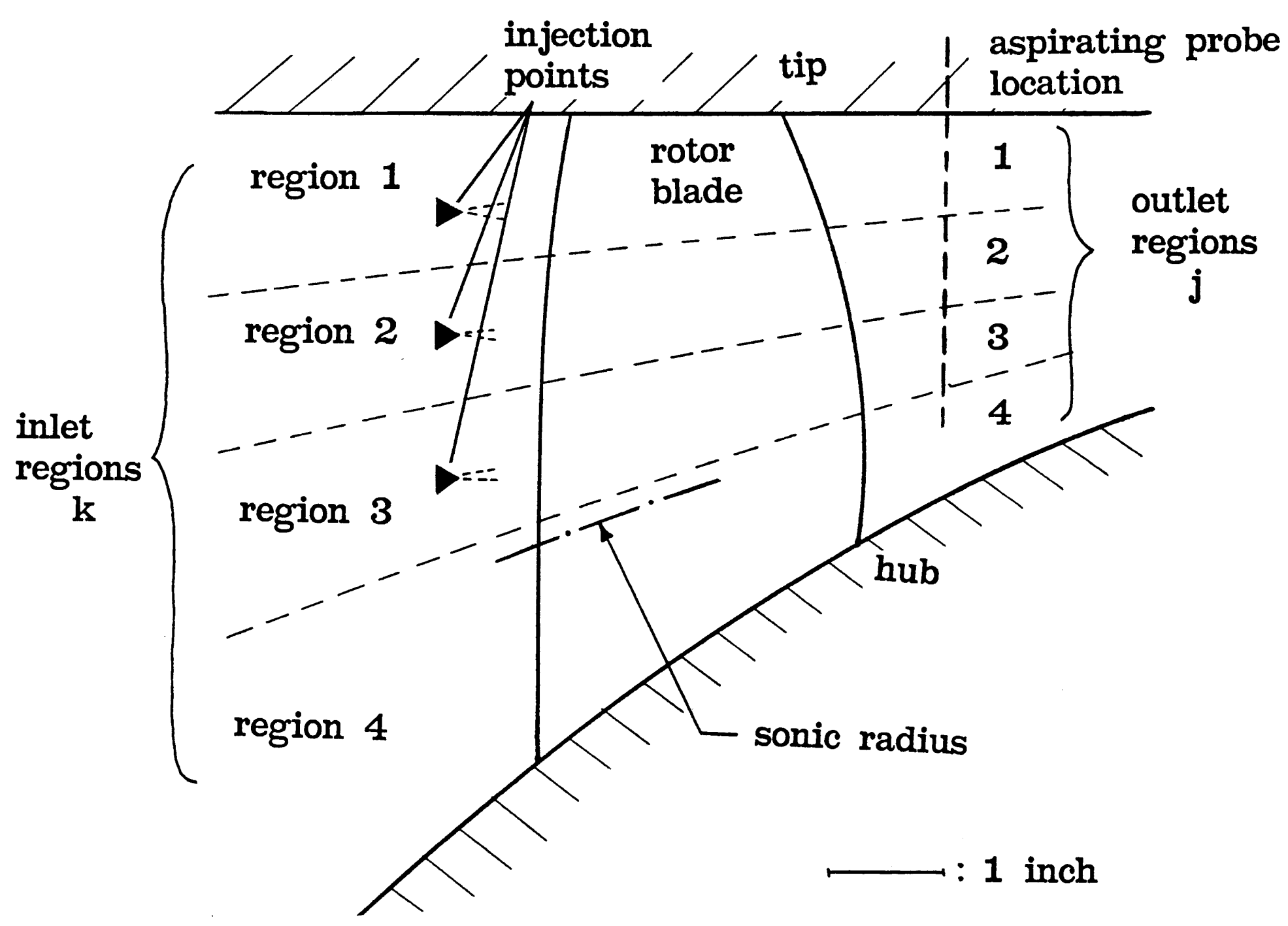

Figure 6.1: Scale drawing of the rotor geometry showing the inlet and outlet regions 


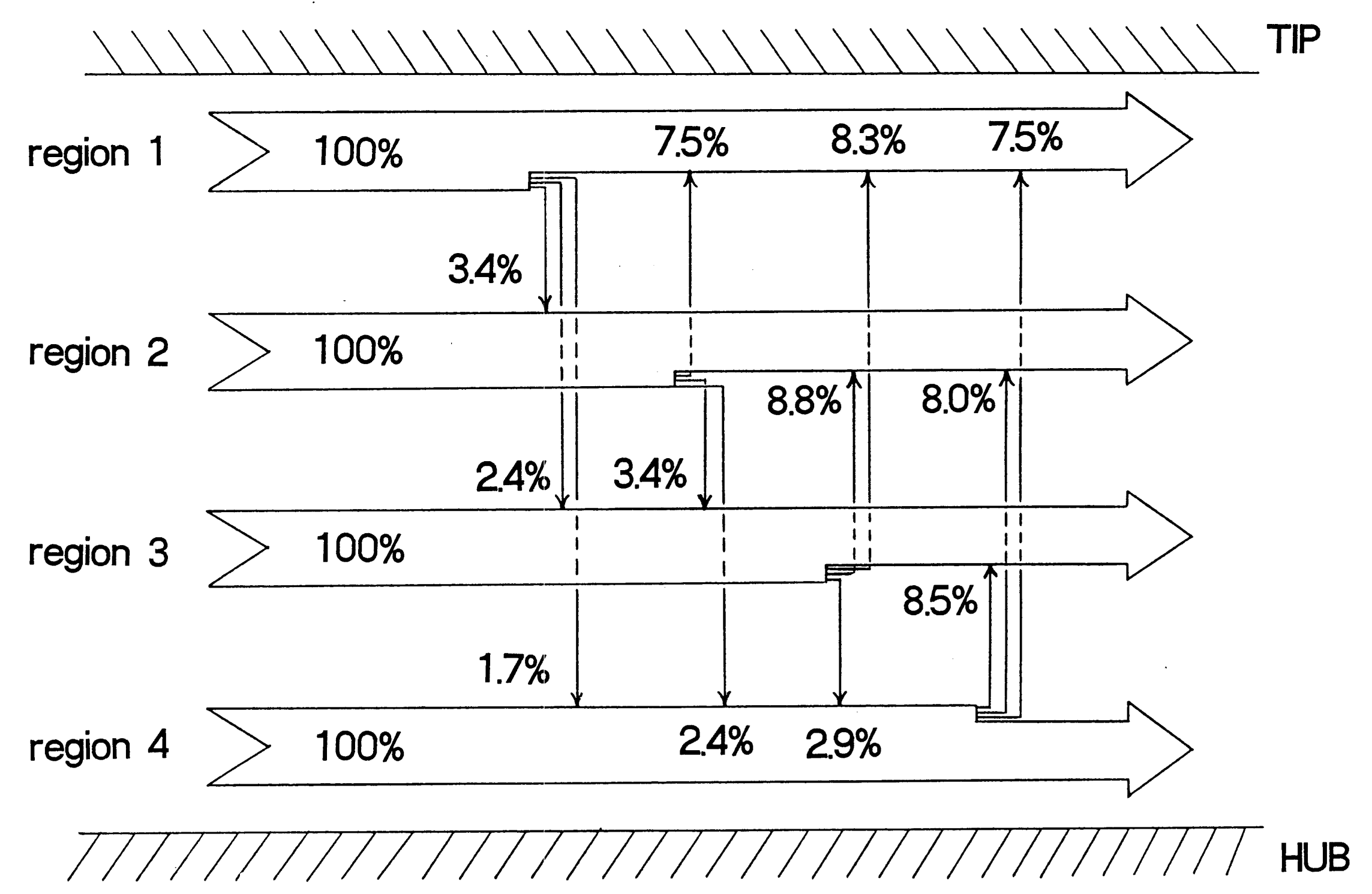

Figure 6.2: Spanwise fluid transport as percentage of the mass flow of each individual inlet region 


\section{RADIAL VARIATION OF MIXING COEFFICIENT}

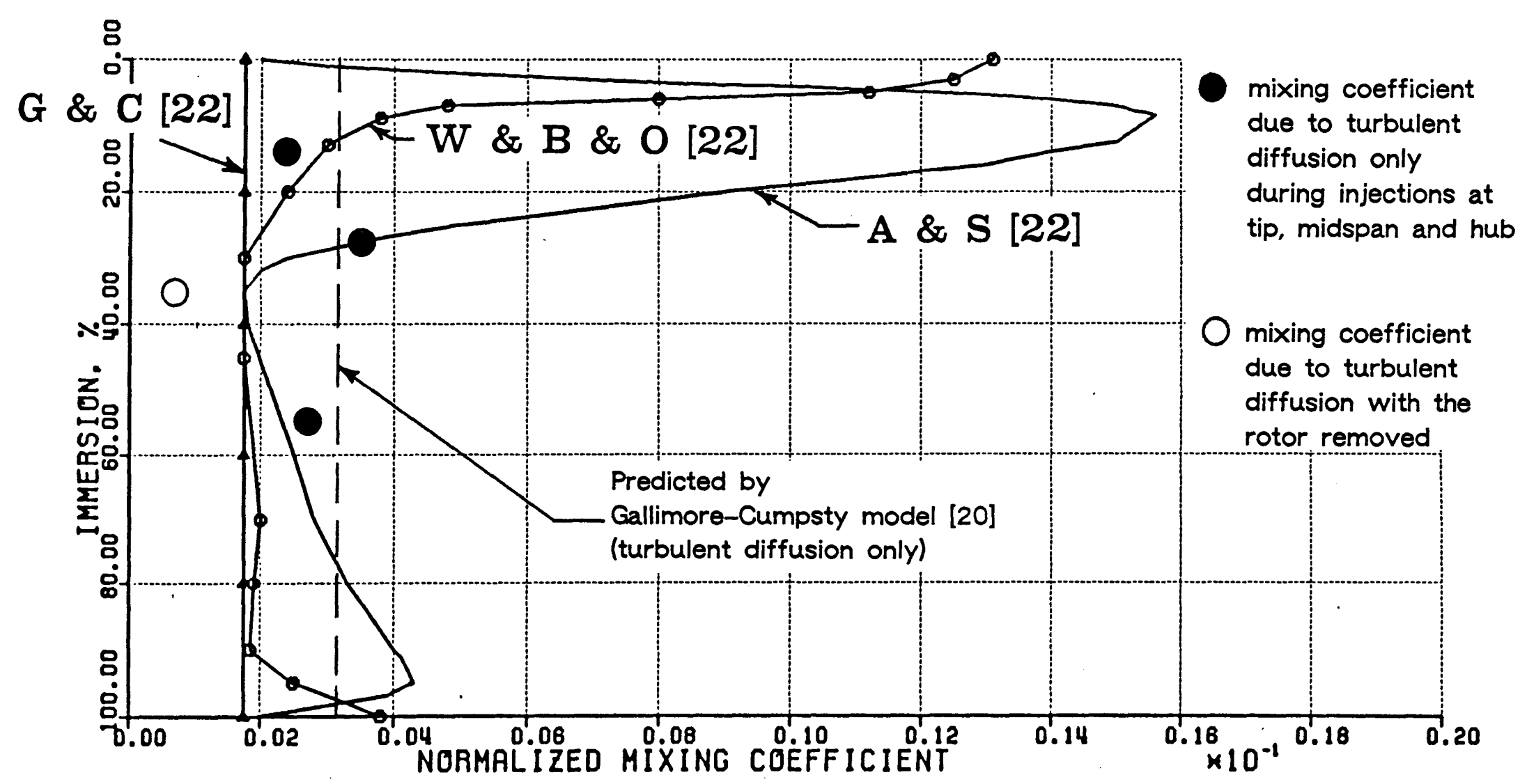

Figure 6.3: Spanwise distribution of time averaged normalized mixing coefficient 


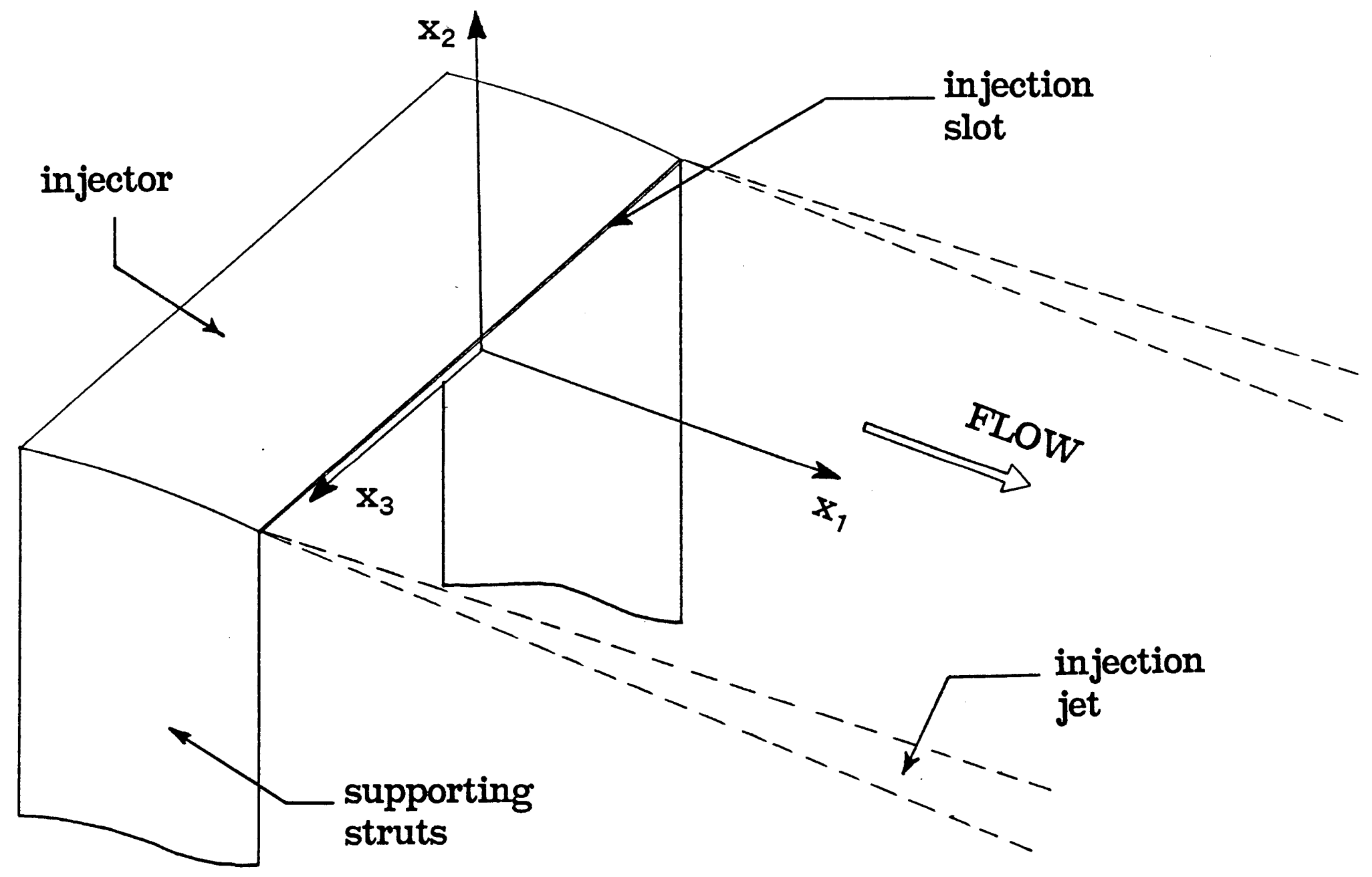

Figure 6.4: Schematic drawing of the coordinates system used in the calculation of the mixing coefficient 


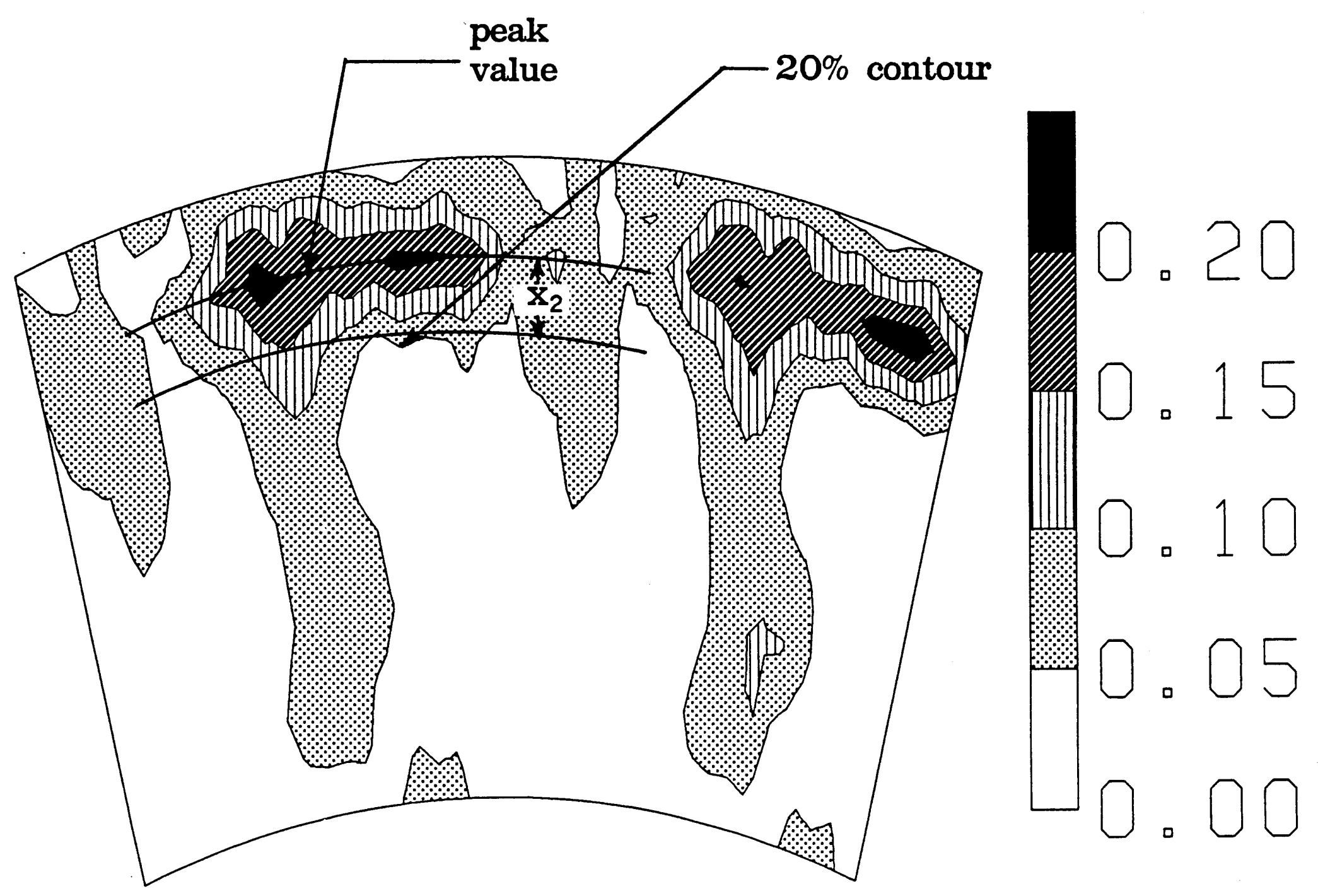

Figure 6.5: Typical concentration contours used in the calculation of the turbulent mixing coefficient 


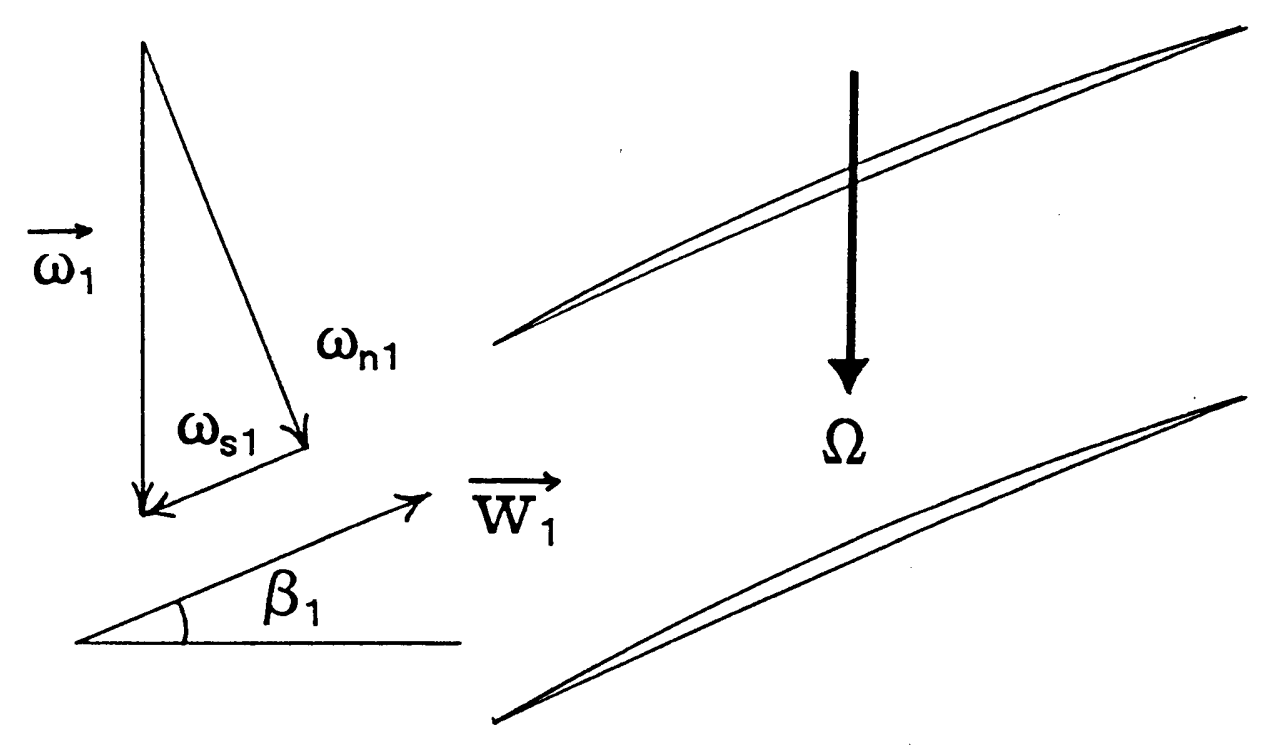

inlet
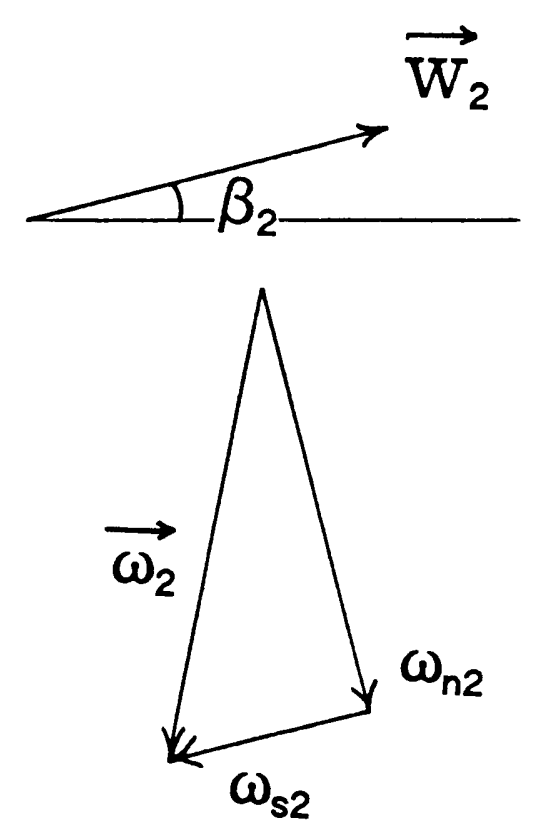

outlet

Figure 7.1: Schematic drawing of rotor geometry, showing the development of secondary flow in the blade passage (streamwise vorticity) 
MEAN ABSOLUTE VELOCITY DISTRIBUTION

MEASURED BY: THE LASER ANEMOMETER

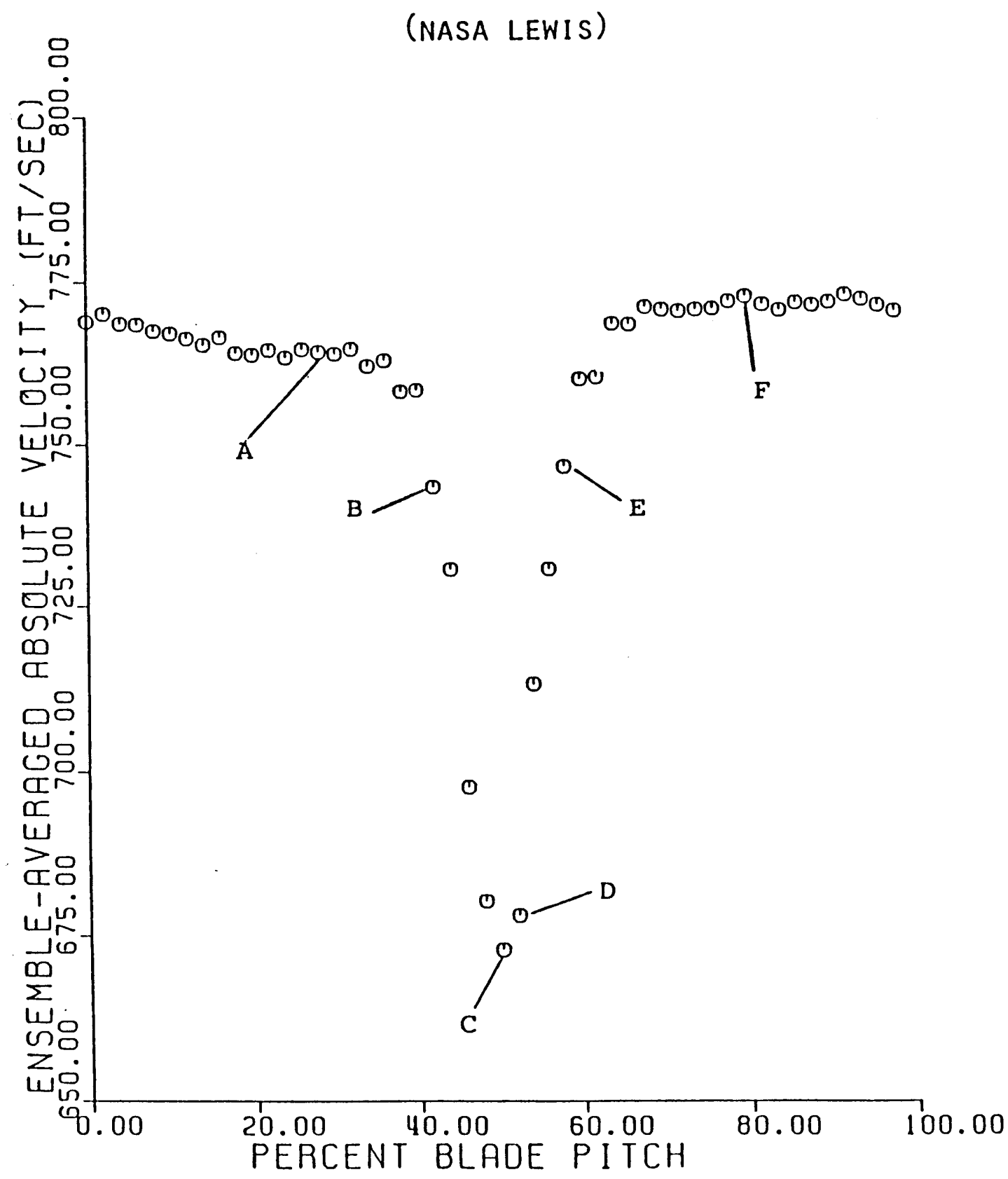

Figure 7.2: Mean rotor exit absolute velocity distribution for NASA Lewis rotor, measured by the laser anemometer at $60 \%$ span, $140 \%$ chord in a plane 42 degrees from the axial direction (from [2]) 

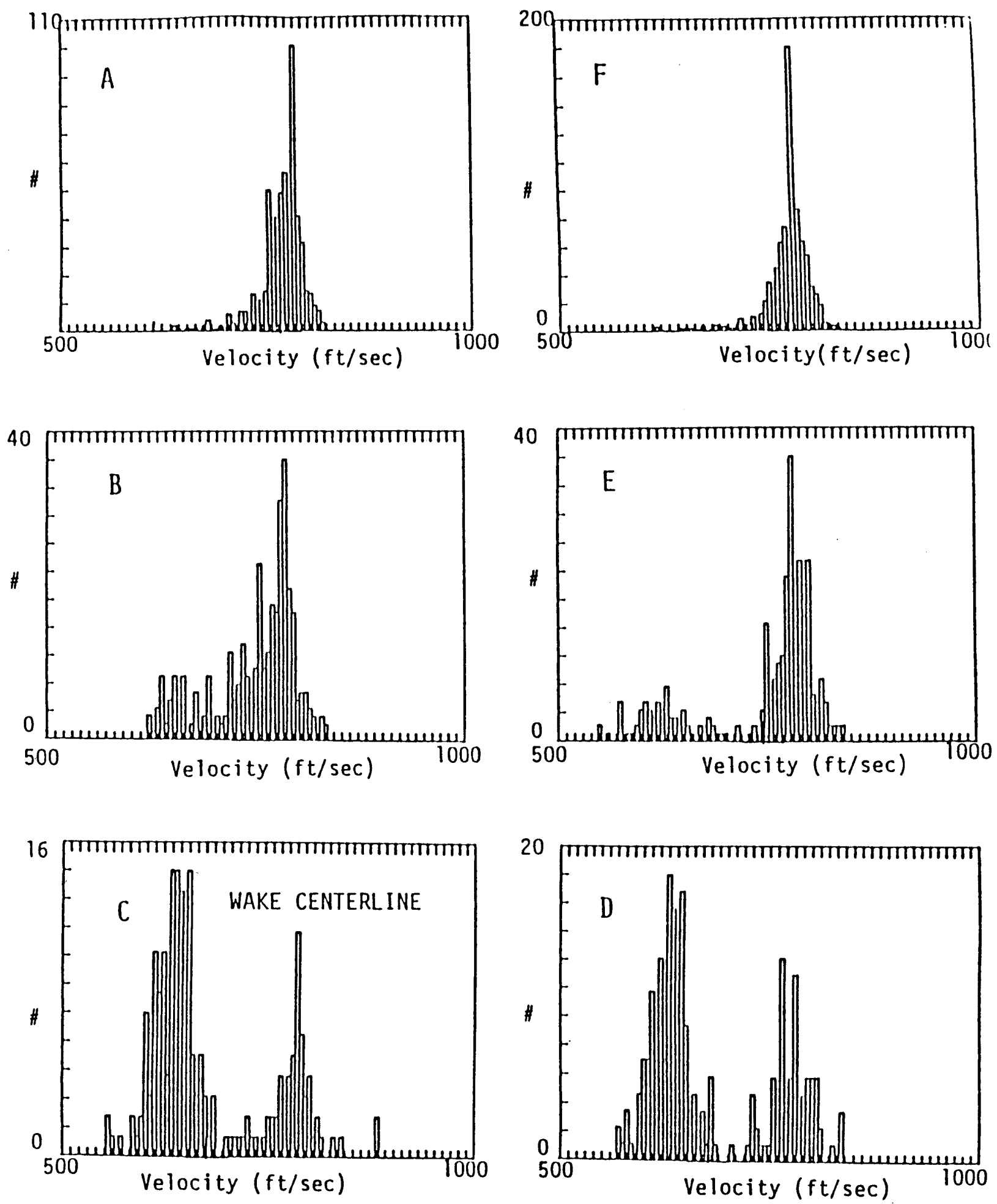

Figure 7.3: Probability density distributions of velocity measured by the L.A. at points A-F in Fig. 7.2 (from [2]) 


\section{GEOMETRY OF ROTOR BLADE VORTEX STREET}

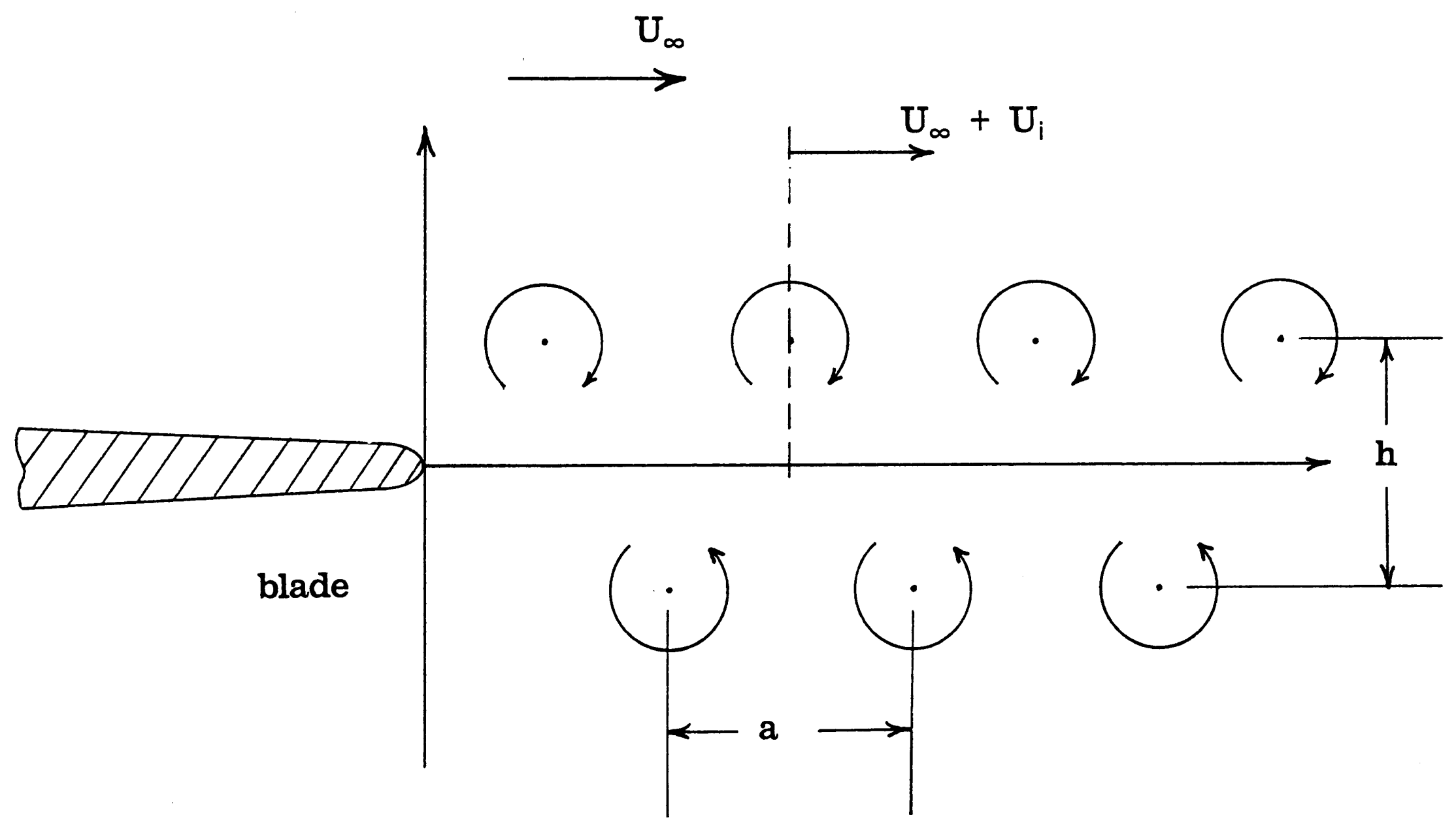

Figure 7.4: Geometry of rotor blade vortex street (from [2]) 
IIP DATA

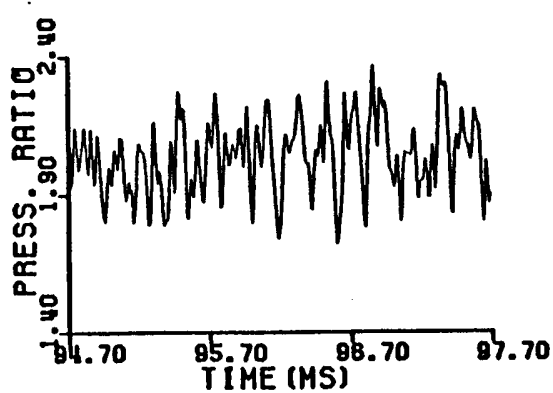

MODEL AT THE. TIP

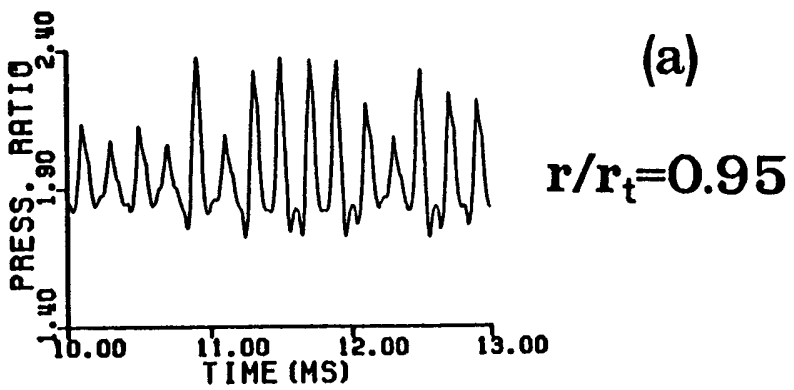

MID DATA

highest peak

MODEL at MIDSPaM a
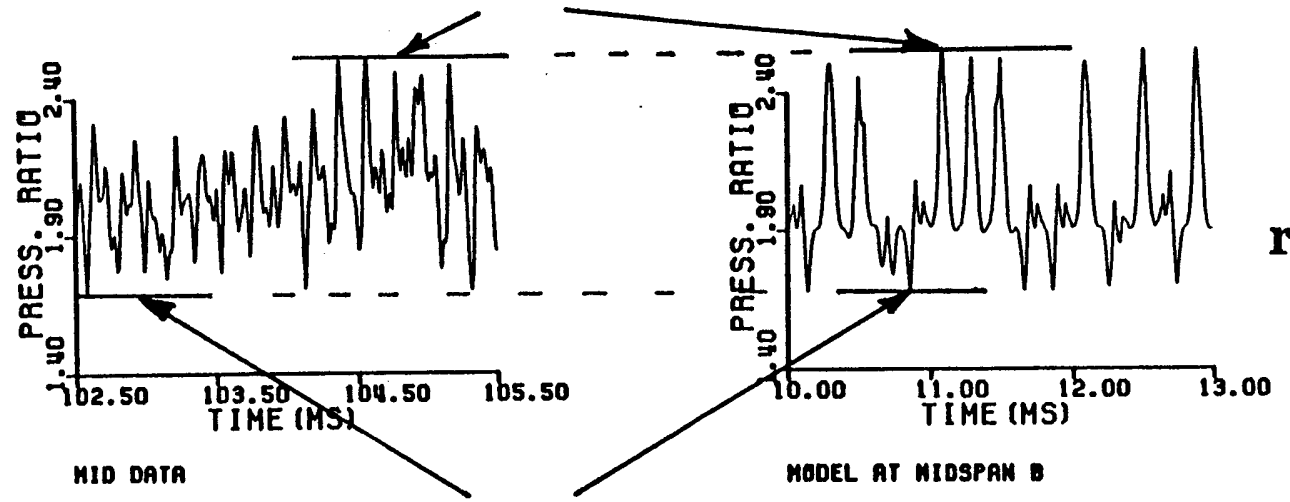

(b)

$\mathbf{r} / \mathbf{r}_{\mathrm{t}}=0.88$
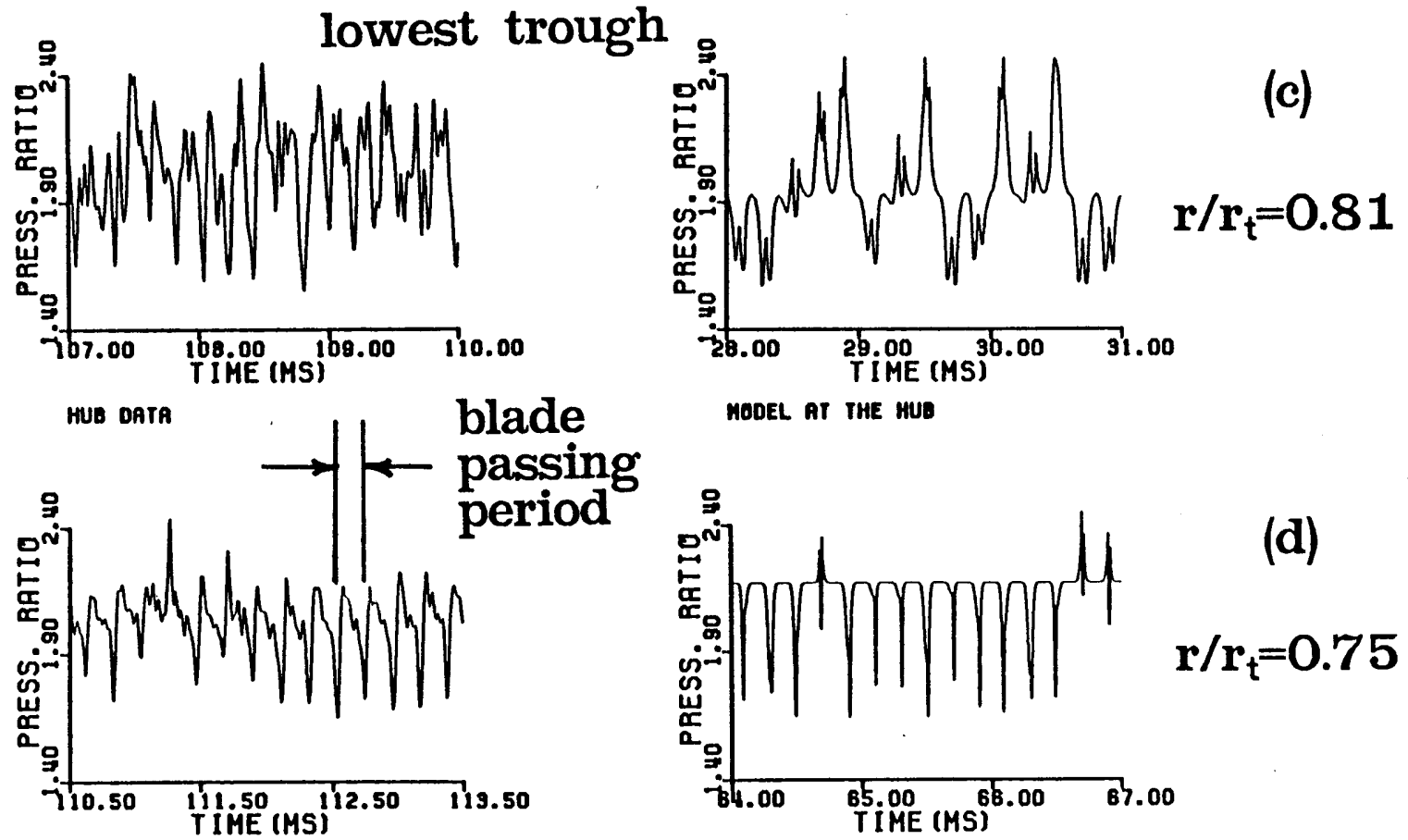
MODEL, AT THE MUS

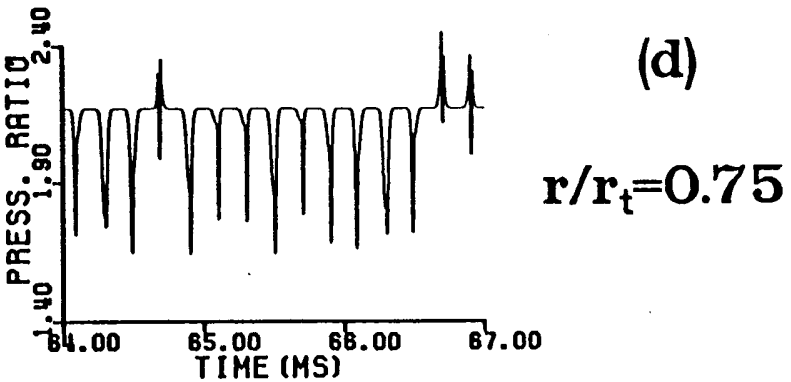

Figure 7.5a: Demonstration of the model fitting procedure at the selected spanwise locations (a), (b), (c) and (d) 


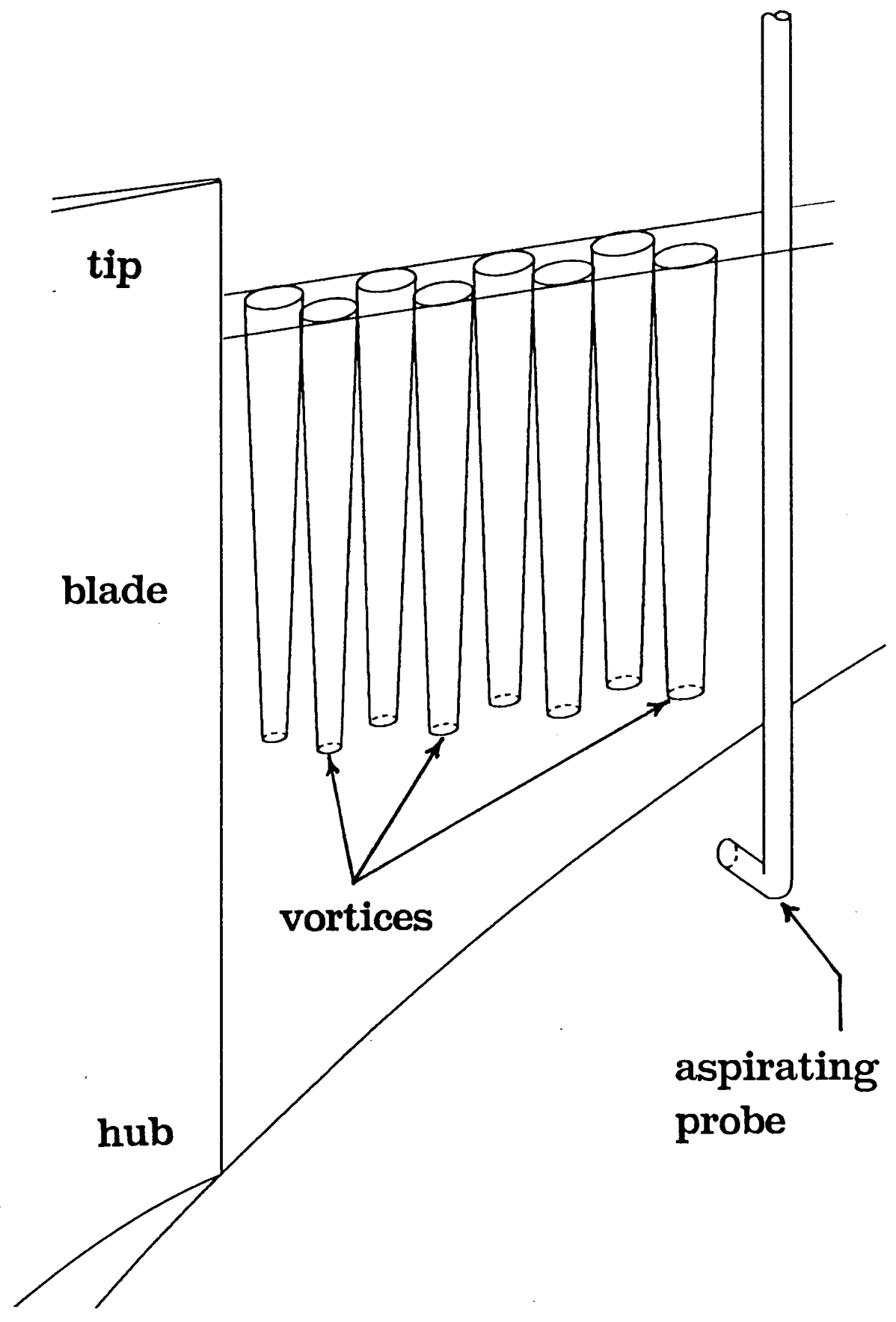

Figure 7.5b: Scaled perspective of the spanwise vortices shed by the rotor blade, as predicted by the vortex model 


\section{BLADE WAKE VORTEX STREET}

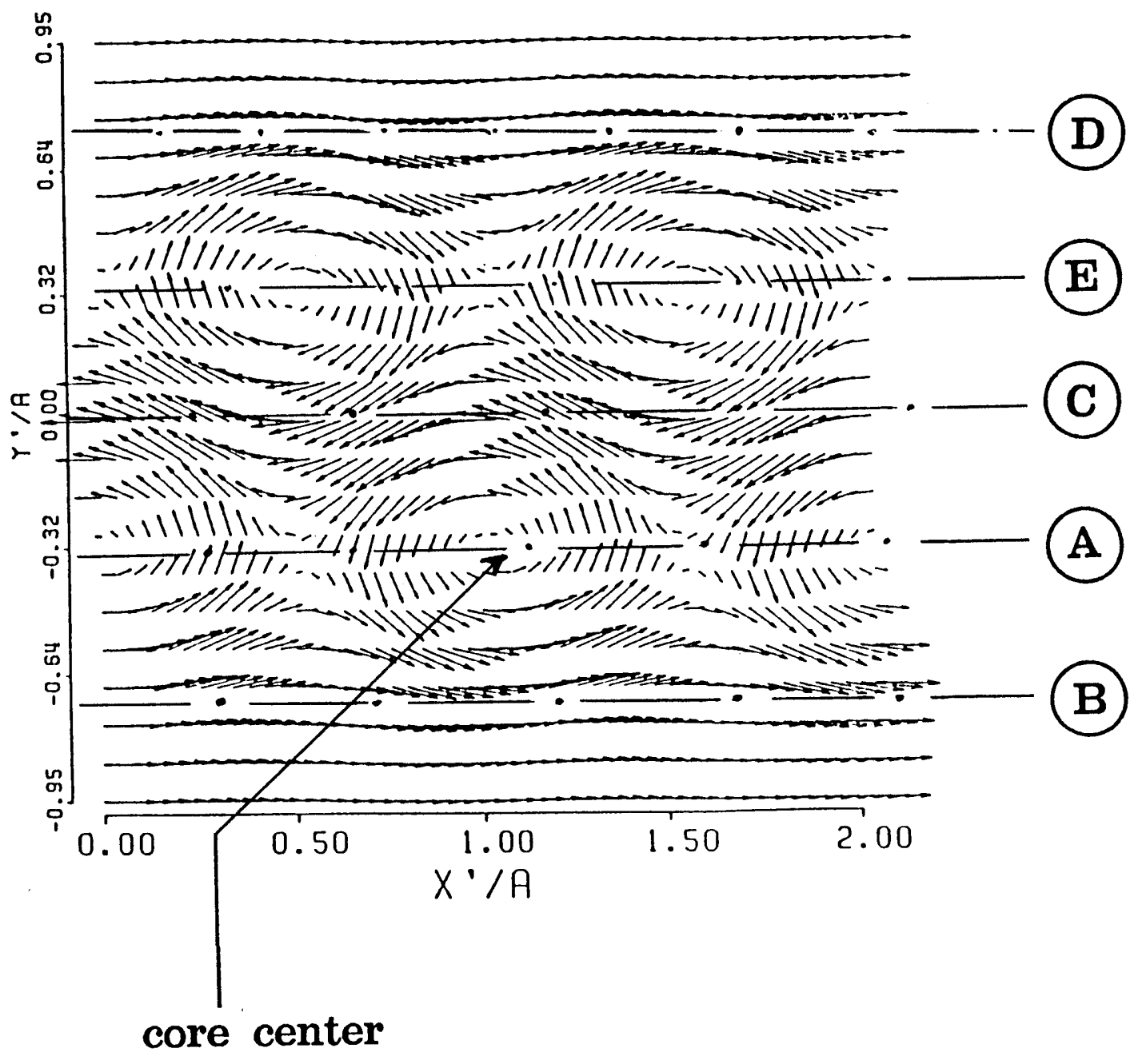

Figure 7.6: Velocity field of blade wake vortex street in the frame of reference moving with the street 


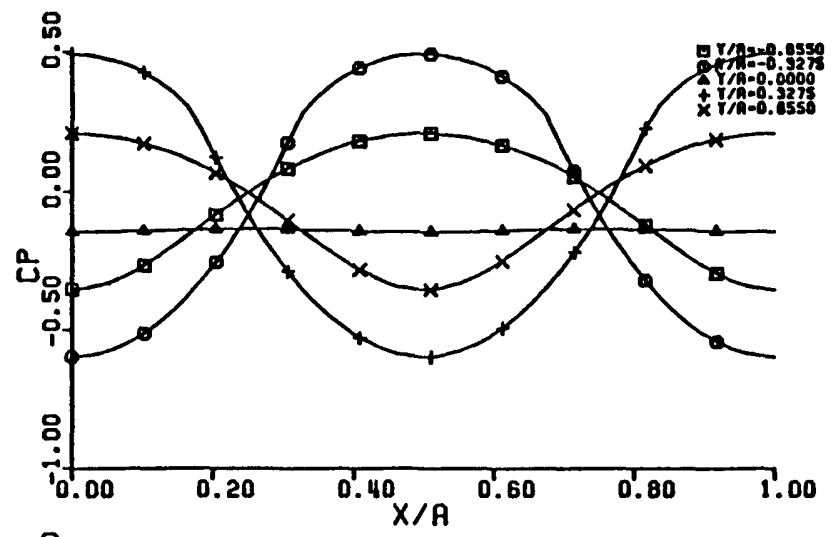

\section{$\mathbf{r} / \mathbf{r}_{\mathrm{t}}=0.95$}

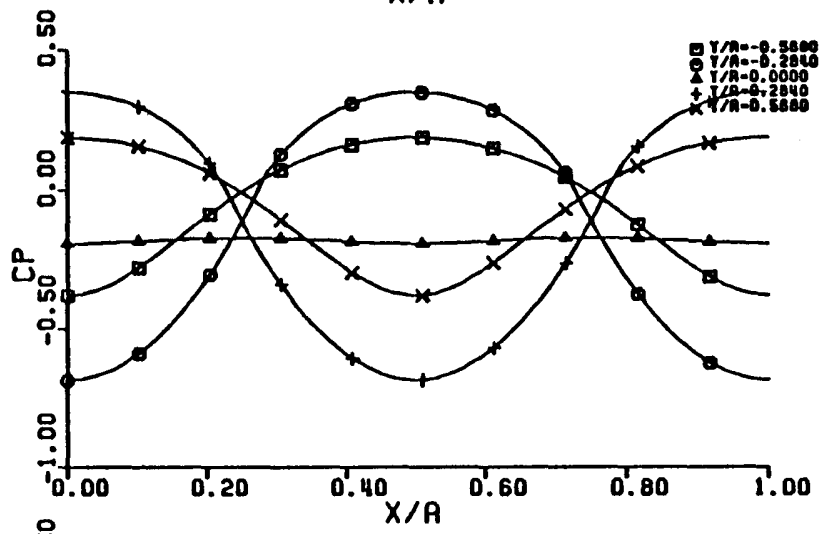

$\mathbf{r} / \mathbf{r}_{\mathrm{t}}=0.88$

from $\{.6\{$

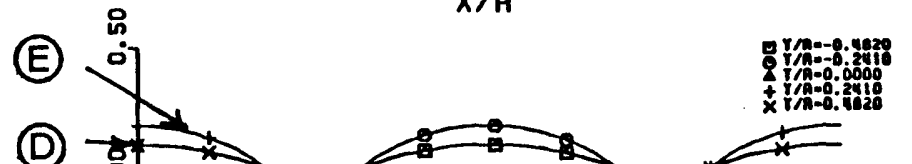

(D)
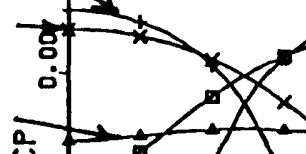

(B)

B

(A)

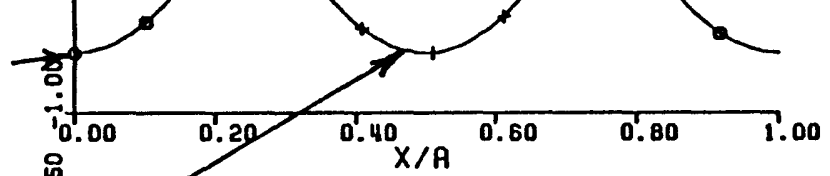

$\mathbf{r} / \mathbf{r}_{\mathrm{t}}=\mathbf{0 . 8 1}$

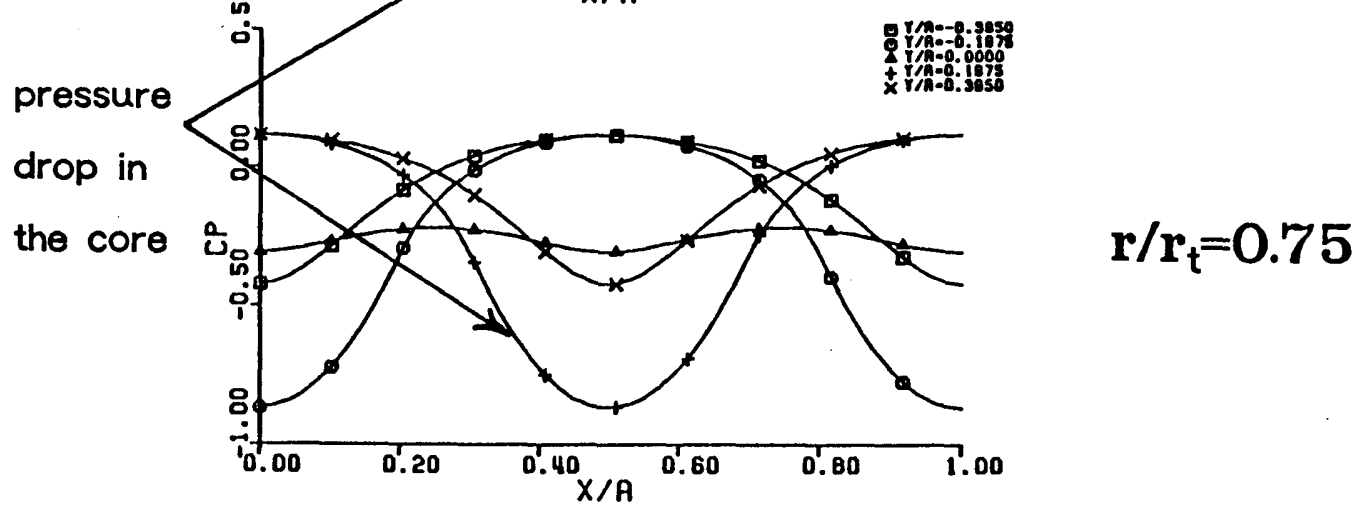

Figure 7.7: Pressure coefficient distribution from vortex model at the four spanwise locations 


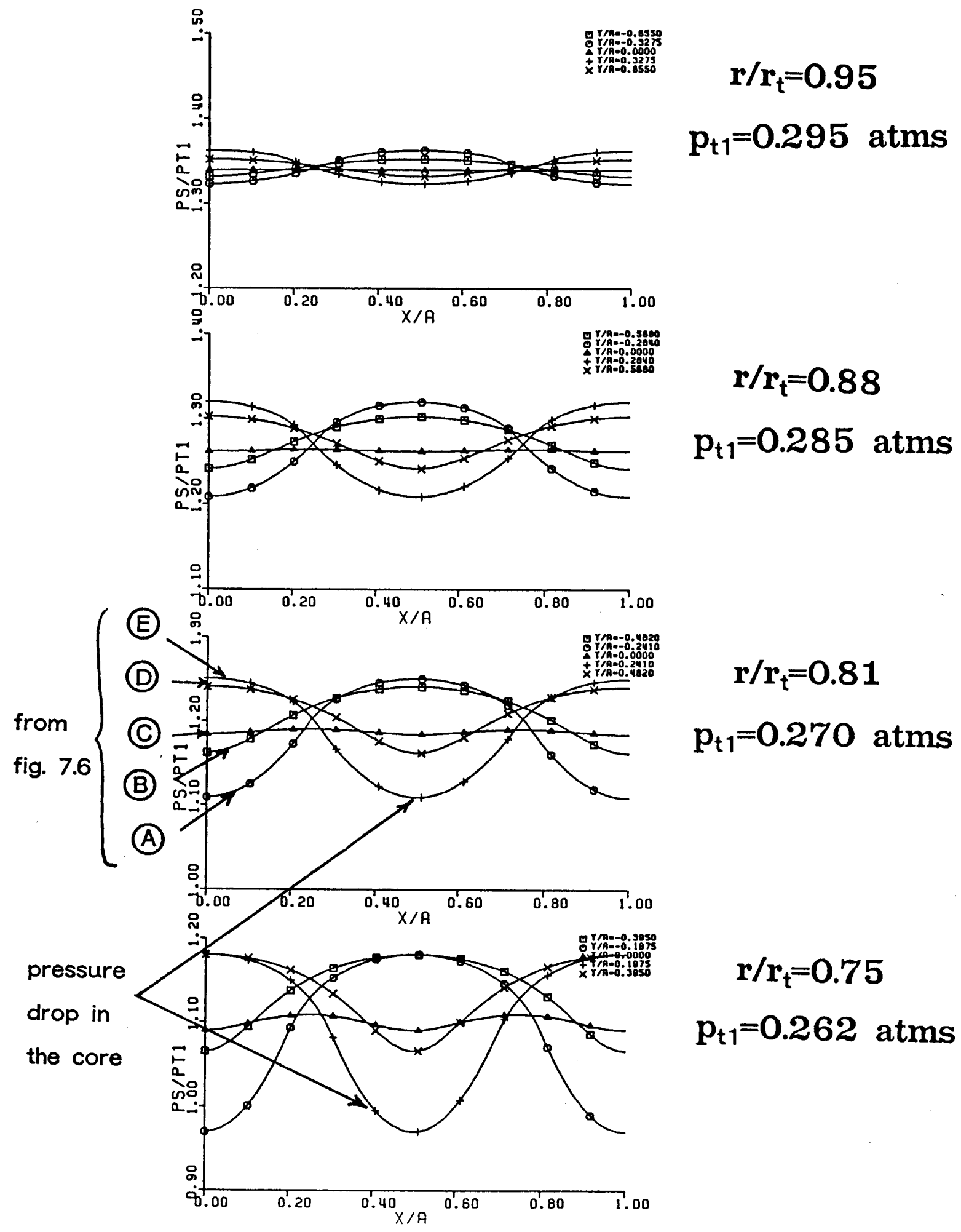

Figure 7.8a: Static pressure distribution nondimensionalized with inlet total pressure from vortex model at four spanwise locations 


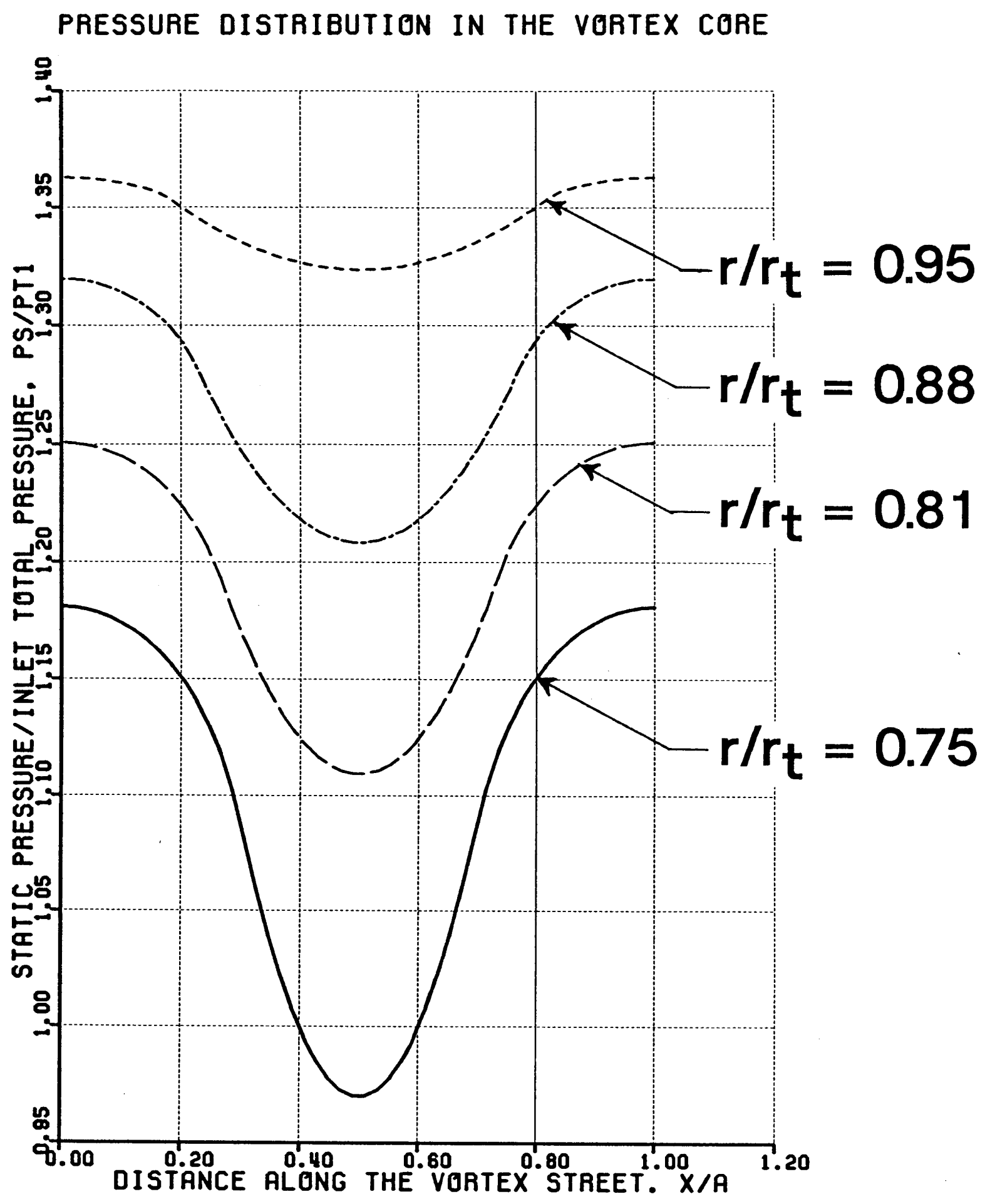

Figure 7.8b: Static pressure distribution in the vortex cores nondimensionalized with inlet total pressure from vortex model at four spanwise locations 


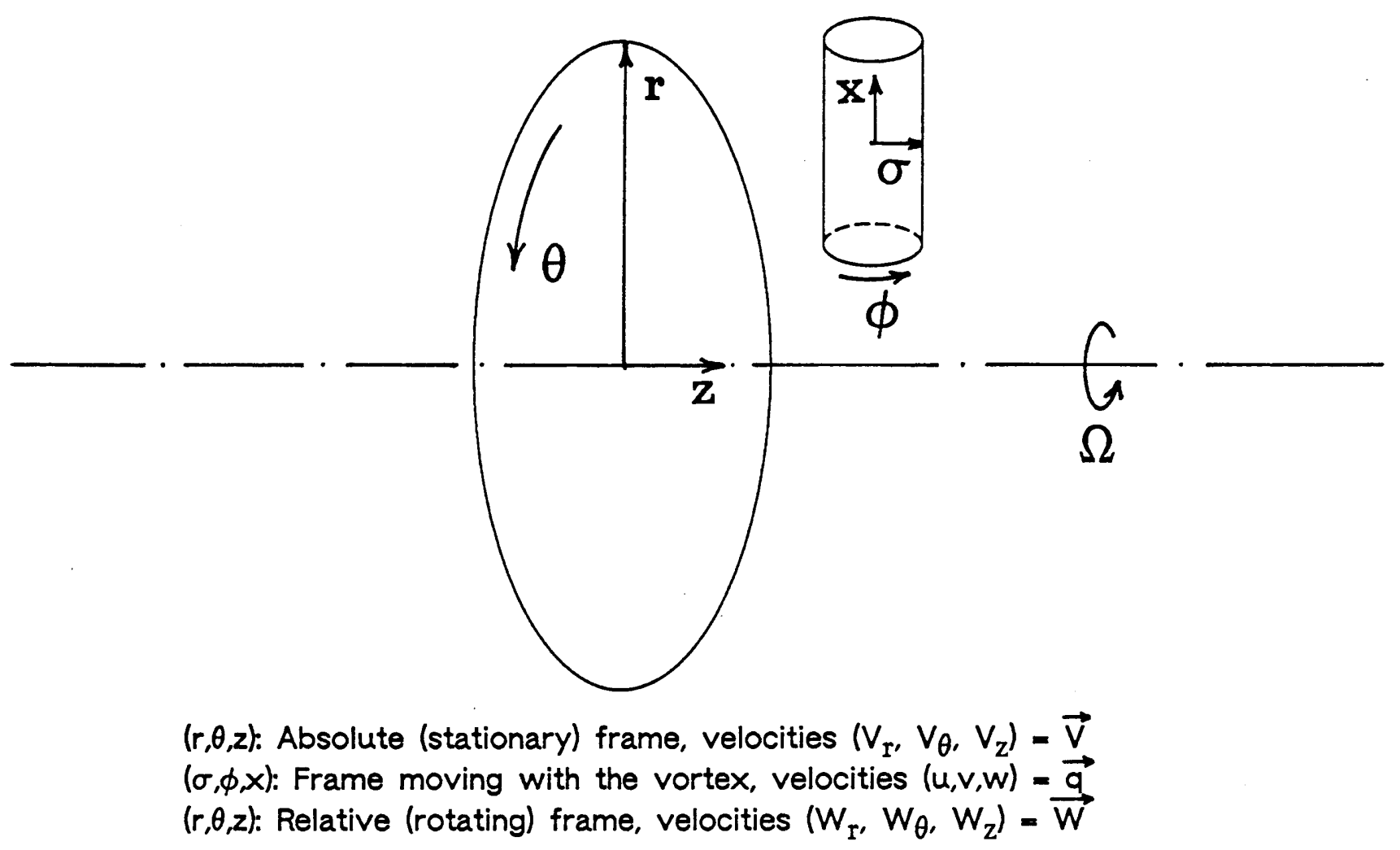

Figure 7.9: Schematic demonstration of the various coordinate systems used in the vortex model 


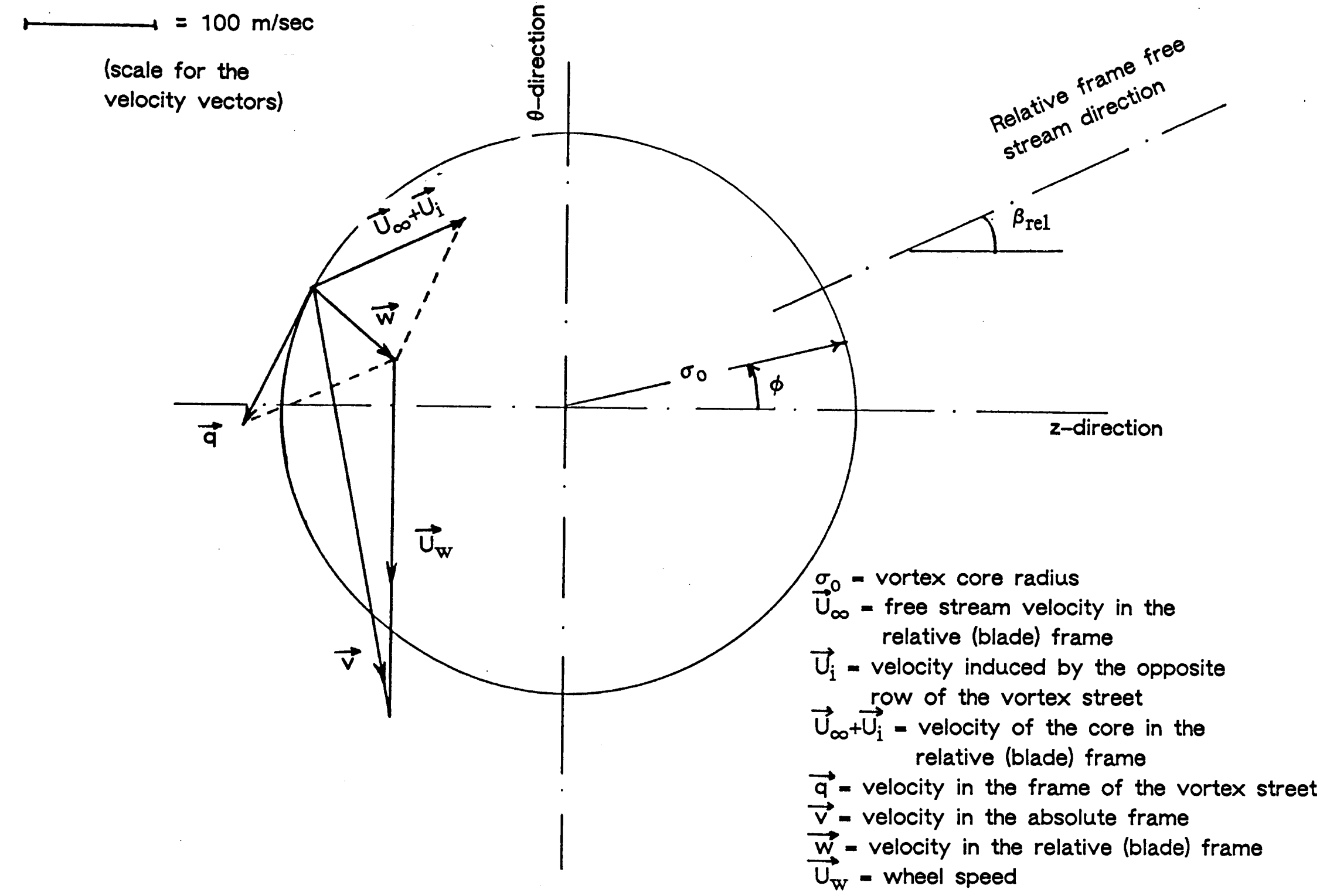

Figure 7.10: Velocity vectors in the core of the vortex 


\section{VORTEX ROTATION}

\section{CLOCKWISE}

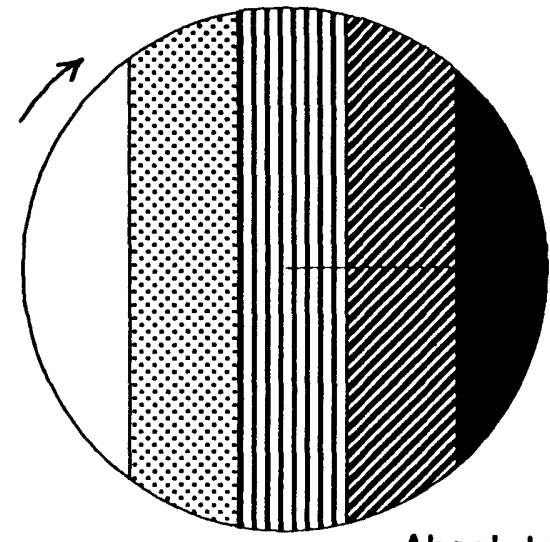

Absolute tangential velocity

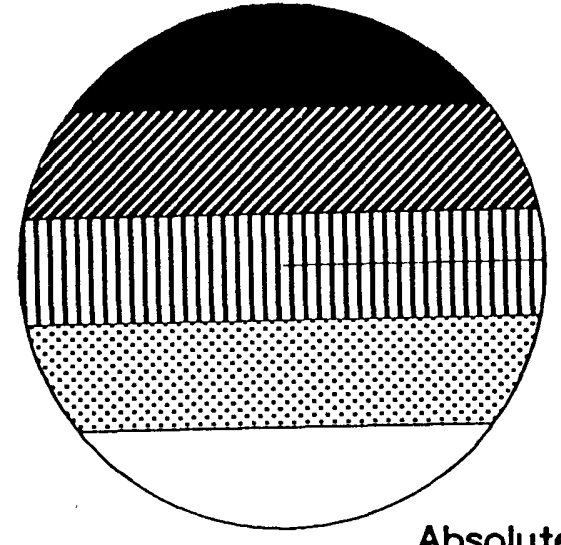

Absolute axial velocity

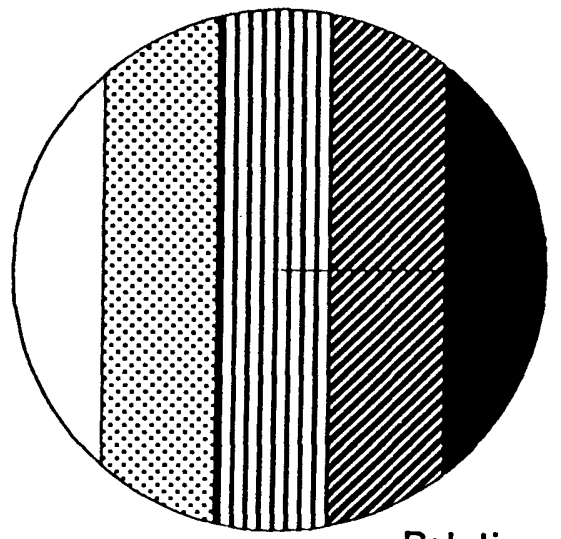

Relative tangential velocity
COUNTERCLOCKWISE
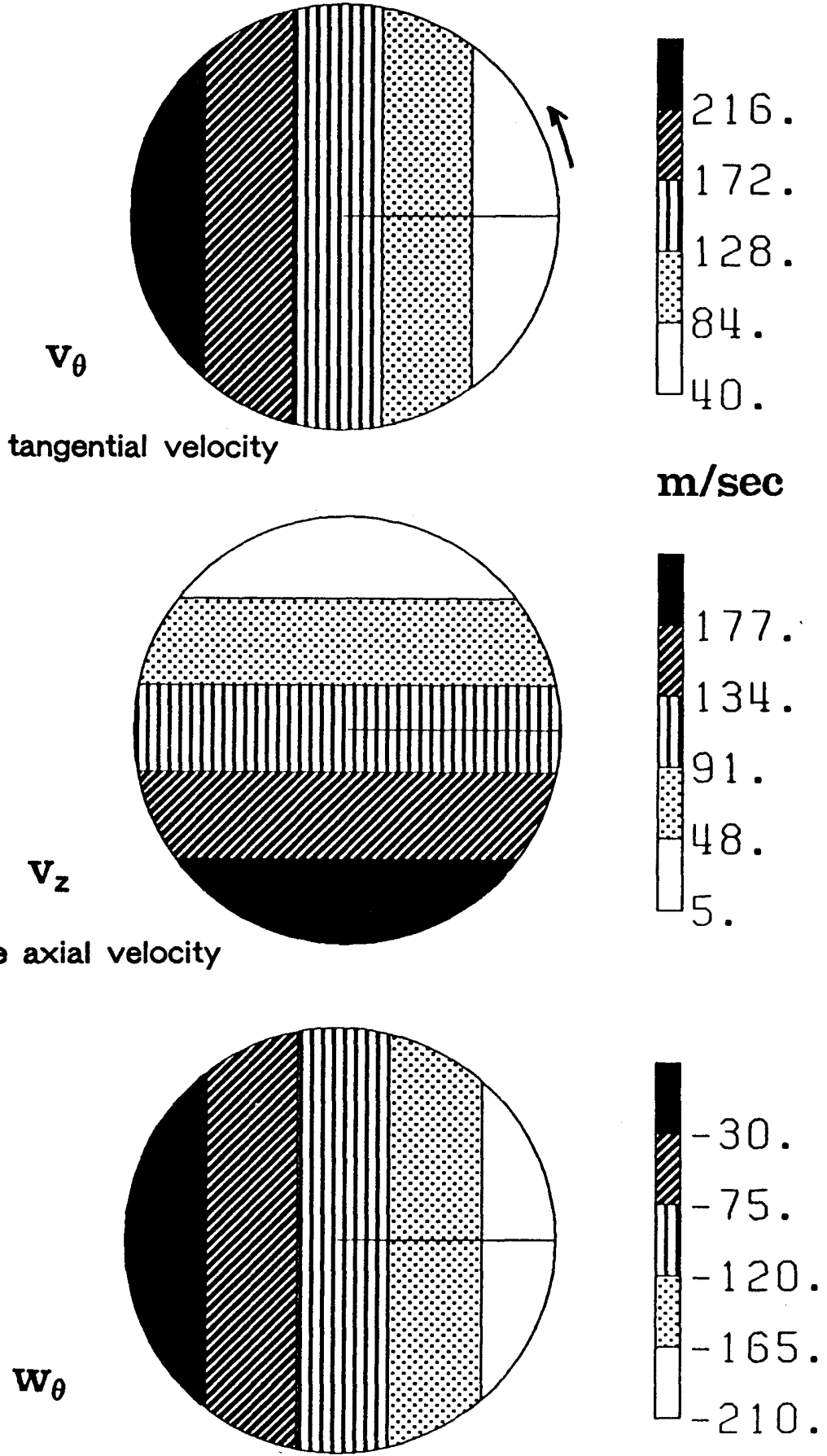

Figure 7.11: Distribution of absolute tangential velocity, absolute axial velocity and relative tangential velocity in the core of the vortex 


\section{VORTEX ROTATION}

\section{CLOCKWISE}
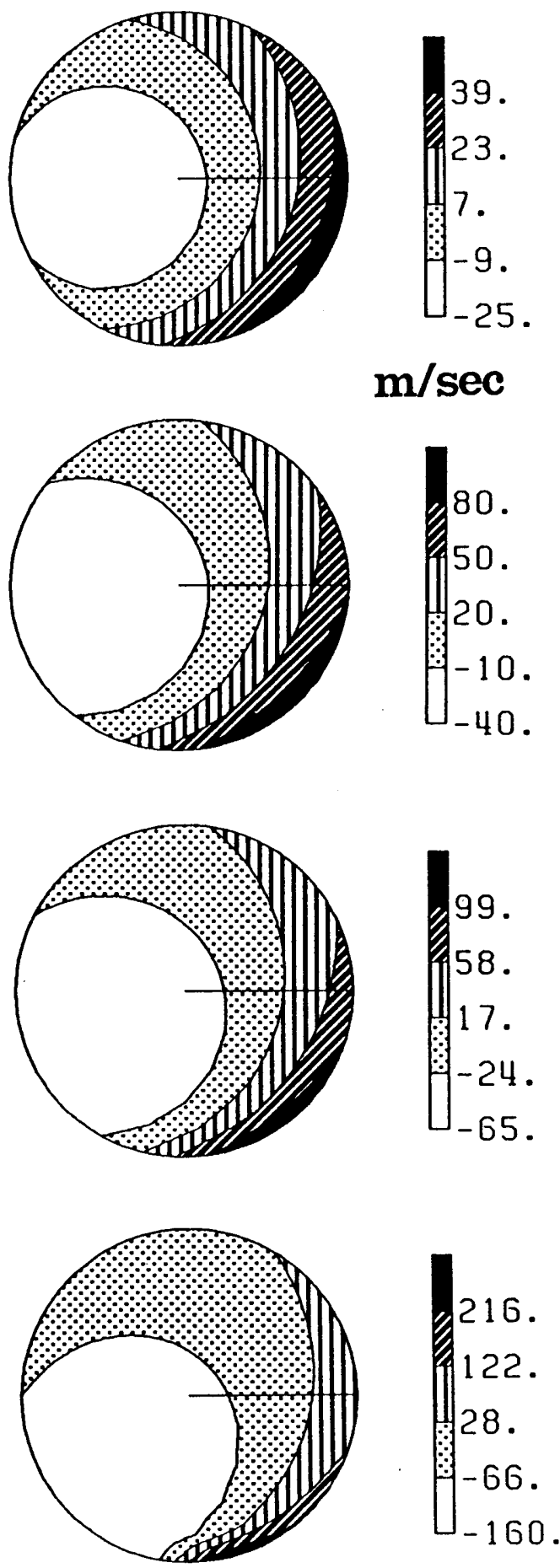

COUNTERCLOCKWISE

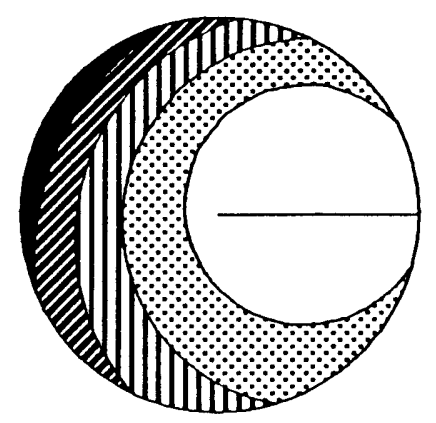

$\mathbf{r} / \mathbf{r}_{\mathrm{t}}=0.95$

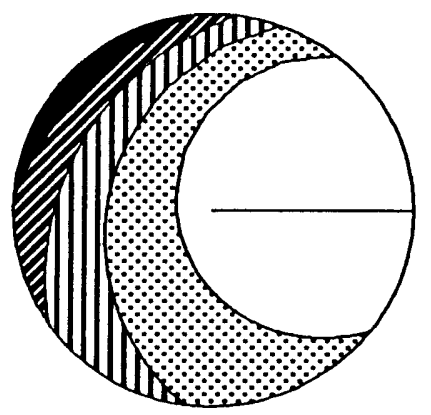

$\mathbf{r} / \mathbf{r}_{\mathrm{t}}=0.88$

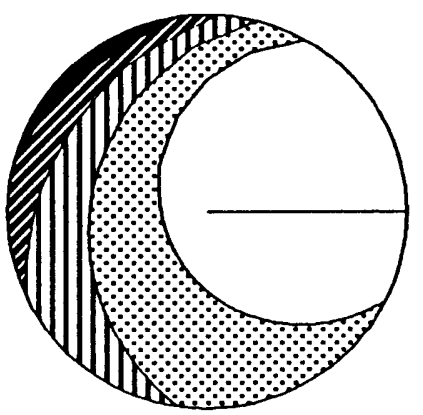

$\mathbf{r} / \mathbf{r}_{\mathrm{t}}=0.81$

(+) : towards tip

$(-)$ : towards hub

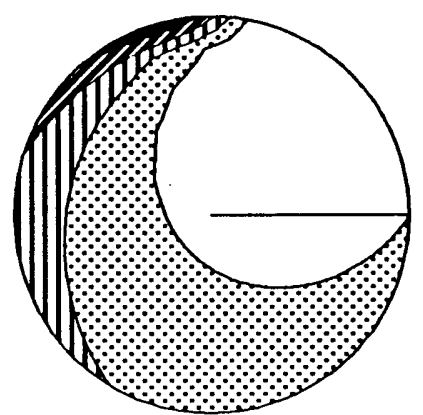

Figure 7.12: Distribution of spanwise velocities in the cores of the vortices at four spanwise locations, as predicted by the vortex model 


\section{CLOCKWISE VORTEX ROTATION}

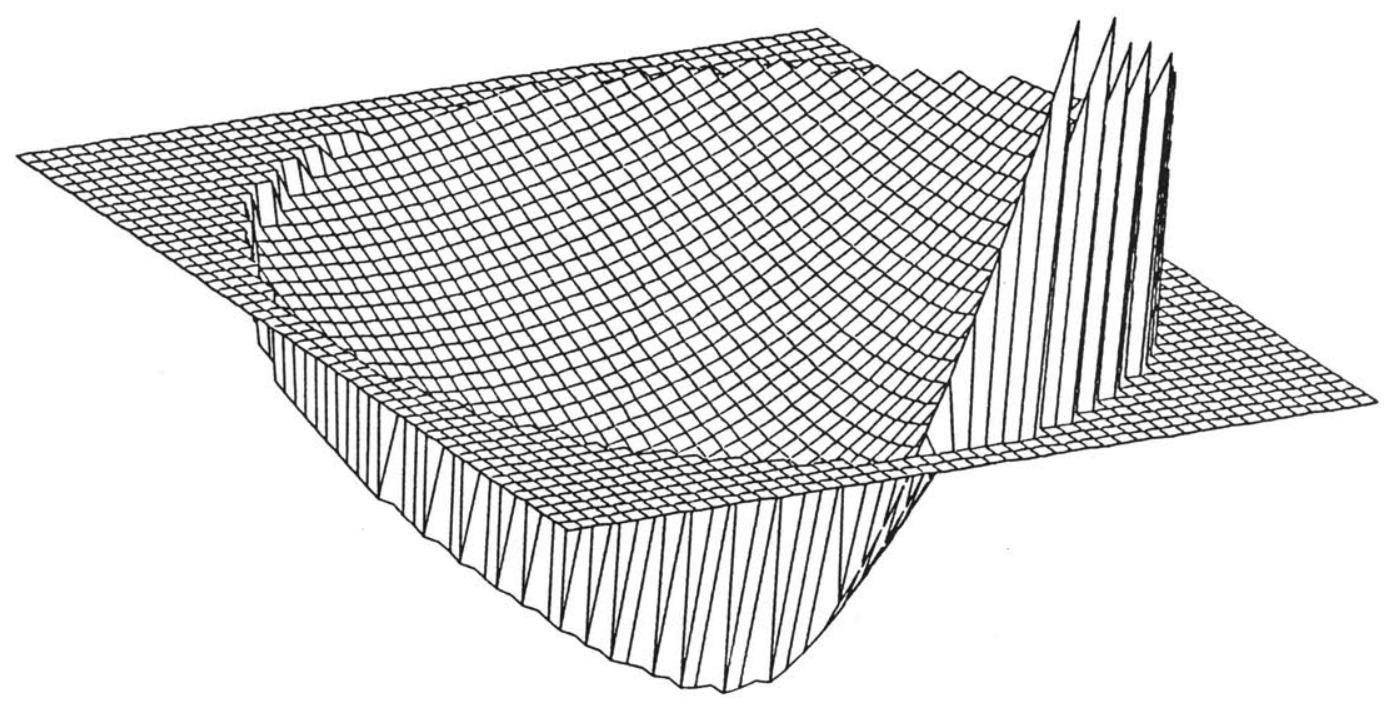

COUNTERCLOCKWISE VORTEX ROTATION

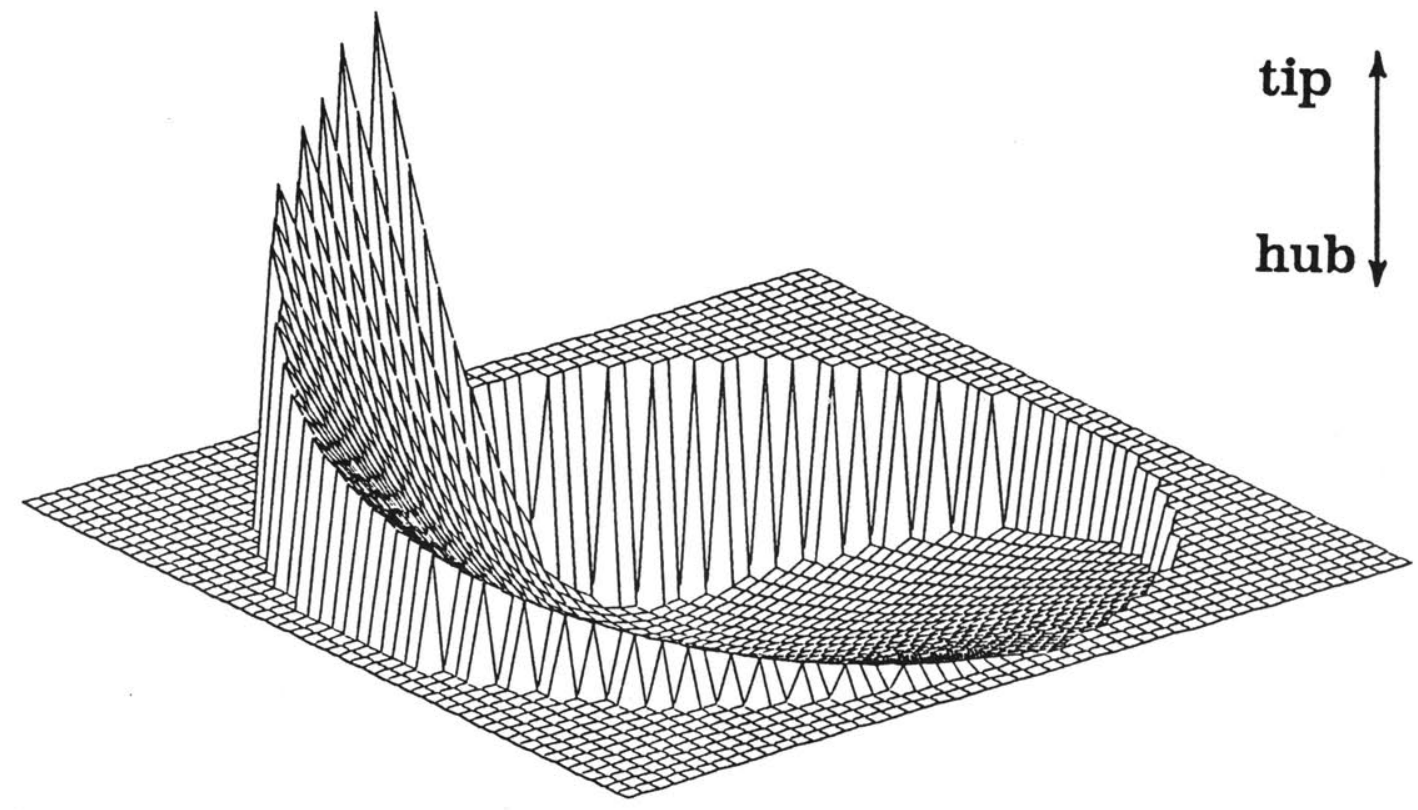

Figure 7.13: Typical distribution of spanwise velocities in the vortex core, as predicted by the vortex model 


\section{SPANWISE VELOCTTIES $(\mathrm{m} / \mathrm{sec}$ )}
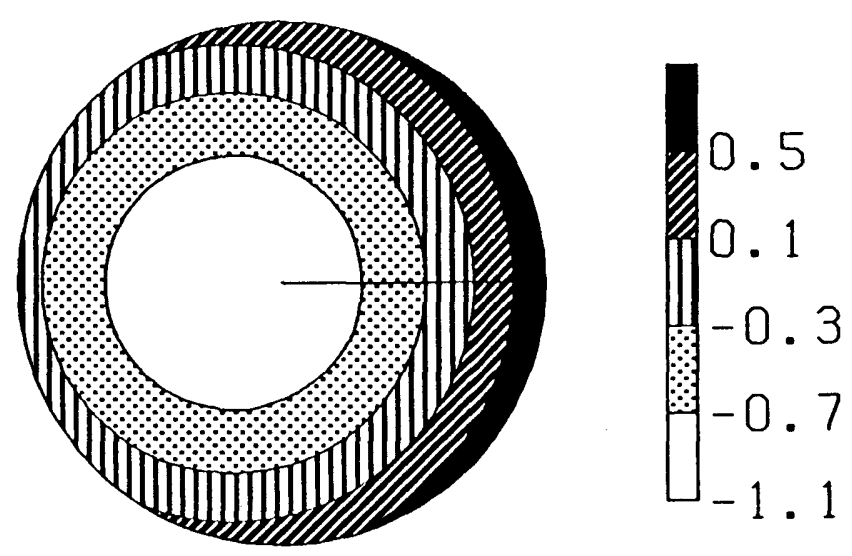

$$
\mathbf{r} / \mathbf{r}_{\mathrm{t}}=0.95
$$

Fully developed vortex immediately after the blade trailing edge
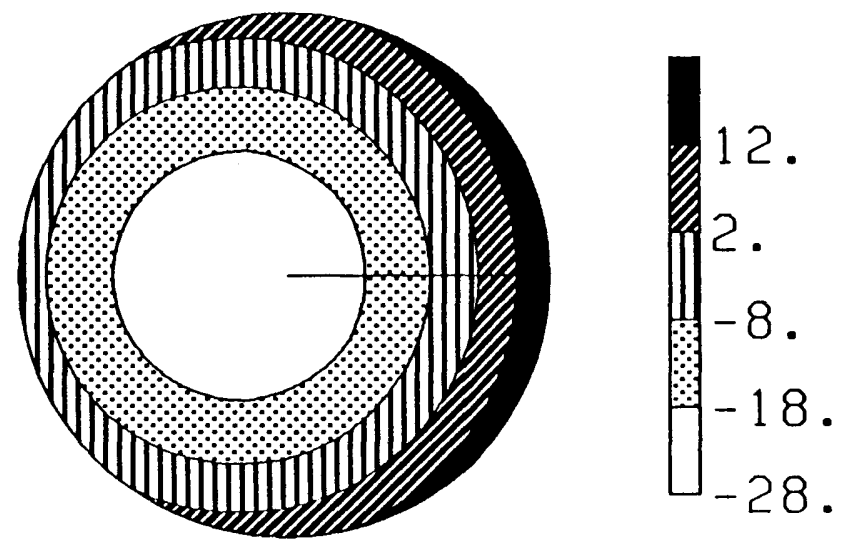

\section{Half the distance} to the probe location
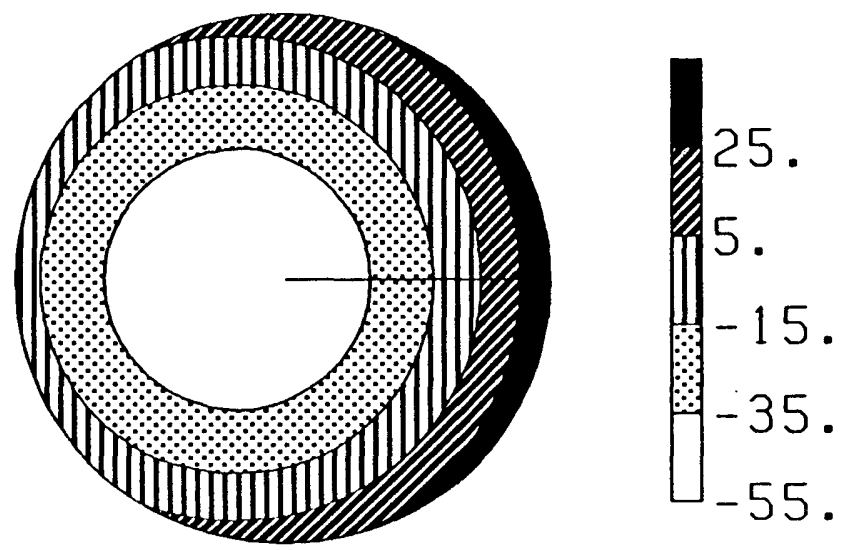

At the probe location

$(+)$ : towards tip $(-)$ : towards hub

Figure 7.14: Evolution of spanwise velocities in the vortex core, during its convection from the trailing edge of the blade to the probe location (calculation at $\mathrm{r} / \mathrm{r}_{\text {tip }}=0.95$ ) 

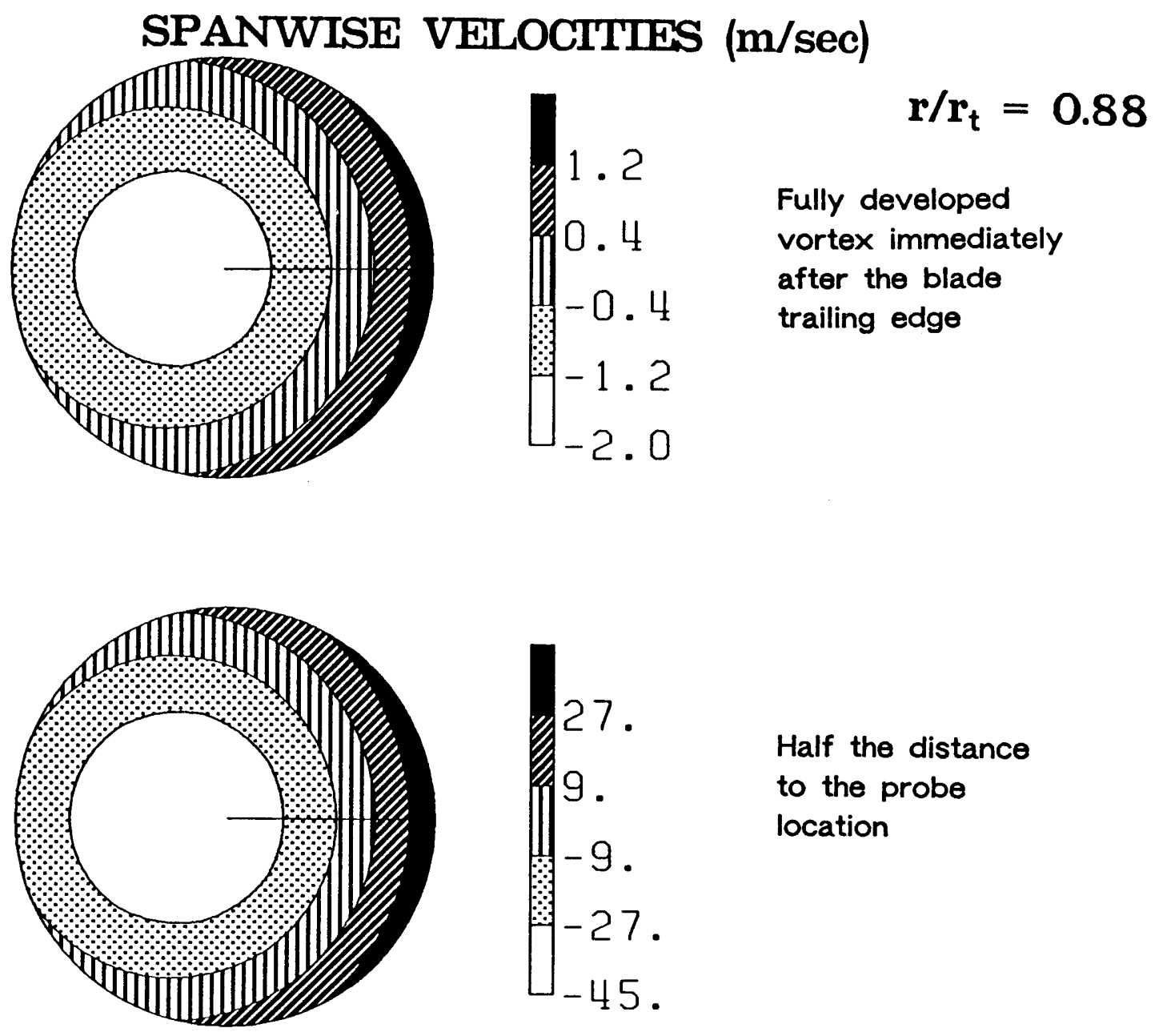
Half the distance to the probe location
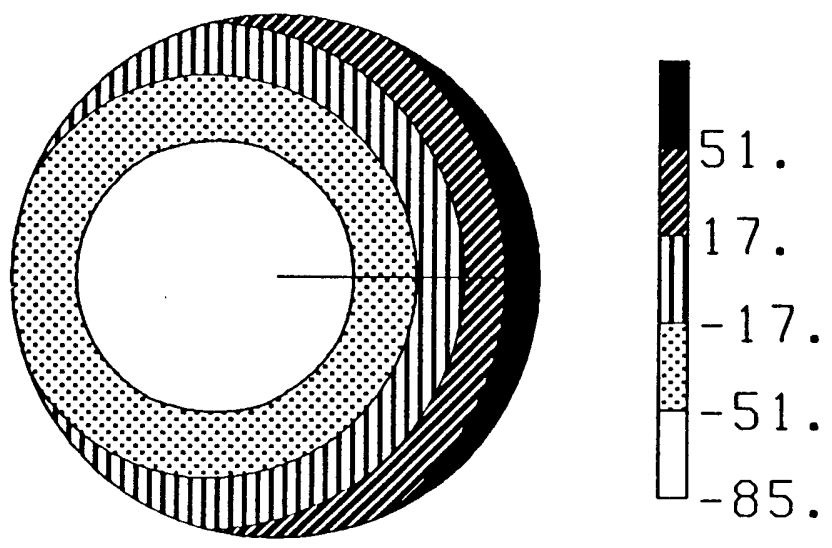

At the probe location

$(+)$ : towards tip (-) : towards hub

Figure 7.15: Evolution of spanwise velocities in the vortex core, during its convection from the trailing edge of the blade to the probe location (calculation at $\mathrm{r} / \mathrm{r}_{\mathrm{tip}}=0.88$ ) 
SPANWISE VELOCTTIES $(\mathrm{m} / \mathrm{sec})$
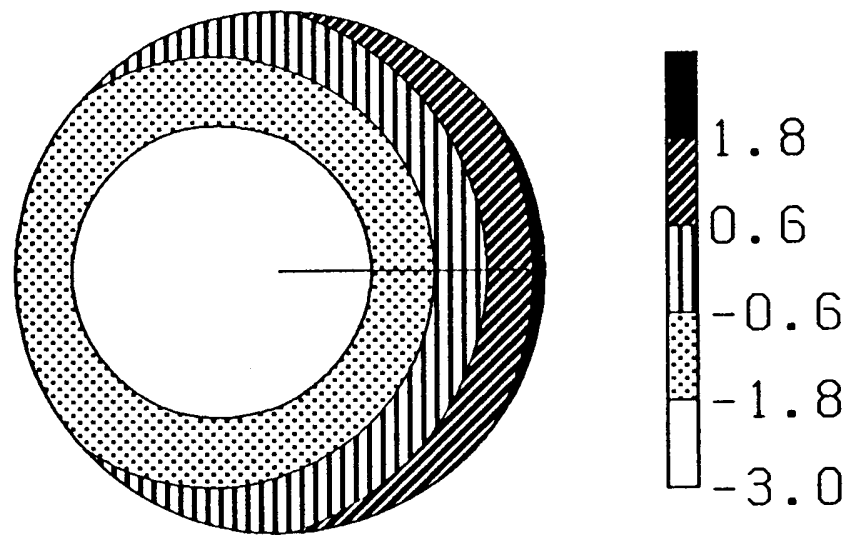

$$
\mathbf{r} / \mathbf{r}_{\mathrm{t}}=0.81
$$

Fully developed vortex immediately after the blade trailing edge
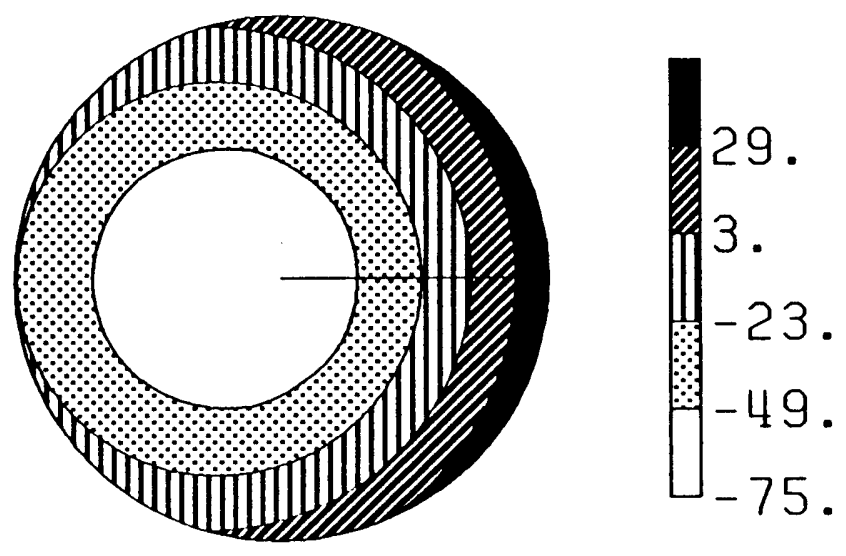

\section{Half the distance} to the probe location
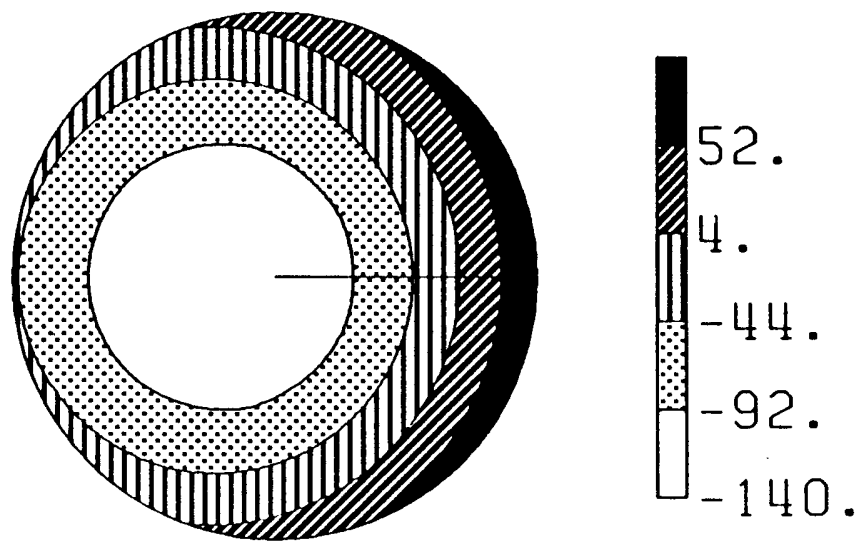

At the probe location

$(+):$ towards tip $(-)$ : towards hub

Figure 7.16: Evolution of spanwise velocities in the vortex core, during its convection from the trailing edge of the blade to the probe location (calculation at $\mathrm{r} / \mathrm{r}_{\mathrm{tip}}=0.81$ ) 


\section{SPANWISE VELOCITIES $(\mathrm{m} / \mathrm{sec})$}
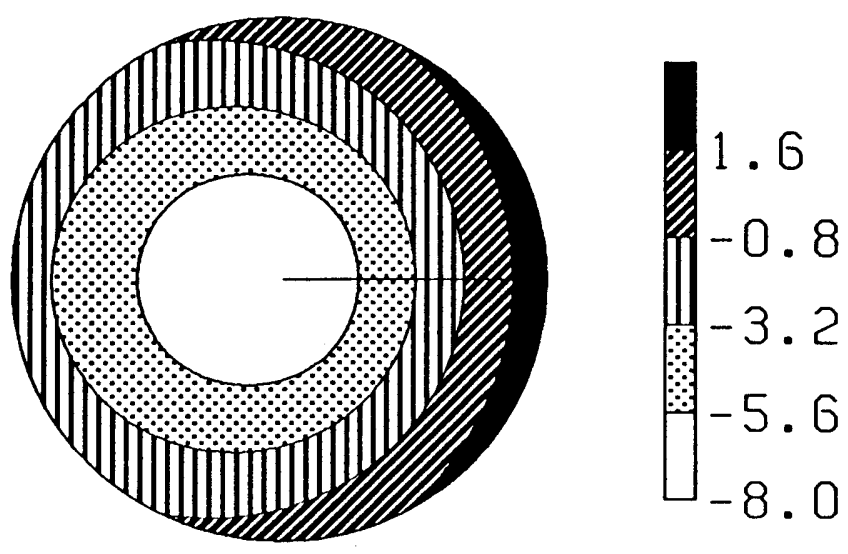

$$
\mathbf{r} / \mathbf{r}_{\mathrm{t}}=0.75
$$

Fully developed

vortex immediately

after the blade

trailing edge
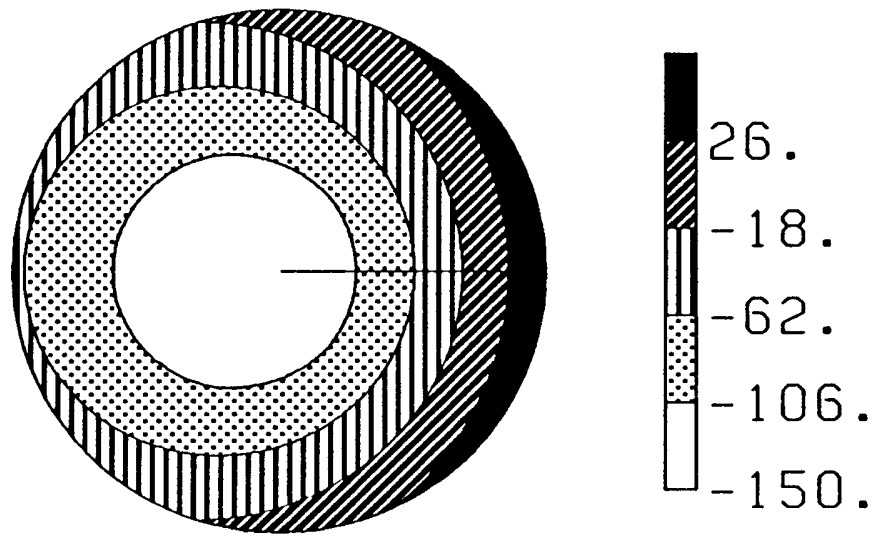

Half the distance to the probe location
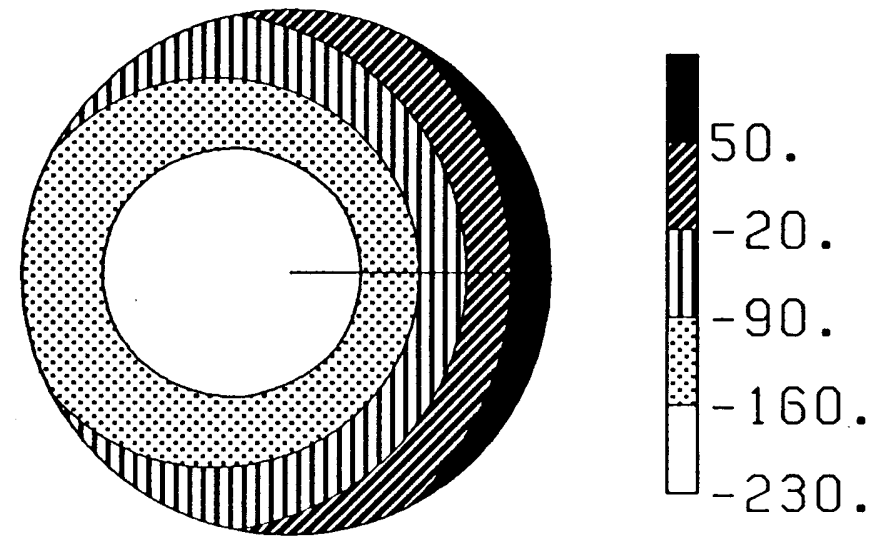

At the probe location

$(+)$ : towards tip

$(-)$ : towards hub

Figure 7.17: Evolution of spanwise velocities in the vortex core, during its convection from the trailing edge of the blade to the probe location (calculation at $r / r_{\text {tip }}=0.75$ ) 


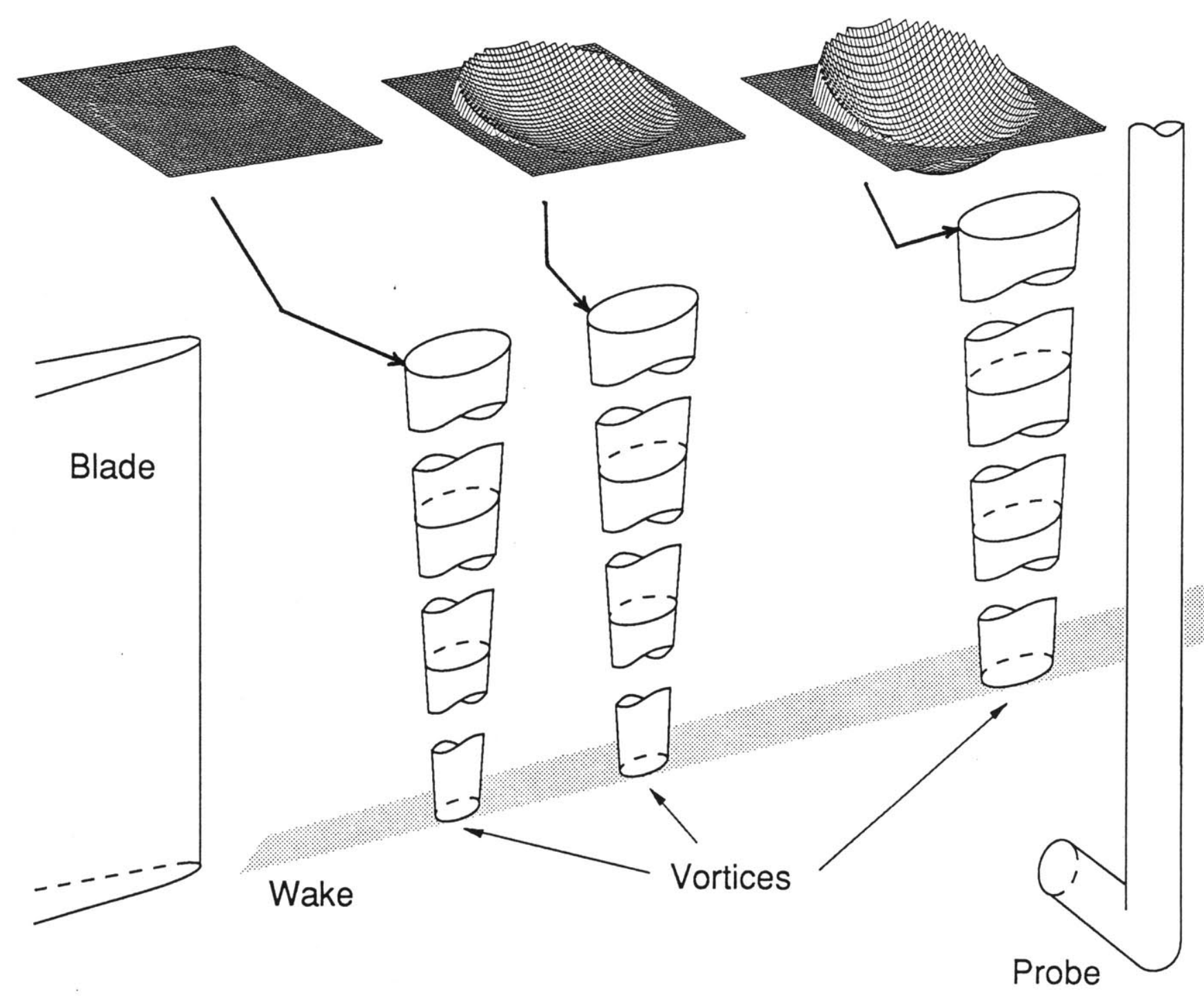

Figure 7.18: Perspective of the evolution of spanwise velocities in the vortex cores, during their convection from the trailing edge of the blade to the probe location (calculation at $r / r_{\text {tip }}=0.95$ ) 


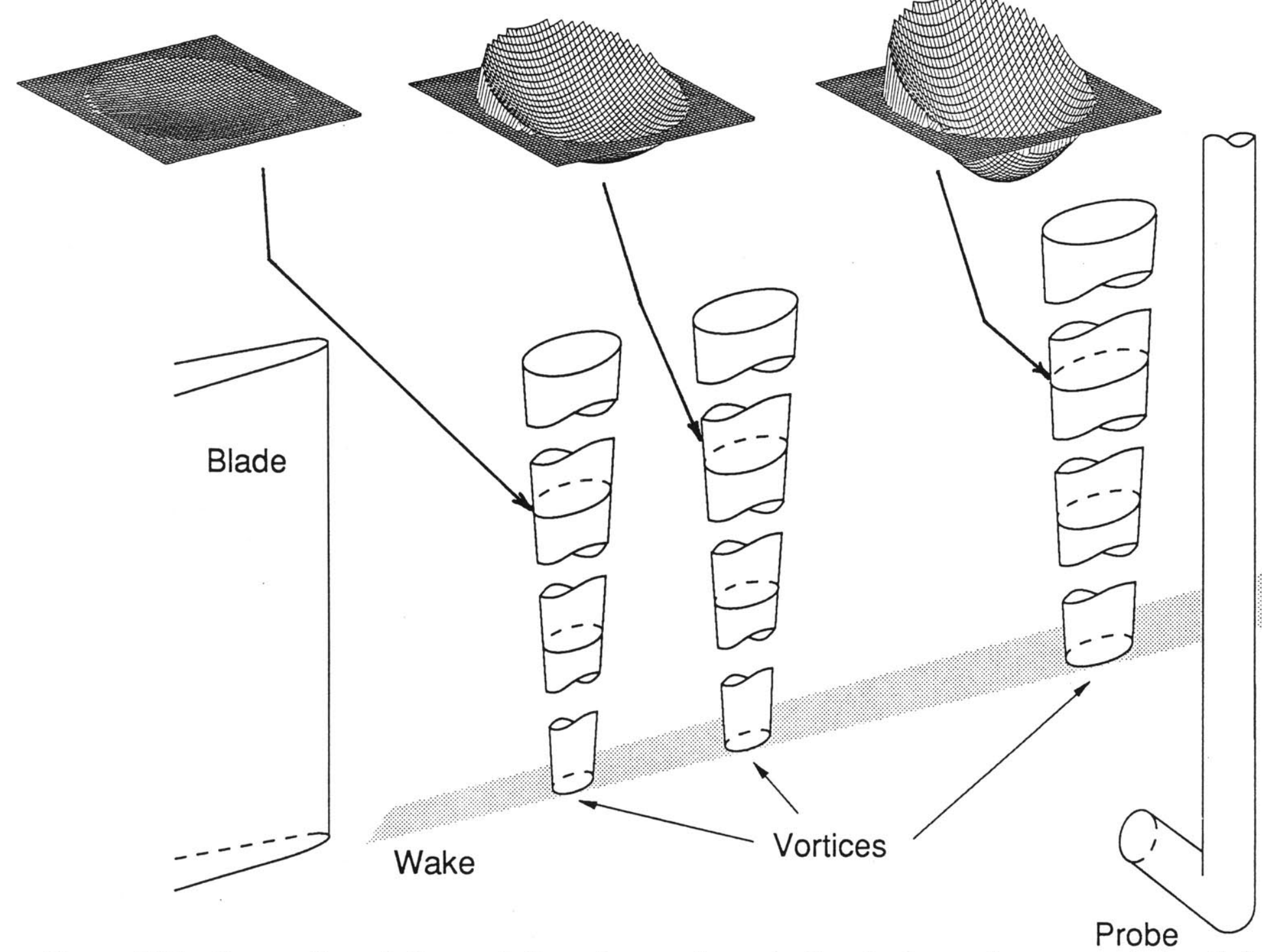

Figure 7.19: Perspective of the evolution of spanwise velocities in the vortex cores, during their convection from the trailing edge of the blade to the probe location (calculation at $r / r_{\text {tip }}=0.88$ ) 


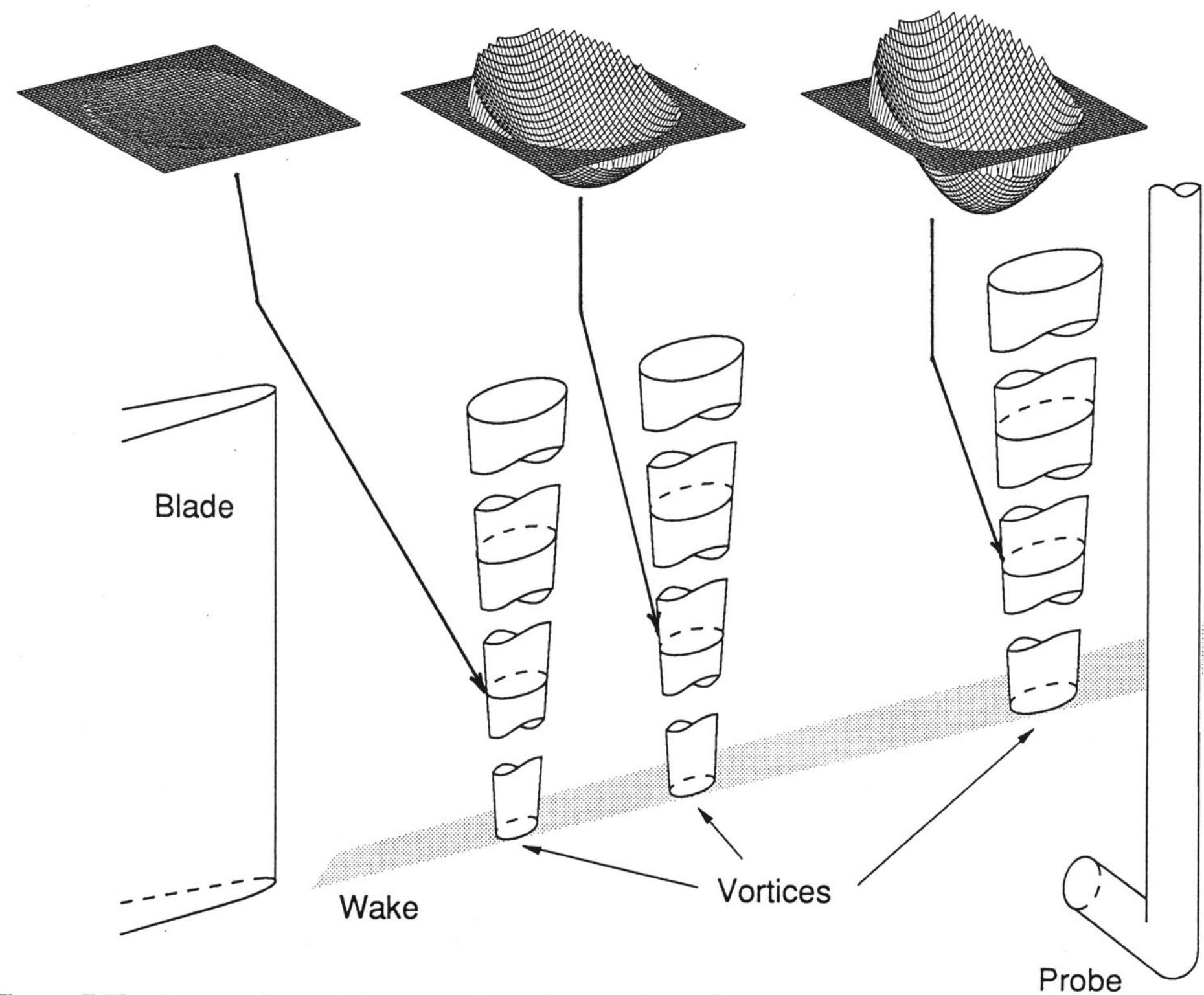

Figure 7.20: Perspective of the evolution of spanwise velocities in the vortex cores, during their convection from the trailing edge of the blade to the probe location (calculation at $r / r_{\text {tip }}=0.81$ ) 


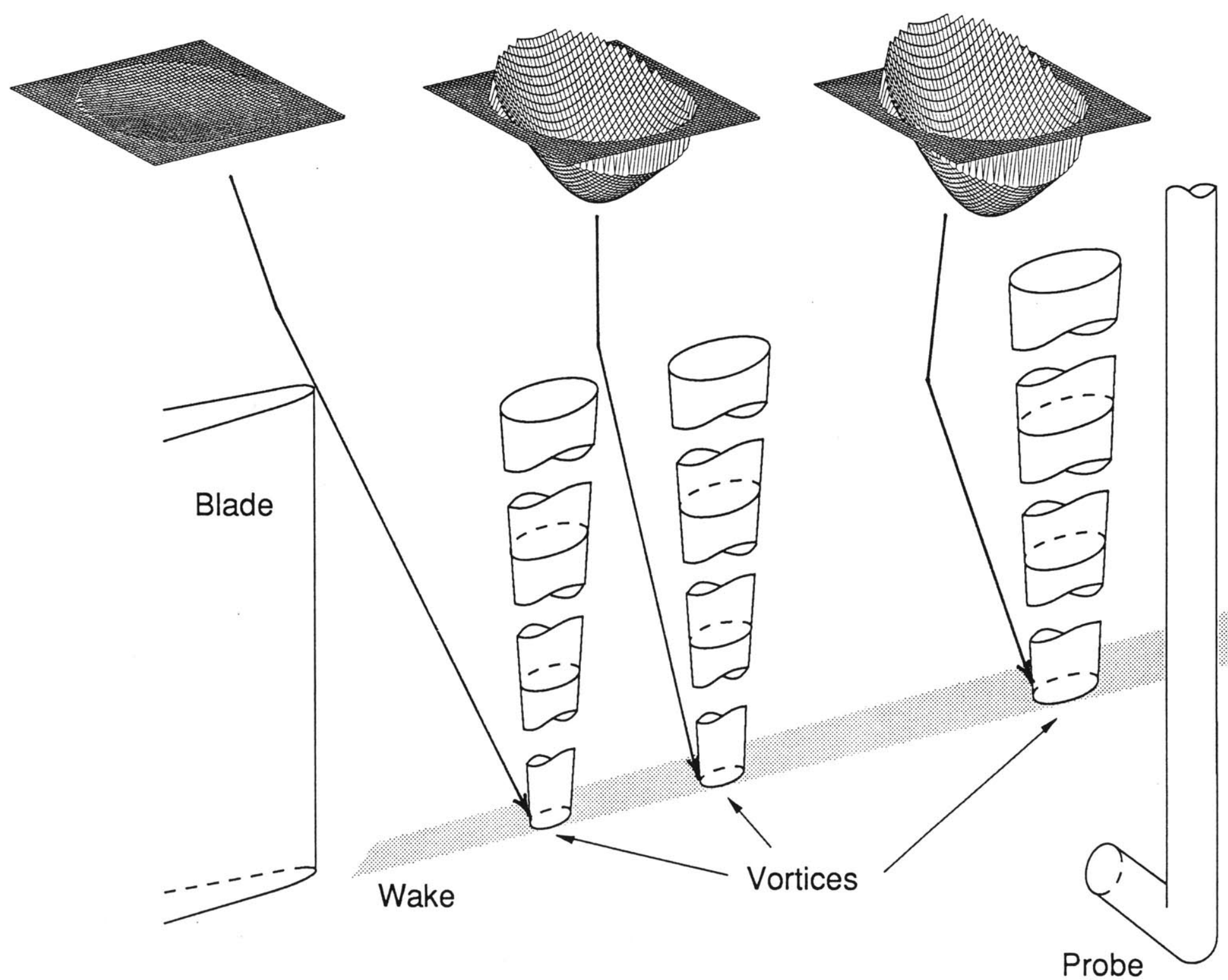

Figure 7.21: Perspective of the evolution of spanwise velocities in the vortex cores, during their convection from the trailing edge of the blade to the probe location (calculation at $r / r_{\text {tip }}=0.75$ ) 

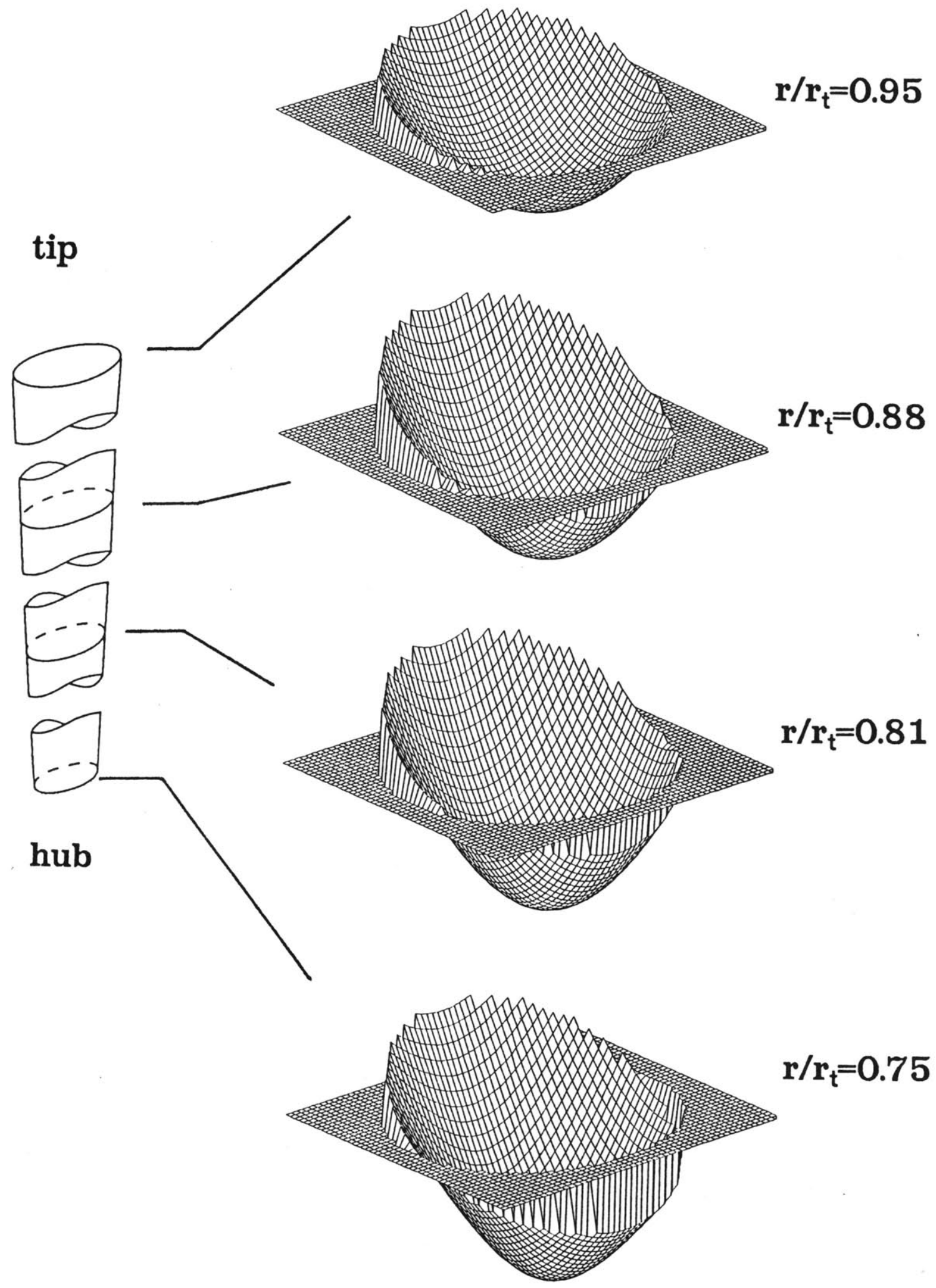

Figure 7.22: Perspective of the spanwise velocities in the vortex cores at four spanwise locations 


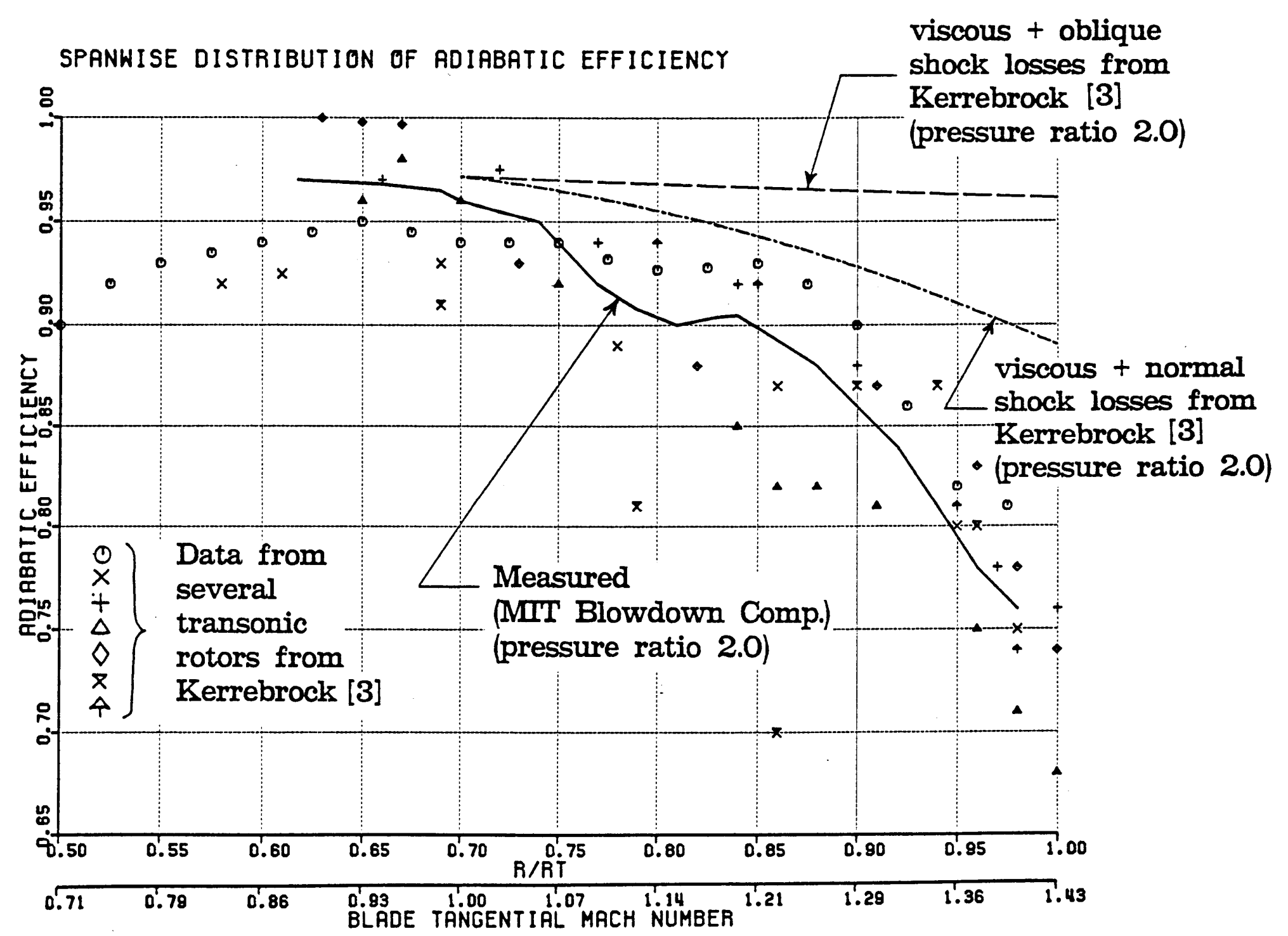

Figure 8.1: Spanwise distribution of adiabatic efficiency for several transonic rotors. Theoretical estimates of losses (from [3]) are given for comparison 


\section{EFFICIENCY REDISTRIBUTION MODEL}

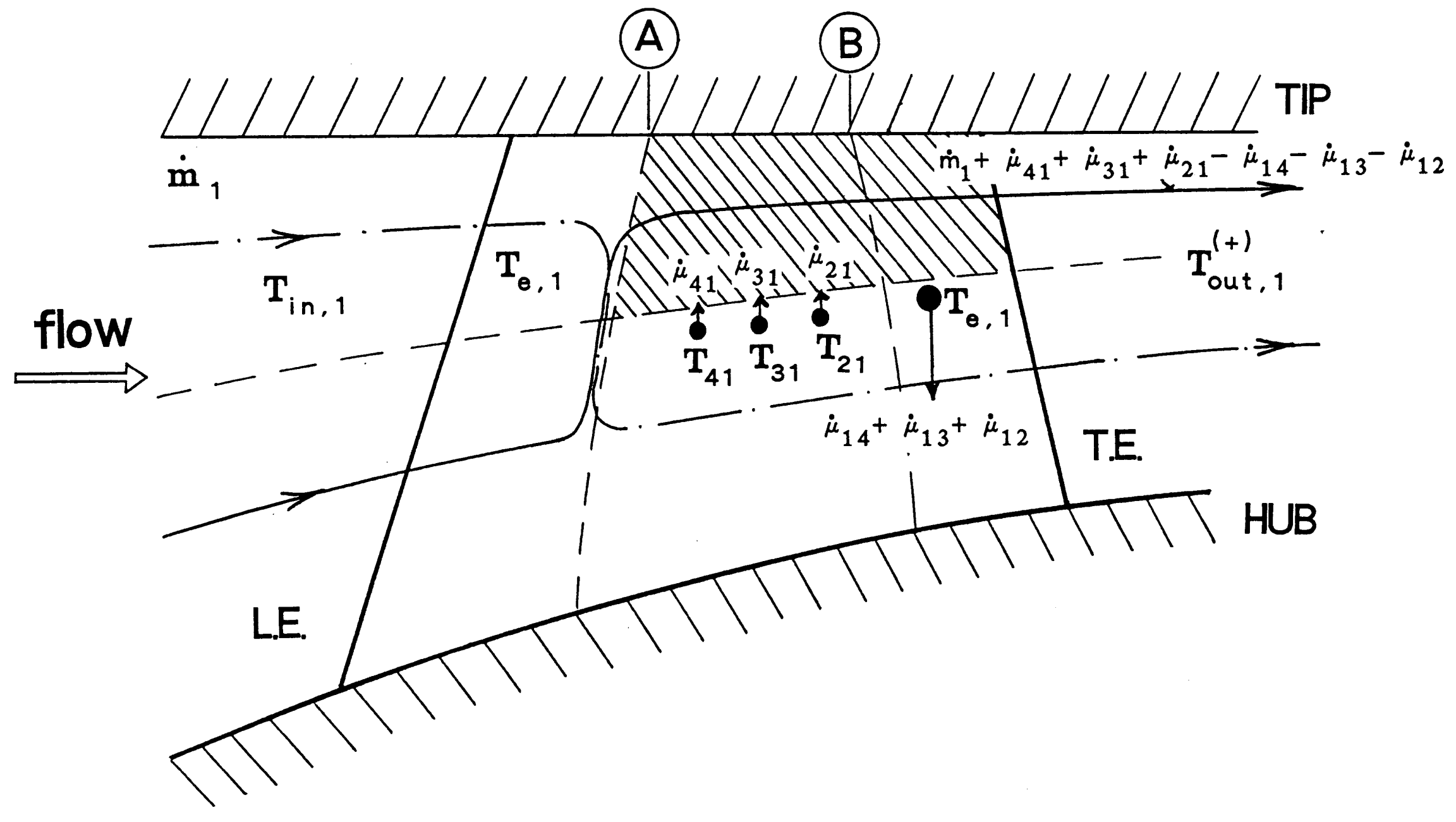

Figure 8.2: Streamtube exchange during spanwise transport within the blade passage (shown only for region 1) 
TRANSPORT ONLY WITHIN THE BLADE PASSAGE ( 4 REGIONS)

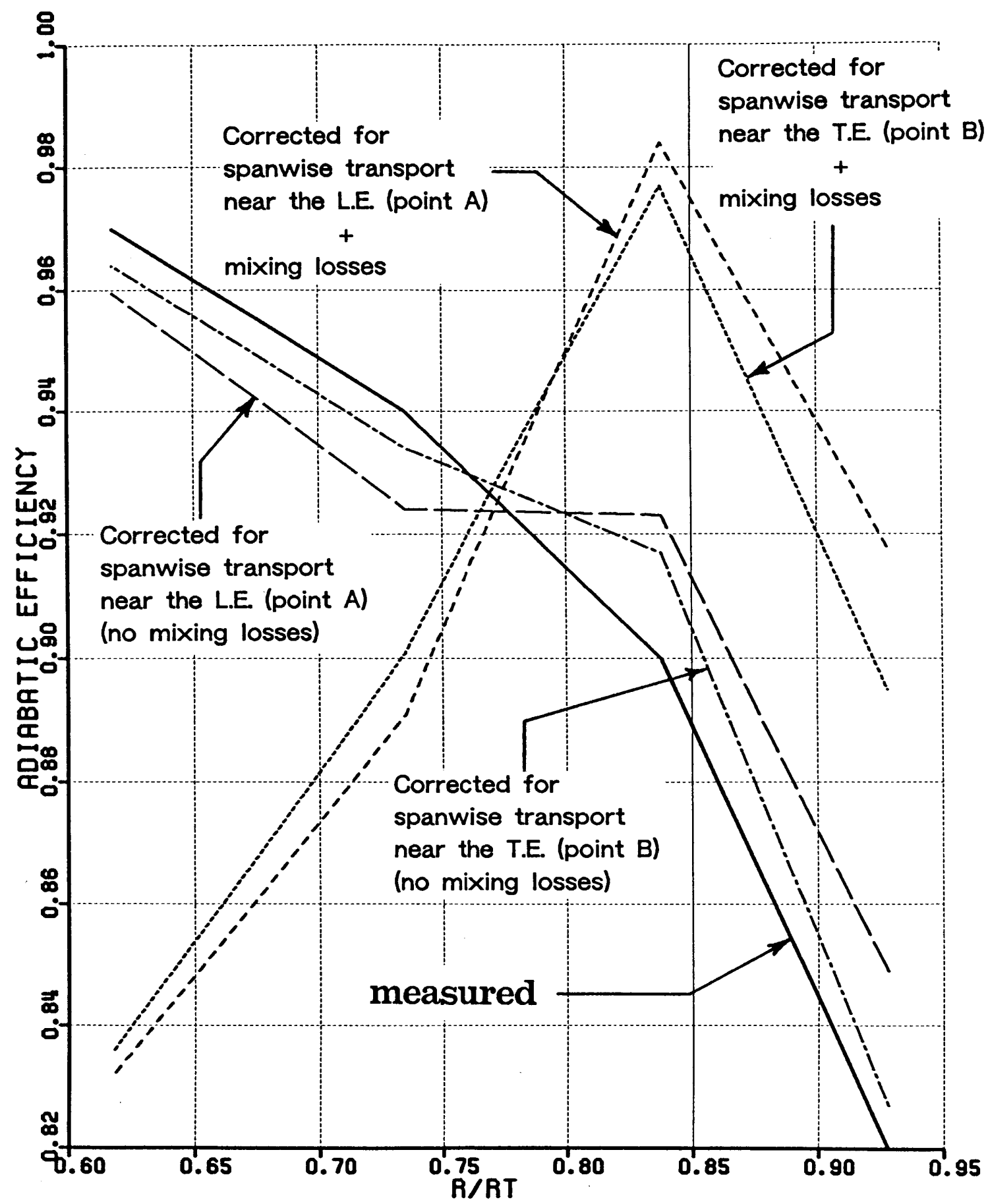

Figure 8.3: Effect of spanwise transport on the spanwise distribution of time averaged adiabatic efficiency, when the transport occurs within the blade passage (4 regions included in the calculations) 
TRANSPORT ONLY WITHIN THE BLADE PASSAGE (3 REGIONS)

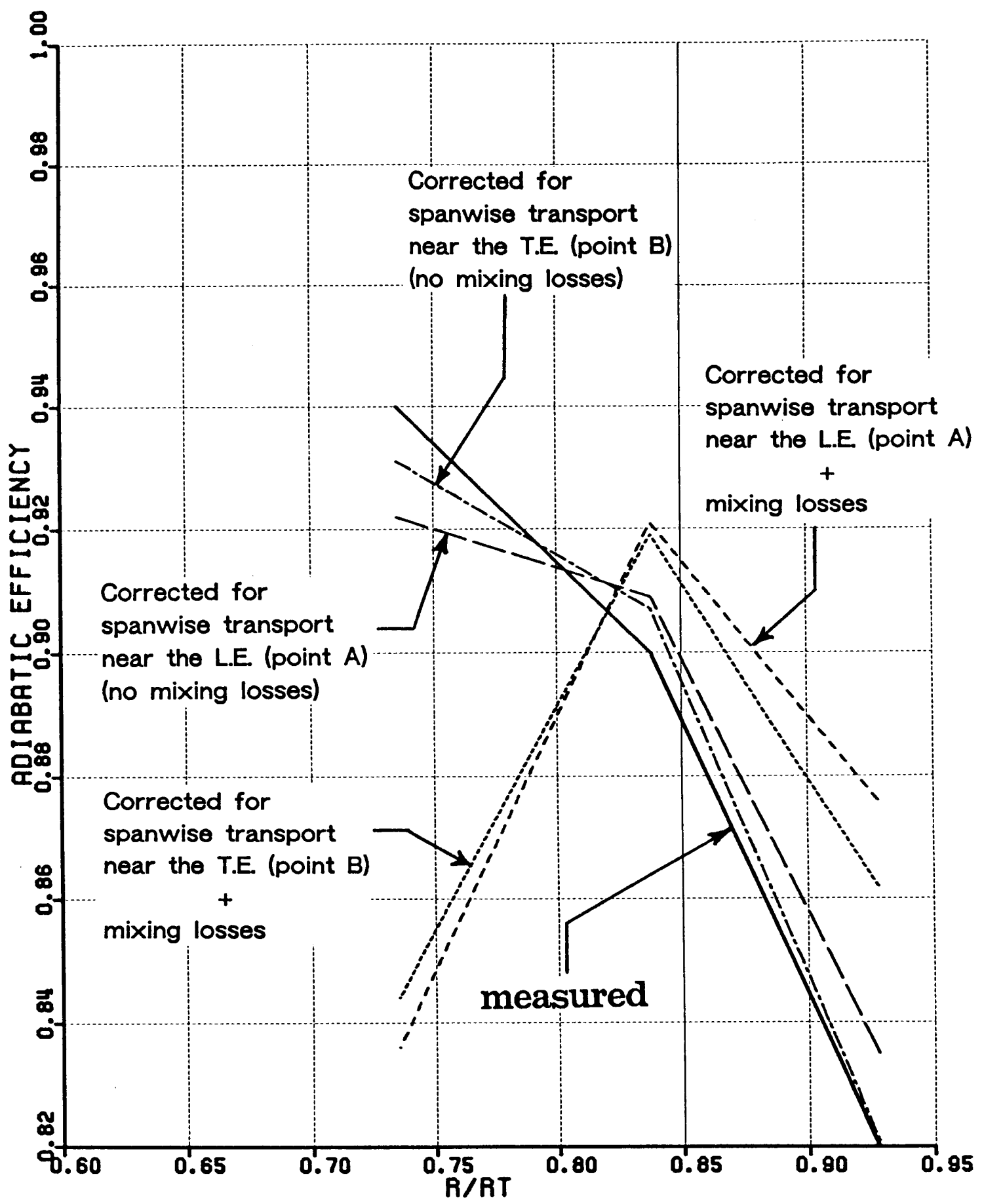

Figure 8.4: Effect of spanwise transport on the spanwise distribution of time averaged adiabatic efficiency, when the transport occurs within the blade passage ( 3 regions included in the calculations) 


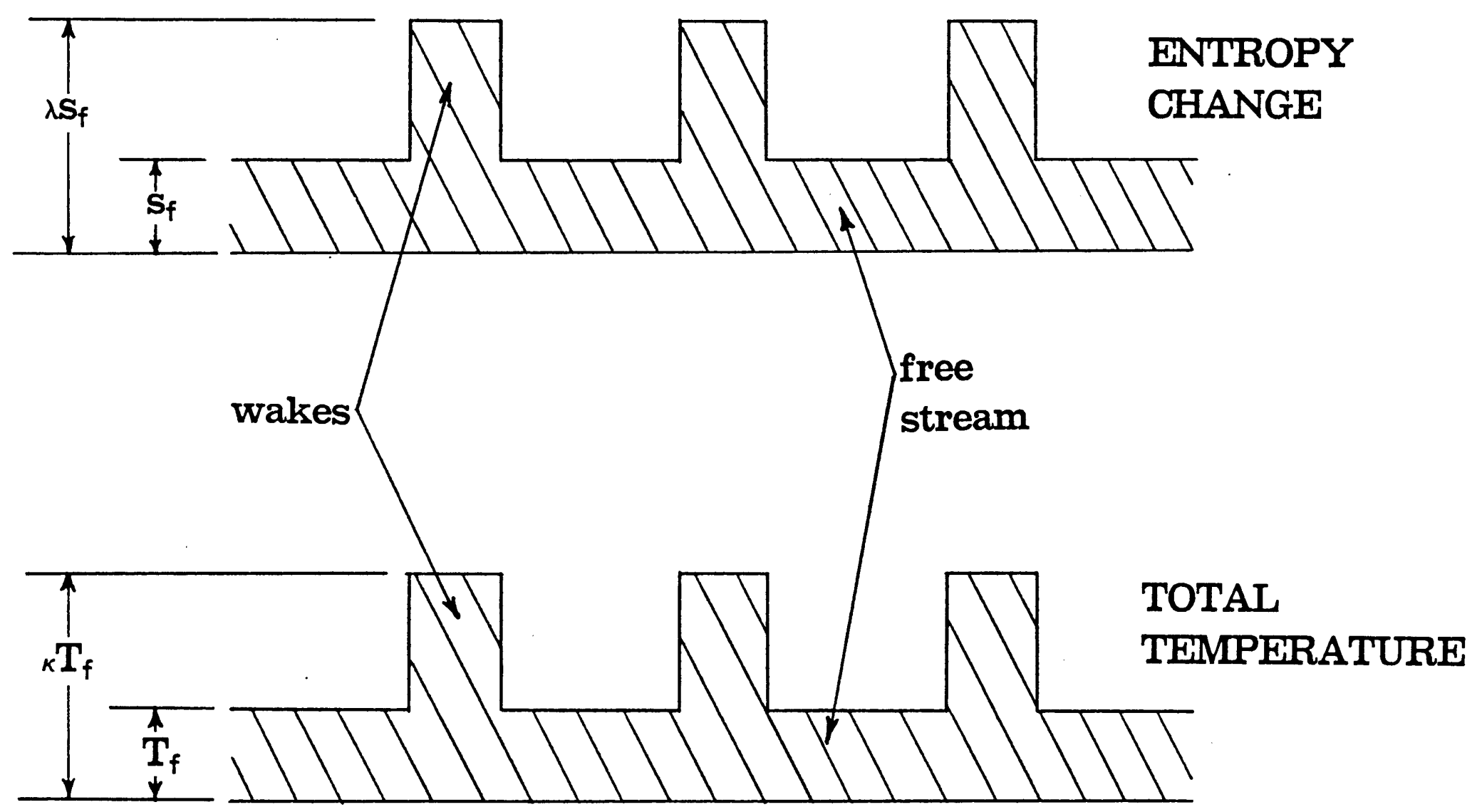

Figure 8.5: Ideal representation of wake and free stream regions for modeling purposes 


\section{TRANSPORT ONLY AFTER THE TRAILING EDGE}

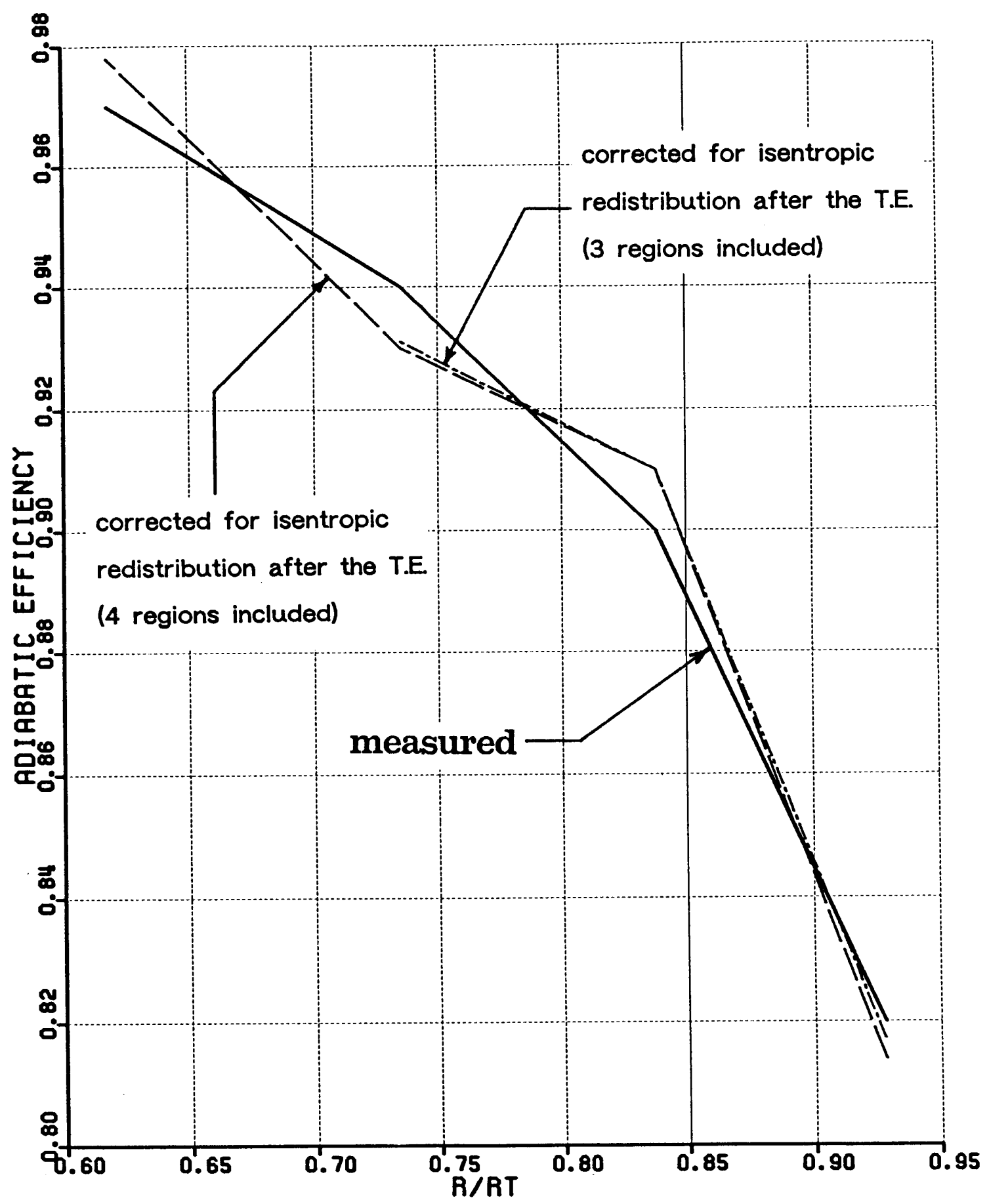

Figure 8.6: Effect of isentropic spanwise redistribution of the flow after the trailing edge of the blade, on the spanwise distribution of adiabatic efficiency 


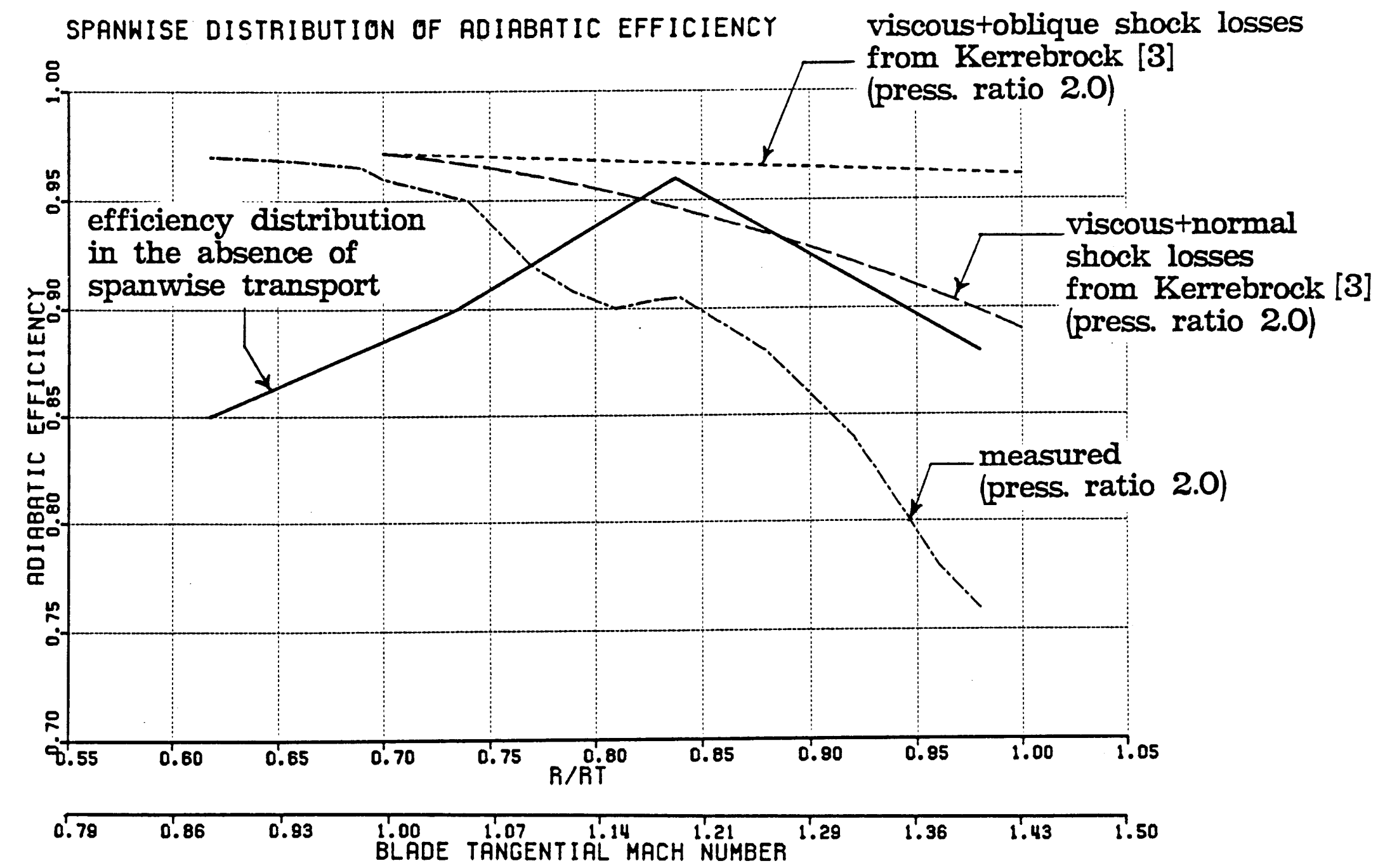

Figure 8.7: Comparison between measured, predicted and corrected spanwise distributions of time averaged adiabatic efficiency 


\section{VORTEX ROTATION}

\section{CLOCKWISE}

COUNTERCLOCKWISE
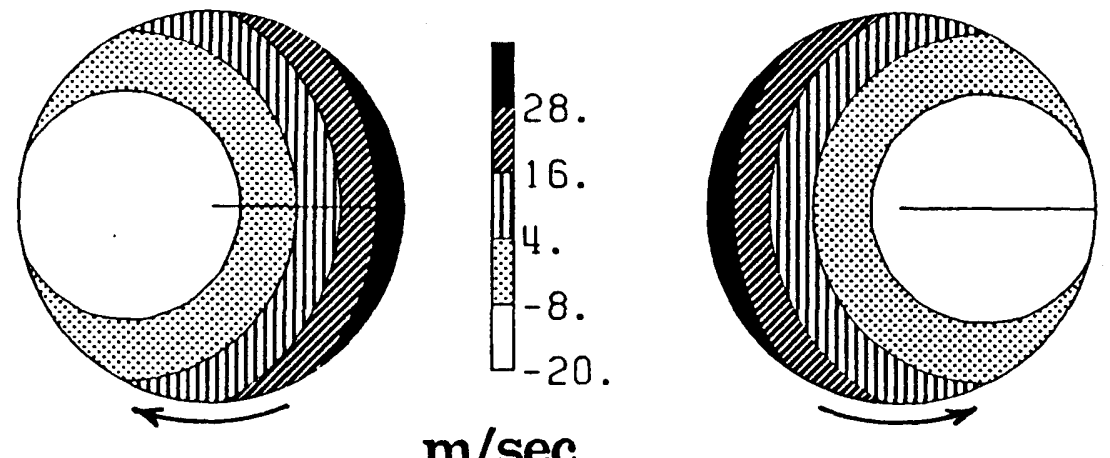

$\mathbf{r} / \mathbf{r}_{\mathrm{t}}=0.95$
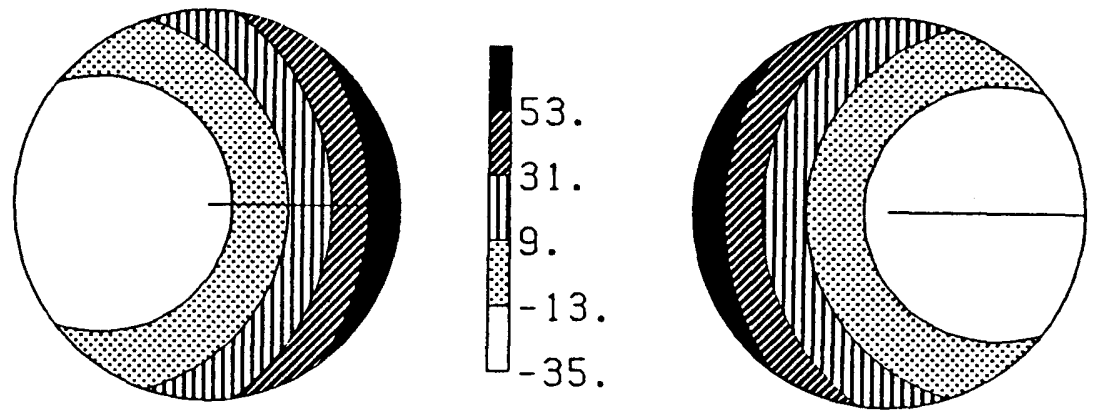

$\mathbf{r} / \mathbf{r}_{\mathrm{t}}=0.88$
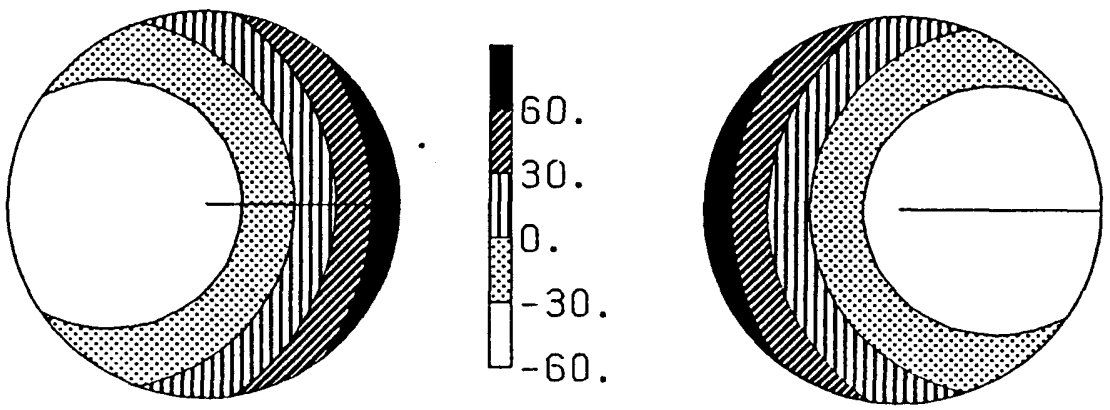

$\mathbf{r} / \mathbf{r}_{\mathrm{t}}=0.81$
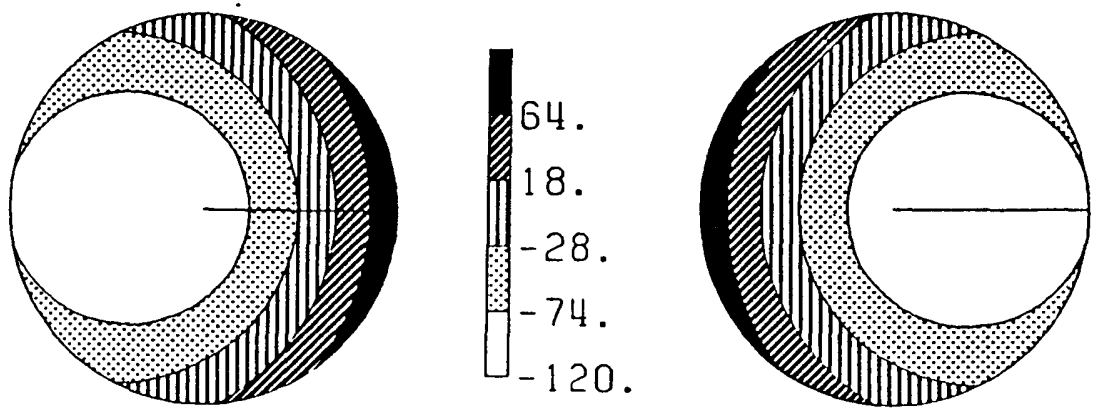

$\mathbf{r} / \mathbf{r}_{\mathrm{t}}=0.75$

Figure A.1: Distribution of spanwise velocities in the cores of the vortices at four spanwise locations, as predicted by the vortex model 


\section{CLOCKWISE VORTEX ROTATION}

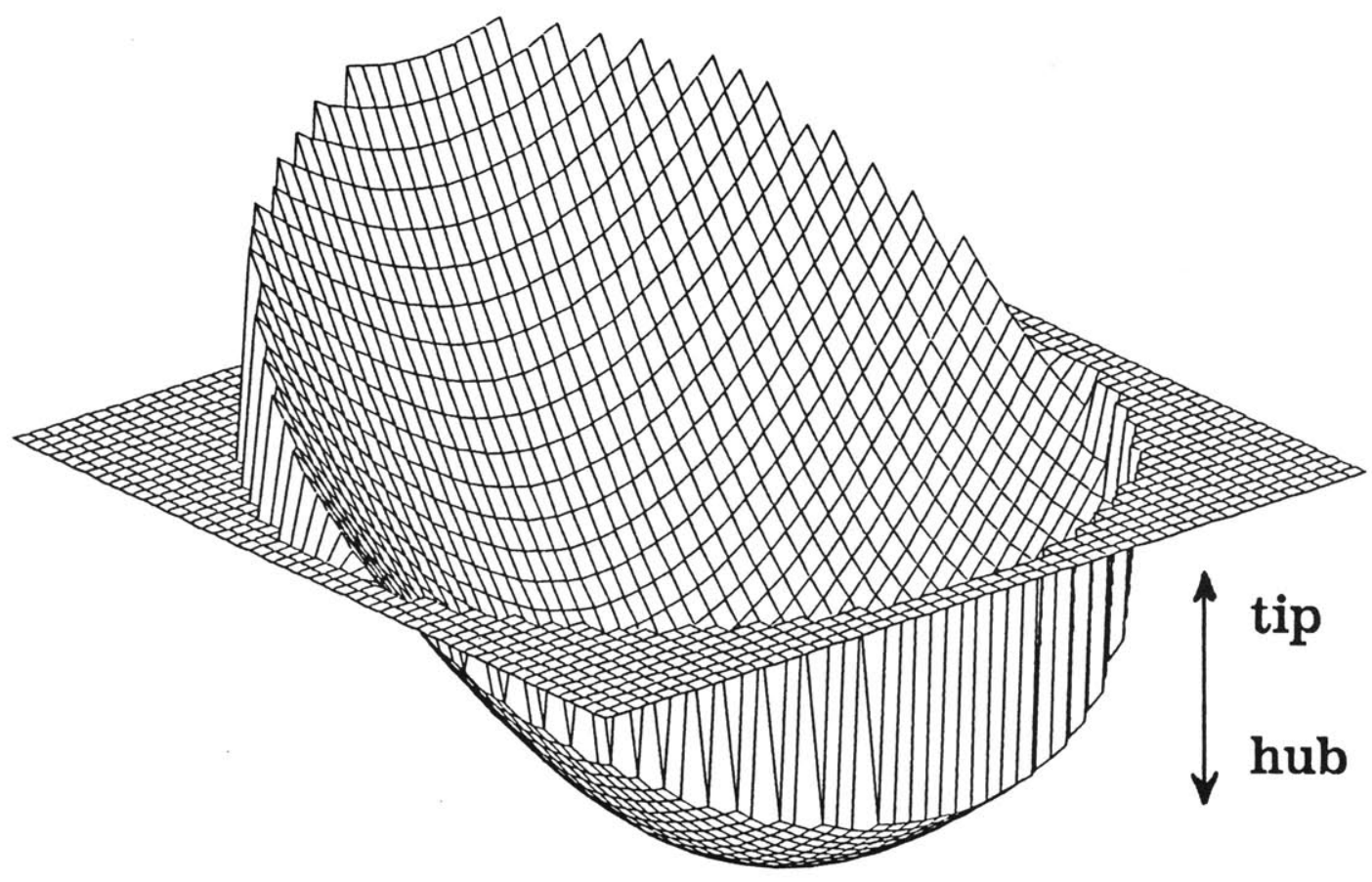

COUNTERCLOCKWISE VORTEX ROTATION

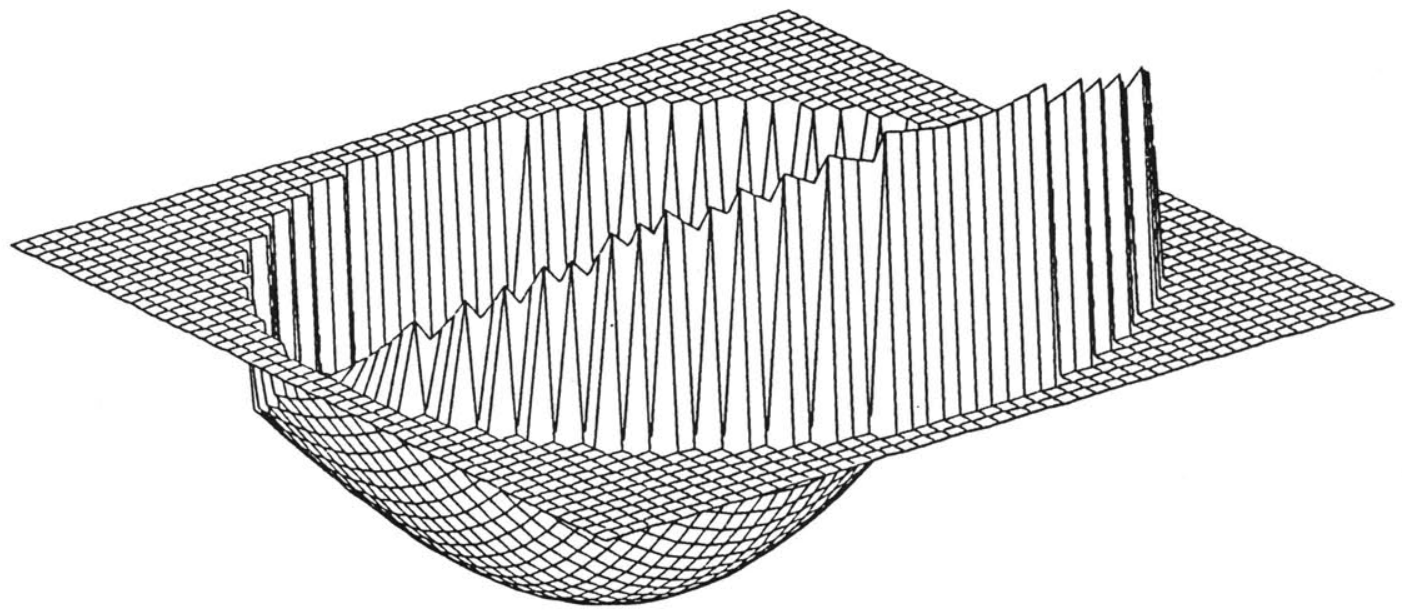

Figure A.2: Typical distribution of spanwise velocities in the vortex core, as predicted by the vortex model 